\title{
Application of Monte Carlo Algorithm to Explore Simplified Molecular-Input Line-Entry System based Molecular Descriptors of BACE1 inhibitors for Therapeutic Application in Alzheimer's Disease
}

\author{
Md Lutful Islam \\ Research Scholar, Department of Computer \\ Science and Engineering, Shri Jagdish Prasad \\ Jhabarmal Tibrewala University, Vidyanagari, \\ Jhunjhunu - 333 001, Rajasthan, India
}

\author{
Gulabchand K. Gupta \\ Seva Sadan College of Arts, Science \& Commerce \\ (Affiliated to University of Mumbai), Seva Sadan \\ Marg, Ulhasnagar - 421003 , Dist. Thane, \\ Maharashtra, India
}

\begin{abstract}
Application of computer science and algorithms are now become an integral part of the drug discovery research. In the current work, well known Monte Carlo (MC) algorithm was used to develop quantitative structure-activity relationship (QSAR) models with molecular descriptors derived from simplified molecular-input line-entry system (SMILES) representation of beta-site APP cleaving enzyme1 (BACE1) inhibitors. A set of BACE1 inhibitors was obtained from the binding database and subsequently divided into training, test, calibration and external sets. The QSAR models were developed from the training set compounds while other sets used to assess the quality of developed models. With- and without-influence of cyclic rings on inhibitory activity were considered to develop the QSAR models. Best QSAR models were selected based on statistical parameters of final models. High correlation and low standard error values of training, test, calibration and external sets undoubtedly suggested that the selected models were robust and efficient enough to predict the inhibitory activity of the molecules. On evaluation of statistical parameters it was revealed that cyclic rings of molecular scaffold significantly contributed to the inhibition of BACE1. The molecular fragments were found to be crucial to increase or decrease the inhibitory activity of the molecules which indicated that models have mechanistic interpretation. Therefore, important molecular fragments explained by the QSAR models can be used to design new and novel BACE1 inhibitors for therapeutic application in Alzheimer's disease.
\end{abstract}

\section{Keywords}

Alzheimer's disease; BACE1 inhibitors; QSAR; Monte Carlo algorithm; SMILES

\section{INTRODUCTION}

Alzheimer's disease (AD) is severe and a progressive neurodegenerative disease categorized by two histopathological hallmarks, namely $\beta$-amyloid plaques and neurofibrillary tangles [1-4]. AD is common cause of senile dementia, accounts for $60-70 \%$ of all dementias and clinically characterized by impairment of memory, disorientation, difficulty in speaking or writing, loss of reasoning skills, and delusions among other symptoms [5]. Direct reason of the AD progression is still doubtful but some studies reported that both genetic and environmental factors are play major role for the $\mathrm{AD}$ [6] development. About 44 million people worldwide are suffering from $A D$ or related dementia in 2017. As per reports, $\mathrm{AD}$ most prominently found in Western Europe and least prevalent in Sub-Saharan Africa. World Health Organization (WHO) data explained that there is about 5.7 million people living in United States of America in 2018 with AD. More than 4 million people have some form of dementia and $\mathrm{AD}$ in India, and it will be double in India by 2030 (The Indian Express, September 21, 2016). With development of $\mathrm{AD}$, there is high risk of several age related diseases including hypertension, dyslipidemia, metabolic syndrome and diabetes. There is no approved chemical agents to treat such devastating disease rather management of the symptoms.

The extracellular accumulation of amyloid plaques collected of the $\beta$-amyloid $(\mathrm{A} \beta)$ peptide characterizes the main reason of $\mathrm{AD}$ [7]. The $\mathrm{A} \beta$ formed due to sequential breakdown of $\beta$ amyloid precursor protein (APP) by two aspartyl protease, beta-site APP cleaving enzyme1 (BACE1) followed by $\gamma$ secretase [8]. Stop or decrease of $A \beta$ production by inhibition of BACE1 enzyme is an ideal approach to control the AD. Researchers from worldwide already proved that BACE1 inhibitors hold great potential as a potential strategy in decreasing $A \beta$ brain concentrations which lead to stop the progression of $\mathrm{AD}[9,10]$. It has been established by the experimental approach that BACE1 enzyme could be clinically feasible with few mechanistic side effects [11-13]. Hence, control production of $A \beta$ through by the inhibition of BACE1 may represent modifying treatment for AD.

In order to develop promising chemical agents for the proper treatment of $\mathrm{AD}$, the present research was deliberated the Monte Carlo (MC) algorithm based quantitative structure activity relationship (QSAR) study to explore critical chemical functionalities and design new lead chemical agents for therapeutic application of AD. The QSAR can be expressed as the statistically validated and mathematical relationship between biological activity and chemical structures in terms of molecular descriptors. Numerical values of chemical or physical properties of the small molecules are called molecular descriptors and used in QSAR model development. Statistically robust QSAR models can give instincts into the decisive structural information of the small molecules which contribute to biological activity [14]. Major division of the molecular descriptors are physico-chemical, topological and electronic, geometric and structural, and simple indicator parameter. Moreover, descriptors are also be characterized on the basis dimensionality such as $0 \mathrm{D}, 1 \mathrm{D}, 2 \mathrm{D}$ or 3D [15]. Obtaining molecular descriptors based on geometry are usually tough and need high computational costs and long computational calculation time. Then $0 \mathrm{D}, 1 \mathrm{D}$ and 2D are termed as the conformation-independent descriptors based on the constitutional and topological molecular features of small molecules have been established as a substitute method 
$[16,17]$. In most cases the descriptors based on molecular graph are used to develop QSAR models [18-20]. But molecules in simplified molecular input-line entry system (SMILES) representation can also be used [21-23] for molecular descriptor generation followed by development of QSAR models. Without any information 3D structure of the molecules the SMILES notation can derived descriptors based on both on the molecular structure and the property under analysis[14]. Therefore, QSAR models can be progressed with SMILES based molecular descriptors [24-26]. Several research groups from industry and academia have already been successfully used SMILES based descriptors to develop robust QSAR models [27-33].

\section{MATERIALS AND METHODS}

\subsection{Dataset}

A set of more than thousand reported BACE1 inhibitors were downloaded from Binding DB (http://www.bindingdb.org/) with inhibition constant $\left(K_{i}\right)$ activity in $\mathrm{nM}$ range. Initially duplicate and without activity molecules were identified and removed. Remaining compounds were considered to verify the Lipinski's rule of five [34] and Viber's [35] rules and only considered molecules those satisfied the above two rules. Finally, 411 molecules were found to be satisfied above criteria and considered for for the study. To QSAR model development the experimental inhibitory activity $\left(K_{i}\right)$ of dataset were converted into logarithm value $\left[p K_{i}=\right.$ $\left.\log \left(\left(1 / K_{i}\right) \times 10^{7}\right)\right]$. Molecules in the entire dataset were randomly divided into training, calibration, test and external sets. Each of the set has specific role during the model development. Compounds present in the training set were considered to develop the model and calibration and test sets used to assess the predictive quality of selected models. Compounds of the external set were unseen during model generation that is no information of validation set was involved for model development. External set finally was used to calculate final estimation of the model. Molecules of all fours sets (training, test, calibration and external) are given in Supplementary file (Table S1 and S2) in SMILES representation with $p K_{i}$.

\subsection{Optimal descriptors}

The SMILES representation of the dataset was used to calculate the molecular descriptor. The SMILES format of chemical structures is one of the widely accepted and helpful molecular file format to calculate the optimal molecular descriptors which are mathematical functions of so-called correlation weights $(\mathrm{CW})$ that is "Descriptors of Correlation Weights" (DCW). The Monte Carlo algorithm was adopted to derive the DCW from the set of BACE1 inhibitors. In the current work, DCW was calculated using two approaches viz. without and with considering the influence of cyclic rings to the $p K_{i}$. For calculation of DCW following expression was used and influence of cyclic rings on inhibitory activity not considered.

$$
\begin{aligned}
D C W_{1}\left(S M I L E S, T, N_{\text {epoch }}\right) & =\alpha \sum C W\left(S_{k}\right)+\beta \sum C W\left(S S_{k}\right)+\gamma \sum C W\left(S_{S S}\right)+\mathrm{x} \cdot C W(N O S P) \\
& +\mathrm{y} \cdot C W(H A L O)+z \cdot C W(B O N D)+t \cdot C W(P A I R)
\end{aligned}
$$

Where, $\mathrm{T}$ indicates threshold and defines as coefficient for classifying various molecular features extracted from SMILES into two classes such as active, in which CW is involved in the modelling process and rare, where $\mathrm{CW}$ is not involved in the modelling process. $\mathrm{N}_{\text {epoch }}$ signifies the number of epochs in Monte Carlo optimization which gives the best statistical results of the calibration set. $S_{k}$ denotes the one symbol separately of the SMILES representation while, the $\mathrm{SS}_{\mathrm{k}}$ and $\mathrm{SSS}_{\mathrm{k}}$ are represented for combination of two or three respectively. NOSP, HALO, BOND and PAIR explain the descriptors based on presence or absence of different elements. NOSP explain the nitrogen, oxygen, sulphur and phosphorus; HALO represents halogen atoms such as fluorine, chlorine, bromine and iodine; BOND offers double (=), triple (\#) or stereochemical bonds (@ or @ @); and PAIR refers the probable grouping of pair atoms and/or SMILES attributes (for example double, triple, and stereochemical bonds) that takes place in the structure together. The $\alpha, \beta, \gamma, \mathrm{x}$, $\mathrm{y}$ and $\mathrm{t}$ are discrete coefficient with values 0 and 1 . Detail calculation of the above descriptors with example can be found in some where else. [31].

The optimal descriptors with influence of cyclic rings can be calculated using following equation (2).

$$
\begin{aligned}
D C W_{2}\left(\operatorname{SMILES}, T, N_{\text {epoch }}\right) & =\alpha \sum C W\left(S_{k}\right)+\beta \sum C W\left(S S_{k}\right)+\gamma \sum C W\left(\mathrm{SSS}_{k}\right)+\mathrm{x} \cdot \mathrm{CW}(\mathrm{NOSP}) \\
& +\mathrm{y} \cdot \mathrm{CW}(\mathrm{HALO})+z \cdot C W(B O N D)+t \cdot C W(P A I R)+C W(\mathrm{C} 3)+C W(C 4) \\
& +C W(C 5)+C W(C 6)+C W(C 7)
\end{aligned}
$$

Where, C3, C4, C5, C6 and C7 are denoted by three-, four-, five-, six- and seven-membered cyclic rings. Details explanation of such descriptors are can be found somewhere else[36].

The well known and widely used MC algorithm was adopted to calculate the $\mathrm{CW}$ which must give the best statistical results for the test set. In order to get preferable threshold ( $\left.\mathrm{T}^{*}\right)$ and number of epochs $\left(\mathrm{N}^{*}\right)$, range of $\mathrm{T}$ and $\mathrm{N}_{\text {epoch }}$ were selected from 1 to 10 and 1 to 20 respectively. The statistical results were analysed and the best $\left(\mathrm{N}^{*}, \mathrm{~T}^{*}\right)$ selected for final model development. The selected best statistics of calibration set makes possible to obtain the endpoint value using numerical values of correlation weights from the training set as follows:

$$
\text { Endpoint }=C_{0}+C_{1} \times \mathrm{DCW}\left(\mathrm{SMILES}, \mathrm{T}, \mathrm{N}_{\text {epoch }}\right)
$$

The endpoint represents the biological activity and, $\mathrm{C}_{0}$ and $\mathrm{C}_{1}$ are constant.

\subsection{Validation}

Validation of any in-silico model is essential step to assess the quality of the model. In this research the QSAR models were validated with the help of a) internal validation using training set compounds; b) external validation using test compounds; and c) Y-scrambling or randomization of data. Several studies $[26,27,29,30,33]$ already used these validation methodologies on SMILES notation optimal descriptor based QSAR models. The cross-validated correlation coefficient $\left(Q^{2}\right)$ was also derived from the predicted activity of training compounds. Model can be explained robust with $Q^{2}>0.5$ [37]. Further to verify the significant predictive capability of the training set molecules, the modified $r^{2}\left(r_{m(L O O)}^{2}\right)[38,39]$ that is $r_{m}^{2}$ was calculated which the measure of the degree of deviation of the predicted activity from the observed ones. In order to check the chance correlation, Y-scrambling given by Ojha and Roy [40] was also performed in which ten probes of calculation were carried out. In one probe of calculation, $X$ and $\mathrm{Y}$ represent the vectors of experiment and the vector of prediction. First of all, exchange of random N1 and random $\mathrm{N} 2$ from row $\mathrm{X}$ (Y is not modified) were performed thousand times. Further, from above probes the $R_{(X, Y)}^{2}$ was calculated and represented as $R_{r}^{2}$. The ${ }^{C} R_{p}^{2}$ was finally calculated according to the equation (3). 


$$
{ }^{C} R_{p}^{2}=R \times\left(R^{2}-R_{r}^{2}\right)^{1 / 2}
$$

Where $R^{2}$ and $R_{r}^{2}$ were utilized from the non-randomized and randomized model respectively. For acceptance of QSAR model the threshold value of ${ }^{C} R_{p}^{2}$ should be greater than 0.5 .

\section{RESULTS AND DISCUSSION}

The SMILES format of 411 BACE1 inhibitors were used to calculate the molecular descriptor followed by QSAR model development using $\mathrm{MC}$ algorithm based CORAL software (http://www.insilico.eu/coral/). The $p K_{i}$ was considered as dependent variable and molecular descriptors as independent variables. In this manuscript, two approaches were implemented to calculate the descriptors such as descriptor generation with- and without-considering the influence of cyclic rings of the molecular systems. During the model development 3 compounds were found to be outlier. Subsequently these three molecules were discarded from the dataset for further study.

\subsection{Selection of optimal $\mathbf{T}$ and $\mathbf{N}_{\text {epoch }}$}

Optimal $\mathrm{T}$ and $\mathrm{N}_{\text {epoch }}$ were identified with help of the "Search for preferable model" option of the CORAL. The threshold values in the range of 1 to 10 and the number of epochs ranging from 1 to 30 were used. The statistical parameters, epoch numbers and corresponding threshold values are given in Tables 1 and 2 in case without- and with-considering the influence of cyclic rings on inhibitory activity respectively. On detailed analysis of the correlation coefficient of training, calibration and test sets, optimal $\mathrm{T}$ and $\mathrm{N}_{\text {epoch }}\left(\mathrm{T}^{*}\right.$ and $\left.\mathrm{N}^{*}{ }_{\text {epoch }}\right)$ were found to be $(6,7)$ and $(6,6)$ in case of without- and withconsidering the influence of cyclic rings on inhibitory activity respectively.

Table 1: Statistical parameters of training, calibration and test sets to search $T^{*}$ and $\mathrm{N}^{*}$ epoch for without influence of cyclic

\begin{tabular}{|c|c|c|c|c|c|c|c|c|}
\hline Epoch no. & $\boldsymbol{R}_{t r}{ }^{2}$ & $s_{t r}$ & $R_{c}^{2}$ & $s_{c}$ & $\boldsymbol{R}_{t s}{ }^{2}$ & $s_{t s}$ & $\boldsymbol{R}_{m a v}{ }^{2}$ & $T$ \\
\hline 2 & 0.622 & 0.689 & 0.607 & 0.864 & 0.610 & 0.779 & 0.574 & 5 \\
\hline 3 & 0.667 & 0.647 & 0.673 & 0.807 & 0.620 & 0.765 & 0.595 & 6 \\
\hline 4 & 0.665 & 0.649 & 0.674 & 0.802 & 0.617 & 0.768 & 0.602 & 9 \\
\hline 5 & 0.700 & 0.614 & 0.714 & 0.756 & 0.601 & 0.783 & 0.587 & 7 \\
\hline 6 & 0.716 & 0.597 & 0.728 & 0.742 & 0.585 & 0.797 & 0.576 & 7 \\
\hline 7 & 0.719 & 0.594 & 0.729 & 0.738 & 0.566 & 0.817 & 0.552 & 7 \\
\hline 8 & 0.723 & 0.590 & 0.745 & 0.728 & 0.569 & 0.814 & 0.555 & 7 \\
\hline 9 & 0.727 & 0.586 & 0.751 & 0.713 & 0.536 & 0.845 & 0.521 & 7 \\
\hline 10 & 0725 & 0.587 & 0.756 & 0.719 & 0.527 & 0.822 & 0.547 & 7 \\
\hline 11 & 0.731 & 0.581 & 0.754 & 0.719 & 0.554 & 0.830 & 0.533 & 7 \\
\hline 12 & 0.730 & 0.583 & 0.745 & 0.710 & 0.543 & 0.840 & 0.516 & 8 \\
\hline 13 & 0.734 & 0.578 & 0.750 & 0.712 & 0.537 & 0.849 & 0.508 & 8 \\
\hline 14 & 0.737 & 0.575 & 0.761 & 0.710 & 0.552 & 0.831 & 0.538 & 7 \\
\hline 15 & 0.729 & 0.58 & 0.776 & 0.701 & 0.517 & 0.866 & 0.492 & 7 \\
\hline 16 & 0.737 & 0.575 & 0.769 & 0.705 & 0.534 & 0.848 & 0.514 & 7 \\
\hline 17 & 0.735 & 0.577 & 0.779 & 0.703 & 0.517 & 0.863 & 0.494 & 7 \\
\hline 18 & 0.735 & 0.577 & 0.774 & 0.702 & 0.515 & 0.866 & 0.494 & 8 \\
\hline 19 & 0.736 & 0.575 & 0.769 & 0.704 & 0.527 & 0.856 & 0.506 & 7 \\
\hline 20 & 0.740 & 0572 & 0.780 & 0.700 & 0.499 & 0.885 & 0.463 & 7 \\
\hline 21 & 0.738 & 0.573 & 0.772 & 0.704 & 0.519 & 0.865 & 0.488 & 9 \\
\hline 22 & 0.735 & 0.577 & 0.769 & 0.702 & 0.513 & 0.872 & 0.486 & 8 \\
\hline 23 & 0.747 & 0.564 & 0.771 & 0.704 & 0.525 & 0.858 & 0.505 & 7 \\
\hline 24 & 0.739 & 0.572 & 0.781 & 0.700 & 0.513 & 0.871 & 0.483 & 7 \\
\hline 25 & 0.737 & 0.575 & 0.777 & 0.687 & 0.507 & 0.880 & 0.464 & 8 \\
\hline 26 & 0.745 & 0.566 & 0.787 & 0.691 & 0.520 & 0.863 & 0.490 & 8 \\
\hline 27 & 0.742 & 0.569 & 0.774 & 0.690 & 0.497 & 0.889 & 0.463 & 8 \\
\hline 28 & 0.740 & 0.571 & 0.775 & 0.695 & 0.794 & 0.888 & 0.464 & 8 \\
\hline 29 & 0.740 & 0.571 & 0.777 & 0.696 & 0.501 & 0.883 & 0.466 & 8 \\
\hline
\end{tabular}


$R_{t r}^{2}$ : Correlation coefficient of training set; $s_{t r}$ : standard error of training set; $R_{c}^{2}$ : Correlation coefficient of calibration set; $s_{c}$ : standard error of calibration set; $R_{t s}{ }^{2}$ : Correlation coefficient of test set; $s_{t s}$ : standard error of test set; $R_{m}{ }^{2} a v$ : Modified correlation coefficient; $T$ : Threshold

Table 2: Statistical parameters of training, calibration and test sets to search $\mathrm{T}^{*}$ and $\mathrm{N}^{*}$ epoch for with influence of cyclic rings

\begin{tabular}{|c|c|c|c|c|c|c|c|c|}
\hline Epoch no. & $\boldsymbol{R}_{t r}{ }^{2}$ & $s_{t r}$ & $R_{c}{ }^{2}$ & $s_{c}$ & $\boldsymbol{R}_{t s}{ }^{2}$ & $s_{t s}$ & $\boldsymbol{R}_{m a v}{ }^{2}$ & $T$ \\
\hline 2 & 0.670 & 0.644 & 0.653 & 0.821 & 0.590 & 0.794 & 0.573 & 3 \\
\hline 3 & 0.664 & 0.650 & 0.662 & 0.809 & 0.615 & 0.770 & 0.596 & 6 \\
\hline 4 & 0.688 & 0.626 & 0.696 & 0.780 & 0.597 & 0.787 & 0.587 & 6 \\
\hline 5 & 0.688 & 0.626 & 0.699 & 0.779 & 0.596 & 0.787 & 0.593 & 9 \\
\hline 6 & 0.721 & 0.592 & 0.732 & 0.729 & 0.584 & 0.799 & 0.569 & 6 \\
\hline 7 & 0.707 & 0.606 & 0.740 & 0.738 & 0.558 & 0.825 & 0.537 & 9 \\
\hline 8 & 0.718 & 0.595 & 0.738 & 0.732 & 0.555 & 0.282 & 0.543 & 9 \\
\hline 9 & 0.713 & 0.600 & 0.748 & 0.730 & 0.548 & 0.836 & 0.519 & 9 \\
\hline 10 & 0.729 & 0.583 & 0.761 & 0.712 & 0.553 & 0.830 & 0.531 & 7 \\
\hline 11 & 0.725 & 0.588 & 0.765 & 0.704 & 0.534 & 0.849 & 0.509 & 7 \\
\hline 12 & 0.734 & 0.578 & 0.763 & 0.708 & 0.531 & 0.851 & 0.508 & 7 \\
\hline 13 & 0.736 & 0.576 & 0.765 & 0.705 & 0.545 & 0.837 & 0.531 & 7 \\
\hline 14 & 0.737 & 0.575 & 0.764 & 0.707 & 0.533 & 0.849 & 0.511 & 7 \\
\hline 15 & 0.739 & 0.573 & 0.762 & 0.700 & 0.537 & 0.847 & 0.510 & 8 \\
\hline 16 & 0.736 & 0.576 & 0.759 & 0.704 & 0.526 & 0.85 & 0.502 & 8 \\
\hline 17 & 0.731 & 0.581 & 0.769 & 0.709 & 0.523 & 0.860 & 0.491 & 7 \\
\hline 18 & 0.738 & 0.573 & 0.781 & 0.694 & 0.501 & 0.881 & 0.474 & 7 \\
\hline 19 & 0.740 & 0.571 & 0.460 & 0.715 & 0.514 & 0.869 & 0.488 & 7 \\
\hline 20 & 0.740 & 0.572 & 0.780 & 0.700 & 0.499 & 0.885 & 0.463 & 7 \\
\hline 21 & 0.737 & 0.574 & 0.777 & 0.695 & 0.505 & 0.877 & 0.481 & 9 \\
\hline 22 & 0.738 & 0.574 & 0.781 & 0.699 & 0.536 & 0.849 & 0.508 & 7 \\
\hline 23 & 0.735 & 0.577 & 0.769 & 0.701 & 0.513 & 0.869 & 0.484 & 7 \\
\hline 24 & 0.751 & 0.559 & 0.760 & 0.698 & 0.516 & 0.875 & 0.461 & 6 \\
\hline 25 & 0.743 & 0.568 & 0.776 & 0.696 & 0.503 & 0.881 & 0.478 & 7 \\
\hline 26 & 0.744 & 0.567 & 0.782 & 0.692 & 0.518 & 0.864 & 0.487 & 7 \\
\hline 27 & 0.742 & 0.569 & 0.774 & 0.690 & 0.497 & 0.889 & 0.463 & 8 \\
\hline 28 & 0.739 & 0.573 & 0.781 & 0.688 & 0.505 & 0.876 & 0.479 & 7 \\
\hline 29 & 0.745 & 0.566 & 0.774 & 0.693 & 0.518 & 0.866 & 0.479 & 6 \\
\hline 30 & 0.738 & 0.573 & 0.785 & 0.692 & 0.500 & 0.882 & 0.471 & 7 \\
\hline
\end{tabular}

$R_{t r}{ }^{2}$ : Correlation coefficient of training set; $s_{t r}$ : standard error of training set; $R_{c}^{2}$ : Correlation coefficient of calibration set; $s_{c}$ : standard error of calibration set; $R_{t s}^{2}$ : Correlation coefficient of test set; $s_{t s}$ : standard error of test set; $R_{m}^{2}$ av : Modified correlation coefficient; $T$ : Threshold

\subsection{Without considering influence of various cyclic rings}

Influence of cyclic ring on the inhibitory activity was not considered and the DCW derived. Subsequently the QSAR model was developed. The best model was selected based on best MC optimization runs.

$$
p K_{i}=0.727( \pm 0.008)+0.024( \pm 0.00007) \times D C W(7,6)
$$

$$
\text { Training set: } \quad \begin{aligned}
n=204 ; & R^{2}=0.711 ; s=0.729 ; F=498 ; \\
& Q^{2}=0.706 ; R_{m}{ }^{2}=0.584 ;{ }^{C} R^{2}{ }_{p}= \\
& 0.709
\end{aligned}
$$




$$
\begin{array}{lc}
\text { Calibration set: } & n=64 ; R^{2}=0.729 ; s=0.742 ; F=167 ; \\
& R_{m}{ }^{2}=0.612 ;{ }^{C} R_{p}{ }^{2}=0.720 \\
\text { Test set: } & n=70 ; R^{2}=0.590 ; s=0.792, F=98 ; R_{m}{ }^{2} \\
& =0.573 ;{ }^{C} R^{2}=0.577 \\
\text { External set: } & n=70 ; R^{2}=0.775 ; s=0.575, F=234 ; \\
& R_{m}{ }^{2}=0.600
\end{array}
$$

The statistical value of the model developed without considering impact of cyclic rings on the inhibitory activity clearly suggested that model was statically robust in nature and capable enough to predict the inhibitory activity of the external set of molecules.

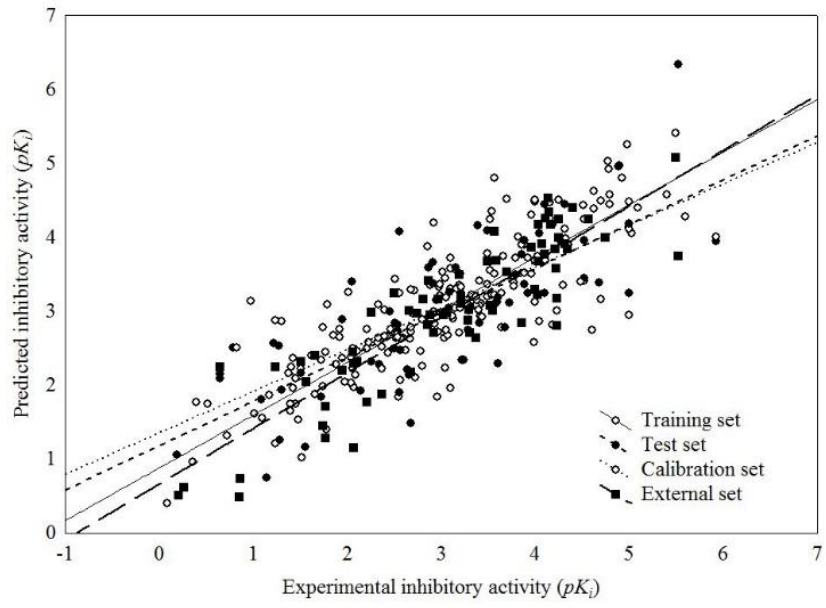

Figure 1: Observed and predicted inhibitory activity as per model developed without considering cyclic rings

The experimental and predicted inhibitory activity according the model are portrayed in the Figure 1 and Table S1 (Supplementary file). To check the closeness between experimental and predicted activity the radar plot was developed and given in Figure 2. The radar plot clearly exhibited the closeness between the experimental and predicted activity.
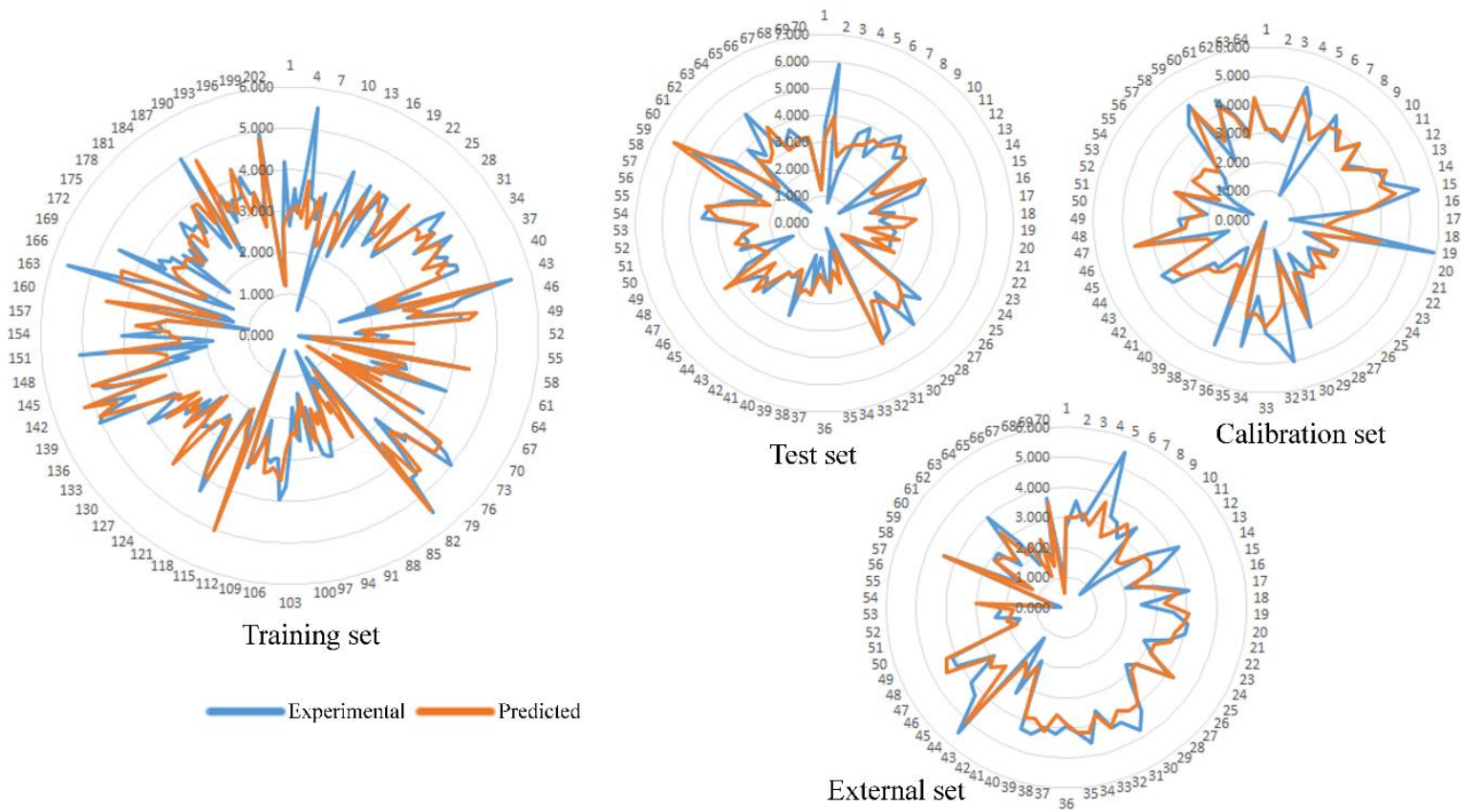

Figure 2: Radar plot showing fitness of observed and predicted inhibitory activity of training, test, calibration and external sets per model developed without considering cyclic rings

The detailed exploration of DCW from the best model developed without considering any influence of cyclic rings on the inhibitory activity explained that components " +++ $+\mathrm{B} 2--\mathrm{B} 3==", "++++\mathrm{Cl}-\mathrm{B} 2=="$ and " $++++\mathrm{S}---\mathrm{B} 2=="$ were found to be positive impact, while $++++\mathrm{F}---\mathrm{B} 2==$ ", "+ $+++\mathrm{F}---\mathrm{S}===$ " and " $++++\mathrm{O}---\mathrm{B} 2==$ " showed negative impact on the inhibitory activity. The "BOND10000000", "BOND10100000" and "BOND11100000" components were also showed negative influence towards the inhibition of BACE1. The components "HALO00000000", "HALO01000000", "HALO01100000" and
"HALO11000000" decrease the $p K_{i}$ but "HALO00100000" showed no significance on $p K_{i}$. On other hand impact of nitrogen, oxygen and sulphur together ("NOSP11100000") showed negative impact towards the $p K_{i}$.

\subsection{With considering influence of various cyclic rings}

The best QSAR model with the influence of cycling rings on inhibitory activity was developed and given below. The best model was selected based on best Monte Carlo optimization runs. 
$p K_{i}=0.645( \pm 0.008)+0.025( \pm 0.0001) \times D C W(6,6)$

Training set: $\quad n=204 ; R^{2}=0.717 ; s=0.597 ; F=511$; $Q^{2}=0.712 ; R_{m}{ }^{2}=0.581 ;{ }^{C} R_{p}^{2}=$ 0.715

Calibration set: $\quad n=64 ; R^{2}=0.723 ; s=0.734 ; F=162$; $R_{m}{ }^{2}=0.598 ;{ }^{C} R_{p}^{2}=0.712$

Test set:

$$
n=70 ; R^{2}=0.539 ; s=0.843 ; F=79 \text {; }
$$$$
R_{m}{ }^{2}=0.512 ;{ }^{C} R_{p}^{2}=0.535
$$

External set:

$$
n=70 ; R^{2}=0.660 ; s=0.621 ; F=52 ; R_{m}{ }^{2}
$$$$
=0.532
$$

The best model was developed with considering the impact of cyclic rings on inhibitory activity with threshold and $\mathrm{N}_{\text {epoch }}$ of 6 and 6 respectively. Values of statistical parameters were recorded and it observed that correlation coefficient of all training, calibration, test and external sets found to be 0.714 , $0.723,0.539$ and 0.660 respectively. The $R_{m}{ }^{2}$ and ${ }^{C} R_{p}^{2}$ were also found to be more than 0.5 for all sets. The cross-validated correlation coefficient $\left(Q^{2}\right)$ of training set was also found to be 0.712 . Therefore, above statistical outcome undoubtedly explained that selected model robust in nature and efficient to predict the biological activity of molecules outside the training set. Experimental and predicted activity as per above model are given in Figure 3 and Table S2 (Supplementary file). Radar plot between the experimental and predicted activity also plotted and given in Figure 4. Radar plot clearly explained the closeness between experimental and predicted activity which substantiated the predictive capability of the model.

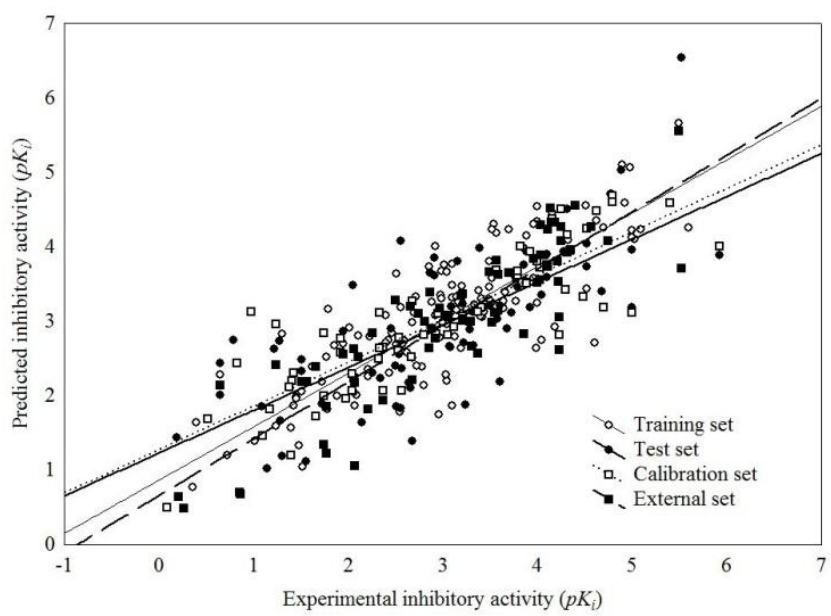

Figure 3: Observed and predicted inhibitory activity as per model developed with considering cyclic rings
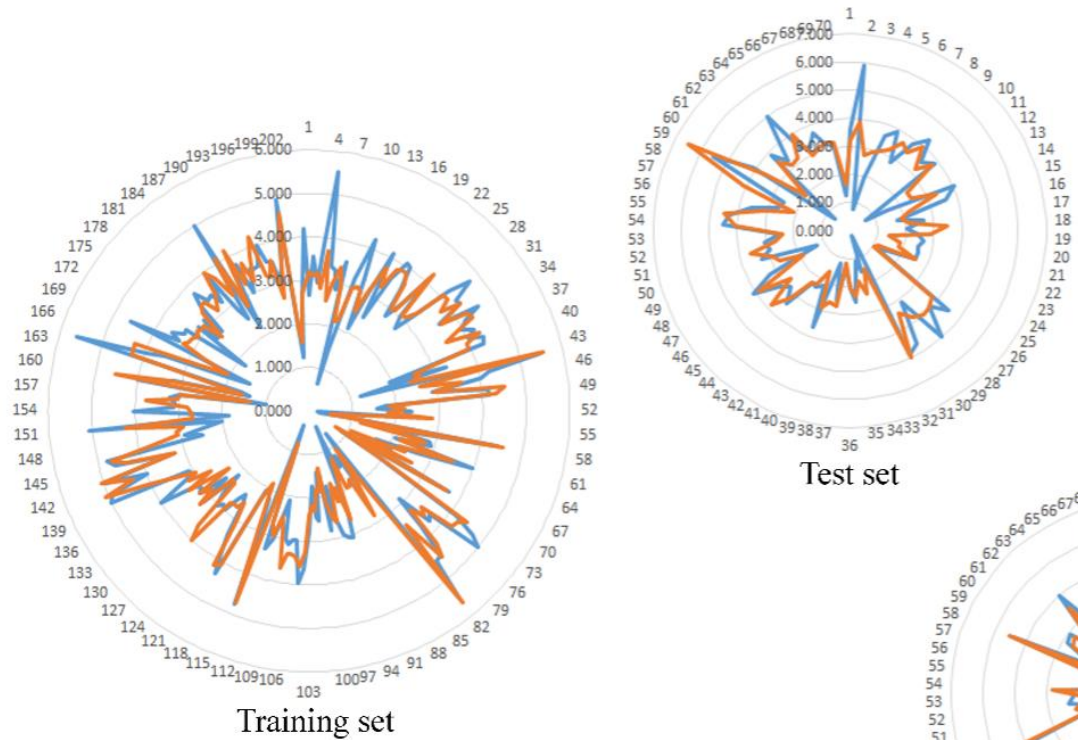

Test set

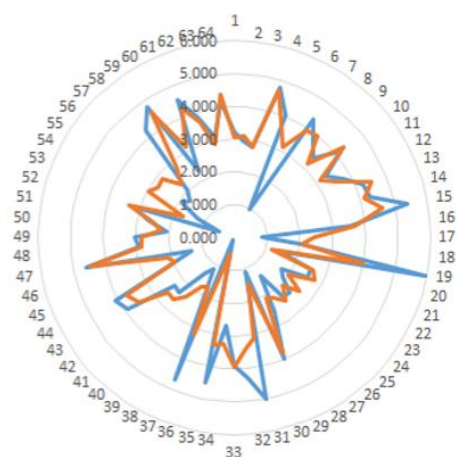

Calibration set

$\Longrightarrow$ Fxperimental 2 Predicted

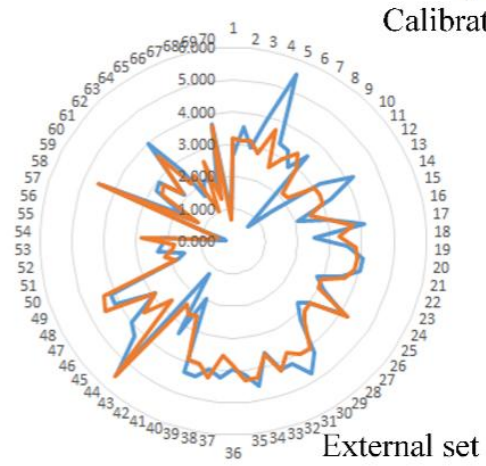

Figure 4: Radar plot showing fitness of observed and predicted inhibitory activity of training, test, calibration and external sets per model developed with considering cyclic rings

To explore the mechanistic interpretation of the model DCW were analysed. The molecular fragments including " $++++\mathrm{F}-$ $-\mathrm{N}===", "++++\mathrm{CL}-\mathrm{-N}===", "++++\mathrm{N}---\mathrm{B} 3=="$ and " $++++\mathrm{S}---\mathrm{B} 3==$ " were found to be crucial for the inhibition of the BACE1 enzyme. Conversly, " ++++ F- - $\mathrm{B} 2==", "++++\mathrm{F}---\mathrm{S}===$ ", " $++++\mathrm{CL}--\mathrm{S}===$ " and " + $+++\mathrm{O}---\mathrm{B} 2==$ " were given negative impact for the inhibitory activity. In case of bonding parameters the "BOND10000000" was found critical for $p K_{i}$ but remaining two parmeters "BOND10100000" and "BOND11100000" not significant to increase or decrease the $p K_{i}$. Presence or absence of halogen atoms were also explored and found that
"HALO10000000" (presence of fluorine) and "HALO11000000" (presence of both fluorine and clrorine) give negative influnece for the inhibition of BACE1.

The influence of cyclic rings of the molecular systems for the inhibition was explored. The statistical parameters of models developed without (Equation 4) and with (Equation 5) considering the cyclic rings were analyses. It was observed improved statistical parameters for the model developed with influence of cyclic ring on the $p K_{i}$. Above observation clearly suggested that cyclic rings in the BACE1 inhibitors were crucial for potential inhibition. 


\section{CONCLUSION}

A large dataset of BACE1 inhibiotors was considered to develop the QSAR models with SMILES based molecular descriptors. The descriptors were calculated using the MC algoritm based CORAL software tool. Two approches such as without and with considering the impact of cyclic rings on the inhibitory activity were considered to develop QSAR model. Both models were assessed using the differentent statitical parameters including $R^{2}, s, Q^{2}, R_{m}{ }^{2},{ }^{C} R_{p}$ etc. All statistical parameters clearly suggested that both models were statitically robust in nature. Predictive cabalibity was adjudged using the external set molcules by predicting the inhibitory activity. High correlation value between experimental and predicted activity of external set explained that models were capable enough to predict the $p K_{i}$ of molecules outside the training set. Statistical parameters obtained from the both models also explained that cyclic rings were crucial of the BACE1 inhihibitors for the potential inhibition of BACE1 enzyme. Different molecular fragments were found to be important either increase or decrease the inhibitory activity which explain that both have mechanistic interpretation. Therefore, important molecular fragmanets explained by both models can play crucial role to identify new potential BACE1 inhibitors for the therapeutic application in Alzheimer's disease.

\section{ACKNOWLEDGEMENT}

One of the authors, Md L. Islam would like to thank Head of the Department, Department of Computer Engineering, MH Saboo Siddik College of Engineering, Byculla, Mumbai for providing research facilities.

\section{REFERENCES}

[1] Santana, I., Farinha, F., Freitas, S., Rodrigues, V., Carvalho, A. [The Epidemiology of Dementia and Alzheimer Disease in Portugal: Estimations of Prevalence and Treatment-Costs]. Acta Med Port. 2015, $28,182-8$.

[2] Alzheimer's, A. 2015 Alzheimer's disease facts and figures. Alzheimers Dement. 2015, 11, 332-84.

[3] Chiang, K., Koo, E.H. Emerging therapeutics for Alzheimer's disease. Annu Rev Pharmacol Toxicol. 2014, 54, 381-405.

[4] Masters, C.L., Bateman, R., Blennow, K., Rowe, C.C., Sperling, R.A., Cummings, J.L. Alzheimer's disease. Nat Rev Dis Primers. 2015, 1, 15056.

[5] Selkoe, D.J. Alzheimer's disease: genes, proteins, and therapy. Physiol Rev. 2001, 81, 741-66.

[6] Ghosh, A.K., Osswald, H.L. BACE1 (beta-secretase) inhibitors for the treatment of Alzheimer's disease. Chem Soc Rev. 2014, 43, 6765-813.

[7] Vassar, R., Bennett, B.D., Babu-Khan, S., Kahn, S., Mendiaz, E.A., Denis, P., et al. Beta-secretase cleavage of Alzheimer's amyloid precursor protein by the transmembrane aspartic protease BACE. Science. 1999, 286, 735-41.

[8] Cui, H., Hung, A.C., Klaver, D.W., Suzuki, T., Freeman, C., Narkowicz, C., et al. Effects of heparin and enoxaparin on APP processing and Abeta production in primary cortical neurons from Tg2576 mice. PLoS One. 2011, 6, e23007.
[9] Citron, M. Beta-secretase as a target for the treatment of Alzheimer's disease. J Neurosci Res. 2002, 70, 373-9.

[10] De Strooper, B., Vassar, R., Golde, T. The secretases: enzymes with therapeutic potential in Alzheimer disease. Nat Rev Neurol. 2010, 6, 99-107.

[11] Luo, Y., Bolon, B., Kahn, S., Bennett, B.D., Babu-Khan, S., Denis, P., et al. Mice deficient in BACE1, the Alzheimer's beta-secretase, have normal phenotype and abolished beta-amyloid generation. Nat Neurosci. 2001, 4, 231-2.

[12] Christensen, M.A., Zhou, W., Qing, H., Lehman, A., Philipsen, S., Song, W. Transcriptional regulation of BACE1, the beta-amyloid precursor protein betasecretase, by Sp1. Mol Cell Biol. 2004, 24, 865-74.

[13] Nishitomi, K., Sakaguchi, G., Horikoshi, Y., Gray, A.J., Maeda, M., Hirata-Fukae, C., et al. BACE1 inhibition reduces endogenous Abeta and alters APP processing in wild-type mice. J Neurochem. 2006, 99, 1555-63.

[14] Zivkovic, J.V., Trutic, N.V., Veselinovic, J.B., Nikolic, G.M., Veselinovic, A.M. Monte Carlo method based QSAR modeling of maleimide derivatives as glycogen synthase kinase-3beta inhibitors. Comp Biol Med. 2015, $64,276-82$.

[15] Roy, K., Kar, S., Das, R.N. A Primer on QSAR/QSPR Modeling Fundamental Concepts, Springer International Publishing, 2015.

[16] Duchowicz, P.R., Comelli, N.C., Ortiz, E.V., Castro, E.A. QSAR study for carcinogenicity in a large set of organic compounds. Current Drug Safety. 2012, 7, 2828.

[17] Talevi, A., Bellera, C.L., Di Ianni, M., Duchowicz, P.R., Bruno-Blanch, L.E., Castro, E.A. An integrated drug development approach applying topological descriptors. Current Comp-Aided Drug Design. 2012, 8, 172-81.

[18] Randic, M., Basak, S.C. A new descriptor for structureproperty and structure-activity correlations. J Chem Inf Comp Sci. 2001, 41, 650-6.

[19] Junkes Bda, S., Arruda, A.C., Yunes, R.A., Porto, L.C., Heinzen, V.E. Semi-empirical topological index: a tool for QSPR/QSAR studies. J Mol Model. 2005, 11, 12834.

[20] Ivanciuc, O. Chemical graphs, molecular matrices and topological indices in chemoinformatics and quantitative structure-activity relationships. Current Comp-Aided Drug Design. 2013, 9, 153-63.

[21] Weininger, D. SMILES, a chemical language and information system. 1. Introduction to methodology and encoding rules. J Chem Inf Comp Sci. 1988, 28, 31-6.

[22] Weininger, D., Weininger, A., Weininger, J.L. SMILES. 2. Algorithm for generation of unique SMILES notation J Chem Inf Comp Sci. 1989, 29, 97-101.

[23] Weininger, D. SMILES. 3. Depict. Graphical depiction of chemical structures. J Chem Inf Comp Sci. 1990, 30, 237-43.

[24] Toropov, A.A., Benfenati, E. SMILES in QSPR/QSAR Modeling: results and perspectives. Curr Drug Discov Technol. 2007, 4, 77-116. 
[25] Toropov, A.A., Benfenati, E. SMILES as an alternative to the graph in QSAR modelling of bee toxicity. Comput Biol Chem. 2007, 31, 57-60.

[26] Veselinovic, A.M., Veselinovic, J.B., Zivkovic, J.V., Nikolic, G.M. Application of SMILES Notation Based Optimal Descriptors in Drug Discovery and Design. Curr Topics Med Chem. 2015, 15, 1768-79.

[27] Toropov, A.A., Toropova, A.P., Diaza, R.G., Benfenati, E., Gini, G. SMILES-based optimal descriptors: QSAR modeling of estrogen receptor binding affinity by correlation balance. Struct Chem. 2012, 23, 529-44.

[28] Veselinovic, A.M., Milosavljevic, J.B., Toropov, A.A., Nikolic, G.M. SMILES-based QSAR models for the calcium channel-antagonistic effect of 1,4dihydropyridines. Archiv der Pharmazie. 2013, 346, 1349.

[29] Veselinovic, A.M., Milosavljevic, J.B., Toropov, A.A., Nikolic, G.M. SMILES-based QSAR model for arylpiperazines as high-affinity 5-HT(1A) receptor ligands using CORAL. European journal of pharmaceutical sciences : official journal of the Euro Fed Pharm Sci. 2013, 48, 532-41.

[30] Veselinovic, J.B., Toropov, A.A., Toropova, A.P., Nikolic, G.M., Veselinovic, A.M. Monte carlo methodbased QSAR modeling of penicillins binding to human serum proteins. Arch Pharm (Weinheim). 2015, 348, 627.

[31] Worachartcheewan, A., Mandi, P., Prachayasittikul, V., Toropova, A.P., Toropov, A.A., Nantasenamat, C. Largescale QSAR study of aromatase inhibitors using SMILES-based descriptors. Chemo Intell Lab Syst. 2014, 138, 120-6.

[32] Li, Q., Ding, X., Si, H., Gao, H. QSAR model based on SMILES of inhibitory rate of 2, 3-diarylpropenoic acids on AKR1C3. Chemo Intell Lab Syst. 2014, 139, 120-6.
[33] Toropova, A.P., Toropov, A.A., Veselinovic, J.B., Miljkovic, F.N., Veselinovic, A.M. QSAR models for HEPT derivates as NNRTI inhibitors based on Monte Carlo method. Europ J Med Chem. 2014, 77, 298-305.

[34] Lipinski, C.A., Lombardo, F., Dominy, B.W., Feeney, P.J. Experimental and computational approaches to estimate solubility and permeability in drug discovery and development settings. Adv Drug Deliv Rev. 2001, 46, 3-26.

[35] Veber, D.F., Johnson, S.R., Cheng, H.Y., Smith, B.R., Ward, K.W., Kopple, K.D. Molecular properties that influence the oral bioavailability of drug candidates. J Med Chem. 2002, 45, 2615-23.

[36] Toropova, M.A., Toropov, A.A., Raska, I., Jr., Raskova, M. Searching therapeutic agents for treatment of Alzheimer disease using the Monte Carlo method. Comput Biol Med. 2015, 64, 148-54.

[37] Kubinyi, H., Hamprecht, F.A., Mietzner, T. Threedimensional quantitative similarity-activity relationships (3D QSiAR) from SEAL similarity matrices. J Med Chem. 1998, 41, 2553-64.

[38] Roy, K., Mitra, I., Kar, S., Ojha, P.K., Das, R.N., Kabir, H. Comparative studies on some metrics for external validation of QSPR models. J Chem Inf Model. 2012, 52, 396-408.

[39] Ojha, P.K., Mitra, I., Das, R.N., Roy, K. Further exploring $\mathrm{rm} 2$ metrics for validation of QSPR models Chemo Intell Lab Syst. 2011, 107, 194-205.

[40] Ojha, P.K., Roy, R. Comparative QSARs for antimalarial endochins: importance of descriptor-thinning and noise reduction prior to feature selection. Chemo Intell Lab Syst. 2011, 109, 146-61. 


\title{
7. APPENDIX
}

\section{Application of Monte Carlo Algorithm to Explore Simplified Molecular-Input Line-Entry System based Molecular Descriptors of BACE1 inhibitors for Therapeutic Application in Alzheimer's Disease}

\author{
Supplementary data
}

Table S1: SMILES, experimental and predicted inhibitory activity of training, test, calibration and external sets used in QSAR model without considering cycling rings

\begin{tabular}{|c|c|c|c|}
\hline \multirow{2}{*}{ Set } & \multirow{2}{*}{ SMILES } & \multicolumn{2}{|c|}{$P k_{i}$} \\
\hline & & ${ }^{1}$ Obs & ${ }^{2}$ Pred \\
\hline \multicolumn{4}{|c|}{ Training set } \\
\hline $\operatorname{Tr} 1$ & $\begin{array}{l}\mathrm{O}=\mathrm{C} 1 \mathrm{OC}([\mathrm{H}])([\mathrm{H}]) \mathrm{C}([\mathrm{H}])([\mathrm{H}]) \mathrm{C}([\mathrm{H}])([\mathrm{H}]) \mathrm{C}([\mathrm{H}])([\mathrm{H}]) \mathrm{N}([\mathrm{H}]) \mathrm{C}(=\mathrm{O}) \mathrm{C}([\mathrm{H}])([\mathrm{H}])[\mathrm{C} @]([\mathrm{H}])(\mathrm{N}( \\
[\mathrm{H}]) \mathrm{C}(=\mathrm{O})[\mathrm{C} @ @]([\mathrm{H}])(\mathrm{N} 1[\mathrm{H}]) \mathrm{C}([\mathrm{H}])(\mathrm{C}([\mathrm{H}])([\mathrm{H}])[\mathrm{H}]) \mathrm{C}([\mathrm{H}])([\mathrm{H}])[\mathrm{H}]) \mathrm{C}(=\mathrm{O}) \mathrm{N}([\mathrm{H}])[\mathrm{C} @ @]([ \\
\mathrm{H}])(\mathrm{C}([\mathrm{H}])([\mathrm{H}]) \mathrm{C}([\mathrm{H}])(\mathrm{C}([\mathrm{H}])([\mathrm{H}])[\mathrm{H}]) \mathrm{C}([\mathrm{H}])([\mathrm{H}])[\mathrm{H}])[\mathrm{C} @ @]([\mathrm{H}])(\mathrm{O}[\mathrm{H}]) \mathrm{C}([\mathrm{H}])([\mathrm{H}])[\mathrm{C} @]([ \\
\mathrm{H}])(\end{array}$ & 2.662 & 3.003 \\
\hline $\operatorname{Tr} 2$ & $\begin{array}{l}\mathrm{O}=\mathrm{C} 1 \mathrm{OC}([\mathrm{H}])([\mathrm{H}]) \mathrm{C}([\mathrm{H}])([\mathrm{H}]) \mathrm{C}([\mathrm{H}])=\mathrm{C}([\mathrm{H}]) \mathrm{C}([\mathrm{H}])([\mathrm{H}]) \mathrm{N}([\mathrm{H}]) \mathrm{C}(=\mathrm{O}) \mathrm{C}([\mathrm{H}])([\mathrm{H}])[\mathrm{C} @([\mathrm{H}]) \\
(\mathrm{N}([\mathrm{H}]) \mathrm{C}(=\mathrm{O})[\mathrm{C} @ @]([\mathrm{H}])(\mathrm{N} 1[\mathrm{H}]) \mathrm{C}([\mathrm{H}])(\mathrm{C}([\mathrm{H}])([\mathrm{H}])[\mathrm{H}]) \mathrm{C}([\mathrm{H}])([\mathrm{H}])[\mathrm{H}]) \mathrm{C}(=\mathrm{O}) \mathrm{N}([\mathrm{H}])[\mathrm{C} @ \\
@]([\mathrm{H}])(\mathrm{C}([\mathrm{H}])([\mathrm{H}]) \mathrm{C}([\mathrm{H}])(\mathrm{C}([\mathrm{H}])([\mathrm{H}])[\mathrm{H}]) \mathrm{C}([\mathrm{H}])([\mathrm{H}])[\mathrm{H}])[\mathrm{C} @ @]([\mathrm{H}])(\mathrm{O}[\mathrm{H}]) \mathrm{C}([\mathrm{H}])([\mathrm{H}])[\mathrm{C} \\
@]([\mathrm{H}]\end{array}$ & 3.547 & 3.009 \\
\hline $\operatorname{Tr} 3$ & $\begin{array}{l}\mathrm{O}=\mathrm{C} 2 \mathrm{~N}([\mathrm{H}])[\mathrm{C} @]([\mathrm{H}])(\mathrm{C}(=\mathrm{O}) \mathrm{N}([\mathrm{H}])[\mathrm{C} @ @]([\mathrm{H}])(\mathrm{C}([\mathrm{H}])([\mathrm{H}]) \mathrm{C}([\mathrm{H}])(\mathrm{C}([\mathrm{H}])([\mathrm{H}])[\mathrm{H}]) \mathrm{C}([\mathrm{H}])( \\
[\mathrm{H}])[\mathrm{H}])[\mathrm{C} @ @]([\mathrm{H}])(\mathrm{O}[\mathrm{H}]) \mathrm{C}([\mathrm{H}])([\mathrm{H}])[\mathrm{C} @]([\mathrm{H}])(\mathrm{C}(=\mathrm{O}) \mathrm{N}([\mathrm{H}])[\mathrm{C} @]([\mathrm{H}])(\mathrm{C}(=\mathrm{O}) \mathrm{N}([\mathrm{H}]) \mathrm{C}([ \\
\mathrm{H}])([\mathrm{H}]) \mathrm{C}=1 \mathrm{C}([\mathrm{H}])=\mathrm{C}([\mathrm{H}]) \mathrm{C}([\mathrm{H}])=\mathrm{C}([\mathrm{H}]) \mathrm{C}=1[\mathrm{H}]) \mathrm{C}([\mathrm{H}])(\mathrm{C}([\mathrm{H}])([\mathrm{H}])[\mathrm{H}]) \mathrm{C}([\mathrm{H}])([\mathrm{H}])[\mathrm{H}]) \mathrm{C}([ \\
\mathrm{H}])([\mathrm{H}]\end{array}$ & 2.966 & 3.165 \\
\hline $\operatorname{Tr} 4$ & $\begin{array}{l}\mathrm{O}=\mathrm{C}(\mathrm{OC}([\mathrm{H}])([\mathrm{H}]) \mathrm{N} 1 \mathrm{~N}=\mathrm{C}(\mathrm{C}([\mathrm{H}])=\mathrm{C} 1 \mathrm{C}([\mathrm{H}])([\mathrm{H}])[\mathrm{H}]) \mathrm{C}([\mathrm{H}])([\mathrm{H}])[\mathrm{H}]) \mathrm{N}([\mathrm{H}])[\mathrm{C} @]([\mathrm{H}])(\mathrm{C}(= \\
\mathrm{O}) \mathrm{N}([\mathrm{H}])[\mathrm{C} @ @]([\mathrm{H}])(\mathrm{C}([\mathrm{H}])([\mathrm{H}]) \mathrm{C}([\mathrm{H}])(\mathrm{C}([\mathrm{H}])([\mathrm{H}])[\mathrm{H}]) \mathrm{C}([\mathrm{H}])([\mathrm{H}])[\mathrm{H}])[\mathrm{C} @ @]([\mathrm{H}])(\mathrm{O}[\mathrm{H}]) \\
\mathrm{C}([\mathrm{H}])([\mathrm{H}])[\mathrm{C} @]([\mathrm{H}])(\mathrm{C}(=\mathrm{O}) \mathrm{N}([\mathrm{H}])[\mathrm{C} @]([\mathrm{H}])(\mathrm{C}(=\mathrm{O}) \mathrm{N}([\mathrm{H}]) \mathrm{C}([\mathrm{H}])([\mathrm{H}]) \mathrm{C}=2 \mathrm{C}([\mathrm{H}])=\mathrm{C}([\mathrm{H}]) \mathrm{C} \\
([\mathrm{H}])=\mathrm{C}([\end{array}$ & 3.854 & 2.836 \\
\hline $\operatorname{Tr} 5$ & $\begin{array}{l}\mathrm{O}=\mathrm{C}(\mathrm{OC}([\mathrm{H}])([\mathrm{H}]) \mathrm{N} 1 \mathrm{~N}=\mathrm{C}(\mathrm{C}([\mathrm{H}])=\mathrm{C} 1 \mathrm{C}([\mathrm{H}])([\mathrm{H}])[\mathrm{H}]) \mathrm{C}([\mathrm{H}])([\mathrm{H}])[\mathrm{H}]) \mathrm{N}([\mathrm{H}])[\mathrm{C} @]([\mathrm{H}])(\mathrm{C}(= \\
\mathrm{O}) \mathrm{N}([\mathrm{H}])[\mathrm{C} @ @]([\mathrm{H}])(\mathrm{C}([\mathrm{H}])([\mathrm{H}]) \mathrm{C}([\mathrm{H}])(\mathrm{C}([\mathrm{H}])([\mathrm{H}])[\mathrm{H}]) \mathrm{C}([\mathrm{H}])([\mathrm{H}])[\mathrm{H}])[\mathrm{C} @ @]([\mathrm{H}])(\mathrm{O}[\mathrm{H}]) \\
\mathrm{C}([\mathrm{H}])([\mathrm{H}])[\mathrm{C} @]([\mathrm{H}])(\mathrm{C}(=\mathrm{O}) \mathrm{N}([\mathrm{H}])[\mathrm{C} @]([\mathrm{H}])(\mathrm{C}(=\mathrm{O}) \mathrm{N}([\mathrm{H}]) \mathrm{C}([\mathrm{H}])([\mathrm{H}]) \mathrm{C}([\mathrm{H}])(\mathrm{C}([\mathrm{H}])([\mathrm{H}])[ \\
\mathrm{H}]) \mathrm{C}([\mathrm{H}\end{array}$ & 5.523 & 3.748 \\
\hline Tr6 & $\begin{array}{l}{[\mathrm{F}] / \mathrm{C} 1=\mathrm{C}(\backslash[\mathrm{H}]) \mathrm{C}([\mathrm{H}])=\mathrm{C}(\mathrm{C}([\mathrm{H}])=\mathrm{C} 1[\mathrm{H}])[\mathrm{C} @]([\mathrm{H}])(\mathrm{N}([\mathrm{H}]) \mathrm{C}(=\mathrm{O}) \mathrm{C} 2=\mathrm{C}([\mathrm{H}]) \mathrm{C}(=\mathrm{C}([\mathrm{H}]) \mathrm{C}(=\mathrm{C} 2} \\
[\mathrm{H}]) \mathrm{C} 3=\mathrm{NN}=\mathrm{C}(\mathrm{O} 3)[\mathrm{C} @ @](\mathrm{C}([\mathrm{H}])([\mathrm{H}])[\mathrm{H}])(\mathrm{C}([\mathrm{H}])([\mathrm{H}]) \mathrm{C}=4 \mathrm{C}([\mathrm{H}])=\mathrm{C}([\mathrm{H}]) \mathrm{C}([\mathrm{H}])=\mathrm{C}([\mathrm{H}]) \mathrm{C}= \\
4[\mathrm{H}])[\mathrm{N}]([\mathrm{H}])([\mathrm{H}])[\mathrm{H}]) \mathrm{N}(\mathrm{C}([\mathrm{H}])([\mathrm{H}])[\mathrm{H}])[\mathrm{S}](=\mathrm{O})(=\mathrm{O}) \mathrm{C}([\mathrm{H}])([\mathrm{H}])[\mathrm{H}]) \mathrm{C}([\mathrm{H}])([\mathrm{H}])[\mathrm{H}]\end{array}$ & 3.373 & 2.634 \\
\hline $\operatorname{Tr} 7$ & $\begin{array}{l}\mathrm{O}=[\mathrm{S}](=\mathrm{O})(\mathrm{N}(\mathrm{C}=2 \mathrm{C}([\mathrm{H}])=\mathrm{C}(\mathrm{C}([\mathrm{H}])=\mathrm{C}([\mathrm{H}])[\mathrm{C} @ @] 1([\mathrm{H}]) \mathrm{C}([\mathrm{H}])([\mathrm{H}])[\mathrm{C} @] 1([\mathrm{H}]) \mathrm{C}([\mathrm{H}])([\mathrm{H}]) \\
[\mathrm{H}]) \mathrm{C}([\mathrm{H}])=\mathrm{C}(\mathrm{C}=2[\mathrm{H}]) \mathrm{C} 3=\mathrm{NN}=\mathrm{C}(\mathrm{O} 3)[\mathrm{C} @ @](\mathrm{C}([\mathrm{H}])([\mathrm{H}])[\mathrm{H}])(\mathrm{C}([\mathrm{H}])([\mathrm{H}]) \mathrm{C}=4 \mathrm{C}([\mathrm{H}])=\mathrm{C}([\mathrm{H}] \\
) \mathrm{C}([\mathrm{H}])=\mathrm{C}([\mathrm{H}]) \mathrm{C}=4[\mathrm{H}])[\mathrm{N}]([\mathrm{H}])([\mathrm{H}])[\mathrm{H}]) \mathrm{C}([\mathrm{H}])([\mathrm{H}]) \mathrm{C}([\mathrm{H}])([\mathrm{H}]) \mathrm{C}([\mathrm{H}])([\mathrm{H}])[\mathrm{H}]) \mathrm{C}([\mathrm{H}])([\mathrm{H}])[ \\
\mathrm{H}]\end{array}$ & 3.285 & 2.874 \\
\hline Tr8 & $\begin{array}{l}\mathrm{O}=\mathrm{C}(\mathrm{C}=1 \mathrm{C}([\mathrm{H}])=\mathrm{C}(\mathrm{C}([\mathrm{H}])=\mathrm{C}(\mathrm{C}=1[\mathrm{H}]) \mathrm{C}(=\mathrm{O}) \mathrm{N}([\mathrm{H}])[\mathrm{C} @ @]([\mathrm{H}])(\mathrm{C}([\mathrm{H}])([\mathrm{H}]) \mathrm{C}([\mathrm{H}])(\mathrm{C}([\mathrm{H}])([ \\
\mathrm{H}])[\mathrm{H}]) \mathrm{C}([\mathrm{H}])([\mathrm{H}])[\mathrm{H}])[\mathrm{C} @ @]([\mathrm{H}])(\mathrm{O}[\mathrm{H}]) \mathrm{C}([\mathrm{H}])([\mathrm{H}])[\mathrm{C} @]([\mathrm{H}])(\mathrm{C}(=\mathrm{O}) \mathrm{N}([\mathrm{H}])[\mathrm{C} @]([\mathrm{H}])(\mathrm{C}( \\
=\mathrm{O}) \mathrm{N}([\mathrm{H}]) \mathrm{C}([\mathrm{H}])(\mathrm{C}([\mathrm{H}])([\mathrm{H}])[\mathrm{H}]) \mathrm{C}([\mathrm{H}])([\mathrm{H}])[\mathrm{H}]) \mathrm{C}([\mathrm{H}])(\mathrm{C}([\mathrm{H}])([\mathrm{H}])[\mathrm{H}]) \mathrm{C}([\mathrm{H}])([\mathrm{H}])[\mathrm{H}]) \mathrm{C}([ \\
\mathrm{H}])([\end{array}$ & 2.866 & 3.413 \\
\hline
\end{tabular}




\begin{tabular}{|c|c|c|c|}
\hline $\operatorname{Tr} 9$ & $\begin{array}{l}\mathrm{O}=\mathrm{C}(\mathrm{N}(\mathrm{C}([\mathrm{H}])([\mathrm{H}])[\mathrm{H}])[\mathrm{C} @ @] 1([\mathrm{H}]) \mathrm{C}([\mathrm{H}])([\mathrm{H}]) \mathrm{C}([\mathrm{H}])([\mathrm{H}]) \mathrm{C}([\mathrm{H}])([\mathrm{H}]) \mathrm{C}([\mathrm{H}])([\mathrm{H}]) \mathrm{Cl}([\mathrm{H}])[ \\
\mathrm{H}]) \mathrm{C}([\mathrm{H}])([\mathrm{H}]) \mathrm{C}([\mathrm{H}])([\mathrm{H}])[\mathrm{C} @ @]([\mathrm{H}])(\mathrm{N} 4 \mathrm{C}(=\mathrm{NC}=3 \mathrm{C}([\mathrm{H}])=\mathrm{C}([\mathrm{H}]) \mathrm{C}(\mathrm{OC}=2 \mathrm{C}([\mathrm{H}])=\mathrm{C}([\mathrm{H}]) \mathrm{C}( \\
[\mathrm{H}])=\mathrm{C}([\mathrm{H}]) \mathrm{C}=2[\mathrm{H}])=\mathrm{C}([\mathrm{H}]) \mathrm{C}=3 \mathrm{C} 4([\mathrm{H}])[\mathrm{H}]) \mathrm{N}([\mathrm{H}])[\mathrm{H}])[\mathrm{C} @ 5([\mathrm{H}]) \mathrm{C}([\mathrm{H}])([\mathrm{H}]) \mathrm{C}([\mathrm{H}])([\mathrm{H}]) \mathrm{C}( \\
[\mathrm{H}])([\mathrm{H}\end{array}$ & 3.523 & 3.079 \\
\hline $\operatorname{Tr} 10$ & $\begin{array}{l}\mathrm{O}=\mathrm{C}(\mathrm{N}([\mathrm{H}])[\mathrm{C} @]([\mathrm{H}])([\mathrm{C} @ @]([\mathrm{H}])(\mathrm{O}[\mathrm{H}]) \mathrm{C}([\mathrm{H}])([\mathrm{H}])[\mathrm{C} @]([\mathrm{H}])(\mathrm{C}(=\mathrm{O}) \mathrm{N}([\mathrm{H}])[\mathrm{C} @]([\mathrm{H}])(\mathrm{C}( \\
=\mathrm{O}) \mathrm{N}([\mathrm{H}]) \mathrm{C}([\mathrm{H}])([\mathrm{H}]) \mathrm{Cl}=\mathrm{C}([\mathrm{H}]) \mathrm{C}([\mathrm{H}])=\mathrm{C}([\mathrm{H}]) \mathrm{C}([\mathrm{H}])=\mathrm{C} 1[\mathrm{H}]) \mathrm{C}([\mathrm{H}])([\mathrm{H}])[\mathrm{H}]) \mathrm{C}([\mathrm{H}])([\mathrm{H}])[\mathrm{H} \\
]) \mathrm{C}([\mathrm{H}])([\mathrm{H}]) \mathrm{C}([\mathrm{H}])(\mathrm{C}([\mathrm{H}])([\mathrm{H}])[\mathrm{H}]) \mathrm{C}([\mathrm{H}])([\mathrm{H}])[\mathrm{H}])[\mathrm{C} @ @]([\mathrm{H}])(\mathrm{N}([\mathrm{H}]) \mathrm{C}(=\mathrm{O}) \mathrm{OC}(\mathrm{C}([\mathrm{H}])([ \\
\mathrm{H}])[\mathrm{H}])\end{array}$ & 0.649 & 2.248 \\
\hline $\operatorname{Tr} 11$ & $\begin{array}{l}\mathrm{O}=\mathrm{C}(\mathrm{N}([\mathrm{H}])[\mathrm{C} @]([\mathrm{H}])([\mathrm{C} @ @]([\mathrm{H}])(\mathrm{O}[\mathrm{H}]) \mathrm{C}([\mathrm{H}])([\mathrm{H}])[\mathrm{C} @]([\mathrm{H}])(\mathrm{C}(=\mathrm{O}) \mathrm{N}([\mathrm{H}])[\mathrm{C} @]([\mathrm{H}])(\mathrm{C}( \\
=\mathrm{O}) \mathrm{N}([\mathrm{H}]) \mathrm{C}([\mathrm{H}])([\mathrm{H}]) \mathrm{Cl}=\mathrm{C}([\mathrm{H}]) \mathrm{C}([\mathrm{H}])=\mathrm{C}([\mathrm{H}]) \mathrm{C}([\mathrm{H}])=\mathrm{C} 1[\mathrm{H}]) \mathrm{C}([\mathrm{H}])(\mathrm{C}([\mathrm{H}])([\mathrm{H}])[\mathrm{H}]) \mathrm{C}([\mathrm{H}]) \\
([\mathrm{H}])[\mathrm{H}]) \mathrm{C}([\mathrm{H}])([\mathrm{H}])[\mathrm{H}]) \mathrm{C}([\mathrm{H}])([\mathrm{H}]) \mathrm{C}([\mathrm{H}])(\mathrm{C}([\mathrm{H}])([\mathrm{H}])[\mathrm{H}]) \mathrm{C}([\mathrm{H}])([\mathrm{H}])[\mathrm{H}])[\mathrm{C} @ @]([\mathrm{H}])(\mathrm{N}([ \\
\mathrm{H}]) \mathrm{C}\end{array}$ & 1.504 & 2.320 \\
\hline $\operatorname{Tr} 12$ & $\begin{array}{l}\mathrm{O}=\mathrm{C}(\mathrm{N}([\mathrm{H}])[\mathrm{C} @]([\mathrm{H}])([\mathrm{C} @ @]([\mathrm{H}])(\mathrm{O}[\mathrm{H}]) \mathrm{C}([\mathrm{H}])([\mathrm{H}])[\mathrm{C} @]([\mathrm{H}])(\mathrm{C}(=\mathrm{O}) \mathrm{N}([\mathrm{H}])[\mathrm{C} @]([\mathrm{H}])(\mathrm{C}( \\
=\mathrm{O}) \mathrm{N}([\mathrm{H}]) \mathrm{C}([\mathrm{H}])([\mathrm{H}]) \mathrm{Cl}=\mathrm{C}([\mathrm{H}]) \mathrm{C}([\mathrm{H}])=\mathrm{C}([\mathrm{H}]) \mathrm{C}([\mathrm{H}])=\mathrm{C} 1[\mathrm{H}]) \mathrm{C}([\mathrm{H}])([\mathrm{H}])[\mathrm{H}]) \mathrm{C}([\mathrm{H}])([\mathrm{H}])[\mathrm{H} \\
]) \mathrm{C}([\mathrm{H}])([\mathrm{H}]) \mathrm{C}([\mathrm{H}])(\mathrm{C}([\mathrm{H}])([\mathrm{H}])[\mathrm{H}]) \mathrm{C}([\mathrm{H}])([\mathrm{H}])[\mathrm{H}])[\mathrm{C} @ @]([\mathrm{H}])(\mathrm{N}([\mathrm{H}]) \mathrm{C}(=\mathrm{O})[\mathrm{C} @ @]([\mathrm{H}])( \\
\mathrm{N}([\mathrm{H}]) \mathrm{C}\end{array}$ & 3.212 & 3.106 \\
\hline $\operatorname{Tr} 13$ & $\begin{array}{l}\mathrm{O}=\mathrm{C}(\mathrm{N}([\mathrm{H}])[\mathrm{C} @]([\mathrm{H}])([\mathrm{C} @ @]([\mathrm{H}])(\mathrm{O}[\mathrm{H}]) \mathrm{C}([\mathrm{H}])([\mathrm{H}])[\mathrm{C} @](([\mathrm{H}])(\mathrm{C}(=\mathrm{O}) \mathrm{N}([\mathrm{H}])[\mathrm{C} @]([\mathrm{H}])(\mathrm{C}( \\
=\mathrm{O}) \mathrm{N}([\mathrm{H}]) \mathrm{C}([\mathrm{H}])([\mathrm{H}]) \mathrm{Cl}=\mathrm{C}([\mathrm{H}]) \mathrm{C}([\mathrm{H}])=\mathrm{C}([\mathrm{H}]) \mathrm{C}([\mathrm{H}])=\mathrm{C} 1[\mathrm{H}]) \mathrm{C}([\mathrm{H}])(\mathrm{C}([\mathrm{H}])([\mathrm{H}])[\mathrm{H}]) \mathrm{C}([\mathrm{H}]) \\
([\mathrm{H}])[\mathrm{H}]) \mathrm{C}([\mathrm{H}])([\mathrm{H}])[\mathrm{H}]) \mathrm{C}([\mathrm{H}])([\mathrm{H}]) \mathrm{C}([\mathrm{H}])(\mathrm{C}([\mathrm{H}])([\mathrm{H}])[\mathrm{H}]) \mathrm{C}([\mathrm{H}])([\mathrm{H}])[\mathrm{H}])[\mathrm{C} @ @]([\mathrm{H}])(\mathrm{N}([ \\
\mathrm{H}]) \mathrm{C}\end{array}$ & 4.229 & 3.178 \\
\hline $\operatorname{Tr} 14$ & $\begin{array}{l}\mathrm{O}=\mathrm{C}(\mathrm{N}([\mathrm{H}])[\mathrm{C} @]([\mathrm{H}])([\mathrm{C} @ @]([\mathrm{H}])(\mathrm{O}[\mathrm{H}]) \mathrm{C}([\mathrm{H}])([\mathrm{H}])[\mathrm{C} @]([\mathrm{H}])(\mathrm{C}(=\mathrm{O}) \mathrm{N}([\mathrm{H}])[\mathrm{C} @]([\mathrm{H}])(\mathrm{C}( \\
=\mathrm{O}) \mathrm{N}([\mathrm{H}]) \mathrm{C}([\mathrm{H}])([\mathrm{H}]) \mathrm{Cl}=\mathrm{C}([\mathrm{H}]) \mathrm{C}([\mathrm{H}])=\mathrm{C}([\mathrm{H}]) \mathrm{C}([\mathrm{H}])=\mathrm{C} 1[\mathrm{H}]) \mathrm{C}([\mathrm{H}])(\mathrm{C}([\mathrm{H}])([\mathrm{H}])[\mathrm{H}]) \mathrm{C}([\mathrm{H}]) \\
([\mathrm{H}])[\mathrm{H}]) \mathrm{C}([\mathrm{H}])([\mathrm{H}])[\mathrm{H}]) \mathrm{C}([\mathrm{H}])([\mathrm{H}]) \mathrm{C}([\mathrm{H}])(\mathrm{C}([\mathrm{H}])([\mathrm{H}])[\mathrm{H}]) \mathrm{C}([\mathrm{H}])([\mathrm{H}])[\mathrm{H}])[\mathrm{C} @ @]([\mathrm{H}])(\mathrm{N}([ \\
\mathrm{H}]) \mathrm{C}\end{array}$ & 3.300 & 3.016 \\
\hline $\operatorname{Tr} 15$ & $\begin{array}{l}\mathrm{O}=\mathrm{C}(\mathrm{C}=1 \mathrm{C}([\mathrm{H}])=\mathrm{C}([\mathrm{H}]) \mathrm{C}([\mathrm{H}])=\mathrm{C}(\mathrm{C}=1[\mathrm{H}]) \mathrm{C}(=\mathrm{O}) \mathrm{N}([\mathrm{H}])[\mathrm{C} @ @]([\mathrm{H}])(\mathrm{C}([\mathrm{H}])([\mathrm{H}]) \mathrm{C}([\mathrm{H}])(\mathrm{C}([ \\
\mathrm{H}])([\mathrm{H}])[\mathrm{H}]) \mathrm{C}([\mathrm{H}])([\mathrm{H}])[\mathrm{H}])[\mathrm{C} @ @]([\mathrm{H}])(\mathrm{O}[\mathrm{H}]) \mathrm{C}([\mathrm{H}])([\mathrm{H}])[\mathrm{C} @]([\mathrm{H}])(\mathrm{C}(=\mathrm{O}) \mathrm{N}([\mathrm{H}])[\mathrm{C} @]([\mathrm{H} \\
])(\mathrm{C}(=\mathrm{O}) \mathrm{N}([\mathrm{H}]) \mathrm{C}([\mathrm{H}])(\mathrm{C}([\mathrm{H}])([\mathrm{H}])[\mathrm{H}]) \mathrm{C}([\mathrm{H}])([\mathrm{H}])[\mathrm{H}]) \mathrm{C}([\mathrm{H}])(\mathrm{C}([\mathrm{H}])([\mathrm{H}])[\mathrm{H}]) \mathrm{C}([\mathrm{H}])([\mathrm{H}])[\mathrm{H}] \\
) \mathrm{C}([\mathrm{H}\end{array}$ & 2.103 & 2.320 \\
\hline $\operatorname{Tr} 16$ & $\begin{array}{l}\mathrm{O}=\mathrm{C} 1 \mathrm{OC}([\mathrm{H}])([\mathrm{H}]) \mathrm{C}([\mathrm{H}])=\mathrm{C}([\mathrm{H}]) \mathrm{C}([\mathrm{H}])([\mathrm{H}]) \mathrm{N}([\mathrm{H}]) \mathrm{C}(=\mathrm{O}) \mathrm{C}([\mathrm{H}])([\mathrm{H}])[\mathrm{C} @]([\mathrm{H}])(\mathrm{N}([\mathrm{H}]) \mathrm{C}(= \\
\mathrm{O})[\mathrm{C} @ @]([\mathrm{H}])(\mathrm{N} 1[\mathrm{H}]) \mathrm{C}([\mathrm{H}])(\mathrm{C}([\mathrm{H}])([\mathrm{H}])[\mathrm{H}]) \mathrm{C}([\mathrm{H}])([\mathrm{H}])[\mathrm{H}]) \mathrm{C}(=\mathrm{O}) \mathrm{N}([\mathrm{H}])[\mathrm{C} @ @]([\mathrm{H}])(\mathrm{C}([ \\
\mathrm{H}])([\mathrm{H}]) \mathrm{C}([\mathrm{H}])(\mathrm{C}([\mathrm{H}])([\mathrm{H}])[\mathrm{H}]) \mathrm{C}([\mathrm{H}])([\mathrm{H}])[\mathrm{H}])[\mathrm{C} @ @]([\mathrm{H}])(\mathrm{O}[\mathrm{H}]) \mathrm{C}([\mathrm{H}])([\mathrm{H}])[\mathrm{C} @]([\mathrm{H}])(\mathrm{C}( \\
=\mathrm{O}) \mathrm{N}([\mathrm{H}\end{array}$ & 3.059 & 2.985 \\
\hline $\operatorname{Tr} 17$ & $\begin{array}{l}\mathrm{O}=\mathrm{C}(\mathrm{N}([\mathrm{H}])[\mathrm{C} @]([\mathrm{H}])([\mathrm{C} @ @]([\mathrm{H}])(\mathrm{O}[\mathrm{H}]) \mathrm{C}([\mathrm{H}])([\mathrm{H}])[\mathrm{C} @](([\mathrm{H}])(\mathrm{C}(=\mathrm{O}) \mathrm{N}([\mathrm{H}])[\mathrm{C} @]([\mathrm{H}])(\mathrm{C}( \\
=\mathrm{O}) \mathrm{N}([\mathrm{H}]) \mathrm{C}([\mathrm{H}])([\mathrm{H}]) \mathrm{Cl}=\mathrm{C}([\mathrm{H}]) \mathrm{C}([\mathrm{H}])=\mathrm{C}([\mathrm{H}]) \mathrm{C}([\mathrm{H}])=\mathrm{C} 1[\mathrm{H}]) \mathrm{C}([\mathrm{H}])(\mathrm{C}([\mathrm{H}])([\mathrm{H}])[\mathrm{H}]) \mathrm{C}([\mathrm{H}]) \\
([\mathrm{H}])[\mathrm{H}]) \mathrm{C}([\mathrm{H}])([\mathrm{H}])[\mathrm{H}]) \mathrm{C}([\mathrm{H}])([\mathrm{H}]) \mathrm{C}([\mathrm{H}])(\mathrm{C}([\mathrm{H}])([\mathrm{H}])[\mathrm{H}]) \mathrm{C}([\mathrm{H}])([\mathrm{H}])[\mathrm{H}])[\mathrm{C} @ @]([\mathrm{H}])(\mathrm{N}([ \\
\mathrm{H}]) \mathrm{C}\end{array}$ & 4.097 & 3.772 \\
\hline $\operatorname{Tr} 18$ & $\begin{array}{l}\mathrm{O}=\mathrm{C}(\mathrm{C}=1 \mathrm{C}([\mathrm{H}])=\mathrm{C}(\mathrm{C}([\mathrm{H}])=\mathrm{C}(\mathrm{C}=1[\mathrm{H}]) \mathrm{C}(=\mathrm{O}) \mathrm{N}([\mathrm{H}])[\mathrm{C} @ @]([\mathrm{H}])(\mathrm{C}([\mathrm{H}])([\mathrm{H}]) \mathrm{C}([\mathrm{H}])(\mathrm{C}([\mathrm{H}])([ \\
\mathrm{H}])[\mathrm{H}]) \mathrm{C}([\mathrm{H}])([\mathrm{H}])[\mathrm{H}])[\mathrm{C} @ @]([\mathrm{H}])(\mathrm{O}[\mathrm{H}]) \mathrm{C}([\mathrm{H}])([\mathrm{H}])[\mathrm{C} @]]([\mathrm{H}])(\mathrm{C}(=\mathrm{O}) \mathrm{N}([\mathrm{H}])[\mathrm{C} @]([\mathrm{H}])(\mathrm{C} \\
=\mathrm{O}) \mathrm{N}([\mathrm{H}]) \mathrm{C}([\mathrm{H}])(\mathrm{C}([\mathrm{H}])([\mathrm{H}])[\mathrm{H}]) \mathrm{C}([\mathrm{H}])([\mathrm{H}])[\mathrm{H}]) \mathrm{C}([\mathrm{H}])(\mathrm{C}([\mathrm{H}])([\mathrm{H}])[\mathrm{H}]) \mathrm{C}([\mathrm{H}])([\mathrm{H}])[\mathrm{H}]) \mathrm{C}([ \\
\mathrm{H}])([\end{array}$ & 2.502 & 3.244 \\
\hline $\operatorname{Tr} 19$ & $\begin{array}{l}\mathrm{O}=\mathrm{C}(\mathrm{C}=1 \mathrm{C}([\mathrm{H}])=\mathrm{C}(\mathrm{C}([\mathrm{H}])=\mathrm{C}(\mathrm{C}=1[\mathrm{H}]) \mathrm{C}(=\mathrm{O}) \mathrm{N}([\mathrm{H}])[\mathrm{C} @ @]([\mathrm{H}])(\mathrm{C}([\mathrm{H}])([\mathrm{H}]) \mathrm{C}([\mathrm{H}])(\mathrm{C}([\mathrm{H}])([ \\
\mathrm{H}])[\mathrm{H}]) \mathrm{C}([\mathrm{H}])([\mathrm{H}])[\mathrm{H}])[\mathrm{C} @ @]([\mathrm{H}])(\mathrm{O}[\mathrm{H}]) \mathrm{C}([\mathrm{H}])([\mathrm{H}])[\mathrm{C} @]([\mathrm{H}])(\mathrm{C}(=\mathrm{O}) \mathrm{N}([\mathrm{H}])[\mathrm{C} @]([\mathrm{H}])(\mathrm{C}( \\
=\mathrm{O}) \mathrm{N}([\mathrm{H}]) \mathrm{C}([\mathrm{H}])(\mathrm{C}([\mathrm{H}])([\mathrm{H}])[\mathrm{H}]) \mathrm{C}([\mathrm{H}])([\mathrm{H}])[\mathrm{H}]) \mathrm{C}([\mathrm{H}])(\mathrm{C}([\mathrm{H}])([\mathrm{H}])[\mathrm{H}]) \mathrm{C}([\mathrm{H}])([\mathrm{H}])[\mathrm{H}]) \mathrm{C}([ \\
\mathrm{H}])([\end{array}$ & 3.569 & 4.073 \\
\hline $\operatorname{Tr} 20$ & $\begin{array}{l}\mathrm{O}=\mathrm{C} 2 \mathrm{~N}(\mathrm{C}(=\mathrm{N}[\mathrm{C} @] 4(\mathrm{C}=1 \mathrm{C}([\mathrm{F}])=\mathrm{C}([\mathrm{H}]) \mathrm{C}([\mathrm{H}])=\mathrm{C}([\mathrm{F}]) \mathrm{C}=1[\mathrm{H}])[\mathrm{C} @ @] 2([\mathrm{H}]) \mathrm{C}([\mathrm{H}])([\mathrm{H}]) \mathrm{N}(/ \mathrm{C} \\
3=\mathrm{N} / \mathrm{C}(=\mathrm{C}(/[\mathrm{F}]) \mathrm{C}(=\mathrm{N} 3) \mathrm{OC}([\mathrm{H}])([\mathrm{H}])[\mathrm{H}]) \mathrm{C}([\mathrm{H}])([\mathrm{H}])[\mathrm{H}]) \mathrm{C} 4([\mathrm{H}])[\mathrm{H}]) \mathrm{N}([\mathrm{H}])[\mathrm{H}]) \mathrm{C}([\mathrm{H}])([\mathrm{H}])[ \\
\mathrm{H}]\end{array}$ & 4.071 & 3.913 \\
\hline
\end{tabular}




\begin{tabular}{|c|c|c|c|}
\hline $\operatorname{Tr} 21$ & $\begin{array}{l}\mathrm{O}=\mathrm{C} 2 \mathrm{~N}(\mathrm{C}(=\mathrm{N}[\mathrm{C} @] 4(\mathrm{C}=1 \mathrm{C}([\mathrm{F}])=\mathrm{C}([\mathrm{H}]) \mathrm{C}([\mathrm{H}])=\mathrm{C}([\mathrm{H}]) \mathrm{C}=1[\mathrm{H}])[\mathrm{C} @ @] 2([\mathrm{H}]) \mathrm{C}([\mathrm{H}])([\mathrm{H}]) \mathrm{N}(/ \\
\mathrm{C} 3=\mathrm{N} / \mathrm{C}(=\mathrm{C}(/[\mathrm{Cl}]) \mathrm{C}(=\mathrm{N} 3) \mathrm{OC}([\mathrm{H}])([\mathrm{H}])[\mathrm{H}]) \mathrm{C}([\mathrm{H}])([\mathrm{H}])[\mathrm{H}]) \mathrm{C} 4([\mathrm{H}])[\mathrm{H}]) \mathrm{N}([\mathrm{H}])[\mathrm{H}]) \mathrm{C}([\mathrm{H}])([\mathrm{H}] \\
)[\mathrm{H}]\end{array}$ & 4.032 & 3.673 \\
\hline $\operatorname{Tr} 22$ & $\begin{array}{l}\mathrm{O}=\mathrm{C} 2 \mathrm{~N}(\mathrm{C}(=\mathrm{N}[\mathrm{C} @] 4(\mathrm{C}=1 \mathrm{C}([\mathrm{F}])=\mathrm{C}([\mathrm{H}]) \mathrm{C}([\mathrm{H}])=\mathrm{C}([\mathrm{H}]) \mathrm{C}=1[\mathrm{~F}])[\mathrm{C} @ @] 2([\mathrm{H}]) \mathrm{C}([\mathrm{H}])([\mathrm{H}]) \mathrm{N} / \mathrm{C} \\
3=\mathrm{N} / \mathrm{C}(=\mathrm{C}([\mathrm{F}]) \mathrm{C}(=\mathrm{N} 3) \mathrm{OC}([\mathrm{H}])([\mathrm{H}])[\mathrm{H}]) \mathrm{C}([\mathrm{H}])([\mathrm{H}])[\mathrm{H}]) \mathrm{C} 4([\mathrm{H}])[\mathrm{H}]) \mathrm{N}([\mathrm{H}])[\mathrm{H}]) \mathrm{C}([\mathrm{H}])([\mathrm{H}])[ \\
\mathrm{H}]\end{array}$ & 3.495 & 3.673 \\
\hline $\operatorname{Tr} 23$ & $\begin{array}{l}\mathrm{O}=\mathrm{C} 2 \mathrm{~N}(\mathrm{C}(=\mathrm{N}[\mathrm{C} @] 4(\mathrm{C}=1 \mathrm{C}([\mathrm{H}])=\mathrm{C}([\mathrm{H}]) \mathrm{C}([\mathrm{H}])=\mathrm{C}([\mathrm{H}]) \mathrm{C}=1[\mathrm{H}])[\mathrm{C} @ @] 2([\mathrm{H}]) \mathrm{C}([\mathrm{H}])([\mathrm{H}]) \mathrm{N}(/ \\
\mathrm{C} 3=\mathrm{N} / \mathrm{C}(=\mathrm{C}(/[\mathrm{F}]) \mathrm{C}(=\mathrm{N} 3) \mathrm{C}([\mathrm{H}])([\mathrm{H}])[\mathrm{H}]) \mathrm{C}([\mathrm{H}])([\mathrm{H}])[\mathrm{H}]) \mathrm{C} 4([\mathrm{H}])[\mathrm{H}]) \mathrm{N}([\mathrm{H}])[\mathrm{H}]) \mathrm{C}([\mathrm{H}])([\mathrm{H}])[ \\
\mathrm{H}]\end{array}$ & 2.812 & 3.157 \\
\hline $\operatorname{Tr} 24$ & $\begin{array}{l}\mathrm{O}=\mathrm{C} 2 \mathrm{~N}(\mathrm{C}(=\mathrm{N}[\mathrm{C} @ 44(\mathrm{C}=1 \mathrm{C}([\mathrm{H}])=\mathrm{C}([\mathrm{H}]) \mathrm{C}([\mathrm{H}])=\mathrm{C}([\mathrm{H}]) \mathrm{C}=1[\mathrm{H}])[\mathrm{C} @ @] 2([\mathrm{H}]) \mathrm{C}([\mathrm{H}])([\mathrm{H}]) \mathrm{N} / \\
\mathrm{C} 3=\mathrm{N} / \mathrm{C}(=\mathrm{C}(/[\mathrm{F}]) \mathrm{C}(=\mathrm{N} 3) \mathrm{C}([\mathrm{H}])([\mathrm{H}]) \mathrm{C}([\mathrm{H}])([\mathrm{H}])[\mathrm{H}]) \mathrm{C}([\mathrm{H}])([\mathrm{H}])[\mathrm{H}]) \mathrm{C} 4([\mathrm{H}])[\mathrm{H}]) \mathrm{N}([\mathrm{H}])[\mathrm{H}]) \mathrm{C} \\
([\mathrm{H}])([\mathrm{H}])[\mathrm{H}]\end{array}$ & 3.215 & 3.181 \\
\hline $\operatorname{Tr} 25$ & $\begin{array}{l}\mathrm{O}=\mathrm{C} 2 \mathrm{~N}(\mathrm{C}(=\mathrm{N}[\mathrm{C} @ 44(\mathrm{C}=1 \mathrm{C}([\mathrm{F}])=\mathrm{C}([\mathrm{H}]) \mathrm{C}([\mathrm{F}])=\mathrm{C}([\mathrm{F}]) \mathrm{C}=1[\mathrm{H}])[\mathrm{C} @ @] 2([\mathrm{H}]) \mathrm{C}([\mathrm{H}])([\mathrm{H}]) \mathrm{N}(/ \mathrm{C} \\
3=\mathrm{N} / \mathrm{C}(=\mathrm{C}(/[\mathrm{F}]) \mathrm{C}(=\mathrm{N} 3) \mathrm{OC}([\mathrm{H}])([\mathrm{H}])[\mathrm{H}]) \mathrm{C}([\mathrm{H}])([\mathrm{H}])[\mathrm{H}]) \mathrm{C} 4([\mathrm{H}])[\mathrm{H}]) \mathrm{N}([\mathrm{H}])[\mathrm{H}]) \mathrm{C}([\mathrm{H}])([\mathrm{H}])[ \\
\mathrm{H}]\end{array}$ & 4.114 & 4.250 \\
\hline $\operatorname{Tr} 26$ & $\begin{array}{l}\mathrm{O}=\mathrm{C} 2 \mathrm{~N}(\mathrm{C}(=\mathrm{N}[\mathrm{C} @] 5(\mathrm{C}=1 \mathrm{C}([\mathrm{H}])=\mathrm{C}([\mathrm{H}]) \mathrm{C}([\mathrm{H}])=\mathrm{C}([\mathrm{H}]) \mathrm{C}=1[\mathrm{H}])[\mathrm{C} @ @] 2([\mathrm{H}]) \mathrm{C}([\mathrm{H}])([\mathrm{H}]) \mathrm{N}(/ \\
\mathrm{C} 3=\mathrm{N} / \mathrm{C}(=\mathrm{C}(/[\mathrm{Cl}]) \mathrm{C}(=\mathrm{N} 3)[\mathrm{C} @ @] 4([\mathrm{H}]) \mathrm{C}([\mathrm{H}])([\mathrm{H}]) \mathrm{C} 4([\mathrm{H}])[\mathrm{H}]) \mathrm{C}([\mathrm{H}])([\mathrm{H}])[\mathrm{H}]) \mathrm{C}([\mathrm{H}])[\mathrm{H}]) \mathrm{N} \\
([\mathrm{H}])[\mathrm{H}]) \mathrm{C}([\mathrm{H}])([\mathrm{H}])[\mathrm{H}]\end{array}$ & 3.027 & 2.953 \\
\hline $\operatorname{Tr} 27$ & $\begin{array}{l}\mathrm{O}=\mathrm{C} 2 \mathrm{~N}(\mathrm{C}(=\mathrm{N}[\mathrm{C} @] 5(\mathrm{C}=1 \mathrm{C}([\mathrm{H}])=\mathrm{C}([\mathrm{H}]) \mathrm{C}([\mathrm{H}])=\mathrm{C}([\mathrm{H}]) \mathrm{C}=1[\mathrm{H}])[\mathrm{C} @ @] 2([\mathrm{H}]) \mathrm{C}([\mathrm{H}])([\mathrm{H}]) \mathrm{N}(/ \\
\mathrm{C} 3=\mathrm{N} / \mathrm{C}(=\mathrm{C}(/[\mathrm{Cl}]) \mathrm{C}(=\mathrm{N} 3)[\mathrm{C} @ @] 4([\mathrm{H}]) \mathrm{C}([\mathrm{H}])([\mathrm{H}]) \mathrm{C} 4([\mathrm{H}])[\mathrm{H}]) \mathrm{C}([\mathrm{H}])([\mathrm{H}]) \mathrm{C}([\mathrm{H}])([\mathrm{H}])[\mathrm{H}]) \mathrm{C} \\
5([\mathrm{H}])[\mathrm{H}]) \mathrm{N}([\mathrm{H}])[\mathrm{H}]) \mathrm{C}([\mathrm{H}])([\mathrm{H}])[\mathrm{H}]\end{array}$ & 2.745 & 2.977 \\
\hline $\operatorname{Tr} 28$ & $\begin{array}{l}\mathrm{O}=\mathrm{C} 2 \mathrm{~N}(\mathrm{C}(=\mathrm{N}[\mathrm{C} @] 4(\mathrm{C}=1 \mathrm{C}([\mathrm{H}])=\mathrm{C}([\mathrm{H}]) \mathrm{C}([\mathrm{F}])=\mathrm{C}([\mathrm{H}]) \mathrm{C}=1[\mathrm{H}])[\mathrm{C} @ @] 2([\mathrm{H}]) \mathrm{C}([\mathrm{H}])([\mathrm{H}]) \mathrm{N}(/ \\
\mathrm{C} 3=\mathrm{N} / \mathrm{C}(=\mathrm{C}(/[\mathrm{F}]) \mathrm{C}(=\mathrm{N} 3) \mathrm{C}([\mathrm{H}])([\mathrm{H}])[\mathrm{H}]) \mathrm{C}([\mathrm{H}])([\mathrm{H}])[\mathrm{H}]) \mathrm{C} 4([\mathrm{H}])[\mathrm{H}]) \mathrm{N}([\mathrm{H}])[\mathrm{H}]) \mathrm{C}([\mathrm{H}])([\mathrm{H}])[ \\
\mathrm{H}]\end{array}$ & 3.194 & 3.493 \\
\hline $\operatorname{Tr} 29$ & $\begin{array}{l}\mathrm{O}=\mathrm{C} 2 \mathrm{~N}(\mathrm{C}(=\mathrm{N}[\mathrm{C} @] 4(\mathrm{C}=1 \mathrm{C}([\mathrm{H}])=\mathrm{C}([\mathrm{F}]) \mathrm{C}([\mathrm{H}])=\mathrm{C}([\mathrm{H}]) \mathrm{C}=1[\mathrm{~F}])[\mathrm{C} @ @] 2([\mathrm{H}]) \mathrm{C}([\mathrm{H}])([\mathrm{H}]) \mathrm{N}(/ \mathrm{C} \\
3=\mathrm{N} / \mathrm{C}(\mathrm{OC}([\mathrm{H}])([\mathrm{H}])[\mathrm{H}])=\mathrm{C}([\mathrm{F}]) \mathrm{C}(=\mathrm{N} 3) \mathrm{OC}([\mathrm{H}])([\mathrm{H}])[\mathrm{H}]) \mathrm{C} 4([\mathrm{H}])[\mathrm{H}]) \mathrm{N}([\mathrm{H}])[\mathrm{H}]) \mathrm{C}([\mathrm{H}])([\mathrm{H}]) \\
{[\mathrm{H}]}\end{array}$ & 4.252 & 3.992 \\
\hline $\operatorname{Tr} 30$ & $\begin{array}{l}\mathrm{O}=\mathrm{C} 2 \mathrm{~N}(\mathrm{C}(=\mathrm{N}[\mathrm{C} @] 4(\mathrm{C}=1 \mathrm{C}([\mathrm{H}])=\mathrm{C}([\mathrm{H}]) \mathrm{C}([\mathrm{F}])=\mathrm{C}([\mathrm{H}]) \mathrm{C}=1[\mathrm{~F}])[\mathrm{C} @ @] 2([\mathrm{H}]) \mathrm{C}([\mathrm{H}])([\mathrm{H}]) \mathrm{N}(/ \mathrm{C} \\
3=\mathrm{N} / \mathrm{C}(\mathrm{OC}([\mathrm{H}])([\mathrm{H}])[\mathrm{H}])=\mathrm{C}([\mathrm{F}]) \mathrm{C}(=\mathrm{N} 3) \mathrm{OC}([\mathrm{H}])([\mathrm{H}])[\mathrm{H}]) \mathrm{C} 4([\mathrm{H}])[\mathrm{H}]) \mathrm{N}([\mathrm{H}])[\mathrm{H}]) \mathrm{C}([\mathrm{H}])([\mathrm{H}]) \\
{[\mathrm{H}]}\end{array}$ & 4.745 & 3.992 \\
\hline $\operatorname{Tr} 31$ & $\begin{array}{l}\mathrm{O}=\mathrm{C} 2 \mathrm{~N}(\mathrm{C}(=\mathrm{N}[\mathrm{C} @] 4(\mathrm{C}=1 \mathrm{C}([\mathrm{H}])=\mathrm{C}([\mathrm{H}]) \mathrm{C}([\mathrm{F}])=\mathrm{C}([\mathrm{H}]) \mathrm{C}=1[\mathrm{H}])[\mathrm{C} @ @] 2([\mathrm{H}]) \mathrm{C}([\mathrm{H}])([\mathrm{H}]) \mathrm{N}(/ \\
\mathrm{C} 3=\mathrm{N} / \mathrm{C}(\mathrm{OC}([\mathrm{H}])([\mathrm{H}])[\mathrm{H}])=\mathrm{C}([\mathrm{F}]) \mathrm{C}(=\mathrm{N} 3) \mathrm{C}([\mathrm{H}])([\mathrm{H}]) \mathrm{C}([\mathrm{H}])([\mathrm{H}])[\mathrm{H}]) \mathrm{C} 4([\mathrm{H}])[\mathrm{H}]) \mathrm{N}([\mathrm{H}])[\mathrm{H}]) \\
\mathrm{C}([\mathrm{H}])([\mathrm{H}])[\mathrm{H}]\end{array}$ & 4.215 & 3.836 \\
\hline $\operatorname{Tr} 32$ & $\begin{array}{l}\mathrm{O}=\mathrm{C} 2 \mathrm{~N}(\mathrm{C}(=\mathrm{N}[\mathrm{C} @] 5(\mathrm{C}=1 \mathrm{C}([\mathrm{F}])=\mathrm{C}([\mathrm{H}]) \mathrm{C}([\mathrm{H}])=\mathrm{C}([\mathrm{F}]) \mathrm{C}=1[\mathrm{H}])[\mathrm{C} @ @] 2([\mathrm{H}]) \mathrm{C}([\mathrm{H}])([\mathrm{H}]) \mathrm{N}(/ \mathrm{C} \\
3=\mathrm{N} / \mathrm{C}(\mathrm{OC}([\mathrm{H}])([\mathrm{H}])[\mathrm{H}])=\mathrm{C}([\mathrm{F}]) \mathrm{C}(=\mathrm{N} 3)[\mathrm{C} @] 4([\mathrm{H}]) \mathrm{C}([\mathrm{H}])([\mathrm{H}]) \mathrm{C} 4([\mathrm{H}])[\mathrm{H}]) \mathrm{C} 5([\mathrm{H}])[\mathrm{H}]) \mathrm{N}([\mathrm{H} \\
])[\mathrm{H}]) \mathrm{C}([\mathrm{H}])([\mathrm{H}])[\mathrm{H}]\end{array}$ & 4.252 & 4.244 \\
\hline $\operatorname{Tr} 33$ & $\begin{array}{l}\mathrm{O}=\mathrm{C} 2 \mathrm{~N}(\mathrm{C}(=\mathrm{N}[\mathrm{C} @] 5(\mathrm{C}=1 \mathrm{C}([\mathrm{H}])=\mathrm{C}([\mathrm{F}]) \mathrm{C}([\mathrm{H}])=\mathrm{C}([\mathrm{H}]) \mathrm{C}=1[\mathrm{~F}])[\mathrm{C} @ @] 2([\mathrm{H}]) \mathrm{C}([\mathrm{H}])([\mathrm{H}]) \mathrm{N}(/ \mathrm{C} \\
3=\mathrm{N} / \mathrm{C}(=\mathrm{C}(/[\mathrm{F}]) \mathrm{C}(=\mathrm{N} 3)[\mathrm{C} @] 4([\mathrm{H}]) \mathrm{C}([\mathrm{H}])([\mathrm{H}]) \mathrm{C} 4([\mathrm{H}])[\mathrm{H}]) \mathrm{C}([\mathrm{H}])([\mathrm{H}])[\mathrm{H}]) \mathrm{C} 5([\mathrm{H}])[\mathrm{H}]) \mathrm{N}([\mathrm{H}] \\
)[\mathrm{H}]) \mathrm{C}([\mathrm{H}])([\mathrm{H}])[\mathrm{H}]\end{array}$ & 3.585 & 3.686 \\
\hline $\operatorname{Tr} 34$ & $\begin{array}{l}\mathrm{O}=\mathrm{C} 2 \mathrm{~N}(\mathrm{C}(=\mathrm{N}[\mathrm{C} @] 5(\mathrm{C}=1 \mathrm{C}([\mathrm{F}])=\mathrm{C}([\mathrm{H}]) \mathrm{C}([\mathrm{F}])=\mathrm{C}([\mathrm{H}]) \mathrm{C}=1[\mathrm{H}])[\mathrm{C} @ @] 2([\mathrm{H}]) \mathrm{C}([\mathrm{H}])([\mathrm{H}]) \mathrm{N}(/ \mathrm{C} \\
3=\mathrm{N} / \mathrm{C}(\mathrm{OC}([\mathrm{H}])([\mathrm{H}])[\mathrm{H}])=\mathrm{C}([\mathrm{F}]) \mathrm{C}(=\mathrm{N} 3)[\mathrm{C} @] 4([\mathrm{H}]) \mathrm{C}([\mathrm{H}])([\mathrm{H}]) \mathrm{C} 4([\mathrm{H}])[\mathrm{H}]) \mathrm{C} 5([\mathrm{H}])[\mathrm{H}]) \mathrm{N}([\mathrm{H} \\
])[\mathrm{H}]) \mathrm{C}([\mathrm{H}])([\mathrm{H}])[\mathrm{H}]\end{array}$ & 4.569 & 4.244 \\
\hline $\operatorname{Tr} 35$ & $\begin{array}{l}\mathrm{O}=\mathrm{C} 2 \mathrm{~N}(\mathrm{C}(=\mathrm{N}[\mathrm{C} @] 5(\mathrm{C}=1 \mathrm{C}([\mathrm{F}])=\mathrm{C}([\mathrm{H}]) \mathrm{C}([\mathrm{F}])=\mathrm{C}([\mathrm{H}]) \mathrm{C}=1[\mathrm{H}])[\mathrm{C} @ @] 2([\mathrm{H}]) \mathrm{C}([\mathrm{H}])([\mathrm{H}]) \mathrm{N}(/ \mathrm{C} \\
3=\mathrm{N} / \mathrm{C}(\mathrm{OC}([\mathrm{H}])([\mathrm{H}])[\mathrm{H}])=\mathrm{C}([\mathrm{Cl}]) \mathrm{C}(=\mathrm{N} 3)[\mathrm{C} @] 4([\mathrm{H}]) \mathrm{C}([\mathrm{H}])([\mathrm{H}]) \mathrm{C} 4([\mathrm{H}])[\mathrm{H}]) \mathrm{C} 5([\mathrm{H}])[\mathrm{H}]) \mathrm{N}([ \\
\mathrm{H}])[\mathrm{H}]) \mathrm{C}([\mathrm{H}])([\mathrm{H}])[\mathrm{H}]\end{array}$ & 4.167 & 4.166 \\
\hline
\end{tabular}




\begin{tabular}{|c|c|c|c|}
\hline $\operatorname{Tr} 36$ & $\begin{array}{l}{[\mathrm{F}] \mathrm{C}=1 \mathrm{C}([\mathrm{H}])=\mathrm{C}([\mathrm{F}]) \mathrm{C}(=\mathrm{C}([\mathrm{H}]) \mathrm{C}=1[\mathrm{H}])[\mathrm{C} @ @] 24 \mathrm{~N}=\mathrm{C}(\mathrm{N}(\mathrm{C}(=\mathrm{O})[\mathrm{C} @] 2([\mathrm{H}]) \mathrm{C}([\mathrm{H}])([\mathrm{H}]) \mathrm{N}(/ \mathrm{C}} \\
3=\mathrm{N} / \mathrm{C}(=\mathrm{C}(/[\mathrm{F}]) \mathrm{C}(=\mathrm{N} 3) \mathrm{OC}([\mathrm{H}])([\mathrm{H}])[\mathrm{H}]) \mathrm{C}([\mathrm{F}])([\mathrm{F}]) \mathrm{C}([\mathrm{H}])([\mathrm{H}])[\mathrm{H}]) \mathrm{C} 4([\mathrm{H}])[\mathrm{H}]) \mathrm{C}([\mathrm{H}])([\mathrm{H}])[ \\
\mathrm{H}]) \mathrm{N}([\mathrm{H}])[\mathrm{H}]\end{array}$ & 3.959 & 3.865 \\
\hline $\operatorname{Tr} 37$ & $\begin{array}{l}\mathrm{O}=\mathrm{C} 2 \mathrm{~N}(\mathrm{C}(=\mathrm{N}[\mathrm{C} @] 4(\mathrm{C}=1 \mathrm{C}([\mathrm{F}])=\mathrm{C}([\mathrm{H}]) \mathrm{C}([\mathrm{H}])=\mathrm{C}([\mathrm{H}]) \mathrm{C}=1[\mathrm{H}])[\mathrm{C} @ @] 2([\mathrm{H}]) \mathrm{C}([\mathrm{H}])([\mathrm{H}]) \mathrm{N}(/ \\
\mathrm{C} 3=\mathrm{N} / \mathrm{C}(=\mathrm{C}([\mathrm{F}]) \mathrm{C}(=\mathrm{N} 3) \mathrm{OC}([\mathrm{H}])([\mathrm{H}])[\mathrm{H}]) \mathrm{C}([\mathrm{H}])([\mathrm{H}])[\mathrm{H}]) \mathrm{C} 4([\mathrm{H}])[\mathrm{H}]) \mathrm{N}([\mathrm{H}])[\mathrm{H}]) \mathrm{C}([\mathrm{H}])([\mathrm{H}]) \\
{[\mathrm{H}]}\end{array}$ & 4.222 & 3.577 \\
\hline $\operatorname{Tr} 38$ & $\begin{array}{l}\mathrm{O}=\mathrm{C} 2 \mathrm{~N}(\mathrm{C}(=\mathrm{N}[\mathrm{C} @] 4(\mathrm{C}=1 \mathrm{C}([\mathrm{F}])=\mathrm{C}([\mathrm{Cl}]) \mathrm{C}([\mathrm{F}])=\mathrm{C}([\mathrm{H}]) \mathrm{C}=1[\mathrm{H}])[\mathrm{C} @ @] 2([\mathrm{H}]) \mathrm{C}([\mathrm{H}])([\mathrm{H}]) \mathrm{N} / \\
\mathrm{C} 3=\mathrm{N} / \mathrm{C}(=\mathrm{C}(/[\mathrm{F}]) \mathrm{C}(=\mathrm{N} 3) \mathrm{OC}([\mathrm{H}])([\mathrm{H}])[\mathrm{H}]) \mathrm{C}([\mathrm{H}])([\mathrm{H}])[\mathrm{H}]) \mathrm{C} 4([\mathrm{H}])[\mathrm{H}]) \mathrm{N}([\mathrm{H}])[\mathrm{H}]) \mathrm{C}([\mathrm{H}])([\mathrm{H}]) \\
{[\mathrm{H}]}\end{array}$ & 4.036 & 4.171 \\
\hline $\operatorname{Tr} 39$ & $\begin{array}{l}\mathrm{O}=\mathrm{C} 2 \mathrm{~N}(\mathrm{C}(=\mathrm{N}[\mathrm{C} @ 44(\mathrm{C}=1 \mathrm{C}([\mathrm{Cl}])=\mathrm{C}([\mathrm{H}]) \mathrm{C}([\mathrm{F}])=\mathrm{C}([\mathrm{H}]) \mathrm{C}=1[\mathrm{H}])[\mathrm{C} @ @] 2([\mathrm{H}]) \mathrm{C}([\mathrm{H}])([\mathrm{H}]) \mathrm{N} / \\
\mathrm{C} 3=\mathrm{N} / \mathrm{C}(=\mathrm{C}(/[\mathrm{F}]) \mathrm{C}(=\mathrm{N} 3) \mathrm{OC}([\mathrm{H}])([\mathrm{H}])[\mathrm{H}]) \mathrm{C}([\mathrm{H}])([\mathrm{H}])[\mathrm{H}]) \mathrm{C} 4([\mathrm{H}])[\mathrm{H}]) \mathrm{N}([\mathrm{H}])[\mathrm{H}]) \mathrm{C}([\mathrm{H}])([\mathrm{H}]) \\
{[\mathrm{H}]}\end{array}$ & 4.347 & 3.835 \\
\hline $\operatorname{Tr} 40$ & $\begin{array}{l}\mathrm{O}=\mathrm{C} 2 \mathrm{~N}(\mathrm{C}(=\mathrm{N}[\mathrm{C} @] 5(\mathrm{C}=1 \mathrm{C}([\mathrm{H}])=\mathrm{C}([\mathrm{H}]) \mathrm{C}([\mathrm{F}])=\mathrm{C}([\mathrm{H}]) \mathrm{C}=1[\mathrm{H}])[\mathrm{C} @ @] 2([\mathrm{H}]) \mathrm{C}([\mathrm{H}])([\mathrm{H}]) \mathrm{N}(/ \\
\mathrm{C} 3=\mathrm{N} / \mathrm{C}(\mathrm{OC}([\mathrm{H}])([\mathrm{H}])[\mathrm{H}])=\mathrm{C}([\mathrm{F}]) \mathrm{C}(=\mathrm{N} 3)[\mathrm{C} @] 4([\mathrm{H}]) \mathrm{C}([\mathrm{H}])([\mathrm{H}]) \mathrm{C} 4([\mathrm{H}])[\mathrm{H}]) \mathrm{C}([\mathrm{H}])[\mathrm{H}]) \mathrm{N}([ \\
\mathrm{H}])[\mathrm{H}]) \mathrm{C}([\mathrm{H}])([\mathrm{H}])[\mathrm{H}]\end{array}$ & 4.319 & 3.908 \\
\hline $\operatorname{Tr} 41$ & $\begin{array}{l}\mathrm{O}=[\mathrm{S}](=\mathrm{O})(\mathrm{C}([\mathrm{H}])([\mathrm{H}])[\mathrm{C} @]([\mathrm{H}])(\mathrm{N}([\mathrm{H}]) \mathrm{C}(=\mathrm{O}) \mathrm{OC}(\mathrm{C}([\mathrm{H}])([\mathrm{H}])[\mathrm{H}])(\mathrm{C}([\mathrm{H}])([\mathrm{H}])[\mathrm{H}]) \mathrm{C}([\mathrm{H}])([ \\
\mathrm{H}])[\mathrm{H}]) \mathrm{C}(=\mathrm{O}) \mathrm{C}([\mathrm{H}])([\mathrm{H}]) \mathrm{N}([\mathrm{H}])[\mathrm{C} @]([\mathrm{H}])([\mathrm{C} @]([\mathrm{H}])(\mathrm{O}[\mathrm{H}]) \mathrm{C}([\mathrm{H}])([\mathrm{H}])[\mathrm{C} @([\mathrm{H}])(\mathrm{C}(=\mathrm{O}) \mathrm{N}( \\
[\mathrm{H}])[\mathrm{C} @]([\mathrm{H}])(\mathrm{C}(=\mathrm{O}) \mathrm{N}([\mathrm{H}]) \mathrm{C}([\mathrm{H}])([\mathrm{H}]) \mathrm{C}=\mathrm{C}([\mathrm{H}]) \mathrm{C}([\mathrm{H}])=\mathrm{C}([\mathrm{H}]) \mathrm{C}([\mathrm{H}])=\mathrm{C} 1[\mathrm{H}]) \mathrm{C}([\mathrm{H}])(\mathrm{C}([\mathrm{H} \\
])([\mathrm{H}\end{array}$ & 1.947 & 2.199 \\
\hline $\operatorname{Tr} 42$ & $\begin{array}{l}\mathrm{O}=\mathrm{C}(\mathrm{N}([\mathrm{H}])[\mathrm{C} @ @]([\mathrm{H}])(\mathrm{C}(=\mathrm{O}) \mathrm{N}([\mathrm{H}])[\mathrm{C} @]([\mathrm{H}])([\mathrm{C} @]([\mathrm{H}])(\mathrm{O}[\mathrm{H}]) \mathrm{C}([\mathrm{H}])([\mathrm{H}])[\mathrm{C} @]([\mathrm{H}])(\mathrm{C}( \\
=\mathrm{O}) \mathrm{N}([\mathrm{H}])[\mathrm{C} @]([\mathrm{H}])(\mathrm{C}(=\mathrm{O}) \mathrm{N}([\mathrm{H}]) \mathrm{C}([\mathrm{H}])([\mathrm{H}]) \mathrm{C} 1=\mathrm{C}([\mathrm{H}]) \mathrm{C}([\mathrm{H}])=\mathrm{C}([\mathrm{H}]) \mathrm{C}([\mathrm{H}])=\mathrm{C} 1[\mathrm{H}]) \mathrm{C}([\mathrm{H}] \\
)(\mathrm{C}([\mathrm{H}])([\mathrm{H}])[\mathrm{H}]) \mathrm{C}([\mathrm{H}])([\mathrm{H}])[\mathrm{H}]) \mathrm{C}([\mathrm{H}])([\mathrm{H}])[\mathrm{H}]) \mathrm{C}([\mathrm{H}])([\mathrm{H}]) \mathrm{C}([\mathrm{H}])(\mathrm{C}([\mathrm{H}])([\mathrm{H}])[\mathrm{H}]) \mathrm{C}([\mathrm{H}])([ \\
\mathrm{H}])[\end{array}$ & 3.301 & 2.715 \\
\hline $\operatorname{Tr} 43$ & $\begin{array}{l}\mathrm{O}=\mathrm{C}(\mathrm{OC}(\mathrm{C}([\mathrm{H}])([\mathrm{H}])[\mathrm{H}])(\mathrm{C}([\mathrm{H}])([\mathrm{H}])[\mathrm{H}]) \mathrm{C}([\mathrm{H}])([\mathrm{H}])[\mathrm{H}]) \mathrm{N}([\mathrm{H}])[\mathrm{C} @ @]([\mathrm{H}])(\mathrm{C}(=\mathrm{O}) \mathrm{N}([\mathrm{H}])[ \\
\mathrm{C} @]([\mathrm{H}])([\mathrm{C} @]([\mathrm{H}])(\mathrm{OH}]) \mathrm{C}([\mathrm{H}])([\mathrm{H}])[\mathrm{C} @]([\mathrm{H}])(\mathrm{C}(=\mathrm{O}) \mathrm{N}([\mathrm{H}])[\mathrm{C} @]([\mathrm{H}])(\mathrm{C}(=\mathrm{O}) \mathrm{N}([\mathrm{H}]) \mathrm{C}([ \\
\mathrm{H}])([\mathrm{H}]) \mathrm{Cl}=\mathrm{C}([\mathrm{H}]) \mathrm{C}([\mathrm{H}])=\mathrm{C}([\mathrm{H}]) \mathrm{C}([\mathrm{H}])=\mathrm{C} 1[\mathrm{H}]) \mathrm{C}([\mathrm{H}])(\mathrm{C}([\mathrm{H}])([\mathrm{H}])[\mathrm{H}]) \mathrm{C}([\mathrm{H}])([\mathrm{H}])[\mathrm{H}]) \mathrm{C}([\mathrm{H} \\
])([\mathrm{H}])\end{array}$ & 1.236 & 2.247 \\
\hline $\operatorname{Tr} 44$ & $\begin{array}{l}\mathrm{O}=\mathrm{C}(\mathrm{N}([\mathrm{H}])[\mathrm{C} @]([\mathrm{H}])(\mathrm{C}(=\mathrm{O}) \mathrm{C}([\mathrm{H}])([\mathrm{H}])[\mathrm{C} @]([\mathrm{H}])(\mathrm{C}(=\mathrm{O}) \mathrm{O}[\mathrm{H}]) \mathrm{C}([\mathrm{H}])([\mathrm{H}]) \mathrm{Cl}=\mathrm{C}([\mathrm{H}]) \mathrm{C}([\mathrm{H} \\
])=\mathrm{C}([\mathrm{H}]) \mathrm{C}([\mathrm{H}])=\mathrm{C} 1[\mathrm{H}]) \mathrm{C}([\mathrm{H}])([\mathrm{H}]) \mathrm{C}([\mathrm{H}])([\mathrm{H}]) \mathrm{C}(=\mathrm{O}) \mathrm{O}[\mathrm{H}])[\mathrm{C} @ @]([\mathrm{H}])(\mathrm{N}([\mathrm{H}]) \mathrm{C}(=\mathrm{O})[\mathrm{C} @ \\
@]([\mathrm{H}])(\mathrm{C}([\mathrm{H}])([\mathrm{H}])[\mathrm{C} @]([\mathrm{H}])(\mathrm{O}[\mathrm{H}])[\mathrm{C} @ @]([\mathrm{H}])(\mathrm{N}([\mathrm{H}]) \mathrm{C}(=\mathrm{O})[\mathrm{C} @ @]([\mathrm{H}])(\mathrm{N}([\mathrm{H}]) \mathrm{C}(=\mathrm{O})[ \\
\mathrm{C} @ @]([\mathrm{H}])(\mathrm{N}([\mathrm{H}]\end{array}$ & 5.495 & 5.079 \\
\hline $\operatorname{Tr} 45$ & $\begin{array}{l}\mathrm{O}=\mathrm{C}(\mathrm{N}([\mathrm{H}])[\mathrm{C} @]([\mathrm{H}])(\mathrm{C}(=\mathrm{O}) \mathrm{N}([\mathrm{H}])[\mathrm{C} @]([\mathrm{H}])([\mathrm{C} @]([\mathrm{H}])(\mathrm{O}[\mathrm{H}]) \mathrm{C}([\mathrm{H}])([\mathrm{H}])[\mathrm{C} @]([\mathrm{H}])(\mathrm{C}(= \\
\mathrm{O}) \mathrm{N}([\mathrm{H}])[\mathrm{C} @]([\mathrm{H}])(\mathrm{C}(=\mathrm{O}) \mathrm{N}([\mathrm{H}]) \mathrm{C}([\mathrm{H}])([\mathrm{H}]) \mathrm{Cl}=\mathrm{C}([\mathrm{H}]) \mathrm{C}([\mathrm{H}])=\mathrm{C}([\mathrm{H}]) \mathrm{C}([\mathrm{H}])=\mathrm{C} 1[\mathrm{H}]) \mathrm{C}([\mathrm{H}]) \\
(\mathrm{C}([\mathrm{H}])([\mathrm{H}])[\mathrm{H}]) \mathrm{C}([\mathrm{H}])([\mathrm{H}])[\mathrm{H}]) \mathrm{C}([\mathrm{H}])([\mathrm{H}])[\mathrm{H}]) \mathrm{C}([\mathrm{H}])([\mathrm{H}]) \mathrm{C}([\mathrm{H}])(\mathrm{C}([\mathrm{H}])([\mathrm{H}])[\mathrm{H}]) \mathrm{C}([\mathrm{H}])([\mathrm{H} \\
])[\mathrm{H}\end{array}$ & 4.229 & 2.805 \\
\hline $\operatorname{Tr} 46$ & $\begin{array}{l}\mathrm{O}=[\mathrm{S}](=\mathrm{O})(\mathrm{C}([\mathrm{H}])([\mathrm{H}])[\mathrm{C} @ @]((\mathrm{H}])(\mathrm{C}(=\mathrm{O}) \mathrm{N}([\mathrm{H}])[\mathrm{C} @]([\mathrm{H}])([\mathrm{C} @]([\mathrm{H}])(\mathrm{O}[\mathrm{H}]) \mathrm{C}([\mathrm{H}])([\mathrm{H}])[\mathrm{C} \\
@]([\mathrm{H}])(\mathrm{C}(=\mathrm{O}) \mathrm{N}([\mathrm{H}])[\mathrm{C} @]([\mathrm{H}])(\mathrm{C}(=\mathrm{O}) \mathrm{N}([\mathrm{H}]) \mathrm{C}([\mathrm{H}])([\mathrm{H}]) \mathrm{Cl}=\mathrm{C}([\mathrm{H}]) \mathrm{C}([\mathrm{H}])=\mathrm{C}([\mathrm{H}]) \mathrm{C}([\mathrm{H}])=\mathrm{C} \\
1[\mathrm{H}]) \mathrm{C}([\mathrm{H}])(\mathrm{C}([\mathrm{H}])([\mathrm{H}])[\mathrm{H}]) \mathrm{C}([\mathrm{H}])([\mathrm{H}])[\mathrm{H}]) \mathrm{C}([\mathrm{H}])([\mathrm{H}])[\mathrm{H}]) \mathrm{C}([\mathrm{H}])([\mathrm{H}]) \mathrm{C}([\mathrm{H}])(\mathrm{C}([\mathrm{H}])([\mathrm{H}])[ \\
\mathrm{H}]) \mathrm{C}\end{array}$ & 4.027 & 3.219 \\
\hline $\operatorname{Tr} 47$ & 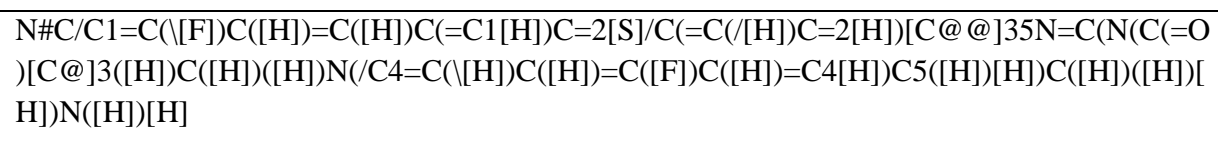 & 2.879 & 2.953 \\
\hline $\operatorname{Tr} 48$ & $\begin{array}{l}\mathrm{O}=[\mathrm{S}] 3(=\mathrm{O}) \mathrm{N}(\mathrm{C}=2 \mathrm{C}([\mathrm{H}])=\mathrm{C}(\mathrm{C}([\mathrm{H}])=\mathrm{Cl} / \mathrm{C}(=\mathrm{C}([\mathrm{H}]) \mathrm{N}(\mathrm{Cl}=2) \mathrm{C}([\mathrm{H}])([\mathrm{H}]) \mathrm{C} 3([\mathrm{H}])[\mathrm{H}]) \mathrm{C}([\mathrm{H}])([ \\
\mathrm{H}]) \mathrm{C}([\mathrm{H}])([\mathrm{H}])[\mathrm{H}]) \mathrm{C}(=\mathrm{O}) \mathrm{N}([\mathrm{H}])[\mathrm{C} @]([\mathrm{H}])(\mathrm{C}([\mathrm{H}])([\mathrm{H}]) \mathrm{N}([\mathrm{H}])[\mathrm{C} @]([\mathrm{H}])(\mathrm{C}(=\mathrm{O}) \mathrm{N}([\mathrm{H}]) \mathrm{C}([\mathrm{H}]) \\
([\mathrm{H}]) \mathrm{C}([\mathrm{H}])(\mathrm{C}([\mathrm{H}])([\mathrm{H}])[\mathrm{H}]) \mathrm{C}([\mathrm{H}])([\mathrm{H}])[\mathrm{H}])[\mathrm{C} @ @]([\mathrm{H}])(\mathrm{O}[\mathrm{H}]) \mathrm{C}([\mathrm{H}])([\mathrm{H}])[\mathrm{H}]) \mathrm{C}([\mathrm{H}])([\mathrm{H}]) \mathrm{C} \\
4=\mathrm{C}(\end{array}$ & 4.137 & 4.524 \\
\hline
\end{tabular}




\begin{tabular}{|c|c|c|c|}
\hline $\operatorname{Tr} 49$ & $\begin{array}{l}\mathrm{O}=[\mathrm{S}] 3(=\mathrm{O}) \mathrm{N}(\mathrm{C}=2 \mathrm{C}([\mathrm{H}])=\mathrm{C}(\mathrm{C}([\mathrm{H}])=\mathrm{C} 1 / \mathrm{C}(=\mathrm{C}(/[\mathrm{H}]) \mathrm{N}(\mathrm{C} 1=2) \mathrm{C}([\mathrm{H}])([\mathrm{H}])[\mathrm{C} @] 34 \mathrm{C}([\mathrm{H}])([\mathrm{H}]) \\
\mathrm{C} 4([\mathrm{H}])[\mathrm{H}]) \mathrm{C}([\mathrm{H}])([\mathrm{H}]) \mathrm{C}([\mathrm{H}])([\mathrm{H}])[\mathrm{H}]) \mathrm{C}(=\mathrm{O}) \mathrm{N}([\mathrm{H}])[\mathrm{C} @]([\mathrm{H}])(\mathrm{C}([\mathrm{H}])([\mathrm{H}]) \mathrm{N}([\mathrm{H}])[\mathrm{C} @]([\mathrm{H}]) \\
(\mathrm{C}(=\mathrm{O}) \mathrm{N}([\mathrm{H}]) \mathrm{C}([\mathrm{H}])([\mathrm{H}]) \mathrm{C}([\mathrm{H}])(\mathrm{C}([\mathrm{H}])([\mathrm{H}])[\mathrm{H}]) \mathrm{C}([\mathrm{H}])([\mathrm{H}])[\mathrm{H}])[\mathrm{C} @ @]([\mathrm{H}])(\mathrm{O}[\mathrm{H}]) \mathrm{C}([\mathrm{H}])([ \\
\mathrm{H}])[\mathrm{H}]\end{array}$ & 4.143 & 4.337 \\
\hline $\operatorname{Tr} 50$ & $\begin{array}{l}\mathrm{O}=\mathrm{C}(\mathrm{N}([\mathrm{H}])[\mathrm{C} @]([\mathrm{H}])([\mathrm{C} @ @]([\mathrm{H}])(\mathrm{O}[\mathrm{H}]) \mathrm{C}([\mathrm{H}])([\mathrm{H}])[\mathrm{C} @]([\mathrm{H}])(\mathrm{C}(=\mathrm{O}) \mathrm{N}([\mathrm{H}]) \mathrm{C}([\mathrm{H}])([\mathrm{H}]) \mathrm{C} \\
([\mathrm{H}])(\mathrm{C}([\mathrm{H}])([\mathrm{H}])[\mathrm{H}]) \mathrm{C}([\mathrm{H}])([\mathrm{H}])[\mathrm{H}]) \mathrm{C}([\mathrm{H}])([\mathrm{H}]) \mathrm{C}([\mathrm{H}])([\mathrm{H}]) \mathrm{C}(=\mathrm{O}) \mathrm{O}[\mathrm{H}]) \mathrm{C}([\mathrm{H}])([\mathrm{H}]) \mathrm{C} 1=\mathrm{C}([ \\
\mathrm{H}]) \mathrm{C}([\mathrm{H}])=\mathrm{C}([\mathrm{H}]) \mathrm{C}([\mathrm{H}])=\mathrm{C} 1[\mathrm{H}]) \mathrm{C}([\mathrm{H}])([\mathrm{H}])[\mathrm{C} @]([\mathrm{H}])(\mathrm{N}([\mathrm{H}]) \mathrm{C}(=\mathrm{O}) \mathrm{C}([\mathrm{H}])([\mathrm{H}]) \mathrm{C}([\mathrm{H}])(\mathrm{C}([\mathrm{H} \\
])([\mathrm{H}\end{array}$ & 1.770 & 1.714 \\
\hline $\operatorname{Tr} 51$ & $\begin{array}{l}\mathrm{O}=\mathrm{C}(\mathrm{N}([\mathrm{H}])[\mathrm{C} @]([\mathrm{H}])([\mathrm{C} @ @]([\mathrm{H}])(\mathrm{O}[\mathrm{H}]) \mathrm{C}([\mathrm{H}])([\mathrm{H}])[\mathrm{C} @]([\mathrm{H}])(\mathrm{C}(=\mathrm{O}) \mathrm{N}([\mathrm{H}]) \mathrm{C}([\mathrm{H}])([\mathrm{H}]) \mathrm{C} \\
([\mathrm{H}])(\mathrm{C}([\mathrm{H}])([\mathrm{H}])[\mathrm{H}]) \mathrm{C}([\mathrm{H}])([\mathrm{H}])[\mathrm{H}]) \mathrm{C}([\mathrm{H}])([\mathrm{H}]) \mathrm{C}([\mathrm{H}])([\mathrm{H}]) \mathrm{OC}(=\mathrm{O}) \mathrm{C}([\mathrm{H}])([\mathrm{H}])[\mathrm{H}]) \mathrm{C}([\mathrm{H}])([ \\
\mathrm{H}]) \mathrm{C} 1=\mathrm{C}([\mathrm{H}]) \mathrm{C}([\mathrm{H}])=\mathrm{C}([\mathrm{H}]) \mathrm{C}([\mathrm{H}])=\mathrm{C} 1[\mathrm{H}]) \mathrm{C}([\mathrm{H}])([\mathrm{H}])[\mathrm{C} @]([\mathrm{H}])(\mathrm{N}([\mathrm{H}]) \mathrm{C}(=\mathrm{O}) \mathrm{C}([\mathrm{H}])([\mathrm{H}]) \mathrm{C} \\
([\mathrm{H}]\end{array}$ & 1.569 & 2.044 \\
\hline $\operatorname{Tr} 52$ & $\begin{array}{l}\mathrm{O}=\mathrm{C}(\mathrm{N}([\mathrm{H}])[\mathrm{C} @ @]([\mathrm{H}])(\mathrm{C}=1 \mathrm{C}([\mathrm{H}])=\mathrm{C}([\mathrm{H}]) \mathrm{C}([\mathrm{H}])=\mathrm{C}([\mathrm{H}]) \mathrm{C}=1[\mathrm{H}]) \mathrm{C}([\mathrm{H}])([\mathrm{H}])[\mathrm{H}]) \mathrm{C} 2=\mathrm{C}([\mathrm{H} \\
]) \mathrm{C}(=\mathrm{C}([\mathrm{H}]) \mathrm{C}(=\mathrm{C} 2[\mathrm{H}]) \mathrm{N}(\mathrm{C}([\mathrm{H}])([\mathrm{H}])[\mathrm{H}])[\mathrm{S}](=\mathrm{O})(=\mathrm{O}) \mathrm{C}([\mathrm{H}])([\mathrm{H}])[\mathrm{H}]) \mathrm{C}(=\mathrm{O}) \mathrm{N}([\mathrm{H}])[\mathrm{C} @]([\mathrm{H}] \\
)([\mathrm{C} @]([\mathrm{H}])(\mathrm{O}[\mathrm{H}]) \mathrm{C}([\mathrm{H}])([\mathrm{H}]) \mathrm{N}([\mathrm{H}]) \mathrm{C}([\mathrm{H}])(\mathrm{C}([\mathrm{H}])([\mathrm{H}])[\mathrm{H}]) \mathrm{C}([\mathrm{H}])([\mathrm{H}])[\mathrm{H}]) \mathrm{C}([\mathrm{H}])([\mathrm{H}]) \mathrm{C} 3= \\
\mathrm{C}([\mathrm{H}]) \mathrm{C}\end{array}$ & 2.372 & 1.883 \\
\hline $\operatorname{Tr} 53$ & $\begin{array}{l}\mathrm{O}=\mathrm{C}(\mathrm{N}([\mathrm{H}])[\mathrm{C} @]([\mathrm{H}])(\mathrm{C} 1=\mathrm{C}([\mathrm{H}]) \mathrm{C}([\mathrm{H}])=\mathrm{C}([\mathrm{H}]) \mathrm{C}([\mathrm{H}])=\mathrm{C} 1[\mathrm{H}]) \mathrm{C}([\mathrm{H}])([\mathrm{H}])[\mathrm{H}]) \mathrm{C} 2=\mathrm{C}([\mathrm{H}]) \mathrm{C} \\
(=\mathrm{C}([\mathrm{H}]) \mathrm{C}(=\mathrm{C} 2[\mathrm{H}]) \mathrm{N}(\mathrm{C}([\mathrm{H}])([\mathrm{H}])[\mathrm{H}])[\mathrm{S}](=\mathrm{O})(=\mathrm{O}) \mathrm{C}([\mathrm{H}])([\mathrm{H}])[\mathrm{H}]) \mathrm{C}(=\mathrm{O}) \mathrm{N}([\mathrm{H}])[\mathrm{C} @]([\mathrm{H}])([ \\
\mathrm{C} @]([\mathrm{H}])(\mathrm{O}[\mathrm{H}]) \mathrm{C}([\mathrm{H}])([\mathrm{H}]) \mathrm{N}([\mathrm{H}]) \mathrm{C}([\mathrm{H}])([\mathrm{H}]) \mathrm{C} 3=\mathrm{C}([\mathrm{H}]) \mathrm{C}(\mathrm{OC}([\mathrm{H}])([\mathrm{H}])[\mathrm{H}])=\mathrm{C}([\mathrm{H}]) \mathrm{C}([\mathrm{H}]) \\
=\mathrm{C} 3[\mathrm{H}]) \mathrm{C}(\end{array}$ & 2.210 & 1.770 \\
\hline $\operatorname{Tr} 54$ & $\begin{array}{l}\mathrm{O}=\mathrm{C}(\mathrm{N}([\mathrm{H}]) \mathrm{C}([\mathrm{H}])([\mathrm{H}]) \mathrm{C}=1 \mathrm{~N}=\mathrm{C}(\mathrm{OC}=1 \mathrm{C}([\mathrm{H}])([\mathrm{H}])[\mathrm{H}]) \mathrm{C}([\mathrm{H}])([\mathrm{H}])[\mathrm{H}]) \mathrm{C} 2=\mathrm{C}([\mathrm{H}]) \mathrm{C}(=\mathrm{C}([\mathrm{H}]) \\
\mathrm{C}(=\mathrm{C} 2[\mathrm{H}]) \mathrm{N}(\mathrm{C}([\mathrm{H}])([\mathrm{H}])[\mathrm{H}])[\mathrm{S}](=\mathrm{O})(=\mathrm{O}) \mathrm{C}([\mathrm{H}])([\mathrm{H}])[\mathrm{H}]) \mathrm{C}(=\mathrm{O}) \mathrm{N}([\mathrm{H}])[\mathrm{C} @]([\mathrm{H}])([\mathrm{C} @]([\mathrm{H}]) \\
(\mathrm{O}[\mathrm{H}]) \mathrm{C}([\mathrm{H}])([\mathrm{H}]) \mathrm{N}([\mathrm{H}]) \mathrm{C}([\mathrm{H}])([\mathrm{H}]) \mathrm{C} 3=\mathrm{C}([\mathrm{H}]) \mathrm{C}(\mathrm{OC}([\mathrm{H}])([\mathrm{H}])[\mathrm{H}])=\mathrm{C}([\mathrm{H}]) \mathrm{C}([\mathrm{H}])=\mathrm{C} 3[\mathrm{H}]) \mathrm{C}( \\
[\mathrm{H}])([\mathrm{H}]\end{array}$ & 2.258 & 2.988 \\
\hline $\operatorname{Tr} 55$ & $\begin{array}{l}\mathrm{O}=\mathrm{C} 3 \mathrm{O} / \mathrm{C} 2=\mathrm{C}(\backslash \mathrm{OC}([\mathrm{H}])([\mathrm{H}]) \mathrm{C}([\mathrm{H}])=\mathrm{C}(\mathrm{C}([\mathrm{H}])([\mathrm{H}]) \mathrm{C}([\mathrm{H}])([\mathrm{H}]) \mathrm{C}([\mathrm{H}])=\mathrm{C}(\mathrm{C}([\mathrm{H}])([\mathrm{H}])[\mathrm{H}]) \mathrm{C}([ \\
\mathrm{H}])([\mathrm{H}])[\mathrm{H}]) \mathrm{C}([\mathrm{H}])([\mathrm{H}])[\mathrm{H}]) \mathrm{C}=1 \mathrm{OC}([\mathrm{H}])=\mathrm{C}([\mathrm{H}]) \mathrm{C}=1 \mathrm{C}([\mathrm{H}])=\mathrm{C} 2 \mathrm{C}([\mathrm{H}])=\mathrm{C} 3[\mathrm{H}]\end{array}$ & 0.208 & 0.512 \\
\hline $\operatorname{Tr} 56$ & $\begin{array}{l}\mathrm{O}=\mathrm{C} 3 \mathrm{OC}=2 \mathrm{C}(\mathrm{OC}([\mathrm{H}])([\mathrm{H}])[\mathrm{C} @ @]([\mathrm{H}])(\mathrm{O}[\mathrm{H}]) \mathrm{C}(\mathrm{O}[\mathrm{H}])(\mathrm{C}([\mathrm{H}])([\mathrm{H}])[\mathrm{H}]) \mathrm{C}([\mathrm{H}])([\mathrm{H}])[\mathrm{H}])=\mathrm{C} 1 \\
\mathrm{OC}([\mathrm{H}])=\mathrm{C}([\mathrm{H}]) \mathrm{C} 1=\mathrm{C}(\mathrm{OC}([\mathrm{H}])([\mathrm{H}])[\mathrm{H}]) \mathrm{C}=2 \mathrm{C}([\mathrm{H}])=\mathrm{C} 3[\mathrm{H}]\end{array}$ & 0.260 & 0.613 \\
\hline $\operatorname{Tr} 57$ & $\begin{array}{l}\mathrm{O}=\mathrm{C} 3 \mathrm{OC}=2 \mathrm{C}(\mathrm{OC}([\mathrm{H}])([\mathrm{H}])[\mathrm{H}])=\mathrm{C} 1 \mathrm{OC}([\mathrm{H}])=\mathrm{C}([\mathrm{H}]) \mathrm{C} 1=\mathrm{C}(\mathrm{OC}([\mathrm{H}])([\mathrm{H}]) \mathrm{C}([\mathrm{H}])=\mathrm{C}(\mathrm{C}([\mathrm{H}])([\mathrm{H} \\
]) \mathrm{C}([\mathrm{H}])([\mathrm{H}]) \mathrm{C}([\mathrm{H}])=\mathrm{C}(\mathrm{C}([\mathrm{H}])([\mathrm{H}])[\mathrm{H}]) \mathrm{C}([\mathrm{H}])([\mathrm{H}])[\mathrm{H}]) \mathrm{C}([\mathrm{H}])([\mathrm{H}])[\mathrm{H}]) \mathrm{C}=2 \mathrm{C}([\mathrm{H}])=\mathrm{C} 3[\mathrm{H}]\end{array}$ & 0.863 & 0.740 \\
\hline $\operatorname{Tr} 58$ & $\begin{array}{l}\mathrm{O}=\mathrm{C}(\mathrm{N}([\mathrm{H}]) \mathrm{C}(=\mathrm{NC}([\mathrm{H}])([\mathrm{H}]) \mathrm{C} 1=\mathrm{C}([\mathrm{H}]) \mathrm{C}([\mathrm{Cl}])=\mathrm{C}(\mathrm{C}([\mathrm{Cl}])=\mathrm{C} 1[\mathrm{H}]) \mathrm{N}([\mathrm{H}]) \mathrm{C}(=\mathrm{O}) \mathrm{C}([\mathrm{H}])([\mathrm{H}]) \\
\mathrm{N} 2 \mathrm{C}([\mathrm{H}])([\mathrm{H}]) \mathrm{C}([\mathrm{H}])([\mathrm{H}]) \mathrm{C} 2([\mathrm{H}])[\mathrm{H}]) \mathrm{N}([\mathrm{H}])[\mathrm{H}]) \mathrm{C}=3 \mathrm{C}(=\mathrm{N}[\mathrm{S}] \mathrm{C}=3 \mathrm{C}([\mathrm{H}])([\mathrm{H}])[\mathrm{H}]) \mathrm{C} 4=\mathrm{C}([\mathrm{H}]) \\
\mathrm{C}([\mathrm{H}])=\mathrm{C}(\mathrm{OC}([\mathrm{H}])([\mathrm{H}])[\mathrm{H}]) \mathrm{C}([\mathrm{H}])=\mathrm{C} 4[\mathrm{H}]\end{array}$ & 4.398 & 4.400 \\
\hline $\operatorname{Tr} 59$ & $\begin{array}{l}\mathrm{O}=\mathrm{C}(\mathrm{N}([\mathrm{H}]) \mathrm{C}(=\mathrm{NC}([\mathrm{H}])([\mathrm{H}]) \mathrm{C}=1 \mathrm{C}([\mathrm{H}])=\mathrm{C}([\mathrm{H}]) \mathrm{C}([\mathrm{H}])=\mathrm{C} 2 \mathrm{C}=1 \mathrm{C}([\mathrm{H}])=\mathrm{C}([\mathrm{H}]) \mathrm{C}([\mathrm{H}])=\mathrm{C} 2[\mathrm{H}]) \\
\mathrm{N}([\mathrm{H}])[\mathrm{H}]) \mathrm{C}=3 \mathrm{C}(=\mathrm{NOC}=3 \mathrm{C}([\mathrm{H}])([\mathrm{H}])[\mathrm{H}]) \mathrm{C} 4=\mathrm{C}([\mathrm{H}]) \mathrm{C}([\mathrm{H}])=\mathrm{C}(\mathrm{OC}([\mathrm{H}])([\mathrm{H}])[\mathrm{H}]) \mathrm{C}([\mathrm{H}])=\mathrm{C} 4[ \\
\mathrm{H}]\end{array}$ & 1.770 & 1.286 \\
\hline Tr60 & $\begin{array}{l}\mathrm{O}=\mathrm{C}(\mathrm{N}([\mathrm{H}]) \mathrm{C}(=\mathrm{NC}([\mathrm{H}])([\mathrm{H}]) \mathrm{C} 1=\mathrm{C}([\mathrm{H}]) \mathrm{C}(=\mathrm{C}([\mathrm{H}]) \mathrm{C}([\mathrm{Cl}])=\mathrm{C} 1[\mathrm{H}]) \mathrm{C}([\mathrm{H}])=\mathrm{C}([\mathrm{H}]) \mathrm{C}([\mathrm{H}])([\mathrm{H}]) \\
\mathrm{C}([\mathrm{H}])([\mathrm{H}]) \mathrm{O}[\mathrm{H}]) \mathrm{N}([\mathrm{H}])[\mathrm{H}]) \mathrm{C}=2 \mathrm{C}(=\mathrm{NOC}=2 \mathrm{C}([\mathrm{H}])([\mathrm{H}])[\mathrm{H}]) \mathrm{C} 3=\mathrm{C}([\mathrm{H}]) \mathrm{C}([\mathrm{H}])=\mathrm{C}(\mathrm{OC}([\mathrm{H}])([\mathrm{H} \\
])[\mathrm{H}]) \mathrm{C}([\mathrm{H}])=\mathrm{C} 3[\mathrm{H}]\end{array}$ & 2.854 & 2.816 \\
\hline $\operatorname{Tr} 61$ & $\begin{array}{l}\mathrm{O}=\mathrm{C}(\mathrm{N}([\mathrm{H}]) \mathrm{C}(=\mathrm{NC}([\mathrm{H}])([\mathrm{H}]) \mathrm{C} 1=\mathrm{C}([\mathrm{H}]) \mathrm{C}([\mathrm{Cl}])=\mathrm{C}(\mathrm{C}([\mathrm{Cl}])=\mathrm{C} 1[\mathrm{H}]) \mathrm{N}([\mathrm{H}])[\mathrm{H}]) \mathrm{N}([\mathrm{H}])[\mathrm{H}]) \mathrm{C}=2 \\
\mathrm{C}(=\mathrm{NOC}=2 \mathrm{C}([\mathrm{H}])([\mathrm{H}])[\mathrm{H}]) \mathrm{C} 3=\mathrm{C}([\mathrm{H}]) \mathrm{C}([\mathrm{H}])=\mathrm{C}(\mathrm{OC}([\mathrm{H}])([\mathrm{H}])[\mathrm{H}]) \mathrm{C}([\mathrm{H}])=\mathrm{C} 3[\mathrm{H}]\end{array}$ & 2.921 & 2.713 \\
\hline Tr62 & $\begin{array}{l}\mathrm{O}=\mathrm{C}(\mathrm{N}([\mathrm{H}]) \mathrm{C}(=\mathrm{NC}([\mathrm{H}])([\mathrm{H}]) \mathrm{C} 1=\mathrm{C}([\mathrm{H}]) \mathrm{C}(=\mathrm{C}([\mathrm{H}]) \mathrm{C}([\mathrm{Cl}])=\mathrm{C} 1[\mathrm{H}]) \mathrm{C}([\mathrm{H}])=\mathrm{C}([\mathrm{H}]) \mathrm{C}([\mathrm{H}])([\mathrm{H}]) \\
\mathrm{C}([\mathrm{H}])([\mathrm{H}]) \mathrm{C}([\mathrm{H}])([\mathrm{H}])[\mathrm{H}]) \mathrm{N}([\mathrm{H}])[\mathrm{H}]) \mathrm{C}=2 \mathrm{C}(=\mathrm{NOC}=2 \mathrm{C}([\mathrm{H}])([\mathrm{H}])[\mathrm{H}]) \mathrm{C} 3=\mathrm{C}([\mathrm{H}]) \mathrm{C}([\mathrm{H}])=\mathrm{C}(\mathrm{O} \\
\mathrm{C}([\mathrm{H}])([\mathrm{H}])[\mathrm{H}]) \mathrm{C}([\mathrm{H}])=\mathrm{C} 3[\mathrm{H}]\end{array}$ & 2.071 & 2.293 \\
\hline Tr63 & $\begin{array}{l}\mathrm{O}=\mathrm{C}(\mathrm{N}([\mathrm{H}]) \mathrm{C}(=\mathrm{NC}([\mathrm{H}])([\mathrm{H}]) \mathrm{C} 1=\mathrm{C}([\mathrm{H}]) \mathrm{C}(=\mathrm{C}(\mathrm{C}([\mathrm{Cl}])=\mathrm{C} 1[\mathrm{H}]) \mathrm{N}([\mathrm{H}]) \mathrm{C}(=\mathrm{O}) \mathrm{C}([\mathrm{H}])([\mathrm{H}]) \mathrm{N}([\mathrm{H}] \\
) \mathrm{C}([\mathrm{H}])([\mathrm{H}]) \mathrm{C} 2=\mathrm{C}([\mathrm{H}]) \mathrm{C}([\mathrm{H}])=\mathrm{C}([\mathrm{H}]) \mathrm{C}([\mathrm{H}])=\mathrm{C} 2[\mathrm{H}]) \mathrm{C}([\mathrm{H}])([\mathrm{H}])[\mathrm{H}]) \mathrm{N}([\mathrm{H}])[\mathrm{H}]) \mathrm{C}=3 \mathrm{C}(=\mathrm{N}[\mathrm{S}]\end{array}$ & 4.000 & 3.296 \\
\hline
\end{tabular}




\begin{tabular}{|c|c|c|c|}
\hline & $\mathrm{C}=3 \mathrm{C}([\mathrm{H}])([\mathrm{H}])[\mathrm{H}]) \mathrm{C} 4=\mathrm{C}([\mathrm{H}]) \mathrm{C}([\mathrm{H}])=\mathrm{C}(\mathrm{OC}([\mathrm{H}])([\mathrm{H}])[\mathrm{H}]) \mathrm{C}([\mathrm{H}])=\mathrm{C} 4[\mathrm{H}]$ & & \\
\hline $\operatorname{Tr} 64$ & $\begin{array}{l}\mathrm{O}=\mathrm{C}(\mathrm{N}([\mathrm{H}]) \mathrm{C}(=\mathrm{NC}([\mathrm{H}])([\mathrm{H}]) \mathrm{C} 1=\mathrm{C}([\mathrm{H}]) \mathrm{C}([\mathrm{Cl}])=\mathrm{C}([\mathrm{H}]) \mathrm{C}([\mathrm{Cl}])=\mathrm{C} 1[\mathrm{H}]) \mathrm{N}([\mathrm{H}])[\mathrm{H}]) \mathrm{C}=2 \mathrm{C}(=\mathrm{N} \\
\mathrm{N}([\mathrm{H}]) \mathrm{C}=2 \mathrm{C}([\mathrm{H}])([\mathrm{H}])[\mathrm{H}]) \mathrm{C} 3=\mathrm{C}([\mathrm{H}]) \mathrm{C}([\mathrm{H}])=\mathrm{C}(\mathrm{OC}([\mathrm{H}])([\mathrm{H}])[\mathrm{H}]) \mathrm{C}([\mathrm{H}])=\mathrm{C} 3[\mathrm{H}]\end{array}$ & 2.678 & 2.179 \\
\hline $\operatorname{Tr} 65$ & $\begin{array}{l}\mathrm{O}=\mathrm{C}(\mathrm{N}([\mathrm{H}]) \mathrm{C}(=\mathrm{NC}([\mathrm{H}])([\mathrm{H}]) \mathrm{C} 1=\mathrm{C}([\mathrm{H}]) \mathrm{C}([\mathrm{Cl}])=\mathrm{C}([\mathrm{H}]) \mathrm{C}([\mathrm{Cl}])=\mathrm{C} 1[\mathrm{H}]) \mathrm{N}([\mathrm{H}])[\mathrm{H}]) \mathrm{C}=2 \mathrm{C}(=\mathrm{N} \\
\mathrm{OC}=2 \mathrm{C}([\mathrm{H}])([\mathrm{H}])[\mathrm{H}]) \mathrm{C} 3=\mathrm{C}([\mathrm{H}]) \mathrm{C}([\mathrm{H}])=\mathrm{C}([\mathrm{F}]) \mathrm{C}([\mathrm{H}])=\mathrm{C} 3[\mathrm{H}]\end{array}$ & 1.658 & 2.395 \\
\hline $\operatorname{Tr} 66$ & $\begin{array}{l}\mathrm{O}=\mathrm{C}(\mathrm{N}([\mathrm{H}]) \mathrm{C}(=\mathrm{NC}([\mathrm{H}])([\mathrm{H}]) \mathrm{C} 1=\mathrm{C}([\mathrm{H}]) \mathrm{C}([\mathrm{Cl}])=\mathrm{C}([\mathrm{H}]) \mathrm{C}([\mathrm{Cl}])=\mathrm{C} 1[\mathrm{H}]) \mathrm{N}([\mathrm{H}])[\mathrm{H}]) \mathrm{C} 2=\mathrm{C}(\mathrm{O} / \mathrm{N} \\
=\mathrm{C} 2 / \mathrm{C}([\mathrm{H}])([\mathrm{H}])[\mathrm{H}]) \mathrm{C} 3=\mathrm{C}([\mathrm{H}]) \mathrm{C}([\mathrm{H}])=\mathrm{C}(\mathrm{OC}([\mathrm{H}])([\mathrm{H}])[\mathrm{H}]) \mathrm{C}([\mathrm{H}])=\mathrm{C} 3[\mathrm{H}]\end{array}$ & 2.066 & 1.147 \\
\hline $\operatorname{Tr} 67$ & $\begin{array}{l}\mathrm{O}=\mathrm{C}(\mathrm{N}([\mathrm{H}]) \mathrm{C}(=\mathrm{NC}([\mathrm{H}])([\mathrm{H}]) \mathrm{C} 1=\mathrm{C}([\mathrm{H}]) \mathrm{C}=2 \mathrm{C}(\mathrm{C}([\mathrm{H}])=\mathrm{C} 1[\mathrm{H}])=\mathrm{C}([\mathrm{H}]) \mathrm{C}([\mathrm{H}])=\mathrm{C}([\mathrm{H}]) \mathrm{C}=2[\mathrm{H}]) \\
\mathrm{N}([\mathrm{H}])[\mathrm{H}]) \mathrm{C}=3 \mathrm{C}(=\mathrm{N}[\mathrm{S}] \mathrm{C}=3 \mathrm{C}([\mathrm{H}])([\mathrm{H}])[\mathrm{H}]) \mathrm{C} 4=\mathrm{C}([\mathrm{H}]) \mathrm{C}([\mathrm{H}])=\mathrm{C}(\mathrm{OC}([\mathrm{H}])([\mathrm{H}])[\mathrm{H}]) \mathrm{C}([\mathrm{H}])=\mathrm{C} \\
4[\mathrm{H}]\end{array}$ & 2.060 & 2.445 \\
\hline $\operatorname{Tr} 68$ & $\begin{array}{l}\mathrm{O}=\mathrm{C}(\mathrm{N}([\mathrm{H}]) \mathrm{C}(=\mathrm{NC}([\mathrm{H}])([\mathrm{H}]) \mathrm{C}=1 \mathrm{C}([\mathrm{H}])=\mathrm{C}([\mathrm{H}]) \mathrm{C}([\mathrm{H}])=\mathrm{C} 2 \mathrm{C}=1 \mathrm{C}([\mathrm{H}])=\mathrm{C}([\mathrm{H}]) \mathrm{C}([\mathrm{H}])=\mathrm{C} 2[\mathrm{H}]) \\
\mathrm{N}([\mathrm{H}])[\mathrm{H}]) \mathrm{C}=3 \mathrm{C}(=\mathrm{NOC}=3 \mathrm{C}([\mathrm{H}])([\mathrm{H}])[\mathrm{H}]) \mathrm{C} 4=\mathrm{C}([\mathrm{H}]) \mathrm{C}([\mathrm{H}])=\mathrm{C}([\mathrm{F}]) \mathrm{C}([\mathrm{H}])=\mathrm{C} 4[\mathrm{H}]\end{array}$ & 1.745 & 1.448 \\
\hline $\operatorname{Tr} 69$ & $\begin{array}{l}\mathrm{O}=\mathrm{C}(\mathrm{N}([\mathrm{H}]) \mathrm{C}(=\mathrm{NC}([\mathrm{H}])([\mathrm{H}]) \mathrm{C} 1=\mathrm{C}([\mathrm{H}]) \mathrm{C}(=\mathrm{C}(\mathrm{C}([\mathrm{Cl}])=\mathrm{C} 1[\mathrm{H}]) \mathrm{N}([\mathrm{H}]) \mathrm{C}(=\mathrm{O}) \mathrm{C}([\mathrm{H}])([\mathrm{H}]) \mathrm{N}([\mathrm{H}] \\
) \mathrm{C}([\mathrm{H}])([\mathrm{H}]) \mathrm{C}([\mathrm{H}])([\mathrm{H}])[\mathrm{H}]) \mathrm{C}([\mathrm{H}])([\mathrm{H}])[\mathrm{H}]) \mathrm{N}([\mathrm{H}])[\mathrm{H}]) \mathrm{C}=2 \mathrm{C}(=\mathrm{N}[\mathrm{S}] \mathrm{C}=2 \mathrm{C}([\mathrm{H}])([\mathrm{H}])[\mathrm{H}]) \mathrm{C} 3= \\
\mathrm{C}([\mathrm{H}]) \mathrm{C}([\mathrm{H}])=\mathrm{C}(\mathrm{OC}([\mathrm{H}])([\mathrm{H}])[\mathrm{H}]) \mathrm{C}([\mathrm{H}])=\mathrm{C} 3[\mathrm{H}]\end{array}$ & 3.699 & 3.530 \\
\hline $\operatorname{Tr} 70$ & $\begin{array}{l}\mathrm{O}=\mathrm{C} 2 \mathrm{C}=1 \mathrm{C}(\mathrm{O}[\mathrm{H}])=\mathrm{C}([\mathrm{H}]) \mathrm{C}(\mathrm{O}[\mathrm{H}])=\mathrm{C}([\mathrm{H}]) \mathrm{C}=1 \mathrm{O} / \mathrm{C}(=\mathrm{C} 2 / \mathrm{O}[\mathrm{H}]) \mathrm{C} 3=\mathrm{C}([\mathrm{H}]) \mathrm{C}([\mathrm{H}])=\mathrm{C}(\mathrm{O}[\mathrm{H}]) \mathrm{C}( \\
\mathrm{O}[\mathrm{H}])=\mathrm{C} 3[\mathrm{H}]\end{array}$ & 0.854 & 0.488 \\
\hline $\operatorname{Tr} 71$ & $\begin{array}{l}{[\mathrm{H}] \mathrm{C}=2 \mathrm{C}(\mathrm{O}[\mathrm{H}])=\mathrm{C} 1 \mathrm{OC}=6 \mathrm{C}(\mathrm{OC} 1=\mathrm{C} 3 \mathrm{C}=2 \mathrm{OC}=4 / \mathrm{C} 3=\mathrm{C}(/ \mathrm{O}[\mathrm{H}]) \mathrm{C}([\mathrm{H}])=\mathrm{C}(\mathrm{O}[\mathrm{H}]) \mathrm{C}=4 \mathrm{O} / \mathrm{C} 5=\mathrm{C}(\backslash[} \\
\mathrm{H}]) \mathrm{C}(\mathrm{O}[\mathrm{H}])=\mathrm{C}([\mathrm{H}]) \mathrm{C}(\mathrm{O}[\mathrm{H}])=\mathrm{C} 5[\mathrm{H}])=\mathrm{C}(\mathrm{O}[\mathrm{H}]) \mathrm{C}([\mathrm{H}])=\mathrm{C}(\mathrm{O}[\mathrm{H}]) \mathrm{C}=6 \mathrm{OC}=7 \mathrm{C}([\mathrm{H}])=\mathrm{C}(\mathrm{O}[\mathrm{H}]) \mathrm{C}([ \\
\mathrm{H}])=\mathrm{C}(\mathrm{O}[\mathrm{H}]) \mathrm{C}=7[\mathrm{H}]\end{array}$ & 1.886 & 2.278 \\
\hline $\operatorname{Tr} 72$ & $\begin{array}{l}\mathrm{O}=\mathrm{C}(/ \mathrm{C} 1=\mathrm{C}(\backslash \mathrm{H}]) \mathrm{C}(=\mathrm{C}([\mathrm{H}]) \mathrm{C}(=\mathrm{C} 1[\mathrm{H}]) \mathrm{C}(=\mathrm{O}) \mathrm{N} 2[\mathrm{C} @]([\mathrm{H}])(\mathrm{C}([\mathrm{H}])([\mathrm{H}]) \mathrm{C}([\mathrm{H}])([\mathrm{H}]) \mathrm{C} 2([\mathrm{H}])[ \\
\mathrm{H}]) \mathrm{C}([\mathrm{H}])([\mathrm{H}]) \mathrm{OC}([\mathrm{H}])([\mathrm{H}])[\mathrm{H}]) \mathrm{C}([\mathrm{H}])([\mathrm{H}])[\mathrm{H}]) \mathrm{N}([\mathrm{H}])[\mathrm{C} @]([\mathrm{H}])([\mathrm{C} @]([\mathrm{H}])(\mathrm{O}[\mathrm{H}])[\mathrm{C} @] 3([ \\
\mathrm{H}]) \mathrm{N}([\mathrm{H}]) \mathrm{C}([\mathrm{H}])([\mathrm{H}]) \mathrm{C}([\mathrm{H}])([\mathrm{H}]) \mathrm{N}(\mathrm{C} 3([\mathrm{H}])[\mathrm{H}])[\mathrm{S}](=\mathrm{O})(=\mathrm{O})[\mathrm{C} @ @] 4([\mathrm{H}]) \mathrm{C}([\mathrm{H}])([\mathrm{H}]) \mathrm{C} 4([\mathrm{H}] \\
)[\mathrm{H}]) \mathrm{C}(\end{array}$ & 4.155 & 4.463 \\
\hline $\operatorname{Tr} 73$ & $\begin{array}{l}{[\mathrm{F}] \mathrm{C}=1 \mathrm{C}([\mathrm{H}])=\mathrm{C}(\mathrm{C}([\mathrm{H}])=\mathrm{C}([\mathrm{F}]) \mathrm{C}=1[\mathrm{H}]) \mathrm{C}([\mathrm{H}])([\mathrm{H}])[\mathrm{C} @]([\mathrm{H}])(\mathrm{N}([\mathrm{H}]) \mathrm{C}(=\mathrm{O}) \mathrm{C} 2=\mathrm{C}([\mathrm{H}]) \mathrm{C}(=\mathrm{C}} \\
([\mathrm{H}]) \mathrm{C}(=\mathrm{C} 2[\mathrm{H}]) \mathrm{C}(=\mathrm{O}) \mathrm{N} 3[\mathrm{C} @]([\mathrm{H}])(\mathrm{C}([\mathrm{H}])([\mathrm{H}]) \mathrm{C}([\mathrm{H}])([\mathrm{H}]) \mathrm{C} 3([\mathrm{H}])[\mathrm{H}]) \mathrm{C}([\mathrm{H}])([\mathrm{H}]) \mathrm{OC}([\mathrm{H}])( \\
[\mathrm{H}])[\mathrm{H}]) \mathrm{C}([\mathrm{H}])([\mathrm{H}])[\mathrm{H}])[\mathrm{C} @]([\mathrm{H}])(\mathrm{O}[\mathrm{H}])[\mathrm{C} @] 4([\mathrm{H}]) \mathrm{N}([\mathrm{H}]) \mathrm{C}([\mathrm{H}])([\mathrm{H}]) \mathrm{C}([\mathrm{H}])([\mathrm{H}]) \mathrm{N}(\mathrm{C} 4([\mathrm{H}] \\
)[\mathrm{H}])[\end{array}$ & 4.699 & 4.487 \\
\hline $\operatorname{Tr} 74$ & $\begin{array}{l}{[\mathrm{F}] \mathrm{C}=1 \mathrm{C}([\mathrm{H}])=\mathrm{C}(\mathrm{C}([\mathrm{H}])=\mathrm{C}([\mathrm{F}]) \mathrm{C}=1[\mathrm{H}]) \mathrm{C}([\mathrm{H}])([\mathrm{H}])[\mathrm{C} @]([\mathrm{H}])(\mathrm{N}([\mathrm{H}]) \mathrm{C}(=\mathrm{O}) \mathrm{C} 2=\mathrm{C}([\mathrm{H}]) \mathrm{C}(=\mathrm{C}} \\
([\mathrm{H}]) \mathrm{C}(=\mathrm{C} 2[\mathrm{H}]) \mathrm{C}(=\mathrm{O}) \mathrm{N} 3[\mathrm{C} @]([\mathrm{H}])(\mathrm{C}([\mathrm{H}])([\mathrm{H}]) \mathrm{C}([\mathrm{H}])([\mathrm{H}]) \mathrm{C} 3([\mathrm{H}])[\mathrm{H}]) \mathrm{C}([\mathrm{H}])([\mathrm{H}]) \mathrm{OC}([\mathrm{H}])( \\
[\mathrm{H}])[\mathrm{H}]) \mathrm{C}([\mathrm{H}])([\mathrm{H}])[\mathrm{H}])[\mathrm{C} @]([\mathrm{H}])(\mathrm{O}[\mathrm{H}])[\mathrm{C} @] 4([\mathrm{H}]) \mathrm{N}([\mathrm{H}]) \mathrm{C}([\mathrm{H}])([\mathrm{H}]) \mathrm{C}([\mathrm{H}])([\mathrm{H}]) \mathrm{N}(\mathrm{C} 4([\mathrm{H}] \\
)[\mathrm{H}])[\end{array}$ & 5.000 & 4.481 \\
\hline $\operatorname{Tr} 75$ & $\begin{array}{l}\mathrm{O}=\mathrm{C} 3 \mathrm{~N}(\mathrm{C}([\mathrm{H}])([\mathrm{H}]) \mathrm{C}([\mathrm{H}])([\mathrm{H}]) \mathrm{C}=4 \mathrm{C}([\mathrm{H}])=\mathrm{C}(\mathrm{OC}=2 \mathrm{C}([\mathrm{H}])=\mathrm{C}([\mathrm{H}]) \mathrm{C}=1 \mathrm{~N}=\mathrm{C}(\mathrm{N}(\mathrm{C}([\mathrm{H}])([\mathrm{H}]) \mathrm{C} \\
=1 \mathrm{C}=2[\mathrm{H}])[\mathrm{C} @ @]([\mathrm{H}])(\mathrm{C}([\mathrm{H}])([\mathrm{H}]) \mathrm{C} 3([\mathrm{H}])[\mathrm{H}]) \mathrm{C}([\mathrm{H}])(\mathrm{C}([\mathrm{H}])([\mathrm{H}])[\mathrm{H}]) \mathrm{C}([\mathrm{H}])([\mathrm{H}])[\mathrm{H}]) \mathrm{N}([\mathrm{H} \\
])[\mathrm{H}]) \mathrm{C}([\mathrm{H}])=\mathrm{C}([\mathrm{H}]) \mathrm{C}=4[\mathrm{H}])[\mathrm{C} @] 5([\mathrm{H}]) \mathrm{C}([\mathrm{H}])([\mathrm{H}]) \mathrm{C}([\mathrm{H}])([\mathrm{H}]) \mathrm{OC}([\mathrm{H}])([\mathrm{H}]) \mathrm{C} 5([\mathrm{H}])[\mathrm{H}]\end{array}$ & 3.658 & 3.747 \\
\hline $\operatorname{Tr} 76$ & $\begin{array}{l}\mathrm{O}=\mathrm{C} 3 \mathrm{~N}(\mathrm{C}([\mathrm{H}])([\mathrm{H}]) \mathrm{C}([\mathrm{H}])([\mathrm{H}]) \mathrm{C}=5 \mathrm{C}([\mathrm{H}])=\mathrm{C}(\mathrm{O} / \mathrm{C} 2=\mathrm{C}(\backslash[\mathrm{H}]) \mathrm{C}([\mathrm{H}])=\mathrm{C} 1 \mathrm{~N}=\mathrm{C}(\mathrm{N}(\mathrm{C}([\mathrm{H}])([\mathrm{H}]) \mathrm{C} \\
1=\mathrm{C} 2[\mathrm{H}])[\mathrm{C} @ @]([\mathrm{H}])(\mathrm{C}([\mathrm{H}])([\mathrm{H}]) \mathrm{C} 3([\mathrm{H}])[\mathrm{H}])[\mathrm{C} @ @] 4([\mathrm{H}]) \mathrm{C}([\mathrm{H}])([\mathrm{H}]) \mathrm{C}([\mathrm{H}])([\mathrm{H}]) \mathrm{C}([\mathrm{H}])([ \\
\mathrm{H}]) \mathrm{C}([\mathrm{H}])([\mathrm{H}]) \mathrm{C} 4([\mathrm{H}])[\mathrm{H}]) \mathrm{N}([\mathrm{H}])[\mathrm{H}]) \mathrm{C}([\mathrm{H}])=\mathrm{C}([\mathrm{H}]) \mathrm{C}=5[\mathrm{H}])[\mathrm{C} @ @] 6([\mathrm{H}]) \mathrm{C}([\mathrm{H}])([\mathrm{H}]) \mathrm{C}([\mathrm{H}] \\
)([\mathrm{H}]) \mathrm{OC}([\end{array}$ & 3.770 & 3.475 \\
\hline $\operatorname{Tr} 77$ & $\begin{array}{l}\mathrm{O}=\mathrm{C}(\mathrm{N}([\mathrm{H}])[\mathrm{C} @ @]([\mathrm{H}])(\mathrm{C} 1=\mathrm{C}([\mathrm{H}]) \mathrm{C}([\mathrm{H}])=\mathrm{C}([\mathrm{H}]) \mathrm{C}([\mathrm{H}])=\mathrm{C} 1[\mathrm{H}]) \mathrm{C}([\mathrm{H}])([\mathrm{H}])[\mathrm{H}]) \mathrm{C} 2=\mathrm{C}([\mathrm{H}]) \\
\mathrm{C}(=\mathrm{C}([\mathrm{H}]) \mathrm{C}(=\mathrm{C} 2[\mathrm{H}]) \mathrm{N}(\mathrm{C}([\mathrm{H}])([\mathrm{H}])[\mathrm{H}])[\mathrm{S}](=\mathrm{O})(=\mathrm{O}) \mathrm{C}([\mathrm{H}])([\mathrm{H}])[\mathrm{H}]) \mathrm{C}(=\mathrm{O}) \mathrm{N}([\mathrm{H}])[\mathrm{C} @]([\mathrm{H}])( \\
\mathrm{C}([\mathrm{H}])([\mathrm{H}]) \mathrm{C}([\mathrm{H}])([\mathrm{H}])[\mathrm{C} @ @]([\mathrm{H}])(\mathrm{OC}([\mathrm{H}])([\mathrm{H}])[\mathrm{H}]) \mathrm{C}(=\mathrm{O}) \mathrm{N}([\mathrm{H}])[\mathrm{C} @]([\mathrm{H}])(\mathrm{C}(=\mathrm{O}) \mathrm{N}([\mathrm{H}]) \\
\mathrm{C}([\mathrm{H}])([\mathrm{H}]\end{array}$ & 2.886 & 3.348 \\
\hline $\operatorname{Tr} 78$ & $\begin{array}{l}\mathrm{O}=\mathrm{C}(\mathrm{N}([\mathrm{H}])[\mathrm{C} @ @]([\mathrm{H}])(\mathrm{C}=1 \mathrm{C}([\mathrm{H}])=\mathrm{C}([\mathrm{H}]) \mathrm{C}([\mathrm{H}])=\mathrm{C}([\mathrm{H}]) \mathrm{C}=1[\mathrm{H}]) \mathrm{C}([\mathrm{H}])([\mathrm{H}])[\mathrm{H}]) \mathrm{C}=2 \mathrm{C}([\mathrm{H} \\
])=\mathrm{C}(\mathrm{C}([\mathrm{H}])=\mathrm{C}(\mathrm{C}=2[\mathrm{H}]) \mathrm{N}(\mathrm{C}([\mathrm{H}])([\mathrm{H}])[\mathrm{H}])[\mathrm{S}](=\mathrm{O})(=\mathrm{O}) \mathrm{C}([\mathrm{H}])([\mathrm{H}])[\mathrm{H}]) \mathrm{C}(=\mathrm{O}) \mathrm{N}([\mathrm{H}])[\mathrm{C} @]([\mathrm{H}\end{array}$ & 3.699 & 4.519 \\
\hline
\end{tabular}




\begin{tabular}{|c|c|c|c|}
\hline & $\begin{array}{l}])([\mathrm{C} @ @]([\mathrm{H}])(\mathrm{O}[\mathrm{H}]) \mathrm{C}([\mathrm{H}])([\mathrm{H}])[\mathrm{C} @ @]([\mathrm{H}])(\mathrm{OC}([\mathrm{H}])([\mathrm{H}]) \mathrm{C} 3=\mathrm{C}([\mathrm{H}]) \mathrm{C}([\mathrm{H}])=\mathrm{C}([\mathrm{H}]) \mathrm{C}([\mathrm{H}]) \\
=\mathrm{C} 3[\mathrm{H}]) \mathrm{C}(=\mathrm{O}\end{array}$ & & \\
\hline $\operatorname{Tr} 79$ & $\begin{array}{l}\mathrm{O}=\mathrm{C}(\mathrm{N}([\mathrm{H}])[\mathrm{C} @ @]([\mathrm{H}])(\mathrm{C} 1=\mathrm{C}([\mathrm{H}]) \mathrm{C}([\mathrm{H}])=\mathrm{C}([\mathrm{H}]) \mathrm{C}([\mathrm{H}])=\mathrm{C} 1[\mathrm{H}]) \mathrm{C}([\mathrm{H}])([\mathrm{H}])[\mathrm{H}]) \mathrm{C}=2 \mathrm{C}([\mathrm{H}]) \\
=\mathrm{C}(\mathrm{C}([\mathrm{H}])=\mathrm{C}(\mathrm{C}=2[\mathrm{H}]) \mathrm{N}(\mathrm{C}([\mathrm{H}])([\mathrm{H}])[\mathrm{H}])[\mathrm{S}](=\mathrm{O})(=\mathrm{O}) \mathrm{C}([\mathrm{H}])([\mathrm{H}])[\mathrm{H}]) \mathrm{C}(=\mathrm{O}) \mathrm{N}([\mathrm{H}])[\mathrm{C} @]([\mathrm{H}]) \\
([\mathrm{C} @ @]([\mathrm{H}])(\mathrm{O}[\mathrm{H}]) \mathrm{C}([\mathrm{H}])([\mathrm{H}])[\mathrm{C} @ @]([\mathrm{H}])(\mathrm{OC}([\mathrm{H}])([\mathrm{H}]) \mathrm{C}([\mathrm{H}])([\mathrm{H}]) \mathrm{OC}([\mathrm{H}])([\mathrm{H}])[\mathrm{H}]) \mathrm{C}(= \\
\mathrm{O}) \mathrm{N}([\mathrm{H}])[\mathrm{C}\end{array}$ & 4.509 & 4.434 \\
\hline $\operatorname{Tr} 80$ & $\begin{array}{l}\mathrm{O}=\mathrm{C}(\mathrm{N}([\mathrm{H}])[\mathrm{C} @ @]([\mathrm{H}])(\mathrm{C} 1=\mathrm{C}([\mathrm{H}]) \mathrm{C}([\mathrm{H}])=\mathrm{C}([\mathrm{H}]) \mathrm{C}([\mathrm{H}])=\mathrm{C} 1[\mathrm{H}]) \mathrm{C}([\mathrm{H}])([\mathrm{H}])[\mathrm{H}]) \mathrm{C} 2=\mathrm{C}([\mathrm{H}]) \\
\mathrm{C}(=\mathrm{C}([\mathrm{H}]) \mathrm{C}(=\mathrm{C} 2[\mathrm{H}]) \mathrm{N}(\mathrm{C}([\mathrm{H}])([\mathrm{H}])[\mathrm{H}])[\mathrm{S}](=\mathrm{O})(=\mathrm{O}) \mathrm{C}([\mathrm{H}])([\mathrm{H}])[\mathrm{H}]) \mathrm{C}(=\mathrm{O}) \mathrm{N}([\mathrm{H}])[\mathrm{C} @]([\mathrm{H}])( \\
{[\mathrm{C} @ @]([\mathrm{H}])(\mathrm{O}[\mathrm{H}]) \mathrm{C}([\mathrm{H}])([\mathrm{H}])[\mathrm{C} @ @]([\mathrm{H}])(\mathrm{OC}([\mathrm{H}])([\mathrm{H}]) \mathrm{C}([\mathrm{H}])([\mathrm{H}]) \mathrm{C}([\mathrm{H}])([\mathrm{H}])[\mathrm{H}]) \mathrm{C}(=\mathrm{O})} \\
\mathrm{N}([\mathrm{H}])[\mathrm{C} @]\end{array}$ & 4.523 & 3.413 \\
\hline $\operatorname{Tr} 81$ & $\begin{array}{l}\mathrm{O}=\mathrm{C}(\mathrm{N}([\mathrm{H}])[\mathrm{C} @]([\mathrm{H}])(\mathrm{C}(=\mathrm{O}) \mathrm{N}([\mathrm{H}])[\mathrm{C} @]([\mathrm{H}])(\mathrm{C}(=\mathrm{O}) \mathrm{O}[\mathrm{H}]) \mathrm{C}([\mathrm{H}])([\mathrm{H}]) \mathrm{C} 1=\mathrm{C}([\mathrm{H}]) \mathrm{C}([\mathrm{H}])=\mathrm{C} \\
([\mathrm{H}]) \mathrm{C}([\mathrm{H}])=\mathrm{C} 1[\mathrm{H}]) \mathrm{C}([\mathrm{H}])([\mathrm{H}]) \mathrm{C}([\mathrm{H}])([\mathrm{H}]) \mathrm{C}(=\mathrm{O}) \mathrm{O}[\mathrm{H}])[\mathrm{C} @ @]([\mathrm{H}])(\mathrm{N}([\mathrm{H}]) \mathrm{C}(=\mathrm{O})[\mathrm{C} @ @]([ \\
\mathrm{H}])(\mathrm{C}([\mathrm{H}])([\mathrm{H}])[\mathrm{C} @]([\mathrm{H}])(\mathrm{O}[\mathrm{H}])[\mathrm{C} @ @]([\mathrm{H}])(\mathrm{N}([\mathrm{H}]) \mathrm{C}(=\mathrm{O})[\mathrm{C} @ @]([\mathrm{H}])(\mathrm{N}([\mathrm{H}]) \mathrm{C}(=\mathrm{O})[\mathrm{C} @ \\
@]([\mathrm{H}])(\mathrm{N}([\mathrm{H}]) \mathrm{C}(=\mathrm{O}\end{array}$ & 5.495 & 5.409 \\
\hline $\operatorname{Tr} 82$ & $\begin{array}{l}\mathrm{O}=\mathrm{C}(\mathrm{OC}(\mathrm{C}([\mathrm{H}])([\mathrm{H}])[\mathrm{H}])(\mathrm{C}([\mathrm{H}])([\mathrm{H}])[\mathrm{H}]) \mathrm{C}([\mathrm{H}])([\mathrm{H}])[\mathrm{H}]) \mathrm{N}([\mathrm{H}])[\mathrm{C} @]([\mathrm{H}])(\mathrm{C}(=\mathrm{O}) \mathrm{N}([\mathrm{H}])[\mathrm{C} \\
@]([\mathrm{H}])([\mathrm{C} @]([\mathrm{H}])(\mathrm{O}[\mathrm{H}]) \mathrm{C}([\mathrm{H}])([\mathrm{H}])[\mathrm{C} @]([\mathrm{H}])(\mathrm{C}(=\mathrm{O}) \mathrm{N}([\mathrm{H}])[\mathrm{C} @]([\mathrm{H}])(\mathrm{C}(=\mathrm{O}) \mathrm{N}([\mathrm{H}]) \mathrm{C}([\mathrm{H}] \\
)([\mathrm{H}]) \mathrm{C} 1=\mathrm{C}([\mathrm{H}]) \mathrm{C}([\mathrm{H}])=\mathrm{C}([\mathrm{H}]) \mathrm{C}([\mathrm{H}])=\mathrm{C} 1[\mathrm{H}]) \mathrm{C}([\mathrm{H}])([\mathrm{H}])[\mathrm{H}]) \mathrm{C}([\mathrm{H}])([\mathrm{H}])[\mathrm{H}]) \mathrm{C}([\mathrm{H}])([\mathrm{H}]) \mathrm{C}([ \\
\mathrm{H}])(\mathrm{C}\end{array}$ & 0.649 & 2.241 \\
\hline $\operatorname{Tr} 83$ & $\begin{array}{l}\mathrm{O}=\mathrm{C} 3 \mathrm{~N}([\mathrm{H}])[\mathrm{C} @]([\mathrm{H}])(\mathrm{C}(=\mathrm{O}) \mathrm{N}([\mathrm{H}])[\mathrm{C} @]([\mathrm{H}])([\mathrm{C} @]([\mathrm{H}])(\mathrm{O}[\mathrm{H}]) \mathrm{C}([\mathrm{H}])([\mathrm{H}]) \mathrm{N}([\mathrm{H}]) \mathrm{C}([\mathrm{H}])([ \\
\mathrm{H}]) \mathrm{C} 1=\mathrm{C}([\mathrm{H}]) \mathrm{C}(=\mathrm{C}([\mathrm{H}]) \mathrm{C}([\mathrm{H}])=\mathrm{C} 1[\mathrm{H}]) \mathrm{N}(\mathrm{C}([\mathrm{H}])([\mathrm{H}])[\mathrm{H}]) \mathrm{C}([\mathrm{H}])([\mathrm{H}])[\mathrm{H}]) \mathrm{C}([\mathrm{H}])([\mathrm{H}]) \mathrm{C} 2=\mathrm{C}([ \\
\mathrm{H}]) \mathrm{C}([\mathrm{H}])=\mathrm{C}([\mathrm{H}]) \mathrm{C}([\mathrm{H}])=\mathrm{C} 2[\mathrm{H}]) \mathrm{C}([\mathrm{H}])([\mathrm{H}])[\mathrm{C} @ @] 3([\mathrm{H}]) \mathrm{C}([\mathrm{H}])([\mathrm{H}]) \mathrm{C} 4=\mathrm{C}([\mathrm{H}]) \mathrm{C}([\mathrm{H}])=\mathrm{C}([ \\
\mathrm{H}]) \mathrm{C}([\mathrm{H}]\end{array}$ & 1.522 & 1.019 \\
\hline $\operatorname{Tr} 84$ & $\begin{array}{l}\mathrm{O}=\mathrm{C} 3 \mathrm{~N}([\mathrm{H}])[\mathrm{C} @]([\mathrm{H}])(\mathrm{C}(=\mathrm{O}) \mathrm{N}([\mathrm{H}])[\mathrm{C} @([\mathrm{H}])([\mathrm{C} @]([\mathrm{H}])(\mathrm{O}[\mathrm{H}]) \mathrm{C}([\mathrm{H}])([\mathrm{H}]) \mathrm{N}([\mathrm{H}]) \mathrm{C}([\mathrm{H}])([ \\
\mathrm{H}]) \mathrm{C} 1=\mathrm{C}([\mathrm{H}]) \mathrm{C}(=\mathrm{C}([\mathrm{H}]) \mathrm{C}([\mathrm{H}])=\mathrm{C} 1[\mathrm{H}]) \mathrm{N}(\mathrm{C}([\mathrm{H}])([\mathrm{H}])[\mathrm{H}]) \mathrm{C}([\mathrm{H}])([\mathrm{H}])[\mathrm{H}]) \mathrm{C}([\mathrm{H}])([\mathrm{H}]) \mathrm{C} 2=\mathrm{C}([ \\
\mathrm{H}]) \mathrm{C}([\mathrm{H}])=\mathrm{C}([\mathrm{H}]) \mathrm{C}([\mathrm{H}])=\mathrm{C} 2[\mathrm{H}]) \mathrm{C}([\mathrm{H}])([\mathrm{H}]) \mathrm{N} 3 \mathrm{C}([\mathrm{H}])([\mathrm{H}]) \mathrm{C} 4=\mathrm{C}([\mathrm{H}]) \mathrm{C}([\mathrm{H}])=\mathrm{C}([\mathrm{F}]) \mathrm{C}([\mathrm{H}])= \\
\mathrm{C} 4[\mathrm{H}]\end{array}$ & 2.087 & 2.135 \\
\hline $\operatorname{Tr} 85$ & $\begin{array}{l}\mathrm{O}=\mathrm{C} 3 \mathrm{~N}([\mathrm{H}])[\mathrm{C} @]([\mathrm{H}])(\mathrm{C}(=\mathrm{O}) \mathrm{N}([\mathrm{H}])[\mathrm{C} @([\mathrm{H}])([\mathrm{C} @]([\mathrm{H}])(\mathrm{O}[\mathrm{H}]) \mathrm{C}([\mathrm{H}])([\mathrm{H}]) \mathrm{N}([\mathrm{H}]) \mathrm{C}([\mathrm{H}])([ \\
\mathrm{H}]) \mathrm{C} 1=\mathrm{C}([\mathrm{H}]) \mathrm{C}(=\mathrm{C}([\mathrm{H}]) \mathrm{C}([\mathrm{H}])=\mathrm{C} 1[\mathrm{H}]) \mathrm{N}(\mathrm{C}([\mathrm{H}])([\mathrm{H}]))[\mathrm{H}]) \mathrm{C}([\mathrm{H}])([\mathrm{H}]))[\mathrm{H}]) \mathrm{C}([\mathrm{H}])([\mathrm{H}]) \mathrm{C} 2=\mathrm{C}([ \\
\mathrm{H}]) \mathrm{C}([\mathrm{H}])=\mathrm{C}([\mathrm{H}]) \mathrm{C}([\mathrm{H}])=\mathrm{C} 2[\mathrm{H}]) \mathrm{C}([\mathrm{H}])([\mathrm{H}]) \mathrm{N} 3 \mathrm{C}([\mathrm{H}])([\mathrm{H}]) \mathrm{C} 4=\mathrm{C}([\mathrm{H}]) \mathrm{C}([\mathrm{H}])=\mathrm{C}(\mathrm{C}([\mathrm{H}])=\mathrm{C} 4[ \\
\mathrm{H}]) \mathrm{C}([\mathrm{F}]\end{array}$ & 1.305 & 2.861 \\
\hline Tr86 & $\begin{array}{l}\mathrm{O}=\mathrm{C} 3 \mathrm{~N}([\mathrm{H}])[\mathrm{C} @](\mathrm{C}(=\mathrm{O}) \mathrm{N}([\mathrm{H}])[\mathrm{C} @]([\mathrm{H}])([\mathrm{C} @]([\mathrm{H}])(\mathrm{O}[\mathrm{H}]) \mathrm{C}([\mathrm{H}])([\mathrm{H}]) \mathrm{N}([\mathrm{H}]) \mathrm{C}([\mathrm{H}])([\mathrm{H}]) \mathrm{C} \\
1=\mathrm{C}([\mathrm{H}]) \mathrm{C}(=\mathrm{C}([\mathrm{H}]) \mathrm{C}([\mathrm{H}])=\mathrm{C} 1[\mathrm{H}]) \mathrm{N}(\mathrm{C}([\mathrm{H}])([\mathrm{H}])[\mathrm{H}]) \mathrm{C}([\mathrm{H}])([\mathrm{H}])[\mathrm{H}]) \mathrm{C}([\mathrm{H}])([\mathrm{H}]) \mathrm{C} 2=\mathrm{C}([\mathrm{H}]) \mathrm{C} \\
([\mathrm{H}])=\mathrm{C}([\mathrm{H}]) \mathrm{C}([\mathrm{H}])=\mathrm{C} 2[\mathrm{H}])(\mathrm{C}([\mathrm{H}])([\mathrm{H}]) \mathrm{N} 3 \mathrm{C}([\mathrm{H}])([\mathrm{H}]) \mathrm{C} 4=\mathrm{C}([\mathrm{H}]) \mathrm{C}([\mathrm{H}])=\mathrm{C}([\mathrm{H}]) \mathrm{C}([\mathrm{H}])=\mathrm{C} 4[ \\
\mathrm{H}]) \mathrm{C}([\mathrm{H}]\end{array}$ & 1.170 & 1.871 \\
\hline Tr87 & $\begin{array}{l}\mathrm{O}=\mathrm{C} 3 \mathrm{~N}([\mathrm{H}])[\mathrm{C} @]([\mathrm{H}])(\mathrm{C}(=\mathrm{O}) \mathrm{N}([\mathrm{H}])[\mathrm{C} @]([\mathrm{H}])([\mathrm{C} @]([\mathrm{H}])(\mathrm{O}[\mathrm{H}]) \mathrm{C}([\mathrm{H}])([\mathrm{H}]) \mathrm{N}([\mathrm{H}]) \mathrm{C}([\mathrm{H}])([ \\
\mathrm{H}]) \mathrm{C} 1=\mathrm{C}([\mathrm{H}]) \mathrm{C}(=\mathrm{C}([\mathrm{H}]) \mathrm{C}([\mathrm{H}])=\mathrm{C} 1[\mathrm{H}]) \mathrm{N}(\mathrm{C}([\mathrm{H}])([\mathrm{H}])[\mathrm{H}]) \mathrm{C}([\mathrm{H}])([\mathrm{H}])[\mathrm{H}]) \mathrm{C}([\mathrm{H}])([\mathrm{H}]) \mathrm{C} 2=\mathrm{C}([ \\
\mathrm{H}]) \mathrm{C}([\mathrm{H}])=\mathrm{C}([\mathrm{H}]) \mathrm{C}([\mathrm{H}])=\mathrm{C} 2[\mathrm{H}]) \mathrm{C}([\mathrm{H}])([\mathrm{H}]) \mathrm{N} 3 \mathrm{C}([\mathrm{H}])([\mathrm{H}]) \mathrm{C}([\mathrm{H}])([\mathrm{H}]) \mathrm{O} / \mathrm{C} 4=\mathrm{C}(\mathrm{V}[\mathrm{H}]) \mathrm{C}([\mathrm{H}])= \\
\mathrm{C}([\mathrm{H}])\end{array}$ & 1.454 & 2.093 \\
\hline $\operatorname{Tr} 88$ & $\begin{array}{l}\mathrm{O}=\mathrm{C} 3 \mathrm{~N}([\mathrm{H}])[\mathrm{C} @]([\mathrm{H}])(\mathrm{C}(=\mathrm{O}) \mathrm{N}([\mathrm{H}])[\mathrm{C} @]([\mathrm{H}])([\mathrm{C} @]([\mathrm{H}])(\mathrm{O}[\mathrm{H}]) \mathrm{C}([\mathrm{H}])([\mathrm{H}]) \mathrm{N}([\mathrm{H}]) \mathrm{C}([\mathrm{H}])([ \\
\mathrm{H}]) \mathrm{C} 1=\mathrm{C}([\mathrm{H}]) \mathrm{C}(=\mathrm{C}([\mathrm{H}]) \mathrm{C}([\mathrm{H}])=\mathrm{C} 1[\mathrm{H}]) \mathrm{N}(\mathrm{C}([\mathrm{H}])([\mathrm{H}])[\mathrm{H}]) \mathrm{C}([\mathrm{H}])([\mathrm{H}])[\mathrm{H}]) \mathrm{C}([\mathrm{H}])([\mathrm{H}]) \mathrm{C} 2=\mathrm{C}([ \\
\mathrm{H}]) \mathrm{C}([\mathrm{H}])=\mathrm{C}([\mathrm{H}]) \mathrm{C}([\mathrm{H}])=\mathrm{C} 2[\mathrm{H}]) \mathrm{C}([\mathrm{H}])([\mathrm{H}]) \mathrm{N} 3 / \mathrm{C} 4=\mathrm{C}(\backslash[\mathrm{H}]) \mathrm{C}([\mathrm{Br}])=\mathrm{C}([\mathrm{H}]) \mathrm{C}([\mathrm{Br}])=\mathrm{C} 4[\mathrm{H}]\end{array}$ & 1.456 & 1.744 \\
\hline Tr89 & $\begin{array}{l}\mathrm{O}=\mathrm{C} 3 \mathrm{~N}([\mathrm{H}])[\mathrm{C} @]([\mathrm{H}])(\mathrm{C}(=\mathrm{O}) \mathrm{N}([\mathrm{H}])[\mathrm{C} @]([\mathrm{H}])([\mathrm{C} @]([\mathrm{H}])(\mathrm{O}[\mathrm{H}]) \mathrm{C}([\mathrm{H}])([\mathrm{H}]) \mathrm{N}([\mathrm{H}]) \mathrm{C}([\mathrm{H}])([ \\
\mathrm{H}]) \mathrm{C} 1=\mathrm{C}([\mathrm{H}]) \mathrm{C}(=\mathrm{C}([\mathrm{H}]) \mathrm{C}([\mathrm{H}])=\mathrm{C} 1[\mathrm{H}]) \mathrm{N}(\mathrm{C}([\mathrm{H}])([\mathrm{H}])[\mathrm{H}]) \mathrm{C}([\mathrm{H}])([\mathrm{H}])[\mathrm{H}]) \mathrm{C}([\mathrm{H}])([\mathrm{H}]) \mathrm{C} 2=\mathrm{C}([ \\
\mathrm{H}]) \mathrm{C}([\mathrm{H}])=\mathrm{C}([\mathrm{H}]) \mathrm{C}([\mathrm{H}])=\mathrm{C} 2[\mathrm{H}]) \mathrm{C}([\mathrm{H}])([\mathrm{H}]) \mathrm{N} 3[\mathrm{H}]\end{array}$ & 0.721 & 1.318 \\
\hline $\operatorname{Tr} 90$ & $\begin{array}{l}\mathrm{O}=\mathrm{C} 3 \mathrm{~N}([\mathrm{H}])[\mathrm{C} @]([\mathrm{H}])(\mathrm{C}(=\mathrm{O}) \mathrm{N}([\mathrm{H}])[\mathrm{C} @]([\mathrm{H}])([\mathrm{C} @]([\mathrm{H}])(\mathrm{O}[\mathrm{H}]) \mathrm{C}([\mathrm{H}])([\mathrm{H}]) \mathrm{N}([\mathrm{H}]) \mathrm{C}([\mathrm{H}])([ \\
\mathrm{H}]) \mathrm{C} 1=\mathrm{C}([\mathrm{H}]) \mathrm{C}(=\mathrm{C}([\mathrm{H}]) \mathrm{C}([\mathrm{H}])=\mathrm{C} 1[\mathrm{H}]) \mathrm{N}(\mathrm{C}([\mathrm{H}])([\mathrm{H}])[\mathrm{H}]) \mathrm{C}([\mathrm{H}])([\mathrm{H}])[\mathrm{H}]) \mathrm{C}([\mathrm{H}])([\mathrm{H}]) \mathrm{C} 2=\mathrm{C}([ \\
\mathrm{H}]) \mathrm{C}([\mathrm{H}])=\mathrm{C}([\mathrm{H}]) \mathrm{C}([\mathrm{H}])=\mathrm{C} 2[\mathrm{H}]) \mathrm{C}([\mathrm{H}])([\mathrm{H}]) \mathrm{N} 3 \mathrm{C}([\mathrm{H}])([\mathrm{H}]) \mathrm{C}([\mathrm{H}])([\mathrm{H}]) \mathrm{C}([\mathrm{H}])([\mathrm{H}]) \mathrm{C}([\mathrm{H}])([\mathrm{H}\end{array}$ & 0.398 & 1.774 \\
\hline
\end{tabular}




\begin{tabular}{|c|c|c|c|}
\hline & ]) $[\mathrm{H}]$ & & \\
\hline $\operatorname{Tr} 91$ & $\begin{array}{l}\mathrm{O}=\mathrm{C} 1 \mathrm{~N}(\mathrm{C}(=\mathrm{N}[\mathrm{C} @ @] 1(\mathrm{C}=2 \mathrm{C}([\mathrm{H}])=\mathrm{C}([\mathrm{H}]) \mathrm{C}([\mathrm{H}])=\mathrm{C}([\mathrm{H}]) \mathrm{C}=2[\mathrm{H}]) \mathrm{C} 3=\mathrm{C}([\mathrm{H}]) \mathrm{C}(=\mathrm{C}([\mathrm{H}]) \mathrm{C}([\mathrm{H}] \\
)=\mathrm{C} 3[\mathrm{H}]) \mathrm{C}=4 / \mathrm{N}=\mathrm{C}(/[\mathrm{H}]) \mathrm{C}([\mathrm{H}])=\mathrm{NC}=4[\mathrm{H}]) \mathrm{N}([\mathrm{H}])[\mathrm{H}]) \mathrm{C}([\mathrm{H}])([\mathrm{H}])[\mathrm{H}]\end{array}$ & 2.509 & 2.696 \\
\hline $\operatorname{Tr} 92$ & $\begin{array}{l}\mathrm{O}=\mathrm{C} 1 \mathrm{~N}(\mathrm{C}(=\mathrm{N}[\mathrm{C} @] 1(\mathrm{C}=2 \mathrm{C}([\mathrm{H}])=\mathrm{C}([\mathrm{H}]) \mathrm{C}([\mathrm{H}])=\mathrm{C}([\mathrm{H}]) \mathrm{C}=2[\mathrm{H}]) \mathrm{C} 3=\mathrm{C}([\mathrm{H}]) \mathrm{C}(=\mathrm{C}([\mathrm{H}]) \mathrm{C}([\mathrm{H}])= \\
\mathrm{C} 3[\mathrm{H}]) \mathrm{C} 4=\mathrm{C}([\mathrm{H}]) \mathrm{C}(\mathrm{OC}([\mathrm{H}])([\mathrm{H}])[\mathrm{H}])=\mathrm{C}([\mathrm{H}]) \mathrm{C}([\mathrm{H}])=\mathrm{C} 4[\mathrm{H}]) \mathrm{N}([\mathrm{H}])[\mathrm{H}]) \mathrm{C}([\mathrm{H}])([\mathrm{H}])[\mathrm{H}]\end{array}$ & 3.097 & 1.964 \\
\hline $\operatorname{Tr} 93$ & $\begin{array}{l}\mathrm{O}=\mathrm{C} 1 \mathrm{~N}(\mathrm{C}(=\mathrm{N}[\mathrm{C} @] 1(\mathrm{C}=2 \mathrm{C}([\mathrm{H}])=\mathrm{C}([\mathrm{H}]) \mathrm{C}([\mathrm{H}])=\mathrm{C}([\mathrm{H}]) \mathrm{C}=2[\mathrm{H}]) \mathrm{C} 3=\mathrm{C}([\mathrm{H}]) \mathrm{C}(=\mathrm{C}([\mathrm{H}]) \mathrm{C}([\mathrm{H}])= \\
\mathrm{C} 3[\mathrm{H}]) \mathrm{C} 4=\mathrm{C}([\mathrm{H}]) \mathrm{N}=\mathrm{C}([\mathrm{H}]) \mathrm{C}([\mathrm{Cl}])=\mathrm{C} 4[\mathrm{H}]) \mathrm{N}([\mathrm{H}])[\mathrm{H}]) \mathrm{C}([\mathrm{H}])([\mathrm{H}])[\mathrm{H}]\end{array}$ & 3.046 & 2.239 \\
\hline $\operatorname{Tr} 94$ & $\begin{array}{l}\mathrm{O}=\mathrm{C} 1 \mathrm{~N}(\mathrm{C}(=\mathrm{N}[\mathrm{C} @] 1(\mathrm{C}=2 \mathrm{C}([\mathrm{H}])=\mathrm{C}([\mathrm{H}]) \mathrm{C}([\mathrm{H}])=\mathrm{C}([\mathrm{H}]) \mathrm{C}=2[\mathrm{H}]) \mathrm{C} 3=\mathrm{C}([\mathrm{H}]) \mathrm{C}(=\mathrm{C}([\mathrm{H}]) \mathrm{C}([\mathrm{H}])= \\
\mathrm{C} 3[\mathrm{H}]) \mathrm{C} 4=\mathrm{C}([\mathrm{H}]) \mathrm{N}=\mathrm{C}([\mathrm{H}]) \mathrm{C}([\mathrm{H}])=\mathrm{C} 4[\mathrm{H}]) \mathrm{N}([\mathrm{H}])[\mathrm{H}]) \mathrm{C}([\mathrm{H}])([\mathrm{H}])[\mathrm{H}]\end{array}$ & 2.959 & 1.844 \\
\hline $\operatorname{Tr} 95$ & $\begin{array}{l}\mathrm{O}=\mathrm{C} 1 \mathrm{~N}(\mathrm{C}(=\mathrm{N}[\mathrm{C} @ @] 1(\mathrm{C}=2 \mathrm{C}([\mathrm{H}])=\mathrm{C}([\mathrm{H}]) \mathrm{C}([\mathrm{H}])=\mathrm{C}([\mathrm{H}]) \mathrm{C}=2[\mathrm{H}]) \mathrm{C} 3=\mathrm{C}([\mathrm{H}]) \mathrm{C}([\mathrm{Cl}])=\mathrm{C}([\mathrm{H}]) \\
\mathrm{C}([\mathrm{H}])=\mathrm{C} 3[\mathrm{H}]) \mathrm{N}([\mathrm{H}])[\mathrm{H}]) \mathrm{C}([\mathrm{H}])([\mathrm{H}])[\mathrm{H}]\end{array}$ & 2.523 & 2.636 \\
\hline $\operatorname{Tr} 96$ & $\begin{array}{l}\mathrm{O}=\mathrm{C} 1 \mathrm{~N}(\mathrm{C}(=\mathrm{N}[\mathrm{C} @ @] 1(\mathrm{C}=2 \mathrm{C}([\mathrm{H}])=\mathrm{C}([\mathrm{H}]) \mathrm{C}([\mathrm{H}])=\mathrm{C}([\mathrm{H}]) \mathrm{C}=2[\mathrm{H}]) \mathrm{C} 3=\mathrm{C}([\mathrm{H}]) \mathrm{C}(=\mathrm{C}([\mathrm{H}]) \mathrm{C}([\mathrm{H}] \\
)=\mathrm{C} 3[\mathrm{H}]) \mathrm{C} 4=\mathrm{C}([\mathrm{H}]) \mathrm{N}=\mathrm{C}([\mathrm{H}]) \mathrm{C}([\mathrm{H}])=\mathrm{C} 4[\mathrm{H}]) \mathrm{N}([\mathrm{H}])[\mathrm{H}]) \mathrm{C}([\mathrm{H}])([\mathrm{H}])[\mathrm{H}]\end{array}$ & 1.770 & 2.444 \\
\hline Tr97 & $\begin{array}{l}\mathrm{O}=\mathrm{C} 1 \mathrm{~N}(\mathrm{C}(=\mathrm{N}[\mathrm{C} @ @] 1(/ \mathrm{C} 2=\mathrm{C}(\backslash[\mathrm{H}]) \mathrm{C}(=\mathrm{C}([\mathrm{H}]) \mathrm{C}([\mathrm{H}])=\mathrm{C} 2[\mathrm{H}]) \mathrm{C} 3=\mathrm{C}([\mathrm{H}]) \mathrm{N}=\mathrm{C}([\mathrm{H}]) \mathrm{C}([\mathrm{F}])=\mathrm{C} 3 \\
[\mathrm{H}])[\mathrm{C} @ @] 4([\mathrm{H}]) \mathrm{C}([\mathrm{H}])([\mathrm{H}]) \mathrm{C} 4([\mathrm{H}])[\mathrm{H}]) \mathrm{N}([\mathrm{H}])[\mathrm{H}]) \mathrm{C}([\mathrm{H}])([\mathrm{H}])[\mathrm{H}]\end{array}$ & 2.796 & 2.257 \\
\hline $\operatorname{Tr} 98$ & $\begin{array}{l}\mathrm{O}=\mathrm{C} 1 \mathrm{~N}(\mathrm{C}(=\mathrm{N}[\mathrm{C} @] 1(/ \mathrm{C} 2=\mathrm{C}(\backslash[\mathrm{H}]) \mathrm{C}([\mathrm{H}])=\mathrm{C}([\mathrm{H}]) \mathrm{C}([\mathrm{H}])=\mathrm{C} 2[\mathrm{H}]) \mathrm{C} 3=\mathrm{C}([\mathrm{H}]) \mathrm{C}(=\mathrm{C}([\mathrm{H}]) \mathrm{C}([\mathrm{H}])= \\
\mathrm{C} 3[\mathrm{H}]) \mathrm{C} 4=\mathrm{C}([\mathrm{H}]) \mathrm{C}([\mathrm{H}])=\mathrm{C}([\mathrm{H}]) \mathrm{C}([\mathrm{H}])=\mathrm{C} 4[\mathrm{H}]) \mathrm{N}([\mathrm{H}])[\mathrm{H}]) \mathrm{C}([\mathrm{H}])([\mathrm{H}])[\mathrm{H}]\end{array}$ & 1.481 & 1.532 \\
\hline $\operatorname{Tr} 99$ & $\begin{array}{l}\mathrm{O}=\mathrm{C} 1 \mathrm{~N}(\mathrm{C}(=\mathrm{N}[\mathrm{C} @] 1(\mathrm{C}=2 \mathrm{C}([\mathrm{H}])=\mathrm{C}([\mathrm{H}]) \mathrm{C}([\mathrm{H}])=\mathrm{C}([\mathrm{H}]) \mathrm{C}=2[\mathrm{H}]) \mathrm{C} 3=\mathrm{C}([\mathrm{H}]) \mathrm{C}(=\mathrm{C}([\mathrm{H}]) \mathrm{C}([\mathrm{H}])= \\
\mathrm{C} 3[\mathrm{H}]) \mathrm{C} 4=\mathrm{C}([\mathrm{H}]) \mathrm{C}([\mathrm{H}])=\mathrm{C}(\mathrm{OC}([\mathrm{H}])([\mathrm{H}])[\mathrm{H}]) \mathrm{C}([\mathrm{H}])=\mathrm{C} 4[\mathrm{H}]) \mathrm{N}([\mathrm{H}])[\mathrm{H}]) \mathrm{C}([\mathrm{H}])([\mathrm{H}])[\mathrm{H}]\end{array}$ & 1.420 & 1.964 \\
\hline $\operatorname{Tr} 100$ & $\begin{array}{l}\mathrm{O}=\mathrm{C} 3 \mathrm{~N}([\mathrm{H}])[\mathrm{C} @]([\mathrm{H}])(\mathrm{C}(=\mathrm{O}) \mathrm{N}([\mathrm{H}])[\mathrm{C} @([\mathrm{H}])([\mathrm{C} @]([\mathrm{H}])(\mathrm{O}[\mathrm{H}]) \mathrm{C}([\mathrm{H}])([\mathrm{H}]) \mathrm{N}([\mathrm{H}]) \mathrm{C}([\mathrm{H}])([ \\
\mathrm{H}]) \mathrm{C} 1=\mathrm{C}([\mathrm{H}]) \mathrm{C}(=\mathrm{C}([\mathrm{H}]) \mathrm{C}([\mathrm{H}])=\mathrm{C} 1[\mathrm{H}]) \mathrm{N}(\mathrm{C}([\mathrm{H}])([\mathrm{H}])[\mathrm{H}]) \mathrm{C}([\mathrm{H}])([\mathrm{H}])[\mathrm{H}]) \mathrm{C}([\mathrm{H}])([\mathrm{H}]) \mathrm{C} 2=\mathrm{C}([ \\
\mathrm{H}]) \mathrm{C}([\mathrm{H}])=\mathrm{C}([\mathrm{H}]) \mathrm{C}([\mathrm{H}])=\mathrm{C} 2[\mathrm{H}]) \mathrm{C}([\mathrm{H}])([\mathrm{H}]) \mathrm{N} 3 \mathrm{C}([\mathrm{H}])([\mathrm{H}]) \mathrm{C} 4=\mathrm{C}([\mathrm{H}]) \mathrm{C}([\mathrm{H}])=\mathrm{C}([\mathrm{H}]) \mathrm{C}([\mathrm{H}])= \\
\mathrm{C} 4[\mathrm{H}]\end{array}$ & 2.547 & 1.847 \\
\hline $\operatorname{Tr} 101$ & $\begin{array}{l}\mathrm{O}=\mathrm{C} 1 \mathrm{~N}(\mathrm{C}(=\mathrm{N}[\mathrm{C} @ @] 1(/ \mathrm{C} 2=\mathrm{C}(\backslash[\mathrm{H}]) \mathrm{C}([\mathrm{H}])=\mathrm{C}([\mathrm{H}]) \mathrm{C}([\mathrm{H}])=\mathrm{C} 2[\mathrm{H}]) \mathrm{C} 3=\mathrm{C}([\mathrm{H}]) \mathrm{C}(\mathrm{C} \# \mathrm{~N})=\mathrm{C}([\mathrm{H}]) \\
\mathrm{C}([\mathrm{H}])=\mathrm{C} 3[\mathrm{H}]) \mathrm{N}([\mathrm{H}])[\mathrm{H}]) \mathrm{C}([\mathrm{H}])([\mathrm{H}])[\mathrm{H}]\end{array}$ & 2.432 & 2.432 \\
\hline $\operatorname{Tr} 102$ & $\begin{array}{l}\mathrm{O}=\mathrm{C} 1 \mathrm{~N}(\mathrm{C}(=\mathrm{N}[\mathrm{C} @] 1(\mathrm{C}=2 \mathrm{C}([\mathrm{H}])=\mathrm{C}([\mathrm{H}]) \mathrm{C}([\mathrm{H}])=\mathrm{C}([\mathrm{H}]) \mathrm{C}=2[\mathrm{H}]) \mathrm{C} 3=\mathrm{C}([\mathrm{H}]) \mathrm{C}(=\mathrm{C}([\mathrm{H}]) \mathrm{C}([\mathrm{H}])= \\
\mathrm{C} 3[\mathrm{H}]) \mathrm{C} 4=\mathrm{NC}([\mathrm{H}])=\mathrm{C}([\mathrm{H}]) \mathrm{C}([\mathrm{H}])=\mathrm{C} 4[\mathrm{H}]) \mathrm{N}([\mathrm{H}])[\mathrm{H}]) \mathrm{C}([\mathrm{H}])([\mathrm{H}])[\mathrm{H}]\end{array}$ & 1.721 & 2.336 \\
\hline $\operatorname{Tr} 103$ & $\begin{array}{l}\mathrm{O}=\mathrm{C}(\mathrm{N}([\mathrm{C} @ @] 1([\mathrm{H}]) \mathrm{C}([\mathrm{H}])([\mathrm{H}]) \mathrm{C}([\mathrm{H}])([\mathrm{H}]) \mathrm{C}([\mathrm{H}])([\mathrm{H}]) \mathrm{C}([\mathrm{H}])([\mathrm{H}]) \mathrm{C} 1([\mathrm{H}])[\mathrm{H}]) \mathrm{C}([\mathrm{H}])([\mathrm{H}])[ \\
\mathrm{H}]) \mathrm{C}([\mathrm{H}])([\mathrm{H}]) \mathrm{C}([\mathrm{H}])([\mathrm{H}])[\mathrm{C} @]([\mathrm{H}])(\mathrm{N} 4 \mathrm{C}(=\mathrm{N} / \mathrm{C} 3=\mathrm{C}(\backslash[\mathrm{H}]) \mathrm{C}([\mathrm{H}])=\mathrm{C}(\mathrm{O} / \mathrm{C} 2=\mathrm{C}(\backslash[\mathrm{H}]) \mathrm{C}([\mathrm{H}])=\mathrm{C} \\
([\mathrm{H}]) \mathrm{C}([\mathrm{H}])=\mathrm{C} 2[\mathrm{H}]) \mathrm{C}([\mathrm{H}])=\mathrm{C} 3 \mathrm{C} 4([\mathrm{H}])[\mathrm{H}]) \mathrm{N}([\mathrm{H}])[\mathrm{H}]) \mathrm{C} 5=\mathrm{C}([\mathrm{H}]) \mathrm{C}([\mathrm{H}])=\mathrm{C}([\mathrm{H}]) \mathrm{C}([\mathrm{H}])=\mathrm{C} 5[\mathrm{H} \\
]\end{array}$ & 2.785 & 2.466 \\
\hline $\operatorname{Tr} 104$ & $\begin{array}{l}\mathrm{O}=\mathrm{C}(\mathrm{N}([\mathrm{C} @ @] 1([\mathrm{H}]) \mathrm{C}([\mathrm{H}])([\mathrm{H}]) \mathrm{C}([\mathrm{H}])([\mathrm{H}]) \mathrm{C}([\mathrm{H}])([\mathrm{H}]) \mathrm{C}([\mathrm{H}])([\mathrm{H}]) \mathrm{C} 1([\mathrm{H}])[\mathrm{H}]) \mathrm{C}([\mathrm{H}])([\mathrm{H}]) \\
\mathrm{C} 2=\mathrm{C}([\mathrm{H}]) \mathrm{N}([\mathrm{H}]) \mathrm{N}=\mathrm{C} 2[\mathrm{H}]) \mathrm{C}([\mathrm{H}])([\mathrm{H}]) \mathrm{C}([\mathrm{H}])([\mathrm{H}])[\mathrm{C} @]([\mathrm{H}])(\mathrm{N} 5 \mathrm{C}(=\mathrm{NC}=4 \mathrm{C}([\mathrm{H}])=\mathrm{C}([\mathrm{H}]) \mathrm{C}( \\
\mathrm{O} / \mathrm{C} 3=\mathrm{C}([\mathrm{H}]) \mathrm{C}([\mathrm{H}])=\mathrm{C}([\mathrm{H}]) \mathrm{C}([\mathrm{H}])=\mathrm{C} 3[\mathrm{H}])=\mathrm{C}([\mathrm{H}]) \mathrm{C}=4 \mathrm{C} 5([\mathrm{H}])[\mathrm{H}]) \mathrm{N}([\mathrm{H}])[\mathrm{H}])[\mathrm{C} @ @] 6([\mathrm{H}]) \\
\mathrm{C}([\mathrm{H}])([\mathrm{H}])\end{array}$ & 3.638 & 2.778 \\
\hline $\operatorname{Tr} 105$ & $\begin{array}{l}\mathrm{O}=\mathrm{C}(\mathrm{N}([\mathrm{C} @ @] 1([\mathrm{H}]) \mathrm{C}([\mathrm{H}])([\mathrm{H}]) \mathrm{C}([\mathrm{H}])([\mathrm{H}]) \mathrm{C}([\mathrm{H}])([\mathrm{H}]) \mathrm{C}([\mathrm{H}])([\mathrm{H}]) \mathrm{C} 1([\mathrm{H}])[\mathrm{H}]) \mathrm{C}([\mathrm{H}])([\mathrm{H}]) \\
\mathrm{C} 2=\mathrm{C}([\mathrm{H}]) \mathrm{N}(/ \mathrm{N}=\mathrm{C} 2 /[\mathrm{H}]) \mathrm{C}([\mathrm{H}])([\mathrm{H}])[\mathrm{H}]) \mathrm{C}([\mathrm{H}])([\mathrm{H}]) \mathrm{C}([\mathrm{H}])([\mathrm{H}])[\mathrm{C} @]([\mathrm{H}])(\mathrm{N} 5 \mathrm{C}(=\mathrm{NC}=4 \mathrm{C}([\mathrm{H} \\
])=\mathrm{C}([\mathrm{H}]) \mathrm{C}(\mathrm{O} / \mathrm{C} 3=\mathrm{C}(\mathrm{V}]) \mathrm{H}([\mathrm{H}])=\mathrm{C}([\mathrm{H}]) \mathrm{C}([\mathrm{H}])=\mathrm{C} 3[\mathrm{H}])=\mathrm{C}([\mathrm{H}]) \mathrm{C}=4 \mathrm{C} 5([\mathrm{H}])[\mathrm{H}]) \mathrm{N}([\mathrm{H}])[\mathrm{H}])[ \\
\mathrm{C} @ @] 6([\mathrm{H}\end{array}$ & 3.959 & 3.505 \\
\hline $\operatorname{Tr} 106$ & $\begin{array}{l}\mathrm{O}=\mathrm{C}(\mathrm{N}([\mathrm{C} @ @] 1([\mathrm{H}]) \mathrm{C}([\mathrm{H}])([\mathrm{H}]) \mathrm{C}([\mathrm{H}])([\mathrm{H}]) \mathrm{C}([\mathrm{H}])([\mathrm{H}]) \mathrm{C}([\mathrm{H}])([\mathrm{H}]) \mathrm{C} 1([\mathrm{H}])[\mathrm{H}]) \mathrm{C}([\mathrm{H}])([\mathrm{H}]) \\
\mathrm{C}([\mathrm{H}])([\mathrm{H}]) \mathrm{C}=2 \mathrm{~N}=\mathrm{C}([\mathrm{S}] \mathrm{C}=2[\mathrm{H}]) \mathrm{C}([\mathrm{H}])([\mathrm{H}])[\mathrm{H}]) \mathrm{C}([\mathrm{H}])([\mathrm{H}]) \mathrm{C}([\mathrm{H}])([\mathrm{H}])[\mathrm{C} @]([\mathrm{H}])(\mathrm{N} 5 \mathrm{C}(=\mathrm{N} \\
\mathrm{C}=4 \mathrm{C}([\mathrm{H}])=\mathrm{C}([\mathrm{H}]) \mathrm{C}(\mathrm{O} / \mathrm{C} 3=\mathrm{C}(1[\mathrm{H}]) \mathrm{C}([\mathrm{H}])=\mathrm{C}([\mathrm{H}]) \mathrm{C}([\mathrm{H}])=\mathrm{C} 3[\mathrm{H}])=\mathrm{C}([\mathrm{H}]) \mathrm{C}=4 \mathrm{C} 5([\mathrm{H}])[\mathrm{H}]) \mathrm{N}([ \\
\mathrm{H}])[\mathrm{H}])[\mathrm{C}\end{array}$ & 2.975 & 3.331 \\
\hline
\end{tabular}




\begin{tabular}{|c|c|c|c|}
\hline $\operatorname{Tr} 107$ & $\begin{array}{l}\mathrm{O}=\mathrm{C}(\mathrm{N}([\mathrm{C} @ @] 1([\mathrm{H}]) \mathrm{C}([\mathrm{H}])([\mathrm{H}]) \mathrm{C}([\mathrm{H}])([\mathrm{H}]) \mathrm{C}([\mathrm{H}])([\mathrm{H}]) \mathrm{C}([\mathrm{H}])([\mathrm{H}]) \mathrm{C} 1([\mathrm{H}])[\mathrm{H}])[\mathrm{C} @ @]([\mathrm{H}] \\
)(\mathrm{C}=2 \mathrm{~N}=\mathrm{C}([\mathrm{S}] \mathrm{C}=2[\mathrm{H}]) \mathrm{C}([\mathrm{H}])([\mathrm{H}])[\mathrm{H}]) \mathrm{C}([\mathrm{H}])([\mathrm{H}])[\mathrm{H}]) \mathrm{C}([\mathrm{H}])([\mathrm{H}]) \mathrm{C}([\mathrm{H}])([\mathrm{H}])[\mathrm{C} @]([\mathrm{H}])(\mathrm{N} 5 \\
\mathrm{C}(=\mathrm{NC}=4 \mathrm{C}([\mathrm{H}])=\mathrm{C}([\mathrm{H}]) \mathrm{C}(\mathrm{O} / \mathrm{C} 3=\mathrm{C}(\backslash[\mathrm{H}]) \mathrm{C}([\mathrm{H}])=\mathrm{C}([\mathrm{H}]) \mathrm{C}([\mathrm{H}])=\mathrm{C} 3[\mathrm{H}])=\mathrm{C}([\mathrm{H}]) \mathrm{C}=4 \mathrm{C} 5([\mathrm{H}])[\mathrm{H} \\
]) \mathrm{N}([\mathrm{H}])[\mathrm{H}\end{array}$ & 2.983 & 3.211 \\
\hline $\operatorname{Tr} 108$ & $\begin{array}{l}\mathrm{O}=\mathrm{C}(\mathrm{N}([\mathrm{C} @ @] 1([\mathrm{H}]) \mathrm{C}([\mathrm{H}])([\mathrm{H}]) \mathrm{C}([\mathrm{H}])([\mathrm{H}]) \mathrm{C}([\mathrm{H}])([\mathrm{H}]) \mathrm{C}([\mathrm{H}])([\mathrm{H}]) \mathrm{C} 1([\mathrm{H}])[\mathrm{H}]) \mathrm{C}([\mathrm{H}])([\mathrm{H}]) \\
\mathrm{C}=2 \mathrm{~N}=\mathrm{C}([\mathrm{S}] \mathrm{C}=2[\mathrm{H}]) \mathrm{C}([\mathrm{H}])([\mathrm{H}])[\mathrm{H}]) \mathrm{C}([\mathrm{H}])([\mathrm{H}]) \mathrm{C}([\mathrm{H}])([\mathrm{H}])[\mathrm{C} @]([\mathrm{H}])(\mathrm{N} 5 \mathrm{C}(=\mathrm{NC}=4 \mathrm{C}([\mathrm{H}])= \\
\mathrm{C}([\mathrm{H}]) \mathrm{C}(\mathrm{O} / \mathrm{C} 3=\mathrm{C}(\backslash[\mathrm{H}]) \mathrm{C}([\mathrm{H}])=\mathrm{C}([\mathrm{H}]) \mathrm{C}([\mathrm{H}])=\mathrm{C} 3[\mathrm{H}])=\mathrm{C}([\mathrm{H}]) \mathrm{C}=4 \mathrm{C} 5([\mathrm{H}])[\mathrm{H}]) \mathrm{N}([\mathrm{H}])[\mathrm{H}])[\mathrm{C} @ \\
@] 6([\mathrm{H}]) \mathrm{C}(\end{array}$ & 3.102 & 3.307 \\
\hline Tr109 & $\begin{array}{l}\mathrm{O}=\mathrm{C}(\mathrm{OC}([\mathrm{H}])([\mathrm{H}])[\mathrm{C} @]([\mathrm{H}])(\mathrm{N} 3 \mathrm{C}(=\mathrm{N} / \mathrm{C} 2=\mathrm{C}(\backslash[\mathrm{H}]) \mathrm{C}([\mathrm{H}])=\mathrm{C}(\mathrm{O} / \mathrm{C} 1=\mathrm{C}(\backslash \mathrm{H}]) \mathrm{C}([\mathrm{H}])=\mathrm{C}([\mathrm{H}]) \mathrm{C}( \\
[\mathrm{H}])=\mathrm{C} 1[\mathrm{H}]) \mathrm{C}([\mathrm{H}])=\mathrm{C} 2 \mathrm{C} 3([\mathrm{H}])[\mathrm{H}]) \mathrm{N}([\mathrm{H}])[\mathrm{H}])[\mathrm{C} @] 4([\mathrm{H}]) \mathrm{C}([\mathrm{H}])([\mathrm{H}]) \mathrm{OC}([\mathrm{H}])([\mathrm{H}]) \mathrm{C}([\mathrm{H}])([\mathrm{H} \\
]) \mathrm{C} 4([\mathrm{H}])[\mathrm{H}]) \mathrm{N}([\mathrm{C} @ @] 5([\mathrm{H}]) \mathrm{C}([\mathrm{H}])([\mathrm{H}]) \mathrm{C}([\mathrm{H}])([\mathrm{H}]) \mathrm{C}([\mathrm{H}])([\mathrm{H}]) \mathrm{C}([\mathrm{H}])([\mathrm{H}]) \mathrm{C} 5([\mathrm{H}]))[\mathrm{H}]) \mathrm{C}([ \\
\mathrm{H}])([\mathrm{H}]\end{array}$ & 2.983 & 3.368 \\
\hline $\operatorname{Tr} 110$ & $\begin{array}{l}\mathrm{O}=\mathrm{C}(\mathrm{OC}([\mathrm{H}])([\mathrm{H}])[\mathrm{C} @]([\mathrm{H}])(\mathrm{N} 3 \mathrm{C}(=\mathrm{N} / \mathrm{C} 2=\mathrm{C}(\backslash[\mathrm{H}]) \mathrm{C}([\mathrm{H}])=\mathrm{C}(\mathrm{O} / \mathrm{C} 1=\mathrm{C}(\mathrm{VH}]) \mathrm{C}([\mathrm{H}])=\mathrm{C}([\mathrm{H}]) \mathrm{C}( \\
[\mathrm{H}])=\mathrm{C} 1[\mathrm{H}]) \mathrm{C}([\mathrm{H}])=\mathrm{C} 2 \mathrm{C} 3([\mathrm{H}])[\mathrm{H}]) \mathrm{N}([\mathrm{H}])[\mathrm{H}]) \mathrm{C} 4=\mathrm{C}([\mathrm{H}]) \mathrm{C}([\mathrm{H}])=\mathrm{C}([\mathrm{H}]) \mathrm{C}([\mathrm{H}])=\mathrm{C} 4[\mathrm{H}]) \mathrm{N}([\mathrm{C} \\
@ @] 5([\mathrm{H}]) \mathrm{C}([\mathrm{H}])([\mathrm{H}]) \mathrm{C}([\mathrm{H}])([\mathrm{H}]) \mathrm{C}([\mathrm{H}])([\mathrm{H}]) \mathrm{C}([\mathrm{H}])([\mathrm{H}]) \mathrm{C} 5([\mathrm{H}])[\mathrm{H}]) \mathrm{C}([\mathrm{H}])([\mathrm{H}])[\mathrm{H}]\end{array}$ & 2.106 & 2.462 \\
\hline $\operatorname{Tr} 111$ & 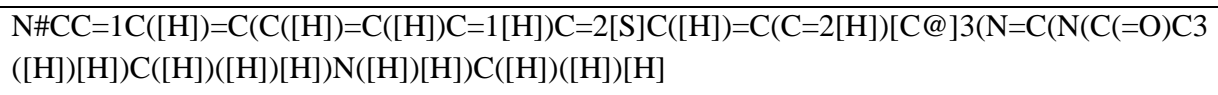 & 2.678 & 2.720 \\
\hline $\operatorname{Tr} 112$ & 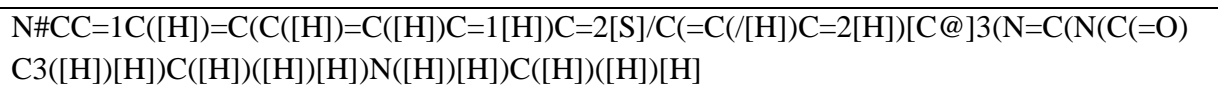 & 3.056 & 3.187 \\
\hline $\operatorname{Tr} 113$ & $\begin{array}{l}\mathrm{O}=\mathrm{C} 3 \mathrm{~N}(\mathrm{C}(=\mathrm{N}[\mathrm{C} @](\mathrm{C}=1 \mathrm{C}([\mathrm{H}])=\mathrm{C}([\mathrm{S}] \mathrm{C}=1[\mathrm{H}]) \mathrm{C} 2=\mathrm{C}([\mathrm{H}]) \mathrm{C}(\mathrm{C \# CC}([\mathrm{H}])([\mathrm{H}])[\mathrm{H}])=\mathrm{C}([\mathrm{H}]) \mathrm{N}=\mathrm{C} \\
2[\mathrm{H}])(\mathrm{C} 3([\mathrm{H}])[\mathrm{H}]) \mathrm{C}([\mathrm{H}])([\mathrm{H}])[\mathrm{H}]) \mathrm{N}([\mathrm{H}])[\mathrm{H}]) \mathrm{C}([\mathrm{H}])([\mathrm{H}])[\mathrm{H}]\end{array}$ & 3.328 & 3.218 \\
\hline $\operatorname{Tr} 114$ & $\begin{array}{l}\mathrm{O}=\mathrm{C} 4 \mathrm{~N}(\mathrm{C}(=\mathrm{N}[\mathrm{C} @ @](/ \mathrm{C} 1=\mathrm{C}(\backslash[\mathrm{H}]) \mathrm{C}([\mathrm{H}])=\mathrm{C}([\mathrm{H}]) \mathrm{C}(=\mathrm{C} 1[\mathrm{H}]) \mathrm{C} 2=\mathrm{C}([\mathrm{H}]) \mathrm{N}=\mathrm{C}([\mathrm{H}]) \mathrm{C}([\mathrm{H}])=\mathrm{C} 2[ \\
\mathrm{H}])(\mathrm{C} 3=\mathrm{C}([\mathrm{H}]) \mathrm{C}([\mathrm{H}])=\mathrm{C}([\mathrm{H}]) \mathrm{C}([\mathrm{H}])=\mathrm{C} 3[\mathrm{H}]) \mathrm{C} 4([\mathrm{H}])[\mathrm{H}]) \mathrm{N}([\mathrm{H}])[\mathrm{H}]) \mathrm{C}([\mathrm{H}])([\mathrm{H}])[\mathrm{H}]\end{array}$ & 0.357 & 0.967 \\
\hline $\operatorname{Tr} 115$ & $\begin{array}{l}\mathrm{O}=\mathrm{C} 3 \mathrm{~N}(\mathrm{C}(=\mathrm{N}[\mathrm{C} @](\mathrm{C}=2[\mathrm{~S}] \mathrm{C}(\mathrm{C}=1 \mathrm{C}([\mathrm{H}])=\mathrm{C}(\mathrm{C \# CC}([\mathrm{H}])([\mathrm{H}])[\mathrm{H}]) \mathrm{C}([\mathrm{H}])=\mathrm{NC}=1[\mathrm{H}])=\mathrm{C}([\mathrm{H}]) \mathrm{C} \\
=2[\mathrm{Cl}])(\mathrm{C} 3([\mathrm{H}])[\mathrm{H}]) \mathrm{C}([\mathrm{H}])([\mathrm{H}])[\mathrm{H}]) \mathrm{N}([\mathrm{H}])[\mathrm{H}]) \mathrm{C}([\mathrm{H}])([\mathrm{H}])[\mathrm{H}]\end{array}$ & 4.770 & 5.025 \\
\hline $\operatorname{Tr} 116$ & $\begin{array}{l}\mathrm{O}=\mathrm{C}(\mathrm{O}[\mathrm{C} @] 2([\mathrm{H}])[\mathrm{C} @ @]([\mathrm{H}])(\mathrm{OC} 1=\mathrm{C}(\mathrm{C}(\mathrm{O}[\mathrm{H}])=\mathrm{C}([\mathrm{H}]) \mathrm{C}(\mathrm{O}[\mathrm{H}])=\mathrm{C} 1[\mathrm{H}]) \mathrm{C} 2([\mathrm{H}])[\mathrm{H}]) \mathrm{C}=3 \mathrm{C}( \\
[\mathrm{H}])=\mathrm{C}(\mathrm{O}[\mathrm{H}]) \mathrm{C}(\mathrm{O}[\mathrm{H}])=\mathrm{C}(\mathrm{O}[\mathrm{H}]) \mathrm{C}=3[\mathrm{H}]) \mathrm{C}=4 \mathrm{C}([\mathrm{H}])=\mathrm{C}(\mathrm{O}[\mathrm{H}]) \mathrm{C}(\mathrm{O}[\mathrm{H}])=\mathrm{C}(\mathrm{O}[\mathrm{H}]) \mathrm{C}=4[\mathrm{H}]\end{array}$ & 2.678 & 2.775 \\
\hline $\operatorname{Tr} 117$ & $\begin{array}{l}\mathrm{O}=\mathrm{C}(\mathrm{N}([\mathrm{H}]) \mathrm{C}([\mathrm{H}])([\mathrm{H}]) \mathrm{C}([\mathrm{H}])([\mathrm{H}]) \mathrm{C}([\mathrm{H}])([\mathrm{H}]) \mathrm{C}([\mathrm{H}])([\mathrm{H}]) \mathrm{C}([\mathrm{H}])([\mathrm{H}]) \mathrm{C}([\mathrm{H}])([\mathrm{H}]) \mathrm{C}([\mathrm{H}])([\mathrm{H}] \\
) \mathrm{C}([\mathrm{H}])([\mathrm{H}]) \mathrm{C}([\mathrm{H}])([\mathrm{H}]) \mathrm{C}([\mathrm{H}])([\mathrm{H}]) \mathrm{N}([\mathrm{H}]) \mathrm{C}=2 \mathrm{C}=1 \mathrm{C}([\mathrm{H}])=\mathrm{C}([\mathrm{H}]) \mathrm{C}([\mathrm{H}])=\mathrm{C}([\mathrm{H}]) \mathrm{C}=1 \mathrm{~N}=\mathrm{C} 3 \mathrm{C} \\
=2 \mathrm{C}([\mathrm{H}])([\mathrm{H}]) \mathrm{C}([\mathrm{H}])([\mathrm{H}]) \mathrm{C}([\mathrm{H}])([\mathrm{H}]) \mathrm{C} 3([\mathrm{H}])[\mathrm{H}]) \mathrm{C}=4 \mathrm{OC}=5 \mathrm{C}(\mathrm{C}(=\mathrm{O}) \mathrm{C}=4[\mathrm{H}])=\mathrm{C}(\mathrm{OC}([\mathrm{H}])([\mathrm{H} \\
])[\mathrm{H}]) \mathrm{C}\end{array}$ & 2.208 & 2.492 \\
\hline $\operatorname{Tr} 118$ & $\begin{array}{l}\mathrm{O}=\mathrm{C}(\mathrm{N}([\mathrm{H}]) \mathrm{C}([\mathrm{H}])([\mathrm{H}]) \mathrm{C}([\mathrm{H}])([\mathrm{H}]) \mathrm{C}([\mathrm{H}])([\mathrm{H}]) \mathrm{C}([\mathrm{H}])([\mathrm{H}]) \mathrm{C}([\mathrm{H}])([\mathrm{H}]) \mathrm{C}([\mathrm{H}])([\mathrm{H}]) \mathrm{C}([\mathrm{H}])([\mathrm{H}] \\
) \mathrm{C}([\mathrm{H}])([\mathrm{H}]) \mathrm{C}([\mathrm{H}])([\mathrm{H}]) \mathrm{C}([\mathrm{H}])([\mathrm{H}]) \mathrm{N}([\mathrm{H}]) \mathrm{C} 2=\mathrm{C} 1 \mathrm{C}([\mathrm{H}])=\mathrm{C}([\mathrm{H}]) \mathrm{C}([\mathrm{H}])=\mathrm{C}([\mathrm{H}]) \mathrm{C} 1=\mathrm{NC} 3=\mathrm{C} 2 \\
\mathrm{C}([\mathrm{H}])([\mathrm{H}]) \mathrm{C}([\mathrm{H}])([\mathrm{H}]) \mathrm{C}([\mathrm{H}])([\mathrm{H}]) \mathrm{C} 3([\mathrm{H}])[\mathrm{H}]) \mathrm{C}=4 \mathrm{OC}=5 \mathrm{C}(\mathrm{C}(=\mathrm{O}) \mathrm{C}=4[\mathrm{H}])=\mathrm{C}(\mathrm{O}[\mathrm{H}]) \mathrm{C}([\mathrm{H}])= \\
\mathrm{C}(\mathrm{OC}([\mathrm{H}\end{array}$ & 2.071 & 1.969 \\
\hline $\operatorname{Tr} 119$ & $\begin{array}{l}\mathrm{O}=\mathrm{C}(\mathrm{N}([\mathrm{H}]) \mathrm{C}([\mathrm{H}])([\mathrm{H}]) \mathrm{C}([\mathrm{H}])([\mathrm{H}]) \mathrm{C}([\mathrm{H}])([\mathrm{H}]) \mathrm{C}([\mathrm{H}])([\mathrm{H}]) \mathrm{C}([\mathrm{H}])([\mathrm{H}]) \mathrm{C}([\mathrm{H}])([\mathrm{H}]) \mathrm{C}([\mathrm{H}])([\mathrm{H}] \\
) \mathrm{C}([\mathrm{H}])([\mathrm{H}]) \mathrm{C}([\mathrm{H}])([\mathrm{H}]) \mathrm{C}([\mathrm{H}])([\mathrm{H}]) \mathrm{N}([\mathrm{H}]) \mathrm{C}=2 \mathrm{C}=1 \mathrm{C}([\mathrm{H}])=\mathrm{C}([\mathrm{H}]) \mathrm{C}([\mathrm{H}])=\mathrm{C}([\mathrm{H}]) \mathrm{C}=1 \mathrm{~N}=\mathrm{C} 3 \mathrm{C} \\
=2 \mathrm{C}([\mathrm{H}])([\mathrm{H}]) \mathrm{C}([\mathrm{H}])([\mathrm{H}]) \mathrm{C}([\mathrm{H}])([\mathrm{H}]) \mathrm{C} 3([\mathrm{H}])[\mathrm{H}]) \mathrm{C}=4 \mathrm{OC}=5 \mathrm{C}(\mathrm{C}(=\mathrm{O}) \mathrm{C}=4[\mathrm{H}])=\mathrm{C}([\mathrm{H}]) \mathrm{C}(\mathrm{OC}([ \\
\mathrm{H}])([\mathrm{H}]\end{array}$ & 2.056 & 2.492 \\
\hline $\operatorname{Tr} 120$ & $\begin{array}{l}\mathrm{O}=\mathrm{C} 2 \mathrm{~N}(/ \mathrm{C}(=\mathrm{N} \backslash[\mathrm{H}]) \mathrm{N}([\mathrm{H}])[\mathrm{C} @] 4(\mathrm{C}=1 \mathrm{C}([\mathrm{F}])=\mathrm{C}([\mathrm{H}]) \mathrm{C}([\mathrm{F}])=\mathrm{C}([\mathrm{H}]) \mathrm{C}=1[\mathrm{H}])[\mathrm{C} @ @] 2([\mathrm{H}]) \mathrm{C}([ \\
\mathrm{H}])([\mathrm{H}]) \mathrm{N}(/ \mathrm{C} 3=\mathrm{N} / \mathrm{C}(\mathrm{OC}([\mathrm{H}])([\mathrm{H}])[\mathrm{H}])=\mathrm{C}([\mathrm{F}]) \mathrm{C}(=\mathrm{N} 3) \mathrm{C}([\mathrm{H}])([\mathrm{H}])[\mathrm{H}]) \mathrm{C} 4([\mathrm{H}])[\mathrm{H}]) \mathrm{C}([\mathrm{H}])([\mathrm{H}]) \\
{[\mathrm{H}]}\end{array}$ & 4.328 & 3.980 \\
\hline $\operatorname{Tr} 121$ & $\begin{array}{l}\mathrm{O}=\mathrm{C} 2 \mathrm{~N}(/ \mathrm{C}(=\mathrm{N} \backslash[\mathrm{H}]) \mathrm{N}([\mathrm{H}])[\mathrm{C} @] 4(\mathrm{C}=1 \mathrm{C}([\mathrm{F}])=\mathrm{C}([\mathrm{H}]) \mathrm{C}([\mathrm{H}])=\mathrm{C}([\mathrm{Cl}]) \mathrm{C}=1[\mathrm{H}])[\mathrm{C} @ @] 2([\mathrm{H}]) \mathrm{C}([ \\
\mathrm{H}])([\mathrm{H}]) \mathrm{N}(/ \mathrm{C} 3=\mathrm{N} / \mathrm{C}(\mathrm{OC}([\mathrm{H}])([\mathrm{H}])[\mathrm{H}])=\mathrm{C}([\mathrm{F}]) \mathrm{C}(=\mathrm{N} 3) \mathrm{C}([\mathrm{H}])([\mathrm{H}])[\mathrm{H}]) \mathrm{C} 4([\mathrm{H}])[\mathrm{H}]) \mathrm{C}([\mathrm{H}])([\mathrm{H}]) \\
{[\mathrm{H}]}\end{array}$ & 3.886 & 3.901 \\
\hline
\end{tabular}




\begin{tabular}{|c|c|c|c|}
\hline $\operatorname{Tr} 122$ & $\begin{array}{l}\mathrm{O}=\mathrm{C}(\mathrm{N}([\mathrm{H}]) \mathrm{C} 3=\mathrm{C}([\mathrm{H}]) \mathrm{C}([\mathrm{H}])=\mathrm{C} 2 \mathrm{OC}([\mathrm{C} @] 4([\mathrm{C} @ @] 1(\mathrm{~N}=\mathrm{C}(\mathrm{OC} 1([\mathrm{H}])[\mathrm{H}]) \mathrm{N}([\mathrm{H}])[\mathrm{H}]) \mathrm{C} 2=\mathrm{C} 3 \\
[\mathrm{H}]) \mathrm{C}([\mathrm{H}])([\mathrm{H}]) \mathrm{OC} 4([\mathrm{H}])[\mathrm{H}])(\mathrm{C}([\mathrm{H}])([\mathrm{H}])[\mathrm{H}]) \mathrm{C}([\mathrm{H}])([\mathrm{H}])[\mathrm{H}]) \mathrm{C}=5 / \mathrm{N}=\mathrm{C}(/[\mathrm{H}]) \mathrm{C}([\mathrm{Cl}])=\mathrm{C}([\mathrm{H}]) \\
\mathrm{C}=5[\mathrm{H}]\end{array}$ & 3.418 & 2.935 \\
\hline $\operatorname{Tr} 123$ & $\begin{array}{l}\mathrm{O}=\mathrm{C}(\mathrm{N}([\mathrm{H}]) \mathrm{C} 3=\mathrm{C}([\mathrm{H}]) \mathrm{C}([\mathrm{H}])=\mathrm{C} 2 \mathrm{OC}([\mathrm{H}])([\mathrm{H}])[\mathrm{C} @] 4([\mathrm{C} @ @] 1(\mathrm{~N}=\mathrm{C}(\mathrm{OC} 1([\mathrm{H}])[\mathrm{H}]) \mathrm{N}([\mathrm{H}])[ \\
\mathrm{H}]) \mathrm{C} 2=\mathrm{C} 3[\mathrm{H}]) \mathrm{C}([\mathrm{H}])([\mathrm{H}]) \mathrm{C} 4([\mathrm{H}])[\mathrm{H}]) \mathrm{C}=5 / \mathrm{N}=\mathrm{C}([\mathrm{H}]) \mathrm{C}([\mathrm{Cl}])=\mathrm{C}([\mathrm{H}]) \mathrm{C}=5[\mathrm{~F}]\end{array}$ & 2.620 & 2.473 \\
\hline $\operatorname{Tr} 124$ & $\begin{array}{l}\mathrm{O}=\mathrm{C}(\mathrm{N}([\mathrm{H}]) \mathrm{C} 3=\mathrm{C}([\mathrm{H}]) \mathrm{C}([\mathrm{H}])=\mathrm{C} 2 \mathrm{OC}([\mathrm{H}])([\mathrm{H}])[\mathrm{C} @] 4([\mathrm{C} @ @] 1(\mathrm{~N}=\mathrm{C}(\mathrm{OC} 1([\mathrm{H}])[\mathrm{H}]) \mathrm{N}([\mathrm{H}])[ \\
\mathrm{H}]) \mathrm{C} 2=\mathrm{C} 3[\mathrm{H}]) \mathrm{C}([\mathrm{H}])([\mathrm{H}]) \mathrm{C} 4([\mathrm{H}])[\mathrm{H}]) \mathrm{C}=5 / \mathrm{N}=\mathrm{C}([\mathrm{H}]) \mathrm{C}([\mathrm{Cl}])=\mathrm{C}([\mathrm{H}]) \mathrm{C}=5[\mathrm{H}]\end{array}$ & 2.423 & 2.659 \\
\hline $\operatorname{Tr} 125$ & $\begin{array}{l}{[\mathrm{H}] / \mathrm{C} 4=\mathrm{C}(/ \mathrm{C}([\mathrm{H}])=\mathrm{C} 1 \mathrm{C}(\mathrm{OC}([\mathrm{C} @] 3([\mathrm{C} @ @] 12 \mathrm{~N}=\mathrm{C}(\mathrm{OC} 2([\mathrm{H}])[\mathrm{H}]) \mathrm{N}([\mathrm{H}])[\mathrm{H}]) \mathrm{C}([\mathrm{H}])([\mathrm{H}]) \mathrm{OC} 3} \\
([\mathrm{H}])[\mathrm{H}])(\mathrm{C}([\mathrm{H}])([\mathrm{H}])[\mathrm{H}]) \mathrm{C}([\mathrm{H}])([\mathrm{H}])[\mathrm{H}])=\mathrm{C} 4[\mathrm{H}]) \mathrm{C} 5=\mathrm{C}([\mathrm{H}]) \mathrm{N}=\mathrm{C}([\mathrm{H}]) \mathrm{C}(\mathrm{OC}([\mathrm{H}])([\mathrm{H}])[\mathrm{H}])= \\
\mathrm{C} 5[\mathrm{H}]\end{array}$ & 2.593 & 2.642 \\
\hline $\operatorname{Tr} 126$ & $\begin{array}{l}{[\mathrm{H}] / \mathrm{C} 4=\mathrm{C}(/ \mathrm{C}([\mathrm{H}])=\mathrm{C} 1 \mathrm{C}(\mathrm{O}[\mathrm{C} @]([\mathrm{H}])([\mathrm{C} @] 3([\mathrm{C} @] 12 \mathrm{~N}=\mathrm{C}(\mathrm{OC} 2([\mathrm{H}])[\mathrm{H}]) \mathrm{N}([\mathrm{H}])[\mathrm{H}]) \mathrm{C}([\mathrm{H}])([} \\
\mathrm{H}]) \mathrm{OC} 3([\mathrm{H}])[\mathrm{H}]) \mathrm{C}([\mathrm{H}])([\mathrm{H}]) \mathrm{C}([\mathrm{H}])([\mathrm{H}])[\mathrm{H}])=\mathrm{C} 4[\mathrm{H}]) \mathrm{C} 5=\mathrm{C}([\mathrm{H}]) \mathrm{N}=\mathrm{C}([\mathrm{H}]) \mathrm{C}(\mathrm{CHCC}([\mathrm{H}])([\mathrm{H}])[ \\
\mathrm{H}])=\mathrm{C} 5[\mathrm{H}]\end{array}$ & 2.971 & 2.719 \\
\hline $\operatorname{Tr} 127$ & $\begin{array}{l}{[\mathrm{H}] / \mathrm{C} 5=\mathrm{C}(/ \mathrm{C}([\mathrm{H}])=\mathrm{C} 1 \mathrm{C}(\mathrm{O}[\mathrm{C} @ @] 4([\mathrm{C} @] 3([\mathrm{C} @ @] 12 \mathrm{~N}=\mathrm{C}(\mathrm{OC} 2([\mathrm{H}])[\mathrm{H}]) \mathrm{N}([\mathrm{H}])[\mathrm{H}]) \mathrm{C}([\mathrm{H}])([} \\
\mathrm{H}]) \mathrm{OC} 3([\mathrm{H}])[\mathrm{H}]) \mathrm{C}([\mathrm{H}])([\mathrm{H}]) \mathrm{C}([\mathrm{H}])([\mathrm{H}]) \mathrm{C} 4([\mathrm{H}])[\mathrm{H}])=\mathrm{C} 5[\mathrm{H}]) \mathrm{C} 6=\mathrm{C}([\mathrm{H}]) \mathrm{N}=\mathrm{C}([\mathrm{H}]) \mathrm{C}(\mathrm{CHCC}([ \\
\mathrm{H}])([\mathrm{H}])[\mathrm{H}])=\mathrm{C} 6[\mathrm{H}]\end{array}$ & 2.921 & 4.190 \\
\hline $\operatorname{Tr} 128$ & $\begin{array}{l}{[\mathrm{H}] / \mathrm{C} 4=\mathrm{C}(/ \mathrm{C}([\mathrm{H}])=\mathrm{C} 1 \mathrm{C}(\mathrm{OC}([\mathrm{C} @] 3([\mathrm{C} @ @] 12 \mathrm{~N}=\mathrm{C}(\mathrm{OC} 2([\mathrm{H}])[\mathrm{H}]) \mathrm{N}([\mathrm{H}])[\mathrm{H}]) \mathrm{C}([\mathrm{H}])([\mathrm{H}]) \mathrm{OC} 3} \\
([\mathrm{H}])[\mathrm{H}])(\mathrm{C}([\mathrm{H}])([\mathrm{H}])[\mathrm{H}]) \mathrm{C}([\mathrm{H}])([\mathrm{H}])[\mathrm{H}])=\mathrm{C} 4[\mathrm{H}]) \mathrm{C} 5=\mathrm{C}([\mathrm{H}]) \mathrm{N}=\mathrm{C}([\mathrm{H}]) \mathrm{C}(\mathrm{CH} \mathrm{CC}([\mathrm{H}])([\mathrm{H}])[\mathrm{H}] \\
=\mathrm{C} 5[\mathrm{H}]\end{array}$ & 3.044 & 3.452 \\
\hline $\operatorname{Tr} 129$ & $\begin{array}{l}{[\mathrm{Cl}] \mathrm{C}=5 \mathrm{C}([\mathrm{H}])=\mathrm{C}(/ \mathrm{C} 3=\mathrm{C}([\mathrm{H}]) \mathrm{C}([\mathrm{H}])=\mathrm{C} 2 \mathrm{OC}([\mathrm{C} @] 4([\mathrm{C} @ @] 11(\mathrm{~N}=\mathrm{C}(\mathrm{OC} 1([\mathrm{H}])[\mathrm{H}]) \mathrm{N}([\mathrm{H}])[\mathrm{H}]} \\
) \mathrm{C} 2=\mathrm{C} 3[\mathrm{H}]) \mathrm{C}([\mathrm{H}])([\mathrm{H}]) \mathrm{OC} 4([\mathrm{H}])[\mathrm{H}])(\mathrm{C}([\mathrm{H}])([\mathrm{H}])[\mathrm{H}]) \mathrm{C}([\mathrm{H}])([\mathrm{H}])[\mathrm{H}]) \mathrm{C}([\mathrm{H}])=\mathrm{C}([\mathrm{F}]) \mathrm{C}=5[\mathrm{H}]\end{array}$ & 2.726 & 2.107 \\
\hline $\operatorname{Tr} 130$ & $\begin{array}{l}\mathrm{O}=\mathrm{C}(\mathrm{N}([\mathrm{H}]) \mathrm{C} 3=\mathrm{C}([\mathrm{H}]) \mathrm{C}([\mathrm{H}])=\mathrm{C} 2 \mathrm{OC}([\mathrm{H}])([\mathrm{H}])[\mathrm{C} @] 4([\mathrm{C} @ @] 1(\mathrm{~N}=\mathrm{C}(\mathrm{OC} 1([\mathrm{H}])[\mathrm{H}]) \mathrm{N}([\mathrm{H}])[ \\
\mathrm{H}]) \mathrm{C} 2=\mathrm{C} 3[\mathrm{H}]) \mathrm{C}([\mathrm{H}])([\mathrm{H}]) \mathrm{C} 4([\mathrm{H}])[\mathrm{H}]) \mathrm{C} 5=\mathrm{NC}([\mathrm{H}])=\mathrm{C}([\mathrm{Br}]) \mathrm{C}([\mathrm{H}])=\mathrm{N} 5\end{array}$ & 2.206 & 2.762 \\
\hline $\operatorname{Tr} 131$ & $\begin{array}{l}\mathrm{O}=\mathrm{C}(\mathrm{N}([\mathrm{H}]) \mathrm{C} 3=\mathrm{C}([\mathrm{H}]) \mathrm{C}([\mathrm{H}])=\mathrm{C} 2 \mathrm{OC}([\mathrm{C} @] 4([\mathrm{C} @ @] 1(\mathrm{~N}=\mathrm{C}(\mathrm{OC} 1([\mathrm{H}])[\mathrm{H}]) \mathrm{N}([\mathrm{H}])[\mathrm{H}]) \mathrm{C} 2=\mathrm{C} 3 \\
[\mathrm{H}]) \mathrm{C}([\mathrm{H}])([\mathrm{H}]) \mathrm{OC} 4([\mathrm{H}])[\mathrm{H}])(\mathrm{C}([\mathrm{H}])([\mathrm{H}])[\mathrm{H}]) \mathrm{C}([\mathrm{H}])([\mathrm{H}])[\mathrm{H}]) \mathrm{C} 5=\mathrm{NC}([\mathrm{H}])=\mathrm{C}([\mathrm{Cl}]) \mathrm{C}([\mathrm{H}])=\mathrm{C} \\
5 \mathrm{C}([\mathrm{H}])([\mathrm{H}])[\mathrm{H}]\end{array}$ & 3.190 & 3.356 \\
\hline $\operatorname{Tr} 132$ & $\begin{array}{l}\mathrm{O}=\mathrm{C}(\mathrm{N}([\mathrm{H}]) \mathrm{C} 3=\mathrm{C}([\mathrm{H}]) \mathrm{C}([\mathrm{H}])=\mathrm{C} 2 \mathrm{OC}([\mathrm{H}])([\mathrm{H}])[\mathrm{C} @] 4([\mathrm{C} @ @] 1(\mathrm{~N}=\mathrm{C}(\mathrm{OC} 1([\mathrm{H}])[\mathrm{H}]) \mathrm{N}([\mathrm{H}])[ \\
\mathrm{H}]) \mathrm{C} 2=\mathrm{C} 3[\mathrm{H}]) \mathrm{C}([\mathrm{H}])([\mathrm{H}]) \mathrm{C} 4([\mathrm{H}])[\mathrm{H}]) \mathrm{C}=5 / \mathrm{N}=\mathrm{C}([/ \mathrm{H}]) \mathrm{C}(\mathrm{CHN})=\mathrm{C}([\mathrm{H}]) \mathrm{C}=5[\mathrm{H}]\end{array}$ & 2.466 & 2.762 \\
\hline $\operatorname{Tr} 133$ & $\begin{array}{l}\mathrm{O}=\mathrm{C}(\mathrm{N}([\mathrm{H}]) \mathrm{C} 3=\mathrm{C}([\mathrm{H}]) \mathrm{C}([\mathrm{H}])=\mathrm{C} 2 \mathrm{OC}([\mathrm{C} @] 4([\mathrm{C} @ @] 1(\mathrm{~N}=\mathrm{C}(\mathrm{OC} 1([\mathrm{H}])[\mathrm{H}]) \mathrm{N}([\mathrm{H}])[\mathrm{H}]) \mathrm{C} 2=\mathrm{C} 3 \\
[\mathrm{H}]) \mathrm{C}([\mathrm{H}])([\mathrm{H}]) \mathrm{OC} 4([\mathrm{H}])[\mathrm{H}])(\mathrm{C}([\mathrm{H}])([\mathrm{H}])[\mathrm{H}]) \mathrm{C}([\mathrm{H}])([\mathrm{H}])[\mathrm{H}]) \mathrm{C}=5 / \mathrm{N}=\mathrm{C}(/[\mathrm{H}]) \mathrm{C}(\mathrm{CHN})=\mathrm{C}([\mathrm{H}] \\
\mathrm{C}=5[\mathrm{H}]\end{array}$ & 3.143 & 3.038 \\
\hline $\operatorname{Tr} 134$ & $\begin{array}{l}{[\mathrm{H}] / \mathrm{C} 5=\mathrm{C}(/ \mathrm{C}([\mathrm{H}])=\mathrm{C} 1 \mathrm{C}(\mathrm{O}[\mathrm{C} @]([\mathrm{H}])([\mathrm{C} @] 3([\mathrm{C} @] 12 \mathrm{~N}=\mathrm{C}(\mathrm{OC} 2([\mathrm{H}])[\mathrm{H}]) \mathrm{N}([\mathrm{H}])[\mathrm{H}]) \mathrm{C}([\mathrm{H}])([} \\
\mathrm{H}]) \mathrm{OC} 3([\mathrm{H}])[\mathrm{H}]) \mathrm{C}([\mathrm{H}])([\mathrm{H}]) \mathrm{C} 4=\mathrm{C}([\mathrm{H}]) \mathrm{C}([\mathrm{H}])=\mathrm{C}([\mathrm{H}]) \mathrm{C}([\mathrm{H}])=\mathrm{C} 4[\mathrm{H}])=\mathrm{C} 5[\mathrm{H}]) \mathrm{C} 6=\mathrm{C}([\mathrm{H}]) \mathrm{N}= \\
\mathrm{C}([\mathrm{H}]) \mathrm{N}=\mathrm{C} 6[\mathrm{H}]\end{array}$ & 3.036 & 2.635 \\
\hline $\operatorname{Tr} 135$ & $\begin{array}{l}{[\mathrm{H}] / \mathrm{C} 5=\mathrm{C}(/ \mathrm{C}([\mathrm{H}])=\mathrm{C} 1 \mathrm{C}(\mathrm{O}[\mathrm{C} @]([\mathrm{H}])([\mathrm{C} @] 3([\mathrm{C} @] 12 \mathrm{~N}=\mathrm{C}(\mathrm{OC} 2([\mathrm{H}])[\mathrm{H}]) \mathrm{N}([\mathrm{H}])[\mathrm{H}]) \mathrm{C}([\mathrm{H}])([} \\
\mathrm{H}]) \mathrm{OC} 3([\mathrm{H}])[\mathrm{H}]) \mathrm{C}([\mathrm{H}])([\mathrm{H}]) \mathrm{C} 4=\mathrm{C}([\mathrm{H}]) \mathrm{C}([\mathrm{H}])=\mathrm{C}([\mathrm{H}]) \mathrm{C}([\mathrm{H}])=\mathrm{C} 4[\mathrm{H}])=\mathrm{C} 5[\mathrm{H}]) \mathrm{C} 6=\mathrm{C}([\mathrm{H}]) \mathrm{N}= \\
\mathrm{C}([\mathrm{H}]) \mathrm{C}(\mathrm{CH} \mathrm{CC}([\mathrm{H}])([\mathrm{H}])[\mathrm{H}])=\mathrm{C} 6[\mathrm{H}]\end{array}$ & 3.148 & 3.566 \\
\hline $\operatorname{Tr} 136$ & $\begin{array}{l}\mathrm{O}=\mathrm{C}(\mathrm{N}([\mathrm{H}]) \mathrm{C} 3=\mathrm{C}([\mathrm{H}]) \mathrm{C}([\mathrm{H}])=\mathrm{C} 2 \mathrm{OC}([\mathrm{C} @] 4([\mathrm{C} @ @] 1(\mathrm{~N}=\mathrm{C}(\mathrm{OC} 1([\mathrm{H}])[\mathrm{H}]) \mathrm{N}([\mathrm{H}])[\mathrm{H}]) \mathrm{C} 2=\mathrm{C} 3 \\
[\mathrm{H}]) \mathrm{C}([\mathrm{H}])([\mathrm{H}]) \mathrm{OC} 4([\mathrm{H}])[\mathrm{H}])(\mathrm{C}([\mathrm{H}])([\mathrm{H}])[\mathrm{H}]) \mathrm{C}([\mathrm{H}])([\mathrm{H}])[\mathrm{H}]) \mathrm{C}=5 / \mathrm{N}=\mathrm{C}(/[\mathrm{H}]) \mathrm{C}([\mathrm{Br}])=\mathrm{C}([\mathrm{H}]) \\
\mathrm{C}=5[\mathrm{H}]\end{array}$ & 3.991 & 2.575 \\
\hline $\operatorname{Tr} 137$ & $\begin{array}{l}\mathrm{O}=\mathrm{C}(\mathrm{N}([\mathrm{H}]) \mathrm{C} 3=\mathrm{C}([\mathrm{H}]) \mathrm{C}([\mathrm{H}])=\mathrm{C} 2 \mathrm{OC}([\mathrm{C} @] 4([\mathrm{C} @ @] 1(\mathrm{~N}=\mathrm{C}(\mathrm{OC} 1([\mathrm{H}])[\mathrm{H}]) \mathrm{N}([\mathrm{H}])[\mathrm{H}]) \mathrm{C} 2=\mathrm{C} 3 \\
[\mathrm{H}]) \mathrm{C}([\mathrm{H}])([\mathrm{H}]) \mathrm{C} 4([\mathrm{H}])[\mathrm{H}])(\mathrm{C}([\mathrm{H}])([\mathrm{H}])[\mathrm{H}]) \mathrm{C}([\mathrm{H}])([\mathrm{H}])[\mathrm{H}]) \mathrm{C} 5=\mathrm{NC}([\mathrm{H}])=\mathrm{C}([\mathrm{Cl}]) \mathrm{C}([\mathrm{H}])=\mathrm{C} 5[ \\
\mathrm{H}]\end{array}$ & 3.423 & 3.097 \\
\hline
\end{tabular}




\begin{tabular}{|c|c|c|c|}
\hline $\operatorname{Tr} 138$ & $\begin{array}{l}\mathrm{O}=\mathrm{C}(\mathrm{N}([\mathrm{H}]) \mathrm{C} 3=\mathrm{C}([\mathrm{H}]) \mathrm{C}([\mathrm{H}])=\mathrm{C} 2 \mathrm{OC}([\mathrm{C} @] 4([\mathrm{C} @ @] 1(\mathrm{~N}=\mathrm{C}(\mathrm{OC} 1([\mathrm{H}])[\mathrm{H}]) \mathrm{N}([\mathrm{H}])[\mathrm{H}]) \mathrm{C} 2=\mathrm{C} 3 \\
[\mathrm{H}]) \mathrm{C}([\mathrm{H}])([\mathrm{H}]) \mathrm{C} 4([\mathrm{H}])[\mathrm{H}])(\mathrm{C}([\mathrm{H}])([\mathrm{H}])[\mathrm{H}]) \mathrm{C}([\mathrm{H}])([\mathrm{H}])[\mathrm{H}]) \mathrm{C} 5=\mathrm{NC}([\mathrm{H}])=\mathrm{C}([\mathrm{F}]) \mathrm{C}([\mathrm{H}])=\mathrm{C} 5[ \\
\mathrm{H}]\end{array}$ & 3.039 & 2.990 \\
\hline $\operatorname{Tr} 139$ & $\begin{array}{l}\mathrm{O}=\mathrm{C}(\mathrm{N}([\mathrm{H}]) \mathrm{C} 3=\mathrm{C}([\mathrm{H}]) \mathrm{C}([\mathrm{H}])=\mathrm{C} 2 \mathrm{OC}([\mathrm{C} @] 4([\mathrm{C} @ @] 1(\mathrm{~N}=\mathrm{C}(\mathrm{OC} 1([\mathrm{H}])[\mathrm{H}]) \mathrm{N}([\mathrm{H}])[\mathrm{H}]) \mathrm{C} 2=\mathrm{C} 3 \\
[\mathrm{H}]) \mathrm{C}([\mathrm{H}])([\mathrm{H}]) \mathrm{OC} 4([\mathrm{H}])[\mathrm{H}])(\mathrm{C}([\mathrm{H}])([\mathrm{H}])[\mathrm{H}]) \mathrm{C}([\mathrm{H}])([\mathrm{H}])[\mathrm{H}]) \mathrm{C}=5 / \mathrm{N}=\mathrm{C}(/[\mathrm{H}]) \mathrm{C}(=\mathrm{NC}=5[\mathrm{H}]) \mathrm{O} \\
\mathrm{C}([\mathrm{H}])([\mathrm{H}])[\mathrm{H}]\end{array}$ & 3.101 & 3.578 \\
\hline $\operatorname{Tr} 140$ & $\begin{array}{l}\mathrm{O}=\mathrm{C}(/ \mathrm{C} 1=\mathrm{N} / \mathrm{C}([\mathrm{H}])=\mathrm{C}([\mathrm{Cl}]) \mathrm{C}([\mathrm{H}])=\mathrm{C} 1[\mathrm{H}]) \mathrm{N}([\mathrm{H}]) \mathrm{C} 2=\mathrm{C}([\mathrm{H}]) \mathrm{C}(=\mathrm{C}([\mathrm{F}]) \mathrm{C}([\mathrm{H}])=\mathrm{C} 2[\mathrm{H}])[\mathrm{C} @ 3] \\
(\mathrm{N}([\mathrm{H}]) \mathrm{C}(=\mathrm{N}[\mathrm{H}]) \mathrm{N}([\mathrm{S}](=\mathrm{O})(=\mathrm{O}) \mathrm{C} 3([\mathrm{H}])[\mathrm{H}]) \mathrm{C}([\mathrm{H}])([\mathrm{H}])[\mathrm{H}]) \mathrm{C}([\mathrm{H}])([\mathrm{H}])[\mathrm{H}]\end{array}$ & 5.023 & 4.057 \\
\hline $\operatorname{Tr} 141$ & $\begin{array}{l}\mathrm{O}=\mathrm{C}(/ \mathrm{C} 1=\mathrm{N} / \mathrm{C}([\mathrm{H}])=\mathrm{C}(\mathrm{C}([\mathrm{H}])=\mathrm{C} 1[\mathrm{H}]) \mathrm{C}([\mathrm{F}])([\mathrm{F}])[\mathrm{F}]) \mathrm{N}([\mathrm{H}]) \mathrm{C} 2=\mathrm{C}([\mathrm{H}]) \mathrm{C}(=\mathrm{C}([\mathrm{F}]) \mathrm{C}([\mathrm{H}])=\mathrm{C} 2[ \\
\mathrm{H}])[\mathrm{C} @] 3(\mathrm{~N}([\mathrm{H}]) \mathrm{C}(=\mathrm{N}[\mathrm{H}]) \mathrm{N}([\mathrm{S}](=\mathrm{O})(=\mathrm{O}) \mathrm{C} 3([\mathrm{H}])[\mathrm{H}]) \mathrm{C}([\mathrm{H}])([\mathrm{H}])[\mathrm{H}]) \mathrm{C}([\mathrm{H}])([\mathrm{H}])[\mathrm{H}]\end{array}$ & 4.899 & 4.970 \\
\hline $\operatorname{Tr} 142$ & $\begin{array}{l}\mathrm{O}=\mathrm{C}(/ \mathrm{C} 1=\mathrm{N} / \mathrm{C}([\mathrm{H}])=\mathrm{C}(/ \mathrm{N}=\mathrm{C} 1 /[\mathrm{H}]) \mathrm{C}([\mathrm{H}])([\mathrm{H}])[\mathrm{H}]) \mathrm{N}([\mathrm{H}]) \mathrm{C} 2=\mathrm{C}([\mathrm{H}]) \mathrm{C}(=\mathrm{C}([\mathrm{F}]) \mathrm{C}([\mathrm{H}])=\mathrm{C} 2[\mathrm{H}] \\
)[\mathrm{C} @] 3(\mathrm{~N}([\mathrm{H}]) \mathrm{C}(=\mathrm{N}[\mathrm{H}]) \mathrm{N}([\mathrm{S}](=\mathrm{O})(=\mathrm{O}) \mathrm{C} 3([\mathrm{H}])[\mathrm{H}]) \mathrm{C}([\mathrm{H}])([\mathrm{H}])[\mathrm{H}]) \mathrm{C}([\mathrm{H}])([\mathrm{H}])[\mathrm{H}]\end{array}$ & 4.000 & 4.478 \\
\hline $\operatorname{Tr} 143$ & $\begin{array}{l}\mathrm{O}=\mathrm{C}(/ \mathrm{C} 1=\mathrm{N} / \mathrm{C}([\mathrm{H}])=\mathrm{C}(\mathrm{N}=\mathrm{C} 1 /[\mathrm{H}]) \mathrm{OC}([\mathrm{H}])([\mathrm{H}])[\mathrm{H}]) \mathrm{N}([\mathrm{H}]) \mathrm{C} 2=\mathrm{C}([\mathrm{H}]) \mathrm{C}(=\mathrm{C}([\mathrm{F}]) \mathrm{C}([\mathrm{F}])=\mathrm{C} 2[\mathrm{H} \\
])[\mathrm{C} @] 3(\mathrm{~N}([\mathrm{H}]) \mathrm{C}(=\mathrm{N}[\mathrm{H}]) \mathrm{N}([\mathrm{S}](=\mathrm{O})(=\mathrm{O}) \mathrm{C} 3([\mathrm{H}])[\mathrm{H}]) \mathrm{C}([\mathrm{H}])([\mathrm{H}])[\mathrm{H}]) \mathrm{C}([\mathrm{H}])([\mathrm{H}])[\mathrm{H}]\end{array}$ & 4.979 & 5.258 \\
\hline $\operatorname{Tr} 144$ & $\begin{array}{l}\mathrm{O}=[\mathrm{S}] 3(=\mathrm{O}) \mathrm{N}(/ \mathrm{C}(=\mathrm{N})[\mathrm{H}]) \mathrm{N}([\mathrm{H}])[\mathrm{C} @](\mathrm{C}=1[\mathrm{~S}] \mathrm{C}([\mathrm{H}])=\mathrm{C}(\mathrm{C}=1[\mathrm{H}]) \mathrm{C} 2=\mathrm{C}([\mathrm{H}]) \mathrm{C}([\mathrm{H}])=\mathrm{C}([\mathrm{F}]) \mathrm{C}( \\
\mathrm{C \# N})=\mathrm{C} 2[\mathrm{H}])(\mathrm{C} 3([\mathrm{H}])[\mathrm{H}]) \mathrm{C}([\mathrm{H}])([\mathrm{H}])[\mathrm{H}]) \mathrm{C}([\mathrm{H}])([\mathrm{H}])[\mathrm{H}]\end{array}$ & 3.337 & 3.145 \\
\hline $\operatorname{Tr} 145$ & $\begin{array}{l}\mathrm{O}=[\mathrm{S}] 3(=\mathrm{O}) \mathrm{N}(/ \mathrm{C}(=\mathrm{N} \backslash[\mathrm{H}]) \mathrm{N}([\mathrm{H}])[\mathrm{C} @](\mathrm{C}=1[\mathrm{~S}] / \mathrm{C}(=\mathrm{C}(/[\mathrm{H}]) \mathrm{C}=1[\mathrm{Cl}]) \mathrm{C} 2=\mathrm{C}([\mathrm{H}]) \mathrm{N}=\mathrm{C}([\mathrm{H}]) \mathrm{C}(\mathrm{C \#} \\
\mathrm{CC}([\mathrm{H}])([\mathrm{H}])[\mathrm{H}])=\mathrm{C} 2[\mathrm{H}])(\mathrm{C} 3([\mathrm{H}])[\mathrm{H}]) \mathrm{C}([\mathrm{H}])([\mathrm{H}])[\mathrm{H}]) \mathrm{C}([\mathrm{H}])([\mathrm{H}])[\mathrm{H}]\end{array}$ & 4.622 & 4.388 \\
\hline $\operatorname{Tr} 146$ & $\begin{array}{l}\mathrm{O}=\mathrm{C}(/ \mathrm{C} 1=\mathrm{N} / \mathrm{C}([\mathrm{H}])=\mathrm{C}(/ \mathrm{N}=\mathrm{C} 1 /[\mathrm{H}]) \mathrm{OC}([\mathrm{H}])([\mathrm{H}])[\mathrm{H}]) \mathrm{N}([\mathrm{H}]) \mathrm{C} 2=\mathrm{C}([\mathrm{H}]) \mathrm{C}(=\mathrm{C}([\mathrm{F}]) \mathrm{C}([\mathrm{H}])=\mathrm{C} 2[ \\
\mathrm{H}])[\mathrm{C} @] 3(\mathrm{~N}([\mathrm{H}]) \mathrm{C}(=\mathrm{N}[\mathrm{H}]) \mathrm{N}([\mathrm{S}](=\mathrm{O})(=\mathrm{O}) \mathrm{C} 3([\mathrm{H}])[\mathrm{H}]) \mathrm{C}([\mathrm{H}])([\mathrm{H}])[\mathrm{H}]) \mathrm{C}([\mathrm{H}])([\mathrm{H}])[\mathrm{H}]\end{array}$ & 4.785 & 4.922 \\
\hline $\operatorname{Tr} 147$ & $\begin{array}{l}{[\mathrm{F}] \mathrm{C}=1 \mathrm{C}([\mathrm{H}])=\mathrm{C}(\mathrm{C}([\mathrm{H}])=\mathrm{C}([\mathrm{F}]) \mathrm{C}=1[\mathrm{H}]) \mathrm{C}([\mathrm{H}])([\mathrm{H}])[\mathrm{C} @]([\mathrm{H}])(\mathrm{N}([\mathrm{H}]) \mathrm{C}(=\mathrm{O}) \mathrm{C} 2=\mathrm{C}([\mathrm{H}]) \mathrm{C}(=\mathrm{C}} \\
([\mathrm{H}]) \mathrm{C}(=\mathrm{C} 2[\mathrm{H}]) \mathrm{C}([\mathrm{H}])([\mathrm{H}])[\mathrm{H}]) \mathrm{C}(=\mathrm{O}) \mathrm{N}(\mathrm{C}([\mathrm{H}])([\mathrm{H}]) \mathrm{C}([\mathrm{H}])([\mathrm{H}]) \mathrm{C}([\mathrm{H}])([\mathrm{H}])[\mathrm{H}]) \mathrm{C}([\mathrm{H}])([\mathrm{H}]) \mathrm{C} \\
([\mathrm{H}])([\mathrm{H}]) \mathrm{C}([\mathrm{H}])([\mathrm{H}])[\mathrm{H}])[\mathrm{C} @]([\mathrm{H}])(\mathrm{O}[\mathrm{H}])[\mathrm{C} @] 3([\mathrm{H}]) \mathrm{N}([\mathrm{H}]) \mathrm{C}([\mathrm{H}])([\mathrm{H}]) \mathrm{C}([\mathrm{H}])([\mathrm{H}]) \mathrm{N}(\mathrm{C} 3([ \\
\mathrm{H}])[\end{array}$ & 2.509 & 3.437 \\
\hline $\operatorname{Tr} 148$ & $\begin{array}{l}{[\mathrm{F}] \mathrm{C}=1 \mathrm{C}([\mathrm{H}])=\mathrm{C}(\mathrm{C}([\mathrm{H}])=\mathrm{C}([\mathrm{F}]) \mathrm{C}=1[\mathrm{H}]) \mathrm{C}([\mathrm{H}])([\mathrm{H}])[\mathrm{C} @]([\mathrm{H}])(\mathrm{N}([\mathrm{H}]) \mathrm{C}(=\mathrm{O}) \mathrm{C} 2=\mathrm{C}([\mathrm{H}]) \mathrm{C}(=\mathrm{C}} \\
([\mathrm{H}]) \mathrm{C}(=\mathrm{C} 2[\mathrm{H}]) \mathrm{C}([\mathrm{H}])([\mathrm{H}])[\mathrm{H}]) \mathrm{C}(=\mathrm{O}) \mathrm{N}(\mathrm{C}([\mathrm{H}])([\mathrm{H}]) \mathrm{C}([\mathrm{H}])([\mathrm{H}]) \mathrm{C}([\mathrm{H}])([\mathrm{H}])[\mathrm{H}]) \mathrm{C}([\mathrm{H}])([\mathrm{H}]) \mathrm{C} \\
([\mathrm{H}])([\mathrm{H}]) \mathrm{C}([\mathrm{H}])([\mathrm{H}])[\mathrm{H}])[\mathrm{C} @]([\mathrm{H}])(\mathrm{O}[\mathrm{H}])[\mathrm{C} @] 3([\mathrm{H}]) \mathrm{N}([\mathrm{H}]) \mathrm{C}([\mathrm{H}])([\mathrm{H}]) \mathrm{C}([\mathrm{H}])([\mathrm{H}]) \mathrm{N}(\mathrm{C} 3([ \\
\mathrm{H}])[\end{array}$ & 2.921 & 2.993 \\
\hline $\operatorname{Tr} 149$ & $\begin{array}{l}{[\mathrm{F}] \mathrm{C}=1 \mathrm{C}([\mathrm{H}])=\mathrm{C}(\mathrm{C}([\mathrm{H}])=\mathrm{C}([\mathrm{F}]) \mathrm{C}=1[\mathrm{H}]) \mathrm{C}([\mathrm{H}])([\mathrm{H}])[\mathrm{C} @]([\mathrm{H}])(\mathrm{N}([\mathrm{H}]) \mathrm{C}(=\mathrm{O}) \mathrm{C} 2=\mathrm{C}([\mathrm{H}]) \mathrm{C}(=\mathrm{C}} \\
([\mathrm{H}]) \mathrm{C}(=\mathrm{C} 2[\mathrm{H}]) \mathrm{C}([\mathrm{H}])([\mathrm{H}])[\mathrm{H}]) \mathrm{C}(=\mathrm{O}) \mathrm{N}(\mathrm{C}([\mathrm{H}])([\mathrm{H}]) \mathrm{C}([\mathrm{H}])([\mathrm{H}]) \mathrm{C}([\mathrm{H}])([\mathrm{H}])[\mathrm{H}]) \mathrm{C}([\mathrm{H}])([\mathrm{H}]) \mathrm{C} \\
([\mathrm{H}])([\mathrm{H}]) \mathrm{C}([\mathrm{H}])([\mathrm{H}])[\mathrm{H}])[\mathrm{C} @]([\mathrm{H}])(\mathrm{O}[\mathrm{H}])[\mathrm{C} @] 3([\mathrm{H}]) \mathrm{N}([\mathrm{H}]) \mathrm{C}([\mathrm{H}])([\mathrm{H}]) \mathrm{C}([\mathrm{H}])([\mathrm{H}]) \mathrm{N}(\mathrm{C} 3([ \\
\mathrm{H}])[\end{array}$ & 2.398 & 3.017 \\
\hline $\operatorname{Tr} 150$ & $\begin{array}{l}{[\mathrm{F}] \mathrm{C}=1 \mathrm{C}([\mathrm{H}])=\mathrm{C}(\mathrm{C}([\mathrm{H}])=\mathrm{C}([\mathrm{F}]) \mathrm{C}=1[\mathrm{H}]) \mathrm{C}([\mathrm{H}])([\mathrm{H}])[\mathrm{C} @]([\mathrm{H}])(\mathrm{N}([\mathrm{H}]) \mathrm{C}(=\mathrm{O}) \mathrm{C} 2=\mathrm{C}([\mathrm{H}]) \mathrm{C}(=\mathrm{C}} \\
([\mathrm{H}]) \mathrm{C}(=\mathrm{C} 2[\mathrm{H}]) \mathrm{C}([\mathrm{H}])([\mathrm{H}])[\mathrm{H}]) \mathrm{C}(=\mathrm{O}) \mathrm{N}(\mathrm{C}([\mathrm{H}])([\mathrm{H}]) \mathrm{C}([\mathrm{H}])([\mathrm{H}]) \mathrm{C}([\mathrm{H}])([\mathrm{H}])[\mathrm{H}]) \mathrm{C}([\mathrm{H}])([\mathrm{H}]) \mathrm{C} \\
([\mathrm{H}])([\mathrm{H}]) \mathrm{C}([\mathrm{H}])([\mathrm{H}])[\mathrm{H}])[\mathrm{C} @]([\mathrm{H}])(\mathrm{O}[\mathrm{H}])[\mathrm{C} @] 3([\mathrm{H}]) \mathrm{N}([\mathrm{H}]) \mathrm{C}([\mathrm{H}])([\mathrm{H}]) \mathrm{C}([\mathrm{H}])([\mathrm{H}]) \mathrm{N}(\mathrm{C} 3([ \\
\mathrm{H}])[\end{array}$ & 2.013 & 3.262 \\
\hline $\operatorname{Tr} 151$ & $\begin{array}{l}\mathrm{O}=\mathrm{C}(\mathrm{N}([\mathrm{H}])[\mathrm{C} @]([\mathrm{H}])([\mathrm{C} @ @]([\mathrm{H}])(\mathrm{O}[\mathrm{H}]) \mathrm{C}([\mathrm{H}])([\mathrm{H}])[\mathrm{C} @]([\mathrm{H}])(\mathrm{C}(=\mathrm{O}) \mathrm{N}([\mathrm{H}])[\mathrm{C} @]([\mathrm{H}])(\mathrm{C} \\
=\mathrm{O}) \mathrm{N}([\mathrm{H}]) \mathrm{C}([\mathrm{H}])([\mathrm{H}]) \mathrm{C}([\mathrm{H}])(\mathrm{C}([\mathrm{H}])([\mathrm{H}])[\mathrm{H}]) \mathrm{C}([\mathrm{H}])([\mathrm{H}])[\mathrm{H}]) \mathrm{C}([\mathrm{H}])(\mathrm{C}([\mathrm{H}])([\mathrm{H}])[\mathrm{H}]) \mathrm{C}([\mathrm{H}])([ \\
\mathrm{H}])[\mathrm{H}]) \mathrm{C}([\mathrm{H}])([\mathrm{H}])[\mathrm{H}]) \mathrm{C}([\mathrm{H}])([\mathrm{H}]) \mathrm{C}([\mathrm{H}])(\mathrm{C}([\mathrm{H}])([\mathrm{H}])[\mathrm{H}]) \mathrm{C}([\mathrm{H}])([\mathrm{H}])[\mathrm{H}])[\mathrm{C} @ @]([\mathrm{H}])(\mathrm{N}([\mathrm{H} \\
]\end{array}$ & 5.097 & 4.395 \\
\hline $\operatorname{Tr} 152$ & $\begin{array}{l}\mathrm{O}=\mathrm{C}(\mathrm{N}([\mathrm{H}]) \mathrm{C}([\mathrm{H}])([\mathrm{H}]) \mathrm{Cl}=\mathrm{C}([\mathrm{H}]) \mathrm{C}([\mathrm{H}])=\mathrm{C}([\mathrm{H}]) \mathrm{C}([\mathrm{H}])=\mathrm{Cl}[\mathrm{H}])[\mathrm{C} @]([\mathrm{H}])(\mathrm{OC}([\mathrm{H}])([\mathrm{H}])[\mathrm{H}] \\
) \mathrm{C}([\mathrm{H}])([\mathrm{H}])[\mathrm{C} @]([\mathrm{H}])(\mathrm{O}[\mathrm{H}])[\mathrm{C} @ @([\mathrm{H}])(\mathrm{N}([\mathrm{H}]) \mathrm{C}(=\mathrm{O}) \mathrm{C} 2=\mathrm{C}([\mathrm{H}]) \mathrm{C}(=\mathrm{C}([\mathrm{H}]) \mathrm{C}(=\mathrm{C} 2[\mathrm{H}]) \mathrm{C}(= \\
\mathrm{O}) \mathrm{N}([\mathrm{H}])[\mathrm{C} @ @]([\mathrm{H}])(\mathrm{C} 3=\mathrm{C}([\mathrm{H}]) \mathrm{C}([\mathrm{H}])=\mathrm{C}([\mathrm{H}]) \mathrm{C}([\mathrm{H}])=\mathrm{C} 3[\mathrm{H}]) \mathrm{C}([\mathrm{H}])([\mathrm{H}])[\mathrm{H}]) \mathrm{N}(\mathrm{C}([\mathrm{H}])([\mathrm{H}] \\
)[\mathrm{S}](=\mathrm{O})(=\mathrm{O})\end{array}$ & 1.854 & 2.670 \\
\hline
\end{tabular}




\begin{tabular}{|c|c|c|c|}
\hline $\operatorname{Tr} 153$ & $\begin{array}{l}\mathrm{O}=\mathrm{C}(\mathrm{N}([\mathrm{H}]) \mathrm{C}([\mathrm{H}])([\mathrm{H}]) \mathrm{C}([\mathrm{H}])(\mathrm{C}([\mathrm{H}])([\mathrm{H}])[\mathrm{H}]) \mathrm{C}([\mathrm{H}])([\mathrm{H}])[\mathrm{H}])[\mathrm{C} @]([\mathrm{H}])(\mathrm{OC}([\mathrm{H}])([\mathrm{H}])[\mathrm{H}]) \\
\mathrm{C}([\mathrm{H}])([\mathrm{H}])[\mathrm{C} @]([\mathrm{H}])(\mathrm{O}[\mathrm{H}])[\mathrm{C} @ @]([\mathrm{H}])(\mathrm{N}([\mathrm{H}]) \mathrm{C}(=\mathrm{O}) \mathrm{C} 1=\mathrm{C}([\mathrm{H}]) \mathrm{C}(=\mathrm{C}([\mathrm{H}]) \mathrm{C}(=\mathrm{C} 1[\mathrm{H}]) \mathrm{C}(= \\
\mathrm{O}) \mathrm{N}([\mathrm{H}])[\mathrm{C} @ @]([\mathrm{H}])(\mathrm{C} 2=\mathrm{C}([\mathrm{H}]) \mathrm{C}([\mathrm{H}])=\mathrm{C}([\mathrm{H}]) \mathrm{C}([\mathrm{H}])=\mathrm{C} 2[\mathrm{H}]) \mathrm{C}([\mathrm{H}])([\mathrm{H}])[\mathrm{H}]) \mathrm{N}(\mathrm{C}([\mathrm{H}])([\mathrm{H}] \\
)[\mathrm{S}](=\mathrm{O})(=\end{array}$ & 2.398 & 2.657 \\
\hline $\operatorname{Tr} 154$ & $\begin{array}{l}\mathrm{O}=\mathrm{C}(\mathrm{N}([\mathrm{H}])[\mathrm{C} @]([\mathrm{H}])(\mathrm{C}(=\mathrm{O}) \mathrm{N}([\mathrm{H}]) \mathrm{C}([\mathrm{H}])([\mathrm{H}]) \mathrm{Cl}=\mathrm{C}([\mathrm{H}]) \mathrm{C}([\mathrm{H}])=\mathrm{C}([\mathrm{H}]) \mathrm{C}([\mathrm{H}])=\mathrm{C} 1[\mathrm{H}]) \mathrm{C}([ \\
\mathrm{H}])(\mathrm{C}([\mathrm{H}])([\mathrm{H}])[\mathrm{H}]) \mathrm{C}([\mathrm{H}])([\mathrm{H}])[\mathrm{H}])[\mathrm{C} @]([\mathrm{H}])(\mathrm{OC}([\mathrm{H}])([\mathrm{H}])[\mathrm{H}]) \mathrm{C}([\mathrm{H}])([\mathrm{H}])[\mathrm{C} @]([\mathrm{H}])(\mathrm{O}[\mathrm{H}] \\
)[\mathrm{C} @ @]([\mathrm{H}])(\mathrm{N}([\mathrm{H}]) \mathrm{C}(=\mathrm{O}) \mathrm{C} 2=\mathrm{C}([\mathrm{H}]) \mathrm{C}(=\mathrm{C}([\mathrm{H}]) \mathrm{C}(=\mathrm{C} 2[\mathrm{H}]) \mathrm{C}(=\mathrm{O}) \mathrm{N}([\mathrm{H}])[\mathrm{C} @ @]([\mathrm{H}])(\mathrm{C} 3=\mathrm{C}( \\
[\mathrm{H}]) \mathrm{C}([\mathrm{H}])=\end{array}$ & 4.046 & 2.867 \\
\hline $\operatorname{Tr} 155$ & $\begin{array}{l}\mathrm{O}=\mathrm{C}(\mathrm{N}([\mathrm{H}])[\mathrm{C} @]([\mathrm{H}])(\mathrm{C}(=\mathrm{O}) \mathrm{N}([\mathrm{H}]) \mathrm{C}([\mathrm{H}])([\mathrm{H}]) \mathrm{C} 1=\mathrm{C}([\mathrm{H}]) \mathrm{C}([\mathrm{H}])=\mathrm{C}([\mathrm{H}]) \mathrm{C}([\mathrm{H}])=\mathrm{C} 1[\mathrm{H}]) \mathrm{C}([ \\
\mathrm{H}])(\mathrm{C}([\mathrm{H}])([\mathrm{H}])[\mathrm{H}]) \mathrm{C}([\mathrm{H}])([\mathrm{H}])[\mathrm{H}])[\mathrm{C} @]([\mathrm{H}])(\mathrm{OC}([\mathrm{H}])([\mathrm{H}])[\mathrm{H}]) \mathrm{C}([\mathrm{H}])([\mathrm{H}])[\mathrm{C} @]([\mathrm{H}])(\mathrm{OH}] \\
)[\mathrm{C} @ @]([\mathrm{H}])(\mathrm{N}([\mathrm{H}]) \mathrm{C}(=\mathrm{O}) \mathrm{C} 2=\mathrm{C}([\mathrm{H}]) \mathrm{C}(=\mathrm{C}([\mathrm{H}]) \mathrm{C}(=\mathrm{C} 2[\mathrm{H}]) \mathrm{C}(=\mathrm{O}) \mathrm{N}([\mathrm{H}])[\mathrm{C} @ @]([\mathrm{H}])(\mathrm{C} 3=\mathrm{C}( \\
[\mathrm{H}]) \mathrm{C}([\mathrm{H}])=\end{array}$ & 3.125 & 2.903 \\
\hline $\operatorname{Tr} 156$ & $\begin{array}{l}\mathrm{O}=\mathrm{C}(\mathrm{N}([\mathrm{H}])[\mathrm{C} @]([\mathrm{H}])(\mathrm{C}(=\mathrm{O}) \mathrm{N}([\mathrm{H}]) \mathrm{C}([\mathrm{H}])([\mathrm{H}]) \mathrm{C}=1 \mathrm{C}([\mathrm{H}])=\mathrm{C}([\mathrm{H}]) \mathrm{C}([\mathrm{H}])=\mathrm{C}([\mathrm{H}]) \mathrm{C}=1[\mathrm{H}]) \mathrm{C} \\
[\mathrm{H}])(\mathrm{C}([\mathrm{H}])([\mathrm{H}])[\mathrm{H}]) \mathrm{C}([\mathrm{H}])([\mathrm{H}])[\mathrm{H}])[\mathrm{C} @]([\mathrm{H}])(\mathrm{OC}([\mathrm{H}])([\mathrm{H}])[\mathrm{H}]) \mathrm{C}([\mathrm{H}])([\mathrm{H}])[\mathrm{C} @]([\mathrm{H}])(\mathrm{O}[\mathrm{H} \\
])[\mathrm{C} @ @([\mathrm{H}])(\mathrm{N}([\mathrm{H}]) \mathrm{C}(=\mathrm{O}) \mathrm{C} 2=\mathrm{C}([\mathrm{H}]) \mathrm{C}(=\mathrm{C}([\mathrm{H}]) \mathrm{C}(=\mathrm{C} 2[\mathrm{H}]) \mathrm{C}(=\mathrm{O}) \mathrm{N}([\mathrm{H}])[\mathrm{C} @ @]([\mathrm{H}])(\mathrm{C} 3=\mathrm{C} \\
([\mathrm{H}]) \mathrm{C}([\mathrm{H}])\end{array}$ & 3.097 & 3.714 \\
\hline $\operatorname{Tr} 157$ & $\begin{array}{l}\mathrm{O}=\mathrm{C}(\mathrm{N}([\mathrm{H}])[\mathrm{C} @]([\mathrm{H}])(\mathrm{C}(=\mathrm{O}) \mathrm{N}([\mathrm{H}]) \mathrm{C}([\mathrm{H}])([\mathrm{H}]) \mathrm{Cl}=\mathrm{C}([\mathrm{H}]) \mathrm{C}([\mathrm{H}])=\mathrm{C}([\mathrm{H}]) \mathrm{C}([\mathrm{H}])=\mathrm{C} 1[\mathrm{H}]) \mathrm{C}([ \\
\mathrm{H}])(\mathrm{C}([\mathrm{H}])([\mathrm{H}])[\mathrm{H}]) \mathrm{C}([\mathrm{H}])([\mathrm{H}])[\mathrm{H}])[\mathrm{C} @]([\mathrm{H}])(\mathrm{OC}([\mathrm{H}])([\mathrm{H}])[\mathrm{H}]) \mathrm{C}([\mathrm{H}])([\mathrm{H}])[\mathrm{C} @]([\mathrm{H}])(\mathrm{OH}] \\
)[\mathrm{C} @ @]([\mathrm{H}])(\mathrm{N}([\mathrm{H}]) \mathrm{C}(=\mathrm{O}) \mathrm{C} 2=\mathrm{C}([\mathrm{H}]) \mathrm{C}(=\mathrm{C}([\mathrm{H}]) \mathrm{C}(=\mathrm{C} 2[\mathrm{H}]) \mathrm{C}(=\mathrm{O}) \mathrm{N}([\mathrm{H}])[\mathrm{C} @ @]([\mathrm{H}])(\mathrm{C} 3=\mathrm{C}( \\
[\mathrm{H}]) \mathrm{C}([\mathrm{H}])=\end{array}$ & 3.222 & 3.149 \\
\hline $\operatorname{Tr} 158$ & $\begin{array}{l}\mathrm{O}=\mathrm{C}(\mathrm{N}([\mathrm{H}]) \mathrm{C} 1=\mathrm{C}([\mathrm{H}]) \mathrm{C}([\mathrm{H}])=\mathrm{C}([\mathrm{F}]) \mathrm{C}([\mathrm{H}])=\mathrm{C} 1[\mathrm{H}])[\mathrm{C} @]([\mathrm{H}])(\mathrm{OC}([\mathrm{H}])([\mathrm{H}])[\mathrm{H}]) \mathrm{C}([\mathrm{H}])([\mathrm{H}]) \\
{[\mathrm{C} @]([\mathrm{H}])(\mathrm{O}[\mathrm{H}])[\mathrm{C} @ @]([\mathrm{H}])(\mathrm{N}([\mathrm{H}]) \mathrm{C}(=\mathrm{O}) \mathrm{C} 2=\mathrm{C}([\mathrm{H}]) \mathrm{C}(=\mathrm{C}([\mathrm{H}]) \mathrm{C}(=\mathrm{C} 2[\mathrm{H}]) \mathrm{C}(=\mathrm{O}) \mathrm{N}([\mathrm{H}])[\mathrm{C}} \\
@ @([\mathrm{H}])(\mathrm{C} 3=\mathrm{C}([\mathrm{H}]) \mathrm{C}([\mathrm{H}])=\mathrm{C}([\mathrm{H}]) \mathrm{C}([\mathrm{H}])=\mathrm{C} 3[\mathrm{H}]) \mathrm{C}([\mathrm{H}])([\mathrm{H}])[\mathrm{H}]) \mathrm{N}(\mathrm{C}([\mathrm{H}])([\mathrm{H}])[\mathrm{S}](=\mathrm{O})(= \\
\mathrm{O}) \mathrm{C}([\mathrm{H}])([\mathrm{H}])\end{array}$ & 3.000 & 2.982 \\
\hline $\operatorname{Tr} 159$ & $\begin{array}{l}\mathrm{O}=\mathrm{C}(\mathrm{N}([\mathrm{H}]) \mathrm{Cl}=\mathrm{C}([\mathrm{H}]) \mathrm{C}(=\mathrm{C}(\mathrm{C}([\mathrm{H}])=\mathrm{C} 1[\mathrm{H}]) \mathrm{N}(\mathrm{C}([\mathrm{H}])([\mathrm{H}])[\mathrm{H}]) \mathrm{C}([\mathrm{H}])([\mathrm{H}])[\mathrm{H}]) \mathrm{C}(=\mathrm{O}) \mathrm{N}([\mathrm{H}]) \\
\mathrm{C}([\mathrm{H}])([\mathrm{H}]) \mathrm{C}([\mathrm{H}])([\mathrm{H}]) \mathrm{C}([\mathrm{H}])([\mathrm{H}]) \mathrm{OC}([\mathrm{H}])([\mathrm{H}]) \mathrm{C}([\mathrm{H}])([\mathrm{H}])[\mathrm{H}]) \mathrm{N}([\mathrm{H}]) \mathrm{C} 2=\mathrm{C}([\mathrm{H}]) \mathrm{C}([\mathrm{Cl}])=\mathrm{C}( \\
[\mathrm{H}]) \mathrm{C}([\mathrm{H}])=\mathrm{C} 2[\mathrm{H}]\end{array}$ & 1.009 & 1.620 \\
\hline $\operatorname{Tr} 160$ & $\begin{array}{l}\mathrm{O}=\mathrm{C}(\mathrm{N}([\mathrm{H}])[\mathrm{C} @]([\mathrm{H}])(\mathrm{C}(=\mathrm{O}) \mathrm{N}([\mathrm{H}])[\mathrm{C} @]([\mathrm{H}])([\mathrm{C} @]([\mathrm{H}])(\mathrm{O}[\mathrm{H}]) \mathrm{C}([\mathrm{H}])([\mathrm{H}])[\mathrm{C} @]([\mathrm{H}])(\mathrm{C}(= \\
\mathrm{O}) \mathrm{N}([\mathrm{H}])[\mathrm{C} @([\mathrm{H}])(\mathrm{C}(=\mathrm{O}) \mathrm{N}([\mathrm{H}])[\mathrm{C} @]([\mathrm{H}])(\mathrm{C}(=\mathrm{O}) \mathrm{N}([\mathrm{H}])[\mathrm{C} @([\mathrm{H}])(\mathrm{C}(=\mathrm{O}) \mathrm{O}[\mathrm{H}]) \mathrm{C}([\mathrm{H}])([\mathrm{H}] \\
) \mathrm{Cl}=\mathrm{C}([\mathrm{H}]) \mathrm{C}([\mathrm{H}])=\mathrm{C}([\mathrm{H}]) \mathrm{C}([\mathrm{H}])=\mathrm{Cl}[\mathrm{H}]) \mathrm{C}([\mathrm{H}])([\mathrm{H}]) \mathrm{C}([\mathrm{H}])([\mathrm{H}]) \mathrm{C}(=\mathrm{O}) \mathrm{O}[\mathrm{H}]) \mathrm{C}([\mathrm{H}])([\mathrm{H}])[\mathrm{H}]) \\
\mathrm{C}([\mathrm{H}])([\mathrm{H}]\end{array}$ & 4.000 & 4.508 \\
\hline $\operatorname{Tr} 161$ & $\begin{array}{l}\mathrm{O}=\mathrm{C}(\mathrm{N}([\mathrm{H}])[\mathrm{C} @]([\mathrm{H}])(\mathrm{C}(=\mathrm{O}) \mathrm{N}([\mathrm{H}])[\mathrm{C} @]([\mathrm{H}])([\mathrm{C} @]([\mathrm{H}])(\mathrm{O}[\mathrm{H}]) \mathrm{C}([\mathrm{H}])([\mathrm{H}])[\mathrm{C} @]([\mathrm{H}])(\mathrm{C}(= \\
\mathrm{O}) \mathrm{N}([\mathrm{H}])[\mathrm{C} @]([\mathrm{H}])(\mathrm{C}(=\mathrm{O}) \mathrm{N}([\mathrm{H}])[\mathrm{C} @]([\mathrm{H}])(\mathrm{C}(=\mathrm{O}) \mathrm{N}([\mathrm{H}])[\mathrm{C} @([\mathrm{H}])(\mathrm{C}(=\mathrm{O}) \mathrm{O}[\mathrm{H}]) \mathrm{C}([\mathrm{H}])([\mathrm{H}] \\
) \mathrm{Cl}=\mathrm{C}([\mathrm{H}]) \mathrm{C}([\mathrm{H}])=\mathrm{C}([\mathrm{H}]) \mathrm{C}([\mathrm{H}])=\mathrm{Cl}[\mathrm{H}]) \mathrm{C}([\mathrm{H}])([\mathrm{H}]) \mathrm{C}([\mathrm{H}])([\mathrm{H}]) \mathrm{C}(=\mathrm{O}) \mathrm{O}[\mathrm{H}]) \mathrm{C}([\mathrm{H}])([\mathrm{H}])[\mathrm{H}]) \\
\mathrm{C}([\mathrm{H}])([\mathrm{H}]\end{array}$ & 3.167 & 3.308 \\
\hline $\operatorname{Tr} 162$ & $\begin{array}{l}\mathrm{O}=\mathrm{C}(\mathrm{N}([\mathrm{H}]) \mathrm{C}(=\mathrm{NC}([\mathrm{H}])([\mathrm{H}]) \mathrm{C} 1=\mathrm{C}([\mathrm{H}]) \mathrm{C}([\mathrm{H}])=\mathrm{C}([\mathrm{H}]) \mathrm{C}([\mathrm{H}])=\mathrm{C} 1[\mathrm{H}]) \mathrm{N}([\mathrm{H}])[\mathrm{H}]) \mathrm{C}=2 \mathrm{C}(=\mathrm{NO} \\
\mathrm{C}=2 \mathrm{C}([\mathrm{H}])([\mathrm{H}])[\mathrm{H}]) \mathrm{C} 3=\mathrm{C}([\mathrm{H}]) \mathrm{C}([\mathrm{H}])=\mathrm{C}(\mathrm{OC}([\mathrm{H}])([\mathrm{H}])) \mathrm{H}]) \mathrm{C}([\mathrm{H}])=\mathrm{C} 3[\mathrm{H}]\end{array}$ & 1.398 & 1.742 \\
\hline $\operatorname{Tr} 163$ & $\begin{array}{l}\mathrm{O}=\mathrm{C}(\mathrm{N}([\mathrm{H}]) \mathrm{C}(=\mathrm{NC}([\mathrm{H}])([\mathrm{H}]) \mathrm{C} 1=\mathrm{C}([\mathrm{H}]) \mathrm{C}([\mathrm{Cl}])=\mathrm{C}([\mathrm{Cl}]) \mathrm{C}([\mathrm{H}])=\mathrm{C} 1[\mathrm{H}]) \mathrm{N}([\mathrm{H}])[\mathrm{H}]) \mathrm{C}=2 \mathrm{C}(=\mathrm{N} \\
\mathrm{OC}=2 \mathrm{C}([\mathrm{H}])([\mathrm{H}])[\mathrm{H}]) \mathrm{C} 3=\mathrm{C}([\mathrm{H}]) \mathrm{C}([\mathrm{H}])=\mathrm{C}([\mathrm{F}]) \mathrm{C}([\mathrm{H}])=\mathrm{C} 3[\mathrm{H}]\end{array}$ & 1.620 & 2.395 \\
\hline $\operatorname{Tr} 164$ & $\begin{array}{l}\mathrm{O}=\mathrm{C}(\mathrm{N}([\mathrm{H}])[\mathrm{C} @(([\mathrm{H}])(\mathrm{C}(=\mathrm{O}) \mathrm{N}([\mathrm{H}])[\mathrm{C} @]([\mathrm{H}])([\mathrm{C} @ @]([\mathrm{H}])(\mathrm{O}[\mathrm{H}]) \mathrm{C}([\mathrm{H}])([\mathrm{H}])[\mathrm{C} @]([\mathrm{H}])(\mathrm{C}( \\
=\mathrm{O}) \mathrm{N}([\mathrm{H}])[\mathrm{C} @([\mathrm{H}])(\mathrm{C}(=\mathrm{O}) \mathrm{N}([\mathrm{H}]) \mathrm{C}([\mathrm{H}])([\mathrm{H}]) \mathrm{C}([\mathrm{H}])(\mathrm{C}([\mathrm{H}])([\mathrm{H}])[\mathrm{H}]) \mathrm{C}([\mathrm{H}])([\mathrm{H}])[\mathrm{H}]) \mathrm{C}([\mathrm{H}]) \\
(\mathrm{C}([\mathrm{H}])([\mathrm{H}])[\mathrm{H}]) \mathrm{C}([\mathrm{H}])([\mathrm{H}])[\mathrm{H}]) \mathrm{C}([\mathrm{H}])([\mathrm{H}])[\mathrm{H}]) \mathrm{C}([\mathrm{H}])([\mathrm{H}]) \mathrm{C}([\mathrm{H}])(\mathrm{C}([\mathrm{H}])([\mathrm{H}])[\mathrm{H}]) \mathrm{C}([\mathrm{H}])([\mathrm{H} \\
]\end{array}$ & 5.602 & 4.275 \\
\hline $\operatorname{Tr} 165$ & $\begin{array}{l}\mathrm{O}=\mathrm{C}(\mathrm{N}([\mathrm{H}])[\mathrm{C} @]([\mathrm{H}])(\mathrm{C}(=\mathrm{O}) \mathrm{N}([\mathrm{H}])[\mathrm{C} @]([\mathrm{H}])([\mathrm{C} @ @]([\mathrm{H}])(\mathrm{O}[\mathrm{H}]) \mathrm{C}([\mathrm{H}])([\mathrm{H}])[\mathrm{C} @]([\mathrm{H}])(\mathrm{C}( \\
=\mathrm{O}) \mathrm{N}([\mathrm{H}])[\mathrm{C} @([\mathrm{H}])(\mathrm{C}(=\mathrm{O}) \mathrm{N}([\mathrm{H}]) \mathrm{C}([\mathrm{H}])([\mathrm{H}]) \mathrm{C}([\mathrm{H}])(\mathrm{C}([\mathrm{H}])([\mathrm{H}])[\mathrm{H}]) \mathrm{C}([\mathrm{H}])([\mathrm{H}])[\mathrm{H}]) \mathrm{C}([\mathrm{H}]) \\
(\mathrm{C}([\mathrm{H}])([\mathrm{H}])[\mathrm{H}]) \mathrm{C}([\mathrm{H}])([\mathrm{H}])[\mathrm{H}]) \mathrm{C}([\mathrm{H}])([\mathrm{H}])[\mathrm{H}]) \mathrm{C}([\mathrm{H}])([\mathrm{H}]) \mathrm{C}([\mathrm{H}])(\mathrm{C}([\mathrm{H}])([\mathrm{H}])[\mathrm{H}]) \mathrm{C}([\mathrm{H}])([\mathrm{H} \\
]\end{array}$ & 3.959 & 4.299 \\
\hline
\end{tabular}




\begin{tabular}{|c|c|c|c|}
\hline $\operatorname{Tr} 166$ & $\begin{array}{l}\mathrm{O}=\mathrm{C}(\mathrm{N}([\mathrm{H}])[\mathrm{C} @]([\mathrm{H}])(\mathrm{C}(=\mathrm{O}) \mathrm{N}([\mathrm{H}])[\mathrm{C} @]([\mathrm{H}])([\mathrm{C} @ @]([\mathrm{H}])(\mathrm{O}[\mathrm{H}]) \mathrm{C}([\mathrm{H}])([\mathrm{H}])[\mathrm{C} @]([\mathrm{H}])(\mathrm{C}( \\
=\mathrm{O}) \mathrm{N}([\mathrm{H}])[\mathrm{C} @([\mathrm{H}])(\mathrm{C}(=\mathrm{O}) \mathrm{N}([\mathrm{H}]) \mathrm{C}([\mathrm{H}])([\mathrm{H}]) \mathrm{C}([\mathrm{H}])(\mathrm{C}([\mathrm{H}])([\mathrm{H}])[\mathrm{H}]) \mathrm{C}([\mathrm{H}])([\mathrm{H}])[\mathrm{H}]) \mathrm{C}([\mathrm{H}]) \\
(\mathrm{C}([\mathrm{H}])([\mathrm{H}])[\mathrm{H}]) \mathrm{C}([\mathrm{H}])([\mathrm{H}])[\mathrm{H}]) \mathrm{C}([\mathrm{H}])([\mathrm{H}])[\mathrm{H}]) \mathrm{C}([\mathrm{H}])([\mathrm{H}]) \mathrm{C}([\mathrm{H}])(\mathrm{C}([\mathrm{H}])([\mathrm{H}])[\mathrm{H}]) \mathrm{C}([\mathrm{H}])([\mathrm{H} \\
]\end{array}$ & 3.538 & 4.347 \\
\hline $\operatorname{Tr} 167$ & $\begin{array}{l}\mathrm{O}=[\mathrm{S}](=\mathrm{O})(\mathrm{C}([\mathrm{H}])([\mathrm{H}])[\mathrm{C} @ @]([\mathrm{H}])(\mathrm{C}(=\mathrm{O}) \mathrm{N}([\mathrm{H}])[\mathrm{C} @]([\mathrm{H}])([\mathrm{C} @]([\mathrm{H}])(\mathrm{O}[\mathrm{H}]) \mathrm{C}([\mathrm{H}])([\mathrm{H}])[\mathrm{C} \\
@]([\mathrm{H}])(\mathrm{C}(=\mathrm{O}) \mathrm{N}([\mathrm{H}])[\mathrm{C} @]([\mathrm{H}])(\mathrm{C}(=\mathrm{O}) \mathrm{N}([\mathrm{H}]) \mathrm{C}([\mathrm{H}])([\mathrm{H}]) \mathrm{Cl}=\mathrm{C}([\mathrm{H}]) \mathrm{C}([\mathrm{H}])=\mathrm{C}([\mathrm{H}]) \mathrm{C}([\mathrm{H}])=\mathrm{C} \\
1[\mathrm{H}]) \mathrm{C}([\mathrm{H}])(\mathrm{C}([\mathrm{H}])([\mathrm{H}])[\mathrm{H}]) \mathrm{C}([\mathrm{H}])([\mathrm{H}])[\mathrm{H}]) \mathrm{C}([\mathrm{H}])([\mathrm{H}])[\mathrm{H}]) \mathrm{C}([\mathrm{H}])([\mathrm{H}]) \mathrm{C}([\mathrm{H}])(\mathrm{C}([\mathrm{H}])([\mathrm{H}])[ \\
\mathrm{H}]) \mathrm{C}\end{array}$ & 1.947 & 2.361 \\
\hline $\operatorname{Tr} 168$ & $\begin{array}{l}\mathrm{O}=\mathrm{C}(\mathrm{N}([\mathrm{H}])[\mathrm{C} @]([\mathrm{H}])([\mathrm{C} @]([\mathrm{H}])(\mathrm{O}[\mathrm{H}]) \mathrm{C}([\mathrm{H}])([\mathrm{H}])[\mathrm{C} @]([\mathrm{H}])(\mathrm{C}(=\mathrm{O}) \mathrm{N}([\mathrm{H}])[\mathrm{C} @]([\mathrm{H}])(\mathrm{C}(= \\
\mathrm{O}) \mathrm{N}([\mathrm{H}]) \mathrm{C}([\mathrm{H}])([\mathrm{H}]) \mathrm{Cl}=\mathrm{C}([\mathrm{H}]) \mathrm{C}([\mathrm{H}])=\mathrm{C}([\mathrm{H}]) \mathrm{C}([\mathrm{H}])=\mathrm{C} 1[\mathrm{H}]) \mathrm{C}([\mathrm{H}])(\mathrm{C}([\mathrm{H}])([\mathrm{H}])[\mathrm{H}]) \mathrm{C}([\mathrm{H}])([ \\
\mathrm{H}])[\mathrm{H}]) \mathrm{C}([\mathrm{H}])([\mathrm{H}])[\mathrm{H}]) \mathrm{C}([\mathrm{H}])([\mathrm{H}]) \mathrm{C}([\mathrm{H}])(\mathrm{C}([\mathrm{H}])([\mathrm{H}])[\mathrm{H}]) \mathrm{C}([\mathrm{H}])([\mathrm{H}])[\mathrm{H}])[\mathrm{C} @ @]([\mathrm{H}])(\mathrm{N}([\mathrm{H} \\
]) \mathrm{C}(\end{array}$ & 1.504 & 2.224 \\
\hline $\operatorname{Tr} 169$ & $\begin{array}{l}\mathrm{O}=\mathrm{C}(\mathrm{N}([\mathrm{H}])[\mathrm{C} @ @]([\mathrm{H}])(\mathrm{C}(=\mathrm{O}) \mathrm{N}([\mathrm{H}])[\mathrm{C} @]([\mathrm{H}])([\mathrm{C} @]([\mathrm{H}])(\mathrm{O}[\mathrm{H}]) \mathrm{C}([\mathrm{H}])([\mathrm{H}])[\mathrm{C} @]([\mathrm{H}])(\mathrm{C} \\
=\mathrm{O}) \mathrm{N}([\mathrm{H}])[\mathrm{C} @]([\mathrm{H}])(\mathrm{C}(=\mathrm{O}) \mathrm{N}([\mathrm{H}]) \mathrm{C}([\mathrm{H}])([\mathrm{H}]) \mathrm{C}=\mathrm{C}([\mathrm{H}]) \mathrm{C}([\mathrm{H}])=\mathrm{C}([\mathrm{H}]) \mathrm{C}([\mathrm{H}])=\mathrm{C} 1[\mathrm{H}]) \mathrm{C}([\mathrm{H}] \\
)(\mathrm{C}([\mathrm{H}])([\mathrm{H}])[\mathrm{H}]) \mathrm{C}([\mathrm{H}])([\mathrm{H}])[\mathrm{H}]) \mathrm{C}([\mathrm{H}])([\mathrm{H}])[\mathrm{H}]) \mathrm{C}([\mathrm{H}])([\mathrm{H}]) \mathrm{C}([\mathrm{H}])(\mathrm{C}([\mathrm{H}])([\mathrm{H}])[\mathrm{H}]) \mathrm{C}([\mathrm{H}])([ \\
\mathrm{H}])[\end{array}$ & 4.602 & 2.739 \\
\hline $\operatorname{Tr} 170$ & $\begin{array}{l}\mathrm{O}=\mathrm{C} 1 \mathrm{~N}(/ \mathrm{C}(=\mathrm{N} /[\mathrm{H}]) \mathrm{N}([\mathrm{H}])[\mathrm{C} @] 1(\mathrm{C}([\mathrm{H}])([\mathrm{H}]) \mathrm{C}([\mathrm{H}])([\mathrm{H}])[\mathrm{C} @ @] 2([\mathrm{H}]) \mathrm{C}([\mathrm{H}])([\mathrm{H}]) \mathrm{C}([\mathrm{H}])([\mathrm{H} \\
]) \mathrm{C}([\mathrm{H}])([\mathrm{H}]) \mathrm{C}([\mathrm{H}])([\mathrm{H}]) \mathrm{C} 2([\mathrm{H}])[\mathrm{H}]) \mathrm{C}([\mathrm{H}])([\mathrm{H}])[\mathrm{C} @] 4([\mathrm{H}]) \mathrm{C}([\mathrm{H}])([\mathrm{H}])[\mathrm{C} @]([\mathrm{H}])(\mathrm{N}([\mathrm{H}]) \mathrm{C}( \\
=\mathrm{O}) \mathrm{C} 3=\mathrm{C}([\mathrm{H}]) \mathrm{C}([\mathrm{H}])=\mathrm{NC}([\mathrm{H}])=\mathrm{C} 3[\mathrm{H}]) \mathrm{C}([\mathrm{H}])([\mathrm{H}]) \mathrm{C}([\mathrm{H}])([\mathrm{H}]) \mathrm{C} 4([\mathrm{H}])[\mathrm{H}]) \mathrm{C}([\mathrm{H}])([\mathrm{H}])[\mathrm{H}]\end{array}$ & 3.409 & 3.083 \\
\hline $\operatorname{Tr} 171$ & $\begin{array}{l}\mathrm{O}=\mathrm{C} 1 \mathrm{~N}(/ \mathrm{C}(=\mathrm{N} \backslash[\mathrm{H}]) \mathrm{N}([\mathrm{H}])[\mathrm{C} @] 1(\mathrm{C}([\mathrm{H}])([\mathrm{H}]) \mathrm{C}([\mathrm{H}])([\mathrm{H}])[\mathrm{C} @ @] 2([\mathrm{H}]) \mathrm{C}([\mathrm{H}])([\mathrm{H}]) \mathrm{C}([\mathrm{H}])([\mathrm{H} \\
]) \mathrm{C}([\mathrm{H}])([\mathrm{H}]) \mathrm{C}([\mathrm{H}])([\mathrm{H}]) \mathrm{C} 2([\mathrm{H}])[\mathrm{H}]) \mathrm{C}([\mathrm{H}])([\mathrm{H}])[\mathrm{C} @] 4([\mathrm{H}]) \mathrm{C}([\mathrm{H}])([\mathrm{H}])[\mathrm{C} @]([\mathrm{H}])(\mathrm{N}([\mathrm{H}])[\mathrm{C} \\
@ @] 3([\mathrm{H}]) \mathrm{C}([\mathrm{H}])([\mathrm{H}]) \mathrm{C}([\mathrm{H}])([\mathrm{H}]) \mathrm{C}([\mathrm{H}])([\mathrm{H}]) \mathrm{C}([\mathrm{H}])([\mathrm{H}]) \mathrm{C} 3([\mathrm{H}])[\mathrm{H}]) \mathrm{C}([\mathrm{H}])([\mathrm{H}]) \mathrm{C}([\mathrm{H}])([\mathrm{H}] \\
) \mathrm{C}\end{array}$ & 3.638 & 3.244 \\
\hline $\operatorname{Tr} 172$ & $\begin{array}{l}\mathrm{O}=\mathrm{C} 1 \mathrm{~N}(/ \mathrm{C}(=\mathrm{N} \backslash[\mathrm{H}]) \mathrm{N}([\mathrm{H}])[\mathrm{C} @] 1(\mathrm{C}([\mathrm{H}])([\mathrm{H}]) \mathrm{C}([\mathrm{H}])([\mathrm{H}])[\mathrm{C} @ @] 2([\mathrm{H}]) \mathrm{C}([\mathrm{H}])([\mathrm{H}]) \mathrm{C}([\mathrm{H}])([\mathrm{H} \\
]) \mathrm{C}([\mathrm{H}])([\mathrm{H}]) \mathrm{C}([\mathrm{H}])([\mathrm{H}]) \mathrm{C} 2([\mathrm{H}])[\mathrm{H}]) \mathrm{C}([\mathrm{H}])([\mathrm{H}])[\mathrm{C} @] 4([\mathrm{H}]) \mathrm{C}([\mathrm{H}])([\mathrm{H}])[\mathrm{C} @]([\mathrm{H}])(\mathrm{N}([\mathrm{H}])[\mathrm{C} \\
@] 3([\mathrm{H}]) \mathrm{C}([\mathrm{H}])([\mathrm{H}]) \mathrm{C}([\mathrm{H}])([\mathrm{H}]) \mathrm{C}([\mathrm{H}])([\mathrm{H}]) \mathrm{C} 3([\mathrm{H}])[\mathrm{H}]) \mathrm{C}([\mathrm{H}])([\mathrm{H}]) \mathrm{C}([\mathrm{H}])([\mathrm{H}]) \mathrm{C} 4([\mathrm{H}])[\mathrm{H}]) \mathrm{C} \\
(\end{array}$ & 3.367 & 3.076 \\
\hline $\operatorname{Tr} 173$ & $\begin{array}{l}\mathrm{O}=\mathrm{C}(\mathrm{N}([\mathrm{H}])[\mathrm{C} @]([\mathrm{H}])(\mathrm{C}(=\mathrm{O}) \mathrm{N}([\mathrm{H}])[\mathrm{C} @]((\mathrm{H}])([\mathrm{C} @ @]([\mathrm{H}])(\mathrm{O}[\mathrm{H}]) \mathrm{C}([\mathrm{H}])([\mathrm{H}])[\mathrm{C} @]([\mathrm{H}])(\mathrm{C}( \\
=\mathrm{O}) \mathrm{N}([\mathrm{H}])[\mathrm{C} @([\mathrm{H}])(\mathrm{C}(=\mathrm{O}) \mathrm{N}([\mathrm{H}]) \mathrm{C}([\mathrm{H}])([\mathrm{H}]) \mathrm{C}([\mathrm{H}])(\mathrm{C}([\mathrm{H}])([\mathrm{H}])[\mathrm{H}]) \mathrm{C}([\mathrm{H}])([\mathrm{H}])[\mathrm{H}]) \mathrm{C}([\mathrm{H}]) \\
(\mathrm{C}([\mathrm{H}])([\mathrm{H}])[\mathrm{H}]) \mathrm{C}([\mathrm{H}])([\mathrm{H}])[\mathrm{H}]) \mathrm{C}([\mathrm{H}])([\mathrm{H}])[\mathrm{H}]) \mathrm{C}([\mathrm{H}])([\mathrm{H}]) \mathrm{C}([\mathrm{H}])(\mathrm{C}([\mathrm{H}])([\mathrm{H}])[\mathrm{H}]) \mathrm{C}([\mathrm{H}])([\mathrm{H} \\
]\end{array}$ & 3.398 & 3.020 \\
\hline $\operatorname{Tr} 174$ & $\begin{array}{l}\mathrm{O}=\mathrm{C}(\mathrm{N}([\mathrm{H}])[\mathrm{C} @(([\mathrm{H}])(\mathrm{C}(=\mathrm{O}) \mathrm{N}([\mathrm{H}])[\mathrm{C} @]([\mathrm{H}])([\mathrm{C} @ @]([\mathrm{H}])(\mathrm{O}[\mathrm{H}]) \mathrm{C}([\mathrm{H}])([\mathrm{H}])[\mathrm{C} @]([\mathrm{H}])(\mathrm{C}( \\
=\mathrm{O}) \mathrm{N}([\mathrm{H}])[\mathrm{C} @([\mathrm{H}])(\mathrm{C}(=\mathrm{O}) \mathrm{N}([\mathrm{H}]) \mathrm{C}([\mathrm{H}])([\mathrm{H}]) \mathrm{C}([\mathrm{H}])(\mathrm{C}([\mathrm{H}])([\mathrm{H}])[\mathrm{H}]) \mathrm{C}([\mathrm{H}])([\mathrm{H}])[\mathrm{H}]) \mathrm{C}([\mathrm{H}]) \\
(\mathrm{C}([\mathrm{H}])([\mathrm{H}])[\mathrm{H}]) \mathrm{C}([\mathrm{H}])([\mathrm{H}])[\mathrm{H}]) \mathrm{C}([\mathrm{H}])([\mathrm{H}])[\mathrm{H}]) \mathrm{C}([\mathrm{H}])([\mathrm{H}]) \mathrm{C}([\mathrm{H}])(\mathrm{C}([\mathrm{H}])([\mathrm{H}])[\mathrm{H}]) \mathrm{C}([\mathrm{H}])([\mathrm{H} \\
]\end{array}$ & 1.785 & 3.092 \\
\hline $\operatorname{Tr} 175$ & $\begin{array}{l}\mathrm{O}=\mathrm{C}(\mathrm{N}([\mathrm{H}])[\mathrm{C} @]([\mathrm{H}])(\mathrm{C}(=\mathrm{O}) \mathrm{N}([\mathrm{H}])[\mathrm{C} @]((\mathrm{H}])([\mathrm{C} @ @]([\mathrm{H}])(\mathrm{O}[\mathrm{H}]) \mathrm{C}([\mathrm{H}])([\mathrm{H}])[\mathrm{C} @]([\mathrm{H}])(\mathrm{C}( \\
=\mathrm{O}) \mathrm{N}([\mathrm{H}])[\mathrm{C} @([\mathrm{H}])(\mathrm{C}(=\mathrm{O}) \mathrm{N}([\mathrm{H}]) \mathrm{C}([\mathrm{H}])([\mathrm{H}]) \mathrm{C}([\mathrm{H}])(\mathrm{C}([\mathrm{H}])([\mathrm{H}])[\mathrm{H}]) \mathrm{C}([\mathrm{H}])([\mathrm{H}])[\mathrm{H}]) \mathrm{C}([\mathrm{H}]) \\
(\mathrm{C}([\mathrm{H}])([\mathrm{H}])[\mathrm{H}]) \mathrm{C}([\mathrm{H}])([\mathrm{H}])[\mathrm{H}]) \mathrm{C}([\mathrm{H}])([\mathrm{H}])[\mathrm{H}]) \mathrm{C}([\mathrm{H}])([\mathrm{H}]) \mathrm{C}([\mathrm{H}])(\mathrm{C}([\mathrm{H}])([\mathrm{H}])[\mathrm{H}]) \mathrm{C}([\mathrm{H}])([\mathrm{H} \\
]\end{array}$ & 3.201 & 3.044 \\
\hline $\operatorname{Tr} 176$ & $\begin{array}{l}\mathrm{O}=\mathrm{C}(\mathrm{N}([\mathrm{H}])[\mathrm{C} @]([\mathrm{H}])(\mathrm{C}(=\mathrm{O}) \mathrm{N}([\mathrm{H}])[\mathrm{C} @]([\mathrm{H}])([\mathrm{C} @ @]([\mathrm{H}])(\mathrm{O}[\mathrm{H}]) \mathrm{C}([\mathrm{H}])([\mathrm{H}])[\mathrm{C} @]([\mathrm{H}])(\mathrm{C}( \\
=\mathrm{O}) \mathrm{N}([\mathrm{H}])[\mathrm{C} @([\mathrm{H}])(\mathrm{C}(=\mathrm{O}) \mathrm{N}([\mathrm{H}]) \mathrm{C}([\mathrm{H}])([\mathrm{H}]) \mathrm{C}([\mathrm{H}])(\mathrm{C}([\mathrm{H}])([\mathrm{H}])[\mathrm{H}]) \mathrm{C}([\mathrm{H}])([\mathrm{H}])[\mathrm{H}]) \mathrm{C}([\mathrm{H}]) \\
(\mathrm{C}([\mathrm{H}])([\mathrm{H}])[\mathrm{H}]) \mathrm{C}([\mathrm{H}])([\mathrm{H}])[\mathrm{H}]) \mathrm{C}([\mathrm{H}])([\mathrm{H}])[\mathrm{H}]) \mathrm{C}([\mathrm{H}])([\mathrm{H}]) \mathrm{C}([\mathrm{H}])(\mathrm{C}([\mathrm{H}])([\mathrm{H}])[\mathrm{H}]) \mathrm{C}([\mathrm{H}])([\mathrm{H} \\
]\end{array}$ & 2.697 & 2.966 \\
\hline $\operatorname{Tr} 177$ & $\begin{array}{l}\mathrm{O}=\mathrm{C}(\mathrm{N}([\mathrm{H}])[\mathrm{C} @]([\mathrm{H}])(\mathrm{C}(=\mathrm{O}) \mathrm{N}([\mathrm{H}])[\mathrm{C} @]([\mathrm{H}])([\mathrm{C} @ @]([\mathrm{H}])(\mathrm{O}[\mathrm{H}]) \mathrm{C}([\mathrm{H}])([\mathrm{H}])[\mathrm{C} @]([\mathrm{H}])(\mathrm{C}( \\
=\mathrm{O}) \mathrm{N}([\mathrm{H}])[\mathrm{C} @]([\mathrm{H}])(\mathrm{C}(=\mathrm{O}) \mathrm{N}([\mathrm{H}]) \mathrm{C}([\mathrm{H}])([\mathrm{H}]) \mathrm{C}([\mathrm{H}])(\mathrm{C}([\mathrm{H}])([\mathrm{H}])[\mathrm{H}]) \mathrm{C}([\mathrm{H}])([\mathrm{H}])[\mathrm{H}]) \mathrm{C}([\mathrm{H}]) \\
(\mathrm{C}([\mathrm{H}])([\mathrm{H}])[\mathrm{H}]) \mathrm{C}([\mathrm{H}])([\mathrm{H}])[\mathrm{H}]) \mathrm{C}([\mathrm{H}])([\mathrm{H}])[\mathrm{H}]) \mathrm{C}([\mathrm{H}])([\mathrm{H}]) \mathrm{C}([\mathrm{H}])(\mathrm{C}([\mathrm{H}])([\mathrm{H}])[\mathrm{H}]) \mathrm{C}([\mathrm{H}])([\mathrm{H} \\
]\end{array}$ & 3.194 & 2.714 \\
\hline
\end{tabular}




\begin{tabular}{|c|c|c|c|}
\hline $\operatorname{Tr} 178$ & $\begin{array}{l}\mathrm{O}=[\mathrm{S}](=\mathrm{O})(\mathrm{C}([\mathrm{H}])([\mathrm{H}])[\mathrm{C} @]([\mathrm{H}])(\mathrm{N}([\mathrm{H}]) \mathrm{C}(=\mathrm{O})[\mathrm{C} @]([\mathrm{H}])(\mathrm{C}([\mathrm{H}])([\mathrm{H}]) \mathrm{N} 1 \mathrm{~N}=\mathrm{C}(\mathrm{C}([\mathrm{H}])=\mathrm{C} 1 \mathrm{C}( \\
[\mathrm{H}])([\mathrm{H}])[\mathrm{H}]) \mathrm{C}([\mathrm{H}])([\mathrm{H}])[\mathrm{H}]) \mathrm{C}([\mathrm{H}])([\mathrm{H}])[\mathrm{H}]) \mathrm{C}(=\mathrm{O}) \mathrm{N}([\mathrm{H}])[\mathrm{C} @]([\mathrm{H}])([\mathrm{C} @ @]([\mathrm{H}])(\mathrm{O}[\mathrm{H}]) \mathrm{C}([ \\
\mathrm{H}])([\mathrm{H}])[\mathrm{C} @]([\mathrm{H}])(\mathrm{C}(=\mathrm{O}) \mathrm{N}([\mathrm{H}])[\mathrm{C} @]([\mathrm{H}])(\mathrm{C}(=\mathrm{O}) \mathrm{N}([\mathrm{H}]) \mathrm{C}([\mathrm{H}])([\mathrm{H}]) \mathrm{C}([\mathrm{H}])(\mathrm{C}([\mathrm{H}])([\mathrm{H}])[\mathrm{H}]) \\
\mathrm{C}([\mathrm{H}])(\end{array}$ & 2.717 & 3.278 \\
\hline $\operatorname{Tr} 179$ & $\begin{array}{l}\mathrm{O}=\mathrm{C}(\mathrm{N}([\mathrm{H}])[\mathrm{C} @]([\mathrm{H}])(\mathrm{C}(=\mathrm{O}) \mathrm{N}([\mathrm{H}])[\mathrm{C} @]([\mathrm{H}])([\mathrm{C} @ @]([\mathrm{H}])(\mathrm{O}[\mathrm{H}]) \mathrm{C}([\mathrm{H}])([\mathrm{H}])[\mathrm{C} @]([\mathrm{H}])(\mathrm{C}( \\
=\mathrm{O}) \mathrm{N}([\mathrm{H}])[\mathrm{C} @]([\mathrm{H}])(\mathrm{C}(=\mathrm{O}) \mathrm{N}([\mathrm{H}]) \mathrm{C}([\mathrm{H}])([\mathrm{H}]) \mathrm{C}([\mathrm{H}])(\mathrm{C}([\mathrm{H}])([\mathrm{H}])[\mathrm{H}]) \mathrm{C}([\mathrm{H}])([\mathrm{H}])[\mathrm{H}]) \mathrm{C}([\mathrm{H}]) \\
(\mathrm{C}([\mathrm{H}])([\mathrm{H}])[\mathrm{H}]) \mathrm{C}([\mathrm{H}])([\mathrm{H}])[\mathrm{H}]) \mathrm{C}([\mathrm{H}])([\mathrm{H}])[\mathrm{H}]) \mathrm{C}([\mathrm{H}])([\mathrm{H}]) \mathrm{C}([\mathrm{H}])(\mathrm{C}([\mathrm{H}])([\mathrm{H}])[\mathrm{H}]) \mathrm{C}([\mathrm{H}])([\mathrm{H} \\
]\end{array}$ & 3.495 & 3.369 \\
\hline $\operatorname{Tr} 180$ & $\begin{array}{l}\mathrm{O}=\mathrm{C}(\mathrm{N}([\mathrm{H}])[\mathrm{C} @]([\mathrm{H}])(\mathrm{C}(=\mathrm{O}) \mathrm{N}([\mathrm{H}])[\mathrm{C} @]([\mathrm{H}])([\mathrm{C} @ @]([\mathrm{H}])(\mathrm{O}[\mathrm{H}]) \mathrm{C}([\mathrm{H}])([\mathrm{H}])[\mathrm{C} @]([\mathrm{H}])(\mathrm{C}( \\
=\mathrm{O}) \mathrm{N}([\mathrm{H}])[\mathrm{C} @]([\mathrm{H}])(\mathrm{C}(=\mathrm{O}) \mathrm{N}([\mathrm{H}]) \mathrm{C}([\mathrm{H}])([\mathrm{H}]) \mathrm{C}([\mathrm{H}])(\mathrm{C}([\mathrm{H}])([\mathrm{H}])[\mathrm{H}]) \mathrm{C}([\mathrm{H}])([\mathrm{H}])[\mathrm{H}]) \mathrm{C}([\mathrm{H}]) \\
(\mathrm{C}([\mathrm{H}])([\mathrm{H}])[\mathrm{H}]) \mathrm{C}([\mathrm{H}])([\mathrm{H}])[\mathrm{H}]) \mathrm{C}([\mathrm{H}])([\mathrm{H}])[\mathrm{H}]) \mathrm{C}([\mathrm{H}])([\mathrm{H}]) \mathrm{C}([\mathrm{H}])(\mathrm{C}([\mathrm{H}])([\mathrm{H}])[\mathrm{H}]) \mathrm{C}([\mathrm{H}])([\mathrm{H} \\
]\end{array}$ & 3.770 & 3.393 \\
\hline $\operatorname{Tr} 181$ & $\begin{array}{l}\mathrm{O}=\mathrm{C} 1 \mathrm{~N}(/ \mathrm{C}(=\mathrm{N} \backslash[\mathrm{H}]) \mathrm{N}([\mathrm{H}])[\mathrm{C} @] 1(\mathrm{C}([\mathrm{H}])([\mathrm{H}]) \mathrm{C}([\mathrm{H}])([\mathrm{H}])[\mathrm{C} @ @] 2([\mathrm{H}]) \mathrm{C}([\mathrm{H}])([\mathrm{H}]) \mathrm{C}([\mathrm{H}])([\mathrm{H} \\
]) \mathrm{C}([\mathrm{H}])([\mathrm{H}]) \mathrm{C}([\mathrm{H}])([\mathrm{H}]) \mathrm{C} 2([\mathrm{H}])[\mathrm{H}]) \mathrm{C}([\mathrm{H}])([\mathrm{H}])[\mathrm{C} @ @] 4([\mathrm{H}]) \mathrm{C}([\mathrm{H}])([\mathrm{H}]) \mathrm{N}(\mathrm{C}(=\mathrm{O}) \mathrm{C}([\mathrm{H}])([\mathrm{H} \\
])[\mathrm{C} @ 3([\mathrm{H}]) \mathrm{C}([\mathrm{H}])([\mathrm{H}]) \mathrm{C}([\mathrm{H}])([\mathrm{H}]) \mathrm{C}([\mathrm{H}])([\mathrm{H}]) \mathrm{C} 3([\mathrm{H}])[\mathrm{H}]) \mathrm{C}([\mathrm{H}])([\mathrm{H}]) \mathrm{C} 4([\mathrm{H}])[\mathrm{H}]) \mathrm{C}([\mathrm{H}])([ \\
\mathrm{H}]\end{array}$ & 3.432 & 2.921 \\
\hline $\operatorname{Tr} 182$ & $\begin{array}{l}\mathrm{O}=\mathrm{C} 1 \mathrm{~N}(/ \mathrm{C}(=\mathrm{N} /[\mathrm{H}]) \mathrm{N}([\mathrm{H}])[\mathrm{C} @] 1(\mathrm{C}([\mathrm{H}])([\mathrm{H}]) \mathrm{C}([\mathrm{H}])([\mathrm{H}])[\mathrm{C} @ @ @)([\mathrm{H}]) \mathrm{C}([\mathrm{H}])([\mathrm{H}]) \mathrm{C}([\mathrm{H}])([\mathrm{H} \\
]) \mathrm{C}([\mathrm{H}])([\mathrm{H}]) \mathrm{C}([\mathrm{H}])([\mathrm{H}]) \mathrm{C} 2([\mathrm{H}])[\mathrm{H}]) \mathrm{C}([\mathrm{H}])([\mathrm{H}])[\mathrm{C} @] 3([\mathrm{H}]) \mathrm{C}([\mathrm{H}])([\mathrm{H}]) \mathrm{N}(\mathrm{C}(=\mathrm{O}) \mathrm{C}([\mathrm{H}])([\mathrm{H}]) \\
\mathrm{C}([\mathrm{H}])([\mathrm{H}]) \mathrm{C}([\mathrm{H}])([\mathrm{H}]) \mathrm{C}([\mathrm{H}])([\mathrm{H}])[\mathrm{H}]) \mathrm{C}([\mathrm{H}])([\mathrm{H}]) \mathrm{C}([\mathrm{H}])([\mathrm{H}]) \mathrm{C} 3([\mathrm{H}])(\mathrm{H}]) \mathrm{C}([\mathrm{H}])([\mathrm{H}])[\mathrm{H}]\end{array}$ & 3.495 & 3.191 \\
\hline $\operatorname{Tr} 183$ & $\begin{array}{l}\mathrm{O}=[\mathrm{S}](=\mathrm{O})(\mathrm{N} 3 \mathrm{C}([\mathrm{H}])([\mathrm{H}])[\mathrm{C} @ @]([\mathrm{H}])(\mathrm{C}([\mathrm{H}])([\mathrm{H}])[\mathrm{C} @] 1(\mathrm{~N}([\mathrm{H}]) \mathrm{C}(=\mathrm{N}[\mathrm{H}]) \mathrm{N}(\mathrm{Cl}=\mathrm{O}) \mathrm{C}([\mathrm{H}])([\mathrm{I} \\
\mathrm{H}])[\mathrm{H}]) \mathrm{C}([\mathrm{H}])([\mathrm{H}]) \mathrm{C}([\mathrm{H}])([\mathrm{H}])[\mathrm{C} @ @] 2([\mathrm{H}]) \mathrm{C}([\mathrm{H}])([\mathrm{H}]) \mathrm{C}([\mathrm{H}])([\mathrm{H}]) \mathrm{C}([\mathrm{H}])([\mathrm{H}]) \mathrm{C}([\mathrm{H}])([\mathrm{H}]) \mathrm{C} \\
2([\mathrm{H}])[\mathrm{H}]) \mathrm{C}([\mathrm{H}])([\mathrm{H}]) \mathrm{C}([\mathrm{H}])([\mathrm{H}]) \mathrm{C} 3([\mathrm{H}])[\mathrm{H}]) \mathrm{C}([\mathrm{H}])([\mathrm{H}]) \mathrm{C}([\mathrm{H}])([\mathrm{H}]) \mathrm{C}([\mathrm{H}])([\mathrm{H}]) \mathrm{C}([\mathrm{H}])([\mathrm{H}]))\end{array}$ & 3.201 & 3.509 \\
\hline $\operatorname{Tr} 184$ & $\begin{array}{l}\mathrm{O}=\mathrm{C} 1 \mathrm{~N}(/ \mathrm{C}(=\mathrm{N} \backslash[\mathrm{H}]) \mathrm{N}([\mathrm{H}])[\mathrm{C} @] 1(\mathrm{C}([\mathrm{H}])([\mathrm{H}]) \mathrm{C}([\mathrm{H}])([\mathrm{H}])[\mathrm{C} @ @] 2([\mathrm{H}]) \mathrm{C}([\mathrm{H}])([\mathrm{H}]) \mathrm{C}([\mathrm{H}])([\mathrm{H} \\
]) \mathrm{C}([\mathrm{H}])([\mathrm{H}]) \mathrm{C}([\mathrm{H}])([\mathrm{H}]) \mathrm{C} 2([\mathrm{H}])[\mathrm{H}]) \mathrm{C}([\mathrm{H}])([\mathrm{H}])[\mathrm{C} @] 5([\mathrm{H}]) \mathrm{C}([\mathrm{H}])([\mathrm{H}])[\mathrm{C}]([\mathrm{H}])(\mathrm{N}([\mathrm{H}]) \mathrm{C}( \\
=\mathrm{O}) \mathrm{N}([\mathrm{H}]) \mathrm{C} 3=\mathrm{NC}=4 \mathrm{C}(/ \mathrm{N}=\mathrm{C} 3 /[\mathrm{H}])=\mathrm{C}([\mathrm{H}]) \mathrm{C}([\mathrm{H}])=\mathrm{C}([\mathrm{H}]) \mathrm{C}=4[\mathrm{H}]) \mathrm{C}([\mathrm{H}])([\mathrm{H}]) \mathrm{C}([\mathrm{H}])([\mathrm{H}]) \mathrm{C} 5( \\
[\mathrm{H}])[\mathrm{H}]\end{array}$ & 3.854 & 3.900 \\
\hline $\operatorname{Tr} 185$ & $\begin{array}{l}\mathrm{O}=\mathrm{C} 1 \mathrm{~N}(/ \mathrm{C}(=\mathrm{N} \backslash[\mathrm{H}]) \mathrm{N}([\mathrm{H}])[\mathrm{C} @] 1(\mathrm{C}([\mathrm{H}])([\mathrm{H}]) \mathrm{C}([\mathrm{H}])([\mathrm{H}])[\mathrm{C} @ @] 2([\mathrm{H}]) \mathrm{C}([\mathrm{H}])([\mathrm{H}]) \mathrm{C}([\mathrm{H}])([\mathrm{H} \\
]) \mathrm{C}([\mathrm{H}])([\mathrm{H}]) \mathrm{C}([\mathrm{H}])([\mathrm{H}]) \mathrm{C}([\mathrm{H}])[\mathrm{H}]) \mathrm{C}([\mathrm{H}])([\mathrm{H}])[\mathrm{C} @] 4([\mathrm{H}]) \mathrm{C}([\mathrm{H}])([\mathrm{H}])[\mathrm{C} @]([\mathrm{H}])(\mathrm{N}([\mathrm{H}]) \mathrm{C}( \\
=\mathrm{O}) \mathrm{N}([\mathrm{H}])[\mathrm{C} @ @] 3([\mathrm{H}]) \mathrm{C}([\mathrm{H}])([\mathrm{H}]) \mathrm{C}([\mathrm{H}])([\mathrm{H}]) \mathrm{C}([\mathrm{H}])([\mathrm{H}]) \mathrm{C}([\mathrm{H}])([\mathrm{H}]) \mathrm{C} 3([\mathrm{H}])[\mathrm{H}]) \mathrm{C}([\mathrm{H}])([ \\
\mathrm{H}]) \mathrm{C}\end{array}$ & 2.860 & 3.874 \\
\hline $\operatorname{Tr} 186$ & $\begin{array}{l}\mathrm{O}=\mathrm{C} 1 \mathrm{~N}(/ \mathrm{C}(=\mathrm{N} /[\mathrm{H}]) \mathrm{N}([\mathrm{H}])[\mathrm{C} @] 1(\mathrm{C}([\mathrm{H}])([\mathrm{H}]) \mathrm{C}([\mathrm{H}])([\mathrm{H}])[\mathrm{C} @ @] 2([\mathrm{H}]) \mathrm{C}([\mathrm{H}])([\mathrm{H}]) \mathrm{C}([\mathrm{H}])([\mathrm{H} \\
]) \mathrm{C}([\mathrm{H}])([\mathrm{H}]) \mathrm{C}([\mathrm{H}])([\mathrm{H}]) \mathrm{C} 2([\mathrm{H}])[\mathrm{H}]) \mathrm{C}([\mathrm{H}])([\mathrm{H}])[\mathrm{C} @] 4([\mathrm{H}]) \mathrm{C}([\mathrm{H}])([\mathrm{H}])[\mathrm{C} @]([\mathrm{H}])(\mathrm{N}([\mathrm{H}]) \mathrm{C}( \\
=\mathrm{O}) \mathrm{N}([\mathrm{H}]) \mathrm{C} 3=\mathrm{C}(\mathrm{OC}([\mathrm{H}])([\mathrm{H}])[\mathrm{H}]) \mathrm{C}([\mathrm{H}])=\mathrm{C}([\mathrm{H}]) \mathrm{C}([\mathrm{H}])=\mathrm{C} 3[\mathrm{H}]) \mathrm{C}([\mathrm{H}])([\mathrm{H}]) \mathrm{C}([\mathrm{H}])([\mathrm{H}]) \mathrm{C} 4([ \\
\mathrm{H}])[\mathrm{H}\end{array}$ & 2.559 & 3.239 \\
\hline $\operatorname{Tr} 187$ & $\begin{array}{l}\mathrm{O}=\mathrm{C} 1 \mathrm{~N}(/ \mathrm{C}(=\mathrm{N} \backslash[\mathrm{H}]) \mathrm{N}([\mathrm{H}])[\mathrm{C} @] 1(\mathrm{C}([\mathrm{H}])([\mathrm{H}]) \mathrm{C}([\mathrm{H}])([\mathrm{H}])[\mathrm{C} @] 2([\mathrm{H}]) \mathrm{C}([\mathrm{H}])([\mathrm{H}]) \mathrm{C}([\mathrm{H}])([\mathrm{H}]) \\
\mathrm{C}([\mathrm{H}])([\mathrm{H}]) \mathrm{C}([\mathrm{H}])([\mathrm{H}]) \mathrm{C} 2([\mathrm{H}])[\mathrm{H}]) \mathrm{C}([\mathrm{H}])([\mathrm{H}])[\mathrm{C} @] 4([\mathrm{H}]) \mathrm{C}([\mathrm{H}])([\mathrm{H}])[\mathrm{C} @]([\mathrm{H}])(\mathrm{N}([\mathrm{H}]) \mathrm{C}(= \\
\mathrm{O}) \mathrm{N}([\mathrm{H}]) \mathrm{C} 3=\mathrm{C}([\mathrm{H}]) \mathrm{C}([\mathrm{H}])=\mathrm{C}([\mathrm{Cl}]) \mathrm{C}([\mathrm{H}])=\mathrm{C} 3[\mathrm{H}]) \mathrm{C}([\mathrm{H}])([\mathrm{H}]) \mathrm{C}([\mathrm{H}])([\mathrm{H}]) \mathrm{C} 4([\mathrm{H}])[\mathrm{H}]) \mathrm{C}([\mathrm{H}])( \\
{[\mathrm{H}]}\end{array}$ & 5.000 & 4.115 \\
\hline $\operatorname{Tr} 188$ & $\begin{array}{l}\mathrm{O}=\mathrm{C} 1 \mathrm{~N}(/ \mathrm{C}(=\mathrm{N} \backslash[\mathrm{H}]) \mathrm{N}([\mathrm{H}])[\mathrm{C} @] 1(\mathrm{C}([\mathrm{H}])([\mathrm{H}]) \mathrm{C}([\mathrm{H}])([\mathrm{H}])[\mathrm{C} @ @] 2([\mathrm{H}]) \mathrm{C}([\mathrm{H}])([\mathrm{H}]) \mathrm{C}([\mathrm{H}])([\mathrm{H} \\
]) \mathrm{C}([\mathrm{H}])([\mathrm{H}]) \mathrm{C}([\mathrm{H}])([\mathrm{H}]) \mathrm{C} 2([\mathrm{H}])[\mathrm{H}]) \mathrm{C}([\mathrm{H}])([\mathrm{H}])[\mathrm{C} @] 4([\mathrm{H}]) \mathrm{C}([\mathrm{H}])([\mathrm{H}])[\mathrm{C} @]([\mathrm{H}])(\mathrm{N}([\mathrm{H}]) \mathrm{C}( \\
=\mathrm{O}) \mathrm{C}([\mathrm{H}])([\mathrm{H}]) \mathrm{C} 3=\mathrm{C}([\mathrm{H}]) \mathrm{C}([\mathrm{H}])=\mathrm{C}([\mathrm{H}]) \mathrm{C}([\mathrm{H}])=\mathrm{C} 3[\mathrm{H}]) \mathrm{C}([\mathrm{H}])([\mathrm{H}]) \mathrm{C}([\mathrm{H}])([\mathrm{H}]) \mathrm{C} 4([\mathrm{H}])[\mathrm{H}]) \mathrm{C} \\
([\mathrm{H}]\end{array}$ & 2.233 & 2.585 \\
\hline $\operatorname{Tr} 189$ & $\begin{array}{l}\mathrm{O}=\mathrm{C} 1 \mathrm{~N}(/ \mathrm{C}(=\mathrm{N} \backslash[\mathrm{H}]) \mathrm{N}([\mathrm{H}])[\mathrm{C} @ @] 1(\mathrm{C}([\mathrm{H}])([\mathrm{H}])[\mathrm{C} @ @] 2([\mathrm{H}]) \mathrm{C}([\mathrm{H}])([\mathrm{H}]) \mathrm{C}([\mathrm{H}])([\mathrm{H}]) \mathrm{C}([\mathrm{H}])( \\
[\mathrm{H}]) \mathrm{C}([\mathrm{H}])([\mathrm{H}]) \mathrm{C} 2([\mathrm{H}])[\mathrm{H}]) \mathrm{C}([\mathrm{H}])([\mathrm{H}]) \mathrm{C}([\mathrm{H}])([\mathrm{H}])[\mathrm{C} @ @]([\mathrm{H}]) \mathrm{C}([\mathrm{H}])([\mathrm{H}]) \mathrm{C}([\mathrm{H}])([\mathrm{H}]) \mathrm{C}([ \\
\mathrm{H}])([\mathrm{H}]) \mathrm{C}([\mathrm{H}])([\mathrm{H}]) \mathrm{C} 3([\mathrm{H}])(\mathrm{H}]) \mathrm{C}([\mathrm{H}])([\mathrm{H}]) \mathrm{C} 4=\mathrm{C}([\mathrm{H}]) \mathrm{C}([\mathrm{H}])) \mathrm{C}(\mathrm{C}([\mathrm{H}])=\mathrm{C} 4[\mathrm{H}]) \mathrm{C}([\mathrm{H}])([\mathrm{H}]) \\
\mathrm{N} 5 \mathrm{C}(=\mathrm{O}\end{array}$ & 3.569 & 4.795 \\
\hline $\operatorname{Tr} 190$ & $\mathrm{O}=\mathrm{C} 1 \mathrm{~N}(/ \mathrm{C}(=\mathrm{N} /[\mathrm{H}]) \mathrm{N}([\mathrm{H}])[\mathrm{C} @] 1(\mathrm{C}([\mathrm{H}])([\mathrm{H}]) \mathrm{C}([\mathrm{H}])([\mathrm{H}])[\mathrm{C} @ @] 2([\mathrm{H}]) \mathrm{C}([\mathrm{H}])([\mathrm{H}]) \mathrm{C}([\mathrm{H}])([\mathrm{H}$ & 3.770 & 3.335 \\
\hline
\end{tabular}




\begin{tabular}{|c|c|c|c|}
\hline & $\begin{array}{l}]) \mathrm{C}([\mathrm{H}])([\mathrm{H}]) \mathrm{C}([\mathrm{H}])([\mathrm{H}]) \mathrm{C} 2([\mathrm{H}])[\mathrm{H}]) \mathrm{C}([\mathrm{H}])([\mathrm{H}])[\mathrm{C} @ @] 3([\mathrm{H}]) \mathrm{C}([\mathrm{H}])([\mathrm{H}]) \mathrm{N}(\mathrm{C}(=\mathrm{O}) \mathrm{C}([\mathrm{H}])([\mathrm{H} \\
]) \mathrm{C}([\mathrm{H}])([\mathrm{H}]) \mathrm{C}([\mathrm{H}])([\mathrm{H}]) \mathrm{C}([\mathrm{H}])([\mathrm{H}])[\mathrm{H}]) \mathrm{C}([\mathrm{H}])([\mathrm{H}]) \mathrm{C}([\mathrm{H}])([\mathrm{H}]) \mathrm{C} 3([\mathrm{H}])[\mathrm{H}]) \mathrm{C}([\mathrm{H}])([\mathrm{H}])[\mathrm{H}]\end{array}$ & & \\
\hline $\operatorname{Tr} 191$ & $\begin{array}{l}\mathrm{O}=\mathrm{C} 1 \mathrm{~N}(/ \mathrm{C}(=\mathrm{N} \backslash[\mathrm{H}]) \mathrm{N}([\mathrm{H}])[\mathrm{C} @] 1(\mathrm{C}([\mathrm{H}])([\mathrm{H}]) \mathrm{C}([\mathrm{H}])([\mathrm{H}])[\mathrm{C} @ @] 2([\mathrm{H}]) \mathrm{C}([\mathrm{H}])([\mathrm{H}]) \mathrm{C}([\mathrm{H}])([\mathrm{H} \\
]) \mathrm{C}([\mathrm{H}])([\mathrm{H}]) \mathrm{C}([\mathrm{H}])([\mathrm{H}]) \mathrm{C} 2([\mathrm{H}])[\mathrm{H}]) \mathrm{C}([\mathrm{H}])([\mathrm{H}])[\mathrm{C} @] 4([\mathrm{H}]) \mathrm{C}([\mathrm{H}])([\mathrm{H}]) \mathrm{N}(\mathrm{C}(=\mathrm{O}) \mathrm{C}([\mathrm{H}])([\mathrm{H}]) \\
{[\mathrm{C} @ 3([\mathrm{H}]) \mathrm{C}([\mathrm{H}])([\mathrm{H}]) \mathrm{C}([\mathrm{H}])([\mathrm{H}]) \mathrm{C}([\mathrm{H}])([\mathrm{H}]) \mathrm{C} 3([\mathrm{H}])[\mathrm{H}]) \mathrm{C}([\mathrm{H}])([\mathrm{H}]) \mathrm{C}([\mathrm{H}])([\mathrm{H}]) \mathrm{C} 4([\mathrm{H}])[\mathrm{H}]} \\
)\end{array}$ & 3.013 & 3.527 \\
\hline $\operatorname{Tr} 192$ & $\begin{array}{l}\mathrm{O}=\mathrm{C} 1 \mathrm{~N}(/ \mathrm{C}(=\mathrm{N} \backslash[\mathrm{H}]) \mathrm{N}([\mathrm{H}])[\mathrm{C} @] 1(\mathrm{C}([\mathrm{H}])([\mathrm{H}]) \mathrm{C}([\mathrm{H}])([\mathrm{H}])[\mathrm{C} @ @] 2([\mathrm{H}]) \mathrm{C}([\mathrm{H}])([\mathrm{H}]) \mathrm{C}([\mathrm{H}])([\mathrm{H} \\
]) \mathrm{C}([\mathrm{H}])([\mathrm{H}]) \mathrm{C}([\mathrm{H}])([\mathrm{H}]) \mathrm{C} 2([\mathrm{H}])[\mathrm{H}]) \mathrm{C}([\mathrm{H}])([\mathrm{H}])[\mathrm{C} @ 3]([\mathrm{H}]) \mathrm{C}([\mathrm{H}])([\mathrm{H}]) \mathrm{N}(\mathrm{C}(=\mathrm{O}) \mathrm{OC}(\mathrm{C}([\mathrm{H}])( \\
[\mathrm{H}])[\mathrm{H}])(\mathrm{C}([\mathrm{H}])([\mathrm{H}])[\mathrm{H}]) \mathrm{C}([\mathrm{H}])([\mathrm{H}])[\mathrm{H}]) \mathrm{C}([\mathrm{H}])([\mathrm{H}]) \mathrm{C}([\mathrm{H}])([\mathrm{H}]) \mathrm{C} 3([\mathrm{H}])[\mathrm{H}]) \mathrm{C}([\mathrm{H}])([\mathrm{H}])[\mathrm{H}]\end{array}$ & 3.538 & 3.215 \\
\hline $\operatorname{Tr} 193$ & $\begin{array}{l}\mathrm{O}=[\mathrm{S}] 2(=\mathrm{O}) \mathrm{N}(/ \mathrm{C}(=\mathrm{N} /[\mathrm{H}]) \mathrm{N}([\mathrm{H}])[\mathrm{C} @] 4(/ \mathrm{C} 1=\mathrm{C}([\mathrm{H}]) \mathrm{C}([\mathrm{F}])=\mathrm{C}([\mathrm{F}]) \mathrm{C}([\mathrm{H}])=\mathrm{C} 1[\mathrm{~F}])[\mathrm{C} @ @] 2([\mathrm{H} \\
]) \mathrm{C}([\mathrm{H}])([\mathrm{H}]) \mathrm{N}(/ \mathrm{C} 3=\mathrm{N} / \mathrm{C}(=\mathrm{C}(/[\mathrm{F}]) \mathrm{C}(=\mathrm{N} 3) \mathrm{OC}([\mathrm{H}])([\mathrm{H}])[\mathrm{H}]) \mathrm{C}([\mathrm{H}])([\mathrm{H}])[\mathrm{H}]) \mathrm{C} 4([\mathrm{H}])[\mathrm{H}]) \mathrm{C}([\mathrm{H}] \\
)([\mathrm{H}])[\mathrm{H}]\end{array}$ & 3.495 & 3.781 \\
\hline $\operatorname{Tr} 194$ & $\begin{array}{l}\mathrm{O}=[\mathrm{S}] 2(=\mathrm{O}) \mathrm{N}(/ \mathrm{C}(=\mathrm{N} /[\mathrm{H}]) \mathrm{N}([\mathrm{H}])[\mathrm{C} @] 4(/ \mathrm{C} 1=\mathrm{C}([\mathrm{H}]) \mathrm{C}([\mathrm{H}])=\mathrm{C}([\mathrm{F}]) \mathrm{C}([\mathrm{H}])=\mathrm{C} 1[\mathrm{~F}])[\mathrm{C} @] 2([\mathrm{H}]) \\
\mathrm{C}([\mathrm{H}])([\mathrm{H}]) \mathrm{N}(/ \mathrm{C} 3=\mathrm{N} / \mathrm{C}(=\mathrm{C}(/[\mathrm{F}]) \mathrm{C}(=\mathrm{N} 3) \mathrm{OC}([\mathrm{H}])([\mathrm{H}])[\mathrm{H}]) \mathrm{C}([\mathrm{H}])([\mathrm{H}])[\mathrm{H}]) \mathrm{C} 4([\mathrm{H}])[\mathrm{H}]) \mathrm{C}([\mathrm{H}])( \\
[\mathrm{H}])[\mathrm{H}]\end{array}$ & 3.523 & 4.249 \\
\hline $\operatorname{Tr} 195$ & $\begin{array}{l}\mathrm{O}=[\mathrm{S}] 2(=\mathrm{O}) \mathrm{N}(/ \mathrm{C}(=\mathrm{N} /[\mathrm{H}]) \mathrm{N}([\mathrm{H}])[\mathrm{C} @] 4(/ \mathrm{C} 1=\mathrm{C}([\mathrm{H}]) \mathrm{C}([\mathrm{F}])=\mathrm{C}([\mathrm{H}]) \mathrm{C}([\mathrm{H}])=\mathrm{C} 1[\mathrm{~F}])[\mathrm{C} @ @] 2([ \\
\mathrm{H}]) \mathrm{C}([\mathrm{H}])([\mathrm{H}]) \mathrm{N}(/ \mathrm{C} 3=\mathrm{N} / \mathrm{C}(\mathrm{OC}([\mathrm{H}])([\mathrm{H}])[\mathrm{H}])=\mathrm{C}([\mathrm{H}]) \mathrm{C}(=\mathrm{C} 3[\mathrm{H}]) \mathrm{C}([\mathrm{H}])([\mathrm{H}]) \mathrm{OC}([\mathrm{H}])([\mathrm{H}])[\mathrm{H}]) \\
\mathrm{C} 4([\mathrm{H}])[\mathrm{H}]) \mathrm{C}([\mathrm{H}])([\mathrm{H}])[\mathrm{H}]\end{array}$ & 4.000 & 3.121 \\
\hline $\operatorname{Tr} 196$ & $\begin{array}{l}\mathrm{O}=[\mathrm{S}] 2(=\mathrm{O}) \mathrm{N}(/ \mathrm{C}(=\mathrm{N} /[\mathrm{H}]) \mathrm{N}([\mathrm{H}])[\mathrm{C} @ @] 4(/ \mathrm{C} 1=\mathrm{C}([\mathrm{H}]) \mathrm{C}([\mathrm{H}])=\mathrm{C}([\mathrm{H}]) \mathrm{C}([\mathrm{H}])=\mathrm{C} 1[\mathrm{H}])[\mathrm{C} @] 2([ \\
\mathrm{H}]) \mathrm{C}([\mathrm{H}])([\mathrm{H}]) \mathrm{N}(/ \mathrm{C} 3=\mathrm{N} / \mathrm{C}(\mathrm{OC}([\mathrm{H}])([\mathrm{H}])[\mathrm{H}])=\mathrm{C}([\mathrm{H}]) \mathrm{C}(=\mathrm{C} 3[\mathrm{H}]) \mathrm{C}([\mathrm{H}])([\mathrm{H}]) \mathrm{OC}([\mathrm{H}])([\mathrm{H}])[\mathrm{H}]) \\
\mathrm{C} 4([\mathrm{H}])[\mathrm{H}]) \mathrm{C}([\mathrm{H}])([\mathrm{H}])[\mathrm{H}]\end{array}$ & 3.569 & 3.223 \\
\hline $\operatorname{Tr} 197$ & $\begin{array}{l}\mathrm{O}=[\mathrm{S}] 2(=\mathrm{O}) \mathrm{N}(/ \mathrm{C}(=\mathrm{N} /[\mathrm{H}]) \mathrm{N}([\mathrm{H}])[\mathrm{C} @ @] 4(/ \mathrm{C} 1=\mathrm{C}([\mathrm{H}]) \mathrm{C}([\mathrm{H}])=\mathrm{C}([\mathrm{H}]) \mathrm{C}([\mathrm{H}])=\mathrm{C} 1[\mathrm{H}])[\mathrm{C} @] 2([ \\
\mathrm{H}]) \mathrm{C}([\mathrm{H}])([\mathrm{H}]) \mathrm{N}(/ \mathrm{C} 3=\mathrm{N} / \mathrm{C}(\mathrm{OC}([\mathrm{H}])([\mathrm{H}])[\mathrm{H}])=\mathrm{C}([\mathrm{F}]) \mathrm{C}(=\mathrm{N} 3) \mathrm{OC}([\mathrm{H}])([\mathrm{H}])[\mathrm{H}]) \mathrm{C} 4([\mathrm{H}])[\mathrm{H}]) \mathrm{C}([ \\
\mathrm{H}])([\mathrm{H}])[\mathrm{H}]\end{array}$ & 3.523 & 3.553 \\
\hline $\operatorname{Tr} 198$ & $\begin{array}{l}\mathrm{O}=[\mathrm{S}] 2(=\mathrm{O}) \mathrm{N}(/ \mathrm{C}(=\mathrm{N} /[\mathrm{H}]) \mathrm{N}([\mathrm{H}])[\mathrm{C} @ @] 4(/ \mathrm{C} 1=\mathrm{C}([\mathrm{H}]) \mathrm{C}([\mathrm{H}])=\mathrm{C}([\mathrm{H}]) \mathrm{C}([\mathrm{H}])=\mathrm{C} 1[\mathrm{H}])[\mathrm{C} @] 2([ \\
\mathrm{H}]) \mathrm{C}([\mathrm{H}])([\mathrm{H}]) \mathrm{N}(/ \mathrm{C} 3=\mathrm{N} / \mathrm{C}(\mathrm{OC}([\mathrm{H}])([\mathrm{H}])[\mathrm{H}])=\mathrm{C}([\mathrm{H}]) \mathrm{C}([\mathrm{H}])=\mathrm{C} 3[\mathrm{H}]) \mathrm{C} 4([\mathrm{H}])[\mathrm{H}]) \mathrm{C}([\mathrm{H}])([\mathrm{H}])[ \\
\mathrm{H}]\end{array}$ & 3.066 & 2.677 \\
\hline $\operatorname{Tr} 199$ & $\begin{array}{l}\mathrm{O}=[\mathrm{S}] 2(=\mathrm{O}) \mathrm{N}(/ \mathrm{C}(=\mathrm{N} /[\mathrm{H}]) \mathrm{N}([\mathrm{H}])[\mathrm{C} @ @] 5(/ \mathrm{C} 1=\mathrm{C}([\mathrm{H}]) \mathrm{C}([\mathrm{H}])=\mathrm{C}([\mathrm{H}]) \mathrm{C}([\mathrm{H}])=\mathrm{C} 1[\mathrm{H}])[\mathrm{C} @] 2([ \\
\mathrm{H}]) \mathrm{C}([\mathrm{H}])([\mathrm{H}]) \mathrm{N}(/ \mathrm{C} 3=\mathrm{N} / \mathrm{C}(=\mathrm{C}([\mathrm{F}]) \mathrm{C}(=\mathrm{N} 3) \mathrm{OC}([\mathrm{H}])([\mathrm{H}])[\mathrm{H}])[\mathrm{C} @ @] 4([\mathrm{H}]) \mathrm{C}([\mathrm{H}])([\mathrm{H}]) \mathrm{C} 4([\mathrm{H} \\
])[\mathrm{H}]) \mathrm{C} 5([\mathrm{H}])[\mathrm{H}]) \mathrm{C}([\mathrm{H}])([\mathrm{H}])[\mathrm{H}]\end{array}$ & 3.377 & 3.145 \\
\hline $\operatorname{Tr} 200$ & $\begin{array}{l}\mathrm{O}=[\mathrm{S}] 3(=\mathrm{O}) \mathrm{N}(/ \mathrm{C}(=\mathrm{N} /[\mathrm{H}]) \mathrm{N}([\mathrm{H}])[\mathrm{C} @] 5(/ \mathrm{Cl}=\mathrm{C}([\mathrm{H}]) \mathrm{C}(=\mathrm{C}([\mathrm{H}]) \mathrm{C}([\mathrm{H}])=\mathrm{C} 1[\mathrm{~F}]) \mathrm{N}([\mathrm{H}]) \mathrm{C}(=\mathrm{O}) \mathrm{C} \\
2=\mathrm{NC}([\mathrm{H}])=\mathrm{C}([\mathrm{F}]) \mathrm{C}([\mathrm{H}])=\mathrm{C} 2[\mathrm{H}])[\mathrm{C} @] 3([\mathrm{H}]) \mathrm{C}([\mathrm{H}])([\mathrm{H}]) \mathrm{N}(/ \mathrm{C} 4=\mathrm{N} / \mathrm{C}(\mathrm{OC}([\mathrm{H}])([\mathrm{H}])[\mathrm{H}])=\mathrm{C}([\mathrm{F} \\
]) \mathrm{C}(=\mathrm{N} 4) \mathrm{C}([\mathrm{H}])([\mathrm{H}])[\mathrm{H}]) \mathrm{C}([\mathrm{H}])[\mathrm{H}]) \mathrm{C}([\mathrm{H}])([\mathrm{H}])[\mathrm{H}]\end{array}$ & 4.921 & 4.802 \\
\hline $\operatorname{Tr} 201$ & $\begin{array}{l}\mathrm{O}=\mathrm{C}(\mathrm{C}=1 \mathrm{C}([\mathrm{H}])=\mathrm{C}(\mathrm{C}([\mathrm{H}])=\mathrm{C}(\mathrm{C}=1[\mathrm{H}]) \mathrm{C}(=\mathrm{O}) \mathrm{N}([\mathrm{H}])[\mathrm{C} @]([\mathrm{H}])([\mathrm{C} @ @]([\mathrm{H}])(\mathrm{O}[\mathrm{H}]) \mathrm{C}([\mathrm{H}])([\mathrm{H} \\
]) \mathrm{N}([\mathrm{H}])[\mathrm{C} @]([\mathrm{H}])(\mathrm{C}(=\mathrm{O}) \mathrm{N}([\mathrm{H}]) \mathrm{C}([\mathrm{H}])([\mathrm{H}]) \mathrm{C}([\mathrm{H}])(\mathrm{C}([\mathrm{H}])([\mathrm{H}])[\mathrm{H}]) \mathrm{C}([\mathrm{H}])([\mathrm{H}])[\mathrm{H}])[\mathrm{C} @ @]([ \\
\mathrm{H}])(\mathrm{O}[\mathrm{H}]) \mathrm{C}([\mathrm{H}])([\mathrm{H}])[\mathrm{H}]) \mathrm{C}([\mathrm{H}])([\mathrm{H}]) \mathrm{C} 2=\mathrm{C}([\mathrm{H}]) \mathrm{C}([\mathrm{H}])=\mathrm{C}([\mathrm{H}]) \mathrm{C}([\mathrm{H}])=\mathrm{C} 2[\mathrm{H}]) \mathrm{C}([\mathrm{H}])([\mathrm{H}])[\mathrm{H}] \\
) \mathrm{N}([\mathrm{C} @]\end{array}$ & 1.781 & 1.397 \\
\hline $\operatorname{Tr} 202$ & $\begin{array}{l}\mathrm{O}=\mathrm{C}(\mathrm{C}=1 \mathrm{C}([\mathrm{H}])=\mathrm{C}([\mathrm{H}]) \mathrm{C}([\mathrm{H}])=\mathrm{C}(\mathrm{C}=1[\mathrm{H}]) \mathrm{C}(=\mathrm{O}) \mathrm{N}([\mathrm{H}])[\mathrm{C} @]([\mathrm{H}])([\mathrm{C} @ @]([\mathrm{H}])(\mathrm{O}[\mathrm{H}]) \mathrm{C}([\mathrm{H}] \\
)([\mathrm{H}]) \mathrm{N}([\mathrm{H}])[\mathrm{C} @]([\mathrm{H}])(\mathrm{C}(=\mathrm{O}) \mathrm{N}([\mathrm{H}]) \mathrm{C}([\mathrm{H}])([\mathrm{H}]) \mathrm{C}([\mathrm{H}])(\mathrm{C}([\mathrm{H}])([\mathrm{H}])[\mathrm{H}]) \mathrm{C}([\mathrm{H}])([\mathrm{H}])[\mathrm{H}])[\mathrm{C} @ \\
@]([\mathrm{H}])(\mathrm{O}[\mathrm{H}]) \mathrm{C}([\mathrm{H}])([\mathrm{H}])[\mathrm{H}]) \mathrm{C}([\mathrm{H}])([\mathrm{H}]) \mathrm{C}=\mathrm{C}([\mathrm{H}]) \mathrm{C}([\mathrm{H}])=\mathrm{C}([\mathrm{H}]) \mathrm{C}([\mathrm{H}])=\mathrm{C} 2[\mathrm{H}]) \mathrm{N}([\mathrm{C} @]([ \\
\mathrm{H}])(\mathrm{C} 3=\mathrm{C}(\end{array}$ & 1.236 & 1.211 \\
\hline $\operatorname{Tr} 203$ & $\begin{array}{l}\mathrm{O}=\mathrm{C}(\mathrm{C}=1 \mathrm{C}([\mathrm{H}])=\mathrm{C}([\mathrm{H}]) \mathrm{C}([\mathrm{H}])=\mathrm{C}(\mathrm{C}=1[\mathrm{H}]) \mathrm{C}(=\mathrm{O}) \mathrm{N}([\mathrm{H}])[\mathrm{C} @]([\mathrm{H}])([\mathrm{C} @ @]([\mathrm{H}])(\mathrm{O}[\mathrm{H}]) \mathrm{C}([\mathrm{H}] \\
)([\mathrm{H}]) \mathrm{N}([\mathrm{H}])[\mathrm{C} @]([\mathrm{H}])(\mathrm{C}(=\mathrm{O}) \mathrm{N}([\mathrm{H}]) \mathrm{C}([\mathrm{H}])([\mathrm{H}]) \mathrm{C}([\mathrm{H}])(\mathrm{C}([\mathrm{H}])([\mathrm{H}])[\mathrm{H}]) \mathrm{C}([\mathrm{H}])([\mathrm{H}])[\mathrm{H}])[\mathrm{C} @ \\
@]([\mathrm{H}])(\mathrm{O}[\mathrm{H}]) \mathrm{C}([\mathrm{H}])([\mathrm{H}]) \mathrm{C}([\mathrm{H}])([\mathrm{H}]) \mathrm{C}([\mathrm{H}])([\mathrm{H}])[\mathrm{H}]) \mathrm{C}([\mathrm{H}])([\mathrm{H}]) \mathrm{C} 2=\mathrm{C}([\mathrm{H}]) \mathrm{C}([\mathrm{H}])=\mathrm{C}([\mathrm{H}]) \mathrm{C} \\
([\mathrm{H}])=\mathrm{C}\end{array}$ & 2.573 & 2.627 \\
\hline
\end{tabular}




\begin{tabular}{|c|c|c|c|}
\hline $\operatorname{Tr} 204$ & $\begin{array}{l}\mathrm{O}=\mathrm{C}(\mathrm{C}=1 \mathrm{C}([\mathrm{H}])=\mathrm{C}(\mathrm{C}([\mathrm{H}])=\mathrm{C}(\mathrm{C}=1[\mathrm{H}]) \mathrm{C}(=\mathrm{O}) \mathrm{N}([\mathrm{H}])[\mathrm{C} @]([\mathrm{H}])([\mathrm{C} @ @]([\mathrm{H}])(\mathrm{O}[\mathrm{H}]) \mathrm{C}([\mathrm{H}])([\mathrm{H} \\
]) \mathrm{N}([\mathrm{H}])[\mathrm{C} @]([\mathrm{H}])(\mathrm{C}(=\mathrm{O}) \mathrm{N}([\mathrm{H}]) \mathrm{C}([\mathrm{H}])([\mathrm{H}]) \mathrm{C}([\mathrm{H}])(\mathrm{C}([\mathrm{H}])([\mathrm{H}])[\mathrm{H}]) \mathrm{C}([\mathrm{H}])([\mathrm{H}])[\mathrm{H}])[\mathrm{C} @ @]([ \\
\mathrm{H}])(\mathrm{O}[\mathrm{H}]) \mathrm{C}([\mathrm{H}])([\mathrm{H}]) \mathrm{C}([\mathrm{H}])([\mathrm{H}]) \mathrm{C}([\mathrm{H}])([\mathrm{H}])[\mathrm{H}]) \mathrm{C}([\mathrm{H}])([\mathrm{H}]) \mathrm{C} 2=\mathrm{C}([\mathrm{H}]) \mathrm{C}([\mathrm{H}])=\mathrm{C}([\mathrm{H}]) \mathrm{C}([\mathrm{H}]) \\
=\mathrm{C} 2[\mathrm{H}]\end{array}$ & 4.183 & 2.813 \\
\hline \multicolumn{4}{|c|}{ Test set } \\
\hline Ts1 & 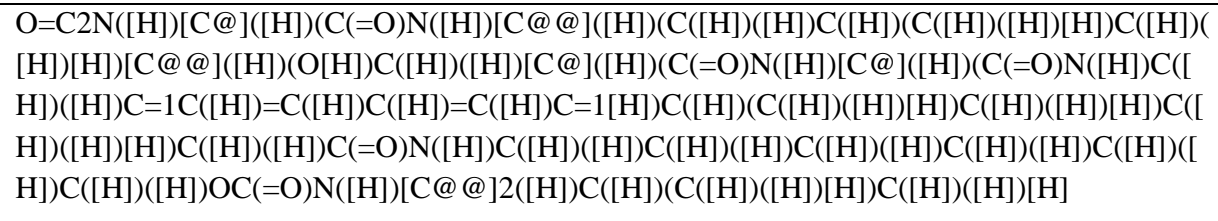 & 3.600 & 3.189 \\
\hline Ts2 & $\begin{array}{l}\mathrm{O}=\mathrm{C}(\mathrm{OC}([\mathrm{H}])([\mathrm{H}]) \mathrm{C}=1 \mathrm{~N}=\mathrm{C}(\mathrm{OC}=1 \mathrm{C}([\mathrm{H}])([\mathrm{H}])[\mathrm{H}]) \mathrm{C}([\mathrm{H}])([\mathrm{H}])[\mathrm{H}]) \mathrm{N}([\mathrm{H}])[\mathrm{C} @]([\mathrm{H}])(\mathrm{C}(=\mathrm{O}) \mathrm{N} \\
([\mathrm{H}])[\mathrm{C} @ @](([\mathrm{H}])(\mathrm{C}([\mathrm{H}])([\mathrm{H}]) \mathrm{C}([\mathrm{H}])(\mathrm{C}([\mathrm{H}])([\mathrm{H}])[\mathrm{H}]) \mathrm{C}([\mathrm{H}])([\mathrm{H}])[\mathrm{H}])[\mathrm{C} @ @]([\mathrm{H}])(\mathrm{O}[\mathrm{H}]) C([ \\
\mathrm{H}])([\mathrm{H}])[\mathrm{C} @]([\mathrm{H}])(\mathrm{C}(=\mathrm{O}) \mathrm{N}([\mathrm{H}])[\mathrm{C} @]([\mathrm{H}])(\mathrm{C}(=\mathrm{O}) \mathrm{N}([\mathrm{H}]) \mathrm{C}([\mathrm{H}])([\mathrm{H}]) \mathrm{C}([\mathrm{H}])(\mathrm{C}([\mathrm{H}])([\mathrm{H}])[\mathrm{H}]) \\
\mathrm{C}([\mathrm{H}])([\mathrm{H}])[\mathrm{H}]) \mathrm{C}([\mathrm{H}])(\mathrm{C}([\mathrm{H}])([\mathrm{H}])[\mathrm{H}]) \mathrm{C}([\mathrm{H}])([\mathrm{H}])[\mathrm{H}]) \mathrm{C}([\mathrm{H}])([\mathrm{H}])[\mathrm{H}]) C([\mathrm{H}])([\mathrm{H}])[\mathrm{S}](=\mathrm{O})(= \\
\mathrm{O}) \mathrm{C}([\mathrm{H}])([\mathrm{H}])[\mathrm{H}]\end{array}$ & 5.921 & 3.940 \\
\hline Ts3 & $\begin{array}{l}\mathrm{O}=\mathrm{C}(/ \mathrm{C} 1=\mathrm{C}([\mathrm{H}]) \mathrm{C}(=\mathrm{C}([\mathrm{H}]) \mathrm{C}(=\mathrm{C} 1[\mathrm{H}]) \mathrm{C} 2=\mathrm{NN}=\mathrm{C}(\mathrm{O} 2)[\mathrm{C} @ @](\mathrm{C}([\mathrm{H}])([\mathrm{H}])[\mathrm{H}])(\mathrm{C}([\mathrm{H}])([\mathrm{H}]) \mathrm{C} \\
=3 \mathrm{C}([\mathrm{H}])=\mathrm{C}([\mathrm{H}]) \mathrm{C}([\mathrm{H}])=\mathrm{C}([\mathrm{H}]) \mathrm{C}=3[\mathrm{H}])[\mathrm{N}]([\mathrm{H}])([\mathrm{H}])[\mathrm{H}]) \mathrm{N}(\mathrm{C}([\mathrm{H}])([\mathrm{H}])[\mathrm{H}])[\mathrm{S}](=\mathrm{O})(=\mathrm{O}) \mathrm{C}([ \\
\mathrm{H}])([\mathrm{H}])[\mathrm{H}]) \mathrm{C}([\mathrm{H}])(\mathrm{C}([\mathrm{H}])([\mathrm{H}])[\mathrm{H}]) \mathrm{C}([\mathrm{H}])([\mathrm{H}])[\mathrm{H}]\end{array}$ & 0.783 & 2.509 \\
\hline Ts4 & $\begin{array}{l}\mathrm{O}=\mathrm{C}(\mathrm{N}([\mathrm{H}])[\mathrm{C} @]([\mathrm{H}])([\mathrm{C} @ @]([\mathrm{H}])(\mathrm{O}[\mathrm{H}]) \mathrm{C}([\mathrm{H}])([\mathrm{H}])[\mathrm{C} @]([\mathrm{H}])(\mathrm{C}(=\mathrm{O}) \mathrm{N}([\mathrm{H}])[\mathrm{C} @]([\mathrm{H}])(\mathrm{C}( \\
=\mathrm{O}) \mathrm{N}([\mathrm{H}]) \mathrm{C}([\mathrm{H}])([\mathrm{H}]) \mathrm{Cl}=\mathrm{C}([\mathrm{H}]) \mathrm{C}([\mathrm{H}])=\mathrm{C}([\mathrm{H}]) \mathrm{C}([\mathrm{H}])=\mathrm{C} 1[\mathrm{H}]) \mathrm{C}([\mathrm{H}])(\mathrm{C}([\mathrm{H}])([\mathrm{H}])(\mathrm{H}]) \mathrm{C}([\mathrm{H}]) \\
([\mathrm{H}])[\mathrm{H}]) \mathrm{C}([\mathrm{H}])([\mathrm{H}])[\mathrm{H}]) \mathrm{C}([\mathrm{H}])([\mathrm{H}]) \mathrm{C}([\mathrm{H}])(\mathrm{C}([\mathrm{H}])([\mathrm{H}])[\mathrm{H}]) \mathrm{C}([\mathrm{H}])([\mathrm{H}])[\mathrm{H}])[\mathrm{C} @ @ @]([\mathrm{H}])(\mathrm{N}([\mathrm{I} \\
\mathrm{H}]) \mathrm{C}(=\mathrm{O}) \mathrm{OC}(\mathrm{C}([\mathrm{H}])([\mathrm{H}])[\mathrm{H}])(\mathrm{C}([\mathrm{H}])([\mathrm{H}])[\mathrm{H}]) \mathrm{C}([\mathrm{H}])([\mathrm{H}])[\mathrm{H}]) \mathrm{C}([\mathrm{H}])([\mathrm{H}])[\mathrm{S}](=\mathrm{O})(=\mathrm{O}) \mathrm{C}([\mathrm{H}]) \\
([\mathrm{H}])[\mathrm{H}]\end{array}$ & 1.947 & 2.890 \\
\hline Ts5 & $\begin{array}{l}\mathrm{O}=\mathrm{C}(\mathrm{OC}([\mathrm{H}])([\mathrm{H}]) \mathrm{C}([\mathrm{H}])=\mathrm{C}([\mathrm{H}])[\mathrm{H}]) \mathrm{N}([\mathrm{H}])[\mathrm{C} @]([\mathrm{H}])(\mathrm{C}(=\mathrm{O}) \mathrm{N}([\mathrm{H}])[\mathrm{C} @]([\mathrm{H}])(\mathrm{C}(=\mathrm{O}) \mathrm{N}([\mathrm{H}] \\
)[\mathrm{C} @ @]([\mathrm{H}])(\mathrm{C}([\mathrm{H}])([\mathrm{H}]) \mathrm{C}([\mathrm{H}])(\mathrm{C}([\mathrm{H}])([\mathrm{H}])[\mathrm{H}]) \mathrm{C}([\mathrm{H}])([\mathrm{H}])[\mathrm{H}])[\mathrm{C} @ @]([\mathrm{H}])(\mathrm{O}[\mathrm{H}]) \mathrm{C}([\mathrm{H}])([ \\
\mathrm{H}])[\mathrm{C} @]([\mathrm{H}])(\mathrm{C}(=\mathrm{O}) \mathrm{N}([\mathrm{H}])[\mathrm{C} @([\mathrm{H}])(\mathrm{C}(=\mathrm{O}) \mathrm{N}([\mathrm{H}]) \mathrm{C}([\mathrm{H}])([\mathrm{H}]) \mathrm{C}=1 \mathrm{C}([\mathrm{H}])=\mathrm{C}([\mathrm{H}]) \mathrm{C}([\mathrm{H}])=\mathrm{C}( \\
[\mathrm{H}]) \mathrm{C}=1[\mathrm{H}]) \mathrm{C}([\mathrm{H}])(\mathrm{C}([\mathrm{H}])([\mathrm{H}])[\mathrm{H}]) \mathrm{C}([\mathrm{H}])([\mathrm{H}])[\mathrm{H}]) \mathrm{C}([\mathrm{H}])([\mathrm{H}])[\mathrm{H}]) \mathrm{C}([\mathrm{H}])([\mathrm{H}]) \mathrm{C}(=\mathrm{O}) \mathrm{N}([\mathrm{H}]) \\
\mathrm{C}([\mathrm{H}])([\mathrm{H}]) \mathrm{C}([\mathrm{H}])=\mathrm{C}([\mathrm{H}])[\mathrm{H}]) \mathrm{C}([\mathrm{H}])(\mathrm{C}([\mathrm{H}])([\mathrm{H}])[\mathrm{H}]) \mathrm{C}([\mathrm{H}])([\mathrm{H}])[\mathrm{H}]\end{array}$ & 3.575 & 3.106 \\
\hline Ts6 & $\begin{array}{l}\mathrm{O}=\mathrm{C} 2 \mathrm{~N}(\mathrm{C}(=\mathrm{N}[\mathrm{C} @] 4(\mathrm{C}=1 \mathrm{C}([\mathrm{H}])=\mathrm{C}([\mathrm{H}]) \mathrm{C}([\mathrm{H}])=\mathrm{C}([\mathrm{H}]) \mathrm{C}=1[\mathrm{H}])[\mathrm{C} @ @] 2([\mathrm{H}]) \mathrm{C}([\mathrm{H}])([\mathrm{H}]) \mathrm{N}(/ \\
\mathrm{C} 3=\mathrm{N} / \mathrm{C}(=\mathrm{C}(/[\mathrm{F}]) \mathrm{C}(=\mathrm{N} 3) \mathrm{OC}([\mathrm{H}])([\mathrm{H}])[\mathrm{H}]) \mathrm{C}([\mathrm{H}])([\mathrm{H}])[\mathrm{H}]) \mathrm{C} 4([\mathrm{H}])[\mathrm{H}]) \mathrm{N}([\mathrm{H}])[\mathrm{H}]) \mathrm{C}([\mathrm{H}])([\mathrm{H}]) \\
{[\mathrm{H}]}\end{array}$ & 3.921 & 3.241 \\
\hline Ts7 & $\begin{array}{l}\mathrm{O}=\mathrm{C} 2 \mathrm{~N}(\mathrm{C}(=\mathrm{N}[\mathrm{C} @] 4(\mathrm{C}=1 \mathrm{C}([\mathrm{H}])=\mathrm{C}([\mathrm{H}]) \mathrm{C}([\mathrm{H}])=\mathrm{C}([\mathrm{H}]) \mathrm{C}=1[\mathrm{H}])[\mathrm{C} @ @] 2([\mathrm{H}]) \mathrm{C}([\mathrm{H}])([\mathrm{H}]) \mathrm{N}(/ \\
\mathrm{C} 3=\mathrm{N} / \mathrm{C}(\mathrm{OC}([\mathrm{H}])([\mathrm{H}]) \mathrm{C}([\mathrm{H}])([\mathrm{H}])[\mathrm{H}])=\mathrm{C}([\mathrm{F}]) \mathrm{C}(=\mathrm{N} 3) \mathrm{OC}([\mathrm{H}])([\mathrm{H}])[\mathrm{H}]) \mathrm{C} 4([\mathrm{H}])[\mathrm{H}]) \mathrm{N}([\mathrm{H}])[\mathrm{H} \\
]) \mathrm{C}([\mathrm{H}])([\mathrm{H}])[\mathrm{H}]\end{array}$ & 2.863 & 3.584 \\
\hline Ts8 & $\begin{array}{l}\mathrm{O}=\mathrm{C} 2 \mathrm{~N}(\mathrm{C}(=\mathrm{N}[\mathrm{C} @] 4(\mathrm{C}=1 \mathrm{C}([\mathrm{H}])=\mathrm{C}([\mathrm{H}]) \mathrm{C}([\mathrm{H}])=\mathrm{C}([\mathrm{H}]) \mathrm{C}=1[\mathrm{H}])[\mathrm{C} @ @] 2([\mathrm{H}]) \mathrm{C}([\mathrm{H}])([\mathrm{H}]) \mathrm{N}(/ \\
\mathrm{C} 3=\mathrm{N} / \mathrm{C}(\mathrm{OC}([\mathrm{H}])([\mathrm{H}])[\mathrm{H}])=\mathrm{C}([\mathrm{F}]) \mathrm{C}(=\mathrm{N} 3) \mathrm{C}([\mathrm{H}])([\mathrm{H}]) \mathrm{C}([\mathrm{H}])([\mathrm{H}])[\mathrm{H}]) \mathrm{C} 4([\mathrm{H}])[\mathrm{H}]) \mathrm{N}([\mathrm{H}])[\mathrm{H}]) \\
\mathrm{C}([\mathrm{H}])([\mathrm{H}])[\mathrm{H}]\end{array}$ & 3.796 & 3.500 \\
\hline Ts9 & $\begin{array}{l}\mathrm{O}=\mathrm{C} 2 \mathrm{~N}(\mathrm{C}(=\mathrm{N}[\mathrm{C} @ 4) 4(\mathrm{C}=1 \mathrm{C}([\mathrm{H}])=\mathrm{C}([\mathrm{H}]) \mathrm{C}([\mathrm{F}])=\mathrm{C}([\mathrm{H}]) \mathrm{C}=1[\mathrm{~F}])[\mathrm{C} @ @] 2([\mathrm{H}]) \mathrm{C}([\mathrm{H}])([\mathrm{H}]) \mathrm{N}(\mathrm{C} \\
3=\mathrm{N} / \mathrm{C}(\mathrm{OC}([\mathrm{H}])([\mathrm{H}])[\mathrm{H}])=\mathrm{C}([\mathrm{F}]) \mathrm{C}(=\mathrm{N} 3) \mathrm{C}([\mathrm{H}])([\mathrm{H}]) \mathrm{C}([\mathrm{H}])([\mathrm{H}])[\mathrm{H}]) \mathrm{C} 4([\mathrm{H}])[\mathrm{H}]) \mathrm{N}([\mathrm{H}])[\mathrm{H}]) \mathrm{C}( \\
[\mathrm{H}])([\mathrm{H}])[\mathrm{H}]\end{array}$ & 4.276 & 3.932 \\
\hline Ts10 & $\begin{array}{l}{[\mathrm{F}] / \mathrm{Cl}=\mathrm{C}(\mathrm{N}=\mathrm{C}(/ \mathrm{N}=\mathrm{C} 1 / \mathrm{OC}([\mathrm{H}])([\mathrm{H}])[\mathrm{H}]) \mathrm{N} 3 \mathrm{C}([\mathrm{H}])([\mathrm{H}])[\mathrm{C} @ @] 2(\mathrm{~N}=\mathrm{C}(\mathrm{N}(\mathrm{C}(=\mathrm{O})[\mathrm{C} @] 2([\mathrm{H}])} \\
\mathrm{C} 3([\mathrm{H}])[\mathrm{H}]) \mathrm{C}([\mathrm{H}])([\mathrm{H}])[\mathrm{H}]) \mathrm{N}([\mathrm{H}])[\mathrm{H}]) \mathrm{C} 4=\mathrm{C}([\mathrm{H}]) \mathrm{C}([\mathrm{H}])=\mathrm{C}([\mathrm{F}]) \mathrm{C}([\mathrm{H}])=\mathrm{C} 4[\mathrm{~F}]) \mathrm{C}([\mathrm{F}])([\mathrm{F}])[\mathrm{F}]\end{array}$ & 3.495 & 4.088 \\
\hline Ts11 & $\begin{array}{l}\mathrm{O}=\mathrm{C} 2 \mathrm{~N}(\mathrm{C}(=\mathrm{N}[\mathrm{C} @] 4(\mathrm{C}=1 \mathrm{C}([\mathrm{H}])=\mathrm{C}(\mathrm{CN}) \mathrm{C}([\mathrm{H}])=\mathrm{C}([\mathrm{H}]) \mathrm{C}=1[\mathrm{H}])[\mathrm{C} @ @] 2([\mathrm{H}]) \mathrm{C}([\mathrm{H}])([\mathrm{H}]) \mathrm{N} / \\
\mathrm{C} 3=\mathrm{N} / \mathrm{C}(=\mathrm{C}(/[\mathrm{F}]) \mathrm{C}(=\mathrm{N} 3) \mathrm{OC}([\mathrm{H}])([\mathrm{H}])[\mathrm{H}]) \mathrm{C}([\mathrm{H}])([\mathrm{H}])[\mathrm{H}]) \mathrm{C} 4([\mathrm{H}])[\mathrm{H}]) \mathrm{N}([\mathrm{H}])[\mathrm{H}]) \mathrm{C}([\mathrm{H}])([\mathrm{H}]) \\
{[\mathrm{H}]}\end{array}$ & 3.854 & 3.763 \\
\hline Ts12 & $\begin{array}{l}\mathrm{O}=\mathrm{C}(\mathrm{OC}(\mathrm{C}([\mathrm{H}])([\mathrm{H}])[\mathrm{H}])(\mathrm{C}([\mathrm{H}])([\mathrm{H}])[\mathrm{H}]) \mathrm{C}([\mathrm{H}])([\mathrm{H}])[\mathrm{H}]) \mathrm{N}([\mathrm{H}])[\mathrm{C} @]([\mathrm{H}])(\mathrm{C}(=\mathrm{O}) \mathrm{C}([\mathrm{H}])([\mathrm{H} \\
]) \mathrm{N}([\mathrm{H}])[\mathrm{C} @]([\mathrm{H}])([\mathrm{C} @]]([\mathrm{H}])(\mathrm{O}[\mathrm{H}]) \mathrm{C}([\mathrm{H}])([\mathrm{H}])[\mathrm{C} @]([\mathrm{H}])(\mathrm{C}(=\mathrm{O}) \mathrm{N}([\mathrm{H}])[\mathrm{C} @]([\mathrm{H}])(\mathrm{C}(=\mathrm{O}) \mathrm{N}\end{array}$ & 0.649 & 2.091 \\
\hline
\end{tabular}




\begin{tabular}{|c|c|c|c|}
\hline & $\begin{array}{l}([\mathrm{H}]) \mathrm{C}([\mathrm{H}])([\mathrm{H}]) \mathrm{C} 1=\mathrm{C}([\mathrm{H}]) \mathrm{C}([\mathrm{H}])=\mathrm{C}([\mathrm{H}]) \mathrm{C}([\mathrm{H}])=\mathrm{C} 1[\mathrm{H}]) \mathrm{C}([\mathrm{H}])([\mathrm{H}])[\mathrm{H}]) \mathrm{C}([\mathrm{H}])([\mathrm{H}])[\mathrm{H}]) \mathrm{C}([ \\
\mathrm{H}])([\mathrm{H}]) \mathrm{C}([\mathrm{H}])(\mathrm{C}([\mathrm{H}])([\mathrm{H}])[\mathrm{H}]) \mathrm{C}([\mathrm{H}])([\mathrm{H}])[\mathrm{H}]) \mathrm{C}([\mathrm{H}])([\mathrm{H}]) \mathrm{C}(=\mathrm{O}) \mathrm{N}([\mathrm{H}])[\mathrm{H}]\end{array}$ & & \\
\hline Ts13 & $\begin{array}{l}\mathrm{O}=\mathrm{C}(\mathrm{OC}(\mathrm{C}([\mathrm{H}])([\mathrm{H}])[\mathrm{H}])(\mathrm{C}([\mathrm{H}])([\mathrm{H}])[\mathrm{H}]) \mathrm{C}([\mathrm{H}])([\mathrm{H}])[\mathrm{H}]) \mathrm{N}([\mathrm{H}])[\mathrm{C} @]([\mathrm{H}])(\mathrm{C}(=\mathrm{O}) \mathrm{C}([\mathrm{H}])([\mathrm{H} \\
]) \mathrm{N}([\mathrm{H}])[\mathrm{C} @]([\mathrm{H}])([\mathrm{C} @]([\mathrm{H}])(\mathrm{O}[\mathrm{H}]) \mathrm{C}([\mathrm{H}])([\mathrm{H}])[\mathrm{C} @]([\mathrm{H}])(\mathrm{C}(=\mathrm{O}) \mathrm{N}([\mathrm{H}])[\mathrm{C} @]([\mathrm{H}])(\mathrm{C}(=\mathrm{O}) \mathrm{N} \\
([\mathrm{H}]) \mathrm{C}([\mathrm{H}])([\mathrm{H}]) \mathrm{C} 1=\mathrm{C}([\mathrm{H}]) \mathrm{C}([\mathrm{H}])=\mathrm{C}([\mathrm{H}]) \mathrm{C}([\mathrm{H}])=\mathrm{C} 1[\mathrm{H}]) \mathrm{C}([\mathrm{H}])(\mathrm{C}([\mathrm{H}])([\mathrm{H}])[\mathrm{H}]) \mathrm{C}([\mathrm{H}])([\mathrm{H}])[ \\
\mathrm{H}]) \mathrm{C}([\mathrm{H}])([\mathrm{H}])[\mathrm{H}]) \mathrm{C}([\mathrm{H}])([\mathrm{H}]) \mathrm{C}([\mathrm{H}])(\mathrm{C}([\mathrm{H}])([\mathrm{H}])[\mathrm{H}]) \mathrm{C}([\mathrm{H}])([\mathrm{H}])[\mathrm{H}]) \mathrm{C}([\mathrm{H}])([\mathrm{H}]) \mathrm{C}(=\mathrm{O}) \mathrm{N}([\mathrm{H} \\
])[\mathrm{H}]\end{array}$ & 1.504 & 2.163 \\
\hline Ts14 & $\begin{array}{l}\mathrm{NC} / \mathrm{C} 1=\mathrm{C}(\backslash[\mathrm{H}]) \mathrm{C}(=\mathrm{C}([\mathrm{H}]) \mathrm{C}([\mathrm{H}])=\mathrm{C} 1[\mathrm{H}]) \mathrm{C} 2=\mathrm{C}([\mathrm{H}])[\mathrm{S}] / \mathrm{C}(=\mathrm{C} 2 /[\mathrm{H}])[\mathrm{C} @ @] 35 \mathrm{~N}=\mathrm{C}(\mathrm{N}(\mathrm{C}(=\mathrm{O})[ \\
\mathrm{C} @] 3([\mathrm{H}]) \mathrm{C}([\mathrm{H}])([\mathrm{H}]) \mathrm{N}(/ \mathrm{C} 4=\mathrm{N} / \mathrm{C}([\mathrm{H}])=\mathrm{C}(\mathrm{C}([\mathrm{H}])=\mathrm{N} 4) \mathrm{C}([\mathrm{H}])([\mathrm{H}])[\mathrm{H}]) \mathrm{C} 5([\mathrm{H}])[\mathrm{H}]) \mathrm{C}([\mathrm{H}])([\mathrm{H} \\
])[\mathrm{H}]) \mathrm{N}([\mathrm{H}])[\mathrm{H}]\end{array}$ & 4.046 & 4.058 \\
\hline Ts15 & $\begin{array}{l}\mathrm{O}=[\mathrm{S}](=\mathrm{O})(\mathrm{N}(\mathrm{C}=1 \mathrm{C}([\mathrm{H}])=\mathrm{C}(\mathrm{C}([\mathrm{H}])=\mathrm{C}(\mathrm{C}=1[\mathrm{H}]) \mathrm{C}(=\mathrm{O}) \mathrm{N}([\mathrm{H}])[\mathrm{C} @]([\mathrm{H}])(\mathrm{C}([\mathrm{H}])([\mathrm{H}]) \mathrm{N}([\mathrm{H}])[\mathrm{C} \\
@]([\mathrm{H}])(\mathrm{C}(=\mathrm{O}) \mathrm{N}([\mathrm{H}]) \mathrm{C}([\mathrm{H}])([\mathrm{H}]) \mathrm{C}([\mathrm{H}])(\mathrm{C}([\mathrm{H}])([\mathrm{H}])[\mathrm{H}]) \mathrm{C}([\mathrm{H}])([\mathrm{H}])[\mathrm{H}])[\mathrm{C} @ @]([\mathrm{H}])(\mathrm{OC}([\mathrm{H} \\
])([\mathrm{H}])[\mathrm{H}]) \mathrm{C}([\mathrm{H}])([\mathrm{H}])[\mathrm{H}]) \mathrm{C}([\mathrm{H}])([\mathrm{H}]) \mathrm{C} 2=\mathrm{C}([\mathrm{H}]) \mathrm{C}([\mathrm{H}])=\mathrm{C}([\mathrm{H}]) \mathrm{C}([\mathrm{H}])=\mathrm{C} 2[\mathrm{H}]) \mathrm{C}(=\mathrm{O}) \mathrm{N}([\mathrm{H}])[ \\
\mathrm{C} @ @]([\mathrm{H}])(\mathrm{C} 3=\mathrm{C}([\mathrm{H}]) \mathrm{C}([\mathrm{H}])=\mathrm{C}([\mathrm{H}]) \mathrm{C}([\mathrm{H}])=\mathrm{C} 3[\mathrm{H}]) \mathrm{C}([\mathrm{H}])([\mathrm{H}])[\mathrm{H}]) \mathrm{C}([\mathrm{H}])([\mathrm{H}])[\mathrm{H}]) \mathrm{C}([\mathrm{H}])([ \\
\mathrm{H}])[\mathrm{H}]\end{array}$ & 3.602 & 2.291 \\
\hline Ts16 & $\begin{array}{l}\mathrm{O}=\mathrm{C}(\mathrm{N}([\mathrm{H}])[\mathrm{C} @]([\mathrm{H}])([\mathrm{C} @ @]([\mathrm{H}])(\mathrm{O}[\mathrm{H}]) \mathrm{C}([\mathrm{H}])([\mathrm{H}])[\mathrm{C} @([\mathrm{H}])(\mathrm{C}(=\mathrm{O}) \mathrm{N}([\mathrm{H}]) \mathrm{C}([\mathrm{H}])([\mathrm{H}]) \mathrm{C} \\
([\mathrm{H}])(\mathrm{C}([\mathrm{H}])([\mathrm{H}])[\mathrm{H}]) \mathrm{C}([\mathrm{H}])([\mathrm{H}])[\mathrm{H}]) \mathrm{C}([\mathrm{H}])([\mathrm{H}]) \mathrm{C}([\mathrm{H}])([\mathrm{H}]) \mathrm{O}[\mathrm{H}]) \mathrm{C}([\mathrm{H}])([\mathrm{H}]) \mathrm{C} 1=\mathrm{C}([\mathrm{H}]) \mathrm{C}([ \\
\mathrm{H}])=\mathrm{C}([\mathrm{H}]) \mathrm{C}([\mathrm{H}])=\mathrm{C} 1[\mathrm{H}]) \mathrm{C}([\mathrm{H}])([\mathrm{H}])[\mathrm{C} @]([\mathrm{H}])(\mathrm{N}([\mathrm{H}]) \mathrm{C}(=\mathrm{O}) \mathrm{C}([\mathrm{H}])([\mathrm{H}]) \mathrm{C}([\mathrm{H}])(\mathrm{C}([\mathrm{H}])([\mathrm{H}]) \\
[\mathrm{H}]) \mathrm{C}([\mathrm{H}])([\mathrm{H}])[\mathrm{H}]) \mathrm{C}(=\mathrm{O}) \mathrm{O}[\mathrm{H}]\end{array}$ & 1.721 & 1.846 \\
\hline Ts17 & $\begin{array}{l}\mathrm{O}=\mathrm{C}(\mathrm{N}([\mathrm{H}])[\mathrm{C} @ @]([\mathrm{H}])(\mathrm{C} 1=\mathrm{C}([\mathrm{H}]) \mathrm{C}([\mathrm{H}])=\mathrm{C}([\mathrm{H}]) \mathrm{C}([\mathrm{H}])=\mathrm{C} 1[\mathrm{H}]) \mathrm{C}([\mathrm{H}])([\mathrm{H}])[\mathrm{H}]) \mathrm{C} 2=\mathrm{C}([\mathrm{H}]) \\
\mathrm{C}(=\mathrm{C}([\mathrm{H}]) \mathrm{C}(=\mathrm{C} 2[\mathrm{H}]) \mathrm{N}(\mathrm{C}([\mathrm{H}])([\mathrm{H}])[\mathrm{H}])[\mathrm{S}](=\mathrm{O})(=\mathrm{O}) \mathrm{C}([\mathrm{H}])([\mathrm{H}])[\mathrm{H}]) \mathrm{C}(=\mathrm{O}) \mathrm{N}([\mathrm{H}])[\mathrm{C} @]([\mathrm{H}])( \\
{[\mathrm{C} @([\mathrm{H}])(\mathrm{O}[\mathrm{H}]) \mathrm{C}([\mathrm{H}])([\mathrm{H}]) \mathrm{N}([\mathrm{H}]) \mathrm{C}([\mathrm{H}])([\mathrm{H}]) \mathrm{C} 3=\mathrm{C}([\mathrm{H}]) \mathrm{C} 4=\mathrm{C}(\mathrm{C}([\mathrm{H}])=\mathrm{C} 3[\mathrm{H}]) \mathrm{C}([\mathrm{H}])=\mathrm{C}([\mathrm{H}} \\
]) \mathrm{N} 4[\mathrm{H}]) \mathrm{C}([\mathrm{H}])([\mathrm{H}]) \mathrm{C} 5=\mathrm{C}([\mathrm{H}]) \mathrm{C}([\mathrm{H}])=\mathrm{C}([\mathrm{H}]) \mathrm{C}([\mathrm{H}])=\mathrm{C} 5[\mathrm{H}]\end{array}$ & 2.638 & 2.214 \\
\hline Ts 18 & $\begin{array}{l}\mathrm{O}=\mathrm{C}(\mathrm{N}([\mathrm{H}])[\mathrm{C} @]([\mathrm{H}])(\mathrm{C}=1 \mathrm{~N}=\mathrm{C}(\mathrm{OC}=1 \mathrm{C}([\mathrm{H}])([\mathrm{H}])[\mathrm{H}]) \mathrm{C}([\mathrm{H}])([\mathrm{H}])[\mathrm{H}]) \mathrm{C}([\mathrm{H}])([\mathrm{H}])[\mathrm{H}]) \mathrm{C}=2 \mathrm{C} \\
([\mathrm{H}])=\mathrm{C}(\mathrm{C}([\mathrm{H}])=\mathrm{C}(\mathrm{C}=2[\mathrm{H}]) \mathrm{N}(\mathrm{C}([\mathrm{H}])([\mathrm{H}])[\mathrm{H}])[\mathrm{S}](=\mathrm{O})(=\mathrm{O}) \mathrm{C}([\mathrm{H}])([\mathrm{H}])[\mathrm{H}]) \mathrm{C}(=\mathrm{O}) \mathrm{N}([\mathrm{H}])[\mathrm{C} @] \\
([\mathrm{H}])([\mathrm{C} @]([\mathrm{H}])(\mathrm{O}[\mathrm{H}]) \mathrm{C}([\mathrm{H}])([\mathrm{H}]) \mathrm{N}([\mathrm{H}]) \mathrm{C}([\mathrm{H}])([\mathrm{H}]) \mathrm{C} 3=\mathrm{C}([\mathrm{H}]) \mathrm{C}(\mathrm{OC}([\mathrm{H}])([\mathrm{H}])[\mathrm{H}])=\mathrm{C}([\mathrm{H}]) \\
\mathrm{C}([\mathrm{H}])=\mathrm{C} 3[\mathrm{H}]) \mathrm{C}([\mathrm{H}])([\mathrm{H}]) \mathrm{C} 4=\mathrm{C}([\mathrm{H}]) \mathrm{C}([\mathrm{H}])=\mathrm{C}([\mathrm{H}]) \mathrm{C}([\mathrm{H}])=\mathrm{C} 4[\mathrm{H}]\end{array}$ & 2.049 & 3.397 \\
\hline Ts19 & $\begin{array}{l}\mathrm{O}=\mathrm{C} 2 \mathrm{~N}([\mathrm{H}]) \mathrm{C} 1=\mathrm{C}([\mathrm{H}]) \mathrm{C}([\mathrm{H}])=\mathrm{C}([\mathrm{H}]) \mathrm{C}([\mathrm{H}])=\mathrm{C} 1 \mathrm{~N}([\mathrm{H}]) \mathrm{C} 3=\mathrm{C} 2 \mathrm{C}([\mathrm{H}])=\mathrm{C}([\mathrm{H}]) \mathrm{C}(=\mathrm{C} 3[\mathrm{H}]) \mathrm{C}(= \\
\mathrm{O}) \mathrm{N}([\mathrm{H}]) \mathrm{C} 4=\mathrm{N} / \mathrm{C}(=\mathrm{C}(/[\mathrm{H}]) \mathrm{N} 4[\mathrm{H}]) \mathrm{C} 5=\mathrm{C}([\mathrm{H}]) \mathrm{C}([\mathrm{H}])=\mathrm{NC}([\mathrm{H}])=\mathrm{C} 5[\mathrm{H}]\end{array}$ & 2.458 & 2.990 \\
\hline Ts20 & $\begin{array}{l}\mathrm{O}=\mathrm{C} 2 \mathrm{~N}([\mathrm{H}]) \mathrm{C} 1=\mathrm{C}([\mathrm{H}]) \mathrm{C}([\mathrm{H}])=\mathrm{C}([\mathrm{H}]) \mathrm{C}([\mathrm{H}])=\mathrm{C} 1 \mathrm{~N}([\mathrm{H}]) \mathrm{C} 3=\mathrm{C} 2 \mathrm{C}([\mathrm{H}])=\mathrm{C}([\mathrm{H}]) \mathrm{C}(=\mathrm{C} 3[\mathrm{H}]) \mathrm{C}(= \\
\mathrm{O}) \mathrm{N}([\mathrm{H}]) \mathrm{C} 4=\mathrm{C}([\mathrm{H}]) \mathrm{C}([\mathrm{H}])=\mathrm{C}([\mathrm{F}]) \mathrm{C}([\mathrm{H}])=\mathrm{C} 4[\mathrm{H}]\end{array}$ & 2.676 & 1.490 \\
\hline Ts21 & $\begin{array}{l}\mathrm{O}=\mathrm{C} 2 \mathrm{~N}([\mathrm{H}]) \mathrm{C} 1=\mathrm{C}([\mathrm{H}]) \mathrm{C}([\mathrm{H}])=\mathrm{C}([\mathrm{H}]) \mathrm{C}([\mathrm{H}])=\mathrm{C} 1 \mathrm{~N}([\mathrm{H}]) \mathrm{C}=3 / \mathrm{C} 2=\mathrm{C}(/[\mathrm{H}]) \mathrm{C}([\mathrm{H}])=\mathrm{C}(\mathrm{C}=3[\mathrm{H}]) \mathrm{C} \\
(=\mathrm{O}) \mathrm{N}([\mathrm{H}]) \mathrm{C} 4=\mathrm{NC}=5 \mathrm{C}(\mathrm{N} 4[\mathrm{H}])=\mathrm{C}([\mathrm{H}]) \mathrm{N}=\mathrm{C}([\mathrm{H}]) \mathrm{C}=5[\mathrm{H}]\end{array}$ & 2.510 & 2.846 \\
\hline Ts22 & $\begin{array}{l}\mathrm{O}=\mathrm{C}(\mathrm{N}([\mathrm{H}]) \mathrm{C}(=\mathrm{NC}([\mathrm{H}])([\mathrm{H}]) \mathrm{C} 1=\mathrm{C}([\mathrm{H}]) \mathrm{C}(=\mathrm{C}([\mathrm{H}]) \mathrm{C}(=\mathrm{C} 1[\mathrm{H}]) \mathrm{C}([\mathrm{H}])([\mathrm{H}])[\mathrm{H}]) \mathrm{C}([\mathrm{H}])([\mathrm{H}])[\mathrm{H}] \\
) \mathrm{N}([\mathrm{H}])[\mathrm{H}]) \mathrm{C}=2 \mathrm{C}(=\mathrm{NOC}=2 \mathrm{C}([\mathrm{H}])([\mathrm{H}])[\mathrm{H}]) \mathrm{C} 3=\mathrm{C}([\mathrm{H}]) \mathrm{C}([\mathrm{H}])=\mathrm{C}(\mathrm{OC}([\mathrm{H}])([\mathrm{H}])[\mathrm{H}]) \mathrm{C}([\mathrm{H}])=\mathrm{C} 3 \\
{[\mathrm{H}]}\end{array}$ & 2.553 & 1.898 \\
\hline Ts 23 & $\begin{array}{l}\mathrm{O}=\mathrm{C}(\mathrm{N}([\mathrm{H}]) \mathrm{C}(=\mathrm{NC}([\mathrm{H}])([\mathrm{H}]) \mathrm{C} 1=\mathrm{C}([\mathrm{H}]) \mathrm{C}([\mathrm{Cl}])=\mathrm{C}([\mathrm{H}]) \mathrm{C}([\mathrm{Cl}])=\mathrm{C} 1[\mathrm{H}]) \mathrm{N}([\mathrm{H}])[\mathrm{H}]) \mathrm{C}=2 \mathrm{C}(=\mathrm{N}[ \\
\mathrm{S}] \mathrm{C}=2 \mathrm{C}([\mathrm{H}])([\mathrm{H}])[\mathrm{H}]) \mathrm{C} 3=\mathrm{C}([\mathrm{H}]) \mathrm{C}([\mathrm{H}])=\mathrm{C}(\mathrm{OC}([\mathrm{H}])([\mathrm{H}])[\mathrm{H}]) \mathrm{C}([\mathrm{H}])=\mathrm{C} 3[\mathrm{H}]\end{array}$ & 2.538 & 2.816 \\
\hline Ts24 & $\begin{array}{l}\mathrm{O}=\mathrm{C}(\mathrm{N}([\mathrm{H}]) \mathrm{C}(=\mathrm{NC}([\mathrm{H}])([\mathrm{H}]) \mathrm{C}=1 \mathrm{C}([\mathrm{H}])=\mathrm{C}([\mathrm{H}]) \mathrm{C}([\mathrm{H}])=\mathrm{C} 2 \mathrm{C}=1 \mathrm{C}([\mathrm{H}])=\mathrm{C}([\mathrm{H}]) \mathrm{C}([\mathrm{H}])=\mathrm{C} 2[\mathrm{H}]) \\
\mathrm{N}([\mathrm{H}])[\mathrm{H}]) \mathrm{C}=3 \mathrm{C}(=\mathrm{NOC}=3 \mathrm{C}([\mathrm{H}])([\mathrm{H}])[\mathrm{H}]) \mathrm{C} 4=\mathrm{C}([\mathrm{H}]) \mathrm{C}([\mathrm{H}])=\mathrm{C}([\mathrm{H}]) \mathrm{C}([\mathrm{H}])=\mathrm{C} 4[\mathrm{H}]\end{array}$ & 1.553 & 1.160 \\
\hline Ts25 & $\begin{array}{l}{[\mathrm{H}] \mathrm{C}=5 \mathrm{C}(\mathrm{O}[\mathrm{H}])=\mathrm{C}(\mathrm{O} / \mathrm{C} 2=\mathrm{C}(\backslash[\mathrm{H}]) \mathrm{C}(\mathrm{O}[\mathrm{H}])=\mathrm{C} 1 \mathrm{OC} 3=\mathrm{C}(\mathrm{O} / \mathrm{C} 1=\mathrm{C} 2 \backslash[\mathrm{H}]) \mathrm{C}(\mathrm{O}[\mathrm{H}])=\mathrm{C}([\mathrm{H}]) \mathrm{C}(\mathrm{O}[\mathrm{H}]} \\
)=\mathrm{C} 3 \mathrm{O} / \mathrm{C} 4=\mathrm{C}(\backslash[\mathrm{H}]) \mathrm{C}(\mathrm{O}[\mathrm{H}])=\mathrm{C}([\mathrm{H}]) \mathrm{C}(\mathrm{O}[\mathrm{H}])=\mathrm{C} 4[\mathrm{H}]) \mathrm{C}(\mathrm{O}[\mathrm{H}])=\mathrm{C}([\mathrm{H}]) \mathrm{C}=5 \mathrm{O}[\mathrm{H}]\end{array}$ & 1.143 & 0.753 \\
\hline Ts26 & $\begin{array}{l}{[\mathrm{F}] \mathrm{C}=1 \mathrm{C}([\mathrm{H}])=\mathrm{C}(\mathrm{C}([\mathrm{H}])=\mathrm{C}([\mathrm{F}]) \mathrm{C}=1[\mathrm{H}]) \mathrm{C}([\mathrm{H}])([\mathrm{H}])[\mathrm{C} @(([\mathrm{H}])(\mathrm{N}([\mathrm{H}]) \mathrm{C}(=\mathrm{O}) \mathrm{C}=2 \mathrm{C}([\mathrm{H}])=\mathrm{C}(\mathrm{C}} \\
([\mathrm{H}])=\mathrm{C}(\mathrm{C}=2[\mathrm{H}]) \mathrm{C}([\mathrm{H}])([\mathrm{H}])[\mathrm{H}]) \mathrm{C}(=\mathrm{O}) \mathrm{N}(\mathrm{C}([\mathrm{H}])([\mathrm{H}]) \mathrm{C}([\mathrm{H}])([\mathrm{H}]) \mathrm{C}([\mathrm{H}])([\mathrm{H}])[\mathrm{H}]) \mathrm{C}([\mathrm{H}])([\mathrm{H}]) \\
\mathrm{C}([\mathrm{H}])([\mathrm{H}]) \mathrm{C}([\mathrm{H}])([\mathrm{H}])[\mathrm{H}])[\mathrm{C} @]([\mathrm{H}])(\mathrm{O}[\mathrm{H}])[\mathrm{C} @] 3([\mathrm{H}]) \mathrm{N}([\mathrm{H}]) \mathrm{C}([\mathrm{H}])([\mathrm{H}]) \mathrm{C}([\mathrm{H}])([\mathrm{H}]) \mathrm{N}(\mathrm{C} 3(\end{array}$ & 4.523 & 3.444 \\
\hline
\end{tabular}




\begin{tabular}{|c|c|c|c|}
\hline & $[\mathrm{H}])[\mathrm{H}])[\mathrm{S}](=\mathrm{O})(=\mathrm{O}) \mathrm{C} 4=\mathrm{C}([\mathrm{H}]) \mathrm{C}([\mathrm{H}])=\mathrm{C}([\mathrm{H}]) \mathrm{C}([\mathrm{H}])=\mathrm{C} 4[\mathrm{H}]$ & & \\
\hline Ts27 & $\begin{array}{l}{[\mathrm{F}] \mathrm{C}=1 \mathrm{C}([\mathrm{H}])=\mathrm{C}(\mathrm{C}([\mathrm{H}])=\mathrm{C}([\mathrm{F}]) \mathrm{C}=1[\mathrm{H}]) \mathrm{C}([\mathrm{H}])([\mathrm{H}])[\mathrm{C} @]([\mathrm{H}])(\mathrm{N}([\mathrm{H}]) \mathrm{C}(=\mathrm{O}) \mathrm{C} 2=\mathrm{C}([\mathrm{H}]) \mathrm{C}(=\mathrm{C}} \\
([\mathrm{H}]) \mathrm{C}(=\mathrm{C} 2[\mathrm{H}]) \mathrm{C}(=\mathrm{O}) \mathrm{N} 3[\mathrm{C} @]([\mathrm{H}])(\mathrm{C}([\mathrm{H}])([\mathrm{H}]) \mathrm{C}([\mathrm{H}])([\mathrm{H}]) \mathrm{C} 3([\mathrm{H}])[\mathrm{H}]) \mathrm{C}([\mathrm{H}])([\mathrm{H}]) \mathrm{OC}([\mathrm{H}])( \\
[\mathrm{H}])[\mathrm{H}]) \mathrm{C}([\mathrm{H}])([\mathrm{H}])[\mathrm{H}])[\mathrm{C} @]([\mathrm{H}])(\mathrm{O}[\mathrm{H}])[\mathrm{C} @]([\mathrm{H}]) \mathrm{N}([\mathrm{H}]) \mathrm{C}([\mathrm{H}])([\mathrm{H}]) \mathrm{C}([\mathrm{H}])([\mathrm{H}]) \mathrm{N}(\mathrm{C} 4([\mathrm{H}] \\
)[\mathrm{H}])[\mathrm{S}](=\mathrm{O})(=\mathrm{O}) \mathrm{C}([\mathrm{H}])([\mathrm{H}])[\mathrm{H}]\end{array}$ & 3.886 & 3.959 \\
\hline Ts 28 & $\begin{array}{l}{[\mathrm{F}] \mathrm{C}=1 \mathrm{C}([\mathrm{H}])=\mathrm{C}(\mathrm{C}([\mathrm{H}])=\mathrm{C}([\mathrm{F}]) \mathrm{C}=1[\mathrm{H}]) \mathrm{C}([\mathrm{H}])([\mathrm{H}])[\mathrm{C} @]([\mathrm{H}])(\mathrm{N}([\mathrm{H}]) \mathrm{C}(=\mathrm{O}) \mathrm{C} 2=\mathrm{C}([\mathrm{H}]) \mathrm{C}(=\mathrm{C}} \\
([\mathrm{H}]) \mathrm{C}(=\mathrm{C} 2[\mathrm{H}]) \mathrm{C}(=\mathrm{O}) \mathrm{N} 3[\mathrm{C} @]([\mathrm{H}])(\mathrm{C}([\mathrm{H}])([\mathrm{H}]) \mathrm{C}([\mathrm{H}])([\mathrm{H}]) \mathrm{C} 3([\mathrm{H}])[\mathrm{H}]) \mathrm{C}([\mathrm{H}])([\mathrm{H}]) \mathrm{OC}([\mathrm{H}])( \\
[\mathrm{H}])[\mathrm{H}]) \mathrm{C}([\mathrm{H}])([\mathrm{H}])[\mathrm{H}])[\mathrm{C} @]([\mathrm{H}])(\mathrm{O}[\mathrm{H}])[\mathrm{C} @] 4([\mathrm{H}]) \mathrm{N}([\mathrm{H}]) \mathrm{C}([\mathrm{H}])([\mathrm{H}]) \mathrm{C}([\mathrm{H}])([\mathrm{H}]) \mathrm{N}(\mathrm{C} 4([\mathrm{H}] \\
)[\mathrm{H}])[\mathrm{S}](=\mathrm{O})(=\mathrm{O}) \mathrm{C}([\mathrm{H}])([\mathrm{H}]) \mathrm{C} 5=\mathrm{C}([\mathrm{H}]) \mathrm{C}([\mathrm{H}])=\mathrm{C}([\mathrm{H}]) \mathrm{C}([\mathrm{H}])=\mathrm{C} 5[\mathrm{H}]\end{array}$ & 5.000 & 4.187 \\
\hline Ts29 & $\begin{array}{l}\mathrm{O}=\mathrm{C}(\mathrm{N}([\mathrm{H}])[\mathrm{C} @ @]([\mathrm{H}])(\mathrm{C} 1=\mathrm{C}([\mathrm{H}]) \mathrm{C}([\mathrm{H}])=\mathrm{C}([\mathrm{H}]) \mathrm{C}([\mathrm{H}])=\mathrm{C} 1[\mathrm{H}]) \mathrm{C}([\mathrm{H}])([\mathrm{H}])[\mathrm{H}]) \mathrm{C} 2=\mathrm{C}([\mathrm{H}]) \\
\mathrm{C}(=\mathrm{C}([\mathrm{H}]) \mathrm{C}(=\mathrm{C} 2[\mathrm{H}]) \mathrm{N}(\mathrm{C}([\mathrm{H}])([\mathrm{H}])[\mathrm{H}])[\mathrm{S}](=\mathrm{O})(=\mathrm{O}) \mathrm{C}([\mathrm{H}])([\mathrm{H}])[\mathrm{H}]) \mathrm{C}(=\mathrm{O}) \mathrm{N}([\mathrm{H}])[\mathrm{C} @]([\mathrm{H}])( \\
{[\mathrm{C} @ @]([\mathrm{H}])(\mathrm{N}=[\mathrm{N}]=[\mathrm{N}]) \mathrm{C}([\mathrm{H}])([\mathrm{H}])[\mathrm{C} @ @]([\mathrm{H}])(\mathrm{OC}([\mathrm{H}])([\mathrm{H}])[\mathrm{H}]) \mathrm{C}(=\mathrm{O}) \mathrm{N}([\mathrm{H}])[\mathrm{C} @]([\mathrm{H}])(} \\
\mathrm{C}(=\mathrm{O}) \mathrm{N}([\mathrm{H}]) \mathrm{C}([\mathrm{H}])([\mathrm{H}]) \mathrm{C} 3=\mathrm{C}([\mathrm{H}]) \mathrm{C}([\mathrm{H}])=\mathrm{C}([\mathrm{H}]) \mathrm{C}([\mathrm{H}])=\mathrm{C} 3[\mathrm{H}]) \mathrm{C}([\mathrm{H}])(\mathrm{C}([\mathrm{H}])([\mathrm{H}]))[\mathrm{H}]) \mathrm{C}([ \\
\mathrm{H}])([\mathrm{H}])[\mathrm{H}]) \mathrm{C}([\mathrm{H}])([\mathrm{H}]) \mathrm{O} / \mathrm{C} 4=\mathrm{C}(\backslash[\mathrm{H}]) \mathrm{C}([\mathrm{F}])=\mathrm{C}([\mathrm{H}]) \mathrm{C}([\mathrm{F}])=\mathrm{C} 4[\mathrm{H}]\end{array}$ & 3.155 & 3.587 \\
\hline Ts 30 & $\begin{array}{l}\mathrm{O}=\mathrm{C}(\mathrm{N}([\mathrm{H}])[\mathrm{C} @ @]([\mathrm{H}])(\mathrm{C} 1=\mathrm{C}([\mathrm{H}]) \mathrm{C}([\mathrm{H}])=\mathrm{C}([\mathrm{H}]) \mathrm{C}([\mathrm{H}])=\mathrm{C} 1[\mathrm{H}]) \mathrm{C}([\mathrm{H}])([\mathrm{H}])[\mathrm{H}]) \mathrm{C} 2=\mathrm{C}([\mathrm{H}]) \\
\mathrm{C}(=\mathrm{C}([\mathrm{H}]) \mathrm{C}(=\mathrm{C} 2[\mathrm{H}]) \mathrm{N}(\mathrm{C}([\mathrm{H}])([\mathrm{H}])[\mathrm{H}])[\mathrm{S}](=\mathrm{O})(=\mathrm{O}) \mathrm{C}([\mathrm{H}])([\mathrm{H}])[\mathrm{H}]) \mathrm{C}(=\mathrm{O}) \mathrm{N}([\mathrm{H}])[\mathrm{C} @]([\mathrm{H}])( \\
{[\mathrm{C} @ @]([\mathrm{H}])(\mathrm{O}[\mathrm{H}]) \mathrm{C}([\mathrm{H}])([\mathrm{H}])[\mathrm{C} @ @]([\mathrm{H}])(\mathrm{OC}([\mathrm{H}])([\mathrm{H}]) \mathrm{C}([\mathrm{H}])([\mathrm{H}])[\mathrm{H}]) \mathrm{C}(=\mathrm{O}) \mathrm{N}([\mathrm{H}])[\mathrm{C} @]} \\
([\mathrm{H}])(\mathrm{C}(=\mathrm{O}) \mathrm{N}([\mathrm{H}]) \mathrm{C}([\mathrm{H}])([\mathrm{H}]) \mathrm{C} 3=\mathrm{C}([\mathrm{H}]) \mathrm{C}([\mathrm{H}])=\mathrm{C}([\mathrm{H}]) \mathrm{C}([\mathrm{H}])=\mathrm{C} 3[\mathrm{H}]) \mathrm{C}([\mathrm{H}])(\mathrm{C}([\mathrm{H}])([\mathrm{H}])[\mathrm{H} \\
]) \mathrm{C}([\mathrm{H}])([\mathrm{H}])[\mathrm{H}]) \mathrm{C}([\mathrm{H}])([\mathrm{H}]) \mathrm{O} / \mathrm{C} 4=\mathrm{C}(\mathrm{V}[\mathrm{H}]) \mathrm{C}([\mathrm{F}])=\mathrm{C}([\mathrm{H}]) \mathrm{C}([\mathrm{F}])=\mathrm{C} 4[\mathrm{H}]\end{array}$ & 4.678 & 3.390 \\
\hline Ts 31 & $\begin{array}{l}\mathrm{O}=\mathrm{C}(\mathrm{N}([\mathrm{H}])[\mathrm{C} @ @]([\mathrm{H}])(\mathrm{C} 1=\mathrm{C}([\mathrm{H}]) \mathrm{C}([\mathrm{H}])=\mathrm{C}([\mathrm{H}]) \mathrm{C}([\mathrm{H}])=\mathrm{C} 1[\mathrm{H}]) \mathrm{C}([\mathrm{H}])([\mathrm{H}])[\mathrm{H}]) \mathrm{C}=2 \mathrm{C}([\mathrm{H}]) \\
=\mathrm{C}(\mathrm{C}([\mathrm{H}])=\mathrm{C}(\mathrm{C}=2[\mathrm{H}]) \mathrm{N}(\mathrm{C}([\mathrm{H}])([\mathrm{H}])[\mathrm{H}])[\mathrm{S}](=\mathrm{O})(=\mathrm{O}) \mathrm{C}([\mathrm{H}])([\mathrm{H}])[\mathrm{H}]) \mathrm{C}(=\mathrm{O}) \mathrm{N}([\mathrm{H}])[\mathrm{C} @]([\mathrm{H}]) \\
([\mathrm{C} @ @]([\mathrm{H}])(\mathrm{O}[\mathrm{H}]) \mathrm{C}([\mathrm{H}])([\mathrm{H}])[\mathrm{C} @ @](([\mathrm{H}])(\mathrm{OC}([\mathrm{H}])([\mathrm{H}])[\mathrm{C} @ @] 3([\mathrm{H}]) \mathrm{C}([\mathrm{H}])([\mathrm{H}]) \mathrm{C} 3([\mathrm{H}])[ \\
\mathrm{H}]) \mathrm{C}(=\mathrm{O}) \mathrm{N}([\mathrm{H}])[\mathrm{C} @]([\mathrm{H}])(\mathrm{C}(=\mathrm{O}) \mathrm{N}([\mathrm{H}]) \mathrm{C}([\mathrm{H}])([\mathrm{H}]) \mathrm{C} 4=\mathrm{C}([\mathrm{H}]) \mathrm{C}([\mathrm{H}])=\mathrm{C}([\mathrm{H}]) \mathrm{C}([\mathrm{H}])=\mathrm{C} 4[\mathrm{H}]) \\
\mathrm{C}([\mathrm{H}])(\mathrm{C}([\mathrm{H}])([\mathrm{H}])[\mathrm{H}]) \mathrm{C}([\mathrm{H}])([\mathrm{H}])[\mathrm{H}]) \mathrm{C}([\mathrm{H}])([\mathrm{H}]) \mathrm{O} / \mathrm{C} 5=\mathrm{C}(1 \mathrm{H}]) \mathrm{C}([\mathrm{F}])=\mathrm{C}([\mathrm{H}]) \mathrm{C}([\mathrm{F}])=\mathrm{C} 5[\mathrm{H}]\end{array}$ & 4.886 & 4.950 \\
\hline Ts 32 & $\begin{array}{l}\mathrm{O}=\mathrm{C} 1 \mathrm{C} 4=\mathrm{C}(\mathrm{O} / \mathrm{C}(=\mathrm{C} 1 / \mathrm{C}([\mathrm{H}])([\mathrm{H}]) \mathrm{C}([\mathrm{H}])=\mathrm{C}(\mathrm{C}([\mathrm{H}])([\mathrm{H}])[\mathrm{H}]) \mathrm{C}([\mathrm{H}])([\mathrm{H}])[\mathrm{H}]) \mathrm{C}=2 \mathrm{C}([\mathrm{H}])=\mathrm{C}([ \\
\mathrm{H}]) \mathrm{C}(\mathrm{O}[\mathrm{H}])=\mathrm{C}([\mathrm{H}]) \mathrm{C}=2 \mathrm{O}[\mathrm{H}]) \mathrm{C} 3=\mathrm{C}(\mathrm{OC}(\mathrm{C}([\mathrm{H}])=\mathrm{C} 3[\mathrm{H}])(\mathrm{C}([\mathrm{H}])([\mathrm{H}])[\mathrm{H}]) \mathrm{C}([\mathrm{H}])([\mathrm{H}])[\mathrm{H}]) \mathrm{C}([ \\
\mathrm{H}])=\mathrm{C} 4 \mathrm{O}[\mathrm{H}]\end{array}$ & 0.193 & 1.058 \\
\hline Ts 33 & $\begin{array}{l}\mathrm{O}=\mathrm{C}(\mathrm{OC}(\mathrm{C}([\mathrm{H}])([\mathrm{H}])[\mathrm{H}])(\mathrm{C}([\mathrm{H}])([\mathrm{H}])[\mathrm{H}]) \mathrm{C}([\mathrm{H}])([\mathrm{H}])[\mathrm{H}]) \mathrm{N}([\mathrm{H}])[\mathrm{C} @]([\mathrm{H}])(\mathrm{C}(=\mathrm{O}) \mathrm{N}([\mathrm{H}])[\mathrm{C} \\
@]([\mathrm{H}])([\mathrm{C} @]([\mathrm{H}])(\mathrm{O}[\mathrm{H}]) \mathrm{C}([\mathrm{H}])([\mathrm{H}])[\mathrm{C} @]([\mathrm{H}])(\mathrm{C}(=\mathrm{O}) \mathrm{N}([\mathrm{H}])[\mathrm{C} @]([\mathrm{H}])(\mathrm{C}(=\mathrm{O}) \mathrm{N}([\mathrm{H}]) \mathrm{C}([\mathrm{H}] \\
)([\mathrm{H}]) \mathrm{C} 1=\mathrm{C}([\mathrm{H}]) \mathrm{C}([\mathrm{H}])=\mathrm{C}([\mathrm{H}]) \mathrm{C}([\mathrm{H}])=\mathrm{C} 1[\mathrm{H}]) \mathrm{C}([\mathrm{H}])(\mathrm{C}([\mathrm{H}])([\mathrm{H}])[\mathrm{H}]) \mathrm{C}([\mathrm{H}])([\mathrm{H}])[\mathrm{H}]) \mathrm{C}([\mathrm{H}])( \\
[\mathrm{H}])[\mathrm{H}]) \mathrm{C}([\mathrm{H}])([\mathrm{H}]) \mathrm{C}([\mathrm{H}])(\mathrm{C}([\mathrm{H}])([\mathrm{H}])[\mathrm{H}]) \mathrm{C}([\mathrm{H}])([\mathrm{H}])[\mathrm{H}]) \mathrm{C}([\mathrm{H}])([\mathrm{H}]) \mathrm{C}(=\mathrm{O}) \mathrm{N}([\mathrm{H}])[\mathrm{H}]\end{array}$ & 1.504 & 2.313 \\
\hline Ts34 & $\begin{array}{l}\mathrm{O}=\mathrm{C} 3 \mathrm{~N}([\mathrm{H}])[\mathrm{C} @]([\mathrm{H}])(\mathrm{C}(=\mathrm{O}) \mathrm{N}([\mathrm{H}])[\mathrm{C} @]([\mathrm{H}])([\mathrm{C} @]([\mathrm{H}])(\mathrm{O}[\mathrm{H}]) \mathrm{C}([\mathrm{H}])([\mathrm{H}]) \mathrm{N}([\mathrm{H}]) \mathrm{C}([\mathrm{H}])([ \\
\mathrm{H}]) \mathrm{C} 1=\mathrm{C}([\mathrm{H}]) \mathrm{C}(=\mathrm{C}([\mathrm{H}]) \mathrm{C}([\mathrm{H}])=\mathrm{C} 1[\mathrm{H}]) \mathrm{N}(\mathrm{C}([\mathrm{H}])([\mathrm{H}])[\mathrm{H}]) \mathrm{C}([\mathrm{H}])([\mathrm{H}])[\mathrm{H}]) \mathrm{C}([\mathrm{H}])([\mathrm{H}]) \mathrm{C} 2=\mathrm{C}([ \\
\mathrm{H}]) \mathrm{C}([\mathrm{H}])=\mathrm{C}([\mathrm{H}]) \mathrm{C}([\mathrm{H}])=\mathrm{C} 2[\mathrm{H}]) \mathrm{C}([\mathrm{H}])([\mathrm{H}]) \mathrm{N} 3 / \mathrm{C} 4=\mathrm{C}(\backslash[\mathrm{H}]) \mathrm{C}([\mathrm{H}])=\mathrm{C}([\mathrm{H}]) \mathrm{C}([\mathrm{H}])=\mathrm{C} 4[\mathrm{H}]\end{array}$ & 1.087 & 1.805 \\
\hline Ts 35 & $\begin{array}{l}\mathrm{O}=\mathrm{C} 3 \mathrm{~N}([\mathrm{H}])[\mathrm{C} @]([\mathrm{H}])(\mathrm{C}(=\mathrm{O}) \mathrm{N}([\mathrm{H}])[\mathrm{C} @]([\mathrm{H}])([\mathrm{C} @]([\mathrm{H}])(\mathrm{O}[\mathrm{H}]) \mathrm{C}([\mathrm{H}])([\mathrm{H}]) \mathrm{N}([\mathrm{H}]) \mathrm{C}([\mathrm{H}])([ \\
\mathrm{H}]) \mathrm{C} 1=\mathrm{C}([\mathrm{H}]) \mathrm{C}(=\mathrm{C}([\mathrm{H}]) \mathrm{C}([\mathrm{H}])=\mathrm{C} 1[\mathrm{H}]) \mathrm{N}(\mathrm{C}([\mathrm{H}])([\mathrm{H}])[\mathrm{H}]) \mathrm{C}([\mathrm{H}])([\mathrm{H}])[\mathrm{H}]) \mathrm{C}([\mathrm{H}])([\mathrm{H}]) \mathrm{C} 2=\mathrm{C}([ \\
\mathrm{H}]) \mathrm{C}([\mathrm{H}])=\mathrm{C}([\mathrm{H}]) \mathrm{C}([\mathrm{H}])=\mathrm{C} 2[\mathrm{H}]) \mathrm{C}([\mathrm{H}])([\mathrm{H}]) \mathrm{N} 3 \mathrm{C}([\mathrm{H}])([\mathrm{H}]) \mathrm{C} 4=\mathrm{C}([\mathrm{H}]) \mathrm{C}([\mathrm{H}])=\mathrm{C}([\mathrm{F}]) \mathrm{C}([\mathrm{F}])= \\
\mathrm{C} 4[\mathrm{H}]\end{array}$ & 2.565 & 2.471 \\
\hline Ts 36 & $\begin{array}{l}\mathrm{O}=\mathrm{C} 3 \mathrm{~N}([\mathrm{H}])[\mathrm{C} @]([\mathrm{H}])(\mathrm{C}(=\mathrm{O}) \mathrm{N}([\mathrm{H}])[\mathrm{C} @([\mathrm{H}])([\mathrm{C} @]([\mathrm{H}])(\mathrm{O}[\mathrm{H}]) \mathrm{C}([\mathrm{H}])([\mathrm{H}]) \mathrm{N}([\mathrm{H}]) \mathrm{C}([\mathrm{H}])([ \\
\mathrm{H}]) \mathrm{C} 1=\mathrm{C}([\mathrm{H}]) \mathrm{C}(=\mathrm{C}([\mathrm{H}]) \mathrm{C}([\mathrm{H}])=\mathrm{C} 1[\mathrm{H}]) \mathrm{N}(\mathrm{C}([\mathrm{H}])([\mathrm{H}])[\mathrm{H}]) \mathrm{C}([\mathrm{H}])([\mathrm{H}])[\mathrm{H}]) \mathrm{C}([\mathrm{H}])([\mathrm{H}]) \mathrm{C} 2=\mathrm{C}([ \\
\mathrm{H}]) \mathrm{C}([\mathrm{H}])=\mathrm{C}([\mathrm{H}]) \mathrm{C}([\mathrm{H}])=\mathrm{C} 2[\mathrm{H}]) \mathrm{C}([\mathrm{H}])([\mathrm{H}]) \mathrm{N} 3 \mathrm{C}([\mathrm{H}])([\mathrm{H}]) \mathrm{C} 4=\mathrm{C}([\mathrm{H}]) \mathrm{C}([\mathrm{H}])=\mathrm{C}([\mathrm{F}]) \mathrm{C}([\mathrm{H}])= \\
\mathrm{C} 4[\mathrm{~F}]\end{array}$ & 2.062 & 2.231 \\
\hline Ts 37 & $\begin{array}{l}\mathrm{O}=\mathrm{C}(\mathrm{N}([\mathrm{H}])[\mathrm{C} @ @]([\mathrm{H}])(\mathrm{C}([\mathrm{H}])([\mathrm{H}]) \mathrm{C} 1=\mathrm{C}([\mathrm{H}]) \mathrm{C}([\mathrm{H}])=\mathrm{C}([\mathrm{H}]) \mathrm{C}([\mathrm{H}])=\mathrm{C} 1[\mathrm{H}])[\mathrm{C} @]([\mathrm{H}])(\mathrm{O}[ \\
\mathrm{H}]) \mathrm{C}([\mathrm{H}])([\mathrm{H}]) \mathrm{N}([\mathrm{H}]) \mathrm{C}([\mathrm{H}])([\mathrm{H}]) \mathrm{C} 2=\mathrm{C}([\mathrm{H}]) \mathrm{C}([\mathrm{H}])=\mathrm{C}([\mathrm{H}]) \mathrm{C}(=\mathrm{C} 2[\mathrm{H}]) \mathrm{N}(\mathrm{C}([\mathrm{H}])([\mathrm{H}])[\mathrm{H}]) \mathrm{C}([\mathrm{H} \\
])([\mathrm{H}])[\mathrm{H}])[\mathrm{C} @ 3] 3(\mathrm{~N}([\mathrm{H}]) \mathrm{C}(=\mathrm{O}) \mathrm{N}(\mathrm{C} 3([\mathrm{H}])[\mathrm{H}]) \mathrm{C}([\mathrm{H}])([\mathrm{H}]) \mathrm{C} 4=\mathrm{C}([\mathrm{H}]) \mathrm{C}([\mathrm{H}])=\mathrm{C}([\mathrm{H}]) \mathrm{C}([\mathrm{H}])=\mathrm{C} \\
4[\mathrm{H}]) \mathrm{C}([\mathrm{H}])([\mathrm{H}]) \mathrm{C}=5 \mathrm{C}([\mathrm{H}])=\mathrm{C}([\mathrm{H}]) \mathrm{C}([\mathrm{H}])=\mathrm{C}([\mathrm{H}]) \mathrm{C}=5[\mathrm{H}]\end{array}$ & 1.301 & 1.932 \\
\hline Ts38 & $\mathrm{O}=\mathrm{C} 1 \mathrm{~N}(\mathrm{C}(=\mathrm{N}[\mathrm{C} @ @] 1(\mathrm{C}=2 \mathrm{C}([\mathrm{H}])=\mathrm{C}([\mathrm{H}]) \mathrm{C}([\mathrm{H}])=\mathrm{C}([\mathrm{H}]) \mathrm{C}=2[\mathrm{H}]) \mathrm{C} 3=\mathrm{C}([\mathrm{H}]) \mathrm{C}(=\mathrm{C}([\mathrm{H}]) \mathrm{C}([\mathrm{H}]$ & 2.538 & 2.732 \\
\hline
\end{tabular}




\begin{tabular}{|c|c|c|c|}
\hline & )$=\mathrm{C} 3[\mathrm{H}]) \mathrm{C} 4=\mathrm{C}([\mathrm{H}]) \mathrm{N}=\mathrm{C}([\mathrm{H}]) \mathrm{C}([\mathrm{F}])=\mathrm{C} 4[\mathrm{H}]) \mathrm{N}([\mathrm{H}])[\mathrm{H}]) \mathrm{C}([\mathrm{H}])([\mathrm{H}])[\mathrm{H}]$ & & \\
\hline Ts39 & $\begin{array}{l}\mathrm{O}=\mathrm{C} 1 \mathrm{~N}(\mathrm{C}(=\mathrm{N}[\mathrm{C} @ @] 1(\mathrm{C}=2 \mathrm{C}([\mathrm{H}])=\mathrm{C}([\mathrm{H}]) \mathrm{C}([\mathrm{H}])=\mathrm{C}([\mathrm{H}]) \mathrm{C}=2[\mathrm{H}]) \mathrm{C} 3=\mathrm{C}([\mathrm{H}]) \mathrm{C}(=\mathrm{C}([\mathrm{H}]) \mathrm{C}([\mathrm{H}] \\
)=\mathrm{C} 3[\mathrm{H}]) \mathrm{C} 4=\mathrm{C}([\mathrm{H}]) \mathrm{C}(\mathrm{OC}([\mathrm{H}])([\mathrm{H}])[\mathrm{H}])=\mathrm{C}([\mathrm{H}]) \mathrm{C}([\mathrm{H}])=\mathrm{C} 4[\mathrm{H}]) \mathrm{N}([\mathrm{H}])[\mathrm{H}]) \mathrm{C}([\mathrm{H}])([\mathrm{H}])[\mathrm{H}]\end{array}$ & 1.222 & 2.565 \\
\hline Ts 40 & $\begin{array}{l}\mathrm{O}=\mathrm{C} 1 \mathrm{~N}(\mathrm{C}(=\mathrm{N}[\mathrm{C} @ @] 1(\mathrm{C}=2 \mathrm{C}([\mathrm{H}])=\mathrm{C}(\mathrm{C}([\mathrm{H}])=\mathrm{C}([\mathrm{H}]) \mathrm{C}=2[\mathrm{H}]) \mathrm{C} 3=\mathrm{C}([\mathrm{H}]) \mathrm{C}([\mathrm{Cl}])=\mathrm{C}([\mathrm{H}]) \mathrm{N}=\mathrm{C} \\
3[\mathrm{H}])[\mathrm{C} @ @] 4([\mathrm{H}]) \mathrm{C}([\mathrm{H}])([\mathrm{H}]) \mathrm{C} 4([\mathrm{H}])[\mathrm{H}]) \mathrm{N}([\mathrm{H}])[\mathrm{H}]) \mathrm{C}([\mathrm{H}])([\mathrm{H}])[\mathrm{H}]\end{array}$ & 3.678 & 2.779 \\
\hline Ts41 & $\begin{array}{l}\mathrm{O}=\mathrm{C} 1 \mathrm{~N}(\mathrm{C}(=\mathrm{N}[\mathrm{C} @] 1(\mathrm{C}=2 \mathrm{C}([\mathrm{H}])=\mathrm{C}([\mathrm{H}]) \mathrm{C}([\mathrm{H}])=\mathrm{C}([\mathrm{H}]) \mathrm{C}=2[\mathrm{H}]) \mathrm{C} 3=\mathrm{C}([\mathrm{H}]) \mathrm{C}([\mathrm{F}])=\mathrm{C}([\mathrm{H}]) \mathrm{C}([ \\
\mathrm{H}])=\mathrm{C} 3[\mathrm{H}]) \mathrm{N}([\mathrm{H}])[\mathrm{H}]) \mathrm{C}([\mathrm{H}])([\mathrm{H}])[\mathrm{H}]\end{array}$ & 2.143 & 1.927 \\
\hline Ts42 & $\begin{array}{l}\mathrm{O}=\mathrm{C} 1 \mathrm{~N}(\mathrm{C}(=\mathrm{N}[\mathrm{C} @ @] 1(/ \mathrm{C} 2=\mathrm{C}(\backslash[\mathrm{H}]) \mathrm{C}([\mathrm{H}])=\mathrm{C}([\mathrm{H}]) \mathrm{C}([\mathrm{H}])=\mathrm{C} 2[\mathrm{H}]) \mathrm{C} 3=\mathrm{C}([\mathrm{H}]) \mathrm{C}(=\mathrm{C}([\mathrm{H}]) \mathrm{C}([\mathrm{H}] \\
)=\mathrm{C} 3[\mathrm{H}]) \mathrm{C} 4=\mathrm{C}([\mathrm{H}]) \mathrm{C}(\mathrm{OC}([\mathrm{H}])([\mathrm{H}]) \mathrm{C}([\mathrm{H}])([\mathrm{H}])[\mathrm{H}])=\mathrm{C}([\mathrm{H}]) \mathrm{C}([\mathrm{H}])=\mathrm{C} 4[\mathrm{H}]) \mathrm{N}([\mathrm{H}])[\mathrm{H}]) \mathrm{C}([\mathrm{H}])( \\
[\mathrm{H}])[\mathrm{H}]\end{array}$ & 2.337 & 2.282 \\
\hline Ts43 & $\begin{array}{l}\mathrm{O}=\mathrm{C} 1 \mathrm{~N}(\mathrm{C}(=\mathrm{N}[\mathrm{C} @ @] 1(\mathrm{C}=2 \mathrm{C}([\mathrm{H}])=\mathrm{C}([\mathrm{H}]) \mathrm{C}([\mathrm{H}])=\mathrm{C}([\mathrm{H}]) \mathrm{C}=2[\mathrm{H}]) \mathrm{C} 3=\mathrm{C}([\mathrm{H}]) \mathrm{C}(=\mathrm{C}([\mathrm{H}]) \mathrm{C}([\mathrm{H}] \\
)=\mathrm{C} 3[\mathrm{H}]) \mathrm{C}([\mathrm{H}])([\mathrm{H}])[\mathrm{H}]) \mathrm{N}([\mathrm{H}])[\mathrm{H}]) \mathrm{C}([\mathrm{H}])([\mathrm{H}])[\mathrm{H}]\end{array}$ & 2.260 & 2.318 \\
\hline Ts44 & $\begin{array}{l}\mathrm{O}=\mathrm{C}(\mathrm{N}([\mathrm{C} @ @] 1([\mathrm{H}]) \mathrm{C}([\mathrm{H}])([\mathrm{H}]) \mathrm{C}([\mathrm{H}])([\mathrm{H}]) \mathrm{C}([\mathrm{H}])([\mathrm{H}]) \mathrm{C}([\mathrm{H}])([\mathrm{H}]) \mathrm{C} 1([\mathrm{H}])[\mathrm{H}]) \mathrm{C}([\mathrm{H}])([\mathrm{H}]) \\
\mathrm{C} 2=\mathrm{C}(\mathrm{N}([\mathrm{H}]) \mathrm{N}=\mathrm{C} 2[\mathrm{H}]) \mathrm{C}([\mathrm{H}])([\mathrm{H}])[\mathrm{H}]) \mathrm{C}([\mathrm{H}])([\mathrm{H}]) \mathrm{C}([\mathrm{H}])([\mathrm{H}])[\mathrm{C} @]([\mathrm{H}])(\mathrm{N} 5 \mathrm{C}(=\mathrm{NC}=4 \mathrm{C}([\mathrm{H}] \\
)=\mathrm{C}([\mathrm{H}]) \mathrm{C}(\mathrm{O} / \mathrm{C} 3=\mathrm{C}(1[\mathrm{H}]) \mathrm{C}([\mathrm{H}])=\mathrm{C}([\mathrm{H}]) \mathrm{C}([\mathrm{H}])=\mathrm{C} 3[\mathrm{H}])=\mathrm{C}([\mathrm{H}]) \mathrm{C}=4 \mathrm{C} 5([\mathrm{H}])[\mathrm{H}]) \mathrm{N}([\mathrm{H}])[\mathrm{H}])[\mathrm{C} \\
@ @] 6([\mathrm{H}]) \mathrm{C}([\mathrm{H}])([\mathrm{H}]) \mathrm{C}([\mathrm{H}])([\mathrm{H}]) \mathrm{C}([\mathrm{H}])([\mathrm{H}]) \mathrm{C}([\mathrm{H}])([\mathrm{H}]) \mathrm{C} 6([\mathrm{H}])[\mathrm{H}]\end{array}$ & 3.409 & 2.844 \\
\hline Ts45 & $\begin{array}{l}\mathrm{O}=\mathrm{C}(\mathrm{N}([\mathrm{C} @ @] 1([\mathrm{H}]) \mathrm{C}([\mathrm{H}])([\mathrm{H}]) \mathrm{C}([\mathrm{H}])([\mathrm{H}]) \mathrm{C}([\mathrm{H}])([\mathrm{H}]) \mathrm{C}([\mathrm{H}])([\mathrm{H}]) \mathrm{C} 1([\mathrm{H}])[\mathrm{H}]) \mathrm{C}([\mathrm{H}])([\mathrm{H}])[ \\
\mathrm{H}]) \mathrm{C}([\mathrm{H}])([\mathrm{H}]) \mathrm{C}([\mathrm{H}])([\mathrm{H}])[\mathrm{C} @]([\mathrm{H}])(\mathrm{N} 4 \mathrm{C}(=\mathrm{N} / \mathrm{C} 3=\mathrm{C}(\backslash[\mathrm{H}]) \mathrm{C}([\mathrm{H}])=\mathrm{C}(\mathrm{OC}=2 \mathrm{C}([\mathrm{H}])=\mathrm{C}([\mathrm{H}]) \mathrm{C}([ \\
\mathrm{H}])=\mathrm{C}([\mathrm{H}]) \mathrm{C}=2[\mathrm{H}]) \mathrm{C}([\mathrm{H}])=\mathrm{C} 3 \mathrm{C} 4([\mathrm{H}])[\mathrm{H}]) \mathrm{N}([\mathrm{H}])[\mathrm{H}])[\mathrm{C} @] 5([\mathrm{H}]) \mathrm{C}([\mathrm{H}])([\mathrm{H}]) \mathrm{OC}([\mathrm{H}])([\mathrm{H}]) \mathrm{C}( \\
[\mathrm{H}])([\mathrm{H}]) \mathrm{C} 5([\mathrm{H}])[\mathrm{H}]\end{array}$ & 2.914 & 3.655 \\
\hline Ts46 & $\begin{array}{l}\mathrm{O}=\mathrm{C}(\mathrm{OC}([\mathrm{H}])([\mathrm{H}])[\mathrm{C} @]([\mathrm{H}])(\mathrm{N} 3 \mathrm{C}(=\mathrm{N} / \mathrm{C} 2=\mathrm{C}(\mathrm{VH}]) \mathrm{C}([\mathrm{H}])=\mathrm{C}(\mathrm{O} / \mathrm{C} 1=\mathrm{C}(\backslash[\mathrm{H}]) \mathrm{C}([\mathrm{H}])=\mathrm{C}([\mathrm{H}]) \mathrm{C}( \\
[\mathrm{H}])=\mathrm{C} 1[\mathrm{H}]) \mathrm{C}([\mathrm{H}])=\mathrm{C} 2 \mathrm{C} 3([\mathrm{H}])[\mathrm{H}]) \mathrm{N}([\mathrm{H}])[\mathrm{H}])[\mathrm{C} @ @] 4([\mathrm{H}]) \mathrm{C}([\mathrm{H}])([\mathrm{H}]) \mathrm{C}([\mathrm{H}])([\mathrm{H}]) \mathrm{C}([\mathrm{H}])([ \\
\mathrm{H}]) \mathrm{C}([\mathrm{H}])([\mathrm{H}]) \mathrm{C} 4([\mathrm{H}])[\mathrm{H}]) \mathrm{N}([\mathrm{H}])[\mathrm{C} @ @] 5([\mathrm{H}]) \mathrm{C}([\mathrm{H}])([\mathrm{H}]) \mathrm{C}([\mathrm{H}])([\mathrm{H}]) \mathrm{C}([\mathrm{H}])([\mathrm{H}]) \mathrm{C}([\mathrm{H}])([\mathrm{H} \\
]) \mathrm{C} 5([\mathrm{H}])[\mathrm{H}]\end{array}$ & 3.222 & 2.341 \\
\hline Ts47 & $\begin{array}{l}\mathrm{O}=\mathrm{C} 3 \mathrm{~N}(\mathrm{C}(=\mathrm{N}[\mathrm{C} @](\mathrm{C}=1 \mathrm{C}([\mathrm{H}])=\mathrm{C}([\mathrm{S}] \mathrm{C}=1[\mathrm{Cl}]) \mathrm{C}=2 \mathrm{C}([\mathrm{H}])=\mathrm{C}(\mathrm{CCC}([\mathrm{H}])([\mathrm{H}])[\mathrm{H}]) \mathrm{C}([\mathrm{H}])=\mathrm{NC} \\
=2[\mathrm{H}])(\mathrm{C} 3([\mathrm{H}])[\mathrm{H}]) \mathrm{C}([\mathrm{H}])([\mathrm{H}])[\mathrm{H}]) \mathrm{N}([\mathrm{H}])[\mathrm{H}]) \mathrm{C}([\mathrm{H}])([\mathrm{H}])[\mathrm{H}]\end{array}$ & 4.097 & 4.443 \\
\hline Ts 48 & $\begin{array}{l}\mathrm{NCC}=1 \mathrm{C}([\mathrm{H}])=\mathrm{C}(\mathrm{C}([\mathrm{H}])=\mathrm{C}([\mathrm{H}]) \mathrm{C}=1[\mathrm{H}]) \mathrm{C}=2 \mathrm{C}([\mathrm{H}])=\mathrm{C}([\mathrm{S}] \mathrm{C}=2[\mathrm{H}])[\mathrm{C} @] 3(\mathrm{~N}=\mathrm{C}(\mathrm{N}(\mathrm{C}(=\mathrm{O}) \mathrm{C} 3( \\
[\mathrm{H}])[\mathrm{H}]) \mathrm{C}([\mathrm{H}])([\mathrm{H}])[\mathrm{H}]) \mathrm{N}([\mathrm{H}])[\mathrm{H}]) \mathrm{C}([\mathrm{H}])([\mathrm{H}])[\mathrm{H}]\end{array}$ & 3.244 & 2.341 \\
\hline Ts49 & $\begin{array}{l}\mathrm{O}=\mathrm{C}(\mathrm{O}[\mathrm{C} @] 2([\mathrm{H}])[\mathrm{C} @ @]([\mathrm{H}])(\mathrm{OC} 1=\mathrm{C}(\mathrm{C}(\mathrm{O}[\mathrm{H}])=\mathrm{C}([\mathrm{H}]) \mathrm{C}(\mathrm{O}[\mathrm{H}])=\mathrm{C} 1[\mathrm{H}]) \mathrm{C} 2([\mathrm{H}])[\mathrm{H}]) \mathrm{C} 3=\mathrm{C}( \\
[\mathrm{H}]) \mathrm{C}(\mathrm{O}[\mathrm{H}])=\mathrm{C}(\mathrm{O}[\mathrm{H}]) \mathrm{C}([\mathrm{H}])=\mathrm{C} 3[\mathrm{H}]) \mathrm{C}=4 \mathrm{C}([\mathrm{H}])=\mathrm{C}(\mathrm{O}[\mathrm{H}]) \mathrm{C}(\mathrm{O}[\mathrm{H}])=\mathrm{C}(\mathrm{O}[\mathrm{H}]) \mathrm{C}=4[\mathrm{H}]\end{array}$ & 1.276 & 2.529 \\
\hline Ts50 & $\begin{array}{l}\mathrm{O}=\mathrm{C}(\mathrm{N}([\mathrm{H}]) \mathrm{C} 3=\mathrm{C}([\mathrm{H}]) \mathrm{C}([\mathrm{H}])=\mathrm{C} 2 \mathrm{OC}([\mathrm{C} @] 4([\mathrm{C} @ @] 1(\mathrm{~N}=\mathrm{C}(\mathrm{OC} 1([\mathrm{H}])[\mathrm{H}]) \mathrm{N}([\mathrm{H}])[\mathrm{H}]) \mathrm{C} 2=\mathrm{C} 3 \\
[\mathrm{H}]) \mathrm{C}([\mathrm{H}])([\mathrm{H}]) \mathrm{OC} 4([\mathrm{H}])[\mathrm{H}])(\mathrm{C}([\mathrm{H}])([\mathrm{H}])[\mathrm{H}]) \mathrm{C}([\mathrm{H}])([\mathrm{H}])[\mathrm{H}]) \mathrm{C}=5 / \mathrm{N}=\mathrm{C}(/[\mathrm{H}]) \mathrm{C}([\mathrm{Cl}])=\mathrm{C}([\mathrm{H}]) \\
\mathrm{C}=5[\mathrm{~F}]\end{array}$ & 3.288 & 2.749 \\
\hline Ts51 & $\begin{array}{l}{[\mathrm{H}] / \mathrm{C} 4=\mathrm{C}(/ \mathrm{C}([\mathrm{H}])=\mathrm{C} 1 \mathrm{C}(\mathrm{OC}([\mathrm{H}])([\mathrm{H}])[\mathrm{C} @] 3([\mathrm{C} @ @] 12 \mathrm{~N}=\mathrm{C}(\mathrm{OC} 2([\mathrm{H}])[\mathrm{H}]) \mathrm{N}([\mathrm{H}])[\mathrm{H}]) \mathrm{C}([\mathrm{H}])} \\
([\mathrm{H}]) \mathrm{OC} 3([\mathrm{H}])[\mathrm{H}])=\mathrm{C} 4[\mathrm{H}]) \mathrm{C} 5=\mathrm{C}([\mathrm{H}]) \mathrm{N}=\mathrm{C}([\mathrm{H}]) \mathrm{C}(\mathrm{CCC}([\mathrm{H}])([\mathrm{H}])[\mathrm{H}])=\mathrm{C} 5[\mathrm{H}]\end{array}$ & 2.910 & 3.381 \\
\hline Ts52 & $\begin{array}{l}\mathrm{O}=\mathrm{C}(\mathrm{N}([\mathrm{H}]) \mathrm{C} 3=\mathrm{C}([\mathrm{H}]) \mathrm{C}([\mathrm{H}])=\mathrm{C} 2 \mathrm{OC}([\mathrm{C} @] 4([\mathrm{C} @ @] 1(\mathrm{~N}=\mathrm{C}(\mathrm{OC} 1([\mathrm{H}])[\mathrm{H}]) \mathrm{N}([\mathrm{H}])[\mathrm{H}]) \mathrm{C} 2=\mathrm{C} 3 \\
[\mathrm{H}]) \mathrm{C}([\mathrm{H}])([\mathrm{H}]) \mathrm{OC} 4([\mathrm{H}])[\mathrm{H}])(\mathrm{C}([\mathrm{H}])([\mathrm{H}])[\mathrm{H}]) \mathrm{C}([\mathrm{H}])([\mathrm{H}])[\mathrm{H}]) \mathrm{C} 5=\mathrm{NC}([\mathrm{H}])=\mathrm{C}([\mathrm{Br}]) \mathrm{C}([\mathrm{H}])=\mathrm{N} \\
5\end{array}$ & 3.078 & 3.038 \\
\hline Ts53 & $\begin{array}{l}\mathrm{O}=\mathrm{C}(\mathrm{N}([\mathrm{H}]) \mathrm{C} 3=\mathrm{C}([\mathrm{H}]) \mathrm{C}([\mathrm{H}])=\mathrm{C} 2 \mathrm{OC}([\mathrm{H}])([\mathrm{H}])[\mathrm{C} @ @] 4([\mathrm{C} @ @] 1(\mathrm{~N}=\mathrm{C}(\mathrm{OC} 1([\mathrm{H}])[\mathrm{H}]) \mathrm{N}([\mathrm{H}]) \\
[\mathrm{H}]) \mathrm{C} 2=\mathrm{C} 3[\mathrm{H}]) \mathrm{C}([\mathrm{H}])([\mathrm{H}]) \mathrm{C} 4([\mathrm{H}])[\mathrm{H}]) \mathrm{C} 5=\mathrm{NC}([\mathrm{H}])=\mathrm{C}([\mathrm{Cl}]) \mathrm{C}([\mathrm{H}])=\mathrm{C} 5 \mathrm{C}([\mathrm{H}])([\mathrm{H}])[\mathrm{H}]\end{array}$ & 2.504 & 2.498 \\
\hline Ts54 & $\begin{array}{l}\mathrm{O}=\mathrm{C}(/ \mathrm{C} 1=\mathrm{N} / \mathrm{C}([\mathrm{H}])=\mathrm{C}(\mathrm{C}([\mathrm{H}])=\mathrm{C} 1[\mathrm{H}]) \mathrm{C}([\mathrm{H}])([\mathrm{H}])[\mathrm{H}]) \mathrm{N}([\mathrm{H}]) \mathrm{C} 2=\mathrm{C}([\mathrm{H}]) \mathrm{C}(=\mathrm{C}([\mathrm{F}]) \mathrm{C}([\mathrm{H}])=\mathrm{C} 2 \\
[\mathrm{H}])[\mathrm{C} @] 3(\mathrm{~N}([\mathrm{H}]) \mathrm{C}(=\mathrm{N}[\mathrm{H}]) \mathrm{N}([\mathrm{S}](=\mathrm{O})(=\mathrm{O}) \mathrm{C} 3([\mathrm{H}])[\mathrm{H}]) \mathrm{C}([\mathrm{H}])([\mathrm{H}])[\mathrm{H}]) \mathrm{C}([\mathrm{H}])([\mathrm{H}])[\mathrm{H}]\end{array}$ & 4.523 & 3.961 \\
\hline
\end{tabular}




\begin{tabular}{|c|c|c|c|}
\hline Ts55 & $\begin{array}{l}\mathrm{O}=\mathrm{C}(/ \mathrm{C} 1=\mathrm{N} / \mathrm{C}([\mathrm{H}])=\mathrm{C}([\mathrm{F}]) \mathrm{C}([\mathrm{H}])=\mathrm{C} 1[\mathrm{H}]) \mathrm{N}([\mathrm{H}]) \mathrm{C} 2=\mathrm{C}([\mathrm{F}]) \mathrm{C}(=\mathrm{C}([\mathrm{F}]) \mathrm{C}([\mathrm{H}])=\mathrm{C} 2[\mathrm{H}])[\mathrm{C} @ 3( \\
\mathrm{N}([\mathrm{H}]) \mathrm{C}(=\mathrm{N}[\mathrm{H}]) \mathrm{N}([\mathrm{S}](=\mathrm{O})(=\mathrm{O}) \mathrm{C} 3([\mathrm{H}])[\mathrm{H}]) \mathrm{C}([\mathrm{H}])([\mathrm{H}])[\mathrm{H}]) \mathrm{C}([\mathrm{H}])([\mathrm{H}])[\mathrm{H}]\end{array}$ & 4.312 & 4.448 \\
\hline Ts56 & $\begin{array}{l}\mathrm{O}=[\mathrm{S}] 3(=\mathrm{O}) \mathrm{N}(/ \mathrm{C}(=\mathrm{N} \backslash[\mathrm{H}]) \mathrm{N}([\mathrm{H}])[\mathrm{C} @](\mathrm{C}=1[\mathrm{~S}] \mathrm{C}([\mathrm{H}])=\mathrm{C}(\mathrm{C}=1[\mathrm{H}]) \mathrm{C} 2=\mathrm{C}([\mathrm{H}]) \mathrm{C}([\mathrm{H}])=\mathrm{C}([\mathrm{H}]) \mathrm{C}( \\
\mathrm{CN})=\mathrm{C} 2[\mathrm{H}])(\mathrm{C} 3([\mathrm{H}])[\mathrm{H}]) \mathrm{C}([\mathrm{H}])([\mathrm{H}])[\mathrm{H}]) \mathrm{C}([\mathrm{H}])([\mathrm{H}])[\mathrm{H}]\end{array}$ & 3.592 & 3.122 \\
\hline Ts57 & $\begin{array}{l}\mathrm{O}=\mathrm{C}(\mathrm{N}([\mathrm{H}])[\mathrm{C} @] 1([\mathrm{H}]) \mathrm{C}([\mathrm{H}])([\mathrm{H}]) \mathrm{C} 1([\mathrm{H}])[\mathrm{H}])[\mathrm{C} @]([\mathrm{H}])(\mathrm{OC}([\mathrm{H}])([\mathrm{H}])[\mathrm{H}]) \mathrm{C}([\mathrm{H}])([\mathrm{H}])[\mathrm{C} @ \\
]([\mathrm{H}])(\mathrm{O}[\mathrm{H}])[\mathrm{C} @ @]([\mathrm{H}])(\mathrm{N}([\mathrm{H}]) \mathrm{C}(=\mathrm{O}) \mathrm{C}=2 \mathrm{C}([\mathrm{H}])=\mathrm{C}([\mathrm{H}]) \mathrm{C}([\mathrm{H}])=\mathrm{C}(\mathrm{C}=2[\mathrm{H}]) \mathrm{C}(=\mathrm{O}) \mathrm{N}(\mathrm{C}([\mathrm{H}]) \\
([\mathrm{H}]) \mathrm{C}([\mathrm{H}])([\mathrm{H}]) \mathrm{C}([\mathrm{H}])([\mathrm{H}])[\mathrm{H}]) \mathrm{C}([\mathrm{H}])([\mathrm{H}]) \mathrm{C}([\mathrm{H}])([\mathrm{H}]) \mathrm{C}([\mathrm{H}])([\mathrm{H}])[\mathrm{H}]) \mathrm{C}([\mathrm{H}])([\mathrm{H}]) \mathrm{O} / \mathrm{C} 3=\mathrm{C}(\backslash \\
[\mathrm{H}]) \mathrm{C}([\mathrm{F}])=\mathrm{C}([\mathrm{H}]) \mathrm{C}([\mathrm{F}])=\mathrm{C} 3[\mathrm{H}]\end{array}$ & 2.658 & 2.141 \\
\hline Ts58 & $\begin{array}{l}\mathrm{O}=\mathrm{C}(\mathrm{N}([\mathrm{H}]) \mathrm{C}(=\mathrm{NC}([\mathrm{H}])([\mathrm{H}]) \mathrm{C} 1=\mathrm{C}([\mathrm{H}]) \mathrm{C}([\mathrm{Cl}])=\mathrm{C}(\mathrm{C}([\mathrm{Cl}])=\mathrm{C} 1[\mathrm{H}]) \mathrm{N}([\mathrm{H}])[\mathrm{S}](=\mathrm{O})(=\mathrm{O}) \mathrm{C}([\mathrm{H}]) \\
([\mathrm{H}])[\mathrm{H}]) \mathrm{N}([\mathrm{H}])[\mathrm{H}]) \mathrm{C}=2 \mathrm{C}(=\mathrm{N}[\mathrm{S}] \mathrm{C}=2 \mathrm{C}([\mathrm{H}])([\mathrm{H}])[\mathrm{H}]) \mathrm{C} 3=\mathrm{C}([\mathrm{H}]) \mathrm{C}([\mathrm{H}])=\mathrm{C}(\mathrm{OC}([\mathrm{H}])([\mathrm{H}])[\mathrm{H}]) \\
\mathrm{C}([\mathrm{H}])=\mathrm{C} 3[\mathrm{H}]\end{array}$ & 2.553 & 4.082 \\
\hline Ts59 & $\begin{array}{l}\mathrm{O}=\mathrm{C}(\mathrm{N}([\mathrm{H}])[\mathrm{C} @]([\mathrm{H}])(\mathrm{C}(=\mathrm{O}) \mathrm{N}([\mathrm{H}])[\mathrm{C} @(([\mathrm{H}])(\mathrm{C}(=\mathrm{O}) \mathrm{O}[\mathrm{H}]) \mathrm{C}([\mathrm{H}])([\mathrm{H}]) \mathrm{C} 1=\mathrm{C}([\mathrm{H}]) \mathrm{C}([\mathrm{H}])=\mathrm{C} \\
([\mathrm{H}]) \mathrm{C}([\mathrm{H}])=\mathrm{C} 1[\mathrm{H}]) \mathrm{C}([\mathrm{H}])([\mathrm{H}]) \mathrm{C}([\mathrm{H}])([\mathrm{H}]) \mathrm{C}(=\mathrm{O}) \mathrm{O}[\mathrm{H}])[\mathrm{C} @ @]([\mathrm{H}])(\mathrm{N}([\mathrm{H}]) \mathrm{C}(=\mathrm{O})[\mathrm{C} @ @]([ \\
\mathrm{H}])(\mathrm{N}([\mathrm{H}]) \mathrm{C}([\mathrm{H}])([\mathrm{H}])[\mathrm{C} @ @]([\mathrm{H}])(\mathrm{O}[\mathrm{H}]) \mathrm{C}(=\mathrm{O})[\mathrm{C} @ @]([\mathrm{H}])(\mathrm{N}([\mathrm{H}]) \mathrm{C}(=\mathrm{O})[\mathrm{C} @ @]([\mathrm{H}])(\mathrm{N}([ \\
\mathrm{H}]) \mathrm{C}(=\mathrm{O})[\mathrm{C} @ @]([\mathrm{H}])(\mathrm{N}([\mathrm{H}]) \mathrm{C}(=\mathrm{O})[\mathrm{C} @ @]([\mathrm{H}])(\mathrm{N}([\mathrm{H}])[\mathrm{H}]) \mathrm{C}([\mathrm{H}])([\mathrm{H}]) \mathrm{C}([\mathrm{H}])([\mathrm{H}]) \mathrm{C}(=\mathrm{O}) \mathrm{O}[ \\
\mathrm{H}]) \mathrm{C}([\mathrm{H}])([\mathrm{H}]) \mathrm{C}([\mathrm{H}])(\mathrm{C}([\mathrm{H}])([\mathrm{H}])[\mathrm{H}]) \mathrm{C}([\mathrm{H}])([\mathrm{H}])[\mathrm{H}]) \mathrm{C}([\mathrm{H}])([\mathrm{H}]) \mathrm{C}(=\mathrm{O}) \mathrm{O}[\mathrm{H}]) \mathrm{C}([\mathrm{H}])([\mathrm{H}]) \mathrm{C}([ \\
\mathrm{H}])(\mathrm{C}([\mathrm{H}])([\mathrm{H}])[\mathrm{H}]) \mathrm{C}([\mathrm{H}])([\mathrm{H}])[\mathrm{H}]) \mathrm{C}([\mathrm{H}])([\mathrm{H}])[\mathrm{H}]) \mathrm{C}([\mathrm{H}])(\mathrm{C}([\mathrm{H}])([\mathrm{H}])[\mathrm{H}]) \mathrm{C}([\mathrm{H}])([\mathrm{H}])[\mathrm{H}] \sim\end{array}$ & 5.523 & 6.340 \\
\hline Ts60 & $\begin{array}{l}\mathrm{O}=[\mathrm{S}](=\mathrm{O})(\mathrm{C}([\mathrm{H}])([\mathrm{H}]) \mathrm{C}([\mathrm{H}])([\mathrm{H}])[\mathrm{C} @ @]([\mathrm{H}])(\mathrm{C}(=\mathrm{O}) \mathrm{N}([\mathrm{H}])[\mathrm{C} @([\mathrm{H}])([\mathrm{C} @([\mathrm{H}])(\mathrm{O}[\mathrm{H}]) \mathrm{C}( \\
[\mathrm{H}])([\mathrm{H}])[\mathrm{C} @(([\mathrm{H}])(\mathrm{C}(=\mathrm{O}) \mathrm{N}([\mathrm{H}])[\mathrm{C} @]([\mathrm{H}])(\mathrm{C}(=\mathrm{O}) \mathrm{N}([\mathrm{H}]) \mathrm{C}([\mathrm{H}])([\mathrm{H}]) \mathrm{C} 1=\mathrm{C}([\mathrm{H}]) \mathrm{C}([\mathrm{H}])=\mathrm{C}([ \\
\mathrm{H}]) \mathrm{C}([\mathrm{H}])=\mathrm{C} 1[\mathrm{H}]) \mathrm{C}([\mathrm{H}])(\mathrm{C}([\mathrm{H}])([\mathrm{H}])[\mathrm{H}]) \mathrm{C}([\mathrm{H}])([\mathrm{H}])[\mathrm{H}]) \mathrm{C}([\mathrm{H}])([\mathrm{H}])[\mathrm{H}]) \mathrm{C}([\mathrm{H}])([\mathrm{H}]) \mathrm{C}([\mathrm{H}])( \\
\mathrm{C}([\mathrm{H}])([\mathrm{H}])[\mathrm{H}]) \mathrm{C}([\mathrm{H}])([\mathrm{H}])[\mathrm{H}]) \mathrm{N}([\mathrm{H}]) \mathrm{C}(=\mathrm{O})[\mathrm{C} @ @]([\mathrm{H}])(\mathrm{N}([\mathrm{H}]) \mathrm{C}(=\mathrm{O}) \mathrm{OC}(\mathrm{C}([\mathrm{H}])([\mathrm{H}])[\mathrm{H}])( \\
\mathrm{C}([\mathrm{H}])([\mathrm{H}])[\mathrm{H}]) \mathrm{C}([\mathrm{H}])([\mathrm{H}])[\mathrm{H}]) \mathrm{C}([\mathrm{H}])(\mathrm{C}([\mathrm{H}])([\mathrm{H}])[\mathrm{H}]) \mathrm{C}([\mathrm{H}])([\mathrm{H}])[\mathrm{H}]) \mathrm{C}([\mathrm{H}])([\mathrm{H}])[\mathrm{H}]\end{array}$ & 4.097 & 3.243 \\
\hline Ts61 & $\begin{array}{l}\mathrm{O}=\mathrm{C}(\mathrm{N}([\mathrm{H}])[\mathrm{C} @]([\mathrm{H}])([\mathrm{C} @]([\mathrm{H}])(\mathrm{O}[\mathrm{H}]) \mathrm{C}([\mathrm{H}])([\mathrm{H}])[\mathrm{C} @]([\mathrm{H}])(\mathrm{C}(=\mathrm{O}) \mathrm{N}([\mathrm{H}])[\mathrm{C} @]([\mathrm{H}])(\mathrm{C}(= \\
\mathrm{O}) \mathrm{N}([\mathrm{H}]) \mathrm{C}([\mathrm{H}])([\mathrm{H}]) \mathrm{C} 1=\mathrm{C}([\mathrm{H}]) \mathrm{C}([\mathrm{H}])=\mathrm{C}([\mathrm{H}]) \mathrm{C}([\mathrm{H}])=\mathrm{C} 1[\mathrm{H}]) \mathrm{C}([\mathrm{H}])([\mathrm{H}])[\mathrm{H}]) \mathrm{C}([\mathrm{H}])([\mathrm{H}])[\mathrm{H}]) \\
\mathrm{C}([\mathrm{H}])([\mathrm{H}]) \mathrm{C}([\mathrm{H}])(\mathrm{C}([\mathrm{H}])([\mathrm{H}])[\mathrm{H}]) \mathrm{C}([\mathrm{H}])([\mathrm{H}])[\mathrm{H}])[\mathrm{C} @ @]([\mathrm{H}])(\mathrm{N}([\mathrm{H}]) \mathrm{C}(=\mathrm{O}) \mathrm{OC}(\mathrm{C}([\mathrm{H}])([\mathrm{H}] \\
)[\mathrm{H}])(\mathrm{C}([\mathrm{H}])([\mathrm{H}])[\mathrm{H}]) \mathrm{C}([\mathrm{H}])([\mathrm{H}])[\mathrm{H}]) \mathrm{C}(=\mathrm{O}) \mathrm{N}([\mathrm{H}])[\mathrm{H}]\end{array}$ & 0.649 & 2.152 \\
\hline Ts62 & $\begin{array}{l}\mathrm{O}=\mathrm{C} 1 \mathrm{~N}(/ \mathrm{C}(=\mathrm{N} \backslash[\mathrm{H}]) \mathrm{N}([\mathrm{H}])[\mathrm{C} @] 1(\mathrm{C}([\mathrm{H}])([\mathrm{H}]) \mathrm{C}([\mathrm{H}])([\mathrm{H}])[\mathrm{C} @] 2([\mathrm{H}]) \mathrm{C}([\mathrm{H}])([\mathrm{H}]) \mathrm{C}([\mathrm{H}])([\mathrm{H}]) \\
\mathrm{C}([\mathrm{H}])([\mathrm{H}]) \mathrm{C}([\mathrm{H}])([\mathrm{H}]) \mathrm{C} 2([\mathrm{H}])[\mathrm{H}]) \mathrm{C}([\mathrm{H}])([\mathrm{H}])[\mathrm{C} @] 4([\mathrm{H}]) \mathrm{C}([\mathrm{H}])([\mathrm{H}])[\mathrm{C} @]([\mathrm{H}])(\mathrm{N}([\mathrm{H}]) \mathrm{C}(= \\
\mathrm{O}) \mathrm{C} 3=\mathrm{C}([\mathrm{H}]) \mathrm{C}([\mathrm{H}])=\mathrm{C}([\mathrm{H}]) \mathrm{C}([\mathrm{H}])=\mathrm{C} 3[\mathrm{H}]) \mathrm{C}([\mathrm{H}])([\mathrm{H}]) \mathrm{C}([\mathrm{H}])([\mathrm{H}]) \mathrm{C} 4([\mathrm{H}])[\mathrm{H}]) \mathrm{C}([\mathrm{H}])([\mathrm{H}])[\mathrm{H} \\
]\end{array}$ & 3.886 & 3.365 \\
\hline Ts63 & $\begin{array}{l}\mathrm{O}=\mathrm{C} 1 \mathrm{~N}(/ \mathrm{C}(=\mathrm{N} \backslash[\mathrm{H}]) \mathrm{N}([\mathrm{H}])[\mathrm{C} @] 1(\mathrm{C}([\mathrm{H}])([\mathrm{H}]) \mathrm{C}([\mathrm{H}])([\mathrm{H}])[\mathrm{C} @ @] 2([\mathrm{H}]) \mathrm{C}([\mathrm{H}])([\mathrm{H}]) \mathrm{C}([\mathrm{H}])([\mathrm{H} \\
]) \mathrm{C}([\mathrm{H}])([\mathrm{H}]) \mathrm{C}([\mathrm{H}])([\mathrm{H}]) \mathrm{C} 2([\mathrm{H}])[\mathrm{H}]) \mathrm{C}([\mathrm{H}])([\mathrm{H}])[\mathrm{C} @] 4([\mathrm{H}]) \mathrm{C}([\mathrm{H}])([\mathrm{H}])[\mathrm{C} @]([\mathrm{H}])(\mathrm{N}([\mathrm{H}]) \mathrm{C}([ \\
\mathrm{H}])([\mathrm{H}])[\mathrm{C} @] 3([\mathrm{H}]) \mathrm{C}([\mathrm{H}])([\mathrm{H}]) \mathrm{C} 3([\mathrm{H}])[\mathrm{H}]) \mathrm{C}([\mathrm{H}])([\mathrm{H}]) \mathrm{C}([\mathrm{H}])([\mathrm{H}]) \mathrm{C} 4([\mathrm{H}])[\mathrm{H}]) \mathrm{C}([\mathrm{H}])([\mathrm{H}])[ \\
\mathrm{H}]\end{array}$ & 3.319 & 3.052 \\
\hline Ts64 & $\begin{array}{l}\mathrm{O}=\mathrm{C} 1 \mathrm{~N}(/ \mathrm{C}(=\mathrm{N} /[\mathrm{H}]) \mathrm{N}([\mathrm{H}])[\mathrm{C} @] 1(\mathrm{C}([\mathrm{H}])([\mathrm{H}]) \mathrm{C}([\mathrm{H}])([\mathrm{H}])[\mathrm{C} @ @] 2([\mathrm{H}]) \mathrm{C}([\mathrm{H}])([\mathrm{H}]) \mathrm{C}([\mathrm{H}])([\mathrm{H} \\
]) \mathrm{C}([\mathrm{H}])([\mathrm{H}]) \mathrm{C}([\mathrm{H}])([\mathrm{H}]) \mathrm{C} 2([\mathrm{H}])[\mathrm{H}]) \mathrm{C}([\mathrm{H}])([\mathrm{H}])[\mathrm{C} @ 4([\mathrm{H}]) \mathrm{C}([\mathrm{H}])([\mathrm{H}])[\mathrm{C} @]([\mathrm{H}])(\mathrm{N}([\mathrm{H}]) \mathrm{C}( \\
=\mathrm{O}) \mathrm{N}([\mathrm{H}]) \mathrm{C} 3=\mathrm{C}([\mathrm{H}]) \mathrm{C}([\mathrm{H}])=\mathrm{C}(\mathrm{OC}([\mathrm{H}])([\mathrm{H}])[\mathrm{H}]) \mathrm{C}([\mathrm{H}])=\mathrm{C} 3[\mathrm{H}]) \mathrm{C}([\mathrm{H}])([\mathrm{H}]) \mathrm{C}([\mathrm{H}])([\mathrm{H}]) \mathrm{C} 4([ \\
\mathrm{H}])[\mathrm{H}]) \mathrm{C}([\mathrm{H}])([\mathrm{H}])[\mathrm{H}]\end{array}$ & 5.000 & 3.239 \\
\hline Ts65 & $\begin{array}{l}\mathrm{O}=\mathrm{C} 1 \mathrm{~N}(/ \mathrm{C}(=\mathrm{N} \backslash[\mathrm{H}]) \mathrm{N}([\mathrm{H}])[\mathrm{C} @] 1(\mathrm{C}([\mathrm{H}])([\mathrm{H}]) \mathrm{C}([\mathrm{H}])([\mathrm{H}])[\mathrm{C} @] 2([\mathrm{H}]) \mathrm{C}([\mathrm{H}])([\mathrm{H}]) \mathrm{C}([\mathrm{H}])([\mathrm{H}]) \\
\mathrm{C}([\mathrm{H}])([\mathrm{H}]) \mathrm{C}([\mathrm{H}])([\mathrm{H}]) \mathrm{C} 2([\mathrm{H}])[\mathrm{H}]) \mathrm{C}([\mathrm{H}])([\mathrm{H}])[\mathrm{C} @ @] 3([\mathrm{H}]) \mathrm{C}([\mathrm{H}])([\mathrm{H}]) \mathrm{N}(\mathrm{C}(=\mathrm{O}) \mathrm{OC}(\mathrm{C}([\mathrm{H}])( \\
[\mathrm{H}])[\mathrm{H}])(\mathrm{C}([\mathrm{H}])([\mathrm{H}])[\mathrm{H}]) \mathrm{C}([\mathrm{H}])([\mathrm{H}])[\mathrm{H}]) \mathrm{C}([\mathrm{H}])([\mathrm{H}]) \mathrm{C}([\mathrm{H}])([\mathrm{H}]) \mathrm{C} 3([\mathrm{H}])[\mathrm{H}]) \mathrm{C}([\mathrm{H}])([\mathrm{H}])[\mathrm{H}]\end{array}$ & 3.387 & 4.164 \\
\hline Ts66 & $\begin{array}{l}\mathrm{O}=[\mathrm{S}] 2(=\mathrm{O}) \mathrm{N}(/ \mathrm{C}(=\mathrm{N} /[\mathrm{H}]) \mathrm{N}([\mathrm{H}])[\mathrm{C} @] 4(/ \mathrm{C} 1=\mathrm{C}(\backslash[\mathrm{H}]) \mathrm{C}([\mathrm{H}])=\mathrm{C}([\mathrm{H}]) \mathrm{C}([\mathrm{H}])=\mathrm{C} 1[\mathrm{H}])[\mathrm{C} @ @] 2([ \\
\mathrm{H}]) \mathrm{C}([\mathrm{H}])([\mathrm{H}]) \mathrm{N}(/ \mathrm{C} 3=\mathrm{N} / \mathrm{C}(=\mathrm{C}(/[\mathrm{F}]) \mathrm{C}(=\mathrm{N} 3) \mathrm{OC}([\mathrm{H}])([\mathrm{H}])[\mathrm{H}]) \mathrm{C}([\mathrm{H}])([\mathrm{H}])[\mathrm{H}]) \mathrm{C} 4([\mathrm{H}])[\mathrm{H}]) \mathrm{C}([ \\
\mathrm{H}])([\mathrm{H}])[\mathrm{H}]\end{array}$ & 3.292 & 3.012 \\
\hline Ts67 & $\begin{array}{l}\mathrm{O}=[\mathrm{S}] 2(=\mathrm{O}) \mathrm{N}(/ \mathrm{C}(=\mathrm{N} /[\mathrm{H}]) \mathrm{N}([\mathrm{H}])[\mathrm{C} @] 4(/ \mathrm{C} 1=\mathrm{C}(\backslash[\mathrm{H}]) \mathrm{C}([\mathrm{H}])=\mathrm{C}([\mathrm{H}]) \mathrm{C}([\mathrm{H}])=\mathrm{C} 1[\mathrm{~F}])[\mathrm{C} @ @] 2([ \\
\mathrm{H}]) \mathrm{C}([\mathrm{H}])([\mathrm{H}]) \mathrm{N}(/ \mathrm{C} 3=\mathrm{N} / \mathrm{C}(=\mathrm{C}(/[\mathrm{F}]) \mathrm{C}(=\mathrm{N} 3) \mathrm{OC}([\mathrm{H}])([\mathrm{H}])[\mathrm{H}]) \mathrm{C}([\mathrm{H}])([\mathrm{H}])[\mathrm{H}]) \mathrm{C} 4([\mathrm{H}])[\mathrm{H}]) \mathrm{C}([ \\
\mathrm{H}])([\mathrm{H}])[\mathrm{H}]\end{array}$ & 3.721 & 3.108 \\
\hline
\end{tabular}




\begin{tabular}{|c|c|c|c|}
\hline Ts68 & $\begin{array}{l}\mathrm{O}=[\mathrm{S}] 2(=\mathrm{O}) \mathrm{N}(/ \mathrm{C}(=\mathrm{N} /[\mathrm{H}]) \mathrm{N}([\mathrm{H}])[\mathrm{C} @ @] 4(/ \mathrm{C} 1=\mathrm{C}([\mathrm{H}]) \mathrm{C}([\mathrm{H}])=\mathrm{C}([\mathrm{H}]) \mathrm{C}([\mathrm{H}])=\mathrm{C} 1[\mathrm{H}])[\mathrm{C} @] 2([ \\
\mathrm{H}]) \mathrm{C}([\mathrm{H}])([\mathrm{H}]) \mathrm{N}(/ \mathrm{C} 3=\mathrm{N} / \mathrm{C}(\mathrm{OC}([\mathrm{H}])([\mathrm{H}])[\mathrm{H}])=\mathrm{C}([\mathrm{H}]) \mathrm{C}(=\mathrm{C} 3[\mathrm{H}]) \mathrm{C}([\mathrm{H}])([\mathrm{H}]) \mathrm{OC}([\mathrm{H}])([\mathrm{H}]) \mathrm{C}([ \\
\mathrm{H}])([\mathrm{H}])[\mathrm{H}]) \mathrm{C} 4([\mathrm{H}])[\mathrm{H}]) \mathrm{C}([\mathrm{H}])([\mathrm{H}])[\mathrm{H}]\end{array}$ & 3.215 & 3.247 \\
\hline Ts69 & $\begin{array}{l}\mathrm{O}=[\mathrm{S}] 2(=\mathrm{O}) \mathrm{N}(/ \mathrm{C}(=\mathrm{N} /[\mathrm{H}]) \mathrm{N}([\mathrm{H}])[\mathrm{C} @ @] 4(/ \mathrm{C} 1=\mathrm{C}([\mathrm{H}]) \mathrm{C}([\mathrm{H}])=\mathrm{C}([\mathrm{H}]) \mathrm{C}([\mathrm{H}])=\mathrm{Cl} 1[\mathrm{H}])[\mathrm{C} @] 2([ \\
\mathrm{H}]) \mathrm{C}([\mathrm{H}])([\mathrm{H}]) \mathrm{N}(/ \mathrm{C} 3=\mathrm{N} / \mathrm{C}(=\mathrm{C}(/[\mathrm{F}]) \mathrm{C}(=\mathrm{N} 3) \mathrm{OC}([\mathrm{H}])([\mathrm{H}])[\mathrm{H}]) \mathrm{C}([\mathrm{H}])([\mathrm{H}]) \mathrm{C}([\mathrm{H}])([\mathrm{H}])[\mathrm{H}]) \mathrm{C} 4([ \\
\mathrm{H}])[\mathrm{H}]) \mathrm{C}([\mathrm{H}])([\mathrm{H}])[\mathrm{H}]\end{array}$ & 3.086 & 3.259 \\
\hline Ts70 & $\begin{array}{l}\mathrm{O}=\mathrm{C}(\mathrm{C}=1 \mathrm{C}([\mathrm{H}])=\mathrm{C}([\mathrm{H}]) \mathrm{C}([\mathrm{H}])=\mathrm{C}(\mathrm{C}=1[\mathrm{H}]) \mathrm{C}(=\mathrm{O}) \mathrm{N}([\mathrm{H}])[\mathrm{C} @]([\mathrm{H}])([\mathrm{C} @ @]([\mathrm{H}])(\mathrm{O}[\mathrm{H}]) \mathrm{C}([\mathrm{H}] \\
)([\mathrm{H}]) \mathrm{N}([\mathrm{H}])[\mathrm{C} @]([\mathrm{H}])(\mathrm{C}(=\mathrm{O}) \mathrm{N}([\mathrm{H}]) \mathrm{C}([\mathrm{H}])([\mathrm{H}]) \mathrm{C}([\mathrm{H}])(\mathrm{C}([\mathrm{H}])([\mathrm{H}])[\mathrm{H}]) \mathrm{C}([\mathrm{H}])([\mathrm{H}])[\mathrm{H}])[\mathrm{C} @ \\
@]([\mathrm{H}])(\mathrm{O}[\mathrm{H}]) \mathrm{C}([\mathrm{H}])([\mathrm{H}]) \mathrm{C}([\mathrm{H}])([\mathrm{H}]) \mathrm{C}([\mathrm{H}])([\mathrm{H}])[\mathrm{H}]) \mathrm{C}([\mathrm{H}])([\mathrm{H}]) \mathrm{C} 2=\mathrm{C}([\mathrm{H}]) \mathrm{C}([\mathrm{H}])=\mathrm{C}([\mathrm{H}]) \mathrm{C} \\
([\mathrm{H}])=\mathrm{C} 2[\mathrm{H}]) \mathrm{N}([\mathrm{C} @]]([\mathrm{H}])(\mathrm{C} 3=\mathrm{C}([\mathrm{H}]) \mathrm{C}([\mathrm{H}])=\mathrm{C}([\mathrm{H}]) \mathrm{C}([\mathrm{H}])=\mathrm{C} 3[\mathrm{H}]) \mathrm{C}([\mathrm{H}])([\mathrm{H}])[\mathrm{H}]) \mathrm{C}([\mathrm{H}])([ \\
\mathrm{H}])[\mathrm{H}]\end{array}$ & 1.280 & 1.258 \\
\hline \multicolumn{4}{|c|}{ Calibration set } \\
\hline Calib1 & $\begin{array}{l}\mathrm{O}=\mathrm{C}(\mathrm{OC}([\mathrm{H}])([\mathrm{H}]) \mathrm{C}([\mathrm{H}])([\mathrm{H}]) \mathrm{C}([\mathrm{H}])=\mathrm{C}([\mathrm{H}])[\mathrm{H}]) \mathrm{N}([\mathrm{H}])[\mathrm{C} @]([\mathrm{H}])(\mathrm{C}(=\mathrm{O}) \mathrm{N}([\mathrm{H}])[\mathrm{C} @]([\mathrm{H}])( \\
\mathrm{C}(=\mathrm{O}) \mathrm{N}([\mathrm{H}])[\mathrm{C} @ @]([\mathrm{H}])(\mathrm{C}([\mathrm{H}])([\mathrm{H}]) \mathrm{C}([\mathrm{H}])(\mathrm{C}([\mathrm{H}])([\mathrm{H}])[\mathrm{H}]) \mathrm{C}([\mathrm{H}])([\mathrm{H}])[\mathrm{H}])[\mathrm{C} @ @]([\mathrm{H}])(\mathrm{O} \\
[\mathrm{H}]) \mathrm{C}([\mathrm{H}])([\mathrm{H}])[\mathrm{C} @]([\mathrm{H}])(\mathrm{C}(=\mathrm{O}) \mathrm{N}([\mathrm{H}])[\mathrm{C} @]([\mathrm{H}])(\mathrm{C}(=\mathrm{O}) \mathrm{N}([\mathrm{H}]) \mathrm{C}([\mathrm{H}])([\mathrm{H}]) \mathrm{C}=1 \mathrm{C}([\mathrm{H}])=\mathrm{C}([ \\
\mathrm{H}]) \mathrm{C}([\mathrm{H}])\end{array}$ & 3.212 & 3.130 \\
\hline Calib2 & $\begin{array}{l}\mathrm{O}=\mathrm{C}(\mathrm{OC}([\mathrm{H}])([\mathrm{H}]) \mathrm{C}([\mathrm{H}])([\mathrm{H}]) \mathrm{C}([\mathrm{H}])([\mathrm{H}]) \mathrm{C}([\mathrm{H}])) \mathrm{C}([\mathrm{H}])[\mathrm{H}]) \mathrm{N}([\mathrm{H}])[\mathrm{C} @]([\mathrm{H}])(\mathrm{C}(=\mathrm{O}) \mathrm{N}([\mathrm{H}])[ \\
\mathrm{C}]([\mathrm{H}])(\mathrm{C}(=\mathrm{O}) \mathrm{N}([\mathrm{H}])[\mathrm{C} @ @]([\mathrm{H}])(\mathrm{C}([\mathrm{H}])([\mathrm{H}]) \mathrm{C}([\mathrm{H}])(\mathrm{C}([\mathrm{H}])([\mathrm{H}])[\mathrm{H}]) \mathrm{C}([\mathrm{H}])([\mathrm{H}])[\mathrm{H}])[\mathrm{C} @ \\
@]([\mathrm{H}])(\mathrm{O}[\mathrm{H}]) \mathrm{C}([\mathrm{H}])([\mathrm{H}])[\mathrm{C} @]([\mathrm{H}])(\mathrm{C}(=\mathrm{O}) \mathrm{N}([\mathrm{H}])[\mathrm{C} @]([\mathrm{H}])(\mathrm{C}(=\mathrm{O}) \mathrm{N}([\mathrm{H}]) \mathrm{C}([\mathrm{H}])([\mathrm{H}]) \mathrm{C}=1 \mathrm{C} \\
([\mathrm{H}])=\mathrm{C}\end{array}$ & 2.950 & 3.154 \\
\hline Calib3 & $\begin{array}{l}\mathrm{O}=\mathrm{C}(/ \mathrm{C} 1=\mathrm{C}([\mathrm{H}]) \mathrm{C}([\mathrm{H}])=\mathrm{C}([\mathrm{H}]) \mathrm{C}(=\mathrm{C} 1[\mathrm{H}]) \mathrm{C}([\mathrm{H}])([\mathrm{H}]) \mathrm{N} 4 \mathrm{C}(=\mathrm{NC}=3 \mathrm{C}([\mathrm{H}])=\mathrm{C}([\mathrm{H}]) \mathrm{C}(\mathrm{OC}=2 \mathrm{C}( \\
[\mathrm{H}])=\mathrm{C}([\mathrm{H}]) \mathrm{C}([\mathrm{H}])=\mathrm{C}([\mathrm{H}]) \mathrm{C}=2[\mathrm{H}])=\mathrm{C}([\mathrm{H}]) \mathrm{C}=3 \mathrm{C} 4([\mathrm{H}])[\mathrm{H}]) \mathrm{N}([\mathrm{H}])[\mathrm{H}]) \mathrm{N}(\mathrm{C}([\mathrm{H}])([\mathrm{H}])[\mathrm{H}]) \mathrm{C} 5( \\
[\mathrm{H}]) \mathrm{C}([\mathrm{H}])([\mathrm{H}]) \mathrm{C}([\mathrm{H}])([\mathrm{H}]) \mathrm{C}([\mathrm{H}])([\mathrm{H}]) \mathrm{C}([\mathrm{H}])([\mathrm{H}]) \mathrm{C}([[\mathrm{H}])[\mathrm{H}]\end{array}$ & 2.801 & 2.889 \\
\hline Calib4 & $\begin{array}{l}\mathrm{O}=\mathrm{C}(\mathrm{O}[\mathrm{H}]) \mathrm{C}([\mathrm{H}])([\mathrm{H}]) \mathrm{C}([\mathrm{H}])([\mathrm{H}])[\mathrm{C} @ @]((\mathrm{H}])(\mathrm{C}(=\mathrm{O}) \mathrm{N}([\mathrm{H}])[\mathrm{C} @]([\mathrm{H}])(\mathrm{C}(=\mathrm{O}) \mathrm{N}([\mathrm{H}])[\mathrm{C} @]([ \\
\mathrm{H}])(\mathrm{C}(=\mathrm{O}) \mathrm{N}([\mathrm{H}])[\mathrm{C} @]([\mathrm{H}])([\mathrm{C} @ @]([\mathrm{H}])(\mathrm{O}[\mathrm{H}]) \mathrm{C}([\mathrm{H}])([\mathrm{H}])[\mathrm{C} @]([\mathrm{H}])(\mathrm{C}(=\mathrm{O}) \mathrm{N}([\mathrm{H}])[\mathrm{C} @]([\mathrm{H} \\
])(\mathrm{C}(=\mathrm{O}) \mathrm{N}([\mathrm{H}])[\mathrm{C} @]([\mathrm{H}])(\mathrm{C}(=\mathrm{O}) \mathrm{N}([\mathrm{H}])[\mathrm{C} @]([\mathrm{H}])(\mathrm{C}(=\mathrm{O}) \mathrm{O}[\mathrm{H}]) \mathrm{C}([\mathrm{H}])([\mathrm{H}]) \mathrm{C} 1=\mathrm{C}([\mathrm{H}]) \mathrm{C}([\mathrm{H}]) \\
=\mathrm{C}([\mathrm{H}]) \mathrm{C}([\mathrm{H}]\end{array}$ & 4.796 & 4.442 \\
\hline Calib5 & $\begin{array}{l}\mathrm{O}=\mathrm{C}(\mathrm{N}([\mathrm{H}])[\mathrm{C} @]([\mathrm{H}])([\mathrm{C} @ @]((\mathrm{H}])(\mathrm{O}[\mathrm{H}]) \mathrm{C}([\mathrm{H}])([\mathrm{H}])[\mathrm{C} @]([\mathrm{H}])(\mathrm{C}(=\mathrm{O}) \mathrm{N}([\mathrm{H}])[\mathrm{C} @])((\mathrm{H}])(\mathrm{C}( \\
=\mathrm{O}) \mathrm{N}([\mathrm{H}]) \mathrm{C}([\mathrm{H}])([\mathrm{H}]) \mathrm{Cl}=\mathrm{C}([\mathrm{H}]) \mathrm{C}([\mathrm{H}])=\mathrm{C}([\mathrm{H}]) \mathrm{C}([\mathrm{H}])=\mathrm{Cl}[\mathrm{H}]) \mathrm{C}([\mathrm{H}])(\mathrm{C}([\mathrm{H}])([\mathrm{H}])[\mathrm{H}]) \mathrm{C}([\mathrm{H}]) \\
([\mathrm{H}])[\mathrm{H}]) \mathrm{C}([\mathrm{H}])([\mathrm{H}])[\mathrm{H}]) \mathrm{C}([\mathrm{H}])([\mathrm{H}]) \mathrm{C}([\mathrm{H}])(\mathrm{C}([\mathrm{H}])([\mathrm{H}])[\mathrm{H}]) \mathrm{C}([\mathrm{H}])([\mathrm{H}])[\mathrm{H}])[\mathrm{C} @ @]([\mathrm{H}])(\mathrm{N}([ \\
\mathrm{H}]) \mathrm{C}\end{array}$ & 4.027 & 3.748 \\
\hline Calib6 & $\begin{array}{l}\mathrm{O}=\mathrm{C}(\mathrm{N}([\mathrm{H}])[\mathrm{C} @]([\mathrm{H}])([\mathrm{C} @ @]((\mathrm{H}])(\mathrm{O}[\mathrm{H}]) \mathrm{C}([\mathrm{H}])([\mathrm{H}])[\mathrm{C} @]([\mathrm{H}])(\mathrm{C}(=\mathrm{O}) \mathrm{N}([\mathrm{H}])[\mathrm{C} @](([\mathrm{H}])(\mathrm{C}( \\
=\mathrm{O}) \mathrm{N}([\mathrm{H}]) \mathrm{C}([\mathrm{H}])([\mathrm{H}]) \mathrm{Cl}=\mathrm{C}([\mathrm{H}]) \mathrm{C}([\mathrm{H}])=\mathrm{C}([\mathrm{H}]) \mathrm{C}([\mathrm{H}])=\mathrm{Cl}[\mathrm{H}]) \mathrm{C}([\mathrm{H}])(\mathrm{C}([\mathrm{H}])([\mathrm{H}])[\mathrm{H}]) \mathrm{C}([\mathrm{H}]) \\
([\mathrm{H}])[\mathrm{H}]) \mathrm{C}([\mathrm{H}])([\mathrm{H}]) \mathrm{C}([\mathrm{H}])(\mathrm{C}([\mathrm{H}])([\mathrm{H}])[\mathrm{H}]) \mathrm{C}([\mathrm{H}])([\mathrm{H}])[\mathrm{H}]) \mathrm{C}([\mathrm{H}])([\mathrm{H}]) \mathrm{C}([\mathrm{H}])(\mathrm{C}([\mathrm{H}])([\mathrm{H}])[\mathrm{H} \\
])\end{array}$ & 0.979 & 3.135 \\
\hline Calib7 & $\begin{array}{l}\mathrm{O}=\mathrm{C} 2 \mathrm{~N}(\mathrm{C}(=\mathrm{N}[\mathrm{C} @] 4(\mathrm{C}=1 \mathrm{C}([\mathrm{H}])=\mathrm{C}([\mathrm{H}]) \mathrm{C}([\mathrm{F}])=\mathrm{C}([\mathrm{H}]) \mathrm{C}=1[\mathrm{H}])[\mathrm{C} @ @] 2([\mathrm{H}]) \mathrm{C}([\mathrm{H}])([\mathrm{H}]) \mathrm{N}(/ \\
\mathrm{C} 3=\mathrm{N} / \mathrm{C}(\mathrm{OC}([\mathrm{H}])([\mathrm{H}])[\mathrm{H}])=\mathrm{C}([\mathrm{F}]) \mathrm{C}(=\mathrm{N} 3) \mathrm{OC}([\mathrm{H}])([\mathrm{H}])[\mathrm{H}]) \mathrm{C} 4([\mathrm{H}])[\mathrm{H}]) \mathrm{N}([\mathrm{H}])[\mathrm{H}]) \mathrm{C}([\mathrm{H}])([\mathrm{H} \\
])[\mathrm{H}]\end{array}$ & 4.357 & 3.896 \\
\hline Calib8 & $\begin{array}{l}\mathrm{O}=\mathrm{C} 2 \mathrm{~N}(\mathrm{C}(=\mathrm{N}[\mathrm{C} @] 4(\mathrm{C}=1 \mathrm{C}([\mathrm{F}])=\mathrm{C}([\mathrm{H}]) \mathrm{C}([\mathrm{F}])=\mathrm{C}([\mathrm{H}]) \mathrm{C}=1[\mathrm{~F}])[\mathrm{C} @ @] 2([\mathrm{H}]) \mathrm{C}([\mathrm{H}])([\mathrm{H}]) \mathrm{N}(/ \mathrm{C} \\
3=\mathrm{N} / \mathrm{C}(=\mathrm{C}(/[\mathrm{F}]) \mathrm{C}(=\mathrm{N} 3) \mathrm{OC}([\mathrm{H}])([\mathrm{H}])[\mathrm{H}]) \mathrm{C}([\mathrm{H}])([\mathrm{H}])[\mathrm{H}]) \mathrm{C} 4([\mathrm{H}])[\mathrm{H}]) \mathrm{N}([\mathrm{H}])[\mathrm{H}]) \mathrm{C}([\mathrm{H}])([\mathrm{H}])[ \\
\mathrm{H}]\end{array}$ & 3.824 & 4.010 \\
\hline Calib9 & $\begin{array}{l}\mathrm{O}=\mathrm{C} 2 \mathrm{~N}(\mathrm{C}(=\mathrm{N}[\mathrm{C} @] 4(/ \mathrm{C} 1=\mathrm{C}([\mathrm{H}]) \mathrm{C}([\mathrm{Cl}])=\mathrm{C}([\mathrm{H}]) \mathrm{C}([\mathrm{H}])=\mathrm{C} 1[\mathrm{H}])[\mathrm{C} @ @] 2([\mathrm{H}]) \mathrm{C}([\mathrm{H}])([\mathrm{H}]) \mathrm{N} / \\
\mathrm{C} 3=\mathrm{N} / \mathrm{C}(\mathrm{OC}([\mathrm{H}])([\mathrm{H}])[\mathrm{H}])=\mathrm{C}([\mathrm{F}]) \mathrm{C}(=\mathrm{N} 3) \mathrm{C}([\mathrm{H}])([\mathrm{H}])[\mathrm{H}]) \mathrm{C} 4([\mathrm{H}])[\mathrm{H}]) \mathrm{N}([\mathrm{H}])[\mathrm{H}]) \mathrm{C}([\mathrm{H}])([\mathrm{H}])[ \\
\mathrm{H}]\end{array}$ & 3.409 & 3.409 \\
\hline Calib10 & $\begin{array}{l}\mathrm{O}=\mathrm{C} 2 \mathrm{~N}(\mathrm{C}(=\mathrm{N}[\mathrm{C} @] 5(\mathrm{C}=1 \mathrm{C}([\mathrm{F}])=\mathrm{C}([\mathrm{H}]) \mathrm{C}([\mathrm{H}])=\mathrm{C}([\mathrm{F}]) \mathrm{C}=1[\mathrm{H}])[\mathrm{C} @ @] 2([\mathrm{H}]) \mathrm{C}([\mathrm{H}])([\mathrm{H}]) \mathrm{N}(/ \mathrm{C} \\
3=\mathrm{N} / \mathrm{C}(\mathrm{OC}([\mathrm{H}])([\mathrm{H}])[\mathrm{H}])=\mathrm{C}([\mathrm{Cl}]) \mathrm{C}(=\mathrm{N} 3)[\mathrm{C} @] 4([\mathrm{H}]) \mathrm{C}([\mathrm{H}])([\mathrm{H}]) \mathrm{C} 4([\mathrm{H}])[\mathrm{H}]) \mathrm{C} 5([\mathrm{H}])[\mathrm{H}]) \mathrm{N}([\end{array}$ & 4.187 & 4.166 \\
\hline
\end{tabular}




\begin{tabular}{|c|c|c|c|}
\hline & $\mathrm{H}])[\mathrm{H}]) \mathrm{C}([\mathrm{H}])([\mathrm{H}])[\mathrm{H}]$ & & \\
\hline Calib11 & $\begin{array}{l}\mathrm{O}=\mathrm{C} 2 \mathrm{~N}(\mathrm{C}(=\mathrm{N}[\mathrm{C} @] 5(\mathrm{C}=1 \mathrm{C}([\mathrm{H}])=\mathrm{C}(\mathrm{C \# N}) \mathrm{C}([\mathrm{H}])=\mathrm{C}([\mathrm{H}]) \mathrm{C}=1[\mathrm{H}])[\mathrm{C} @ @] 2([\mathrm{H}]) \mathrm{C}([\mathrm{H}])([\mathrm{H}]) \mathrm{N}( \\
/ \mathrm{C} 3=\mathrm{N} / \mathrm{C}(=\mathrm{C}(/[\mathrm{F}]) \mathrm{C}(=\mathrm{N} 3)[\mathrm{C} @ @] 4([\mathrm{H}]) \mathrm{C}([\mathrm{H}])([\mathrm{H}]) \mathrm{C} 4([\mathrm{H}])[\mathrm{H}]) \mathrm{C}([\mathrm{H}])([\mathrm{H}])[\mathrm{H}]) \mathrm{C} 5([\mathrm{H}])[\mathrm{H}]) \mathrm{N} \\
([\mathrm{H}])[\mathrm{H}]) \mathrm{C}([\mathrm{H}])([\mathrm{H}])[\mathrm{H}]\end{array}$ & 3.367 & 3.193 \\
\hline Calib12 & $\begin{array}{l}{[\mathrm{F}] \mathrm{C}=1 \mathrm{C}([\mathrm{H}])=\mathrm{C}([\mathrm{F}]) \mathrm{C}(=\mathrm{C}([\mathrm{H}]) \mathrm{C}=1[\mathrm{H}])[\mathrm{C} @ @] 24 \mathrm{~N}=\mathrm{C}(\mathrm{N}(\mathrm{C}(=\mathrm{O})[\mathrm{C} @] 2([\mathrm{H}]) \mathrm{C}([\mathrm{H}])([\mathrm{H}]) \mathrm{N}(/ \mathrm{C}} \\
3=\mathrm{N} / \mathrm{C}(=\mathrm{C}(/[\mathrm{F}]) \mathrm{C}(=\mathrm{N} 3) \mathrm{OC}([\mathrm{H}])([\mathrm{H}])[\mathrm{H}]) \mathrm{C}([\mathrm{H}])([\mathrm{H}]) \mathrm{O}[\mathrm{H}]) \mathrm{C} 4([\mathrm{H}])[\mathrm{H}]) \mathrm{C}([\mathrm{H}])([\mathrm{H}])[\mathrm{H}]) \mathrm{N}([\mathrm{H}]) \\
{[\mathrm{H}]}\end{array}$ & 3.796 & 3.715 \\
\hline Calib13 & $\begin{array}{l}\mathrm{O}=\mathrm{C} 2 \mathrm{~N}(\mathrm{C}(=\mathrm{N}[\mathrm{C} @] 4(\mathrm{C}=1 \mathrm{C}([\mathrm{F}])=\mathrm{C}([\mathrm{H}]) \mathrm{C}([\mathrm{F}])=\mathrm{C}([\mathrm{F}]) \mathrm{C}=1[\mathrm{H}])[\mathrm{C} @ @] 2([\mathrm{H}]) \mathrm{C}([\mathrm{H}])([\mathrm{H}]) \mathrm{N}(/ \mathrm{C} \\
3=\mathrm{N} / \mathrm{C}(\mathrm{OC}([\mathrm{H}])([\mathrm{H}])[\mathrm{H}])=\mathrm{C}([\mathrm{F}]) \mathrm{C}(=\mathrm{N} 3) \mathrm{C}([\mathrm{H}])([\mathrm{H}]) \mathrm{C}([\mathrm{H}])([\mathrm{H}])[\mathrm{H}]) \mathrm{C} 4([\mathrm{H}])[\mathrm{H}]) \mathrm{N}([\mathrm{H}])[\mathrm{H}]) \mathrm{C}( \\
[\mathrm{H}])([\mathrm{H}])[\mathrm{H}]\end{array}$ & 4.252 & 4.508 \\
\hline Calib14 & $\begin{array}{l}\mathrm{O}=\mathrm{C}(/ \mathrm{C} 1=\mathrm{N} / \mathrm{C}([\mathrm{H}])=\mathrm{C}([\mathrm{F}]) \mathrm{C}([\mathrm{H}])=\mathrm{C} 1[\mathrm{H}]) \mathrm{N}([\mathrm{H}]) \mathrm{C} 2=\mathrm{C}([\mathrm{H}]) \mathrm{C}(=\mathrm{C}([\mathrm{F}]) \mathrm{C}([\mathrm{H}])=\mathrm{C} 2[\mathrm{H}])[\mathrm{C} @] 3( \\
\mathrm{N}([\mathrm{H}]) \mathrm{C}(=\mathrm{N}[\mathrm{H}]) \mathrm{N}([\mathrm{S}](=\mathrm{O})(=\mathrm{O}) \mathrm{C} 3([\mathrm{H}])[\mathrm{H}]) \mathrm{C}([\mathrm{H}])([\mathrm{H}])[\mathrm{H}]) \mathrm{C}([\mathrm{H}])([\mathrm{H}])[\mathrm{H}]\end{array}$ & 4.312 & 4.112 \\
\hline Calib15 & $\begin{array}{l}\mathrm{O}=[\mathrm{S}] 3(=\mathrm{O}) \mathrm{N}(\mathrm{C}=2 \mathrm{C}([\mathrm{H}])=\mathrm{C}(\mathrm{C}([\mathrm{H}])=\mathrm{C} 1 / \mathrm{C}(=\mathrm{C}(/[\mathrm{H}]) \mathrm{N}(\mathrm{C} 1=2) \mathrm{C}([\mathrm{H}])([\mathrm{H}]) \mathrm{C} 3([\mathrm{H}])[\mathrm{H}]) \mathrm{C}([\mathrm{H}])([ \\
\mathrm{H}]) \mathrm{C}([\mathrm{H}])([\mathrm{H}])[\mathrm{H}]) \mathrm{C}(=\mathrm{O}) \mathrm{N}([\mathrm{H}])[\mathrm{C} @]([\mathrm{H}])(\mathrm{C}([\mathrm{H}])([\mathrm{H}]) \mathrm{N}([\mathrm{H}])[\mathrm{C} @]([\mathrm{H}])(\mathrm{C}(=\mathrm{O}) \mathrm{N}([\mathrm{H}]) \mathrm{C}([\mathrm{H}]) \\
([\mathrm{H}]) \mathrm{C}([\mathrm{H}])(\mathrm{C}([\mathrm{H}])([\mathrm{H}])[\mathrm{H}]) \mathrm{C}([\mathrm{H}])([\mathrm{H}])[\mathrm{H}])[\mathrm{C} @ @]([\mathrm{H}])(\mathrm{O}[\mathrm{H}]) \mathrm{C}([\mathrm{H}])([\mathrm{H}]) \mathrm{C}([\mathrm{H}])([\mathrm{H}]) \mathrm{C}([\mathrm{H}] \\
)([\mathrm{H}\end{array}$ & 5.398 & 4.572 \\
\hline Calib16 & $\begin{array}{l}\mathrm{O}=\mathrm{C}(\mathrm{N}([\mathrm{H}])[\mathrm{C} @ @]([\mathrm{H}])(\mathrm{C}=1 \mathrm{C}([\mathrm{H}])=\mathrm{C}([\mathrm{H}]) \mathrm{C}([\mathrm{H}])=\mathrm{C}([\mathrm{H}]) \mathrm{C}=1[\mathrm{H}]) \mathrm{C}([\mathrm{H}])([\mathrm{H}])[\mathrm{H}]) \mathrm{C} 2=\mathrm{C}([\mathrm{H} \\
]) \mathrm{C}(=\mathrm{C}([\mathrm{H}]) \mathrm{C}(=\mathrm{C} 2[\mathrm{H}]) \mathrm{N}(\mathrm{C}([\mathrm{H}])([\mathrm{H}])[\mathrm{H}])[\mathrm{S}](=\mathrm{O})(=\mathrm{O}) \mathrm{C}([\mathrm{H}])([\mathrm{H}])[\mathrm{H}]) \mathrm{C}(=\mathrm{O}) \mathrm{N}([\mathrm{H}])[\mathrm{C} @]([\mathrm{H}] \\
)([\mathrm{C} @]([\mathrm{H}])(\mathrm{O}[\mathrm{H}]) \mathrm{C}([\mathrm{H}])([\mathrm{H}]) \mathrm{N}([\mathrm{H}]) \mathrm{C}([\mathrm{H}])([\mathrm{H}]) \mathrm{C} 3=\mathrm{C}([\mathrm{H}]) \mathrm{C} 4=\mathrm{C}(\mathrm{C}([\mathrm{H}])=\mathrm{C} 3[\mathrm{H}]) \mathrm{C}([\mathrm{H}])=\mathrm{C}([ \\
\mathrm{H}]) \mathrm{N} 4[\mathrm{~S}](=\end{array}$ & 3.569 & 3.504 \\
\hline Calib17 & $\begin{array}{l}\mathrm{O}=\mathrm{C}(\mathrm{OC}([\mathrm{H}])([\mathrm{H}]) \mathrm{C}=1 \mathrm{~N}=\mathrm{C}(\mathrm{OC}=1 \mathrm{C}([\mathrm{H}])([\mathrm{H}])[\mathrm{H}]) \mathrm{C}([\mathrm{H}])([\mathrm{H}])[\mathrm{H}]) \mathrm{N}([\mathrm{H}])[\mathrm{C} @]([\mathrm{H}])(\mathrm{C}(=\mathrm{O}) \mathrm{N} \\
([\mathrm{H}])[\mathrm{C} @ @]([\mathrm{H}])(\mathrm{C}([\mathrm{H}])([\mathrm{H}]) \mathrm{C} 2=\mathrm{C}([\mathrm{H}]) \mathrm{C}([\mathrm{H}])=\mathrm{C}([\mathrm{H}]) \mathrm{C}([\mathrm{H}])=\mathrm{C} 2[\mathrm{H}])[\mathrm{C} @]([\mathrm{H}])(\mathrm{O}[\mathrm{H}]) \mathrm{C}([\mathrm{H} \\
])([\mathrm{H}]) \mathrm{N}([\mathrm{H}]) \mathrm{C}([\mathrm{H}])([\mathrm{H}]) \mathrm{C} 3=\mathrm{C}([\mathrm{H}]) \mathrm{C}(\mathrm{OC}([\mathrm{H}])([\mathrm{H}])[\mathrm{H}])=\mathrm{C}([\mathrm{H}]) \mathrm{C}([\mathrm{H}])=\mathrm{C} 3[\mathrm{H}]) \mathrm{C}([\mathrm{H}])([\mathrm{H}])[\mathrm{S} \\
](=\mathrm{O})(=\mathrm{O})\end{array}$ & 0.824 & 2.502 \\
\hline Calib18 & $\begin{array}{l}\mathrm{O}=\mathrm{C}(\mathrm{N}([\mathrm{H}])[\mathrm{C} @ @]([\mathrm{H}])(\mathrm{C}=1 \mathrm{C}([\mathrm{H}])=\mathrm{C}([\mathrm{H}]) \mathrm{C}([\mathrm{H}])=\mathrm{C}([\mathrm{H}]) \mathrm{C}=1[\mathrm{H}]) \mathrm{C}([\mathrm{H}])([\mathrm{H}])[\mathrm{H}]) \mathrm{C} 2=\mathrm{C}([\mathrm{H} \\
]) \mathrm{C}(=\mathrm{C}([\mathrm{H}]) \mathrm{C}(=\mathrm{C} 2[\mathrm{H}]) \mathrm{N}(\mathrm{C}([\mathrm{H}])([\mathrm{H}])[\mathrm{H}])[\mathrm{S}](=\mathrm{O})(=\mathrm{O}) \mathrm{C}([\mathrm{H}])([\mathrm{H}])[\mathrm{H}]) \mathrm{C}(=\mathrm{O}) \mathrm{N}([\mathrm{H}])[\mathrm{C} @]([\mathrm{H}] \\
)([\mathrm{C} @]([\mathrm{H}])(\mathrm{O}[\mathrm{H}]) \mathrm{C}([\mathrm{H}])([\mathrm{H}]) \mathrm{N}([\mathrm{H}]) \mathrm{C}([\mathrm{H}])([\mathrm{H}]) \mathrm{C} 3=\mathrm{C}([\mathrm{H}]) \mathrm{C}(\mathrm{OC}([\mathrm{H}])([\mathrm{H}])[\mathrm{H}])=\mathrm{C}([\mathrm{H}]) \mathrm{C}([\mathrm{H} \\
])=\mathrm{C} 3[\mathrm{H}])\end{array}$ & 2.038 & 2.033 \\
\hline Calib19 & $\begin{array}{l}\mathrm{O}=\mathrm{C}(\mathrm{OC}([\mathrm{H}])([\mathrm{H}]) \mathrm{C}=1 \mathrm{~N}=\mathrm{C}(\mathrm{OC}=1 \mathrm{C}([\mathrm{H}])([\mathrm{H}])[\mathrm{H}]) \mathrm{C}([\mathrm{H}])([\mathrm{H}])[\mathrm{H}]) \mathrm{N}([\mathrm{H}])[\mathrm{C} @]([\mathrm{H}])(\mathrm{C}(=\mathrm{O}) \mathrm{N} \\
([\mathrm{H}])[\mathrm{C} @]([\mathrm{H}])([\mathrm{C} @ @]([\mathrm{H}])(\mathrm{O}[\mathrm{H}]) \mathrm{C}([\mathrm{H}])([\mathrm{H}])[\mathrm{C} @([\mathrm{H}])(\mathrm{C}(=\mathrm{O}) \mathrm{N}([\mathrm{H}])[\mathrm{C} @]([\mathrm{H}])(\mathrm{C}(=\mathrm{O}) \mathrm{N}([ \\
\mathrm{H}]) \mathrm{C}(\mathrm{C}([\mathrm{H}])([\mathrm{H}])[\mathrm{H}])(\mathrm{C}([\mathrm{H}])([\mathrm{H}])[\mathrm{H}]) \mathrm{C}([\mathrm{H}])([\mathrm{H}])[\mathrm{H}]) \mathrm{C}([\mathrm{H}])(\mathrm{C}([\mathrm{H}])([\mathrm{H}])[\mathrm{H}]) \mathrm{C}([\mathrm{H}])([\mathrm{H}])[\mathrm{H}]) \\
\mathrm{C}([\mathrm{H}]\end{array}$ & 5.921 & 4.006 \\
\hline Calib20 & $\begin{array}{l}\mathrm{O}=\mathrm{C}(\mathrm{N}([\mathrm{H}]) \mathrm{C}(=\mathrm{NC}([\mathrm{H}])([\mathrm{H}]) \mathrm{C} 1=\mathrm{C}([\mathrm{H}]) \mathrm{C}([\mathrm{Cl}])=\mathrm{C}([\mathrm{H}]) \mathrm{C}([\mathrm{Cl}])=\mathrm{C} 1[\mathrm{H}]) \mathrm{N}([\mathrm{H}])[\mathrm{H}]) \mathrm{C} 2=\mathrm{C}(\mathrm{N}(/ \\
\mathrm{N}=\mathrm{C} 2 / \mathrm{C}([\mathrm{H}])([\mathrm{H}])[\mathrm{H}]) \mathrm{C}([\mathrm{H}])([\mathrm{H}])[\mathrm{H}]) \mathrm{C} 3=\mathrm{C}([\mathrm{H}]) \mathrm{C}([\mathrm{H}])=\mathrm{C}(\mathrm{OC}([\mathrm{H}])([\mathrm{H}])[\mathrm{H}]) \mathrm{C}([\mathrm{H}])=\mathrm{C} 3[\mathrm{H}]\end{array}$ & 1.398 & 1.656 \\
\hline Calib21 & $\begin{array}{l}\mathrm{O}=\mathrm{C} 2 \mathrm{~N}([\mathrm{H}]) \mathrm{C} 1=\mathrm{C}([\mathrm{H}]) \mathrm{C}([\mathrm{H}])=\mathrm{C}([\mathrm{H}]) \mathrm{C}([\mathrm{H}])=\mathrm{C} 1 \mathrm{~N}([\mathrm{H}]) \mathrm{C}=3 / \mathrm{C} 2=\mathrm{C}(/[\mathrm{H}]) \mathrm{C}([\mathrm{H}])=\mathrm{C}(\mathrm{C}=3[\mathrm{H}]) \mathrm{C} \\
(=\mathrm{O}) \mathrm{N}([\mathrm{H}]) \mathrm{C} 4=\mathrm{C}([\mathrm{H}]) \mathrm{C}([\mathrm{H}])=\mathrm{NC}([\mathrm{H}])=\mathrm{C} 4[\mathrm{H}]\end{array}$ & 2.530 & 2.691 \\
\hline Calib22 & $\begin{array}{l}\mathrm{O}=\mathrm{C} 2 \mathrm{~N}([\mathrm{H}]) \mathrm{C}=1 \mathrm{C}([\mathrm{H}])=\mathrm{C}([\mathrm{H}]) \mathrm{C}([\mathrm{H}])=\mathrm{C}([\mathrm{H}]) \mathrm{C}=1 \mathrm{~N}([\mathrm{H}]) \mathrm{C} 3=\mathrm{C} 2 \mathrm{C}([\mathrm{H}])=\mathrm{C}([\mathrm{H}]) \mathrm{C}(=\mathrm{C} 3[\mathrm{H}]) \mathrm{C}( \\
=\mathrm{O}) \mathrm{N}([\mathrm{H}]) \mathrm{C} 4=\mathrm{N} / \mathrm{C}(=\mathrm{C}(/[\mathrm{H}]) \mathrm{N} 4[\mathrm{H}]) \mathrm{C} 5=\mathrm{C}([\mathrm{H}]) \mathrm{C}([\mathrm{H}])=\mathrm{C}([\mathrm{F}]) \mathrm{C}([\mathrm{H}])=\mathrm{C} 5[\mathrm{H}]\end{array}$ & 2.533 & 2.654 \\
\hline Calib23 & $\begin{array}{l}\mathrm{O}=\mathrm{C}(\mathrm{N}([\mathrm{H}]) \mathrm{C}(=\mathrm{NC}([\mathrm{H}])([\mathrm{H}]) \mathrm{C} 1=\mathrm{C}([\mathrm{H}]) \mathrm{C}(=\mathrm{C}([\mathrm{H}]) \mathrm{C}([\mathrm{Cl}])=\mathrm{C} 1[\mathrm{H}]) \mathrm{C} 2=\mathrm{C}([\mathrm{H}]) \mathrm{N}=\mathrm{C}([\mathrm{H}]) \mathrm{C}([\mathrm{H}] \\
)=\mathrm{C} 2[\mathrm{H}]) \mathrm{N}([\mathrm{H}])[\mathrm{H}]) \mathrm{C}=3 \mathrm{C}(=\mathrm{NOC}=3 \mathrm{C}([\mathrm{H}])([\mathrm{H}])[\mathrm{H}]) \mathrm{C} 4=\mathrm{C}([\mathrm{H}]) \mathrm{C}([\mathrm{H}])=\mathrm{C}(\mathrm{OC}([\mathrm{H}])([\mathrm{H}])[\mathrm{H}]) \mathrm{C}( \\
[\mathrm{H}])=\mathrm{C} 4[\mathrm{H}]\end{array}$ & 1.745 & 1.988 \\
\hline Calib24 & $\begin{array}{l}\mathrm{O}=\mathrm{C}(\mathrm{N}([\mathrm{H}]) \mathrm{C}(=\mathrm{NC}([\mathrm{H}])([\mathrm{H}]) \mathrm{C} 1=\mathrm{C}([\mathrm{H}]) \mathrm{C}(=\mathrm{C}([\mathrm{H}]) \mathrm{C}([\mathrm{Cl}])=\mathrm{C} 1[\mathrm{H}]) \mathrm{C}=2 \mathrm{C}([\mathrm{H}])=\mathrm{C}([\mathrm{H}])[\mathrm{S}] \mathrm{C}=2 \\
[\mathrm{H}]) \mathrm{N}([\mathrm{H}])[\mathrm{H}]) \mathrm{C}=3 \mathrm{C}(=\mathrm{NOC}=3 \mathrm{C}([\mathrm{H}])([\mathrm{H}])[\mathrm{H}]) \mathrm{C} 4=\mathrm{C}([\mathrm{H}]) \mathrm{C}([\mathrm{H}])=\mathrm{C}(\mathrm{OC}([\mathrm{H}])([\mathrm{H}])[\mathrm{H}]) \mathrm{C}([\mathrm{H}]) \\
=\mathrm{C} 4[\mathrm{H}]\end{array}$ & 1.921 & 2.654 \\
\hline
\end{tabular}




\begin{tabular}{|c|c|c|c|}
\hline Calib25 & $\begin{array}{l}\mathrm{O}=\mathrm{C}(\mathrm{N}([\mathrm{H}]) \mathrm{C}(=\mathrm{NC}([\mathrm{H}])([\mathrm{H}]) \mathrm{C} 1=\mathrm{C}([\mathrm{H}]) \mathrm{C}(=\mathrm{C}([\mathrm{H}]) \mathrm{C}([\mathrm{Cl}])=\mathrm{C} 1[\mathrm{H}]) \mathrm{C}([\mathrm{H}])=\mathrm{C}([\mathrm{H}])[\mathrm{H}]) \mathrm{N}([\mathrm{H}])[ \\
\mathrm{H}]) \mathrm{C}=2 \mathrm{C}(=\mathrm{NOC}=2 \mathrm{C}([\mathrm{H}])([\mathrm{H}])[\mathrm{H}]) \mathrm{C} 3=\mathrm{C}([\mathrm{H}]) \mathrm{C}([\mathrm{H}])=\mathrm{C}(\mathrm{OC}([\mathrm{H}])([\mathrm{H}])[\mathrm{H}]) \mathrm{C}([\mathrm{H}])=\mathrm{C} 3[\mathrm{H}]\end{array}$ & 2.367 & 2.222 \\
\hline Calib26 & $\begin{array}{l}\mathrm{O}=\mathrm{C}(\mathrm{N}([\mathrm{H}]) \mathrm{C}(=\mathrm{NC}([\mathrm{H}])([\mathrm{H}]) \mathrm{C} 1=\mathrm{C}([\mathrm{H}]) \mathrm{C}([\mathrm{Cl}])=\mathrm{C}(\mathrm{O}[\mathrm{H}]) \mathrm{C}([\mathrm{Cl}])=\mathrm{C} 1[\mathrm{H}]) \mathrm{N}([\mathrm{H}])[\mathrm{H}]) \mathrm{C}=2 \mathrm{C}(= \\
\mathrm{NOC}=2 \mathrm{C}([\mathrm{H}])([\mathrm{H}])[\mathrm{H}]) \mathrm{C} 3=\mathrm{C}([\mathrm{H}]) \mathrm{C}([\mathrm{H}])=\mathrm{C}(\mathrm{OC}([\mathrm{H}])([\mathrm{H}])[\mathrm{H}]) \mathrm{C}([\mathrm{H}])=\mathrm{C} 3[\mathrm{H}]\end{array}$ & 2.319 & 2.575 \\
\hline Calib27 & $\begin{array}{l}\mathrm{O}=\mathrm{C}(\mathrm{N}([\mathrm{H}]) \mathrm{C}(=\mathrm{NC}([\mathrm{H}])([\mathrm{H}]) \mathrm{C} 1=\mathrm{C}([\mathrm{H}]) \mathrm{C}([\mathrm{Cl}])=\mathrm{C}([\mathrm{H}]) \mathrm{C}([\mathrm{Cl}])=\mathrm{C} 1[\mathrm{H}]) \mathrm{N}([\mathrm{H}])[\mathrm{H}]) \mathrm{C}=2 \mathrm{C}(=\mathrm{N} \\
\mathrm{OC}=2 \mathrm{C}([\mathrm{H}])([\mathrm{H}])[\mathrm{H}]) \mathrm{C} 3=\mathrm{C}([\mathrm{H}]) \mathrm{C}([\mathrm{H}])=\mathrm{C}([\mathrm{H}]) \mathrm{C}([\mathrm{H}])=\mathrm{C} 3[\mathrm{H}]\end{array}$ & 1.409 & 2.245 \\
\hline Calib28 & $\begin{array}{l}\mathrm{O}=\mathrm{C}(\mathrm{N}([\mathrm{H}]) \mathrm{C}(=\mathrm{NC}([\mathrm{H}])([\mathrm{H}]) \mathrm{C} 1=\mathrm{C}([\mathrm{H}]) \mathrm{C}([\mathrm{Br}])=\mathrm{C}([\mathrm{H}]) \mathrm{C}([\mathrm{Cl}])=\mathrm{C} 1[\mathrm{H}]) \mathrm{N}([\mathrm{H}])[\mathrm{H}]) \mathrm{C}=2 \mathrm{C}(=\mathrm{N} \\
\mathrm{OC}=2 \mathrm{C}([\mathrm{H}])([\mathrm{H}])[\mathrm{H}]) \mathrm{C} 3=\mathrm{C}([\mathrm{H}]) \mathrm{C}([\mathrm{H}])=\mathrm{C}(\mathrm{OC}([\mathrm{H}])([\mathrm{H}])[\mathrm{H}]) \mathrm{C}([\mathrm{H}])=\mathrm{C} 3[\mathrm{H}]\end{array}$ & 2.569 & 2.071 \\
\hline Calib29 & $\begin{array}{l}\mathrm{O}=\mathrm{C}(\mathrm{N}([\mathrm{H}]) \mathrm{C}(=\mathrm{NC}([\mathrm{H}])([\mathrm{H}]) \mathrm{C} 1=\mathrm{C}([\mathrm{H}]) \mathrm{C}([\mathrm{Cl}])=\mathrm{C}(\mathrm{C}([\mathrm{Cl}])=\mathrm{C} 1[\mathrm{H}]) \mathrm{N}([\mathrm{H}]) \mathrm{C}(=\mathrm{O}) \mathrm{C}([\mathrm{H}])([\mathrm{H}]) \\
\mathrm{N}([\mathrm{H}]) \mathrm{C}([\mathrm{H}])([\mathrm{H}]) \mathrm{C}([\mathrm{H}])([\mathrm{H}])[\mathrm{H}]) \mathrm{N}([\mathrm{H}])[\mathrm{H}]) \mathrm{C}=2 \mathrm{C}(=\mathrm{N}[\mathrm{S}] \mathrm{C}=2 \mathrm{C}([\mathrm{H}])([\mathrm{H}])[\mathrm{H}]) \mathrm{C} 3=\mathrm{C}([\mathrm{H}]) \mathrm{C}([ \\
\mathrm{H}])=\mathrm{C}(\mathrm{OC}([\mathrm{H}])([\mathrm{H}])[\mathrm{H}]) \mathrm{C}([\mathrm{H}])=\mathrm{C} 3[\mathrm{H}]\end{array}$ & 4.000 & 3.685 \\
\hline Calib30 & $\begin{array}{l}{[\mathrm{H}] \mathrm{C}=2 \mathrm{C}(\mathrm{O}[\mathrm{H}])=\mathrm{C}([\mathrm{H}]) \mathrm{C}=1 \mathrm{OC}=3 \mathrm{C}(\mathrm{OC}=1 \mathrm{C}=2 \mathrm{O}[\mathrm{H}])=\mathrm{C}([\mathrm{H}]) \mathrm{C}(\mathrm{O}[\mathrm{H}])=\mathrm{C} 4 \mathrm{OC} 5=\mathrm{C}(\mathrm{OC}=34) \mathrm{C}(} \\
\mathrm{O}[\mathrm{H}])=\mathrm{C}([\mathrm{H}]) \mathrm{C}(\mathrm{O}[\mathrm{H}])=\mathrm{C} 5[\mathrm{H}]\end{array}$ & 1.097 & 1.557 \\
\hline Calib31 & $\begin{array}{l}{[\mathrm{F}] \mathrm{C}=1 \mathrm{C}([\mathrm{H}])=\mathrm{C}(\mathrm{C}([\mathrm{H}])=\mathrm{C}([\mathrm{F}]) \mathrm{C}=1[\mathrm{H}]) \mathrm{C}([\mathrm{H}])([\mathrm{H}])[\mathrm{C} @([\mathrm{H}])(\mathrm{N}([\mathrm{H}]) \mathrm{C}(=\mathrm{O}) \mathrm{C} 2=\mathrm{C}([\mathrm{H}]) \mathrm{C}(=\mathrm{C}} \\
([\mathrm{H}]) \mathrm{C}(=\mathrm{C} 2[\mathrm{H}]) \mathrm{C}([\mathrm{H}])([\mathrm{H}])[\mathrm{H}]) \mathrm{C}(=\mathrm{O}) \mathrm{N}(\mathrm{C}([\mathrm{H}])([\mathrm{H}]) \mathrm{C}([\mathrm{H}])([\mathrm{H}]) \mathrm{C}([\mathrm{H}])([\mathrm{H}])[\mathrm{H}]) \mathrm{C}([\mathrm{H}])([\mathrm{H}]) \mathrm{C} \\
([\mathrm{H}])([\mathrm{H}]) \mathrm{C}([\mathrm{H}])([\mathrm{H}])[\mathrm{H}])[\mathrm{C} @]([\mathrm{H}])(\mathrm{O}[\mathrm{H}])[\mathrm{C} @] 3([\mathrm{H}]) \mathrm{N}([\mathrm{H}]) \mathrm{C}([\mathrm{H}])([\mathrm{H}]) \mathrm{C}([\mathrm{H}])([\mathrm{H}]) \mathrm{N}(\mathrm{C} 3([ \\
\mathrm{H}])[\end{array}$ & 5.000 & 2.945 \\
\hline Calib32 & $\begin{array}{l}{[\mathrm{F}] \mathrm{C}=1 \mathrm{C}([\mathrm{H}])=\mathrm{C}(\mathrm{C}([\mathrm{H}])=\mathrm{C}([\mathrm{F}]) \mathrm{C}=1[\mathrm{H}]) \mathrm{C}([\mathrm{H}])([\mathrm{H}])[\mathrm{C} @]([\mathrm{H}])(\mathrm{N}([\mathrm{H}]) \mathrm{C}(=\mathrm{O}) \mathrm{C} 2=\mathrm{C}([\mathrm{H}]) \mathrm{C}(=\mathrm{C}} \\
([\mathrm{H}]) \mathrm{C}(=\mathrm{C} 2[\mathrm{H}]) \mathrm{C}([\mathrm{H}])([\mathrm{H}])[\mathrm{H}]) \mathrm{C}(=\mathrm{O}) \mathrm{N}(\mathrm{C}([\mathrm{H}])([\mathrm{H}]) \mathrm{C}([\mathrm{H}])([\mathrm{H}]) \mathrm{C}([\mathrm{H}])([\mathrm{H}])[\mathrm{H}]) \mathrm{C}([\mathrm{H}])([\mathrm{H}]) \mathrm{C} \\
([\mathrm{H}])([\mathrm{H}]) \mathrm{C}([\mathrm{H}])([\mathrm{H}])[\mathrm{H}])[\mathrm{C} @]([\mathrm{H}])(\mathrm{O}[\mathrm{H}])[\mathrm{C} @] 3([\mathrm{H}]) \mathrm{N}([\mathrm{H}]) \mathrm{C}([\mathrm{H}])([\mathrm{H}]) \mathrm{C}([\mathrm{H}])([\mathrm{H}]) \mathrm{N}(\mathrm{C} 3([ \\
\mathrm{H}])[\end{array}$ & 4.301 & 3.353 \\
\hline Calib33 & $\begin{array}{l}\mathrm{O}=\mathrm{C} 3 \mathrm{~N}(\mathrm{C}([\mathrm{H}])([\mathrm{H}]) \mathrm{C}([\mathrm{H}])([\mathrm{H}]) \mathrm{C}=5 \mathrm{C}([\mathrm{H}])=\mathrm{C}(\mathrm{OC}=2 \mathrm{C}(\mathrm{OC}([\mathrm{H}])([\mathrm{H}])[\mathrm{H}])=\mathrm{C}([\mathrm{H}]) \mathrm{C}=1 \mathrm{~N}=\mathrm{C}(\mathrm{N}( \\
\mathrm{C}([\mathrm{H}])([\mathrm{H}]) \mathrm{C}=1 \mathrm{C}=2[\mathrm{H}])[\mathrm{C} @ @]([\mathrm{H}])(\mathrm{C}([\mathrm{H}])([\mathrm{H}]) \mathrm{C} 3([\mathrm{H}])[\mathrm{H}])[\mathrm{C} @ @] 4([\mathrm{H}]) \mathrm{C}([\mathrm{H}])([\mathrm{H}]) \mathrm{C}([\mathrm{H}] \\
)([\mathrm{H}]) \mathrm{C}([\mathrm{H}])([\mathrm{H}]) \mathrm{C}([\mathrm{H}])([\mathrm{H}]) \mathrm{C} 4([\mathrm{H}])[\mathrm{H}]) \mathrm{N}([\mathrm{H}])[\mathrm{H}]) \mathrm{C}([\mathrm{H}])=\mathrm{C}([\mathrm{H}]) \mathrm{C}=5[\mathrm{H}])[\mathrm{C} @] 6([\mathrm{H}]) \mathrm{C}([\mathrm{H}] \\
)([\mathrm{H}]) \mathrm{C}([\mathrm{H}\end{array}$ & 3.921 & 3.715 \\
\hline Calib34 & $\begin{array}{l}\mathrm{O}=\mathrm{C}(\mathrm{N}([\mathrm{H}])[\mathrm{C} @ @]([\mathrm{H}])(\mathrm{C} 1=\mathrm{C}([\mathrm{H}]) \mathrm{C}([\mathrm{H}])=\mathrm{C}([\mathrm{H}]) \mathrm{C}([\mathrm{H}])=\mathrm{C} 1[\mathrm{H}]) \mathrm{C}([\mathrm{H}])([\mathrm{H}])[\mathrm{H}]) \mathrm{C} 2=\mathrm{C}([\mathrm{H}]) \\
\mathrm{C}(=\mathrm{C}([\mathrm{H}]) \mathrm{C}(=\mathrm{C} 2[\mathrm{H}]) \mathrm{N}(\mathrm{C}([\mathrm{H}])([\mathrm{H}])[\mathrm{H}])[\mathrm{S}](=\mathrm{O})(=\mathrm{O}) \mathrm{C}([\mathrm{H}])([\mathrm{H}])[\mathrm{H}]) \mathrm{C}(=\mathrm{O}) \mathrm{N}([\mathrm{H}])[\mathrm{C} @]([\mathrm{H}])( \\
{[\mathrm{C} @]([\mathrm{H}])(\mathrm{O}[\mathrm{H}]) \mathrm{C}([\mathrm{H}])([\mathrm{H}])[\mathrm{C} @ @]([\mathrm{H}])(\mathrm{OC}([\mathrm{H}])([\mathrm{H}])[\mathrm{H}]) \mathrm{C}(=\mathrm{O}) \mathrm{N}([\mathrm{H}])[\mathrm{C} @]([\mathrm{H}])(\mathrm{C}(=\mathrm{O}) \mathrm{N}} \\
([\mathrm{H}]) \mathrm{C}([\mathrm{H}])\end{array}$ & 2.678 & 3.293 \\
\hline Calib35 & $\begin{array}{l}\mathrm{O}=\mathrm{C}(\mathrm{N}([\mathrm{H}])[\mathrm{C} @ @]([\mathrm{H}])(\mathrm{C} 1=\mathrm{C}([\mathrm{H}]) \mathrm{C}([\mathrm{H}])=\mathrm{C}([\mathrm{H}]) \mathrm{C}([\mathrm{H}])=\mathrm{C} 1[\mathrm{H}]) \mathrm{C}([\mathrm{H}])([\mathrm{H}])[\mathrm{H}]) \mathrm{C} 2=\mathrm{C}([\mathrm{H}]) \\
\mathrm{C}(=\mathrm{C}([\mathrm{H}]) \mathrm{C}(=\mathrm{C} 2[\mathrm{H}]) \mathrm{N}(\mathrm{C}([\mathrm{H}])([\mathrm{H}])[\mathrm{H}])[\mathrm{S}](=\mathrm{O})(=\mathrm{O}) \mathrm{C}([\mathrm{H}])([\mathrm{H}])[\mathrm{H}]) \mathrm{C}(=\mathrm{O}) \mathrm{N}([\mathrm{H}])[\mathrm{C} @]([\mathrm{H}])( \\
{[\mathrm{C} @ @]([\mathrm{H}])(\mathrm{O}[\mathrm{H}]) \mathrm{C}([\mathrm{H}])([\mathrm{H}])[\mathrm{C} @ @]([\mathrm{H}])(\mathrm{OC}([\mathrm{H}])([\mathrm{H}]) \mathrm{C}([\mathrm{H}])=\mathrm{C}([\mathrm{H}])[\mathrm{H}]) \mathrm{C}(=\mathrm{O}) \mathrm{N}([\mathrm{H}])[\mathrm{C}} \\
@]([\mathrm{H}])(\mathrm{C}(=\end{array}$ & 4.481 & 3.396 \\
\hline Calib36 & $\begin{array}{l}\mathrm{O}=\mathrm{C} 1 \mathrm{C}([\mathrm{H}])=\mathrm{C}(\mathrm{OC}=2 / \mathrm{C} 1=\mathrm{C}(/ \mathrm{O}[\mathrm{H}]) \mathrm{C}([\mathrm{H}])=\mathrm{C}(\mathrm{O}[\mathrm{H}]) \mathrm{C}=2[\mathrm{H}]) \mathrm{C} 3=\mathrm{C}(\mathrm{O}[\mathrm{H}]) \mathrm{C}([\mathrm{H}])=\mathrm{C}(\mathrm{O}[\mathrm{H}]) \mathrm{C}( \\
[\mathrm{H}])=\mathrm{C} 3[\mathrm{H}]\end{array}$ & 0.083 & 0.404 \\
\hline Calib37 & $\begin{array}{l}\mathrm{O}=\mathrm{C} 3 \mathrm{~N}([\mathrm{H}])[\mathrm{C} @]([\mathrm{H}])(\mathrm{C}(=\mathrm{O}) \mathrm{N}([\mathrm{H}])[\mathrm{C} @]([\mathrm{H}])([\mathrm{C} @]([\mathrm{H}])(\mathrm{O}[\mathrm{H}]) \mathrm{C}([\mathrm{H}])([\mathrm{H}]) \mathrm{N}([\mathrm{H}]) \mathrm{C}([\mathrm{H}])([ \\
\mathrm{H}]) \mathrm{C} 1=\mathrm{C}([\mathrm{H}]) \mathrm{C}(=\mathrm{C}([\mathrm{H}]) \mathrm{C}([\mathrm{H}])=\mathrm{C} 1[\mathrm{H}]) \mathrm{C}(\mathrm{C}([\mathrm{H}])([\mathrm{H}])[\mathrm{H}])(\mathrm{C}([\mathrm{H}])([\mathrm{H}])[\mathrm{H}]) \mathrm{C}([\mathrm{H}])([\mathrm{H}])[\mathrm{H}]) \mathrm{C}( \\
[\mathrm{H}])([\mathrm{H}]) \mathrm{C} 2=\mathrm{C}([\mathrm{H}]) \mathrm{C}([\mathrm{F}])=\mathrm{C}([\mathrm{H}]) \mathrm{C}([\mathrm{F}])=\mathrm{C} 2[\mathrm{H}]) \mathrm{C}([\mathrm{H}])([\mathrm{H}]) \mathrm{N} 3 \mathrm{C}([\mathrm{H}])([\mathrm{H}]) \mathrm{C} 4=\mathrm{C}([\mathrm{H}]) \mathrm{C}([\mathrm{F}]) \\
=\mathrm{C}([\mathrm{H}\end{array}$ & 4.699 & 3.156 \\
\hline Calib38 & $\begin{array}{l}\mathrm{O}=\mathrm{C} 3 \mathrm{~N}(\mathrm{C}([\mathrm{H}])([\mathrm{H}])[\mathrm{C} @ @]([\mathrm{H}])(\mathrm{C}(=\mathrm{O}) \mathrm{N}([\mathrm{H}])[\mathrm{C} @]([\mathrm{H}])([\mathrm{C} @]([\mathrm{H}])(\mathrm{O}[\mathrm{H}]) \mathrm{C}([\mathrm{H}])([\mathrm{H}]) \mathrm{N}([\mathrm{H}] \\
) \mathrm{C}([\mathrm{H}])([\mathrm{H}]) \mathrm{C} 1=\mathrm{C}([\mathrm{H}]) \mathrm{C}(=\mathrm{C}([\mathrm{H}]) \mathrm{C}([\mathrm{H}])=\mathrm{C} 1[\mathrm{H}]) \mathrm{N}(\mathrm{C}([\mathrm{H}])([\mathrm{H}])[\mathrm{H}]) \mathrm{C}([\mathrm{H}])([\mathrm{H}])[\mathrm{H}]) \mathrm{C}([\mathrm{H}])([\mathrm{H} \\
]) \mathrm{C} 2=\mathrm{C}([\mathrm{H}]) \mathrm{C}([\mathrm{H}])=\mathrm{C}([\mathrm{H}]) \mathrm{C}([\mathrm{H}])=\mathrm{C} 2[\mathrm{H}]) \mathrm{C} 3([\mathrm{H}])[\mathrm{H}]) \mathrm{C}([\mathrm{H}])([\mathrm{H}]) \mathrm{C} 4=\mathrm{C}([\mathrm{H}]) \mathrm{C}([\mathrm{H}])=\mathrm{C}([\mathrm{H}]) \mathrm{C} \\
([\mathrm{H}])=\mathrm{C}\end{array}$ & 1.659 & 1.872 \\
\hline Calib39 & $\begin{array}{l}\mathrm{O}=\mathrm{C} 3 \mathrm{~N}([\mathrm{H}])[\mathrm{C} @]([\mathrm{H}])(\mathrm{C}(=\mathrm{O}) \mathrm{N}([\mathrm{H}])[\mathrm{C} @([\mathrm{H}])([\mathrm{C} @]([\mathrm{H}])(\mathrm{O}[\mathrm{H}]) \mathrm{C}([\mathrm{H}])([\mathrm{H}]) \mathrm{N}([\mathrm{H}]) \mathrm{C}([\mathrm{H}])([ \\
\mathrm{H}]) \mathrm{C} 1=\mathrm{C}([\mathrm{H}]) \mathrm{C}(=\mathrm{C}([\mathrm{H}]) \mathrm{C}([\mathrm{H}])=\mathrm{C} 1[\mathrm{H}]) \mathrm{N}(\mathrm{C}([\mathrm{H}])([\mathrm{H}])[\mathrm{H}]) \mathrm{C}([\mathrm{H}])([\mathrm{H}])[\mathrm{H}]) \mathrm{C}([\mathrm{H}])([\mathrm{H}]) \mathrm{C} 2=\mathrm{C}([ \\
\mathrm{H}]) \mathrm{C}([\mathrm{H}])=\mathrm{C}([\mathrm{H}]) \mathrm{C}([\mathrm{H}])=\mathrm{C} 2[\mathrm{H}]) \mathrm{C}([\mathrm{H}])([\mathrm{H}]) \mathrm{N} 3 \mathrm{C}([\mathrm{H}])([\mathrm{H}]) \mathrm{C}([\mathrm{H}])([\mathrm{H}]) \mathrm{C} 4=\mathrm{C}([\mathrm{H}]) \mathrm{C}([\mathrm{H}])=\mathrm{C}([\end{array}$ & 1.167 & 1.871 \\
\hline
\end{tabular}




\begin{tabular}{|c|c|c|c|}
\hline & $\mathrm{H}]) \mathrm{C}([$ & & \\
\hline Calib40 & $\begin{array}{l}\mathrm{O}=\mathrm{C} 1 \mathrm{~N}(\mathrm{C}(=\mathrm{N}[\mathrm{C} @] 1(\mathrm{C}=2 \mathrm{C}([\mathrm{H}])=\mathrm{C}([\mathrm{H}]) \mathrm{C}([\mathrm{H}])=\mathrm{C}([\mathrm{H}]) \mathrm{C}=2[\mathrm{H}]) \mathrm{C} 3=\mathrm{C}([\mathrm{H}]) \mathrm{C}(=\mathrm{C}([\mathrm{H}]) \mathrm{C}([\mathrm{H}])= \\
\mathrm{C} 3[\mathrm{H}]) \mathrm{C} 4=\mathrm{C}([\mathrm{H}]) \mathrm{C}([\mathrm{H}])=\mathrm{NC}([\mathrm{H}])=\mathrm{C} 4[\mathrm{H}]) \mathrm{N}([\mathrm{H}])[\mathrm{H}]) \mathrm{C}([\mathrm{H}])([\mathrm{H}])[\mathrm{H}]\end{array}$ & 1.420 & 2.360 \\
\hline Calib41 & $\begin{array}{l}\mathrm{O}=\mathrm{C} 1 \mathrm{~N}(\mathrm{C}(=\mathrm{N}[\mathrm{C} @ @] 1(\mathrm{C}=2 \mathrm{C}([\mathrm{H}])=\mathrm{C}([\mathrm{H}]) \mathrm{C}([\mathrm{H}])=\mathrm{C}([\mathrm{H}]) \mathrm{C}=2[\mathrm{H}]) \mathrm{C} 3=\mathrm{C}([\mathrm{H}]) \mathrm{C}(=\mathrm{C}([\mathrm{H}]) \mathrm{C}([\mathrm{H}] \\
)=\mathrm{C} 3[\mathrm{H}]) \mathrm{C} 4=\mathrm{C}([\mathrm{H}]) \mathrm{N}=\mathrm{C}([\mathrm{H}]) \mathrm{C}(=\mathrm{C} 4[\mathrm{H}]) \mathrm{C}([\mathrm{H}])([\mathrm{H}])[\mathrm{H}]) \mathrm{N}([\mathrm{H}])[\mathrm{H}]) \mathrm{C}([\mathrm{H}])([\mathrm{H}])[\mathrm{H}]\end{array}$ & 2.367 & 2.522 \\
\hline Calib42 & $\begin{array}{l}\mathrm{O}=\mathrm{C} 1 \mathrm{~N}(\mathrm{C}(=\mathrm{N}[\mathrm{C} @ @] 1(/ \mathrm{C} 2=\mathrm{C}(\backslash[\mathrm{H}]) \mathrm{C}([\mathrm{H}])=\mathrm{C}([\mathrm{H}]) \mathrm{C}([\mathrm{H}])=\mathrm{C} 2[\mathrm{H}]) \mathrm{C} 3=\mathrm{C}([\mathrm{H}]) \mathrm{C}(=\mathrm{C}([\mathrm{H}]) \mathrm{C}([\mathrm{H}] \\
)=\mathrm{C} 3[\mathrm{H}]) \mathrm{C} 4=\mathrm{C}([\mathrm{H}]) \mathrm{N}=\mathrm{C}([\mathrm{H}]) \mathrm{C}(\mathrm{CHN})=\mathrm{C} 4[\mathrm{H}]) \mathrm{N}([\mathrm{H}])[\mathrm{H}]) \mathrm{C}([\mathrm{H}])([\mathrm{H}])[\mathrm{H}]\end{array}$ & 2.328 & 2.636 \\
\hline Calib43 & $\begin{array}{l}\mathrm{O}=\mathrm{C}(\mathrm{N}([\mathrm{C} @] 1([\mathrm{H}]) \mathrm{C}([\mathrm{H}])([\mathrm{H}]) \mathrm{C}([\mathrm{H}])([\mathrm{H}]) \mathrm{C}([\mathrm{H}])([\mathrm{H}]) \mathrm{C}([\mathrm{H}])([\mathrm{H}]) \mathrm{C} 1([\mathrm{H}])[\mathrm{H}]) \mathrm{C}([\mathrm{H}])([\mathrm{H}]) \mathrm{C}= \\
2 \mathrm{~N}=\mathrm{C}([\mathrm{S}] \mathrm{C}=2[\mathrm{H}]) \mathrm{C}([\mathrm{H}])([\mathrm{H}]) \mathrm{OC}([\mathrm{H}])([\mathrm{H}])[\mathrm{H}]) \mathrm{C}([\mathrm{H}])([\mathrm{H}]) \mathrm{C}([\mathrm{H}])([\mathrm{H}])[\mathrm{C} @]([\mathrm{H}])(\mathrm{N} 5 \mathrm{C}(=\mathrm{N} / \mathrm{C} \\
4=\mathrm{C}(\backslash[\mathrm{H}]) \mathrm{C}([\mathrm{H}])=\mathrm{C}(\mathrm{O} / \mathrm{C} 3=\mathrm{C}(\backslash[\mathrm{H}]) \mathrm{C}([\mathrm{H}])=\mathrm{C}([\mathrm{H}]) \mathrm{C}([\mathrm{H}])=\mathrm{C} 3[\mathrm{H}]) \mathrm{C}([\mathrm{H}])=\mathrm{C} 4 \mathrm{C} 5([\mathrm{H}])[\mathrm{H}]) \mathrm{N}([\mathrm{H}] \\
)[\mathrm{H}])[\end{array}$ & 3.886 & 3.516 \\
\hline Calib44 & $\begin{array}{l}\mathrm{O}=\mathrm{C} 3 \mathrm{~N}(\mathrm{C}(=\mathrm{N}[\mathrm{C} @(\mathrm{C}=2[\mathrm{~S}] \mathrm{C}([\mathrm{H}])=\mathrm{C}(/ \mathrm{C} 1=\mathrm{C}(\backslash[\mathrm{H}]) \mathrm{C}(\mathrm{C \# CC}([\mathrm{H}])([\mathrm{H}])[\mathrm{H}])=\mathrm{C}([\mathrm{H}]) \mathrm{N}=\mathrm{C} 1[\mathrm{H}]) \mathrm{C} \\
=2[\mathrm{H}])(\mathrm{C} 3([\mathrm{H}])[\mathrm{H}]) \mathrm{C}([\mathrm{H}])([\mathrm{H}])[\mathrm{H}]) \mathrm{N}([\mathrm{H}])[\mathrm{H}]) \mathrm{C}([\mathrm{H}])([\mathrm{H}])[\mathrm{H}]\end{array}$ & 4.108 & 3.680 \\
\hline Calib45 & $\begin{array}{l}\mathrm{O}=\mathrm{C}(\mathrm{N}([\mathrm{H}]) \mathrm{C}([\mathrm{H}])([\mathrm{H}]) \mathrm{C}([\mathrm{H}])([\mathrm{H}]) \mathrm{C}([\mathrm{H}])([\mathrm{H}]) \mathrm{C}([\mathrm{H}])([\mathrm{H}]) \mathrm{C}([\mathrm{H}])([\mathrm{H}]) \mathrm{C}([\mathrm{H}])([\mathrm{H}]) \mathrm{C}([\mathrm{H}])([\mathrm{H}] \\
) \mathrm{C}([\mathrm{H}])([\mathrm{H}]) \mathrm{C}([\mathrm{H}])([\mathrm{H}]) \mathrm{C}([\mathrm{H}])([\mathrm{H}]) \mathrm{N}([\mathrm{H}]) \mathrm{C} 2=\mathrm{C} 1 \mathrm{C}([\mathrm{H}])=\mathrm{C}([\mathrm{H}]) \mathrm{C}([\mathrm{H}])=\mathrm{C}([\mathrm{H}]) \mathrm{C} 1=\mathrm{NC} 3=\mathrm{C} 2 \\
\mathrm{C}([\mathrm{H}])([\mathrm{H}]) \mathrm{C}([\mathrm{H}])([\mathrm{H}]) \mathrm{C}([\mathrm{H}])([\mathrm{H}]) \mathrm{C} 3([\mathrm{H}])[\mathrm{H}]) \mathrm{C}=4 \mathrm{OC}=5 \mathrm{C}(\mathrm{C}(=\mathrm{O}) \mathrm{C}=4[\mathrm{H}])=\mathrm{C}(\mathrm{O}[\mathrm{H}]) \mathrm{C}([\mathrm{H}])= \\
\mathrm{C}(\mathrm{O}[\mathrm{H}])\end{array}$ & 1.975 & 2.047 \\
\hline Calib46 & $\begin{array}{l}\mathrm{O}=\mathrm{C}(\mathrm{N}([\mathrm{H}]) \mathrm{C}([\mathrm{H}])([\mathrm{H}]) \mathrm{C}([\mathrm{H}])([\mathrm{H}]) \mathrm{C}([\mathrm{H}])([\mathrm{H}]) \mathrm{C}([\mathrm{H}])([\mathrm{H}]) \mathrm{C}([\mathrm{H}])([\mathrm{H}]) \mathrm{C}([\mathrm{H}])([\mathrm{H}]) \mathrm{C}([\mathrm{H}])([\mathrm{H}] \\
) \mathrm{C}([\mathrm{H}])([\mathrm{H}]) \mathrm{C}([\mathrm{H}])([\mathrm{H}]) \mathrm{C}([\mathrm{H}])([\mathrm{H}]) \mathrm{N}([\mathrm{H}]) \mathrm{C} 2=\mathrm{C} 1 \mathrm{C}([\mathrm{H}])=\mathrm{C}([\mathrm{H}]) \mathrm{C}([\mathrm{Cl}])=\mathrm{C}([\mathrm{H}]) \mathrm{C} 1=\mathrm{NC} 3=\mathrm{C} 2 \\
\mathrm{C}([\mathrm{H}])([\mathrm{H}]) \mathrm{C}([\mathrm{H}])([\mathrm{H}]) \mathrm{C}([\mathrm{H}])([\mathrm{H}]) \mathrm{C} 3([\mathrm{H}])[\mathrm{H}]) \mathrm{C}=4 \mathrm{OC}=5 \mathrm{C}(\mathrm{C}(=\mathrm{O}) \mathrm{C}=4[\mathrm{H}])=\mathrm{C}([\mathrm{H}]) \mathrm{C}(\mathrm{OC}([\mathrm{H}]) \\
([\mathrm{H}])\end{array}$ & 1.374 & 2.160 \\
\hline Calib47 & $\begin{array}{l}\mathrm{O}=[\mathrm{S}] 3(=\mathrm{O}) \mathrm{N}(\mathrm{C}(=\mathrm{N}[\mathrm{C} @ @](\mathrm{C}=1[\mathrm{~S}] / \mathrm{C}(=\mathrm{C}(/[\mathrm{H}]) \mathrm{C}=1[\mathrm{Cl}]) \mathrm{C} 2=\mathrm{C}([\mathrm{H}]) \mathrm{C}(\mathrm{C} \# \mathrm{CC}([\mathrm{H}])([\mathrm{H}])[\mathrm{H}])= \\
\mathrm{C}([\mathrm{H}]) \mathrm{N}=\mathrm{C} 2[\mathrm{H}])(\mathrm{C}([\mathrm{H}])([\mathrm{H}])[\mathrm{H}]) \mathrm{C} 3([\mathrm{H}])[\mathrm{H}]) \mathrm{N}([\mathrm{H}])[\mathrm{H}]) \mathrm{C}([\mathrm{H}])([\mathrm{H}])[\mathrm{H}]\end{array}$ & 4.620 & 4.628 \\
\hline Calib48 & $\begin{array}{l}\mathrm{O}=\mathrm{C}(\mathrm{N}([\mathrm{H}]) \mathrm{C} 3=\mathrm{C}([\mathrm{H}]) \mathrm{C}([\mathrm{H}])=\mathrm{C} 2 \mathrm{OC}([\mathrm{C} @] 4([\mathrm{C} @ @] 1(\mathrm{~N}=\mathrm{C}(\mathrm{OC} 1([\mathrm{H}])[\mathrm{H}]) \mathrm{N}([\mathrm{H}])[\mathrm{H}]) \mathrm{C} 2=\mathrm{C} 3 \\
[\mathrm{H}]) \mathrm{C}([\mathrm{H}])([\mathrm{H}]) \mathrm{OC} 4([\mathrm{H}])[\mathrm{H}])(\mathrm{C}([\mathrm{H}])([\mathrm{H}])[\mathrm{H}]) \mathrm{C}([\mathrm{H}])([\mathrm{H}])[\mathrm{H}]) \mathrm{C}=5 / \mathrm{N}=\mathrm{C}(/[\mathrm{H}]) \mathrm{C}([\mathrm{F}])=\mathrm{C}([\mathrm{H}]) \\
\mathrm{C}=5[\mathrm{H}]\end{array}$ & 2.932 & 2.828 \\
\hline Calib49 & $\begin{array}{l}{[\mathrm{H}] / \mathrm{C} 4=\mathrm{C}(/ \mathrm{C}([\mathrm{H}])=\mathrm{C} 1 \mathrm{C}(\mathrm{O}[\mathrm{C} @]([\mathrm{H}])([\mathrm{C} @] 3([\mathrm{C} @] 12 \mathrm{~N}=\mathrm{C}(\mathrm{OC} 2([\mathrm{H}])[\mathrm{H}]) \mathrm{N}([\mathrm{H}])[\mathrm{H}]) \mathrm{C}([\mathrm{H}])([} \\
\mathrm{H}]) \mathrm{OC} 3([\mathrm{H}])[\mathrm{H}]) \mathrm{C}([\mathrm{H}])([\mathrm{H}]) \mathrm{C}([\mathrm{H}])([\mathrm{H}]) \mathrm{C}([\mathrm{H}])([\mathrm{H}])[\mathrm{H}])=\mathrm{C} 4[\mathrm{H}]) \mathrm{C} 5=\mathrm{C}([\mathrm{H}]) \mathrm{N}=\mathrm{C}([\mathrm{H}]) \mathrm{C}(\mathrm{C \# C} \\
\mathrm{C}([\mathrm{H}])([\mathrm{H}])[\mathrm{H}])=\mathrm{C} 5[\mathrm{H}]\end{array}$ & 3.056 & 2.743 \\
\hline Calib50 & $\begin{array}{l}{[\mathrm{F}] \mathrm{C}([\mathrm{F}])([\mathrm{F}]) \mathrm{C}([\mathrm{H}])([\mathrm{H}]) \mathrm{C}([\mathrm{H}])([\mathrm{H}])[\mathrm{C} @] 3([\mathrm{H}]) \mathrm{O} / \mathrm{C} 1=\mathrm{C}(\backslash[\mathrm{H}]) \mathrm{C}([\mathrm{H}])=\mathrm{C}(\mathrm{C}([\mathrm{H}])=\mathrm{C} 1[\mathrm{C} @ @] 2} \\
(\mathrm{N}=\mathrm{C}(\mathrm{OC} 2([\mathrm{H}])[\mathrm{H}]) \mathrm{N}([\mathrm{H}])[\mathrm{H}])[\mathrm{C} @] 34 \mathrm{C}([\mathrm{H}])([\mathrm{H}]) \mathrm{OC} 4([\mathrm{H}])[\mathrm{H}]) \mathrm{C} 5=\mathrm{C}([\mathrm{H}]) \mathrm{N}=\mathrm{C}([\mathrm{H}]) \mathrm{C}(\mathrm{C \# C} \\
\mathrm{C}([\mathrm{H}])([\mathrm{H}])[\mathrm{H}])=\mathrm{C} 5[\mathrm{H}]\end{array}$ & 2.061 & 2.221 \\
\hline Calib51 & $\begin{array}{l}\mathrm{O}=\mathrm{C}(\mathrm{N}([\mathrm{H}]) \mathrm{C} 3=\mathrm{C}([\mathrm{H}]) \mathrm{C}([\mathrm{H}])=\mathrm{C} 2 \mathrm{OC}([\mathrm{H}])([\mathrm{H}])[\mathrm{C} @ @] 4([\mathrm{C} @ @] 1(\mathrm{~N}=\mathrm{C}(\mathrm{OC} 1([\mathrm{H}])[\mathrm{H}]) \mathrm{N}([\mathrm{H}]) \\
[\mathrm{H}]) \mathrm{C} 2=\mathrm{C} 3[\mathrm{H}]) \mathrm{C}([\mathrm{H}])([\mathrm{H}]) \mathrm{C} 4([\mathrm{H}])[\mathrm{H}]) \mathrm{C} 5=\mathrm{NC}([\mathrm{H}])=\mathrm{C}(\mathrm{C \# N}) \mathrm{C}([\mathrm{H}])=\mathrm{C} 5 \mathrm{C}([\mathrm{H}])([\mathrm{H}])[\mathrm{H}]\end{array}$ & 2.668 & 2.624 \\
\hline Calib52 & $\begin{array}{l}\mathrm{O}=\mathrm{C}(\mathrm{N}([\mathrm{H}]) \mathrm{C} 3=\mathrm{C}([\mathrm{H}]) \mathrm{C}([\mathrm{H}])=\mathrm{C} 2 \mathrm{OC}([\mathrm{C} @] 4([\mathrm{C} @ @] 1(\mathrm{~N}=\mathrm{C}(\mathrm{OC} 1([\mathrm{H}])[\mathrm{H}]) \mathrm{N}([\mathrm{H}])[\mathrm{H}]) \mathrm{C} 2=\mathrm{C} 3 \\
[\mathrm{H}]) \mathrm{C}([\mathrm{H}])([\mathrm{H}]) \mathrm{C} 4([\mathrm{H}])[\mathrm{H}])(\mathrm{C}([\mathrm{H}])([\mathrm{H}])[\mathrm{H}]) \mathrm{C}([\mathrm{H}])([\mathrm{H}])[\mathrm{H}]) \mathrm{C} 5=\mathrm{NC}([\mathrm{H}])=\mathrm{C}(/ \mathrm{N}=\mathrm{C} 5 /[\mathrm{H}]) \mathrm{OC}( \\
[\mathrm{H}])([\mathrm{H}])[\mathrm{H}]\end{array}$ & 3.213 & 3.326 \\
\hline Calib53 & $\begin{array}{l}\mathrm{O}=\mathrm{C}(\mathrm{N}([\mathrm{H}]) \mathrm{C} 3=\mathrm{C}([\mathrm{H}]) \mathrm{C}([\mathrm{H}])=\mathrm{C} 2 \mathrm{OC}([\mathrm{C} @ @] 4([\mathrm{C} @] 1(\mathrm{~N}=\mathrm{C}(\mathrm{OC} 1([\mathrm{H}])[\mathrm{H}]) \mathrm{N}([\mathrm{H}])[\mathrm{H}]) \mathrm{C} 2=\mathrm{C} 3 \\
[\mathrm{H}]) \mathrm{C}([\mathrm{H}])([\mathrm{H}]) \mathrm{OC} 4([\mathrm{H}])[\mathrm{H}])(\mathrm{C}([\mathrm{H}])([\mathrm{H}])[\mathrm{H}]) \mathrm{C}([\mathrm{H}])([\mathrm{H}])[\mathrm{H}]) \mathrm{C}=5 / \mathrm{N}=\mathrm{C}(/[\mathrm{H}]) \mathrm{C}([\mathrm{Cl}])=\mathrm{C}([\mathrm{H}]) \\
\mathrm{C}=5[\mathrm{H}]\end{array}$ & 0.520 & 1.753 \\
\hline Calib54 & $\begin{array}{l}{[\mathrm{F}] \mathrm{C}=1 \mathrm{C}([\mathrm{H}])=\mathrm{C}(\mathrm{C}([\mathrm{H}])=\mathrm{C}([\mathrm{F}]) \mathrm{C}=1[\mathrm{H}]) \mathrm{C}([\mathrm{H}])([\mathrm{H}])[\mathrm{C} @]([\mathrm{H}])(\mathrm{N}([\mathrm{H}]) \mathrm{C}(=\mathrm{O}) \mathrm{C} 2=\mathrm{C}([\mathrm{H}]) \mathrm{C}(=\mathrm{C}} \\
([\mathrm{H}]) \mathrm{C}(=\mathrm{C} 2[\mathrm{H}]) \mathrm{C}([\mathrm{H}])([\mathrm{H}])[\mathrm{H}]) \mathrm{C}(=\mathrm{O}) \mathrm{N}(\mathrm{C}([\mathrm{H}])([\mathrm{H}]) \mathrm{C}([\mathrm{H}])([\mathrm{H}]) \mathrm{C}([\mathrm{H}])([\mathrm{H}])[\mathrm{H}]) \mathrm{C}([\mathrm{H}])([\mathrm{H}]) \mathrm{C} \\
([\mathrm{H}])([\mathrm{H}]) \mathrm{C}([\mathrm{H}])([\mathrm{H}])[\mathrm{H}])[\mathrm{C} @]([\mathrm{H}])(\mathrm{O}[\mathrm{H}])[\mathrm{C} @] 3([\mathrm{H}]) \mathrm{N}([\mathrm{H}]) \mathrm{C}([\mathrm{H}])([\mathrm{H}]) \mathrm{C}([\mathrm{H}])([\mathrm{H}]) \mathrm{N}(\mathrm{C} 3([ \\
\mathrm{H}])[\end{array}$ & 1.237 & 2.873 \\
\hline
\end{tabular}




\begin{tabular}{|c|c|c|c|}
\hline Calib55 & $\begin{array}{l}{[\mathrm{F}] \mathrm{C}=1 \mathrm{C}([\mathrm{H}])=\mathrm{C}(\mathrm{C}([\mathrm{H}])=\mathrm{C}([\mathrm{F}]) \mathrm{C}=1[\mathrm{H}]) \mathrm{C}([\mathrm{H}])([\mathrm{H}])[\mathrm{C} @]([\mathrm{H}])(\mathrm{N}([\mathrm{H}]) \mathrm{C}(=\mathrm{O}) \mathrm{C} 2=\mathrm{C}([\mathrm{H}]) \mathrm{C}(=\mathrm{C}} \\
([\mathrm{H}]) \mathrm{C}(=\mathrm{C} 2[\mathrm{H}]) \mathrm{C}([\mathrm{H}])([\mathrm{H}])[\mathrm{H}]) \mathrm{C}(=\mathrm{O}) \mathrm{N}(\mathrm{C}([\mathrm{H}])([\mathrm{H}]) \mathrm{C}([\mathrm{H}])([\mathrm{H}]) \mathrm{C}([\mathrm{H}])([\mathrm{H}])[\mathrm{H}]) \mathrm{C}([\mathrm{H}])([\mathrm{H}]) \mathrm{C} \\
([\mathrm{H}])([\mathrm{H}]) \mathrm{C}([\mathrm{H}])([\mathrm{H}])[\mathrm{H}])[\mathrm{C} @]([\mathrm{H}])(\mathrm{O}[\mathrm{H}])[\mathrm{C} @]([\mathrm{H}]) \mathrm{N}([\mathrm{H}]) \mathrm{C}([\mathrm{H}])([\mathrm{H}]) \mathrm{C}([\mathrm{H}])([\mathrm{H}]) \mathrm{N}(\mathrm{C} 3([\mathrm{~L} \\
\mathrm{H}])[\end{array}$ & 1.921 & 2.753 \\
\hline Calib56 & 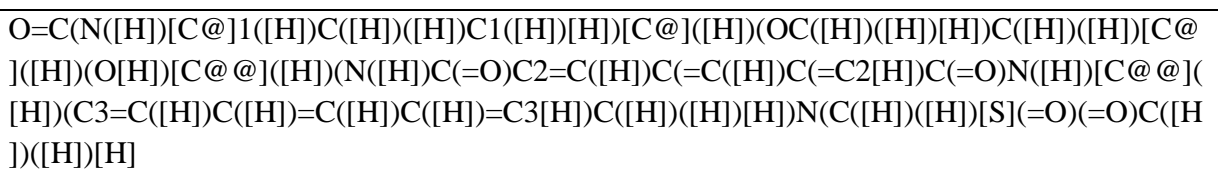 & 1.745 & 2.783 \\
\hline Calib57 & $\begin{array}{l}\mathrm{O}=\mathrm{C}(\mathrm{N}([\mathrm{H}]) \mathrm{C}(=\mathrm{NC}([\mathrm{H}])([\mathrm{H}]) \mathrm{Cl}=\mathrm{C}([\mathrm{H}]) \mathrm{C}([\mathrm{Cl}])=\mathrm{C}([\mathrm{Cl}]) \mathrm{C}([\mathrm{H}])=\mathrm{C} 1[\mathrm{H}]) \mathrm{N}([\mathrm{H}])[\mathrm{H}]) \mathrm{C}=2 \mathrm{C}(=\mathrm{N} \\
\mathrm{OC}=2 \mathrm{C}([\mathrm{H}])([\mathrm{H}])[\mathrm{H}]) \mathrm{C} 3=\mathrm{C}([\mathrm{H}]) \mathrm{C}([\mathrm{H}])=\mathrm{C}(\mathrm{OC}([\mathrm{H}])([\mathrm{H}])[\mathrm{H}]) \mathrm{C}([\mathrm{H}])=\mathrm{C} 3[\mathrm{H}]\end{array}$ & 2.041 & 2.371 \\
\hline Calib58 & $\begin{array}{l}\mathrm{O}=\mathrm{C}(\mathrm{N}([\mathrm{H}])[\mathrm{C} @]([\mathrm{H}])([\mathrm{C} @]([\mathrm{H}])(\mathrm{O}[\mathrm{H}]) \mathrm{C}([\mathrm{H}])([\mathrm{H}])[\mathrm{C} @]([\mathrm{H}])(\mathrm{C}(=\mathrm{O}) \mathrm{N}([\mathrm{H}])[\mathrm{C} @]([\mathrm{H}])(\mathrm{C}= \\
\mathrm{O}) \mathrm{N}([\mathrm{H}]) \mathrm{C}([\mathrm{H}])([\mathrm{H}]) \mathrm{Cl}=\mathrm{C}([\mathrm{H}]) \mathrm{C}([\mathrm{H}])=\mathrm{C}([\mathrm{H}]) \mathrm{C}([\mathrm{H}])=\mathrm{Cl}[\mathrm{H}]) \mathrm{C}([\mathrm{H}])(\mathrm{C}([\mathrm{H}])([\mathrm{H}])[\mathrm{H}]) \mathrm{C}([\mathrm{H}])([ \\
\mathrm{H}])[\mathrm{H}]) \mathrm{C}([\mathrm{H}])([\mathrm{H}])[\mathrm{H}]) \mathrm{C}([\mathrm{H}])([\mathrm{H}]) \mathrm{C}([\mathrm{H}])(\mathrm{C}([\mathrm{H}])([\mathrm{H}])[\mathrm{H}]) \mathrm{C}([\mathrm{H}])([\mathrm{H}])[\mathrm{H}])[\mathrm{C} @]([\mathrm{H}])(\mathrm{N}([\mathrm{H}]) \\
\mathrm{C}(=\end{array}$ & 4.229 & 3.009 \\
\hline Calib59 & $\begin{array}{l}\mathrm{O}=\mathrm{C}(\mathrm{O}[\mathrm{H}]) \mathrm{C}([\mathrm{H}])([\mathrm{H}]) \mathrm{C}([\mathrm{H}])([\mathrm{H}])[\mathrm{C} @]([\mathrm{H}])(\mathrm{N}([\mathrm{H}])[\mathrm{H}]) \mathrm{C}(=\mathrm{O}) \mathrm{N}([\mathrm{H}])[\mathrm{C} @ @]([\mathrm{H}])(\mathrm{C}(=\mathrm{O}) \mathrm{N}( \\
[\mathrm{H}])[\mathrm{C} @]([\mathrm{H}])(\mathrm{C}(=\mathrm{O}) \mathrm{N}([\mathrm{H}])[\mathrm{C} @]([\mathrm{H}])([\mathrm{C} @ @]([\mathrm{H}])(\mathrm{O}[\mathrm{H}]) \mathrm{C}([\mathrm{H}])([\mathrm{H}])[\mathrm{C} @]([\mathrm{H}])(\mathrm{C}(=\mathrm{O}) \mathrm{N}([ \\
\mathrm{H}])[\mathrm{C} @]([\mathrm{H}])(\mathrm{C}(=\mathrm{O}) \mathrm{N}([\mathrm{H}])[\mathrm{C} @]([\mathrm{H}])(\mathrm{C}(=\mathrm{O}) \mathrm{N}([\mathrm{H}])[\mathrm{C} @ @]([\mathrm{H}])(\mathrm{C}(=\mathrm{O}) \mathrm{O}[\mathrm{H}]) \mathrm{C}([\mathrm{H}])([\mathrm{H}]) \mathrm{C} 1 \\
=\mathrm{C}([\mathrm{H}]) \mathrm{C}([\mathrm{H}])=\end{array}$ & 4.796 & 4.574 \\
\hline Calib60 & $\begin{array}{l}\mathrm{O}=\mathrm{C}(\mathrm{N}([\mathrm{H}])[\mathrm{C} @]([\mathrm{H}])(\mathrm{C}(=\mathrm{O}) \mathrm{N}([\mathrm{H}])[\mathrm{C} @]([\mathrm{H}])([\mathrm{C} @ @]([\mathrm{H}])(\mathrm{O}[\mathrm{H}]) \mathrm{C}([\mathrm{H}])([\mathrm{H}])[\mathrm{C} @])([\mathrm{H}])(\mathrm{C}( \\
=\mathrm{O}) \mathrm{N}([\mathrm{H}])[\mathrm{C} @]([\mathrm{H}])(\mathrm{C}(=\mathrm{O}) \mathrm{N}([\mathrm{H}]) \mathrm{C}([\mathrm{H}])([\mathrm{H}]) \mathrm{C}([\mathrm{H}])(\mathrm{C}([\mathrm{H}])([\mathrm{H}])[\mathrm{H}]) \mathrm{C}([\mathrm{H}])([\mathrm{H}])[\mathrm{H}]) \mathrm{C}([\mathrm{H}]) \\
(\mathrm{C}([\mathrm{H}])([\mathrm{H}])[\mathrm{H}]) \mathrm{C}([\mathrm{H}])([\mathrm{H}])[\mathrm{H}]) \mathrm{C}([\mathrm{H}])([\mathrm{H}])[\mathrm{H}]) \mathrm{C}([\mathrm{H}])([\mathrm{H}]) \mathrm{C}([\mathrm{H}])(\mathrm{C}([\mathrm{H}])([\mathrm{H}])[\mathrm{H}]) \mathrm{C}([\mathrm{H}])([\mathrm{H} \\
]\end{array}$ & 2.327 & 3.092 \\
\hline Calib61 & $\begin{array}{l}\mathrm{O}=\mathrm{C} 1 \mathrm{~N}(/ \mathrm{C}(=\mathrm{N} \backslash \mathrm{H}]) \mathrm{N}([\mathrm{H}])[\mathrm{C} @] 1(\mathrm{C}([\mathrm{H}])([\mathrm{H}]) \mathrm{C}([\mathrm{H}])([\mathrm{H}])[\mathrm{C} @] 2([\mathrm{H}]) \mathrm{C}([\mathrm{H}])([\mathrm{H}]) \mathrm{C}([\mathrm{H}])([\mathrm{H}]) \\
\mathrm{C}([\mathrm{H}])([\mathrm{H}]) \mathrm{C}([\mathrm{H}])([\mathrm{H}]) \mathrm{C} 2([\mathrm{H}])(\mathrm{H}]) \mathrm{C}([\mathrm{H}])([\mathrm{H}])[\mathrm{C} @] 4([\mathrm{H}]) \mathrm{C}([\mathrm{H}])([\mathrm{H}])[\mathrm{C} @]([\mathrm{H}])(\mathrm{N}([\mathrm{H}]) \mathrm{C}(= \\
\mathrm{O}) \mathrm{N}([\mathrm{H}]) \mathrm{C} 3=\mathrm{C}([\mathrm{H}]) \mathrm{C}([\mathrm{H}])=\mathrm{C}(\mathrm{CHN}) \mathrm{C}([\mathrm{H}])=\mathrm{C} 3[\mathrm{H}]) \mathrm{C}([\mathrm{H}])([\mathrm{H}]) \mathrm{C}([\mathrm{H}])([\mathrm{H}]) \mathrm{C} 4([\mathrm{H}])[\mathrm{H}]) \mathrm{C}([\mathrm{H}]) \\
([\mathrm{H}])\end{array}$ & 4.523 & 4.241 \\
\hline Calib62 & $\begin{array}{l}\mathrm{O}=\mathrm{C} 1 \mathrm{~N}(/ \mathrm{C}(=\mathrm{N} \backslash[\mathrm{H}]) \mathrm{N}([\mathrm{H}])[\mathrm{C} @] 1(\mathrm{C}([\mathrm{H}])([\mathrm{H}]) \mathrm{C}([\mathrm{H}])([\mathrm{H}])[\mathrm{C} @] 2([\mathrm{H}]) \mathrm{C}([\mathrm{H}])([\mathrm{H}]) \mathrm{C}([\mathrm{H}])([\mathrm{H}]) \\
\mathrm{C}([\mathrm{H}])([\mathrm{H}]) \mathrm{C}([\mathrm{H}])([\mathrm{H}]) \mathrm{C} 2([\mathrm{H}])(\mathrm{H}]) \mathrm{C}([\mathrm{H}])([\mathrm{H}])[\mathrm{C} @] 4([\mathrm{H}]) \mathrm{C}([\mathrm{H}])([\mathrm{H}])[\mathrm{C} @]([\mathrm{H}])(\mathrm{N}([\mathrm{H}]) \mathrm{C}(= \\
\mathrm{O}) \mathrm{N}([\mathrm{H}]) \mathrm{C} 3=\mathrm{C}([\mathrm{H}]) \mathrm{C}([\mathrm{H}])=\mathrm{C}([\mathrm{H}]) \mathrm{C}([\mathrm{H}])=\mathrm{C} 3[\mathrm{H}]) \mathrm{C}([\mathrm{H}])([\mathrm{H}]) \mathrm{C}([\mathrm{H}])([\mathrm{H}]) \mathrm{C} 4([\mathrm{H}])[\mathrm{H}]) \mathrm{C}([\mathrm{H}])( \\
[\mathrm{H}])\end{array}$ & 3.796 & 3.719 \\
\hline Calib63 & $\begin{array}{l}\mathrm{O}=\mathrm{C} 1 \mathrm{~N}(/ \mathrm{C}(=\mathrm{N} /[\mathrm{H}]) \mathrm{N}([\mathrm{H}])[\mathrm{C} @] 1(\mathrm{C}([\mathrm{H}])([\mathrm{H}]) \mathrm{C}([\mathrm{H}])([\mathrm{H}])[\mathrm{C} @ @] 2([\mathrm{H}]) \mathrm{C}([\mathrm{H}])([\mathrm{H}]) \mathrm{C}([\mathrm{H}])([\mathrm{H} \\
]) \mathrm{C}([\mathrm{H}])([\mathrm{H}]) \mathrm{C}([\mathrm{H}])([\mathrm{H}]) \mathrm{C} 2([\mathrm{H}])[\mathrm{H}]) \mathrm{C}([\mathrm{H}])([\mathrm{H}])[\mathrm{C} @ 3] 3([\mathrm{H}]) \mathrm{C}([\mathrm{H}])([\mathrm{H}]) \mathrm{N}(\mathrm{C}([\mathrm{H}])([\mathrm{H}]) \mathrm{C}([\mathrm{H}]) \\
([\mathrm{H}]) \mathrm{C} 3([\mathrm{H}])[\mathrm{H}]) \mathrm{C}([\mathrm{H}])([\mathrm{H}]) \mathrm{C}([\mathrm{H}])([\mathrm{H}]) \mathrm{C}([\mathrm{H}])([\mathrm{H}]) \mathrm{C}([\mathrm{H}])([\mathrm{H}]) \mathrm{C}([\mathrm{H}])([\mathrm{H}])[\mathrm{H}]) \mathrm{C}([\mathrm{H}])([\mathrm{H}])\end{array}$ & 3.119 & 2.939 \\
\hline Calib64 & $\begin{array}{l}\mathrm{O}=[\mathrm{S}] 2(=\mathrm{O}) \mathrm{N}(/ \mathrm{C}(=\mathrm{N} /[\mathrm{H}]) \mathrm{N}([\mathrm{H}])[\mathrm{C} @] 4(/ \mathrm{C} 1=\mathrm{C}([\mathrm{H}]) \mathrm{C}([\mathrm{F}])=\mathrm{C}([\mathrm{F}]) \mathrm{C}([\mathrm{H}])=\mathrm{C} 1[\mathrm{~F}])[\mathrm{C} @] 2([\mathrm{H}]) \\
\mathrm{C}([\mathrm{H}])([\mathrm{H}]) \mathrm{N}(/ \mathrm{C} 3=\mathrm{N} / \mathrm{C}(\mathrm{OC}([\mathrm{H}])([\mathrm{H}])[\mathrm{H}])=\mathrm{C}([\mathrm{H}]) \mathrm{C}(=\mathrm{C} 3[\mathrm{H}]) \mathrm{C}([\mathrm{H}])([\mathrm{H}]) \mathrm{OC}([\mathrm{H}])([\mathrm{H}])[\mathrm{H}]) \mathrm{C} 4 \\
([\mathrm{H}])[\mathrm{H}]) \mathrm{C}([\mathrm{H}])([\mathrm{H}])[\mathrm{H}]\end{array}$ & 4.155 & 4.261 \\
\hline \multicolumn{4}{|c|}{ External set } \\
\hline Ext1 & $\begin{array}{l}\mathrm{C} 004 \mathrm{O}=\mathrm{C} 2 \mathrm{~N}([\mathrm{H}])[\mathrm{C} @]([\mathrm{H}])(\mathrm{C}(=\mathrm{O}) \mathrm{N}([\mathrm{H}])[\mathrm{C} @ @]([\mathrm{H}])(\mathrm{C}([\mathrm{H}])([\mathrm{H}]) \mathrm{C}([\mathrm{H}])(\mathrm{C}([\mathrm{H}])([\mathrm{H}]))[\mathrm{H}]) \mathrm{C} \\
([\mathrm{H}])([\mathrm{H}])(\mathrm{H}])[\mathrm{C} @ @]([\mathrm{H}])(\mathrm{O}[\mathrm{H}]) \mathrm{C}([\mathrm{H}])([\mathrm{H}])[\mathrm{C} @]]([\mathrm{H}])(\mathrm{C}(=\mathrm{O}) \mathrm{N}([\mathrm{H}])[\mathrm{C} @]([\mathrm{H}])(\mathrm{C}(=\mathrm{O}) \mathrm{N}([\mathrm{H} \\
]) \mathrm{C}([\mathrm{H}])([\mathrm{H}]) \mathrm{C}=1 \mathrm{C}([\mathrm{H}])=\mathrm{C}([\mathrm{H}]) \mathrm{C}([\mathrm{H}])=\mathrm{C}([\mathrm{H}]) \mathrm{C}=1[\mathrm{H}]) \mathrm{C}([\mathrm{H}])(\mathrm{C}([\mathrm{H}])([\mathrm{H}])(\mathrm{H}]) \mathrm{C}([\mathrm{H}])([\mathrm{H}])[\mathrm{H} \\
]) \mathrm{C}([\mathrm{H}])([\mathrm{H}])[\mathrm{H}]) \mathrm{C}([\mathrm{H}])([\mathrm{H}]) \mathrm{C}(=\mathrm{O}) \mathrm{N}([\mathrm{H}]) \mathrm{C}([\mathrm{H}])([\mathrm{H}]) \mathrm{C}([\mathrm{H}])([\mathrm{H}]) \mathrm{C}([\mathrm{H}])([\mathrm{H}]) \mathrm{C}([\mathrm{H}])([\mathrm{H}]) \mathrm{C}([ \\
\mathrm{H}])([\mathrm{H}]) \mathrm{OC}(=\mathrm{O}) \mathrm{N}([\mathrm{H}])[\mathrm{C} @ @] 2([\mathrm{H}]) \mathrm{C}([\mathrm{H}])(\mathrm{C}([\mathrm{H}])([\mathrm{H}])[\mathrm{H}]) \mathrm{C}([\mathrm{H}])([\mathrm{H}])[\mathrm{H}]\end{array}$ & 2.966 & 3.084 \\
\hline Ext2 & 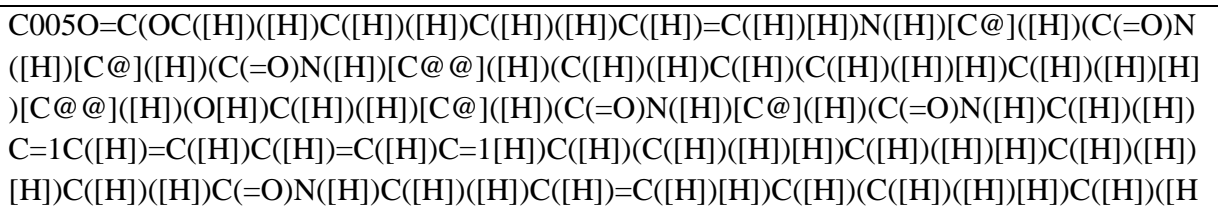 & 2.950 & 3.093 \\
\hline
\end{tabular}




\begin{tabular}{|c|c|c|c|}
\hline & ]) $[\mathrm{H}]$ & & \\
\hline Ext3 & $\begin{array}{l}\mathrm{C} 006 \mathrm{O}=\mathrm{C} 1 \mathrm{OC}([\mathrm{H}])([\mathrm{H}]) \mathrm{C}([\mathrm{H}])([\mathrm{H}]) \mathrm{C}([\mathrm{H}])([\mathrm{H}]) \mathrm{C}([\mathrm{H}])=\mathrm{C}([\mathrm{H}]) \mathrm{C}([\mathrm{H}])([\mathrm{H}]) \mathrm{N}([\mathrm{H}]) \mathrm{C}(=\mathrm{O}) \mathrm{C}([\mathrm{H}] \\
)([\mathrm{H}])[\mathrm{C} @]([\mathrm{H}])(\mathrm{N}([\mathrm{H}]) \mathrm{C}(=\mathrm{O})[\mathrm{C} @ @]([\mathrm{H}])(\mathrm{N} 1[\mathrm{H}]) \mathrm{C}([\mathrm{H}])(\mathrm{C}([\mathrm{H}])([\mathrm{H}])[\mathrm{H}]) \mathrm{C}([\mathrm{H}])([\mathrm{H}])[\mathrm{H}]) \mathrm{C}( \\
=\mathrm{O}) \mathrm{N}([\mathrm{H}])[\mathrm{C} @ @]([\mathrm{H}])(\mathrm{C}([\mathrm{H}])([\mathrm{H}]) \mathrm{C}([\mathrm{H}])(\mathrm{C}([\mathrm{H}])([\mathrm{H}])[\mathrm{H}]) \mathrm{C}([\mathrm{H}])([\mathrm{H}])[\mathrm{H}])[\mathrm{C} @ @]([\mathrm{H}])(\mathrm{O}[\mathrm{H} \\
]) \mathrm{C}([\mathrm{H}])([\mathrm{H}])[\mathrm{C} @]([\mathrm{H}])(\mathrm{C}(=\mathrm{O}) \mathrm{N}([\mathrm{H}])[\mathrm{C} @]([\mathrm{H}])(\mathrm{C}(=\mathrm{O}) \mathrm{N}([\mathrm{H}]) \mathrm{C}([\mathrm{H}])([\mathrm{H}]) \mathrm{C}=2 \mathrm{C}([\mathrm{H}])=\mathrm{C}([\mathrm{H}]) \\
\mathrm{C}([\mathrm{H}])=\mathrm{C}([\mathrm{H}]) \mathrm{C}=2[\mathrm{H}]) \mathrm{C}([\mathrm{H}])(\mathrm{C}([\mathrm{H}])([\mathrm{H}])[\mathrm{H}]) \mathrm{C}([\mathrm{H}])([\mathrm{H}])[\mathrm{H}]) \mathrm{C}([\mathrm{H}])([\mathrm{H}])[\mathrm{H}]\end{array}$ & 3.848 & 3.684 \\
\hline Ext4 & $\begin{array}{l}\mathrm{C} 015 \mathrm{O}=\mathrm{C}(\mathrm{C}=1 \mathrm{C}([\mathrm{H}])=\mathrm{C}(\mathrm{C}([\mathrm{H}])=\mathrm{C}(\mathrm{C}=1[\mathrm{H}]) \mathrm{C}(=\mathrm{O}) \mathrm{N}([\mathrm{H}])[\mathrm{C} @ @]([\mathrm{H}])(\mathrm{C}([\mathrm{H}])([\mathrm{H}]) \mathrm{C}([\mathrm{H}])(\mathrm{C}( \\
[\mathrm{H}])([\mathrm{H}])[\mathrm{H}]) \mathrm{C}([\mathrm{H}])([\mathrm{H}])[\mathrm{H}])[\mathrm{C} @ @]([\mathrm{H}])(\mathrm{O}[\mathrm{H}]) \mathrm{C}([\mathrm{H}])([\mathrm{H}])[\mathrm{C} @]([\mathrm{H}])(\mathrm{C}(=\mathrm{O}) \mathrm{N}([\mathrm{H}])[\mathrm{C} @]([ \\
\mathrm{H}])(\mathrm{C}(=\mathrm{O}) \mathrm{N}([\mathrm{H}]) \mathrm{C}([\mathrm{H}])(\mathrm{C}([\mathrm{H}])([\mathrm{H}])[\mathrm{H}]) \mathrm{C}([\mathrm{H}])([\mathrm{H}])[\mathrm{H}]) \mathrm{C}([\mathrm{H}])(\mathrm{C}([\mathrm{H}])([\mathrm{H}])[\mathrm{H}]) \mathrm{C}([\mathrm{H}])([\mathrm{H}])[ \\
\mathrm{H}]) \mathrm{C}([\mathrm{H}])([\mathrm{H}])[\mathrm{H}]) \mathrm{N}(\mathrm{C}([\mathrm{H}])([\mathrm{H}])[\mathrm{H}])[\mathrm{S}](=\mathrm{O})(=\mathrm{O}) \mathrm{C}([\mathrm{H}])([\mathrm{H}])[\mathrm{H}]) \mathrm{N}([\mathrm{H}]) \mathrm{C}([\mathrm{H}])([\mathrm{H}]) \mathrm{C}=2 \mathrm{~N}= \\
\mathrm{C}(\mathrm{OC}=2 \mathrm{C}([\mathrm{H}])([\mathrm{H}])[\mathrm{H}]) \mathrm{C}([\mathrm{H}])([\mathrm{H}])[\mathrm{H}]\end{array}$ & 4.745 & 3.570 \\
\hline Ext5 & $\begin{array}{l}\mathrm{C} 020 \mathrm{O}=\mathrm{C}(\mathrm{N}(\mathrm{C}([\mathrm{H}])([\mathrm{H}])[\mathrm{H}])[\mathrm{C} @ @] 1([\mathrm{H}]) \mathrm{C}([\mathrm{H}])([\mathrm{H}]) \mathrm{C}([\mathrm{H}])([\mathrm{H}]) \mathrm{C}([\mathrm{H}])([\mathrm{H}]) \mathrm{C}([\mathrm{H}])([\mathrm{H}]) \mathrm{C} 1 \\
([\mathrm{H}])[\mathrm{H}]) \mathrm{C}([\mathrm{H}])([\mathrm{H}]) \mathrm{C}([\mathrm{H}])([\mathrm{H}])[\mathrm{C} @ @]([\mathrm{H}])(\mathrm{N} 4 \mathrm{C}(=\mathrm{NC}=3 \mathrm{C}([\mathrm{H}])=\mathrm{C}([\mathrm{H}]) \mathrm{C}(\mathrm{OC}=2 \mathrm{C}([\mathrm{H}])=\mathrm{C}([ \\
\mathrm{H}]) \mathrm{C}([\mathrm{H}])=\mathrm{C}([\mathrm{H}]) \mathrm{C}=2[\mathrm{H}])=\mathrm{C}([\mathrm{H}]) \mathrm{C}=3 \mathrm{C} 4([\mathrm{H}])[\mathrm{H}]) \mathrm{N}([\mathrm{H}])[\mathrm{H}])[\mathrm{C} @] 5([\mathrm{H}]) \mathrm{C}([\mathrm{H}])([\mathrm{H}]) \mathrm{C}([\mathrm{H}])([ \\
\mathrm{H}]) \mathrm{C}([\mathrm{H}])([\mathrm{H}]) \mathrm{C}([\mathrm{H}])([\mathrm{H}]) \mathrm{C} 5([\mathrm{H}])[\mathrm{H}]\end{array}$ & 3.523 & 3.030 \\
\hline Ext6 & $\begin{array}{l}\mathrm{C} 028 \mathrm{O}=\mathrm{C}(\mathrm{N}([\mathrm{H}])[\mathrm{C} @]([\mathrm{H}])([\mathrm{C} @ @]([\mathrm{H}])(\mathrm{O}[\mathrm{H}]) \mathrm{C}([\mathrm{H}])([\mathrm{H}])[\mathrm{C} @]([\mathrm{H}])(\mathrm{C}(=\mathrm{O}) \mathrm{N}([\mathrm{H}])[\mathrm{C} @]([ \\
\mathrm{H}])(\mathrm{C}(=\mathrm{O}) \mathrm{N}([\mathrm{H}]) \mathrm{C}([\mathrm{H}])([\mathrm{H}]) \mathrm{C} 1=\mathrm{C}([\mathrm{H}]) \mathrm{C}([\mathrm{H}])=\mathrm{C}([\mathrm{H}]) \mathrm{C}([\mathrm{H}])=\mathrm{C} 1[\mathrm{H}]) \mathrm{C}([\mathrm{H}])(\mathrm{C}([\mathrm{H}])([\mathrm{H}])[\mathrm{H}]) \\
\mathrm{C}([\mathrm{H}])([\mathrm{H}])[\mathrm{H}]) \mathrm{C}([\mathrm{H}])([\mathrm{H}])[\mathrm{H}]) \mathrm{C}([\mathrm{H}])([\mathrm{H}]) \mathrm{C}([\mathrm{H}])(\mathrm{C}([\mathrm{H}])([\mathrm{H}])[\mathrm{H}]) \mathrm{C}([\mathrm{H}])([\mathrm{H}])[\mathrm{H}])[\mathrm{C} @ @]([ \\
\mathrm{H}])(\mathrm{N}([\mathrm{H}]) \mathrm{C}(=\mathrm{O})[\mathrm{C} @ @]([\mathrm{H}])(\mathrm{N}([\mathrm{H}]) \mathrm{C}(=\mathrm{O}) \mathrm{OC}(\mathrm{C}([\mathrm{H}])([\mathrm{H}])[\mathrm{H}])(\mathrm{C}([\mathrm{H}])([\mathrm{H}])[\mathrm{H}]) \mathrm{C}([\mathrm{H}])([\mathrm{H}])[ \\
\mathrm{H}]) \mathrm{C}([\mathrm{H}])(\mathrm{C}([\mathrm{H}])([\mathrm{H}])[\mathrm{H}]) \mathrm{C}([\mathrm{H}])([\mathrm{H}])[\mathrm{H}]) \mathrm{C}([\mathrm{H}])([\mathrm{H}]) \mathrm{C}(=\mathrm{O}) \mathrm{N}([\mathrm{H}])[\mathrm{H}]\end{array}$ & 4.229 & 3.904 \\
\hline Ext7 & $\begin{array}{l}\mathrm{C} 109 \mathrm{O}=\mathrm{C}(\mathrm{N}([\mathrm{H}])[\mathrm{C} @ @]([\mathrm{H}])(\mathrm{C}=1 \mathrm{C}([\mathrm{H}])=\mathrm{C}([\mathrm{H}]) \mathrm{C}([\mathrm{H}])=\mathrm{C}([\mathrm{H}]) \mathrm{C}=1[\mathrm{H}]) \mathrm{C}([\mathrm{H}])([\mathrm{H}])[\mathrm{H}]) \mathrm{C} 2= \\
\mathrm{C}([\mathrm{H}]) \mathrm{C}(=\mathrm{C}([\mathrm{H}]) \mathrm{C}(=\mathrm{C} 2[\mathrm{H}]) \mathrm{N}(\mathrm{C}([\mathrm{H}])([\mathrm{H}])[\mathrm{H}])[\mathrm{S}](=\mathrm{O})(=\mathrm{O}) \mathrm{C}([\mathrm{H}])([\mathrm{H}])[\mathrm{H}]) \mathrm{C}(=\mathrm{O}) \mathrm{N}([\mathrm{H}])[\mathrm{C} @ \\
]([\mathrm{H}])([\mathrm{C} @]([\mathrm{H}])(\mathrm{O}[\mathrm{H}]) \mathrm{C}([\mathrm{H}])([\mathrm{H}]) \mathrm{N}([\mathrm{H}]) \mathrm{C}([\mathrm{H}])(\mathrm{C}([\mathrm{H}])([\mathrm{H}])[\mathrm{H}]) \mathrm{C}([\mathrm{H}])([\mathrm{H}])[\mathrm{H}]) \mathrm{C}([\mathrm{H}])([\mathrm{H}]) \\
\mathrm{C} 3=\mathrm{C}([\mathrm{H}]) \mathrm{C}([\mathrm{H}])=\mathrm{C}([\mathrm{H}]) \mathrm{C}([\mathrm{H}])=\mathrm{C} 3[\mathrm{H}]\end{array}$ & 2.372 & 1.766 \\
\hline Ext8 & $\begin{array}{l}\mathrm{C} 115 \mathrm{O}=\mathrm{C}(\mathrm{N}([\mathrm{H}]) \mathrm{C}(=\mathrm{NC}([\mathrm{H}])([\mathrm{H}]) \mathrm{C} 1=\mathrm{C}([\mathrm{H}]) \mathrm{C}([\mathrm{Cl}])=\mathrm{C}([\mathrm{H}]) \mathrm{C}([\mathrm{Cl}])=\mathrm{C} 1[\mathrm{H}]) \mathrm{N}([\mathrm{H}])[\mathrm{H}]) \mathrm{C} 2=\mathrm{C} \\
(\mathrm{N}(/ \mathrm{N}=\mathrm{C} 2 / \mathrm{C}([\mathrm{H}])([\mathrm{H}])[\mathrm{H}]) \mathrm{C}([\mathrm{H}])([\mathrm{H}])[\mathrm{H}]) \mathrm{C} 3=\mathrm{C}([\mathrm{H}]) \mathrm{C}([\mathrm{H}])=\mathrm{C}(\mathrm{OC}([\mathrm{H}])([\mathrm{H}])[\mathrm{H}]) \mathrm{C}([\mathrm{H}])=\mathrm{C} 3[ \\
\mathrm{H}]\end{array}$ & 1.398 & 1.712 \\
\hline Ext9 & $\begin{array}{l}\mathrm{C} 117 \mathrm{O}=\mathrm{C} 3 \mathrm{OC}=2 \mathrm{C}(\mathrm{OC}([\mathrm{H}])([\mathrm{H}])[\mathrm{C} @ @]([\mathrm{H}])(\mathrm{O}[\mathrm{H}]) \mathrm{C}(\mathrm{O}[\mathrm{H}])(\mathrm{C}([\mathrm{H}])([\mathrm{H}])[\mathrm{H}]) \mathrm{C}([\mathrm{H}])([\mathrm{H}])[\mathrm{H}] \\
)=\mathrm{C} 1 \mathrm{OC}([\mathrm{H}])=\mathrm{C}([\mathrm{H}]) \mathrm{C} 1=\mathrm{C}(\mathrm{OC}([\mathrm{H}])([\mathrm{H}])[\mathrm{H}]) \mathrm{C}=2 \mathrm{C}([\mathrm{H}])=\mathrm{C} 3[\mathrm{H}]\end{array}$ & 0.260 & 0.995 \\
\hline Ext10 & $\begin{array}{l}\mathrm{C} 123 \mathrm{O}=\mathrm{C} 2 \mathrm{~N}([\mathrm{H}]) \mathrm{C} 1=\mathrm{C}([\mathrm{H}]) \mathrm{C}([\mathrm{H}])=\mathrm{C}([\mathrm{H}]) \mathrm{C}([\mathrm{H}])=\mathrm{C} 1 \mathrm{~N}([\mathrm{H}]) \mathrm{C} 3=\mathrm{C} 2 \mathrm{C}([\mathrm{H}])=\mathrm{C}([\mathrm{H}]) \mathrm{C}(=\mathrm{C} 3[\mathrm{H}] \\
) \mathrm{C}(=\mathrm{O}) \mathrm{N}([\mathrm{H}]) \mathrm{C} 4=\mathrm{C}([\mathrm{H}]) \mathrm{C}([\mathrm{H}])=\mathrm{C}([\mathrm{F}]) \mathrm{C}([\mathrm{H}])=\mathrm{C} 4[\mathrm{H}]\end{array}$ & 2.676 & 2.639 \\
\hline Ext11 & $\begin{array}{l}\mathrm{C} 124 \mathrm{O}=\mathrm{C}(\mathrm{N}([\mathrm{H}])[\mathrm{C} @ @]([\mathrm{H}])(\mathrm{C} 1=\mathrm{C}([\mathrm{H}]) \mathrm{C}([\mathrm{H}])=\mathrm{C}([\mathrm{F}]) \mathrm{C}([\mathrm{H}])=\mathrm{C} 1[\mathrm{H}]) \mathrm{C}([\mathrm{H}])([\mathrm{H}])[\mathrm{H}]) \mathrm{C}=2 \mathrm{C} \\
([\mathrm{H}])=\mathrm{C}(\mathrm{C}([\mathrm{H}])=\mathrm{C}(\mathrm{C}=2[\mathrm{H}]) \mathrm{N}([\mathrm{H}])[\mathrm{S}](=\mathrm{O})(=\mathrm{O}) \mathrm{C}([\mathrm{H}])([\mathrm{H}])[\mathrm{H}]) \mathrm{C}(=\mathrm{O}) \mathrm{N}([\mathrm{H}])[\mathrm{C} @]([\mathrm{H}])([\mathrm{C} @ \\
@]([\mathrm{H}])(\mathrm{O}[\mathrm{H}]) \mathrm{C}([\mathrm{H}])([\mathrm{H}]) \mathrm{N}([\mathrm{H}])[\mathrm{C} @ @] 3([\mathrm{H}]) \mathrm{C}([\mathrm{H}])([\mathrm{H}]) \mathrm{C} 3([\mathrm{H}])[\mathrm{H}]) \mathrm{C}([\mathrm{H}])([\mathrm{H}]) \mathrm{C} 4=\mathrm{C}([\mathrm{H}] \\
) \mathrm{C}([\mathrm{H}])=\mathrm{C}([\mathrm{H}]) \mathrm{C}([\mathrm{H}])=\mathrm{C} 4[\mathrm{H}]\end{array}$ & 4.000 & 2.982 \\
\hline Ext12 & $\begin{array}{l}\mathrm{C} 134 \mathrm{O}=\mathrm{C}(\mathrm{N}([\mathrm{H}]) \mathrm{C}(=\mathrm{NC}([\mathrm{H}])([\mathrm{H}]) \mathrm{C} 1=\mathrm{C}([\mathrm{H}]) \mathrm{C}(=\mathrm{C}([\mathrm{H}]) \mathrm{C}([\mathrm{Cl}])=\mathrm{C} 1[\mathrm{H}]) \mathrm{C}([\mathrm{H}])=\mathrm{C}([\mathrm{H}]) \mathrm{C}([\mathrm{H}]) \\
([\mathrm{H}]) \mathrm{OC}([\mathrm{H}])([\mathrm{H}])[\mathrm{H}]) \mathrm{N}([\mathrm{H}])[\mathrm{H}]) \mathrm{C}=2 \mathrm{C}(=\mathrm{NOC}=2 \mathrm{C}([\mathrm{H}])([\mathrm{H}])[\mathrm{H}]) \mathrm{C} 3=\mathrm{C}([\mathrm{H}]) \mathrm{C}([\mathrm{H}])=\mathrm{C}(\mathrm{OC}([\mathrm{H} \\
])([\mathrm{H}])[\mathrm{H}]) \mathrm{C}([\mathrm{H}])=\mathrm{C} 3[\mathrm{H}]\end{array}$ & 2.824 & 2.037 \\
\hline Ext13 & $\begin{array}{l}\mathrm{C} 140 \mathrm{O}=\mathrm{C}(\mathrm{N}([\mathrm{H}]) \mathrm{C}(=\mathrm{NC}([\mathrm{H}])([\mathrm{H}]) \mathrm{C} 1=\mathrm{C}([\mathrm{H}]) \mathrm{C}([\mathrm{Cl}])=\mathrm{C}([\mathrm{H}]) \mathrm{C}([\mathrm{Cl}])=\mathrm{C} 1[\mathrm{H}]) \mathrm{N}([\mathrm{H}])[\mathrm{H}]) \mathrm{C}=2 \mathrm{C} \\
(=\mathrm{N}[\mathrm{S}] \mathrm{C}=2 \mathrm{C}([\mathrm{H}])([\mathrm{H}])[\mathrm{H}]) \mathrm{C} 3=\mathrm{C}([\mathrm{H}]) \mathrm{C}([\mathrm{H}])=\mathrm{C}(\mathrm{OC}([\mathrm{H}])([\mathrm{H}])[\mathrm{H}]) \mathrm{C}([\mathrm{H}])=\mathrm{C} 3[\mathrm{H}]\end{array}$ & 2.538 & 2.601 \\
\hline Ext14 & $\begin{array}{l}\mathrm{C} 153[\mathrm{H}] \mathrm{C}=2 \mathrm{C}(\mathrm{O}[\mathrm{H}])=\mathrm{C} 1 \mathrm{OC}=6 \mathrm{C}(\mathrm{OC} 1=\mathrm{C} 3 \mathrm{C}=2 \mathrm{OC}=4 / \mathrm{C} 3=\mathrm{C}(/ \mathrm{O}[\mathrm{H}]) \mathrm{C}([\mathrm{H}])=\mathrm{C}(\mathrm{O}[\mathrm{H}]) \mathrm{C}=4 \mathrm{O} / \mathrm{C} 5 \\
=\mathrm{C}(\backslash[\mathrm{H}]) \mathrm{C}(\mathrm{O}[\mathrm{H}])=\mathrm{C}([\mathrm{H}]) \mathrm{C}(\mathrm{O}[\mathrm{H}])=\mathrm{C} 5[\mathrm{H}])=\mathrm{C}(\mathrm{O}[\mathrm{H}]) \mathrm{C}([\mathrm{H}])=\mathrm{C}(\mathrm{O}[\mathrm{H}]) \mathrm{C}=6 \mathrm{OC}=7 \mathrm{C}([\mathrm{H}])=\mathrm{C}(\mathrm{O}[\mathrm{H} \\
]) \mathrm{C}([\mathrm{H}])=\mathrm{C}(\mathrm{O}[\mathrm{H}]) \mathrm{C}=7[\mathrm{H}]\end{array}$ & 1.886 & 1.258 \\
\hline Ext15 & $\begin{array}{l}\mathrm{C} 158 \mathrm{O}=\mathrm{C}(/ \mathrm{C} 1=\mathrm{C}(1[\mathrm{H}]) \mathrm{C}(=\mathrm{C}([\mathrm{H}]) \mathrm{C}(=\mathrm{C} 1[\mathrm{H}]) \mathrm{C}(=\mathrm{O}) \mathrm{N} 2[\mathrm{C} @]([\mathrm{H}])(\mathrm{C}([\mathrm{H}])([\mathrm{H}]) \mathrm{C}([\mathrm{H}])([\mathrm{H}]) \mathrm{C} 2([ \\
\mathrm{H}])[\mathrm{H}]) \mathrm{C}([\mathrm{H}])([\mathrm{H}]) \mathrm{OC}([\mathrm{H}])([\mathrm{H}])[\mathrm{H}]) \mathrm{C}([\mathrm{H}])([\mathrm{H}])[\mathrm{H}]) \mathrm{N}([\mathrm{H}])[\mathrm{C} @]([\mathrm{H}])([\mathrm{C} @]([\mathrm{H}])(\mathrm{O}[\mathrm{H}])[\mathrm{C} @ \\
] 3([\mathrm{H}]) \mathrm{N}([\mathrm{H}]) \mathrm{C}([\mathrm{H}])([\mathrm{H}]) \mathrm{C}([\mathrm{H}])([\mathrm{H}]) \mathrm{N}(\mathrm{C} 3([\mathrm{H}])[\mathrm{H}])[\mathrm{S}](=\mathrm{O})(=\mathrm{O})[\mathrm{C} @ @] 4([\mathrm{H}]) \mathrm{C}([\mathrm{H}])([\mathrm{H}]) \mathrm{C} 4\end{array}$ & 4.155 & 4.345 \\
\hline
\end{tabular}




\begin{tabular}{|c|c|c|c|}
\hline & $([\mathrm{H}])[\mathrm{H}]) \mathrm{C}([\mathrm{H}])([\mathrm{H}]) \mathrm{C} 5=\mathrm{C}([\mathrm{H}]) \mathrm{C}([\mathrm{F}])=\mathrm{C}([\mathrm{H}]) \mathrm{C}([\mathrm{F}])=\mathrm{C} 5[\mathrm{H}]$ & & \\
\hline Ext16 & $\begin{array}{l}\mathrm{C} 167 \mathrm{O}=\mathrm{C}(\mathrm{N}([\mathrm{H}])[\mathrm{C} @ @]([\mathrm{H}])(\mathrm{C} 1=\mathrm{C}([\mathrm{H}]) \mathrm{C}([\mathrm{H}])=\mathrm{C}([\mathrm{H}]) \mathrm{C}([\mathrm{H}])=\mathrm{C} 1[\mathrm{H}]) \mathrm{C}([\mathrm{H}])([\mathrm{H}])[\mathrm{H}]) \mathrm{C} 2= \\
\mathrm{C}([\mathrm{H}]) \mathrm{C}(=\mathrm{C}([\mathrm{H}]) \mathrm{C}(=\mathrm{C} 2[\mathrm{H}]) \mathrm{N}(\mathrm{C}([\mathrm{H}])([\mathrm{H}])[\mathrm{H}])[\mathrm{S}](=\mathrm{O})(=\mathrm{O}) \mathrm{C}([\mathrm{H}])([\mathrm{H}])[\mathrm{H}]) \mathrm{C}(=\mathrm{O}) \mathrm{N}([\mathrm{H}])[\mathrm{C} @ \\
]([\mathrm{H}])([\mathrm{C} @]([\mathrm{H}])(\mathrm{O}[\mathrm{H}]) \mathrm{C}([\mathrm{H}])([\mathrm{H}])[\mathrm{C} @ @]([\mathrm{H}])(\mathrm{OC}([\mathrm{H}])([\mathrm{H}])[\mathrm{H}]) \mathrm{C}(=\mathrm{O}) \mathrm{N}([\mathrm{H}])[\mathrm{C} @]([\mathrm{H}])(\mathrm{C} \\
(=\mathrm{O}) \mathrm{N}([\mathrm{H}]) \mathrm{C}([\mathrm{H}])([\mathrm{H}]) \mathrm{C} 3=\mathrm{C}([\mathrm{H}]) \mathrm{C}([\mathrm{H}])=\mathrm{C}([\mathrm{H}]) \mathrm{C}([\mathrm{H}])=\mathrm{C} 3[\mathrm{H}]) \mathrm{C}([\mathrm{H}])(\mathrm{C}([\mathrm{H}])([\mathrm{H}])[\mathrm{H}]) \mathrm{C}([\mathrm{H}] \\
)([\mathrm{H}])[\mathrm{H}]) \mathrm{C}([\mathrm{H}])([\mathrm{H}]) \mathrm{O} / \mathrm{C} 4=\mathrm{C}(\backslash \mathrm{H}]) \mathrm{C}([\mathrm{F}])=\mathrm{C}([\mathrm{H}]) \mathrm{C}([\mathrm{F}])=\mathrm{C} 4[\mathrm{H}]\end{array}$ & 2.678 & 3.169 \\
\hline Ext17 & $\begin{array}{l}\mathrm{C} 172 \mathrm{O}=\mathrm{C}(\mathrm{N}([\mathrm{H}])[\mathrm{C} @ @]([\mathrm{H}])(\mathrm{C}=1 \mathrm{C}([\mathrm{H}])=\mathrm{C}([\mathrm{H}]) \mathrm{C}([\mathrm{H}])=\mathrm{C}([\mathrm{H}]) \mathrm{C}=1[\mathrm{H}]) \mathrm{C}([\mathrm{H}])([\mathrm{H}])[\mathrm{H}]) \mathrm{C}=2 \\
\mathrm{C}([\mathrm{H}])=\mathrm{C}(\mathrm{C}([\mathrm{H}])=\mathrm{C}(\mathrm{C}=2[\mathrm{H}]) \mathrm{N}(\mathrm{C}([\mathrm{H}])([\mathrm{H}])[\mathrm{H}])[\mathrm{S}](=\mathrm{O})(=\mathrm{O}) \mathrm{C}([\mathrm{H}])([\mathrm{H}])[\mathrm{H}]) \mathrm{C}(=\mathrm{O}) \mathrm{N}([\mathrm{H}])[\mathrm{C} \\
@]([\mathrm{H}])([\mathrm{C} @ @]([\mathrm{H}])(\mathrm{O}[\mathrm{H}]) \mathrm{C}([\mathrm{H}])([\mathrm{H}])[\mathrm{C} @ @]([\mathrm{H}])(\mathrm{OC}([\mathrm{H}])([\mathrm{H}]) \mathrm{C} 3=\mathrm{C}([\mathrm{H}]) \mathrm{C}([\mathrm{H}])=\mathrm{C}([\mathrm{H}]) \\
\mathrm{C}([\mathrm{H}])=\mathrm{C} 3[\mathrm{H}]) \mathrm{C}(=\mathrm{O}) \mathrm{N}([\mathrm{H}])[\mathrm{C} @]([\mathrm{H}])(\mathrm{C}(=\mathrm{O}) \mathrm{N}([\mathrm{H}]) \mathrm{C}([\mathrm{H}])([\mathrm{H}]) \mathrm{C} 4=\mathrm{C}([\mathrm{H}]) \mathrm{C}([\mathrm{H}])=\mathrm{C}([\mathrm{H}]) \mathrm{C}([ \\
\mathrm{H}])=\mathrm{C} 4[\mathrm{H}]) \mathrm{C}([\mathrm{H}])(\mathrm{C}([\mathrm{H}])([\mathrm{H}])[\mathrm{H}]) \mathrm{C}([\mathrm{H}])([\mathrm{H}])[\mathrm{H}]) \mathrm{C}([\mathrm{H}])([\mathrm{H}]) \mathrm{OC}=5 \mathrm{C}([\mathrm{H}])=\mathrm{C}([\mathrm{F}]) \mathrm{C}([\mathrm{H}])=\mathrm{C}( \\
[\mathrm{F}]) \mathrm{C}=5[\mathrm{H}]\end{array}$ & 3.699 & 3.899 \\
\hline Ext18 & $\begin{array}{l}\mathrm{C} 177 \mathrm{O}=\mathrm{C}(\mathrm{N}([\mathrm{H}]) \mathrm{C} 1=\mathrm{C}([\mathrm{H}]) \mathrm{C}([\mathrm{H}])=\mathrm{C}([\mathrm{H}]) \mathrm{C}([\mathrm{H}])=\mathrm{C} 1[\mathrm{H}])[\mathrm{C} @]([\mathrm{H}])(\mathrm{OC}([\mathrm{H}])([\mathrm{H}])[\mathrm{H}]) \mathrm{C}([\mathrm{H}] \\
)([\mathrm{H}])[\mathrm{C} @]([\mathrm{H}])(\mathrm{O}[\mathrm{H}])[\mathrm{C} @ @]([\mathrm{H}])(\mathrm{N}([\mathrm{H}]) \mathrm{C}(=\mathrm{O}) \mathrm{C} 2=\mathrm{C}([\mathrm{H}]) \mathrm{C}(=\mathrm{C}([\mathrm{H}]) \mathrm{C}(=\mathrm{C} 2[\mathrm{H}]) \mathrm{C}(=\mathrm{O}) \mathrm{N}([ \\
\mathrm{H}])[\mathrm{C} @ @]([\mathrm{H}])(\mathrm{C} 3=\mathrm{C}([\mathrm{H}]) \mathrm{C}([\mathrm{H}])=\mathrm{C}([\mathrm{H}]) \mathrm{C}([\mathrm{H}])=\mathrm{C} 3[\mathrm{H}]) \mathrm{C}([\mathrm{H}])([\mathrm{H}])[\mathrm{H}]) \mathrm{N}(\mathrm{C}([\mathrm{H}])([\mathrm{H}])[\mathrm{S}](= \\
\mathrm{O})(=\mathrm{O}) \mathrm{C}([\mathrm{H}])([\mathrm{H}])[\mathrm{H}]) \mathrm{C}([\mathrm{H}])([\mathrm{H}])[\mathrm{H}]) \mathrm{C}([\mathrm{H}])([\mathrm{H}]) \mathrm{O} / \mathrm{C} 4=\mathrm{C}(\backslash[\mathrm{H}]) \mathrm{C}([\mathrm{F}])=\mathrm{C}([\mathrm{H}]) \mathrm{C}([\mathrm{F}])=\mathrm{C} 4[\mathrm{H}]\end{array}$ & 2.699 & 2.405 \\
\hline Ext19 & $\begin{array}{l}\mathrm{C} 184 \mathrm{O}=\mathrm{C}(\mathrm{OC}(\mathrm{C}([\mathrm{H}])([\mathrm{H}])[\mathrm{H}])(\mathrm{C}([\mathrm{H}])([\mathrm{H}])[\mathrm{H}]) \mathrm{C}([\mathrm{H}])([\mathrm{H}])[\mathrm{H}]) \mathrm{N}([\mathrm{H}])[\mathrm{C} @]([\mathrm{H}])(\mathrm{C}(=\mathrm{O}) \mathrm{N}([\mathrm{H} \\
])[\mathrm{C} @]([\mathrm{H}])([\mathrm{C} @]([\mathrm{H}])(\mathrm{O}[\mathrm{H}]) \mathrm{C}([\mathrm{H}])([\mathrm{H}])[\mathrm{C} @([\mathrm{H}])(\mathrm{C}(=\mathrm{O}) \mathrm{N}([\mathrm{H}])[\mathrm{C} @]([\mathrm{H}])(\mathrm{C}(=\mathrm{O}) \mathrm{N}([\mathrm{H}]) \mathrm{C}( \\
[\mathrm{H}])([\mathrm{H}]) \mathrm{C} 1=\mathrm{C}([\mathrm{H}]) \mathrm{C}([\mathrm{H}])=\mathrm{C}([\mathrm{H}]) \mathrm{C}([\mathrm{H}])=\mathrm{C} 1[\mathrm{H}]) \mathrm{C}([\mathrm{H}])(\mathrm{C}([\mathrm{H}])([\mathrm{H}])[\mathrm{H}]) \mathrm{C}([\mathrm{H}])([\mathrm{H}])[\mathrm{H}]) \mathrm{C}([ \\
\mathrm{H}])([\mathrm{H}])[\mathrm{H}]) \mathrm{C}([\mathrm{H}])([\mathrm{H}]) \mathrm{C}([\mathrm{H}])(\mathrm{C}([\mathrm{H}])([\mathrm{H}])[\mathrm{H}]) \mathrm{C}([\mathrm{H}])([\mathrm{H}])[\mathrm{H}]) \mathrm{C}([\mathrm{H}])([\mathrm{H}]) \mathrm{C}(=\mathrm{O}) \mathrm{N}([\mathrm{H}])[\mathrm{H}]\end{array}$ & 1.504 & 2.150 \\
\hline Ext20 & $\begin{array}{l}\mathrm{C} 201 \mathrm{O}=\mathrm{C} 3 \mathrm{~N}([\mathrm{H}])[\mathrm{C} @]([\mathrm{H}])(\mathrm{C}(=\mathrm{O}) \mathrm{N}([\mathrm{H}])[\mathrm{C} @]([\mathrm{H}])([\mathrm{C} @]([\mathrm{H}])(\mathrm{O}[\mathrm{H}]) \mathrm{C}([\mathrm{H}])([\mathrm{H}]) \mathrm{N}([\mathrm{H}]) \mathrm{C}([ \\
\mathrm{H}])([\mathrm{H}]) \mathrm{C} 1=\mathrm{C}([\mathrm{H}]) \mathrm{C}(=\mathrm{C}([\mathrm{H}]) \mathrm{C}([\mathrm{H}])=\mathrm{C} 1[\mathrm{H}]) \mathrm{N}(\mathrm{C}([\mathrm{H}])([\mathrm{H}])[\mathrm{H}]) \mathrm{C}([\mathrm{H}])([\mathrm{H}])[\mathrm{H}]) \mathrm{C}([\mathrm{H}])([\mathrm{H}]) \mathrm{C} \\
2=\mathrm{C}([\mathrm{H}]) \mathrm{C}([\mathrm{H}])=\mathrm{C}([\mathrm{H}]) \mathrm{C}([\mathrm{H}])=\mathrm{C} 2[\mathrm{H}]) \mathrm{C}([\mathrm{H}])([\mathrm{H}]) \mathrm{N} 3 \mathrm{C}([\mathrm{H}])([\mathrm{H}]) \mathrm{C}([\mathrm{H}])([\mathrm{H}]) \mathrm{C}([\mathrm{H}])([\mathrm{H}]) \mathrm{C}([ \\
\mathrm{H}])([\mathrm{H}])[\mathrm{H}]\end{array}$ & 0.398 & 0.564 \\
\hline Ext21 & $\begin{array}{l}\mathrm{C} 202 \mathrm{O}=\mathrm{C} 1 \mathrm{~N}(\mathrm{C}(=\mathrm{N}[\mathrm{C} @] 1(\mathrm{C}=2 \mathrm{C}([\mathrm{H}])=\mathrm{C}([\mathrm{H}]) \mathrm{C}([\mathrm{H}])=\mathrm{C}([\mathrm{H}]) \mathrm{C}=2[\mathrm{H}]) \mathrm{C} 3=\mathrm{C}([\mathrm{H}]) \mathrm{C}(=\mathrm{C}([\mathrm{H}]) \mathrm{C}( \\
[\mathrm{H}])=\mathrm{C} 3[\mathrm{H}]) \mathrm{C} 4=\mathrm{C}([\mathrm{H}]) \mathrm{C}([\mathrm{H}])=\mathrm{NC}([\mathrm{H}])=\mathrm{C} 4[\mathrm{H}]) \mathrm{N}([\mathrm{H}])[\mathrm{H}]) \mathrm{C}([\mathrm{H}])([\mathrm{H}])[\mathrm{H}]\end{array}$ & 1.420 & 2.498 \\
\hline Ext22 & $\begin{array}{l}\mathrm{C} 208 \mathrm{O}=\mathrm{C} 1 \mathrm{~N}(\mathrm{C}(=\mathrm{N}[\mathrm{C} @] 1(\mathrm{C}=2 \mathrm{C}([\mathrm{H}])=\mathrm{C}([\mathrm{H}]) \mathrm{C}([\mathrm{H}])=\mathrm{C}([\mathrm{H}]) \mathrm{C}=2[\mathrm{H}]) \mathrm{C} 3=\mathrm{C}([\mathrm{H}]) \mathrm{C}(=\mathrm{C}([\mathrm{H}]) \mathrm{C}( \\
[\mathrm{H}])=\mathrm{C} 3[\mathrm{H}]) \mathrm{C} 4=\mathrm{C}([\mathrm{H}]) \mathrm{N}=\mathrm{C}([\mathrm{H}]) \mathrm{C}([\mathrm{Cl}])=\mathrm{C} 4[\mathrm{H}]) \mathrm{N}([\mathrm{H}])[\mathrm{H}]) \mathrm{C}([\mathrm{H}])([\mathrm{H}])[\mathrm{H}]\end{array}$ & 3.046 & 2.972 \\
\hline Ext23 & $\begin{array}{l}\mathrm{C} 212 \mathrm{O}=\mathrm{C} 1 \mathrm{~N}(\mathrm{C}(=\mathrm{N}[\mathrm{C} @ @] 1(\mathrm{C}=2 \mathrm{C}([\mathrm{H}])=\mathrm{C}([\mathrm{H}]) \mathrm{C}([\mathrm{H}])=\mathrm{C}([\mathrm{H}]) \mathrm{C}=2[\mathrm{H}]) \mathrm{C} 3=\mathrm{C}([\mathrm{H}]) \mathrm{C}(=\mathrm{C}([\mathrm{H}]) \\
\mathrm{C}([\mathrm{H}])=\mathrm{C} 3[\mathrm{H}]) \mathrm{C} 4=\mathrm{C}([\mathrm{H}]) \mathrm{N}=\mathrm{C}([\mathrm{H}]) \mathrm{C}([\mathrm{H}])=\mathrm{C} 4[\mathrm{H}]) \mathrm{N}([\mathrm{H}])[\mathrm{H}]) \mathrm{C}([\mathrm{H}])([\mathrm{H}])[\mathrm{H}]\end{array}$ & 1.770 & 2.372 \\
\hline Ext24 & $\begin{array}{l}\mathrm{C} 213 \mathrm{O}=\mathrm{C} 1 \mathrm{~N}(\mathrm{C}(=\mathrm{N}[\mathrm{C} @ @] 1(/ \mathrm{C} 2=\mathrm{C}(\backslash \mathrm{H}]) \mathrm{C}(=\mathrm{C}([\mathrm{H}]) \mathrm{C}([\mathrm{H}])=\mathrm{C} 2[\mathrm{H}]) \mathrm{C} 3=\mathrm{C}([\mathrm{H}]) \mathrm{N}=\mathrm{C}([\mathrm{H}]) \mathrm{C}([\mathrm{F}] \\
)=\mathrm{C} 3[\mathrm{H}])[\mathrm{C} @ @] 4([\mathrm{H}]) \mathrm{C}([\mathrm{H}])([\mathrm{H}]) \mathrm{C} 4([\mathrm{H}])[\mathrm{H}]) \mathrm{N}([\mathrm{H}])[\mathrm{H}]) \mathrm{C}([\mathrm{H}])([\mathrm{H}])[\mathrm{H}]\end{array}$ & 2.796 & 2.549 \\
\hline Ext25 & $\begin{array}{l}\mathrm{C} 219 \mathrm{O}=\mathrm{C} 1 \mathrm{~N}(\mathrm{C}(=\mathrm{N}[\mathrm{C} @] 1(\mathrm{C}=2 \mathrm{C}([\mathrm{H}])=\mathrm{C}([\mathrm{H}]) \mathrm{C}([\mathrm{H}])=\mathrm{C}([\mathrm{H}]) \mathrm{C}=2[\mathrm{H}]) \mathrm{C} 3=\mathrm{C}([\mathrm{H}]) \mathrm{C}([\mathrm{F}])=\mathrm{C}([\mathrm{H}] \\
) \mathrm{C}([\mathrm{H}])=\mathrm{C} 3[\mathrm{H}]) \mathrm{N}([\mathrm{H}])[\mathrm{H}]) \mathrm{C}([\mathrm{H}])([\mathrm{H}])[\mathrm{H}]\end{array}$ & 2.143 & 2.174 \\
\hline Ext26 & $\begin{array}{l}\mathrm{C} 224 \mathrm{O}=\mathrm{C} 1 \mathrm{~N}(\mathrm{C}(=\mathrm{N}[\mathrm{C} @ @] 1(\mathrm{C}=2 \mathrm{C}([\mathrm{H}])=\mathrm{C}([\mathrm{H}]) \mathrm{C}([\mathrm{H}])=\mathrm{C}([\mathrm{H}]) \mathrm{C}=2[\mathrm{H}]) \mathrm{C} 3=\mathrm{C}([\mathrm{H}]) \mathrm{C}(=\mathrm{C}([\mathrm{H}]) \\
\mathrm{C}([\mathrm{H}])=\mathrm{C} 3[\mathrm{H}]) \mathrm{C} 4=\mathrm{C}([\mathrm{H}]) \mathrm{N}=\mathrm{C}([\mathrm{H}]) \mathrm{C}([\mathrm{H}])=\mathrm{C} 4[\mathrm{H}]) \mathrm{N}([\mathrm{H}])[\mathrm{H}]) \mathrm{C}([\mathrm{H}])([\mathrm{H}])[\mathrm{H}]\end{array}$ & 2.276 & 2.372 \\
\hline Ext27 & $\begin{array}{l}\mathrm{C} 229 \mathrm{O}=\mathrm{C}(\mathrm{N}([\mathrm{C} @ @] 1([\mathrm{H}]) \mathrm{C}([\mathrm{H}])([\mathrm{H}]) \mathrm{C}([\mathrm{H}])([\mathrm{H}]) \mathrm{C}([\mathrm{H}])([\mathrm{H}]) \mathrm{C}([\mathrm{H}])([\mathrm{H}]) \mathrm{C} 1([\mathrm{H}])[\mathrm{H}]) \mathrm{C}([\mathrm{H}]) \\
([\mathrm{H}]) \mathrm{C} 2=\mathrm{C}([\mathrm{H}]) \mathrm{N}(/ \mathrm{N}=\mathrm{C} 2 /[\mathrm{H}]) \mathrm{C}([\mathrm{H}])([\mathrm{H}])[\mathrm{H}]) \mathrm{C}([\mathrm{H}])([\mathrm{H}]) \mathrm{C}([\mathrm{H}])([\mathrm{H}])[\mathrm{C} @]([\mathrm{H}])(\mathrm{N} 5 \mathrm{C}(=\mathrm{NC}=4 \\
\mathrm{C}([\mathrm{H}])=\mathrm{C}([\mathrm{H}]) \mathrm{C}(\mathrm{O} / \mathrm{C} 3=\mathrm{C}([\mathrm{H}]) \mathrm{C}([\mathrm{H}])=\mathrm{C}([\mathrm{H}]) \mathrm{C}([\mathrm{H}])=\mathrm{C} 3[\mathrm{H}])=\mathrm{C}([\mathrm{H}]) \mathrm{C}=4 \mathrm{C} 5([\mathrm{H}])[\mathrm{H}]) \mathrm{N}([\mathrm{H}])[ \\
\mathrm{H}])[\mathrm{C} @ @] 6([\mathrm{H}]) \mathrm{C}([\mathrm{H}])([\mathrm{H}]) \mathrm{C}([\mathrm{H}])([\mathrm{H}]) \mathrm{C}([\mathrm{H}])([\mathrm{H}]) \mathrm{C}([\mathrm{H}])([\mathrm{H}]) \mathrm{C} 6([\mathrm{H}])[\mathrm{H}]\end{array}$ & 3.959 & 3.832 \\
\hline Ext28 & $\begin{array}{l}\mathrm{C} 234 \mathrm{O}=\mathrm{C}(\mathrm{N}([\mathrm{C} @ @] 1([\mathrm{H}]) \mathrm{C}([\mathrm{H}])([\mathrm{H}]) \mathrm{C}([\mathrm{H}])([\mathrm{H}]) \mathrm{C}([\mathrm{H}])([\mathrm{H}]) \mathrm{C}([\mathrm{H}])([\mathrm{H}]) \mathrm{C} 1([\mathrm{H}])[\mathrm{H}]) \mathrm{C}([\mathrm{H}]) \\
([\mathrm{H}]) \mathrm{C}=2 \mathrm{~N}=\mathrm{C}([\mathrm{S}] \mathrm{C}=2[\mathrm{H}]) \mathrm{C}([\mathrm{H}])([\mathrm{H}])[\mathrm{H}]) \mathrm{C}([\mathrm{H}])([\mathrm{H}]) \mathrm{C}([\mathrm{H}])([\mathrm{H}])[\mathrm{C} @]([\mathrm{H}])(\mathrm{N} 5 \mathrm{C}(=\mathrm{NC}=4 \mathrm{C}([ \\
\mathrm{H}])=\mathrm{C}([\mathrm{H}]) \mathrm{C}(\mathrm{O} / \mathrm{C} 3=\mathrm{C}([\mathrm{H}]) \mathrm{C}([\mathrm{H}])=\mathrm{C}([\mathrm{H}]) \mathrm{C}([\mathrm{H}])=\mathrm{C} 3[\mathrm{H}])=\mathrm{C}([\mathrm{H}]) \mathrm{C}=4 \mathrm{C} 5([\mathrm{H}])[\mathrm{H}]) \mathrm{N}([\mathrm{H}])[\mathrm{H}]) \\
{[\mathrm{C} @ @] 6([\mathrm{H}]) \mathrm{C}([\mathrm{H}])([\mathrm{H}]) \mathrm{C}([\mathrm{H}])([\mathrm{H}]) \mathrm{C}([\mathrm{H}])([\mathrm{H}]) \mathrm{C}([\mathrm{H}])([\mathrm{H}]) \mathrm{C} 6([\mathrm{H}])[\mathrm{H}]}\end{array}$ & 3.102 & 3.256 \\
\hline Ext29 & $\begin{array}{l}\mathrm{C} 239 \mathrm{O}=\mathrm{C}(\mathrm{OC}([\mathrm{H}])([\mathrm{H}])[\mathrm{C} @]([\mathrm{H}])(\mathrm{N} 3 \mathrm{C}(=\mathrm{N} / \mathrm{C} 2=\mathrm{C}(\backslash \mathrm{H}]) \mathrm{C}([\mathrm{H}])=\mathrm{C}(\mathrm{O} / \mathrm{C} 1=\mathrm{C}(\backslash[\mathrm{H}]) \mathrm{C}([\mathrm{H}])=\mathrm{C}([ \\
\mathrm{H}]) \mathrm{C}([\mathrm{H}])=\mathrm{C} 1[\mathrm{H}]) \mathrm{C}([\mathrm{H}])=\mathrm{C} 2 \mathrm{C} 3([\mathrm{H}])[\mathrm{H}]) \mathrm{N}([\mathrm{H}])[\mathrm{H}])[\mathrm{C} @ @] 4([\mathrm{H}]) \mathrm{C}([\mathrm{H}])([\mathrm{H}]) \mathrm{C}([\mathrm{H}])([\mathrm{H}]) \mathrm{C}([\end{array}$ & 3.420 & 2.813 \\
\hline
\end{tabular}




\begin{tabular}{|c|c|c|c|}
\hline & $\begin{array}{l}\mathrm{H}])([\mathrm{H}]) \mathrm{C}([\mathrm{H}])([\mathrm{H}]) \mathrm{C} 4([\mathrm{H}])[\mathrm{H}]) \mathrm{N}([\mathrm{C} @ @] 5([\mathrm{H}]) \mathrm{C}([\mathrm{H}])([\mathrm{H}]) \mathrm{C}([\mathrm{H}])([\mathrm{H}]) \mathrm{C}([\mathrm{H}])([\mathrm{H}]) \mathrm{C}([\mathrm{H}])([ \\
\mathrm{H}]) \mathrm{C} 5([\mathrm{H}])[\mathrm{H}]) \mathrm{C}([\mathrm{H}])([\mathrm{H}])[\mathrm{H}]\end{array}$ & & \\
\hline Ext30 & $\begin{array}{l}\mathrm{C} 240 \mathrm{O}=\mathrm{C}(\mathrm{OC}([\mathrm{H}])([\mathrm{H}])[\mathrm{C} @]([\mathrm{H}])(\mathrm{N} 3 \mathrm{C}(=\mathrm{N} / \mathrm{C} 2=\mathrm{C}(\backslash[\mathrm{H}]) \mathrm{C}([\mathrm{H}])=\mathrm{C}(\mathrm{O} / \mathrm{C} 1=\mathrm{C}(\backslash[\mathrm{H}]) \mathrm{C}([\mathrm{H}])=\mathrm{C}([ \\
\mathrm{H}]) \mathrm{C}([\mathrm{H}])=\mathrm{C} 1[\mathrm{H}]) \mathrm{C}([\mathrm{H}])=\mathrm{C} 2 \mathrm{C} 3([\mathrm{H}])[\mathrm{H}]) \mathrm{N}([\mathrm{H}])[\mathrm{H}]) \mathrm{C} 4=\mathrm{C}([\mathrm{H}]) \mathrm{C}([\mathrm{H}])=\mathrm{C}([\mathrm{H}]) \mathrm{C}([\mathrm{H}])=\mathrm{C} 4[\mathrm{H}]) \\
\mathrm{N}([\mathrm{C} @ @] 5([\mathrm{H}]) \mathrm{C}([\mathrm{H}])([\mathrm{H}]) \mathrm{C}([\mathrm{H}])([\mathrm{H}]) \mathrm{C}([\mathrm{H}])([\mathrm{H}]) \mathrm{C}([\mathrm{H}])([\mathrm{H}]) \mathrm{C}([\mathrm{H}])[\mathrm{H}]) \mathrm{C}([\mathrm{H}])([\mathrm{H}])[\mathrm{H}]\end{array}$ & 2.106 & 2.360 \\
\hline Ext31 & $\begin{array}{l}\mathrm{C} 246 \mathrm{O}=\mathrm{C} 4 \mathrm{~N}(\mathrm{C}(=\mathrm{N}[\mathrm{C} @ @](\mathrm{C}=1 \mathrm{C}([\mathrm{H}])=\mathrm{C}([\mathrm{H}]) \mathrm{C}([\mathrm{H}])=\mathrm{C}(\mathrm{C}=1[\mathrm{H}]) \mathrm{C} 2=\mathrm{C}([\mathrm{H}]) \mathrm{C}(\mathrm{CN})=\mathrm{C}([\mathrm{H}]) \mathrm{C} \\
([\mathrm{H}])=\mathrm{C} 2[\mathrm{H}])(\mathrm{C} 3=\mathrm{C}([\mathrm{H}]) \mathrm{C}([\mathrm{H}])=\mathrm{C}([\mathrm{H}]) \mathrm{C}([\mathrm{H}])=\mathrm{C} 3[\mathrm{H}]) \mathrm{C} 4([\mathrm{H}])[\mathrm{H}]) \mathrm{N}([\mathrm{H}])[\mathrm{H}]) \mathrm{C}([\mathrm{H}])([\mathrm{H}])[\mathrm{H}]\end{array}$ & 1.022 & 1.487 \\
\hline Ext32 & $\begin{array}{l}\mathrm{C} 252 \mathrm{O}=\mathrm{C}(\mathrm{O}[\mathrm{C} @] 2([\mathrm{H}])[\mathrm{C} @ @]([\mathrm{H}])(\mathrm{OC} 1=\mathrm{C}(\mathrm{C}(\mathrm{O}[\mathrm{H}])=\mathrm{C}([\mathrm{H}]) \mathrm{C}(\mathrm{O}[\mathrm{H}])=\mathrm{C} 1[\mathrm{H}]) \mathrm{C} 2([\mathrm{H}])[\mathrm{H}]) \mathrm{C} \\
=3 \mathrm{C}([\mathrm{H}])=\mathrm{C}(\mathrm{O}[\mathrm{H}]) \mathrm{C}(\mathrm{O}[\mathrm{H}])=\mathrm{C}(\mathrm{O}[\mathrm{H}]) \mathrm{C}=3[\mathrm{H}]) \mathrm{C}=4 \mathrm{C}([\mathrm{H}])=\mathrm{C}(\mathrm{O}[\mathrm{H}]) \mathrm{C}(\mathrm{O}[\mathrm{H}])=\mathrm{C}(\mathrm{O}[\mathrm{H}]) \mathrm{C}=4[\mathrm{H}]\end{array}$ & 2.678 & 1.075 \\
\hline Ext33 & $\begin{array}{l}\mathrm{C} 257 \mathrm{O}=\mathrm{C}(\mathrm{N}([\mathrm{H}]) \mathrm{C}([\mathrm{H}])([\mathrm{H}]) \mathrm{C}([\mathrm{H}])([\mathrm{H}]) \mathrm{C}([\mathrm{H}])([\mathrm{H}]) \mathrm{C}([\mathrm{H}])([\mathrm{H}]) \mathrm{C}([\mathrm{H}])([\mathrm{H}]) \mathrm{C}([\mathrm{H}])([\mathrm{H}]) \mathrm{C}([\mathrm{H}] \\
)([\mathrm{H}]) \mathrm{C}([\mathrm{H}])([\mathrm{H}]) \mathrm{C}([\mathrm{H}])([\mathrm{H}]) \mathrm{C}([\mathrm{H}])([\mathrm{H}]) \mathrm{N}([\mathrm{H}]) \mathrm{C}=2 \mathrm{C}=1 \mathrm{C}([\mathrm{H}])=\mathrm{C}([\mathrm{H}]) \mathrm{C}([\mathrm{H}])=\mathrm{C}([\mathrm{H}]) \mathrm{C}=1 \mathrm{~N}= \\
\mathrm{C} 3 \mathrm{C}=2 \mathrm{C}([\mathrm{H}])([\mathrm{H}]) \mathrm{C}([\mathrm{H}])([\mathrm{H}]) \mathrm{C}([\mathrm{H}])([\mathrm{H}]) \mathrm{C} 3([\mathrm{H}])[\mathrm{H}]) \mathrm{C}=4 \mathrm{OC}=5 \mathrm{C}(\mathrm{C}(=\mathrm{O}) \mathrm{C}=4[\mathrm{H}])=\mathrm{C}([\mathrm{H}]) \mathrm{C}( \\
\mathrm{OC}([\mathrm{H}])([\mathrm{H}])[\mathrm{H}])=\mathrm{C}(\mathrm{OC}([\mathrm{H}])([\mathrm{H}])[\mathrm{H}]) \mathrm{C}=5[\mathrm{H}]\end{array}$ & 2.056 & 2.215 \\
\hline Ext34 & $\begin{array}{l}\mathrm{C} 262 \mathrm{O}=\mathrm{C} 2 \mathrm{~N}(/ \mathrm{C}(=\mathrm{N} /[\mathrm{H}]) \mathrm{N}([\mathrm{H}])[\mathrm{C} @] 4(\mathrm{C}=1 \mathrm{C}([\mathrm{Cl}])=\mathrm{C}([\mathrm{H}]) \mathrm{C}([\mathrm{H}])=\mathrm{C}([\mathrm{F}]) \mathrm{C}=1[\mathrm{H}])[\mathrm{C} @ @] 2([ \\
\mathrm{H}]) \mathrm{C}([\mathrm{H}])([\mathrm{H}]) \mathrm{N}(/ \mathrm{C} 3=\mathrm{N} / \mathrm{C}(\mathrm{OC}([\mathrm{H}])([\mathrm{H}])[\mathrm{H}])=\mathrm{C}([\mathrm{F}]) \mathrm{C}(=\mathrm{N} 3) \mathrm{C}([\mathrm{H}])([\mathrm{H}])[\mathrm{H}]) \mathrm{C} 4([\mathrm{H}])[\mathrm{H}]) \mathrm{C}([\mathrm{H} \\
])([\mathrm{H}])[\mathrm{H}]\end{array}$ & 4.051 & 4.094 \\
\hline Ext35 & $\begin{array}{l}\mathrm{C} 266 \mathrm{O}=\mathrm{C}(\mathrm{N}([\mathrm{H}]) \mathrm{C} 3=\mathrm{C}([\mathrm{H}]) \mathrm{C}([\mathrm{H}])=\mathrm{C} 2 \mathrm{OC}([\mathrm{C} @] 4([\mathrm{C} @ @] 1(\mathrm{~N}=\mathrm{C}(\mathrm{OC} 1([\mathrm{H}])[\mathrm{H}]) \mathrm{N}([\mathrm{H}])[\mathrm{H}]) \mathrm{C} \\
2=\mathrm{C} 3[\mathrm{H}]) \mathrm{C}([\mathrm{H}])([\mathrm{H}]) \mathrm{OC} 4([\mathrm{H}])[\mathrm{H}])(\mathrm{C}([\mathrm{H}])([\mathrm{H}])[\mathrm{H}]) \mathrm{C}([\mathrm{H}])([\mathrm{H}])[\mathrm{H}]) \mathrm{C}=5 / \mathrm{N}=\mathrm{C}(/[\mathrm{H}]) \mathrm{C}([\mathrm{Cl}])=\mathrm{C} \\
([\mathrm{H}]) \mathrm{C}=5[\mathrm{~F}]\end{array}$ & 3.288 & 2.905 \\
\hline Ext36 & $\begin{array}{l}\mathrm{C} 270[\mathrm{H}] / \mathrm{C} 4=\mathrm{C}(/ \mathrm{C}([\mathrm{H}])=\mathrm{C} 1 \mathrm{C}(\mathrm{OC}([\mathrm{C} @] 3([\mathrm{C} @ @] 12 \mathrm{~N}=\mathrm{C}(\mathrm{OC} 2([\mathrm{H}])[\mathrm{H}]) \mathrm{N}([\mathrm{H}])[\mathrm{H}]) \mathrm{C}([\mathrm{H}])([\mathrm{H}] \\
) \mathrm{OC} 3([\mathrm{H}])[\mathrm{H}])(\mathrm{C}([\mathrm{H}])([\mathrm{H}])[\mathrm{H}]) \mathrm{C}([\mathrm{H}])([\mathrm{H}])[\mathrm{H}])=\mathrm{C} 4[\mathrm{H}]) \mathrm{C} 5=\mathrm{C}([\mathrm{H}]) \mathrm{N}=\mathrm{C}([\mathrm{H}]) \mathrm{C}(\mathrm{OC}([\mathrm{H}])([\mathrm{H}])[ \\
\mathrm{H}])=\mathrm{C} 5[\mathrm{H}]\end{array}$ & 2.593 & 2.329 \\
\hline Ext37 & $\begin{array}{l}\mathrm{C} 275[\mathrm{H}] / \mathrm{C} 4=\mathrm{C}(/ \mathrm{C}([\mathrm{H}])=\mathrm{C} 1 \mathrm{C}(\mathrm{OC}([\mathrm{C} @] 3([\mathrm{C} @ @] 12 \mathrm{~N}=\mathrm{C}(\mathrm{OC} 2([\mathrm{H}])[\mathrm{H}]) \mathrm{N}([\mathrm{H}])[\mathrm{H}]) \mathrm{C}([\mathrm{H}])([\mathrm{H}] \\
) \mathrm{OC} 3([\mathrm{H}])[\mathrm{H}])(\mathrm{C}([\mathrm{H}])([\mathrm{H}])[\mathrm{H}]) \mathrm{C}([\mathrm{H}])([\mathrm{H}])[\mathrm{H}])=\mathrm{C} 4[\mathrm{H}]) \mathrm{C} 5=\mathrm{C}([\mathrm{H}]) \mathrm{N}=\mathrm{C}([\mathrm{H}]) \mathrm{C}(\mathrm{CCC}([\mathrm{H}])([\mathrm{H}] \\
)[\mathrm{H}])=\mathrm{C} 5[\mathrm{H}]\end{array}$ & 3.044 & 3.163 \\
\hline Ext38 & $\begin{array}{l}\mathrm{C} 283 \mathrm{O}=\mathrm{C}(\mathrm{N}([\mathrm{H}]) \mathrm{C} 3=\mathrm{C}([\mathrm{H}]) \mathrm{C}([\mathrm{H}])=\mathrm{C} 2 \mathrm{OC}([\mathrm{C} @] 4([\mathrm{C} @ @] 1(\mathrm{~N}=\mathrm{C}(\mathrm{OC} 1([\mathrm{H}])[\mathrm{H}]) \mathrm{N}([\mathrm{H}])[\mathrm{H}]) \mathrm{C} \\
2=\mathrm{C} 3[\mathrm{H}]) \mathrm{C}([\mathrm{H}])([\mathrm{H}]) \mathrm{OC} 4([\mathrm{H}])[\mathrm{H}])(\mathrm{C}([\mathrm{H}])([\mathrm{H}])[\mathrm{H}]) \mathrm{C}([\mathrm{H}])([\mathrm{H}])[\mathrm{H}]) \mathrm{C} 5=\mathrm{NC}([\mathrm{H}])=\mathrm{C}([\mathrm{Cl}]) \mathrm{C}([ \\
\mathrm{H}])=\mathrm{C} 5 \mathrm{C}([\mathrm{H}])([\mathrm{H}])[\mathrm{H}]\end{array}$ & 3.190 & 3.442 \\
\hline Ext39 & $\begin{array}{l}\mathrm{C} 289 \mathrm{O}=\mathrm{C}(\mathrm{N}([\mathrm{H}]) \mathrm{C} 3=\mathrm{C}([\mathrm{H}]) \mathrm{C}([\mathrm{H}])=\mathrm{C} 2 \mathrm{OC}([\mathrm{C} @] 4([\mathrm{C} @ @] 1(\mathrm{~N}=\mathrm{C}(\mathrm{OC} 1([\mathrm{H}])[\mathrm{H}]) \mathrm{N}([\mathrm{H}])[\mathrm{H}]) \mathrm{C} \\
2=\mathrm{C} 3[\mathrm{H}]) \mathrm{C}([\mathrm{H}])([\mathrm{H}]) \mathrm{OC} 4([\mathrm{H}])[\mathrm{H}])(\mathrm{C}([\mathrm{H}])([\mathrm{H}])[\mathrm{H}]) \mathrm{C}([\mathrm{H}])([\mathrm{H}])[\mathrm{H}]) \mathrm{C}=5 / \mathrm{N}=\mathrm{C}(/[\mathrm{H}]) \mathrm{C}([\mathrm{Cl}])=\mathrm{C} \\
([\mathrm{H}]) \mathrm{C}=5[\mathrm{H}]\end{array}$ & 3.987 & 2.848 \\
\hline Ext40 & $\begin{array}{l}\mathrm{C} 294 \mathrm{O}=\mathrm{C}(\mathrm{N}([\mathrm{H}]) \mathrm{C} 3=\mathrm{C}([\mathrm{H}]) \mathrm{C}([\mathrm{H}])=\mathrm{C} 2 \mathrm{OC}([\mathrm{C} @] 4([\mathrm{C} @ @] 1(\mathrm{~N}=\mathrm{C}(\mathrm{OC} 1([\mathrm{H}])[\mathrm{H}]) \mathrm{N}([\mathrm{H}])[\mathrm{H}]) \mathrm{C} \\
2=\mathrm{C} 3[\mathrm{H}]) \mathrm{C}([\mathrm{H}])([\mathrm{H}]) \mathrm{C} 4([\mathrm{H}])[\mathrm{H}])(\mathrm{C}([\mathrm{H}])([\mathrm{H}])[\mathrm{H}]) \mathrm{C}([\mathrm{H}])([\mathrm{H}])[\mathrm{H}]) \mathrm{C} 5=\mathrm{NC}([\mathrm{H}])=\mathrm{C}(/ \mathrm{N}=\mathrm{C} 5 /[\mathrm{H}] \\
) \mathrm{OC}([\mathrm{H}])([\mathrm{H}])[\mathrm{H}]\end{array}$ & 3.213 & 3.098 \\
\hline Ext41 & $\begin{array}{l}\mathrm{C} 295 \mathrm{O}=\mathrm{C}(\mathrm{N}([\mathrm{H}]) \mathrm{C} 3=\mathrm{C}([\mathrm{H}]) \mathrm{C}([\mathrm{H}])=\mathrm{C} 2 \mathrm{OC}([\mathrm{C} @] 4([\mathrm{C} @ @] 1(\mathrm{~N}=\mathrm{C}(\mathrm{OC} 1([\mathrm{H}])[\mathrm{H}]) \mathrm{N}([\mathrm{H}])[\mathrm{H}]) \mathrm{C} \\
2=\mathrm{C} 3[\mathrm{H}]) \mathrm{C}([\mathrm{H}])([\mathrm{H}]) \mathrm{C} 4([\mathrm{H}])[\mathrm{H}])(\mathrm{C}([\mathrm{H}])([\mathrm{H}])[\mathrm{H}]) \mathrm{C}([\mathrm{H}])([\mathrm{H}])[\mathrm{H}]) \mathrm{C} 5=\mathrm{NC}([\mathrm{H}])=\mathrm{C}(/ \mathrm{N}=\mathrm{C} 5 /[\mathrm{H}] \\
) \mathrm{C}([\mathrm{F}])([\mathrm{F}])[\mathrm{H}]\end{array}$ & 3.278 & 3.192 \\
\hline Ext42 & $\begin{array}{l}\mathrm{C} 301 \mathrm{O}=\mathrm{C}(/ \mathrm{C} 1=\mathrm{N} / \mathrm{C}([\mathrm{H}])=\mathrm{C}(/ \mathrm{N}=\mathrm{C} 1 /[\mathrm{H}]) \mathrm{OC}([\mathrm{H}])([\mathrm{H}])[\mathrm{H}]) \mathrm{N}([\mathrm{H}]) \mathrm{C} 2=\mathrm{C}([\mathrm{H}]) \mathrm{C}(=\mathrm{C}([\mathrm{F}]) \mathrm{C}([\mathrm{F}])= \\
\mathrm{C} 2[\mathrm{H}])[\mathrm{C} @ 3(\mathrm{~N}([\mathrm{H}]) \mathrm{C}(=\mathrm{N}[\mathrm{H}]) \mathrm{N}([\mathrm{S}](=\mathrm{O})(=\mathrm{O}) \mathrm{C} 3([\mathrm{H}])[\mathrm{H}]) \mathrm{C}([\mathrm{H}])([\mathrm{H}])[\mathrm{H}]) \mathrm{C}([\mathrm{H}])([\mathrm{H}])[\mathrm{H}]\end{array}$ & 4.979 & 4.840 \\
\hline Ext43 & $\begin{array}{l}\mathrm{C} 304 \mathrm{O}=[\mathrm{S}] 3(=\mathrm{O}) \mathrm{N}(/ \mathrm{C}(=\mathrm{N} \backslash[\mathrm{H}]) \mathrm{N}([\mathrm{H}])[\mathrm{C} @](\mathrm{C}=1[\mathrm{~S}] \mathrm{C}([\mathrm{H}])=\mathrm{C}(\mathrm{C}=1[\mathrm{H}]) \mathrm{C} 2=\mathrm{C}([\mathrm{H}]) \mathrm{C}([\mathrm{H}])=\mathrm{C}([ \\
\mathrm{H}]) \mathrm{C}(\mathrm{CN})=\mathrm{C} 2[\mathrm{H}])(\mathrm{C} 3([\mathrm{H}])[\mathrm{H}]) \mathrm{C}([\mathrm{H}])([\mathrm{H}])[\mathrm{H}]) \mathrm{C}([\mathrm{H}])([\mathrm{H}])[\mathrm{H}]\end{array}$ & 3.592 & 3.245 \\
\hline Ext44 & $\begin{array}{l}\mathrm{C} 308 \mathrm{O}=\mathrm{C}(\mathrm{N}([\mathrm{H}])[\mathrm{C} @]([\mathrm{H}])(\mathrm{C}(=\mathrm{O}) \mathrm{N}([\mathrm{H}])[\mathrm{C} @]([\mathrm{H}])([\mathrm{C} @ @]([\mathrm{H}])(\mathrm{O}[\mathrm{H}]) \mathrm{C}([\mathrm{H}])([\mathrm{H}])[\mathrm{C} @]([ \\
\mathrm{H}])(\mathrm{C}(=\mathrm{O}) \mathrm{N}([\mathrm{H}])[\mathrm{C} @]([\mathrm{H}])(\mathrm{C}(=\mathrm{O}) \mathrm{N}([\mathrm{H}]) \mathrm{C}([\mathrm{H}])([\mathrm{H}]) \mathrm{C} 1=\mathrm{C}([\mathrm{H}]) \mathrm{C}([\mathrm{H}])=\mathrm{C}([\mathrm{H}]) \mathrm{C}([\mathrm{H}])=\mathrm{C} 1[\mathrm{H}] \\
) \mathrm{C}([\mathrm{H}])(\mathrm{C}([\mathrm{H}])([\mathrm{H}])[\mathrm{H}]) \mathrm{C}([\mathrm{H}])([\mathrm{H}])[\mathrm{H}]) \mathrm{C}([\mathrm{H}])([\mathrm{H}])[\mathrm{H}]) \mathrm{C}([\mathrm{H}])([\mathrm{H}]) \mathrm{C}([\mathrm{H}])(\mathrm{C}([\mathrm{H}])([\mathrm{H}])[\mathrm{H}]) \mathrm{C}( \\
[\mathrm{H}])([\mathrm{H}])[\mathrm{H}]) \mathrm{C}([\mathrm{H}])([\mathrm{H}]) \mathrm{C}(=\mathrm{O}) \mathrm{N}([\mathrm{H}]) \mathrm{C}([\mathrm{H}])([\mathrm{H}]) \mathrm{C}([\mathrm{H}])=\mathrm{C}([\mathrm{H}])[\mathrm{H}])[\mathrm{C} @]([\mathrm{H}])(\mathrm{N}([\mathrm{H}]) \mathrm{C}(=\mathrm{O})\end{array}$ & 3.569 & 2.098 \\
\hline
\end{tabular}




\begin{tabular}{|c|c|c|c|}
\hline & $\mathrm{OC}([\mathrm{H}])([\mathrm{H}]) \mathrm{C}([\mathrm{H}])=\mathrm{C}([\mathrm{H}])[\mathrm{H}]) \mathrm{C}([\mathrm{H}])(\mathrm{C}([\mathrm{H}])([\mathrm{H}])[\mathrm{H}]) \mathrm{C}([\mathrm{H}])([\mathrm{H}])[\mathrm{H}]$ & & \\
\hline Ext45 & $\begin{array}{l}\mathrm{C} 312[\mathrm{~F}] \mathrm{C}=1 \mathrm{C}([\mathrm{H}])=\mathrm{C}(\mathrm{C}([\mathrm{H}])=\mathrm{C}([\mathrm{F}]) \mathrm{C}=1[\mathrm{H}]) \mathrm{C}([\mathrm{H}])([\mathrm{H}])[\mathrm{C} @]([\mathrm{H}])(\mathrm{N}([\mathrm{H}]) \mathrm{C}(=\mathrm{O}) \mathrm{C} 2=\mathrm{C}([\mathrm{H}]) \\
\mathrm{C}(=\mathrm{C}([\mathrm{H}]) \mathrm{C}(=\mathrm{C} 2[\mathrm{H}]) \mathrm{C}([\mathrm{H}])([\mathrm{H}])[\mathrm{H}]) \mathrm{C}(=\mathrm{O}) \mathrm{N}(\mathrm{C}([\mathrm{H}])([\mathrm{H}]) \mathrm{C}([\mathrm{H}])([\mathrm{H}]) \mathrm{C}([\mathrm{H}])([\mathrm{H}])[\mathrm{H}]) \mathrm{C}([\mathrm{H}])( \\
[\mathrm{H}]) \mathrm{C}([\mathrm{H}])([\mathrm{H}]) \mathrm{C}([\mathrm{H}])([\mathrm{H}])[\mathrm{H}])[\mathrm{C} @]([\mathrm{H}])(\mathrm{O}[\mathrm{H}])[\mathrm{C} @] 3([\mathrm{H}]) \mathrm{N}([\mathrm{H}]) \mathrm{C}([\mathrm{H}])([\mathrm{H}]) \mathrm{C}([\mathrm{H}])([\mathrm{H}]) \mathrm{N}( \\
\mathrm{C} 3([\mathrm{H}])[\mathrm{H}])[\mathrm{S}](=\mathrm{O})(=\mathrm{O}) \mathrm{C} 4=\mathrm{C}([\mathrm{H}]) \mathrm{C}([\mathrm{H}])=\mathrm{C}([\mathrm{H}]) \mathrm{N}=\mathrm{C} 4[\mathrm{H}]\end{array}$ & 1.237 & 1.453 \\
\hline Ext46 & $\begin{array}{l}\mathrm{C} 316 \mathrm{O}=\mathrm{C}(\mathrm{N}([\mathrm{H}])[\mathrm{C} @]([\mathrm{H}])([\mathrm{C} @ @]([\mathrm{H}])(\mathrm{O}[\mathrm{H}]) \mathrm{C}([\mathrm{H}])([\mathrm{H}])[\mathrm{C} @(([\mathrm{H}])(\mathrm{C}(=\mathrm{O}) \mathrm{N}([\mathrm{H}])[\mathrm{C} @]([ \\
\mathrm{H}])(\mathrm{C}(=\mathrm{O}) \mathrm{N}([\mathrm{H}]) \mathrm{C}([\mathrm{H}])([\mathrm{H}]) \mathrm{C}([\mathrm{H}])(\mathrm{C}([\mathrm{H}])([\mathrm{H}])[\mathrm{H}]) \mathrm{C}([\mathrm{H}])([\mathrm{H}])[\mathrm{H}]) \mathrm{C}([\mathrm{H}])(\mathrm{C}([\mathrm{H}])([\mathrm{H}])[\mathrm{H}]) \mathrm{C} \\
([\mathrm{H}])([\mathrm{H}])[\mathrm{H}]) \mathrm{C}([\mathrm{H}])([\mathrm{H}])[\mathrm{H}]) \mathrm{C}([\mathrm{H}])([\mathrm{H}]) \mathrm{C}([\mathrm{H}])(\mathrm{C}([\mathrm{H}])([\mathrm{H}])[\mathrm{H}]) \mathrm{C}([\mathrm{H}])([\mathrm{H}])[\mathrm{H}])[\mathrm{C} @ @]([\mathrm{H}] \\
)(\mathrm{N}([\mathrm{H}]) \mathrm{C}(=\mathrm{O})[\mathrm{C} @ @](([\mathrm{H}])(\mathrm{N}([\mathrm{H}]) \mathrm{C}(=\mathrm{O}) \mathrm{OC}(\mathrm{C}([\mathrm{H}])([\mathrm{H}])[\mathrm{H}])(\mathrm{C}([\mathrm{H}])([\mathrm{H}])[\mathrm{H}]) \mathrm{C}([\mathrm{H}])([\mathrm{H}])[\mathrm{H}] \\
) \mathrm{C}([\mathrm{H}])(\mathrm{C}([\mathrm{H}])([\mathrm{H}])[\mathrm{H}]) \mathrm{C}([\mathrm{H}])([\mathrm{H}])[\mathrm{H}]) \mathrm{C}([\mathrm{H}])([\mathrm{H}]) \mathrm{C}([\mathrm{H}])([\mathrm{H}])[\mathrm{S}] \mathrm{C}([\mathrm{H}])([\mathrm{H}])[\mathrm{H}]\end{array}$ & 5.097 & 3.649 \\
\hline Ext47 & $\begin{array}{l}\mathrm{C} 321 \mathrm{O}=\mathrm{C}(\mathrm{N}([\mathrm{H}])[\mathrm{C} @]([\mathrm{H}])(\mathrm{C}(=\mathrm{O}) \mathrm{N}([\mathrm{H}]) \mathrm{C}([\mathrm{H}])([\mathrm{H}]) \mathrm{C} 1=\mathrm{C}([\mathrm{H}]) \mathrm{C}([\mathrm{H}])=\mathrm{C}([\mathrm{H}]) \mathrm{C}([\mathrm{H}])=\mathrm{C} 1[\mathrm{H} \\
]) \mathrm{C}([\mathrm{H}])(\mathrm{C}([\mathrm{H}])([\mathrm{H}])[\mathrm{H}]) \mathrm{C}([\mathrm{H}])([\mathrm{H}])[\mathrm{H}])[\mathrm{C} @(([\mathrm{H}])(\mathrm{OC}([\mathrm{H}])([\mathrm{H}])[\mathrm{H}]) \mathrm{C}([\mathrm{H}])([\mathrm{H}])[\mathrm{C} @]([\mathrm{H}])( \\
\mathrm{O}[\mathrm{H}])[\mathrm{C} @ @]([\mathrm{H}])(\mathrm{N}([\mathrm{H}]) \mathrm{C}(=\mathrm{O}) \mathrm{C} 2=\mathrm{C}([\mathrm{H}]) \mathrm{C}(=\mathrm{C}([\mathrm{H}]) \mathrm{C}(=\mathrm{C} 2[\mathrm{H}]) \mathrm{C}(=\mathrm{O}) \mathrm{N}([\mathrm{H}])[\mathrm{C} @ @]([\mathrm{H}])(\mathrm{C} \\
3=\mathrm{C}([\mathrm{H}]) \mathrm{C}([\mathrm{H}])=\mathrm{C}([\mathrm{H}]) \mathrm{C}([\mathrm{H}])=\mathrm{C} 3[\mathrm{H}]) \mathrm{C}([\mathrm{H}])([\mathrm{H}])[\mathrm{H}]) \mathrm{N}(\mathrm{C}([\mathrm{H}])([\mathrm{H}])[\mathrm{S}](=\mathrm{O})(=\mathrm{O}) \mathrm{C}([\mathrm{H}])([\mathrm{H}]) \\
[\mathrm{H}]) \mathrm{C}([\mathrm{H}])([\mathrm{H}])[\mathrm{H}]) \mathrm{C}([\mathrm{H}])([\mathrm{H}]) \mathrm{O} / \mathrm{C} 4=\mathrm{C}(\mathrm{H}]) \mathrm{C}([\mathrm{H}])=\mathrm{C}([\mathrm{Br}]) \mathrm{C}([\mathrm{H}])=\mathrm{C} 4[\mathrm{H}]\end{array}$ & 3.125 & 2.623 \\
\hline Ext48 & $\begin{array}{l}\mathrm{C} 325 \mathrm{O}=\mathrm{C}(\mathrm{N}([\mathrm{H}]) \mathrm{C} 1=\mathrm{C}([\mathrm{H}]) \mathrm{C}([\mathrm{H}])=\mathrm{C}([\mathrm{F}]) \mathrm{C}([\mathrm{H}])=\mathrm{C} 1[\mathrm{H}])[\mathrm{C} @]([\mathrm{H}])(\mathrm{OC}([\mathrm{H}])([\mathrm{H}])[\mathrm{H}]) \mathrm{C}([\mathrm{H}]) \\
([\mathrm{H}])[\mathrm{C} @]([\mathrm{H}])(\mathrm{O}[\mathrm{H}])[\mathrm{C} @ @]([\mathrm{H}])(\mathrm{N}([\mathrm{H}]) \mathrm{C}(=\mathrm{O}) \mathrm{C} 2=\mathrm{C}([\mathrm{H}]) \mathrm{C}(=\mathrm{C}([\mathrm{H}]) \mathrm{C}(=\mathrm{C} 2[\mathrm{H}]) \mathrm{C}(=\mathrm{O}) \mathrm{N}([\mathrm{H} \\
])[\mathrm{C} @ @]([\mathrm{H}])(\mathrm{C} 3=\mathrm{C}([\mathrm{H}]) \mathrm{C}([\mathrm{H}])=\mathrm{C}([\mathrm{H}]) \mathrm{C}([\mathrm{H}])=\mathrm{C} 3[\mathrm{H}]) \mathrm{C}([\mathrm{H}])([\mathrm{H}])[\mathrm{H}]) \mathrm{N}(\mathrm{C}([\mathrm{H}])([\mathrm{H}])[\mathrm{S}](=\mathrm{O} \\
)(=\mathrm{O}) \mathrm{C}([\mathrm{H}])([\mathrm{H}])[\mathrm{H}]) \mathrm{C}([\mathrm{H}])([\mathrm{H}])[\mathrm{H}]) \mathrm{C}([\mathrm{H}])([\mathrm{H}]) \mathrm{O} / \mathrm{C} 4=\mathrm{C}(\backslash \mathrm{H}]) \mathrm{C}([\mathrm{F}])=\mathrm{C}([\mathrm{H}]) \mathrm{C}([\mathrm{F}])=\mathrm{C} 4[\mathrm{H}]\end{array}$ & 3.000 & 2.540 \\
\hline Ext49 & $\begin{array}{l}\mathrm{C} 328 \mathrm{O}=\mathrm{C}(\mathrm{N}([\mathrm{H}])[\mathrm{C} @]([\mathrm{H}])(\mathrm{C}(=\mathrm{O}) \mathrm{N}([\mathrm{H}])[\mathrm{C} @]([\mathrm{H}])([\mathrm{C} @]([\mathrm{H}])(\mathrm{O}[\mathrm{H}]) \mathrm{C}([\mathrm{H}])([\mathrm{H}])[\mathrm{C} @]([\mathrm{H}]) \\
(\mathrm{C}(=\mathrm{O}) \mathrm{N}([\mathrm{H}])[\mathrm{C} @([\mathrm{H}])(\mathrm{C}(=\mathrm{O}) \mathrm{N}([\mathrm{H}])[\mathrm{C} @]([\mathrm{H}])(\mathrm{C}(=\mathrm{O}) \mathrm{N}([\mathrm{H}])[\mathrm{C} @]([\mathrm{H}])(\mathrm{C}(=\mathrm{O}) \mathrm{O}[\mathrm{H}]) \mathrm{C}([\mathrm{H}]) \\
([\mathrm{H}]) \mathrm{C} 1=\mathrm{C}([\mathrm{H}]) \mathrm{C}([\mathrm{H}])=\mathrm{C}([\mathrm{H}]) \mathrm{C}([\mathrm{H}])=\mathrm{C} 1[\mathrm{H}]) \mathrm{C}([\mathrm{H}])([\mathrm{H}]) \mathrm{C}([\mathrm{H}])([\mathrm{H}]) \mathrm{C}(=\mathrm{O}) \mathrm{O}[\mathrm{H}]) \mathrm{C}([\mathrm{H}])([\mathrm{H}]) \\
[\mathrm{H}]) \mathrm{C}([\mathrm{H}])([\mathrm{H}])[\mathrm{H}]) \mathrm{C}([\mathrm{H}])([\mathrm{H}]) \mathrm{C}([\mathrm{H}])(\mathrm{C}([\mathrm{H}])([\mathrm{H}])[\mathrm{H}]) \mathrm{C}([\mathrm{H}])([\mathrm{H}])[\mathrm{H}]) \mathrm{C}([\mathrm{H}])([\mathrm{H}]) \mathrm{C}(=\mathrm{O}) \mathrm{N}([ \\
\mathrm{H}])[\mathrm{H}])[\mathrm{C} @ @]([\mathrm{H}])(\mathrm{N}([\mathrm{H}]) \mathrm{C}(=\mathrm{O})[\mathrm{C} @ @]([\mathrm{H}])(\mathrm{N}([\mathrm{H}])[\mathrm{H}]) \mathrm{C}([\mathrm{H}])([\mathrm{H}]) \mathrm{C}(=\mathrm{O}) \mathrm{O}[\mathrm{H}]) \mathrm{C}([\mathrm{H}])(\mathrm{C}( \\
[\mathrm{H}])([\mathrm{H}])[\mathrm{H}]) \mathrm{C}([\mathrm{H}])([\mathrm{H}])[\mathrm{H}]\end{array}$ & 4.000 & 4.150 \\
\hline Ext50 & $\begin{array}{l}\mathrm{C} 332 \mathrm{O}=\mathrm{C}(\mathrm{N}([\mathrm{H}]) \mathrm{C}(=\mathrm{NC}([\mathrm{H}])([\mathrm{H}]) \mathrm{C} 1=\mathrm{C}([\mathrm{H}]) \mathrm{C}([\mathrm{Cl}])=\mathrm{C}([\mathrm{Cl}]) \mathrm{C}([\mathrm{H}])=\mathrm{C} 1[\mathrm{H}]) \mathrm{N}([\mathrm{H}])[\mathrm{H}]) \mathrm{C}=2 \mathrm{C} \\
(=\mathrm{NOC}=2 \mathrm{C}([\mathrm{H}])([\mathrm{H}])[\mathrm{H}]) \mathrm{C} 3=\mathrm{C}([\mathrm{H}]) \mathrm{C}([\mathrm{H}])=\mathrm{C}([\mathrm{F}]) \mathrm{C}([\mathrm{H}])=\mathrm{C} 3[\mathrm{H}]\end{array}$ & 1.620 & 2.016 \\
\hline Ext51 & $\begin{array}{l}\mathrm{C} 334 \mathrm{O}=\mathrm{C}(\mathrm{N}([\mathrm{H}]) \mathrm{C}(=\mathrm{NC}([\mathrm{H}])([\mathrm{H}]) \mathrm{C} 1=\mathrm{C}([\mathrm{H}]) \mathrm{C}([\mathrm{Cl}])=\mathrm{C}([\mathrm{Cl}]) \mathrm{C}([\mathrm{H}])=\mathrm{C} 1[\mathrm{H}]) \mathrm{N}([\mathrm{H}])[\mathrm{H}]) \mathrm{C}=2 \mathrm{C} \\
(=\mathrm{NOC}=2 \mathrm{C}([\mathrm{H}])([\mathrm{H}])[\mathrm{H}]) \mathrm{C} 3=\mathrm{C}([\mathrm{H}]) \mathrm{C}([\mathrm{H}])=\mathrm{C}([\mathrm{H}]) \mathrm{C}([\mathrm{H}])=\mathrm{C} 3[\mathrm{H}]\end{array}$ & 1.056 & 1.861 \\
\hline Ext52 & $\begin{array}{l}\mathrm{C} 337 \mathrm{O}=\mathrm{C}(\mathrm{N}([\mathrm{H}])[\mathrm{C} @]([\mathrm{H}])(\mathrm{C}(=\mathrm{O}) \mathrm{N}([\mathrm{H}])[\mathrm{C} @]([\mathrm{H}])([\mathrm{C} @ @]([\mathrm{H}])(\mathrm{O}[\mathrm{H}]) \mathrm{C}([\mathrm{H}])([\mathrm{H}])[\mathrm{C} @]([ \\
\mathrm{H}])(\mathrm{C}(=\mathrm{O}) \mathrm{N}([\mathrm{H}])[\mathrm{C} @]([\mathrm{H}])(\mathrm{C}(=\mathrm{O}) \mathrm{N}([\mathrm{H}]) \mathrm{C}([\mathrm{H}])([\mathrm{H}]) \mathrm{C}([\mathrm{H}])(\mathrm{C}([\mathrm{H}])([\mathrm{H}])[\mathrm{H}]) \mathrm{C}([\mathrm{H}])([\mathrm{H}])[\mathrm{H}]) \\
\mathrm{C}([\mathrm{H}])(\mathrm{C}([\mathrm{H}])([\mathrm{H}])[\mathrm{H}]) \mathrm{C}([\mathrm{H}])([\mathrm{H}])[\mathrm{H}]) \mathrm{C}([\mathrm{H}])([\mathrm{H}])[\mathrm{H}]) \mathrm{C}([\mathrm{H}])([\mathrm{H}]) \mathrm{C}([\mathrm{H}])(\mathrm{C}([\mathrm{H}])([\mathrm{H}])[\mathrm{H}]) \mathrm{C}([ \\
\mathrm{H}])([\mathrm{H}])[\mathrm{H}]) \mathrm{C}([\mathrm{H}])([\mathrm{H}]) \mathrm{N}([\mathrm{H}]) \mathrm{C}(=\mathrm{O}) \mathrm{OC}(\mathrm{C}([\mathrm{H}])([\mathrm{H}])[\mathrm{H}])(\mathrm{C}([\mathrm{H}])([\mathrm{H}])[\mathrm{H}]) \mathrm{C}([\mathrm{H}])([\mathrm{H}])[\mathrm{H}])[\mathrm{C} \\
@]([\mathrm{H}])(\mathrm{C}([\mathrm{H}])([\mathrm{H}]) \mathrm{C}=1[\mathrm{~S}] \mathrm{C}([\mathrm{H}])=\mathrm{C}(\mathrm{N}=1) \mathrm{C}([\mathrm{H}])([\mathrm{H}])[\mathrm{H}]) \mathrm{C}([\mathrm{H}])(\mathrm{C}([\mathrm{H}])([\mathrm{H}])[\mathrm{H}]) \mathrm{C}([\mathrm{H}])([\mathrm{H}] \\
)[\mathrm{H}]\end{array}$ & 3.538 & 4.335 \\
\hline Ext53 & $\begin{array}{l}\mathrm{C} 341 \mathrm{O}=\mathrm{C}(\mathrm{N}([\mathrm{H}])[\mathrm{C} @ @]([\mathrm{H}])(\mathrm{C}(=\mathrm{O}) \mathrm{N}([\mathrm{H}])[\mathrm{C} @]([\mathrm{H}])([\mathrm{C} @]([\mathrm{H}])(\mathrm{O}[\mathrm{H}]) \mathrm{C}([\mathrm{H}])([\mathrm{H}])[\mathrm{C} @]([ \\
\mathrm{H}])(\mathrm{C}(=\mathrm{O}) \mathrm{N}([\mathrm{H}])[\mathrm{C} @]([\mathrm{H}])(\mathrm{C}(=\mathrm{O}) \mathrm{N}([\mathrm{H}]) \mathrm{C}([\mathrm{H}])([\mathrm{H}]) \mathrm{C} 1=\mathrm{C}([\mathrm{H}]) \mathrm{C}([\mathrm{H}])=\mathrm{C}([\mathrm{H}]) \mathrm{C}([\mathrm{H}])=\mathrm{C} 1[\mathrm{H}] \\
) \mathrm{C}([\mathrm{H}])(\mathrm{C}([\mathrm{H}])([\mathrm{H}])[\mathrm{H}]) \mathrm{C}([\mathrm{H}])([\mathrm{H}])[\mathrm{H}]) \mathrm{C}([\mathrm{H}])([\mathrm{H}]) \mathrm{C}([\mathrm{H}])(\mathrm{C}([\mathrm{H}])([\mathrm{H}])[\mathrm{H}]) \mathrm{C}([\mathrm{H}])([\mathrm{H}])[\mathrm{H}]) \mathrm{C}( \\
[\mathrm{H}])([\mathrm{H}]) \mathrm{C}([\mathrm{H}])(\mathrm{C}([\mathrm{H}])([\mathrm{H}])[\mathrm{H}]) \mathrm{C}([\mathrm{H}])([\mathrm{H}])[\mathrm{H}]) \mathrm{C}([\mathrm{H}])([\mathrm{H}]) \mathrm{C}([\mathrm{H}])([\mathrm{H}])[\mathrm{S}] \mathrm{C}([\mathrm{H}])([\mathrm{H}])[\mathrm{H}])[\mathrm{C} \\
@ @ @]([\mathrm{H}])(\mathrm{N}([\mathrm{H}]) \mathrm{C}(=\mathrm{O}) \mathrm{OC}(\mathrm{C}([\mathrm{H}])([\mathrm{H}])[\mathrm{H}])(\mathrm{C}([\mathrm{H}])([\mathrm{H}])[\mathrm{H}]) \mathrm{C}([\mathrm{H}])([\mathrm{H}])[\mathrm{H}]) \mathrm{C}([\mathrm{H}])(\mathrm{C}([\mathrm{H}])([ \\
\mathrm{H}])[\mathrm{H}]) \mathrm{C}([\mathrm{H}])([\mathrm{H}])[\mathrm{H}]\end{array}$ & 0.979 & 2.564 \\
\hline Ext54 & $\begin{array}{l}\mathrm{C} 344 \mathrm{O}=\mathrm{C}(\mathrm{N}([\mathrm{H}])[\mathrm{C} @ @]([\mathrm{H}])(\mathrm{C}(=\mathrm{O}) \mathrm{N}([\mathrm{H}])[\mathrm{C} @]([\mathrm{H}])([\mathrm{C} @]([\mathrm{H}])(\mathrm{O}[\mathrm{H}]) \mathrm{C}([\mathrm{H}])([\mathrm{H}])[\mathrm{C} @]([ \\
\mathrm{H}])(\mathrm{C}(=\mathrm{O}) \mathrm{N}([\mathrm{H}])[\mathrm{C} @]([\mathrm{H}])(\mathrm{C}(=\mathrm{O}) \mathrm{N}([\mathrm{H}]) \mathrm{C}([\mathrm{H}])([\mathrm{H}]) \mathrm{C}=\mathrm{C}([\mathrm{H}]) \mathrm{C}([\mathrm{H}])=\mathrm{C}([\mathrm{H}]) \mathrm{C}([\mathrm{H}])=\mathrm{C} 1[\mathrm{H}] \\
) \mathrm{C}([\mathrm{H}])(\mathrm{C}([\mathrm{H}])([\mathrm{H}])[\mathrm{H}]) \mathrm{C}([\mathrm{H}])([\mathrm{H}])[\mathrm{H}]) \mathrm{C}([\mathrm{H}])([\mathrm{H}])[\mathrm{H}]) \mathrm{C}([\mathrm{H}])([\mathrm{H}]) \mathrm{C}([\mathrm{H}])(\mathrm{C}([\mathrm{H}])([\mathrm{H}])[\mathrm{H}]) \mathrm{C}( \\
[\mathrm{H}])([\mathrm{H}])[\mathrm{H}]) \mathrm{C}([\mathrm{H}])([\mathrm{H}])[\mathrm{S}] \mathrm{C}([\mathrm{H}])([\mathrm{H}])[\mathrm{H}])[\mathrm{C} @ @]([\mathrm{H}])(\mathrm{N}([\mathrm{H}]) \mathrm{C}(=\mathrm{O}) \mathrm{OC}(\mathrm{C}([\mathrm{H}])([\mathrm{H}])[\mathrm{H}])(\mathrm{C} \\
([\mathrm{H}])([\mathrm{H}])[\mathrm{H}]) \mathrm{C}([\mathrm{H}])([\mathrm{H}])[\mathrm{H}]) \mathrm{C}([\mathrm{H}])(\mathrm{C}([\mathrm{H}])([\mathrm{H}])[\mathrm{H}]) \mathrm{C}([\mathrm{H}])([\mathrm{H}])[\mathrm{H}]\end{array}$ & 3.301 & 2.442 \\
\hline Ext55 & $\begin{array}{l}\mathrm{C} 348 \mathrm{O}=\mathrm{C}(\mathrm{OC}(\mathrm{C}([\mathrm{H}])([\mathrm{H}])[\mathrm{H}])(\mathrm{C}([\mathrm{H}])([\mathrm{H}])[\mathrm{H}]) \mathrm{C}([\mathrm{H}])([\mathrm{H}])[\mathrm{H}]) \mathrm{N}([\mathrm{H}])[\mathrm{C} @ @]([\mathrm{H}])(\mathrm{C}(=\mathrm{O}) \mathrm{N}( \\
[\mathrm{H}])[\mathrm{C} @]([\mathrm{H}])([\mathrm{C} @]([\mathrm{H}])(\mathrm{O}[\mathrm{H}]) \mathrm{C}([\mathrm{H}])([\mathrm{H}])[\mathrm{C} @]([\mathrm{H}])(\mathrm{C}(=\mathrm{O}) \mathrm{N}([\mathrm{H}])[\mathrm{C} @]([\mathrm{H}])(\mathrm{C}(=\mathrm{O}) \mathrm{N}([\mathrm{H}]\end{array}$ & 3.229 & 2.094 \\
\hline
\end{tabular}




\begin{tabular}{|c|c|c|c|}
\hline & $\begin{array}{l}) \mathrm{C}([\mathrm{H}])([\mathrm{H}]) \mathrm{C} 1=\mathrm{C}([\mathrm{H}]) \mathrm{C}([\mathrm{H}])=\mathrm{C}([\mathrm{H}]) \mathrm{C}([\mathrm{H}])=\mathrm{C} 1[\mathrm{H}]) \mathrm{C}([\mathrm{H}])(\mathrm{C}([\mathrm{H}])([\mathrm{H}])[\mathrm{H}]) \mathrm{C}([\mathrm{H}])([\mathrm{H}])[\mathrm{H}]) \\
\mathrm{C}([\mathrm{H}])([\mathrm{H}])[\mathrm{H}]) \mathrm{C}([\mathrm{H}])([\mathrm{H}]) \mathrm{C}([\mathrm{H}])(\mathrm{C}([\mathrm{H}])([\mathrm{H}])(\mathrm{H}]) \mathrm{C}([\mathrm{H}])([\mathrm{H}])[\mathrm{H}]) \mathrm{C}([\mathrm{H}])([\mathrm{H}]) \mathrm{C}([\mathrm{H}])([\mathrm{H}])[\mathrm{S}] \\
\mathrm{C}([\mathrm{H}])([\mathrm{H}])[\mathrm{H}]\end{array}$ & & \\
\hline Ext56 & $\begin{array}{l}\mathrm{C} 351 \mathrm{O}=\mathrm{C} 1 \mathrm{~N}(/ \mathrm{C}(=\mathrm{N} \backslash[\mathrm{H}]) \mathrm{N}([\mathrm{H}])[\mathrm{C} @] 1(\mathrm{C}([\mathrm{H}])([\mathrm{H}]) \mathrm{C}([\mathrm{H}])([\mathrm{H}])[\mathrm{C} @ @] 2([\mathrm{H}]) \mathrm{C}([\mathrm{H}])([\mathrm{H}]) \mathrm{C}([\mathrm{H} \\
])([\mathrm{H}]) \mathrm{C}([\mathrm{H}])([\mathrm{H}]) \mathrm{C}([\mathrm{H}])([\mathrm{H}]) \mathrm{C} 2([\mathrm{H}])[\mathrm{H}]) \mathrm{C}([\mathrm{H}])([\mathrm{H}])[\mathrm{C} @ 4([\mathrm{H}]) \mathrm{C}([\mathrm{H}])([\mathrm{H}])(\mathrm{C} @]([\mathrm{H}])(\mathrm{N}([ \\
\mathrm{H}]) \mathrm{C}(=\mathrm{O}) \mathrm{C} 3=\mathrm{C}([\mathrm{H}]) \mathrm{C}([\mathrm{H}])=\mathrm{NC}([\mathrm{H}])=\mathrm{C} 3[\mathrm{H}]) \mathrm{C}([\mathrm{H}])([\mathrm{H}]) \mathrm{C}([\mathrm{H}])([\mathrm{H}]) \mathrm{C} 4([\mathrm{H}])[\mathrm{H}]) \mathrm{C}([\mathrm{H}])([\mathrm{H}])[ \\
\mathrm{H}]\end{array}$ & 3.409 & 3.545 \\
\hline Ext57 & $\begin{array}{l}\mathrm{C} 355 \mathrm{O}=\mathrm{C} 1 \mathrm{~N}(/ \mathrm{C}(=\mathrm{N} \backslash[\mathrm{H}]) \mathrm{N}([\mathrm{H}])[\mathrm{C} @] 1(\mathrm{C}([\mathrm{H}])([\mathrm{H}]) \mathrm{C}([\mathrm{H}])([\mathrm{H}])[\mathrm{C} @ @] 2([\mathrm{H}]) \mathrm{C}([\mathrm{H}])([\mathrm{H}]) \mathrm{C}([\mathrm{H} \\
])([\mathrm{H}]) \mathrm{C}([\mathrm{H}])([\mathrm{H}]) \mathrm{C}([\mathrm{H}])([\mathrm{H}]) \mathrm{C} 2([\mathrm{H}])[\mathrm{H}]) \mathrm{C}([\mathrm{H}])([\mathrm{H}])[\mathrm{C} @] 4([\mathrm{H}]) \mathrm{C}([\mathrm{H}])([\mathrm{H}])(\mathrm{C} @]([\mathrm{H}])(\mathrm{N}([ \\
\mathrm{H}])[\mathrm{C} @] 3([\mathrm{H}]) \mathrm{C}([\mathrm{H}])([\mathrm{H}]) \mathrm{C}([\mathrm{H}])([\mathrm{H}]) \mathrm{C}([\mathrm{H}])([\mathrm{H}]) \mathrm{C} 3([\mathrm{H}])[\mathrm{H}]) \mathrm{C}([\mathrm{H}])([\mathrm{H}]) \mathrm{C}([\mathrm{H}])([\mathrm{H}]) \mathrm{C} 4([\mathrm{H}]) \\
[\mathrm{H}]) \mathrm{C}([\mathrm{H}])([\mathrm{H}])[\mathrm{H}]\end{array}$ & 3.367 & 3.801 \\
\hline Ext58 & $\begin{array}{l}\mathrm{C} 359 \mathrm{O}=\mathrm{C}(\mathrm{N}([\mathrm{H}])[\mathrm{C} @]([\mathrm{H}])(\mathrm{C}(=\mathrm{O}) \mathrm{N}([\mathrm{H}])[\mathrm{C} @]([\mathrm{H}])([\mathrm{C} @ @]([\mathrm{H}])(\mathrm{O}[\mathrm{H}]) \mathrm{C}([\mathrm{H}])([\mathrm{H}])[\mathrm{C} @]([ \\
\mathrm{H}])(\mathrm{C}(=\mathrm{O}) \mathrm{N}([\mathrm{H}])[\mathrm{C} @]([\mathrm{H}])(\mathrm{C}(=\mathrm{O}) \mathrm{N}([\mathrm{H}]) \mathrm{C}([\mathrm{H}])([\mathrm{H}]) \mathrm{C}([\mathrm{H}])(\mathrm{C}([\mathrm{H}])([\mathrm{H}])[\mathrm{H}]) \mathrm{C}([\mathrm{H}])([\mathrm{H}])[\mathrm{H}]) \\
\mathrm{C}([\mathrm{H}])(\mathrm{C}([\mathrm{H}])([\mathrm{H}])[\mathrm{H}]) \mathrm{C}([\mathrm{H}])([\mathrm{H}])(\mathrm{H}]) \mathrm{C}([\mathrm{H}])([\mathrm{H}])[\mathrm{H}]) \mathrm{C}([\mathrm{H}])([\mathrm{H}]) \mathrm{C}([\mathrm{H}])(\mathrm{C}([\mathrm{H}])([\mathrm{H}])[\mathrm{H}]) \mathrm{C}([ \\
\mathrm{H}])([\mathrm{H}])[\mathrm{H}]) \mathrm{C}([\mathrm{H}])([\mathrm{H}]) \mathrm{N}([\mathrm{H}]) \mathrm{C}(=\mathrm{O}) \mathrm{OC}(\mathrm{C}([\mathrm{H}])([\mathrm{H}])[\mathrm{H}])(\mathrm{C}([\mathrm{H}])([\mathrm{H}])[\mathrm{H}]) \mathrm{C}([\mathrm{H}])([\mathrm{H}])[\mathrm{H}])[\mathrm{C} \\
@ @]([\mathrm{H}])(\mathrm{C}([\mathrm{H}])([\mathrm{H}]) \mathrm{N} 1 \mathrm{~N}=\mathrm{C}(\mathrm{C}([\mathrm{H}])=\mathrm{C} 1 \mathrm{C}([\mathrm{H}])([\mathrm{H}])[\mathrm{H}]) \mathrm{C}([\mathrm{H}])([\mathrm{H}])[\mathrm{H}]) \mathrm{C}([\mathrm{H}])([\mathrm{H}])[\mathrm{H}]\end{array}$ & 1.785 & 3.338 \\
\hline Ext59 & 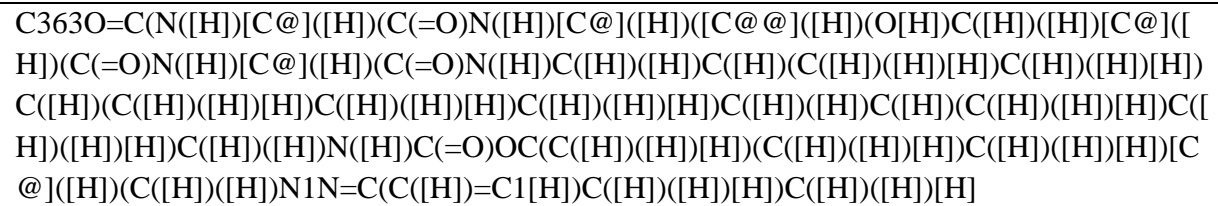 & 2.697 & 3.251 \\
\hline
\end{tabular}

${ }^{1}$ Experimental inhibitory activity; ${ }^{2}$ Predicted inhibitory activity

Table S2: SMILES, experimental and predicted inhibitory activity of training, test, calibration and external sets used in QSAR model with considering cycling rings

\begin{tabular}{|c|c|c|c|}
\hline \multirow{2}{*}{ Set } & \multirow{2}{*}{ SMILES } & \multicolumn{2}{|c|}{$P k_{i}$} \\
\hline & & ${ }^{1}$ Obs & ${ }^{2}$ Pred \\
\hline \multicolumn{4}{|c|}{ Training set } \\
\hline $\operatorname{Tr} 1$ & $\begin{array}{l}\mathrm{O}=\mathrm{C} 1 \mathrm{OC}([\mathrm{H}])([\mathrm{H}]) \mathrm{C}([\mathrm{H}])([\mathrm{H}]) \mathrm{C}([\mathrm{H}])([\mathrm{H}]) \mathrm{C}([\mathrm{H}])([\mathrm{H}]) \mathrm{N}([\mathrm{H}]) \mathrm{C}(=\mathrm{O}) \mathrm{C}([\mathrm{H}])([\mathrm{H}])[\mathrm{C} @ \\
]([\mathrm{H}])(\mathrm{N}([\mathrm{H}]) \mathrm{C}(=\mathrm{O})[\mathrm{C} @ @]([\mathrm{H}])(\mathrm{N} 1[\mathrm{H}]) \mathrm{C}([\mathrm{H}])(\mathrm{C}([\mathrm{H}])([\mathrm{H}])[\mathrm{H}]) \mathrm{C}([\mathrm{H}])([\mathrm{H}])[\mathrm{H}]) \mathrm{C}(= \\
\mathrm{O}) \mathrm{N}([\mathrm{H}])[\mathrm{C} @ @]([\mathrm{H}])(\mathrm{C}([\mathrm{H}])([\mathrm{H}]) \mathrm{C}([\mathrm{H}])(\mathrm{C}([\mathrm{H}])([\mathrm{H}])[\mathrm{H}]) \mathrm{C}([\mathrm{H}])([\mathrm{H}])[\mathrm{H}])[\mathrm{C} @ @]([ \\
\mathrm{H}])(\mathrm{O}[\mathrm{H}]) \mathrm{C}([\mathrm{H}])([\mathrm{H}])[\mathrm{C} @]([\mathrm{H}])(\end{array}$ & 2.662 & 3.194 \\
\hline $\operatorname{Tr} 2$ & $\begin{array}{l}\mathrm{O}=\mathrm{C} 1 \mathrm{OC}([\mathrm{H}])([\mathrm{H}]) \mathrm{C}([\mathrm{H}])([\mathrm{H}]) \mathrm{C}([\mathrm{H}])=\mathrm{C}([\mathrm{H}]) \mathrm{C}([\mathrm{H}])([\mathrm{H}]) \mathrm{N}([\mathrm{H}]) \mathrm{C}(=\mathrm{O}) \mathrm{C}([\mathrm{H}])([\mathrm{H}])[ \\
\mathrm{C} @]([\mathrm{H}])(\mathrm{N}([\mathrm{H}]) \mathrm{C}(=\mathrm{O})[\mathrm{C} @ @]([\mathrm{H}])(\mathrm{N} 1[\mathrm{H}]) \mathrm{C}([\mathrm{H}])(\mathrm{C}([\mathrm{H}])([\mathrm{H}])[\mathrm{H}]) \mathrm{C}([\mathrm{H}])([\mathrm{H}])[\mathrm{H}]) \\
\mathrm{C}(=\mathrm{O}) \mathrm{N}([\mathrm{H}])[\mathrm{C} @ @]([\mathrm{H}])(\mathrm{C}([\mathrm{H}])([\mathrm{H}]) \mathrm{C}([\mathrm{H}])(\mathrm{C}([\mathrm{H}])([\mathrm{H}])[\mathrm{H}]) \mathrm{C}([\mathrm{H}])([\mathrm{H}])[\mathrm{H}])[\mathrm{C} @ \\
@]([\mathrm{H}])(\mathrm{O}[\mathrm{H}]) \mathrm{C}([\mathrm{H}])([\mathrm{H}])[\mathrm{C} @]([\mathrm{H}]\end{array}$ & 3.547 & 3.119 \\
\hline $\operatorname{Tr} 3$ & $\begin{array}{l}\mathrm{O}=\mathrm{C} 2 \mathrm{~N}([\mathrm{H}])[\mathrm{C} @]([\mathrm{H}])(\mathrm{C}(=\mathrm{O}) \mathrm{N}([\mathrm{H}])[\mathrm{C} @ @]([\mathrm{H}])(\mathrm{C}([\mathrm{H}])([\mathrm{H}]) \mathrm{C}([\mathrm{H}])(\mathrm{C}([\mathrm{H}])([\mathrm{H}])[ \\
\mathrm{H}]) \mathrm{C}([\mathrm{H}])([\mathrm{H}])[\mathrm{H}])[\mathrm{C} @ @]([\mathrm{H}])(\mathrm{O}[\mathrm{H}]) \mathrm{C}([\mathrm{H}])([\mathrm{H}])[\mathrm{C} @]([\mathrm{H}])(\mathrm{C}(=\mathrm{O}) \mathrm{N}([\mathrm{H}])[\mathrm{C} @([\mathrm{H} \\
])(\mathrm{C}(=\mathrm{O}) \mathrm{N}([\mathrm{H}]) \mathrm{C}([\mathrm{H}])([\mathrm{H}]) \mathrm{C}=1 \mathrm{C}([\mathrm{H}])=\mathrm{C}([\mathrm{H}]) \mathrm{C}([\mathrm{H}])=\mathrm{C}([\mathrm{H}]) \mathrm{C}=1[\mathrm{H}]) \mathrm{C}([\mathrm{H}])(\mathrm{C}([\mathrm{H}]) \\
([\mathrm{H}])[\mathrm{H}]) \mathrm{C}([\mathrm{H}])([\mathrm{H}])[\mathrm{H}]) \mathrm{C}([\mathrm{H}])([\mathrm{H}]\end{array}$ & 2.966 & 3.168 \\
\hline
\end{tabular}




\begin{tabular}{|c|c|c|c|}
\hline $\operatorname{Tr} 4$ & $\begin{array}{l}\mathrm{O}=\mathrm{C}(\mathrm{OC}([\mathrm{H}])([\mathrm{H}]) \mathrm{N} 1 \mathrm{~N}=\mathrm{C}(\mathrm{C}([\mathrm{H}])=\mathrm{C} 1 \mathrm{C}([\mathrm{H}])([\mathrm{H}])[\mathrm{H}]) \mathrm{C}([\mathrm{H}])([\mathrm{H}])[\mathrm{H}]) \mathrm{N}([\mathrm{H}])[\mathrm{C} @]([ \\
\mathrm{H}])(\mathrm{C}(=\mathrm{O}) \mathrm{N}([\mathrm{H}])[\mathrm{C} @ @]([\mathrm{H}])(\mathrm{C}([\mathrm{H}])([\mathrm{H}]) \mathrm{C}([\mathrm{H}])(\mathrm{C}([\mathrm{H}])([\mathrm{H}])[\mathrm{H}]) \mathrm{C}([\mathrm{H}])([\mathrm{H}])[\mathrm{H}])[ \\
\mathrm{C} @ @]([\mathrm{H}])(\mathrm{O}[\mathrm{H}]) \mathrm{C}([\mathrm{H}])([\mathrm{H}])[\mathrm{C} @]([\mathrm{H}])(\mathrm{C}(=\mathrm{O}) \mathrm{N}([\mathrm{H}])[\mathrm{C} @]([\mathrm{H}])(\mathrm{C}(=\mathrm{O}) \mathrm{N}([\mathrm{H}]) \mathrm{C}([ \\
\mathrm{H}])([\mathrm{H}]) \mathrm{C}=2 \mathrm{C}([\mathrm{H}])=\mathrm{C}([\mathrm{H}]) \mathrm{C}([\mathrm{H}])=\mathrm{C}([\end{array}$ & 3.854 & 2.834 \\
\hline $\operatorname{Tr} 5$ & $\begin{array}{l}\mathrm{O}=\mathrm{C}(\mathrm{OC}([\mathrm{H}])([\mathrm{H}]) \mathrm{N} 1 \mathrm{~N}=\mathrm{C}(\mathrm{C}([\mathrm{H}])=\mathrm{C} 1 \mathrm{C}([\mathrm{H}])([\mathrm{H}])(\mathrm{H}]) \mathrm{C}([\mathrm{H}])([\mathrm{H}])[\mathrm{H}]) \mathrm{N}([\mathrm{H}])[\mathrm{C} @]([ \\
\mathrm{H}])(\mathrm{C}(=\mathrm{O}) \mathrm{N}([\mathrm{H}])[\mathrm{C} @ @](([\mathrm{H}])(\mathrm{C}([\mathrm{H}])([\mathrm{H}]) \mathrm{C}([\mathrm{H}])(\mathrm{C}([\mathrm{H}])([\mathrm{H}])[\mathrm{H}]) \mathrm{C}([\mathrm{H}])([\mathrm{H}])[\mathrm{H}])[ \\
\mathrm{C} @ @]([\mathrm{H}])(\mathrm{O}[\mathrm{H}]) \mathrm{C}([\mathrm{H}])([\mathrm{H}])[\mathrm{C} @]([\mathrm{H}])(\mathrm{C}(=\mathrm{O}) \mathrm{N}([\mathrm{H}])[\mathrm{C} @]([\mathrm{H}])(\mathrm{C}(=\mathrm{O}) \mathrm{N}([\mathrm{H}]) \mathrm{C}([ \\
\mathrm{H}])([\mathrm{H}]) \mathrm{C}([\mathrm{H}])(\mathrm{C}([\mathrm{H}])([\mathrm{H}])[\mathrm{H}]) \mathrm{C}([\mathrm{H}\end{array}$ & 5.523 & 3.702 \\
\hline Tr6 & $\begin{array}{l}[\mathrm{F}] / \mathrm{C} 1=\mathrm{C}(\backslash \mathrm{H}]) \mathrm{C}([\mathrm{H}])=\mathrm{C}(\mathrm{C}([\mathrm{H}])=\mathrm{C} 1[\mathrm{H}])[\mathrm{C} @]([\mathrm{H}])(\mathrm{N}([\mathrm{H}]) \mathrm{C}(=\mathrm{O}) \mathrm{C} 2=\mathrm{C}([\mathrm{H}]) \mathrm{C}(=\mathrm{C}([ \\
\mathrm{H}]) \mathrm{C}(=\mathrm{C} 2[\mathrm{H}]) \mathrm{C} 3=\mathrm{NN}=\mathrm{C}(\mathrm{O} 3)[\mathrm{C} @ @](\mathrm{C}([\mathrm{H}])([\mathrm{H}])[\mathrm{H}])(\mathrm{C}([\mathrm{H}])([\mathrm{H}]) \mathrm{C}=4 \mathrm{C}([\mathrm{H}])=\mathrm{C}([\mathrm{H} \\
]) \mathrm{C}([\mathrm{H}])=\mathrm{C}([\mathrm{H}]) \mathrm{C}=4[\mathrm{H}])[\mathrm{N}+]([\mathrm{H}])([\mathrm{H}])[\mathrm{H}]) \mathrm{N}(\mathrm{C}([\mathrm{H}])([\mathrm{H}])[\mathrm{H}])[\mathrm{S}](=\mathrm{O})(=\mathrm{O}) \mathrm{C}([\mathrm{H}])([ \\
\mathrm{H}])[\mathrm{H}]) \mathrm{C}([\mathrm{H}])([\mathrm{H}])[\mathrm{H}]\end{array}$ & 3.373 & 2.564 \\
\hline $\operatorname{Tr} 7$ & $\begin{array}{l}\mathrm{O}=[\mathrm{S}](=\mathrm{O})(\mathrm{N}(\mathrm{C}=2 \mathrm{C}([\mathrm{H}])=\mathrm{C}(\mathrm{C}([\mathrm{H}])=\mathrm{C}([\mathrm{H}])[\mathrm{C} @ @] 1([\mathrm{H}]) \mathrm{C}([\mathrm{H}])([\mathrm{H}])[\mathrm{C} @] 1([\mathrm{H}]) \mathrm{C}( \\
[\mathrm{H}])([\mathrm{H}])[\mathrm{H}]) \mathrm{C}([\mathrm{H}])=\mathrm{C}(\mathrm{C}=2[\mathrm{H}]) \mathrm{C} 3=\mathrm{NN}=\mathrm{C}(\mathrm{O} 3)[\mathrm{C} @ @](\mathrm{C}([\mathrm{H}])([\mathrm{H}])[\mathrm{H}])(\mathrm{C}([\mathrm{H}])([\mathrm{H}] \\
) \mathrm{C}=4 \mathrm{C}([\mathrm{H}])=\mathrm{C}([\mathrm{H}]) \mathrm{C}([\mathrm{H}])=\mathrm{C}([\mathrm{H}]) \mathrm{C}=4[\mathrm{H}])[\mathrm{N}+]([\mathrm{H}])([\mathrm{H}])[\mathrm{H}]) \mathrm{C}([\mathrm{H}])([\mathrm{H}]) \mathrm{C}([\mathrm{H}])([\mathrm{H} \\
]) \mathrm{C}([\mathrm{H}])([\mathrm{H}])[\mathrm{H}]) \mathrm{C}([\mathrm{H}])([\mathrm{H}])[\mathrm{H}]\end{array}$ & 3.285 & 2.999 \\
\hline $\operatorname{Tr} 8$ & $\begin{array}{l}\mathrm{O}=\mathrm{C}(\mathrm{C}=1 \mathrm{C}([\mathrm{H}])=\mathrm{C}(\mathrm{C}([\mathrm{H}])=\mathrm{C}(\mathrm{C}=1[\mathrm{H}]) \mathrm{C}(=\mathrm{O}) \mathrm{N}([\mathrm{H}])[\mathrm{C} @ @]([\mathrm{H}])(\mathrm{C}([\mathrm{H}])([\mathrm{H}]) \mathrm{C}([\mathrm{H}] \\
)(\mathrm{C}([\mathrm{H}])([\mathrm{H}])[\mathrm{H}]) \mathrm{C}([\mathrm{H}])([\mathrm{H}])[\mathrm{H}])[\mathrm{C} @ @]([\mathrm{H}])(\mathrm{O}[\mathrm{H}]) \mathrm{C}([\mathrm{H}])([\mathrm{H}])[\mathrm{C} @([\mathrm{H}])(\mathrm{C}(=\mathrm{O}) \mathrm{N} \\
([\mathrm{H}])[\mathrm{C} @]([\mathrm{H}])(\mathrm{C}(=\mathrm{O}) \mathrm{N}([\mathrm{H}]) \mathrm{C}([\mathrm{H}])(\mathrm{C}([\mathrm{H}])([\mathrm{H}])[\mathrm{H}]) \mathrm{C}([\mathrm{H}])([\mathrm{H}])[\mathrm{H}]) \mathrm{C}([\mathrm{H}])(\mathrm{C}([\mathrm{H}])( \\
[\mathrm{H}])[\mathrm{H}]) \mathrm{C}([\mathrm{H}])([\mathrm{H}])[\mathrm{H}]) \mathrm{C}([\mathrm{H}])([\end{array}$ & 2.866 & 3.390 \\
\hline $\operatorname{Tr} 9$ & $\begin{array}{l}\mathrm{O}=\mathrm{C}(\mathrm{N}(\mathrm{C}([\mathrm{H}])([\mathrm{H}])[\mathrm{H}])[\mathrm{C} @ @] 1([\mathrm{H}]) \mathrm{C}([\mathrm{H}])([\mathrm{H}]) \mathrm{C}([\mathrm{H}])([\mathrm{H}]) \mathrm{C}([\mathrm{H}])([\mathrm{H}]) \mathrm{C}([\mathrm{H}])([\mathrm{H}] \\
) \mathrm{C} 1([\mathrm{H}])[\mathrm{H}]) \mathrm{C}([\mathrm{H}])([\mathrm{H}]) \mathrm{C}([\mathrm{H}])([\mathrm{H}])[\mathrm{C} @ @]([\mathrm{H}])(\mathrm{N} 4 \mathrm{C}(=\mathrm{NC}=3 \mathrm{C}([\mathrm{H}])=\mathrm{C}([\mathrm{H}]) \mathrm{C}(\mathrm{OC} \\
=2 \mathrm{C}([\mathrm{H}])=\mathrm{C}([\mathrm{H}]) \mathrm{C}([\mathrm{H}])=\mathrm{C}([\mathrm{H}]) \mathrm{C}=2[\mathrm{H}])=\mathrm{C}([\mathrm{H}]) \mathrm{C}=3 \mathrm{C} 4([\mathrm{H}])[\mathrm{H}]) \mathrm{N}([\mathrm{H}])[\mathrm{H}])[\mathrm{C} @] 5([ \\
\mathrm{H}]) \mathrm{C}([\mathrm{H}])([\mathrm{H}]) \mathrm{C}([\mathrm{H}])([\mathrm{H}]) \mathrm{C}([\mathrm{H}])([\mathrm{H}\end{array}$ & 3.523 & 2.984 \\
\hline $\operatorname{Tr} 10$ & $\begin{array}{l}\mathrm{O}=\mathrm{C}(\mathrm{N}([\mathrm{H}])[\mathrm{C} @]([\mathrm{H}])([\mathrm{C} @ @]([\mathrm{H}])(\mathrm{O}[\mathrm{H}]) \mathrm{C}([\mathrm{H}])([\mathrm{H}])[\mathrm{C} @]([\mathrm{H}])(\mathrm{C}(=\mathrm{O}) \mathrm{N}([\mathrm{H}])[\mathrm{C} @ \\
]([\mathrm{H}])(\mathrm{C}(=\mathrm{O}) \mathrm{N}([\mathrm{H}]) \mathrm{C}([\mathrm{H}])([\mathrm{H}]) \mathrm{C} 1=\mathrm{C}([\mathrm{H}]) \mathrm{C}([\mathrm{H}])=\mathrm{C}([\mathrm{H}]) \mathrm{C}([\mathrm{H}])=\mathrm{C} 1[\mathrm{H}]) \mathrm{C}([\mathrm{H}])([\mathrm{H}]) \\
[\mathrm{H}]) \mathrm{C}([\mathrm{H}])([\mathrm{H}])[\mathrm{H}]) \mathrm{C}([\mathrm{H}])([\mathrm{H}]) \mathrm{C}([\mathrm{H}])(\mathrm{C}([\mathrm{H}])([\mathrm{H}])[\mathrm{H}]) \mathrm{C}([\mathrm{H}])([\mathrm{H}])[\mathrm{H}])[\mathrm{C} @ @]([\mathrm{H}]) \\
(\mathrm{N}([\mathrm{H}]) \mathrm{C}(=\mathrm{O}) \mathrm{OC}(\mathrm{C}([\mathrm{H}])([\mathrm{H}])[\mathrm{H}])\end{array}$ & 0.649 & 2.144 \\
\hline $\operatorname{Tr} 11$ & $\begin{array}{l}\mathrm{O}=\mathrm{C}(\mathrm{N}([\mathrm{H}])[\mathrm{C} @]([\mathrm{H}])([\mathrm{C} @ @]([\mathrm{H}])(\mathrm{O}[\mathrm{H}]) \mathrm{C}([\mathrm{H}])([\mathrm{H}])[\mathrm{C} @]([\mathrm{H}])(\mathrm{C}(=\mathrm{O}) \mathrm{N}([\mathrm{H}])[\mathrm{C} @ \\
]([\mathrm{H}])(\mathrm{C}(=\mathrm{O}) \mathrm{N}([\mathrm{H}]) \mathrm{C}([\mathrm{H}])([\mathrm{H}]) \mathrm{C} 1=\mathrm{C}([\mathrm{H}]) \mathrm{C}([\mathrm{H}])=\mathrm{C}([\mathrm{H}]) \mathrm{C}([\mathrm{H}])=\mathrm{C} 1[\mathrm{H}]) \mathrm{C}([\mathrm{H}])(\mathrm{C}([ \\
\mathrm{H}])([\mathrm{H}])[\mathrm{H}]) \mathrm{C}([\mathrm{H}])([\mathrm{H}])[\mathrm{H}]) \mathrm{C}([\mathrm{H}])([\mathrm{H}])[\mathrm{H}]) \mathrm{C}([\mathrm{H}])([\mathrm{H}]) \mathrm{C}([\mathrm{H}])(\mathrm{C}([\mathrm{H}])([\mathrm{H}])[\mathrm{H}]) \mathrm{C}([ \\
\mathrm{H}])([\mathrm{H}])[\mathrm{H}])[\mathrm{C} @ @]([\mathrm{H}])(\mathrm{N}([\mathrm{H}]) \mathrm{C}\end{array}$ & 1.504 & 2.192 \\
\hline $\operatorname{Tr} 12$ & $\begin{array}{l}\mathrm{O}=\mathrm{C}(\mathrm{N}([\mathrm{H}])[\mathrm{C} @]([\mathrm{H}])([\mathrm{C} @ @]([\mathrm{H}])(\mathrm{O}[\mathrm{H}]) \mathrm{C}([\mathrm{H}])([\mathrm{H}])[\mathrm{C} @]([\mathrm{H}])(\mathrm{C}(=\mathrm{O}) \mathrm{N}([\mathrm{H}])[\mathrm{C} @ \\
]([\mathrm{H}])(\mathrm{C}(=\mathrm{O}) \mathrm{N}([\mathrm{H}]) \mathrm{C}([\mathrm{H}])([\mathrm{H}]) \mathrm{C} 1=\mathrm{C}([\mathrm{H}]) \mathrm{C}([\mathrm{H}])=\mathrm{C}([\mathrm{H}]) \mathrm{C}([\mathrm{H}])=\mathrm{C} 1[\mathrm{H}]) \mathrm{C}([\mathrm{H}])([\mathrm{H}]) \\
[\mathrm{H}]) \mathrm{C}([\mathrm{H}])([\mathrm{H}])[\mathrm{H}]) \mathrm{C}([\mathrm{H}])([\mathrm{H}]) \mathrm{C}([\mathrm{H}])(\mathrm{C}([\mathrm{H}])([\mathrm{H}])[\mathrm{H}]) \mathrm{C}([\mathrm{H}])([\mathrm{H}])[\mathrm{H}])[\mathrm{C} @ @]([\mathrm{H}]) \\
(\mathrm{N}([\mathrm{H}]) \mathrm{C}(=\mathrm{O})[\mathrm{C} @ @]([\mathrm{H}])(\mathrm{N}([\mathrm{H}]) \mathrm{C}\end{array}$ & 3.212 & 3.001 \\
\hline $\operatorname{Tr} 13$ & $\begin{array}{l}\mathrm{O}=\mathrm{C}(\mathrm{N}([\mathrm{H}])[\mathrm{C} @]([\mathrm{H}])([\mathrm{C} @ @]([\mathrm{H}])(\mathrm{O}[\mathrm{H}]) \mathrm{C}([\mathrm{H}])([\mathrm{H}])[\mathrm{C} @]([\mathrm{H}])(\mathrm{C}(=\mathrm{O}) \mathrm{N}([\mathrm{H}])[\mathrm{C} @ \\
]([\mathrm{H}])(\mathrm{C}(=\mathrm{O}) \mathrm{N}([\mathrm{H}]) \mathrm{C}([\mathrm{H}])([\mathrm{H}]) \mathrm{C} 1=\mathrm{C}([\mathrm{H}]) \mathrm{C}([\mathrm{H}])=\mathrm{C}([\mathrm{H}]) \mathrm{C}([\mathrm{H}])=\mathrm{C} 1[\mathrm{H}]) \mathrm{C}([\mathrm{H}])(\mathrm{C}([ \\
\mathrm{H}])([\mathrm{H}])[\mathrm{H}]) \mathrm{C}([\mathrm{H}])([\mathrm{H}])[\mathrm{H}]) \mathrm{C}([\mathrm{H}])([\mathrm{H}])[\mathrm{H}]) \mathrm{C}([\mathrm{H}])([\mathrm{H}]) \mathrm{C}([\mathrm{H}])(\mathrm{C}([\mathrm{H}])([\mathrm{H}])[\mathrm{H}]) \mathrm{C}([ \\
\mathrm{H}])([\mathrm{H}])[\mathrm{H}])[\mathrm{C} @ @]([\mathrm{H}])(\mathrm{N}([\mathrm{H}]) \mathrm{C}\end{array}$ & 4.229 & 3.049 \\
\hline $\operatorname{Tr} 14$ & $\begin{array}{l}\mathrm{O}=\mathrm{C}(\mathrm{N}([\mathrm{H}])[\mathrm{C} @]([\mathrm{H}])([\mathrm{C} @ @]([\mathrm{H}])(\mathrm{O}[\mathrm{H}]) \mathrm{C}([\mathrm{H}])([\mathrm{H}])[\mathrm{C} @]([\mathrm{H}])(\mathrm{C}(=\mathrm{O}) \mathrm{N}([\mathrm{H}])[\mathrm{C} @ \\
]([\mathrm{H}])(\mathrm{C}(=\mathrm{O}) \mathrm{N}([\mathrm{H}]) \mathrm{C}([\mathrm{H}])([\mathrm{H}]) \mathrm{Cl}=\mathrm{C}([\mathrm{H}]) \mathrm{C}([\mathrm{H}])=\mathrm{C}([\mathrm{H}]) \mathrm{C}([\mathrm{H}])=\mathrm{C} 1[\mathrm{H}]) \mathrm{C}([\mathrm{H}])(\mathrm{C}([ \\
\mathrm{H}])([\mathrm{H}])[\mathrm{H}]) \mathrm{C}([\mathrm{H}])([\mathrm{H}])[\mathrm{H}]) \mathrm{C}([\mathrm{H}])([\mathrm{H}])[\mathrm{H}]) \mathrm{C}([\mathrm{H}])([\mathrm{H}]) \mathrm{C}([\mathrm{H}])(\mathrm{C}([\mathrm{H}])([\mathrm{H}])[\mathrm{H}]) \mathrm{C}([ \\
\mathrm{H}])([\mathrm{H}])[\mathrm{H}])[\mathrm{C} @ @]([\mathrm{H}])(\mathrm{N}([\mathrm{H}]) \mathrm{C}\end{array}$ & 3.300 & 3.000 \\
\hline
\end{tabular}




\begin{tabular}{|c|c|c|c|}
\hline $\operatorname{Tr} 15$ & $\begin{array}{l}\mathrm{O}=\mathrm{C}(\mathrm{C}=1 \mathrm{C}([\mathrm{H}])=\mathrm{C}([\mathrm{H}]) \mathrm{C}([\mathrm{H}])=\mathrm{C}(\mathrm{C}=1[\mathrm{H}]) \mathrm{C}(=\mathrm{O}) \mathrm{N}([\mathrm{H}])[\mathrm{C} @ @]([\mathrm{H}])(\mathrm{C}([\mathrm{H}])([\mathrm{H}]) \mathrm{C} \\
([\mathrm{H}])(\mathrm{C}([\mathrm{H}])([\mathrm{H}])[\mathrm{H}]) \mathrm{C}([\mathrm{H}])([\mathrm{H}])[\mathrm{H}])[\mathrm{C} @ @]([\mathrm{H}])(\mathrm{OH}]) \mathrm{C}([\mathrm{H}])([\mathrm{H}])[\mathrm{C} @]([\mathrm{H}])(\mathrm{C}(= \\
\mathrm{O}) \mathrm{N}([\mathrm{H}])[\mathrm{C} @]([\mathrm{H}])(\mathrm{C}(=\mathrm{O}) \mathrm{N}([\mathrm{H}]) \mathrm{C}([\mathrm{H}])(\mathrm{C}([\mathrm{H}])([\mathrm{H}])[\mathrm{H}]) \mathrm{C}([\mathrm{H}])([\mathrm{H}])[\mathrm{H}]) \mathrm{C}([\mathrm{H}])(\mathrm{C}([ \\
\mathrm{H}])([\mathrm{H}])[\mathrm{H}]) \mathrm{C}([\mathrm{H}])([\mathrm{H}])[\mathrm{H}]) \mathrm{C}([\mathrm{H}\end{array}$ & 2.103 & 2.525 \\
\hline $\operatorname{Tr} 16$ & $\begin{array}{l}\mathrm{O}=\mathrm{C} 1 \mathrm{OC}([\mathrm{H}])([\mathrm{H}]) \mathrm{C}([\mathrm{H}])=\mathrm{C}([\mathrm{H}]) \mathrm{C}([\mathrm{H}])([\mathrm{H}]) \mathrm{N}([\mathrm{H}]) \mathrm{C}(=\mathrm{O}) \mathrm{C}([\mathrm{H}])([\mathrm{H}])[\mathrm{C} @]([\mathrm{H}])(\mathrm{N} \\
([\mathrm{H}]) \mathrm{C}(=\mathrm{O})[\mathrm{C} @ @]([\mathrm{H}])(\mathrm{N} 1[\mathrm{H}]) \mathrm{C}([\mathrm{H}])(\mathrm{C}([\mathrm{H}])([\mathrm{H}])[\mathrm{H}]) \mathrm{C}([\mathrm{H}])([\mathrm{H}])[\mathrm{H}]) \mathrm{C}(=\mathrm{O}) \mathrm{N}([\mathrm{H}] \\
)[\mathrm{C} @ @]([\mathrm{H}])(\mathrm{C}([\mathrm{H}])([\mathrm{H}]) \mathrm{C}([\mathrm{H}])(\mathrm{C}([\mathrm{H}])([\mathrm{H}])[\mathrm{H}]) \mathrm{C}([\mathrm{H}])([\mathrm{H}])[\mathrm{H}])[\mathrm{C} @ @]([\mathrm{H}])(\mathrm{O}[\mathrm{H}] \\
) \mathrm{C}([\mathrm{H}])([\mathrm{H}])[\mathrm{C} @]([\mathrm{H}])(\mathrm{C}(=\mathrm{O}) \mathrm{N}([\mathrm{H}\end{array}$ & 3.059 & 3.082 \\
\hline $\operatorname{Tr} 17$ & $\begin{array}{l}\mathrm{O}=\mathrm{C}(\mathrm{N}([\mathrm{H}])[\mathrm{C} @]([\mathrm{H}])([\mathrm{C} @ @]([\mathrm{H}])(\mathrm{O}[\mathrm{H}]) \mathrm{C}([\mathrm{H}])([\mathrm{H}])[\mathrm{C} @]([\mathrm{H}])(\mathrm{C}(=\mathrm{O}) \mathrm{N}([\mathrm{H}])[\mathrm{C} @ \\
]([\mathrm{H}])(\mathrm{C}(=\mathrm{O}) \mathrm{N}([\mathrm{H}]) \mathrm{C}([\mathrm{H}])([\mathrm{H}]) \mathrm{C} 1=\mathrm{C}([\mathrm{H}]) \mathrm{C}([\mathrm{H}])=\mathrm{C}([\mathrm{H}]) \mathrm{C}([\mathrm{H}])=\mathrm{C} 1[\mathrm{H}]) \mathrm{C}([\mathrm{H}])(\mathrm{C}([ \\
\mathrm{H}])([\mathrm{H}])[\mathrm{H}]) \mathrm{C}([\mathrm{H}])([\mathrm{H}])[\mathrm{H}]) \mathrm{C}([\mathrm{H}])([\mathrm{H}])[\mathrm{H}]) \mathrm{C}([\mathrm{H}])([\mathrm{H}]) \mathrm{C}([\mathrm{H}])(\mathrm{C}([\mathrm{H}])([\mathrm{H}])[\mathrm{H}]) \mathrm{C}([ \\
\mathrm{H}])([\mathrm{H}])[\mathrm{H}])[\mathrm{C} @ @]([\mathrm{H}])(\mathrm{N}([\mathrm{H}]) \mathrm{C}\end{array}$ & 4.097 & 3.753 \\
\hline $\operatorname{Tr} 18$ & $\begin{array}{l}\mathrm{O}=\mathrm{C}(\mathrm{C}=1 \mathrm{C}([\mathrm{H}])=\mathrm{C}(\mathrm{C}([\mathrm{H}])=\mathrm{C}(\mathrm{C}=1[\mathrm{H}]) \mathrm{C}(=\mathrm{O}) \mathrm{N}([\mathrm{H}])[\mathrm{C} @ @]([\mathrm{H}])(\mathrm{C}([\mathrm{H}])([\mathrm{H}]) \mathrm{C}([\mathrm{H}] \\
)(\mathrm{C}([\mathrm{H}])([\mathrm{H}])[\mathrm{H}]) \mathrm{C}([\mathrm{H}])([\mathrm{H}])[\mathrm{H}])[\mathrm{C} @ @]([\mathrm{H}])(\mathrm{O}[\mathrm{H}]) \mathrm{C}([\mathrm{H}])([\mathrm{H}])[\mathrm{C} @([\mathrm{H}])(\mathrm{C}(=\mathrm{O}) \mathrm{N} \\
([\mathrm{H}])[\mathrm{C} @]([\mathrm{H}])(\mathrm{C}(=\mathrm{O}) \mathrm{N}([\mathrm{H}]) \mathrm{C}([\mathrm{H}])(\mathrm{C}([\mathrm{H}])([\mathrm{H}])[\mathrm{H}]) \mathrm{C}([\mathrm{H}])([\mathrm{H}])[\mathrm{H}]) \mathrm{C}([\mathrm{H}])(\mathrm{C}([\mathrm{H}])( \\
[\mathrm{H}])[\mathrm{H}]) \mathrm{C}([\mathrm{H}])([\mathrm{H}])[\mathrm{H}]) \mathrm{C}([\mathrm{H}])([\end{array}$ & 2.502 & 3.283 \\
\hline $\operatorname{Tr} 19$ & $\begin{array}{l}\mathrm{O}=\mathrm{C}(\mathrm{C}=1 \mathrm{C}([\mathrm{H}])=\mathrm{C}(\mathrm{C}([\mathrm{H}])=\mathrm{C}(\mathrm{C}=1[\mathrm{H}]) \mathrm{C}(=\mathrm{O}) \mathrm{N}([\mathrm{H}])[\mathrm{C} @ @]([\mathrm{H}])(\mathrm{C}([\mathrm{H}])([\mathrm{H}]) \mathrm{C}([\mathrm{H}] \\
)(\mathrm{C}([\mathrm{H}])([\mathrm{H}])[\mathrm{H}]) \mathrm{C}([\mathrm{H}])([\mathrm{H}])[\mathrm{H}])[\mathrm{C} @ @]([\mathrm{H}])(\mathrm{O}[\mathrm{H}]) \mathrm{C}([\mathrm{H}])([\mathrm{H}])[\mathrm{C} @]([\mathrm{H}])(\mathrm{C}(=\mathrm{O}) \mathrm{N} \\
([\mathrm{H}])[\mathrm{C} @]([\mathrm{H}])(\mathrm{C}(=\mathrm{O}) \mathrm{N}([\mathrm{H}]) \mathrm{C}([\mathrm{H}])(\mathrm{C}([\mathrm{H}])([\mathrm{H}])[\mathrm{H}]) \mathrm{C}([\mathrm{H}])([\mathrm{H}])[\mathrm{H}]) \mathrm{C}([\mathrm{H}])(\mathrm{C}([\mathrm{H}])( \\
[\mathrm{H}])[\mathrm{H}]) \mathrm{C}([\mathrm{H}])([\mathrm{H}])[\mathrm{H}]) \mathrm{C}([\mathrm{H}])([\end{array}$ & 3.569 & 3.819 \\
\hline $\operatorname{Tr} 20$ & $\begin{array}{l}\mathrm{O}=\mathrm{C} 2 \mathrm{~N}(\mathrm{C}(=\mathrm{N}[\mathrm{C} @] 4(\mathrm{C}=1 \mathrm{C}([\mathrm{F}])=\mathrm{C}([\mathrm{H}]) \mathrm{C}([\mathrm{H}])=\mathrm{C}([\mathrm{F}]) \mathrm{C}=1[\mathrm{H}])[\mathrm{C} @ @] 2([\mathrm{H}]) \mathrm{C}([\mathrm{H}]) \\
([\mathrm{H}]) \mathrm{N}(/ \mathrm{C} 3=\mathrm{N} / \mathrm{C}(=\mathrm{C}(/[\mathrm{F}]) \mathrm{C}(=\mathrm{N} 3) \mathrm{OC}([\mathrm{H}])([\mathrm{H}])[\mathrm{H}]) \mathrm{C}([\mathrm{H}])([\mathrm{H}])[\mathrm{H}]) \mathrm{C} 4([\mathrm{H}])[\mathrm{H}]) \mathrm{N}([\mathrm{H} \\
])[\mathrm{H}]) \mathrm{C}([\mathrm{H}])([\mathrm{H}])[\mathrm{H}]\end{array}$ & 4.071 & 3.883 \\
\hline $\operatorname{Tr} 21$ & $\begin{array}{l}\mathrm{O}=\mathrm{C} 2 \mathrm{~N}(\mathrm{C}(=\mathrm{N}[\mathrm{C} @] 4(\mathrm{C}=1 \mathrm{C}([\mathrm{F}])=\mathrm{C}([\mathrm{H}]) \mathrm{C}([\mathrm{H}])=\mathrm{C}([\mathrm{H}]) \mathrm{C}=1[\mathrm{H}])[\mathrm{C} @ @] 2([\mathrm{H}]) \mathrm{C}([\mathrm{H}]) \\
([\mathrm{H}]) \mathrm{N}(/ \mathrm{C} 3=\mathrm{N} / \mathrm{C}(=\mathrm{C}(/[\mathrm{Cl}]) \mathrm{C}(=\mathrm{N} 3) \mathrm{OC}([\mathrm{H}])([\mathrm{H}])[\mathrm{H}]) \mathrm{C}([\mathrm{H}])([\mathrm{H}])[\mathrm{H}]) \mathrm{C} 4([\mathrm{H}])[\mathrm{H}]) \mathrm{N}([ \\
\mathrm{H}])[\mathrm{H}]) \mathrm{C}([\mathrm{H}])([\mathrm{H}])[\mathrm{H}]\end{array}$ & 4.032 & 3.883 \\
\hline $\operatorname{Tr} 22$ & $\begin{array}{l}\mathrm{O}=\mathrm{C} 2 \mathrm{~N}(\mathrm{C}(=\mathrm{N}[\mathrm{C} @] 4(\mathrm{C}=1 \mathrm{C}([\mathrm{F}])=\mathrm{C}([\mathrm{H}]) \mathrm{C}([\mathrm{H}])=\mathrm{C}([\mathrm{H}]) \mathrm{C}=1[\mathrm{~F}])[\mathrm{C} @ @] 2([\mathrm{H}]) \mathrm{C}([\mathrm{H}]) \\
([\mathrm{H}]) \mathrm{N}(/ \mathrm{C} 3=\mathrm{N} / \mathrm{C}(=\mathrm{C}(/[\mathrm{F}]) \mathrm{C}(=\mathrm{N} 3) \mathrm{OC}([\mathrm{H}])([\mathrm{H}])[\mathrm{H}]) \mathrm{C}([\mathrm{H}])([\mathrm{H}])[\mathrm{H}]) \mathrm{C} 4([\mathrm{H}])[\mathrm{H}]) \mathrm{N}([\mathrm{H} \\
])[\mathrm{H}]) \mathrm{C}([\mathrm{H}])([\mathrm{H}])[\mathrm{H}]\end{array}$ & 3.495 & 3.658 \\
\hline $\operatorname{Tr} 23$ & $\begin{array}{l}\mathrm{O}=\mathrm{C} 2 \mathrm{~N}(\mathrm{C}(=\mathrm{N}[\mathrm{C} @] 4(\mathrm{C}=1 \mathrm{C}([\mathrm{H}])=\mathrm{C}([\mathrm{H}]) \mathrm{C}([\mathrm{H}])=\mathrm{C}([\mathrm{H}]) \mathrm{C}=1[\mathrm{H}])[\mathrm{C} @ @] 2([\mathrm{H}]) \mathrm{C}([\mathrm{H}] \\
)([\mathrm{H}]) \mathrm{N}(/ \mathrm{C} 3=\mathrm{N} / \mathrm{C}(=\mathrm{C}(/[\mathrm{F}]) \mathrm{C}(=\mathrm{N} 3) \mathrm{C}([\mathrm{H}])([\mathrm{H}])[\mathrm{H}]) \mathrm{C}([\mathrm{H}])([\mathrm{H}])[\mathrm{H}]) \mathrm{C} 4([\mathrm{H}])[\mathrm{H}]) \mathrm{N}([\mathrm{H}] \\
)[\mathrm{H}]) \mathrm{C}([\mathrm{H}])([\mathrm{H}])[\mathrm{H}]\end{array}$ & 2.812 & 2.996 \\
\hline $\operatorname{Tr} 24$ & $\begin{array}{l}\mathrm{O}=\mathrm{C} 2 \mathrm{~N}(\mathrm{C}(=\mathrm{N}[\mathrm{C} @] 4(\mathrm{C}=1 \mathrm{C}([\mathrm{H}])=\mathrm{C}([\mathrm{H}]) \mathrm{C}([\mathrm{H}])=\mathrm{C}([\mathrm{H}]) \mathrm{C}=1[\mathrm{H}])[\mathrm{C} @ @] 2([\mathrm{H}]) \mathrm{C}([\mathrm{H}] \\
)([\mathrm{H}]) \mathrm{N}(/ \mathrm{C} 3=\mathrm{N} / \mathrm{C}(=\mathrm{C}(/[\mathrm{H}]) \mathrm{C}(=\mathrm{N} 3) \mathrm{C}([\mathrm{H}])([\mathrm{H}]) \mathrm{C}([\mathrm{H}])([\mathrm{H}])[\mathrm{H}]) \mathrm{C}([\mathrm{H}])([\mathrm{H}])[\mathrm{H}]) \mathrm{C} 4([\mathrm{H} \\
])[\mathrm{H}]) \mathrm{N}([\mathrm{H}])[\mathrm{H}]) \mathrm{C}([\mathrm{H}])([\mathrm{H}])[\mathrm{H}]\end{array}$ & 3.215 & 3.032 \\
\hline $\operatorname{Tr} 25$ & $\begin{array}{l}\mathrm{O}=\mathrm{C} 2 \mathrm{~N}(\mathrm{C}(=\mathrm{N}[\mathrm{C} @] 4(\mathrm{C}=1 \mathrm{C}([\mathrm{F}])=\mathrm{C}([\mathrm{H}]) \mathrm{C}([\mathrm{F}])=\mathrm{C}([\mathrm{F}]) \mathrm{C}=1[\mathrm{H}])[\mathrm{C} @ @] 2([\mathrm{H}]) \mathrm{C}([\mathrm{H}])( \\
[\mathrm{H}]) \mathrm{N}(/ \mathrm{C} 3=\mathrm{N} / \mathrm{C}(=\mathrm{C}(/[\mathrm{F}]) \mathrm{C}(=\mathrm{N} 3) \mathrm{OC}([\mathrm{H}])([\mathrm{H}])[\mathrm{H}]) \mathrm{C}([\mathrm{H}])([\mathrm{H}])[\mathrm{H}]) \mathrm{C} 4([\mathrm{H}])[\mathrm{H}]) \mathrm{N}([\mathrm{H}] \\
)[\mathrm{H}]) \mathrm{C}([\mathrm{H}])([\mathrm{H}])[\mathrm{H}]\end{array}$ & 4.114 & 4.233 \\
\hline $\operatorname{Tr} 26$ & $\begin{array}{l}\mathrm{O}=\mathrm{C} 2 \mathrm{~N}(\mathrm{C}(=\mathrm{N}[\mathrm{C} @] 5(\mathrm{C}=1 \mathrm{C}([\mathrm{H}])=\mathrm{C}([\mathrm{H}]) \mathrm{C}([\mathrm{H}])=\mathrm{C}([\mathrm{H}]) \mathrm{C}=1[\mathrm{H}])[\mathrm{C} @ @] 2([\mathrm{H}]) \mathrm{C}([\mathrm{H}] \\
)([\mathrm{H}]) \mathrm{N}(/ \mathrm{C} 3=\mathrm{N} / \mathrm{C}(=\mathrm{C}(/[\mathrm{Cl}]) \mathrm{C}(=\mathrm{N} 3)[\mathrm{C} @ @] 4([\mathrm{H}]) \mathrm{C}([\mathrm{H}])([\mathrm{H}]) \mathrm{C} 4([\mathrm{H}])[\mathrm{H}]) \mathrm{C}([\mathrm{H}])([\mathrm{H}] \\
)[\mathrm{H}]) \mathrm{C} 5([\mathrm{H}])[\mathrm{H}]) \mathrm{N}([\mathrm{H}])[\mathrm{H}]) \mathrm{C}([\mathrm{H}])([\mathrm{H}])[\mathrm{H}]\end{array}$ & 3.027 & 3.062 \\
\hline $\operatorname{Tr} 27$ & $\begin{array}{l}\mathrm{O}=\mathrm{C} 2 \mathrm{~N}(\mathrm{C}(=\mathrm{N}[\mathrm{C} @] 5(\mathrm{C}=1 \mathrm{C}([\mathrm{H}])=\mathrm{C}([\mathrm{H}]) \mathrm{C}([\mathrm{H}])=\mathrm{C}([\mathrm{H}]) \mathrm{C}=1[\mathrm{H}])[\mathrm{C} @ @] 2([\mathrm{H}]) \mathrm{C}([\mathrm{H}] \\
)([\mathrm{H}]) \mathrm{N}(/ \mathrm{C} 3=\mathrm{N} / \mathrm{C}(=\mathrm{C}(/[\mathrm{Cl}]) \mathrm{C}(=\mathrm{N} 3)[\mathrm{C} @ @] 4([\mathrm{H}]) \mathrm{C}([\mathrm{H}])([\mathrm{H}]) \mathrm{C} 4([\mathrm{H}])[\mathrm{H}]) \mathrm{C}([\mathrm{H}])([\mathrm{H}]\end{array}$ & 2.745 & 3.099 \\
\hline
\end{tabular}




\begin{tabular}{|c|c|c|c|}
\hline & ) $\mathrm{C}([\mathrm{H}])([\mathrm{H}])[\mathrm{H}]) \mathrm{C}([\mathrm{H}])[\mathrm{H}]) \mathrm{N}([\mathrm{H}])[\mathrm{H}]) \mathrm{C}([\mathrm{H}])([\mathrm{H}])[\mathrm{H}]$ & & \\
\hline $\operatorname{Tr} 28$ & $\begin{array}{l}\mathrm{O}=\mathrm{C} 2 \mathrm{~N}(\mathrm{C}(=\mathrm{N}[\mathrm{C} @] 4(\mathrm{C}=1 \mathrm{C}([\mathrm{H}])=\mathrm{C}([\mathrm{H}]) \mathrm{C}([\mathrm{F}])=\mathrm{C}([\mathrm{H}]) \mathrm{C}=1[\mathrm{H}])[\mathrm{C} @ @] 2([\mathrm{H}]) \mathrm{C}([\mathrm{H}]) \\
([\mathrm{H}]) \mathrm{N}(/ \mathrm{C} 3=\mathrm{N} / \mathrm{C}(=\mathrm{C}(/[\mathrm{F}]) \mathrm{C}(=\mathrm{N} 3) \mathrm{C}([\mathrm{H}])([\mathrm{H}])[\mathrm{H}]) \mathrm{C}([\mathrm{H}])([\mathrm{H}])[\mathrm{H}]) \mathrm{C} 4([\mathrm{H}])[\mathrm{H}]) \mathrm{N}([\mathrm{H}]) \\
[\mathrm{H}]) \mathrm{C}([\mathrm{H}])([\mathrm{H}])[\mathrm{H}]\end{array}$ & 3.194 & 3.346 \\
\hline $\operatorname{Tr} 29$ & $\begin{array}{l}\mathrm{O}=\mathrm{C} 2 \mathrm{~N}(\mathrm{C}(=\mathrm{N}[\mathrm{C} @] 4(\mathrm{C}=1 \mathrm{C}([\mathrm{H}])=\mathrm{C}([\mathrm{F}]) \mathrm{C}([\mathrm{H}])=\mathrm{C}([\mathrm{H}]) \mathrm{C}=1[\mathrm{~F}])[\mathrm{C} @ @] 2([\mathrm{H}]) \mathrm{C}([\mathrm{H}]) \\
([\mathrm{H}]) \mathrm{N}(/ \mathrm{C} 3=\mathrm{N} / \mathrm{C}(\mathrm{OC}([\mathrm{H}])([\mathrm{H}])[\mathrm{H}])=\mathrm{C}([\mathrm{F}]) \mathrm{C}(=\mathrm{N} 3) \mathrm{OC}([\mathrm{H}])([\mathrm{H}])[\mathrm{H}]) \mathrm{C} 4([\mathrm{H}])[\mathrm{H}]) \mathrm{N}([ \\
\mathrm{H}])[\mathrm{H}]) \mathrm{C}([\mathrm{H}])([\mathrm{H}])[\mathrm{H}]\end{array}$ & 4.252 & 4.082 \\
\hline $\operatorname{Tr} 30$ & $\begin{array}{l}\mathrm{O}=\mathrm{C} 2 \mathrm{~N}(\mathrm{C}(=\mathrm{N}[\mathrm{C} @] 4(\mathrm{C}=1 \mathrm{C}([\mathrm{H}])=\mathrm{C}([\mathrm{H}]) \mathrm{C}([\mathrm{F}])=\mathrm{C}([\mathrm{H}]) \mathrm{C}=1[\mathrm{~F}])[\mathrm{C} @ @] 2([\mathrm{H}]) \mathrm{C}([\mathrm{H}]) \\
([\mathrm{H}]) \mathrm{N}(/ \mathrm{C} 3=\mathrm{N} / \mathrm{C}(\mathrm{OC}([\mathrm{H}])([\mathrm{H}])[\mathrm{H}])=\mathrm{C}([\mathrm{F}]) \mathrm{C}(=\mathrm{N} 3) \mathrm{OC}([\mathrm{H}])([\mathrm{H}])[\mathrm{H}]) \mathrm{C} 4([\mathrm{H}])[\mathrm{H}]) \mathrm{N}([ \\
\mathrm{H}])[\mathrm{H}]) \mathrm{C}([\mathrm{H}])([\mathrm{H}])[\mathrm{H}]\end{array}$ & 4.745 & 4.082 \\
\hline $\operatorname{Tr} 31$ & $\begin{array}{l}\mathrm{O}=\mathrm{C} 2 \mathrm{~N}(\mathrm{C}(=\mathrm{N}[\mathrm{C} @] 4(\mathrm{C}=1 \mathrm{C}([\mathrm{H}])=\mathrm{C}([\mathrm{H}]) \mathrm{C}([\mathrm{F}])=\mathrm{C}([\mathrm{H}]) \mathrm{C}=1[\mathrm{H}])[\mathrm{C} @ @] 2([\mathrm{H}]) \mathrm{C}([\mathrm{H}]) \\
([\mathrm{H}]) \mathrm{N}(/ \mathrm{C} 3=\mathrm{N} / \mathrm{C}(\mathrm{OC}([\mathrm{H}])([\mathrm{H}])[\mathrm{H}])=\mathrm{C}([\mathrm{F}]) \mathrm{C}(=\mathrm{N} 3) \mathrm{C}([\mathrm{H}])([\mathrm{H}]) \mathrm{C}([\mathrm{H}])([\mathrm{H}])[\mathrm{H}]) \mathrm{C} 4([\mathrm{H} \\
])[\mathrm{H}]) \mathrm{N}([\mathrm{H}])[\mathrm{H}]) \mathrm{C}([\mathrm{H}])([\mathrm{H}])[\mathrm{H}]\end{array}$ & 4.215 & 3.806 \\
\hline $\operatorname{Tr} 32$ & $\begin{array}{l}\mathrm{O}=\mathrm{C} 2 \mathrm{~N}(\mathrm{C}(=\mathrm{N}[\mathrm{C} @] 5(\mathrm{C}=1 \mathrm{C}([\mathrm{F}])=\mathrm{C}([\mathrm{H}]) \mathrm{C}([\mathrm{H}])=\mathrm{C}([\mathrm{F}]) \mathrm{C}=1[\mathrm{H}])[\mathrm{C} @ @] 2([\mathrm{H}]) \mathrm{C}([\mathrm{H}]) \\
([\mathrm{H}]) \mathrm{N}(/ \mathrm{C} 3=\mathrm{N} / \mathrm{C}(\mathrm{OC}([\mathrm{H}])([\mathrm{H}])[\mathrm{H}])=\mathrm{C}([\mathrm{F}]) \mathrm{C}(=\mathrm{N} 3)[\mathrm{C} @] 4([\mathrm{H}]) \mathrm{C}([\mathrm{H}])([\mathrm{H}]) \mathrm{C} 4([\mathrm{H}])[\mathrm{H} \\
]) \mathrm{C} 5([\mathrm{H}])[\mathrm{H}]) \mathrm{N}([\mathrm{H}])[\mathrm{H}]) \mathrm{C}([\mathrm{H}])([\mathrm{H}])[\mathrm{H}]\end{array}$ & 4.252 & 4.268 \\
\hline $\operatorname{Tr} 33$ & $\begin{array}{l}\mathrm{O}=\mathrm{C} 2 \mathrm{~N}(\mathrm{C}(=\mathrm{N}[\mathrm{C} @] 5(\mathrm{C}=1 \mathrm{C}([\mathrm{H}])=\mathrm{C}([\mathrm{F}]) \mathrm{C}([\mathrm{H}])=\mathrm{C}([\mathrm{H}]) \mathrm{C}=1[\mathrm{~F}])[\mathrm{C} @ @] 2([\mathrm{H}]) \mathrm{C}([\mathrm{H}]) \\
([\mathrm{H}]) \mathrm{N}(/ \mathrm{C} 3=\mathrm{N} / \mathrm{C}(=\mathrm{C}(/[\mathrm{F}]) \mathrm{C}(=\mathrm{N} 3)[\mathrm{C} @] 4([\mathrm{H}]) \mathrm{C}([\mathrm{H}])([\mathrm{H}]) \mathrm{C} 4([\mathrm{H}])[\mathrm{H}]) \mathrm{C}([\mathrm{H}])([\mathrm{H}])[\mathrm{H}] \\
) \mathrm{C}([\mathrm{H}])[\mathrm{H}]) \mathrm{N}([\mathrm{H}])[\mathrm{H}]) \mathrm{C}([\mathrm{H}])([\mathrm{H}])[\mathrm{H}]\end{array}$ & 3.585 & 3.619 \\
\hline $\operatorname{Tr} 34$ & $\begin{array}{l}\mathrm{O}=\mathrm{C} 2 \mathrm{~N}(\mathrm{C}(=\mathrm{N}[\mathrm{C} @] 5(\mathrm{C}=1 \mathrm{C}([\mathrm{F}])=\mathrm{C}([\mathrm{H}]) \mathrm{C}([\mathrm{F}])=\mathrm{C}([\mathrm{H}]) \mathrm{C}=1[\mathrm{H}])[\mathrm{C} @ @] 2([\mathrm{H}]) \mathrm{C}([\mathrm{H}]) \\
([\mathrm{H}]) \mathrm{N}(/ \mathrm{C} 3=\mathrm{N} / \mathrm{C}(\mathrm{OC}([\mathrm{H}])([\mathrm{H}])[\mathrm{H}])=\mathrm{C}([\mathrm{F}]) \mathrm{C}(=\mathrm{N} 3)[\mathrm{C} @] 4([\mathrm{H}]) \mathrm{C}([\mathrm{H}])([\mathrm{H}]) \mathrm{C} 4([\mathrm{H}])[\mathrm{H} \\
]) \mathrm{C} 5([\mathrm{H}])[\mathrm{H}]) \mathrm{N}([\mathrm{H}])[\mathrm{H}]) \mathrm{C}([\mathrm{H}])([\mathrm{H}])[\mathrm{H}]\end{array}$ & 4.569 & 4.268 \\
\hline $\operatorname{Tr} 35$ & $\begin{array}{l}\mathrm{O}=\mathrm{C} 2 \mathrm{~N}(\mathrm{C}(=\mathrm{N}[\mathrm{C} @] 5(\mathrm{C}=1 \mathrm{C}([\mathrm{F}])=\mathrm{C}([\mathrm{H}]) \mathrm{C}([\mathrm{F}])=\mathrm{C}([\mathrm{H}]) \mathrm{C}=1[\mathrm{H}])[\mathrm{C} @ @] 2([\mathrm{H}]) \mathrm{C}([\mathrm{H}]) \\
([\mathrm{H}]) \mathrm{N}(/ \mathrm{C} 3=\mathrm{N} / \mathrm{C}(\mathrm{OC}([\mathrm{H}])([\mathrm{H}])[\mathrm{H}])=\mathrm{C}([\mathrm{Cl}]) \mathrm{C}(=\mathrm{N} 3)[\mathrm{C} @] 4([\mathrm{H}]) \mathrm{C}([\mathrm{H}])([\mathrm{H}]) \mathrm{C} 4([\mathrm{H}])[ \\
\mathrm{H}]) \mathrm{C} 5([\mathrm{H}])[\mathrm{H}]) \mathrm{N}([\mathrm{H}])[\mathrm{H}]) \mathrm{C}([\mathrm{H}])([\mathrm{H}])[\mathrm{H}]\end{array}$ & 4.167 & 4.330 \\
\hline $\operatorname{Tr} 36$ & $\begin{array}{l}{[\mathrm{F}] \mathrm{C}=1 \mathrm{C}([\mathrm{H}])=\mathrm{C}([\mathrm{F}]) \mathrm{C}(=\mathrm{C}([\mathrm{H}]) \mathrm{C}=1[\mathrm{H}])[\mathrm{C} @ @] 24 \mathrm{~N}=\mathrm{C}(\mathrm{N}(\mathrm{C}(=\mathrm{O})[\mathrm{C} @] 2([\mathrm{H}]) \mathrm{C}([\mathrm{H}])} \\
([\mathrm{H}]) \mathrm{N}(/ \mathrm{C} 3=\mathrm{N} / \mathrm{C}(=\mathrm{C}(/[\mathrm{F}]) \mathrm{C}(=\mathrm{N} 3) \mathrm{OC}([\mathrm{H}])([\mathrm{H}])[\mathrm{H}]) \mathrm{C}([\mathrm{F}])([\mathrm{F}]) \mathrm{C}([\mathrm{H}])([\mathrm{H}])[\mathrm{H}]) \mathrm{C} 4([\mathrm{H} \\
])[\mathrm{H}]) \mathrm{C}([\mathrm{H}])([\mathrm{H}])[\mathrm{H}]) \mathrm{N}([\mathrm{H}])[\mathrm{H}]\end{array}$ & 3.959 & 3.845 \\
\hline $\operatorname{Tr} 37$ & $\begin{array}{l}\mathrm{O}=\mathrm{C} 2 \mathrm{~N}(\mathrm{C}(=\mathrm{N}[\mathrm{C} @] 4(\mathrm{C}=1 \mathrm{C}([\mathrm{F}])=\mathrm{C}([\mathrm{H}]) \mathrm{C}([\mathrm{H}])=\mathrm{C}([\mathrm{H}]) \mathrm{C}=1[\mathrm{H}])[\mathrm{C} @ @] 2([\mathrm{H}]) \mathrm{C}([\mathrm{H}]) \\
([\mathrm{H}]) \mathrm{N}(/ \mathrm{C} 3=\mathrm{N} / \mathrm{C}(=\mathrm{C}(/[\mathrm{F}]) \mathrm{C}(=\mathrm{N} 3) \mathrm{OC}([\mathrm{H}])([\mathrm{H}])[\mathrm{H}]) \mathrm{C}([\mathrm{H}])([\mathrm{H}])[\mathrm{H}]) \mathrm{C} 4([\mathrm{H}])[\mathrm{H}]) \mathrm{N}([\mathrm{H} \\
])[\mathrm{H}]) \mathrm{C}([\mathrm{H}])([\mathrm{H}])[\mathrm{H}]\end{array}$ & 4.222 & 3.533 \\
\hline $\operatorname{Tr} 38$ & $\begin{array}{l}\mathrm{O}=\mathrm{C} 2 \mathrm{~N}(\mathrm{C}(=\mathrm{N}[\mathrm{C} @] 4(\mathrm{C}=1 \mathrm{C}([\mathrm{F}])=\mathrm{C}([\mathrm{Cl}]) \mathrm{C}([\mathrm{F}])=\mathrm{C}([\mathrm{H}]) \mathrm{C}=1[\mathrm{H}])[\mathrm{C} @ @] 2([\mathrm{H}]) \mathrm{C}([\mathrm{H}]) \\
([\mathrm{H}]) \mathrm{N}(/ \mathrm{C} 3=\mathrm{N} / \mathrm{C}(=\mathrm{C}(/[\mathrm{F}]) \mathrm{C}(=\mathrm{N} 3) \mathrm{OC}([\mathrm{H}])([\mathrm{H}])[\mathrm{H}]) \mathrm{C}([\mathrm{H}])([\mathrm{H}])[\mathrm{H}]) \mathrm{C} 4([\mathrm{H}])[\mathrm{H}]) \mathrm{N}([\mathrm{H} \\
])[\mathrm{H}]) \mathrm{C}([\mathrm{H}])([\mathrm{H}])[\mathrm{H}]\end{array}$ & 4.036 & 4.295 \\
\hline $\operatorname{Tr} 39$ & $\begin{array}{l}\mathrm{O}=\mathrm{C} 2 \mathrm{~N}(\mathrm{C}(=\mathrm{N}[\mathrm{C} @] 4(\mathrm{C}=1 \mathrm{C}([\mathrm{Cl}])=\mathrm{C}([\mathrm{H}]) \mathrm{C}([\mathrm{F}])=\mathrm{C}([\mathrm{H}]) \mathrm{C}=1[\mathrm{H}])[\mathrm{C} @ @] 2([\mathrm{H}]) \mathrm{C}([\mathrm{H}] \\
)([\mathrm{H}]) \mathrm{N}(/ \mathrm{C} 3=\mathrm{N} / \mathrm{C}(=\mathrm{C}(/[\mathrm{F}]) \mathrm{C}(=\mathrm{N} 3) \mathrm{OC}([\mathrm{H}])([\mathrm{H}])[\mathrm{H}]) \mathrm{C}([\mathrm{H}])([\mathrm{H}])[\mathrm{H}]) \mathrm{C} 4([\mathrm{H}])[\mathrm{H}]) \mathrm{N}([ \\
\mathrm{H}])[\mathrm{H}]) \mathrm{C}([\mathrm{H}])([\mathrm{H}])[\mathrm{H}]\end{array}$ & 4.347 & 3.945 \\
\hline $\operatorname{Tr} 40$ & $\begin{array}{l}\mathrm{O}=\mathrm{C} 2 \mathrm{~N}(\mathrm{C}(=\mathrm{N}[\mathrm{C} @] 5(\mathrm{C}=1 \mathrm{C}([\mathrm{H}])=\mathrm{C}([\mathrm{H}]) \mathrm{C}([\mathrm{F}])=\mathrm{C}([\mathrm{H}]) \mathrm{C}=1[\mathrm{H}])[\mathrm{C} @ @] 2([\mathrm{H}]) \mathrm{C}([\mathrm{H}]) \\
([\mathrm{H}]) \mathrm{N}(/ \mathrm{C} 3=\mathrm{N} / \mathrm{C}(\mathrm{OC}([\mathrm{H}])([\mathrm{H}])[\mathrm{H}])=\mathrm{C}([\mathrm{F}]) \mathrm{C}(=\mathrm{N} 3)[\mathrm{C} @] 4([\mathrm{H}]) \mathrm{C}([\mathrm{H}])([\mathrm{H}]) \mathrm{C} 4([\mathrm{H}])[\mathrm{H} \\
]) \mathrm{C} 5([\mathrm{H}])[\mathrm{H}]) \mathrm{N}([\mathrm{H}])[\mathrm{H}]) \mathrm{C}([\mathrm{H}])([\mathrm{H}])[\mathrm{H}]\end{array}$ & 4.319 & 3.918 \\
\hline
\end{tabular}




\begin{tabular}{|c|c|c|c|}
\hline $\operatorname{Tr} 41$ & $\begin{array}{l}\mathrm{O}=[\mathrm{S}](=\mathrm{O})(\mathrm{C}([\mathrm{H}])([\mathrm{H}])[\mathrm{C} @(([\mathrm{H}])(\mathrm{N}([\mathrm{H}]) \mathrm{C}(=\mathrm{O}) \mathrm{OC}(\mathrm{C}([\mathrm{H}])([\mathrm{H}])[\mathrm{H}])(\mathrm{C}([\mathrm{H}])([\mathrm{H}])[\mathrm{H}] \\
) \mathrm{C}([\mathrm{H}])([\mathrm{H}])[\mathrm{H}]) \mathrm{C}(=\mathrm{O}) \mathrm{C}([\mathrm{H}])([\mathrm{H}]) \mathrm{N}([\mathrm{H}])[\mathrm{C} @([\mathrm{H}])([\mathrm{C} @]([\mathrm{H}])(\mathrm{O}[\mathrm{H}]) \mathrm{C}([\mathrm{H}])([\mathrm{H}])[ \\
\mathrm{C} @]([\mathrm{H}])(\mathrm{C}(=\mathrm{O}) \mathrm{N}([\mathrm{H}])[\mathrm{C} @]([\mathrm{H}])(\mathrm{C}(=\mathrm{O}) \mathrm{N}([\mathrm{H}]) \mathrm{C}([\mathrm{H}])([\mathrm{H}]) \mathrm{C} 1=\mathrm{C}([\mathrm{H}]) \mathrm{C}([\mathrm{H}])=\mathrm{C}([\mathrm{H} \\
]) \mathrm{C}([\mathrm{H}])=\mathrm{C} 1[\mathrm{H}]) \mathrm{C}([\mathrm{H}])(\mathrm{C}([\mathrm{H}])([\mathrm{H}\end{array}$ & 1.947 & 2.559 \\
\hline $\operatorname{Tr} 42$ & $\begin{array}{l}\mathrm{O}=\mathrm{C}(\mathrm{N}([\mathrm{H}])[\mathrm{C} @ @]([\mathrm{H}])(\mathrm{C}(=\mathrm{O}) \mathrm{N}([\mathrm{H}])[\mathrm{C} @]([\mathrm{H}])([\mathrm{C} @]([\mathrm{H}])(\mathrm{O}[\mathrm{H}]) \mathrm{C}([\mathrm{H}])([\mathrm{H}])[\mathrm{C} @ \\
]([\mathrm{H}])(\mathrm{C}(=\mathrm{O}) \mathrm{N}([\mathrm{H}])[\mathrm{C} @]([\mathrm{H}])(\mathrm{C}(=\mathrm{O}) \mathrm{N}([\mathrm{H}]) \mathrm{C}([\mathrm{H}])([\mathrm{H}]) \mathrm{C} 1=\mathrm{C}([\mathrm{H}]) \mathrm{C}([\mathrm{H}])=\mathrm{C}([\mathrm{H}]) \mathrm{C}( \\
[\mathrm{H}])=\mathrm{C} 1[\mathrm{H}]) \mathrm{C}([\mathrm{H}])(\mathrm{C}([\mathrm{H}])([\mathrm{H}])[\mathrm{H}]) \mathrm{C}([\mathrm{H}])([\mathrm{H}])[\mathrm{H}]) \mathrm{C}([\mathrm{H}])([\mathrm{H}])[\mathrm{H}]) \mathrm{C}([\mathrm{H}])([\mathrm{H}]) \mathrm{C}([ \\
\mathrm{H}])(\mathrm{C}([\mathrm{H}])([\mathrm{H}])[\mathrm{H}]) \mathrm{C}([\mathrm{H}])([\mathrm{H}])[\end{array}$ & 3.301 & 2.668 \\
\hline $\operatorname{Tr} 43$ & $\begin{array}{l}\mathrm{O}=\mathrm{C}(\mathrm{OC}(\mathrm{C}([\mathrm{H}])([\mathrm{H}])[\mathrm{H}])(\mathrm{C}([\mathrm{H}])([\mathrm{H}])[\mathrm{H}]) \mathrm{C}([\mathrm{H}])([\mathrm{H}])[\mathrm{H}]) \mathrm{N}([\mathrm{H}])[\mathrm{C} @ @]([\mathrm{H}])(\mathrm{C}(= \\
\mathrm{O}) \mathrm{N}([\mathrm{H}])[\mathrm{C} @]([\mathrm{H}])([\mathrm{C} @]([\mathrm{H}])(\mathrm{O}[\mathrm{H}]) \mathrm{C}([\mathrm{H}])([\mathrm{H}])[\mathrm{C} @([\mathrm{H}])(\mathrm{C}(=\mathrm{O}) \mathrm{N}([\mathrm{H}])[\mathrm{C} @]([\mathrm{H}] \\
)(\mathrm{C}(=\mathrm{O}) \mathrm{N}([\mathrm{H}]) \mathrm{C}([\mathrm{H}])([\mathrm{H}]) \mathrm{C} 1=\mathrm{C}([\mathrm{H}]) \mathrm{C}([\mathrm{H}])=\mathrm{C}([\mathrm{H}]) \mathrm{C}([\mathrm{H}])=\mathrm{C} 1[\mathrm{H}]) \mathrm{C}([\mathrm{H}])(\mathrm{C}([\mathrm{H}])([ \\
\mathrm{H}])[\mathrm{H}]) \mathrm{C}([\mathrm{H}])([\mathrm{H}])[\mathrm{H}]) \mathrm{C}([\mathrm{H}])([\mathrm{H}])\end{array}$ & 1.236 & 2.416 \\
\hline $\operatorname{Tr} 44$ & $\begin{array}{l}\mathrm{O}=\mathrm{C}(\mathrm{N}([\mathrm{H}])[\mathrm{C} @]([\mathrm{H}])(\mathrm{C}(=\mathrm{O}) \mathrm{C}([\mathrm{H}])([\mathrm{H}])[\mathrm{C} @]([\mathrm{H}])(\mathrm{C}(=\mathrm{O}) \mathrm{O}[\mathrm{H}]) \mathrm{C}([\mathrm{H}])([\mathrm{H}]) \mathrm{C} 1=\mathrm{C}( \\
[\mathrm{H}]) \mathrm{C}([\mathrm{H}])=\mathrm{C}([\mathrm{H}]) \mathrm{C}([\mathrm{H}])=\mathrm{C} 1[\mathrm{H}]) \mathrm{C}([\mathrm{H}])([\mathrm{H}]) \mathrm{C}([\mathrm{H}])([\mathrm{H}]) \mathrm{C}(=\mathrm{O}) \mathrm{O}[\mathrm{H}])[\mathrm{C} @ @]([\mathrm{H}])( \\
\mathrm{N}([\mathrm{H}]) \mathrm{C}(=\mathrm{O})[\mathrm{C} @ @]([\mathrm{H}])(\mathrm{C}([\mathrm{H}])([\mathrm{H}])[\mathrm{C} @]([\mathrm{H}])(\mathrm{O}[\mathrm{H}])[\mathrm{C} @ @]([\mathrm{H}])(\mathrm{N}([\mathrm{H}]) \mathrm{C}(=\mathrm{O})[ \\
\mathrm{C} @ @]([\mathrm{H}])(\mathrm{N}([\mathrm{H}]) \mathrm{C}(=\mathrm{O})[\mathrm{C} @ @]([\mathrm{H}])(\mathrm{N}([\mathrm{H}]\end{array}$ & 5.495 & 5.547 \\
\hline $\operatorname{Tr} 45$ & $\begin{array}{l}\mathrm{O}=\mathrm{C}(\mathrm{N}([\mathrm{H}])[\mathrm{C} @]([\mathrm{H}])(\mathrm{C}(=\mathrm{O}) \mathrm{N}([\mathrm{H}])[\mathrm{C} @]([\mathrm{H}])([\mathrm{C} @]([\mathrm{H}])(\mathrm{O}[\mathrm{H}]) \mathrm{C}([\mathrm{H}])([\mathrm{H}])[\mathrm{C} @]( \\
[\mathrm{H}])(\mathrm{C}(=\mathrm{O}) \mathrm{N}([\mathrm{H}])[\mathrm{C} @]([\mathrm{H}])(\mathrm{C}(=\mathrm{O}) \mathrm{N}([\mathrm{H}]) \mathrm{C}([\mathrm{H}])([\mathrm{H}]) \mathrm{C} 1=\mathrm{C}([\mathrm{H}]) \mathrm{C}([\mathrm{H}])=\mathrm{C}([\mathrm{H}]) \mathrm{C}([ \\
\mathrm{H}])=\mathrm{C} 1[\mathrm{H}]) \mathrm{C}([\mathrm{H}])(\mathrm{C}([\mathrm{H}])([\mathrm{H}])[\mathrm{H}]) \mathrm{C}([\mathrm{H}])([\mathrm{H}])[\mathrm{H}]) \mathrm{C}([\mathrm{H}])([\mathrm{H}])[\mathrm{H}]) \mathrm{C}([\mathrm{H}])([\mathrm{H}]) \mathrm{C}([\mathrm{H} \\
])(\mathrm{C}([\mathrm{H}])([\mathrm{H}])[\mathrm{H}]) \mathrm{C}([\mathrm{H}])([\mathrm{H}])[\mathrm{H}\end{array}$ & 4.229 & 2.618 \\
\hline $\operatorname{Tr} 46$ & $\begin{array}{l}\mathrm{O}=[\mathrm{S}](=\mathrm{O})(\mathrm{C}([\mathrm{H}])([\mathrm{H}])[\mathrm{C} @ @]([\mathrm{H}])(\mathrm{C}(=\mathrm{O}) \mathrm{N}([\mathrm{H}])[\mathrm{C} @]([\mathrm{H}])([\mathrm{C} @]([\mathrm{H}])(\mathrm{O}[\mathrm{H}]) \mathrm{C}([\mathrm{H} \\
])([\mathrm{H}])[\mathrm{C} @([\mathrm{H}])(\mathrm{C}(=\mathrm{O}) \mathrm{N}([\mathrm{H}])[\mathrm{C} @]([\mathrm{H}])(\mathrm{C}(=\mathrm{O}) \mathrm{N}([\mathrm{H}]) \mathrm{C}([\mathrm{H}])([\mathrm{H}]) \mathrm{C} 1=\mathrm{C}([\mathrm{H}]) \mathrm{C}([\mathrm{H}] \\
)=\mathrm{C}([\mathrm{H}]) \mathrm{C}([\mathrm{H}])=\mathrm{C} 1[\mathrm{H}]) \mathrm{C}([\mathrm{H}])(\mathrm{C}([\mathrm{H}])([\mathrm{H}])[\mathrm{H}]) \mathrm{C}([\mathrm{H}])([\mathrm{H}])[\mathrm{H}]) \mathrm{C}([\mathrm{H}])([\mathrm{H}])[\mathrm{H}]) \mathrm{C}([\mathrm{H} \\
])([\mathrm{H}]) \mathrm{C}([\mathrm{H}])(\mathrm{C}([\mathrm{H}])([\mathrm{H}])[\mathrm{H}]) \mathrm{C}\end{array}$ & 4.027 & 3.555 \\
\hline $\operatorname{Tr} 47$ & $\begin{array}{l}\mathrm{N} \# \mathrm{C} / \mathrm{C} 1=\mathrm{C}(\backslash[\mathrm{F}]) \mathrm{C}([\mathrm{H}])=\mathrm{C}([\mathrm{H}]) \mathrm{C}(=\mathrm{C} 1[\mathrm{H}]) \mathrm{C}=2[\mathrm{~S}] / \mathrm{C}(=\mathrm{C}(/[\mathrm{H}]) \mathrm{C}=2[\mathrm{H}])[\mathrm{C} @ @] 35 \mathrm{~N}= \\
\mathrm{C}(\mathrm{N}(\mathrm{C}(=\mathrm{O})[\mathrm{C} @] 3([\mathrm{H}]) \mathrm{C}([\mathrm{H}])([\mathrm{H}]) \mathrm{N}(/ \mathrm{C} 4=\mathrm{C}(\backslash \mathrm{H}]) \mathrm{C}([\mathrm{H}])=\mathrm{C}([\mathrm{F}]) \mathrm{C}([\mathrm{H}])=\mathrm{C} 4[\mathrm{H}]) \mathrm{C} 5([ \\
\mathrm{H}])[\mathrm{H}]) \mathrm{C}([\mathrm{H}])([\mathrm{H}])[\mathrm{H}]) \mathrm{N}([\mathrm{H}])[\mathrm{H}]\end{array}$ & 2.879 & 2.896 \\
\hline $\operatorname{Tr} 48$ & $\begin{array}{l}\mathrm{O}=[\mathrm{S}] 3(=\mathrm{O}) \mathrm{N}(\mathrm{C}=2 \mathrm{C}([\mathrm{H}])=\mathrm{C}(\mathrm{C}([\mathrm{H}])=\mathrm{C} 1 / \mathrm{C}(=\mathrm{C}([\mathrm{H}]) \mathrm{N}(\mathrm{C} 1=2) \mathrm{C}([\mathrm{H}])([\mathrm{H}]) \mathrm{C} 3([\mathrm{H}])[\mathrm{H}] \\
) \mathrm{C}([\mathrm{H}])([\mathrm{H}]) \mathrm{C}([\mathrm{H}])([\mathrm{H}])[\mathrm{H}]) \mathrm{C}(=\mathrm{O}) \mathrm{N}([\mathrm{H}])[\mathrm{C} @]([\mathrm{H}])(\mathrm{C}([\mathrm{H}])([\mathrm{H}]) \mathrm{N}([\mathrm{H}])[\mathrm{C} @]([\mathrm{H}])( \\
\mathrm{C}(=\mathrm{O}) \mathrm{N}([\mathrm{H}]) \mathrm{C}([\mathrm{H}])([\mathrm{H}]) \mathrm{C}([\mathrm{H}])(\mathrm{C}([\mathrm{H}])([\mathrm{H}])[\mathrm{H}]) \mathrm{C}([\mathrm{H}])([\mathrm{H}])[\mathrm{H}])[\mathrm{C} @ @]([\mathrm{H}])(\mathrm{O}[\mathrm{H}]) \\
\mathrm{C}([\mathrm{H}])([\mathrm{H}])[\mathrm{H}]) \mathrm{C}([\mathrm{H}])([\mathrm{H}]) \mathrm{C} 4=\mathrm{C}(\end{array}$ & 4.137 & 4.520 \\
\hline $\operatorname{Tr} 49$ & $\begin{array}{l}\mathrm{O}=[\mathrm{S}] 3(=\mathrm{O}) \mathrm{N}(\mathrm{C}=2 \mathrm{C}([\mathrm{H}])=\mathrm{C}(\mathrm{C}([\mathrm{H}])=\mathrm{Cl} / \mathrm{C}(=\mathrm{C}(/[\mathrm{H}]) \mathrm{N}(\mathrm{C} 1=2) \mathrm{C}([\mathrm{H}])([\mathrm{H}])[\mathrm{C} @] 34 \mathrm{C}([ \\
\mathrm{H}])([\mathrm{H}]) \mathrm{C} 4([\mathrm{H}])[\mathrm{H}]) \mathrm{C}([\mathrm{H}])([\mathrm{H}]) \mathrm{C}([\mathrm{H}])([\mathrm{H}])[\mathrm{H}]) \mathrm{C}(=\mathrm{O}) \mathrm{N}([\mathrm{H}])[\mathrm{C} @]([\mathrm{H}])(\mathrm{C}([\mathrm{H}])([\mathrm{H}] \\
) \mathrm{N}([\mathrm{H}])[\mathrm{C} @]([\mathrm{H}])(\mathrm{C}(=\mathrm{O}) \mathrm{N}([\mathrm{H}]) \mathrm{C}([\mathrm{H}])([\mathrm{H}]) \mathrm{C}([\mathrm{H}])(\mathrm{C}([\mathrm{H}])([\mathrm{H}])[\mathrm{H}]) \mathrm{C}([\mathrm{H}])([\mathrm{H}])[\mathrm{H}])[ \\
\mathrm{C} @ @]([\mathrm{H}])(\mathrm{O}[\mathrm{H}]) \mathrm{C}([\mathrm{H}])([\mathrm{H}])[\mathrm{H}]\end{array}$ & 4.143 & 4.331 \\
\hline $\operatorname{Tr} 50$ & $\begin{array}{l}\mathrm{O}=\mathrm{C}(\mathrm{N}([\mathrm{H}])[\mathrm{C} @]([\mathrm{H}])([\mathrm{C} @ @]([\mathrm{H}])(\mathrm{O}[\mathrm{H}]) \mathrm{C}([\mathrm{H}])([\mathrm{H}])[\mathrm{C} @]([\mathrm{H}])(\mathrm{C}(=\mathrm{O}) \mathrm{N}([\mathrm{H}]) \mathrm{C}([ \\
\mathrm{H}])([\mathrm{H}]) \mathrm{C}([\mathrm{H}])(\mathrm{C}([\mathrm{H}])([\mathrm{H}])[\mathrm{H}]) \mathrm{C}([\mathrm{H}])([\mathrm{H}])[\mathrm{H}]) \mathrm{C}([\mathrm{H}])([\mathrm{H}]) \mathrm{C}([\mathrm{H}])([\mathrm{H}]) \mathrm{C}(=\mathrm{O}) \mathrm{O}[\mathrm{H}]) \\
\mathrm{C}([\mathrm{H}])([\mathrm{H}]) \mathrm{C} 1=\mathrm{C}([\mathrm{H}]) \mathrm{C}([\mathrm{H}])=\mathrm{C}([\mathrm{H}]) \mathrm{C}([\mathrm{H}])=\mathrm{C} 1[\mathrm{H}]) \mathrm{C}([\mathrm{H}])([\mathrm{H}])[\mathrm{C} @]([\mathrm{H}])(\mathrm{N}([\mathrm{H}]) \mathrm{C} \\
(=\mathrm{O}) \mathrm{C}([\mathrm{H}])([\mathrm{H}]) \mathrm{C}([\mathrm{H}])(\mathrm{C}([\mathrm{H}])([\mathrm{H}\end{array}$ & 1.770 & 1.859 \\
\hline $\operatorname{Tr} 51$ & $\begin{array}{l}\mathrm{O}=\mathrm{C}(\mathrm{N}([\mathrm{H}])[\mathrm{C} @]([\mathrm{H}])([\mathrm{C} @ @]([\mathrm{H}])(\mathrm{O}[\mathrm{H}]) \mathrm{C}([\mathrm{H}])([\mathrm{H}])[\mathrm{C} @]([\mathrm{H}])(\mathrm{C}(=\mathrm{O}) \mathrm{N}([\mathrm{H}]) \mathrm{C}([ \\
\mathrm{H}])([\mathrm{H}]) \mathrm{C}([\mathrm{H}])(\mathrm{C}([\mathrm{H}])([\mathrm{H}])[\mathrm{H}]) \mathrm{C}([\mathrm{H}])([\mathrm{H}])[\mathrm{H}]) \mathrm{C}([\mathrm{H}])([\mathrm{H}]) \mathrm{C}([\mathrm{H}])([\mathrm{H}]) \mathrm{OC}(=\mathrm{O}) \mathrm{C}([\mathrm{H} \\
])([\mathrm{H}])[\mathrm{H}]) \mathrm{C}([\mathrm{H}])([\mathrm{H}]) \mathrm{Cl}=\mathrm{C}([\mathrm{H}]) \mathrm{C}([\mathrm{H}])=\mathrm{C}([\mathrm{H}]) \mathrm{C}([\mathrm{H}])=\mathrm{C} 1[\mathrm{H}]) \mathrm{C}([\mathrm{H}])([\mathrm{H}])[\mathrm{C} @([\mathrm{H} \\
])(\mathrm{N}([\mathrm{H}]) \mathrm{C}(=\mathrm{O}) \mathrm{C}([\mathrm{H}])([\mathrm{H}]) \mathrm{C}([\mathrm{H}]\end{array}$ & 1.569 & 2.189 \\
\hline
\end{tabular}




\begin{tabular}{|c|c|c|c|}
\hline $\operatorname{Tr} 52$ & $\begin{array}{l}\mathrm{O}=\mathrm{C}(\mathrm{N}([\mathrm{H}])[\mathrm{C} @ @]([\mathrm{H}])(\mathrm{C}=1 \mathrm{C}([\mathrm{H}])=\mathrm{C}([\mathrm{H}]) \mathrm{C}([\mathrm{H}])=\mathrm{C}([\mathrm{H}]) \mathrm{C}=1[\mathrm{H}]) \mathrm{C}([\mathrm{H}])([\mathrm{H}])[\mathrm{H}]) \\
\mathrm{C} 2=\mathrm{C}([\mathrm{H}]) \mathrm{C}(=\mathrm{C}([\mathrm{H}]) \mathrm{C}(=\mathrm{C} 2[\mathrm{H}]) \mathrm{N}(\mathrm{C}([\mathrm{H}])([\mathrm{H}])[\mathrm{H}])[\mathrm{S}](=\mathrm{O})(=\mathrm{O}) \mathrm{C}([\mathrm{H}])([\mathrm{H}])[\mathrm{H}]) \mathrm{C}(= \\
\mathrm{O}) \mathrm{N}([\mathrm{H}])[\mathrm{C} @]([\mathrm{H}])([\mathrm{C} @([\mathrm{H}])(\mathrm{O}[\mathrm{H}]) \mathrm{C}([\mathrm{H}])([\mathrm{H}]) \mathrm{N}([\mathrm{H}]) \mathrm{C}([\mathrm{H}])(\mathrm{C}([\mathrm{H}])([\mathrm{H}])[\mathrm{H}]) \mathrm{C}([ \\
\mathrm{H}])([\mathrm{H}])[\mathrm{H}]) \mathrm{C}([\mathrm{H}])([\mathrm{H}]) \mathrm{C} 3=\mathrm{C}([\mathrm{H}]) \mathrm{C}\end{array}$ & 2.372 & 1.934 \\
\hline $\operatorname{Tr} 53$ & $\begin{array}{l}\mathrm{O}=\mathrm{C}(\mathrm{N}([\mathrm{H}])[\mathrm{C} @]([\mathrm{H}])(\mathrm{C} 1=\mathrm{C}([\mathrm{H}]) \mathrm{C}([\mathrm{H}])=\mathrm{C}([\mathrm{H}]) \mathrm{C}([\mathrm{H}])=\mathrm{C} 1[\mathrm{H}]) \mathrm{C}([\mathrm{H}])([\mathrm{H}])[\mathrm{H}]) \mathrm{C} 2 \\
=\mathrm{C}([\mathrm{H}]) \mathrm{C}(=\mathrm{C}([\mathrm{H}]) \mathrm{C}(=\mathrm{C} 2[\mathrm{H}]) \mathrm{N}(\mathrm{C}([\mathrm{H}])([\mathrm{H}])[\mathrm{H}])[\mathrm{S}](=\mathrm{O})(=\mathrm{O}) \mathrm{C}([\mathrm{H}])([\mathrm{H}])[\mathrm{H}]) \mathrm{C}(=\mathrm{O}) \\
\mathrm{N}([\mathrm{H}])[\mathrm{C} @]([\mathrm{H}])([\mathrm{C} @([\mathrm{H}])(\mathrm{O}[\mathrm{H}]) \mathrm{C}([\mathrm{H}])([\mathrm{H}]) \mathrm{N}([\mathrm{H}]) \mathrm{C}([\mathrm{H}])([\mathrm{H}]) \mathrm{C} 3=\mathrm{C}([\mathrm{H}]) \mathrm{C}(\mathrm{OC}([ \\
\mathrm{H}])([\mathrm{H}])[\mathrm{H}])=\mathrm{C}([\mathrm{H}]) \mathrm{C}([\mathrm{H}])=\mathrm{C} 3[\mathrm{H}]) \mathrm{C}(\end{array}$ & 2.210 & 1.820 \\
\hline $\operatorname{Tr} 54$ & $\begin{array}{l}\mathrm{O}=\mathrm{C}(\mathrm{N}([\mathrm{H}]) \mathrm{C}([\mathrm{H}])([\mathrm{H}]) \mathrm{C}=1 \mathrm{~N}=\mathrm{C}(\mathrm{OC}=1 \mathrm{C}([\mathrm{H}])([\mathrm{H}])[\mathrm{H}]) \mathrm{C}([\mathrm{H}])([\mathrm{H}])[\mathrm{H}]) \mathrm{C} 2=\mathrm{C}([\mathrm{H}]) \mathrm{C} \\
(=\mathrm{C}([\mathrm{H}]) \mathrm{C}(=\mathrm{C} 2[\mathrm{H}]) \mathrm{N}(\mathrm{C}([\mathrm{H}])([\mathrm{H}])[\mathrm{H}])[\mathrm{S}](=\mathrm{O})(=\mathrm{O}) \mathrm{C}([\mathrm{H}])([\mathrm{H}])[\mathrm{H}]) \mathrm{C}(=\mathrm{O}) \mathrm{N}([\mathrm{H}])[\mathrm{C} \\
@]([\mathrm{H}])([\mathrm{C} @]([\mathrm{H}])(\mathrm{O}[\mathrm{H}]) \mathrm{C}([\mathrm{H}])([\mathrm{H}]) \mathrm{N}([\mathrm{H}]) \mathrm{C}([\mathrm{H}])([\mathrm{H}]) \mathrm{C} 3=\mathrm{C}([\mathrm{H}]) \mathrm{C}(\mathrm{OC}([\mathrm{H}])([\mathrm{H}])[ \\
\mathrm{H}])=\mathrm{C}([\mathrm{H}]) \mathrm{C}([\mathrm{H}])=\mathrm{C} 3[\mathrm{H}]) \mathrm{C}([\mathrm{H}])([\mathrm{H}]\end{array}$ & 2.258 & 2.839 \\
\hline $\operatorname{Tr} 55$ & $\begin{array}{l}\mathrm{O}=\mathrm{C} 3 \mathrm{O} / \mathrm{C} 2=\mathrm{C}(1 \mathrm{OC}([\mathrm{H}])([\mathrm{H}]) \mathrm{C}([\mathrm{H}])=\mathrm{C}(\mathrm{C}([\mathrm{H}])([\mathrm{H}]) \mathrm{C}([\mathrm{H}])([\mathrm{H}]) \mathrm{C}([\mathrm{H}])=\mathrm{C}(\mathrm{C}([\mathrm{H}])([\mathrm{H} \\
])[\mathrm{H}]) \mathrm{C}([\mathrm{H}])([\mathrm{H}])[\mathrm{H}]) \mathrm{C}([\mathrm{H}])([\mathrm{H}])[\mathrm{H}]) \mathrm{C}=1 \mathrm{OC}([\mathrm{H}])=\mathrm{C}([\mathrm{H}]) \mathrm{C}=1 \mathrm{C}([\mathrm{H}])=\mathrm{C} 2 \mathrm{C}([\mathrm{H}])=\mathrm{C} \\
3[\mathrm{H}]\end{array}$ & 0.208 & 0.646 \\
\hline $\operatorname{Tr} 56$ & $\begin{array}{l}\mathrm{O}=\mathrm{C} 3 \mathrm{OC}=2 \mathrm{C}(\mathrm{OC}([\mathrm{H}])([\mathrm{H}])[\mathrm{C} @ @]([\mathrm{H}])(\mathrm{O}[\mathrm{H}]) \mathrm{C}(\mathrm{O}[\mathrm{H}])(\mathrm{C}([\mathrm{H}])([\mathrm{H}])[\mathrm{H}]) \mathrm{C}([\mathrm{H}])([\mathrm{H}] \\
)[\mathrm{H}])=\mathrm{C} 1 \mathrm{OC}([\mathrm{H}])=\mathrm{C}([\mathrm{H}]) \mathrm{C} 1=\mathrm{C}(\mathrm{OC}([\mathrm{H}])([\mathrm{H}])[\mathrm{H}]) \mathrm{C}=2 \mathrm{C}([\mathrm{H}])=\mathrm{C} 3[\mathrm{H}]\end{array}$ & 0.260 & 0.486 \\
\hline $\operatorname{Tr} 57$ & $\begin{array}{l}\mathrm{O}=\mathrm{C} 3 \mathrm{OC}=2 \mathrm{C}(\mathrm{OC}([\mathrm{H}])([\mathrm{H}])[\mathrm{H}])=\mathrm{C} 1 \mathrm{OC}([\mathrm{H}])=\mathrm{C}([\mathrm{H}]) \mathrm{C} 1=\mathrm{C}(\mathrm{OC}([\mathrm{H}])([\mathrm{H}]) \mathrm{C}([\mathrm{H}])=\mathrm{C}( \\
\mathrm{C}([\mathrm{H}])([\mathrm{H}]) \mathrm{C}([\mathrm{H}])([\mathrm{H}]) \mathrm{C}([\mathrm{H}])=\mathrm{C}(\mathrm{C}([\mathrm{H}])([\mathrm{H}])[\mathrm{H}]) \mathrm{C}([\mathrm{H}])([\mathrm{H}])[\mathrm{H}]) \mathrm{C}([\mathrm{H}])([\mathrm{H}])[\mathrm{H}]) \mathrm{C} \\
=2 \mathrm{C}([\mathrm{H}])=\mathrm{C} 3[\mathrm{H}]\end{array}$ & 0.863 & 0.677 \\
\hline $\operatorname{Tr} 58$ & $\begin{array}{l}\mathrm{O}=\mathrm{C}(\mathrm{N}([\mathrm{H}]) \mathrm{C}(=\mathrm{NC}([\mathrm{H}])([\mathrm{H}]) \mathrm{C} 1=\mathrm{C}([\mathrm{H}]) \mathrm{C}([\mathrm{Cl}])=\mathrm{C}(\mathrm{C}([\mathrm{Cl}])=\mathrm{C} 1[\mathrm{H}]) \mathrm{N}([\mathrm{H}]) \mathrm{C}(=\mathrm{O}) \mathrm{C}([ \\
\mathrm{H}])([\mathrm{H}]) \mathrm{N} 2 \mathrm{C}([\mathrm{H}])([\mathrm{H}]) \mathrm{C}([\mathrm{H}])([\mathrm{H}]) \mathrm{C} 2([\mathrm{H}])[\mathrm{H}]) \mathrm{N}([\mathrm{H}])[\mathrm{H}]) \mathrm{C}=3 \mathrm{C}(=\mathrm{N}[\mathrm{S}] \mathrm{C}=3 \mathrm{C}([\mathrm{H}])([ \\
\mathrm{H}])[\mathrm{H}]) \mathrm{C} 4=\mathrm{C}([\mathrm{H}]) \mathrm{C}([\mathrm{H}])=\mathrm{C}(\mathrm{OC}([\mathrm{H}])([\mathrm{H}])[\mathrm{H}]) \mathrm{C}([\mathrm{H}])=\mathrm{C} 4[\mathrm{H}]\end{array}$ & 4.398 & 4.548 \\
\hline $\operatorname{Tr} 59$ & $\begin{array}{l}\mathrm{O}=\mathrm{C}(\mathrm{N}([\mathrm{H}]) \mathrm{C}(=\mathrm{NC}([\mathrm{H}])([\mathrm{H}]) \mathrm{C}=1 \mathrm{C}([\mathrm{H}])=\mathrm{C}([\mathrm{H}]) \mathrm{C}([\mathrm{H}])=\mathrm{C} 2 \mathrm{C}=1 \mathrm{C}([\mathrm{H}])=\mathrm{C}([\mathrm{H}]) \mathrm{C}([\mathrm{H}] \\
)=\mathrm{C} 2[\mathrm{H}]) \mathrm{N}([\mathrm{H}])[\mathrm{H}]) \mathrm{C}=3 \mathrm{C}(=\mathrm{NOC}=3 \mathrm{C}([\mathrm{H}])([\mathrm{H}])[\mathrm{H}]) \mathrm{C} 4=\mathrm{C}([\mathrm{H}]) \mathrm{C}([\mathrm{H}])=\mathrm{C}(\mathrm{OC}([\mathrm{H}])([ \\
\mathrm{H}])[\mathrm{H}]) \mathrm{C}([\mathrm{H}])=\mathrm{C} 4[\mathrm{H}]\end{array}$ & 1.770 & 1.221 \\
\hline Tr60 & $\begin{array}{l}\mathrm{O}=\mathrm{C}(\mathrm{N}([\mathrm{H}]) \mathrm{C}(=\mathrm{NC}([\mathrm{H}])([\mathrm{H}]) \mathrm{Cl}=\mathrm{C}([\mathrm{H}]) \mathrm{C}(=\mathrm{C}([\mathrm{H}]) \mathrm{C}([\mathrm{Cl}])=\mathrm{C} 1[\mathrm{H}]) \mathrm{C}([\mathrm{H}])=\mathrm{C}([\mathrm{H}]) \mathrm{C}( \\
[\mathrm{H}])([\mathrm{H}]) \mathrm{C}([\mathrm{H}])([\mathrm{H}]) \mathrm{O}[\mathrm{H}]) \mathrm{N}([\mathrm{H}])[\mathrm{H}]) \mathrm{C}=2 \mathrm{C}(=\mathrm{NOC}=2 \mathrm{C}([\mathrm{H}])([\mathrm{H}])[\mathrm{H}]) \mathrm{C} 3=\mathrm{C}([\mathrm{H}]) \mathrm{C}([ \\
\mathrm{H}])=\mathrm{C}(\mathrm{OC}([\mathrm{H}])([\mathrm{H}])[\mathrm{H}]) \mathrm{C}([\mathrm{H}])=\mathrm{C} 3[\mathrm{H}]\end{array}$ & 2.854 & 2.644 \\
\hline Tr61 & $\begin{array}{l}\mathrm{O}=\mathrm{C}(\mathrm{N}([\mathrm{H}]) \mathrm{C}(=\mathrm{NC}([\mathrm{H}])([\mathrm{H}]) \mathrm{Cl}=\mathrm{C}([\mathrm{H}]) \mathrm{C}([\mathrm{Cl}])=\mathrm{C}(\mathrm{C}([\mathrm{Cl}])=\mathrm{C} 1[\mathrm{H}]) \mathrm{N}([\mathrm{H}])[\mathrm{H}]) \mathrm{N}([\mathrm{H}] \\
)[\mathrm{H}]) \mathrm{C}=2 \mathrm{C}(=\mathrm{NOC}=2 \mathrm{C}([\mathrm{H}])([\mathrm{H}])[\mathrm{H}]) \mathrm{C} 3=\mathrm{C}([\mathrm{H}]) \mathrm{C}([\mathrm{H}])=\mathrm{C}(\mathrm{OC}([\mathrm{H}])([\mathrm{H}])[\mathrm{H}]) \mathrm{C}([\mathrm{H}])= \\
\mathrm{C} 3[\mathrm{H}]\end{array}$ & 2.921 & 2.765 \\
\hline Tr62 & $\begin{array}{l}\mathrm{O}=\mathrm{C}(\mathrm{N}([\mathrm{H}]) \mathrm{C}(=\mathrm{NC}([\mathrm{H}])([\mathrm{H}]) \mathrm{C} 1=\mathrm{C}([\mathrm{H}]) \mathrm{C}(=\mathrm{C}([\mathrm{H}]) \mathrm{C}([\mathrm{Cl}])=\mathrm{C} 1[\mathrm{H}]) \mathrm{C}([\mathrm{H}])=\mathrm{C}([\mathrm{H}]) \mathrm{C}( \\
[\mathrm{H}])([\mathrm{H}]) \mathrm{C}([\mathrm{H}])([\mathrm{H}]) \mathrm{C}([\mathrm{H}])([\mathrm{H}])[\mathrm{H}]) \mathrm{N}([\mathrm{H}])[\mathrm{H}]) \mathrm{C}=2 \mathrm{C}(=\mathrm{NOC}=2 \mathrm{C}([\mathrm{H}])([\mathrm{H}])[\mathrm{H}]) \mathrm{C} 3= \\
\mathrm{C}([\mathrm{H}]) \mathrm{C}([\mathrm{H}])=\mathrm{C}(\mathrm{OC}([\mathrm{H}])([\mathrm{H}])[\mathrm{H}]) \mathrm{C}([\mathrm{H}])=\mathrm{C} 3[\mathrm{H}]\end{array}$ & 2.071 & 2.180 \\
\hline Tr63 & $\begin{array}{l}\mathrm{O}=\mathrm{C}(\mathrm{N}([\mathrm{H}]) \mathrm{C}(=\mathrm{NC}([\mathrm{H}])([\mathrm{H}]) \mathrm{C} 1=\mathrm{C}([\mathrm{H}]) \mathrm{C}(=\mathrm{C}(\mathrm{C}([\mathrm{Cl}])=\mathrm{C} 1[\mathrm{H}]) \mathrm{N}([\mathrm{H}]) \mathrm{C}(=\mathrm{O}) \mathrm{C}([\mathrm{H}])([ \\
\mathrm{H}]) \mathrm{N}([\mathrm{H}]) \mathrm{C}([\mathrm{H}])([\mathrm{H}]) \mathrm{C} 2=\mathrm{C}([\mathrm{H}]) \mathrm{C}([\mathrm{H}])=\mathrm{C}([\mathrm{H}]) \mathrm{C}([\mathrm{H}])=\mathrm{C} 2[\mathrm{H}]) \mathrm{C}([\mathrm{H}])([\mathrm{H}]))[\mathrm{H}]) \mathrm{N}([\mathrm{H} \\
])[\mathrm{H}]) \mathrm{C}=3 \mathrm{C}(=\mathrm{N}[\mathrm{S}] \mathrm{C}=3 \mathrm{C}([\mathrm{H}])([\mathrm{H}])[\mathrm{H}]) \mathrm{C} 4=\mathrm{C}([\mathrm{H}]) \mathrm{C}([\mathrm{H}])=\mathrm{C}(\mathrm{OC}([\mathrm{H}])([\mathrm{H}])[\mathrm{H}]) \mathrm{C}([\mathrm{H}] \\
)=\mathrm{C} 4[\mathrm{H}]\end{array}$ & 4.000 & 3.521 \\
\hline Tr64 & $\begin{array}{l}\mathrm{O}=\mathrm{C}(\mathrm{N}([\mathrm{H}]) \mathrm{C}(=\mathrm{NC}([\mathrm{H}])([\mathrm{H}]) \mathrm{C} 1=\mathrm{C}([\mathrm{H}]) \mathrm{C}([\mathrm{Cl}])=\mathrm{C}([\mathrm{H}]) \mathrm{C}([\mathrm{Cl}])=\mathrm{C} 1[\mathrm{H}]) \mathrm{N}([\mathrm{H}])[\mathrm{H}]) \mathrm{C} \\
=2 \mathrm{C}(=\mathrm{NN}([\mathrm{H}]) \mathrm{C}=2 \mathrm{C}([\mathrm{H}])([\mathrm{H}])[\mathrm{H}]) \mathrm{C} 3=\mathrm{C}([\mathrm{H}]) \mathrm{C}([\mathrm{H}])=\mathrm{C}(\mathrm{OC}([\mathrm{H}])([\mathrm{H}])[\mathrm{H}]) \mathrm{C}([\mathrm{H}])=\mathrm{C} \\
3[\mathrm{H}]\end{array}$ & 2.678 & 2.215 \\
\hline
\end{tabular}




\begin{tabular}{|c|c|c|c|}
\hline Tr65 & $\begin{array}{l}\mathrm{O}=\mathrm{C}(\mathrm{N}([\mathrm{H}]) \mathrm{C}(=\mathrm{NC}([\mathrm{H}])([\mathrm{H}]) \mathrm{C} 1=\mathrm{C}([\mathrm{H}]) \mathrm{C}([\mathrm{Cl}])=\mathrm{C}([\mathrm{H}]) \mathrm{C}([\mathrm{Cl}])=\mathrm{C} 1[\mathrm{H}]) \mathrm{N}([\mathrm{H}])[\mathrm{H}]) \mathrm{C} \\
=2 \mathrm{C}(=\mathrm{NOC}=2 \mathrm{C}([\mathrm{H}])([\mathrm{H}])[\mathrm{H}]) \mathrm{C} 3=\mathrm{C}([\mathrm{H}]) \mathrm{C}([\mathrm{H}])=\mathrm{C}([\mathrm{F}]) \mathrm{C}([\mathrm{H}])=\mathrm{C} 3[\mathrm{H}]\end{array}$ & 1.658 & 2.386 \\
\hline Tr66 & $\begin{array}{l}\mathrm{O}=\mathrm{C}(\mathrm{N}([\mathrm{H}]) \mathrm{C}(=\mathrm{NC}([\mathrm{H}])([\mathrm{H}]) \mathrm{C} 1=\mathrm{C}([\mathrm{H}]) \mathrm{C}([\mathrm{Cl}])=\mathrm{C}([\mathrm{H}]) \mathrm{C}([\mathrm{Cl}])=\mathrm{C} 1[\mathrm{H}]) \mathrm{N}([\mathrm{H}])[\mathrm{H}]) \mathrm{C} \\
2=\mathrm{C}(\mathrm{O} / \mathrm{N}=\mathrm{C} 2 / \mathrm{C}([\mathrm{H}])([\mathrm{H}])[\mathrm{H}]) \mathrm{C} 3=\mathrm{C}([\mathrm{H}]) \mathrm{C}([\mathrm{H}])=\mathrm{C}(\mathrm{OC}([\mathrm{H}])([\mathrm{H}])[\mathrm{H}]) \mathrm{C}([\mathrm{H}])=\mathrm{C} 3[\mathrm{H}]\end{array}$ & 2.066 & 1.054 \\
\hline Tr67 & $\begin{array}{l}\mathrm{O}=\mathrm{C}(\mathrm{N}([\mathrm{H}]) \mathrm{C}(=\mathrm{NC}([\mathrm{H}])([\mathrm{H}]) \mathrm{C} 1=\mathrm{C}([\mathrm{H}]) \mathrm{C}=2 \mathrm{C}(\mathrm{C}([\mathrm{H}])=\mathrm{C} 1[\mathrm{H}])=\mathrm{C}([\mathrm{H}]) \mathrm{C}([\mathrm{H}])=\mathrm{C}([\mathrm{H}] \\
) \mathrm{C}=2[\mathrm{H}]) \mathrm{N}([\mathrm{H}])[\mathrm{H}]) \mathrm{C}=3 \mathrm{C}(=\mathrm{N}[\mathrm{S}] \mathrm{C}=3 \mathrm{C}([\mathrm{H}])([\mathrm{H}])[\mathrm{H}]) \mathrm{C} 4=\mathrm{C}([\mathrm{H}]) \mathrm{C}([\mathrm{H}])=\mathrm{C}(\mathrm{OC}([\mathrm{H}])( \\
[\mathrm{H}])[\mathrm{H}]) \mathrm{C}([\mathrm{H}])=\mathrm{C} 4[\mathrm{H}]\end{array}$ & 2.060 & 2.632 \\
\hline Tr68 & $\begin{array}{l}\mathrm{O}=\mathrm{C}(\mathrm{N}([\mathrm{H}]) \mathrm{C}(=\mathrm{NC}([\mathrm{H}])([\mathrm{H}]) \mathrm{C}=1 \mathrm{C}([\mathrm{H}])=\mathrm{C}([\mathrm{H}]) \mathrm{C}([\mathrm{H}])=\mathrm{C} 2 \mathrm{C}=1 \mathrm{C}([\mathrm{H}])=\mathrm{C}([\mathrm{H}]) \mathrm{C}([\mathrm{H}] \\
)=\mathrm{C} 2[\mathrm{H}]) \mathrm{N}([\mathrm{H}])[\mathrm{H}]) \mathrm{C}=3 \mathrm{C}(=\mathrm{NOC}=3 \mathrm{C}([\mathrm{H}])([\mathrm{H}])[\mathrm{H}]) \mathrm{C} 4=\mathrm{C}([\mathrm{H}]) \mathrm{C}([\mathrm{H}])=\mathrm{C}([\mathrm{F}]) \mathrm{C}([\mathrm{H}]) \\
=\mathrm{C} 4[\mathrm{H}]\end{array}$ & 1.745 & 1.347 \\
\hline Tr69 & $\begin{array}{l}\mathrm{O}=\mathrm{C}(\mathrm{N}([\mathrm{H}]) \mathrm{C}(=\mathrm{NC}([\mathrm{H}])([\mathrm{H}]) \mathrm{C} 1=\mathrm{C}([\mathrm{H}]) \mathrm{C}(=\mathrm{C}(\mathrm{C}([\mathrm{Cl}])=\mathrm{C} 1[\mathrm{H}]) \mathrm{N}([\mathrm{H}]) \mathrm{C}(=\mathrm{O}) \mathrm{C}([\mathrm{H}])([ \\
\mathrm{H}]) \mathrm{N}([\mathrm{H}]) \mathrm{C}([\mathrm{H}])([\mathrm{H}]) \mathrm{C}([\mathrm{H}])([\mathrm{H}])[\mathrm{H}]) \mathrm{C}([\mathrm{H}])([\mathrm{H}])[\mathrm{H}]) \mathrm{N}([\mathrm{H}])[\mathrm{H}]) \mathrm{C}=2 \mathrm{C}(=\mathrm{N}[\mathrm{S}] \mathrm{C}=2 \mathrm{C}( \\
[\mathrm{H}])([\mathrm{H}])[\mathrm{H}]) \mathrm{C} 3=\mathrm{C}([\mathrm{H}]) \mathrm{C}([\mathrm{H}])=\mathrm{C}(\mathrm{OC}([\mathrm{H}])([\mathrm{H}])[\mathrm{H}]) \mathrm{C}([\mathrm{H}])=\mathrm{C} 3[\mathrm{H}]\end{array}$ & 3.699 & 3.653 \\
\hline $\operatorname{Tr} 70$ & $\begin{array}{l}\mathrm{O}=\mathrm{C} 2 \mathrm{C}=1 \mathrm{C}(\mathrm{O}[\mathrm{H}])=\mathrm{C}([\mathrm{H}]) \mathrm{C}(\mathrm{O}[\mathrm{H}])=\mathrm{C}([\mathrm{H}]) \mathrm{C}=1 \mathrm{O} / \mathrm{C}(=\mathrm{C} 2 / \mathrm{O}[\mathrm{H}]) \mathrm{C} 3=\mathrm{C}([\mathrm{H}]) \mathrm{C}([\mathrm{H}])=\mathrm{C} \\
(\mathrm{O}[\mathrm{H}]) \mathrm{C}(\mathrm{O}[\mathrm{H}])=\mathrm{C} 3[\mathrm{H}]\end{array}$ & 0.854 & 0.696 \\
\hline $\operatorname{Tr} 71$ & $\begin{array}{l}{[\mathrm{H}] \mathrm{C}=2 \mathrm{C}(\mathrm{O}[\mathrm{H}])=\mathrm{C} 1 \mathrm{OC}=6 \mathrm{C}(\mathrm{OC} 1=\mathrm{C} 3 \mathrm{C}=2 \mathrm{OC}=4 / \mathrm{C} 3=\mathrm{C}(/ \mathrm{O}[\mathrm{H}]) \mathrm{C}([\mathrm{H}])=\mathrm{C}(\mathrm{O}[\mathrm{H}]) \mathrm{C}=4} \\
\mathrm{O} / \mathrm{C} 5=\mathrm{C}(\backslash[\mathrm{H}]) \mathrm{C}(\mathrm{O}[\mathrm{H}])=\mathrm{C}([\mathrm{H}]) \mathrm{C}(\mathrm{O}[\mathrm{H}])=\mathrm{C} 5[\mathrm{H}])=\mathrm{C}(\mathrm{O}[\mathrm{H}]) \mathrm{C}([\mathrm{H}])=\mathrm{C}(\mathrm{O}[\mathrm{H}]) \mathrm{C}=6 \mathrm{OC}=7 \\
\mathrm{C}([\mathrm{H}])=\mathrm{C}(\mathrm{O}[\mathrm{H}]) \mathrm{C}([\mathrm{H}])=\mathrm{C}(\mathrm{O}[\mathrm{H}]) \mathrm{C}=7[\mathrm{H}]\end{array}$ & 1.886 & 1.992 \\
\hline $\operatorname{Tr} 72$ & $\begin{array}{l}\mathrm{O}=\mathrm{C}(/ \mathrm{C} 1=\mathrm{C}(\backslash[\mathrm{H}]) \mathrm{C}(=\mathrm{C}([\mathrm{H}]) \mathrm{C}(=\mathrm{C} 1[\mathrm{H}]) \mathrm{C}(=\mathrm{O}) \mathrm{N} 2[\mathrm{C} @]([\mathrm{H}])(\mathrm{C}([\mathrm{H}])([\mathrm{H}]) \mathrm{C}([\mathrm{H}])([\mathrm{H}]) \\
\mathrm{C} 2([\mathrm{H}])[\mathrm{H}]) \mathrm{C}([\mathrm{H}])([\mathrm{H}]) \mathrm{OC}([\mathrm{H}])([\mathrm{H}])[\mathrm{H}]) \mathrm{C}([\mathrm{H}])([\mathrm{H}])[\mathrm{H}]) \mathrm{N}([\mathrm{H}])[\mathrm{C} @]([\mathrm{H}])([\mathrm{C} @]([ \\
\mathrm{H}])(\mathrm{O}[\mathrm{H}])[\mathrm{C} @] 3([\mathrm{H}]) \mathrm{N}([\mathrm{H}]) \mathrm{C}([\mathrm{H}])([\mathrm{H}]) \mathrm{C}([\mathrm{H}])([\mathrm{H}]) \mathrm{N}(\mathrm{C} 3([\mathrm{H}])[\mathrm{H}])[\mathrm{S}](=\mathrm{O})(=\mathrm{O})[\mathrm{C} @ \\
@] 4([\mathrm{H}]) \mathrm{C}([\mathrm{H}])([\mathrm{H}]) \mathrm{C} 4([\mathrm{H}])[\mathrm{H}]) \mathrm{C}(\end{array}$ & 4.155 & 4.452 \\
\hline $\operatorname{Tr} 73$ & $\begin{array}{l}{[\mathrm{F}] \mathrm{C}=1 \mathrm{C}([\mathrm{H}])=\mathrm{C}(\mathrm{C}([\mathrm{H}])=\mathrm{C}([\mathrm{F}]) \mathrm{C}=1[\mathrm{H}]) \mathrm{C}([\mathrm{H}])([\mathrm{H}])[\mathrm{C} @]([\mathrm{H}])(\mathrm{N}([\mathrm{H}]) \mathrm{C}(=\mathrm{O}) \mathrm{C} 2=\mathrm{C}([} \\
\mathrm{H}]) \mathrm{C}(=\mathrm{C}([\mathrm{H}]) \mathrm{C}(=\mathrm{C} 2[\mathrm{H}]) \mathrm{C}(=\mathrm{O}) \mathrm{N} 3[\mathrm{C} @]([\mathrm{H}])(\mathrm{C}([\mathrm{H}])([\mathrm{H}]) \mathrm{C}([\mathrm{H}])([\mathrm{H}]) \mathrm{C} 3([\mathrm{H}])[\mathrm{H}]) \mathrm{C}([ \\
\mathrm{H}])([\mathrm{H}]) \mathrm{OC}([\mathrm{H}])([\mathrm{H}])[\mathrm{H}]) \mathrm{C}([\mathrm{H}])([\mathrm{H}])[\mathrm{H}])[\mathrm{C} @]([\mathrm{H}])(\mathrm{O}[\mathrm{H}])[\mathrm{C} @] 4([\mathrm{H}]) \mathrm{N}([\mathrm{H}]) \mathrm{C}([\mathrm{H}] \\
)([\mathrm{H}]) \mathrm{C}([\mathrm{H}])([\mathrm{H}]) \mathrm{N}(\mathrm{C} 4([\mathrm{H}])[\mathrm{H}])[\end{array}$ & 4.699 & 4.251 \\
\hline $\operatorname{Tr} 74$ & $\begin{array}{l}{[\mathrm{F}] \mathrm{C}=1 \mathrm{C}([\mathrm{H}])=\mathrm{C}(\mathrm{C}([\mathrm{H}])=\mathrm{C}([\mathrm{F}]) \mathrm{C}=1[\mathrm{H}]) \mathrm{C}([\mathrm{H}])([\mathrm{H}])[\mathrm{C} @]([\mathrm{H}])(\mathrm{N}([\mathrm{H}]) \mathrm{C}(=\mathrm{O}) \mathrm{C} 2=\mathrm{C}([} \\
\mathrm{H}]) \mathrm{C}(=\mathrm{C}([\mathrm{H}]) \mathrm{C}(=\mathrm{C} 2[\mathrm{H}]) \mathrm{C}(=\mathrm{O}) \mathrm{N} 3[\mathrm{C} @]([\mathrm{H}])(\mathrm{C}([\mathrm{H}])([\mathrm{H}]) \mathrm{C}([\mathrm{H}])([\mathrm{H}]) \mathrm{C} 3([\mathrm{H}])[\mathrm{H}]) \mathrm{C}([ \\
\mathrm{H}])([\mathrm{H}]) \mathrm{OC}([\mathrm{H}])([\mathrm{H}])[\mathrm{H}]) \mathrm{C}([\mathrm{H}])([\mathrm{H}])[\mathrm{H}])[\mathrm{C} @]([\mathrm{H}])(\mathrm{O}[\mathrm{H}])[\mathrm{C} @] 4([\mathrm{H}]) \mathrm{N}([\mathrm{H}]) \mathrm{C}([\mathrm{H}] \\
)([\mathrm{H}]) \mathrm{C}([\mathrm{H}])([\mathrm{H}]) \mathrm{N}(\mathrm{C} 4([\mathrm{H}])[\mathrm{H}])[\end{array}$ & 5.000 & 4.214 \\
\hline $\operatorname{Tr} 75$ & $\begin{array}{l}\mathrm{O}=\mathrm{C} 3 \mathrm{~N}(\mathrm{C}([\mathrm{H}])([\mathrm{H}]) \mathrm{C}([\mathrm{H}])([\mathrm{H}]) \mathrm{C}=4 \mathrm{C}([\mathrm{H}])=\mathrm{C}(\mathrm{OC}=2 \mathrm{C}([\mathrm{H}])=\mathrm{C}([\mathrm{H}]) \mathrm{C}=1 \mathrm{~N}=\mathrm{C}(\mathrm{N}(\mathrm{C}([ \\
\mathrm{H}])([\mathrm{H}]) \mathrm{C}=1 \mathrm{C}=2[\mathrm{H}])[\mathrm{C} @ @]([\mathrm{H}])(\mathrm{C}([\mathrm{H}])([\mathrm{H}]) \mathrm{C} 3([\mathrm{H}])[\mathrm{H}]) \mathrm{C}([\mathrm{H}])(\mathrm{C}([\mathrm{H}])([\mathrm{H}])[\mathrm{H}]) \mathrm{C} \\
([\mathrm{H}])([\mathrm{H}])[\mathrm{H}]) \mathrm{N}([\mathrm{H}])[\mathrm{H}]) \mathrm{C}([\mathrm{H}])=\mathrm{C}([\mathrm{H}]) \mathrm{C}=4[\mathrm{H}])[\mathrm{C} @ 5]([\mathrm{H}]) \mathrm{C}([\mathrm{H}])([\mathrm{H}]) \mathrm{C}([\mathrm{H}])([\mathrm{H}]) \\
\mathrm{OC}([\mathrm{H}])([\mathrm{H}]) \mathrm{C} 5([\mathrm{H}])[\mathrm{H}]\end{array}$ & 3.658 & 3.393 \\
\hline Tr76 & $\begin{array}{l}\mathrm{O}=\mathrm{C} 3 \mathrm{~N}(\mathrm{C}([\mathrm{H}])([\mathrm{H}]) \mathrm{C}([\mathrm{H}])([\mathrm{H}]) \mathrm{C}=5 \mathrm{C}([\mathrm{H}])=\mathrm{C}(\mathrm{O} / \mathrm{C} 2=\mathrm{C}(\mathrm{VH}]) \mathrm{C}([\mathrm{H}])=\mathrm{C} 1 \mathrm{~N}=\mathrm{C}(\mathrm{N}(\mathrm{C}([ \\
\mathrm{H}])([\mathrm{H}]) \mathrm{C} 1=\mathrm{C} 2[\mathrm{H}])[\mathrm{C} @ @]([\mathrm{H}])(\mathrm{C}([\mathrm{H}])([\mathrm{H}]) \mathrm{C} 3([\mathrm{H}])[\mathrm{H}])[\mathrm{C} @ @] 4([\mathrm{H}]) \mathrm{C}([\mathrm{H}])([\mathrm{H}]) \\
\mathrm{C}([\mathrm{H}])([\mathrm{H}]) \mathrm{C}([\mathrm{H}])([\mathrm{H}]) \mathrm{C}([\mathrm{H}])([\mathrm{H}]) \mathrm{C} 4([\mathrm{H}])[\mathrm{H}]) \mathrm{N}([\mathrm{H}])[\mathrm{H}]) \mathrm{C}([\mathrm{H}])=\mathrm{C}([\mathrm{H}]) \mathrm{C}=5[\mathrm{H}])[\mathrm{C} \\
@ @] 6([\mathrm{H}]) \mathrm{C}([\mathrm{H}])([\mathrm{H}]) \mathrm{C}([\mathrm{H}])([\mathrm{H}]) \mathrm{OC}([\end{array}$ & 3.770 & 3.579 \\
\hline Tr77 & $\begin{array}{l}\mathrm{O}=\mathrm{C}(\mathrm{N}([\mathrm{H}])[\mathrm{C} @ @]([\mathrm{H}])(\mathrm{C} 1=\mathrm{C}([\mathrm{H}]) \mathrm{C}([\mathrm{H}])=\mathrm{C}([\mathrm{H}]) \mathrm{C}([\mathrm{H}])=\mathrm{C} 1[\mathrm{H}]) \mathrm{C}([\mathrm{H}])([\mathrm{H}])[\mathrm{H}]) \mathrm{C} \\
2=\mathrm{C}([\mathrm{H}]) \mathrm{C}(=\mathrm{C}([\mathrm{H}]) \mathrm{C}(=\mathrm{C} 2[\mathrm{H}]) \mathrm{N}(\mathrm{C}([\mathrm{H}])([\mathrm{H}])[\mathrm{H}])[\mathrm{S}](=\mathrm{O})(=\mathrm{O}) \mathrm{C}([\mathrm{H}])([\mathrm{H}])[\mathrm{H}]) \mathrm{C}(=\mathrm{O}) \\
\mathrm{N}([\mathrm{H}])[\mathrm{C} @]([\mathrm{H}])(\mathrm{C}([\mathrm{H}])([\mathrm{H}]) \mathrm{C}([\mathrm{H}])([\mathrm{H}])[\mathrm{C} @ @]([\mathrm{H}])(\mathrm{OC}([\mathrm{H}])([\mathrm{H}])[\mathrm{H}]) \mathrm{C}(=\mathrm{O}) \mathrm{N}([ \\
\mathrm{H}])[\mathrm{C} @]([\mathrm{H}])(\mathrm{C}(=\mathrm{O}) \mathrm{N}([\mathrm{H}]) \mathrm{C}([\mathrm{H}])([\mathrm{H}]\end{array}$ & 2.886 & 3.251 \\
\hline
\end{tabular}




\begin{tabular}{|c|c|c|c|}
\hline $\operatorname{Tr} 78$ & $\begin{array}{l}\mathrm{O}=\mathrm{C}(\mathrm{N}([\mathrm{H}])[\mathrm{C} @ @]([\mathrm{H}])(\mathrm{C}=1 \mathrm{C}([\mathrm{H}])=\mathrm{C}([\mathrm{H}]) \mathrm{C}([\mathrm{H}])=\mathrm{C}([\mathrm{H}]) \mathrm{C}=1[\mathrm{H}]) \mathrm{C}([\mathrm{H}])([\mathrm{H}])[\mathrm{H}]) \\
\mathrm{C}=2 \mathrm{C}([\mathrm{H}])=\mathrm{C}(\mathrm{C}([\mathrm{H}])=\mathrm{C}(\mathrm{C}=2[\mathrm{H}]) \mathrm{N}(\mathrm{C}([\mathrm{H}])([\mathrm{H}])[\mathrm{H}])[\mathrm{S}](=\mathrm{O})(=\mathrm{O}) \mathrm{C}([\mathrm{H}])([\mathrm{H}])[\mathrm{H}]) \mathrm{C}( \\
=\mathrm{O}) \mathrm{N}([\mathrm{H}])[\mathrm{C} @]([\mathrm{H}])([\mathrm{C} @ @]([\mathrm{H}])(\mathrm{O}[\mathrm{H}]) \mathrm{C}([\mathrm{H}])([\mathrm{H}])[\mathrm{C} @ @]([\mathrm{H}])(\mathrm{OC}([\mathrm{H}])([\mathrm{H}]) \mathrm{C} 3 \\
=\mathrm{C}([\mathrm{H}]) \mathrm{C}([\mathrm{H}])=\mathrm{C}([\mathrm{H}]) \mathrm{C}([\mathrm{H}])=\mathrm{C} 3[\mathrm{H}]) \mathrm{C}(=\mathrm{O}\end{array}$ & 3.699 & 4.228 \\
\hline $\operatorname{Tr} 79$ & $\begin{array}{l}\mathrm{O}=\mathrm{C}(\mathrm{N}([\mathrm{H}])[\mathrm{C} @ @]([\mathrm{H}])(\mathrm{C} 1=\mathrm{C}([\mathrm{H}]) \mathrm{C}([\mathrm{H}])=\mathrm{C}([\mathrm{H}]) \mathrm{C}([\mathrm{H}])=\mathrm{C} 1[\mathrm{H}]) \mathrm{C}([\mathrm{H}])([\mathrm{H}])[\mathrm{H}]) \mathrm{C} \\
=2 \mathrm{C}([\mathrm{H}])=\mathrm{C}(\mathrm{C}([\mathrm{H}])=\mathrm{C}(\mathrm{C}=2[\mathrm{H}]) \mathrm{N}(\mathrm{C}([\mathrm{H}])([\mathrm{H}])[\mathrm{H}])[\mathrm{S}](=\mathrm{O})(=\mathrm{O}) \mathrm{C}([\mathrm{H}])([\mathrm{H}])[\mathrm{H}]) \mathrm{C}(= \\
\mathrm{O}) \mathrm{N}([\mathrm{H}])[\mathrm{C} @]([\mathrm{H}])([\mathrm{C} @ @]([\mathrm{H}])(\mathrm{O}[\mathrm{H}]) \mathrm{C}([\mathrm{H}])([\mathrm{H}])[\mathrm{C} @ @]([\mathrm{H}])(\mathrm{OC}([\mathrm{H}])([\mathrm{H}]) \mathrm{C}([\mathrm{H} \\
])([\mathrm{H}]) \mathrm{OC}([\mathrm{H}])([\mathrm{H}])[\mathrm{H}]) \mathrm{C}(=\mathrm{O}) \mathrm{N}([\mathrm{H}])[\mathrm{C}\end{array}$ & 4.509 & 4.554 \\
\hline $\operatorname{Tr} 80$ & $\begin{array}{l}\mathrm{O}=\mathrm{C}(\mathrm{N}([\mathrm{H}])[\mathrm{C} @ @]([\mathrm{H}])(\mathrm{C} 1=\mathrm{C}([\mathrm{H}]) \mathrm{C}([\mathrm{H}])=\mathrm{C}([\mathrm{H}]) \mathrm{C}([\mathrm{H}])=\mathrm{C} 1[\mathrm{H}]) \mathrm{C}([\mathrm{H}])([\mathrm{H}])[\mathrm{H}]) \mathrm{C} \\
2=\mathrm{C}([\mathrm{H}]) \mathrm{C}(=\mathrm{C}([\mathrm{H}]) \mathrm{C}(=\mathrm{C} 2[\mathrm{H}]) \mathrm{N}(\mathrm{C}([\mathrm{H}])([\mathrm{H}])[\mathrm{H}])[\mathrm{S}](=\mathrm{O})(=\mathrm{O}) \mathrm{C}([\mathrm{H}])([\mathrm{H}])[\mathrm{H}]) \mathrm{C}(=\mathrm{O}) \\
\mathrm{N}([\mathrm{H}])[\mathrm{C} @]([\mathrm{H}])([\mathrm{C} @ @]([\mathrm{H}])(\mathrm{O}[\mathrm{H}]) \mathrm{C}([\mathrm{H}])([\mathrm{H}])[\mathrm{C} @ @](([\mathrm{H}])(\mathrm{OC}([\mathrm{H}])([\mathrm{H}]) \mathrm{C}([\mathrm{H}])( \\
[\mathrm{H}]) \mathrm{C}([\mathrm{H}])([\mathrm{H}])[\mathrm{H}]) \mathrm{C}(=\mathrm{O}) \mathrm{N}([\mathrm{H}])[\mathrm{C} @]\end{array}$ & 4.523 & 3.437 \\
\hline $\operatorname{Tr} 81$ & $\begin{array}{l}\mathrm{O}=\mathrm{C}(\mathrm{N}([\mathrm{H}])[\mathrm{C} @]([\mathrm{H}])(\mathrm{C}(=\mathrm{O}) \mathrm{N}([\mathrm{H}])[\mathrm{C} @]([\mathrm{H}])(\mathrm{C}(=\mathrm{O}) \mathrm{O}[\mathrm{H}]) \mathrm{C}([\mathrm{H}])([\mathrm{H}]) \mathrm{C} 1=\mathrm{C}([\mathrm{H}]) \\
\mathrm{C}([\mathrm{H}])=\mathrm{C}([\mathrm{H}]) \mathrm{C}([\mathrm{H}])=\mathrm{C} 1[\mathrm{H}]) \mathrm{C}([\mathrm{H}])([\mathrm{H}]) \mathrm{C}([\mathrm{H}])([\mathrm{H}]) \mathrm{C}(=\mathrm{O}) \mathrm{O}[\mathrm{H}])[\mathrm{C} @ @]([\mathrm{H}])(\mathrm{N}([ \\
\mathrm{H}]) \mathrm{C}(=\mathrm{O})[\mathrm{C} @ @]([\mathrm{H}])(\mathrm{C}([\mathrm{H}])([\mathrm{H}])[\mathrm{C} @]([\mathrm{H}])(\mathrm{O}[\mathrm{H}])[\mathrm{C} @ @]([\mathrm{H}])(\mathrm{N}([\mathrm{H}]) \mathrm{C}(=\mathrm{O})[\mathrm{C} @ \\
@]([\mathrm{H}])(\mathrm{N}([\mathrm{H}]) \mathrm{C}(=\mathrm{O})[\mathrm{C} @ @]([\mathrm{H}])(\mathrm{N}([\mathrm{H}]) \mathrm{C}(=\mathrm{O}\end{array}$ & 5.495 & 5.660 \\
\hline $\operatorname{Tr} 82$ & $\begin{array}{l}\mathrm{O}=\mathrm{C}(\mathrm{OC}(\mathrm{C}([\mathrm{H}])([\mathrm{H}])[\mathrm{H}])(\mathrm{C}([\mathrm{H}])([\mathrm{H}])[\mathrm{H}]) \mathrm{C}([\mathrm{H}])([\mathrm{H}])[\mathrm{H}]) \mathrm{N}([\mathrm{H}])[\mathrm{C} @]([\mathrm{H}])(\mathrm{C}(=\mathrm{O}) \\
\mathrm{N}([\mathrm{H}])[\mathrm{C} @]([\mathrm{H}])([\mathrm{C} @]([\mathrm{H}])(\mathrm{O}[\mathrm{H}]) \mathrm{C}([\mathrm{H}])([\mathrm{H}])[\mathrm{C} @]([\mathrm{H}])(\mathrm{C}(=\mathrm{O}) \mathrm{N}([\mathrm{H}])[\mathrm{C} @]([\mathrm{H}])( \\
\mathrm{C}(=\mathrm{O}) \mathrm{N}([\mathrm{H}]) \mathrm{C}([\mathrm{H}])([\mathrm{H}]) \mathrm{C} 1=\mathrm{C}([\mathrm{H}]) \mathrm{C}([\mathrm{H}])=\mathrm{C}([\mathrm{H}]) \mathrm{C}([\mathrm{H}])=\mathrm{C} 1[\mathrm{H}]) \mathrm{C}([\mathrm{H}])([\mathrm{H}])[\mathrm{H}]) \mathrm{C}( \\
[\mathrm{H}])([\mathrm{H}])[\mathrm{H}]) \mathrm{C}([\mathrm{H}])([\mathrm{H}]) \mathrm{C}([\mathrm{H}])(\mathrm{C}\end{array}$ & 0.649 & 2.281 \\
\hline $\operatorname{Tr} 83$ & $\begin{array}{l}\mathrm{O}=\mathrm{C} 3 \mathrm{~N}([\mathrm{H}])[\mathrm{C} @]([\mathrm{H}])(\mathrm{C}(=\mathrm{O}) \mathrm{N}([\mathrm{H}])[\mathrm{C} @]([\mathrm{H}])([\mathrm{C} @]([\mathrm{H}])(\mathrm{O}[\mathrm{H}]) \mathrm{C}([\mathrm{H}])([\mathrm{H}]) \mathrm{N}([\mathrm{H}] \\
) \mathrm{C}([\mathrm{H}])([\mathrm{H}]) \mathrm{C} 1=\mathrm{C}([\mathrm{H}]) \mathrm{C}(=\mathrm{C}([\mathrm{H}]) \mathrm{C}([\mathrm{H}])=\mathrm{C} 1[\mathrm{H}]) \mathrm{N}(\mathrm{C}([\mathrm{H}])([\mathrm{H}])[\mathrm{H}]) \mathrm{C}([\mathrm{H}])([\mathrm{H}])[\mathrm{H}]) \\
\mathrm{C}([\mathrm{H}])([\mathrm{H}]) \mathrm{C} 2=\mathrm{C}([\mathrm{H}]) \mathrm{C}([\mathrm{H}])=\mathrm{C}([\mathrm{H}]) \mathrm{C}([\mathrm{H}])=\mathrm{C} 2[\mathrm{H}]) \mathrm{C}([\mathrm{H}])([\mathrm{H}])[\mathrm{C} @ @] 3([\mathrm{H}]) \mathrm{C}([\mathrm{H}] \\
)([\mathrm{H}]) \mathrm{C} 4=\mathrm{C}([\mathrm{H}]) \mathrm{C}([\mathrm{H}])=\mathrm{C}([\mathrm{H}]) \mathrm{C}([\mathrm{H}]\end{array}$ & 1.522 & 1.049 \\
\hline $\operatorname{Tr} 84$ & $\begin{array}{l}\mathrm{O}=\mathrm{C} 3 \mathrm{~N}([\mathrm{H}])[\mathrm{C} @]([\mathrm{H}])(\mathrm{C}(=\mathrm{O}) \mathrm{N}([\mathrm{H}])[\mathrm{C} @]([\mathrm{H}])([\mathrm{C} @]([\mathrm{H}])(\mathrm{O}[\mathrm{H}]) \mathrm{C}([\mathrm{H}])([\mathrm{H}]) \mathrm{N}([\mathrm{H}] \\
) \mathrm{C}([\mathrm{H}])([\mathrm{H}]) \mathrm{C} 1=\mathrm{C}([\mathrm{H}]) \mathrm{C}(=\mathrm{C}([\mathrm{H}]) \mathrm{C}([\mathrm{H}])=\mathrm{C} 1[\mathrm{H}]) \mathrm{N}(\mathrm{C}([\mathrm{H}])([\mathrm{H}])[\mathrm{H}]) \mathrm{C}([\mathrm{H}])([\mathrm{H}])[\mathrm{H}]) \\
\mathrm{C}([\mathrm{H}])([\mathrm{H}]) \mathrm{C} 2=\mathrm{C}([\mathrm{H}]) \mathrm{C}([\mathrm{H}])=\mathrm{C}([\mathrm{H}]) \mathrm{C}([\mathrm{H}])=\mathrm{C} 2[\mathrm{H}]) \mathrm{C}([\mathrm{H}])([\mathrm{H}]) \mathrm{N} 3 \mathrm{C}([\mathrm{H}])([\mathrm{H}]) \mathrm{C} 4= \\
\mathrm{C}([\mathrm{H}]) \mathrm{C}([\mathrm{H}])=\mathrm{C}([\mathrm{F}]) \mathrm{C}([\mathrm{H}])=\mathrm{C} 4[\mathrm{H}]\end{array}$ & 2.087 & 2.011 \\
\hline $\operatorname{Tr} 85$ & $\begin{array}{l}\mathrm{O}=\mathrm{C} 3 \mathrm{~N}([\mathrm{H}])[\mathrm{C} @]([\mathrm{H}])(\mathrm{C}(=\mathrm{O}) \mathrm{N}([\mathrm{H}])[\mathrm{C} @]([\mathrm{H}])([\mathrm{C} @]([\mathrm{H}])(\mathrm{O}[\mathrm{H}]) \mathrm{C}([\mathrm{H}])([\mathrm{H}]) \mathrm{N}([\mathrm{H}] \\
) \mathrm{C}([\mathrm{H}])([\mathrm{H}]) \mathrm{C} 1=\mathrm{C}([\mathrm{H}]) \mathrm{C}(=\mathrm{C}([\mathrm{H}]) \mathrm{C}([\mathrm{H}])=\mathrm{C} 1[\mathrm{H}]) \mathrm{N}(\mathrm{C}([\mathrm{H}])([\mathrm{H}])[\mathrm{H}]) \mathrm{C}([\mathrm{H}])([\mathrm{H}])[\mathrm{H}]) \\
\mathrm{C}([\mathrm{H}])([\mathrm{H}]) \mathrm{C} 2=\mathrm{C}([\mathrm{H}]) \mathrm{C}([\mathrm{H}])=\mathrm{C}([\mathrm{H}]) \mathrm{C}([\mathrm{H}])=\mathrm{C} 2[\mathrm{H}]) \mathrm{C}([\mathrm{H}])([\mathrm{H}]) \mathrm{N} 3 \mathrm{C}([\mathrm{H}])([\mathrm{H}]) \mathrm{C} 4= \\
\mathrm{C}([\mathrm{H}]) \mathrm{C}([\mathrm{H}])=\mathrm{C}(\mathrm{C}([\mathrm{H}])=\mathrm{C} 4[\mathrm{H}]) \mathrm{C}([\mathrm{F}]\end{array}$ & 1.305 & 2.829 \\
\hline $\operatorname{Tr} 86$ & $\begin{array}{l}\mathrm{O}=\mathrm{C} 3 \mathrm{~N}([\mathrm{H}])[\mathrm{C} @](\mathrm{C}(=\mathrm{O}) \mathrm{N}([\mathrm{H}])[\mathrm{C} @]([\mathrm{H}])([\mathrm{C} @]([\mathrm{H}])(\mathrm{O}[\mathrm{H}]) \mathrm{C}([\mathrm{H}])([\mathrm{H}]) \mathrm{N}([\mathrm{H}]) \mathrm{C}([ \\
\mathrm{H}])([\mathrm{H}]) \mathrm{C} 1=\mathrm{C}([\mathrm{H}]) \mathrm{C}(=\mathrm{C}([\mathrm{H}]) \mathrm{C}([\mathrm{H}])=\mathrm{C} 1[\mathrm{H}]) \mathrm{N}(\mathrm{C}([\mathrm{H}])([\mathrm{H}])[\mathrm{H}]) \mathrm{C}([\mathrm{H}])([\mathrm{H}])[\mathrm{H}]) \mathrm{C}([ \\
\mathrm{H}])([\mathrm{H}]) \mathrm{C} 2=\mathrm{C}([\mathrm{H}]) \mathrm{C}([\mathrm{H}])=\mathrm{C}([\mathrm{H}]) \mathrm{C}([\mathrm{H}])=\mathrm{C} 2[\mathrm{H}])(\mathrm{C}([\mathrm{H}])([\mathrm{H}]) \mathrm{N} 3 \mathrm{C}([\mathrm{H}])([\mathrm{H}]) \mathrm{C} 4=\mathrm{C}([ \\
\mathrm{H}]) \mathrm{C}([\mathrm{H}])=\mathrm{C}([\mathrm{H}]) \mathrm{C}([\mathrm{H}])=\mathrm{C} 4[\mathrm{H}]) \mathrm{C}([\mathrm{H}]\end{array}$ & 1.170 & 1.823 \\
\hline $\operatorname{Tr} 87$ & $\begin{array}{l}\mathrm{O}=\mathrm{C} 3 \mathrm{~N}([\mathrm{H}])[\mathrm{C} @]([\mathrm{H}])(\mathrm{C}(=\mathrm{O}) \mathrm{N}([\mathrm{H}])[\mathrm{C} @]([\mathrm{H}])([\mathrm{C} @]([\mathrm{H}])(\mathrm{O}[\mathrm{H}]) \mathrm{C}([\mathrm{H}])([\mathrm{H}]) \mathrm{N}([\mathrm{H}] \\
) \mathrm{C}([\mathrm{H}])([\mathrm{H}]) \mathrm{C} 1=\mathrm{C}([\mathrm{H}]) \mathrm{C}(=\mathrm{C}([\mathrm{H}]) \mathrm{C}([\mathrm{H}])=\mathrm{C} 1[\mathrm{H}]) \mathrm{N}(\mathrm{C}([\mathrm{H}])([\mathrm{H}])[\mathrm{H}]) \mathrm{C}([\mathrm{H}])([\mathrm{H}])[\mathrm{H}]) \\
\mathrm{C}([\mathrm{H}])([\mathrm{H}]) \mathrm{C} 2=\mathrm{C}([\mathrm{H}]) \mathrm{C}([\mathrm{H}])=\mathrm{C}([\mathrm{H}]) \mathrm{C}([\mathrm{H}])=\mathrm{C} 2[\mathrm{H}]) \mathrm{C}([\mathrm{H}])([\mathrm{H}]) \mathrm{N} 3 \mathrm{C}([\mathrm{H}])([\mathrm{H}]) \mathrm{C}([\mathrm{H} \\
])([\mathrm{H}]) \mathrm{O} / \mathrm{C} 4=\mathrm{C}([\mathrm{H}]) \mathrm{C}([\mathrm{H}])=\mathrm{C}([\mathrm{H}])\end{array}$ & 1.454 & 1.979 \\
\hline $\operatorname{Tr} 88$ & $\begin{array}{l}\mathrm{O}=\mathrm{C} 3 \mathrm{~N}([\mathrm{H}])[\mathrm{C} @]([\mathrm{H}])(\mathrm{C}(=\mathrm{O}) \mathrm{N}([\mathrm{H}])[\mathrm{C} @]([\mathrm{H}])([\mathrm{C} @]([\mathrm{H}])(\mathrm{O}[\mathrm{H}]) \mathrm{C}([\mathrm{H}])([\mathrm{H}]) \mathrm{N}([\mathrm{H}] \\
) \mathrm{C}([\mathrm{H}])([\mathrm{H}]) \mathrm{C} 1=\mathrm{C}([\mathrm{H}]) \mathrm{C}(=\mathrm{C}([\mathrm{H}]) \mathrm{C}([\mathrm{H}])=\mathrm{C} 1[\mathrm{H}]) \mathrm{N}(\mathrm{C}([\mathrm{H}])([\mathrm{H}])[\mathrm{H}]) \mathrm{C}([\mathrm{H}])([\mathrm{H}])[\mathrm{H}]) \\
\mathrm{C}([\mathrm{H}])([\mathrm{H}]) \mathrm{C} 2=\mathrm{C}([\mathrm{H}]) \mathrm{C}([\mathrm{H}])=\mathrm{C}([\mathrm{H}]) \mathrm{C}([\mathrm{H}])=\mathrm{C} 2[\mathrm{H}]) \mathrm{C}([\mathrm{H}])([\mathrm{H}]) \mathrm{N} 3 / \mathrm{C} 4=\mathrm{C}([\mathrm{H}]) \mathrm{C}([\mathrm{B} \\
\mathrm{r}])=\mathrm{C}([\mathrm{H}]) \mathrm{C}([\mathrm{Br}])=\mathrm{C} 4[\mathrm{H}]\end{array}$ & 1.456 & 1.956 \\
\hline
\end{tabular}




\begin{tabular}{|c|c|c|c|}
\hline Tr89 & $\begin{array}{l}\mathrm{O}=\mathrm{C} 3 \mathrm{~N}([\mathrm{H}])[\mathrm{C} @]([\mathrm{H}])(\mathrm{C}(=\mathrm{O}) \mathrm{N}([\mathrm{H}])[\mathrm{C} @]([\mathrm{H}])([\mathrm{C} @]([\mathrm{H}])(\mathrm{O}[\mathrm{H}]) \mathrm{C}([\mathrm{H}])([\mathrm{H}]) \mathrm{N}([\mathrm{H}] \\
) \mathrm{C}([\mathrm{H}])([\mathrm{H}]) \mathrm{C} 1=\mathrm{C}([\mathrm{H}]) \mathrm{C}(=\mathrm{C}([\mathrm{H}]) \mathrm{C}([\mathrm{H}])=\mathrm{C} 1[\mathrm{H}]) \mathrm{N}(\mathrm{C}([\mathrm{H}])([\mathrm{H}])[\mathrm{H}]) \mathrm{C}([\mathrm{H}])([\mathrm{H}])[\mathrm{H}]) \\
\mathrm{C}([\mathrm{H}])([\mathrm{H}]) \mathrm{C} 2=\mathrm{C}([\mathrm{H}]) \mathrm{C}([\mathrm{H}])=\mathrm{C}([\mathrm{H}]) \mathrm{C}([\mathrm{H}])=\mathrm{C} 2[\mathrm{H}]) \mathrm{C}([\mathrm{H}])([\mathrm{H}]) \mathrm{N} 3[\mathrm{H}]\end{array}$ & 0.721 & 1.197 \\
\hline $\operatorname{Tr} 90$ & $\begin{array}{l}\mathrm{O}=\mathrm{C} 3 \mathrm{~N}([\mathrm{H}])[\mathrm{C} @]([\mathrm{H}])(\mathrm{C}(=\mathrm{O}) \mathrm{N}([\mathrm{H}])[\mathrm{C} @]([\mathrm{H}])([\mathrm{C} @]([\mathrm{H}])(\mathrm{O}[\mathrm{H}]) \mathrm{C}([\mathrm{H}])([\mathrm{H}]) \mathrm{N}([\mathrm{H}] \\
) \mathrm{C}([\mathrm{H}])([\mathrm{H}]) \mathrm{C} 1=\mathrm{C}([\mathrm{H}]) \mathrm{C}(=\mathrm{C}([\mathrm{H}]) \mathrm{C}([\mathrm{H}])=\mathrm{C} 1[\mathrm{H}]) \mathrm{N}(\mathrm{C}([\mathrm{H}])([\mathrm{H}])[\mathrm{H}]) \mathrm{C}([\mathrm{H}])([\mathrm{H}])[\mathrm{H}]) \\
\mathrm{C}([\mathrm{H}])([\mathrm{H}]) \mathrm{C} 2=\mathrm{C}([\mathrm{H}]) \mathrm{C}([\mathrm{H}])=\mathrm{C}([\mathrm{H}]) \mathrm{C}([\mathrm{H}])=\mathrm{C} 2[\mathrm{H}]) \mathrm{C}([\mathrm{H}])([\mathrm{H}]) \mathrm{N} 3 \mathrm{C}([\mathrm{H}])([\mathrm{H}]) \mathrm{C}([\mathrm{H} \\
])([\mathrm{H}]) \mathrm{C}([\mathrm{H}])([\mathrm{H}]) \mathrm{C}([\mathrm{H}])([\mathrm{H}])[\mathrm{H}]\end{array}$ & 0.398 & 1.636 \\
\hline $\operatorname{Tr} 91$ & $\begin{array}{l}\mathrm{O}=\mathrm{C} 1 \mathrm{~N}(\mathrm{C}(=\mathrm{N}[\mathrm{C} @ @] 1(\mathrm{C}=2 \mathrm{C}([\mathrm{H}])=\mathrm{C}([\mathrm{H}]) \mathrm{C}([\mathrm{H}])=\mathrm{C}([\mathrm{H}]) \mathrm{C}=2[\mathrm{H}]) \mathrm{C} 3=\mathrm{C}([\mathrm{H}]) \mathrm{C}(=\mathrm{C}([ \\
\mathrm{H}]) \mathrm{C}([\mathrm{H}])=\mathrm{C} 3[\mathrm{H}]) \mathrm{C}=4 / \mathrm{N}=\mathrm{C}(/[\mathrm{H}]) \mathrm{C}([\mathrm{H}])=\mathrm{NC}=4[\mathrm{H}]) \mathrm{N}([\mathrm{H}])[\mathrm{H}]) \mathrm{C}([\mathrm{H}])([\mathrm{H}])[\mathrm{H}]\end{array}$ & 2.509 & 2.685 \\
\hline $\operatorname{Tr} 92$ & $\begin{array}{l}\mathrm{O}=\mathrm{C} 1 \mathrm{~N}(\mathrm{C}(=\mathrm{N}[\mathrm{C} @] 1(\mathrm{C}=2 \mathrm{C}([\mathrm{H}])=\mathrm{C}([\mathrm{H}]) \mathrm{C}([\mathrm{H}])=\mathrm{C}([\mathrm{H}]) \mathrm{C}=2[\mathrm{H}]) \mathrm{C} 3=\mathrm{C}([\mathrm{H}]) \mathrm{C}(=\mathrm{C}([\mathrm{H} \\
]) \mathrm{C}([\mathrm{H}])=\mathrm{C} 3[\mathrm{H}]) \mathrm{C} 4=\mathrm{C}([\mathrm{H}]) \mathrm{C}(\mathrm{OC}([\mathrm{H}])([\mathrm{H}])[\mathrm{H}])=\mathrm{C}([\mathrm{H}]) \mathrm{C}([\mathrm{H}])=\mathrm{C} 4[\mathrm{H}]) \mathrm{N}([\mathrm{H}])[\mathrm{H}]) \mathrm{C} \\
([\mathrm{H}])([\mathrm{H}])[\mathrm{H}]\end{array}$ & 3.097 & 1.864 \\
\hline $\operatorname{Tr} 93$ & $\begin{array}{l}\mathrm{O}=\mathrm{C} 1 \mathrm{~N}(\mathrm{C}(=\mathrm{N}[\mathrm{C} @] 1(\mathrm{C}=2 \mathrm{C}([\mathrm{H}])=\mathrm{C}([\mathrm{H}]) \mathrm{C}([\mathrm{H}])=\mathrm{C}([\mathrm{H}]) \mathrm{C}=2[\mathrm{H}]) \mathrm{C} 3=\mathrm{C}([\mathrm{H}]) \mathrm{C}(=\mathrm{C}([\mathrm{H} \\
]) \mathrm{C}([\mathrm{H}])=\mathrm{C} 3[\mathrm{H}]) \mathrm{C} 4=\mathrm{C}([\mathrm{H}]) \mathrm{N}=\mathrm{C}([\mathrm{H}]) \mathrm{C}([\mathrm{Cl}])=\mathrm{C} 4[\mathrm{H}]) \mathrm{N}([\mathrm{H}])[\mathrm{H}]) \mathrm{C}([\mathrm{H}])([\mathrm{H}])[\mathrm{H}]\end{array}$ & 3.046 & 2.197 \\
\hline $\operatorname{Tr} 94$ & $\begin{array}{l}\mathrm{O}=\mathrm{C} 1 \mathrm{~N}(\mathrm{C}(=\mathrm{N}[\mathrm{C} @] 1(\mathrm{C}=2 \mathrm{C}([\mathrm{H}])=\mathrm{C}([\mathrm{H}]) \mathrm{C}([\mathrm{H}])=\mathrm{C}([\mathrm{H}]) \mathrm{C}=2[\mathrm{H}]) \mathrm{C} 3=\mathrm{C}([\mathrm{H}]) \mathrm{C}(=\mathrm{C}([\mathrm{H} \\
]) \mathrm{C}([\mathrm{H}])=\mathrm{C} 3[\mathrm{H}]) \mathrm{C} 4=\mathrm{C}([\mathrm{H}]) \mathrm{N}=\mathrm{C}([\mathrm{H}]) \mathrm{C}([\mathrm{H}])=\mathrm{C} 4[\mathrm{H}]) \mathrm{N}([\mathrm{H}])[\mathrm{H}]) \mathrm{C}([\mathrm{H}])([\mathrm{H}])[\mathrm{H}]\end{array}$ & 2.959 & 1.747 \\
\hline $\operatorname{Tr} 95$ & $\begin{array}{l}\mathrm{O}=\mathrm{C} 1 \mathrm{~N}(\mathrm{C}(=\mathrm{N}[\mathrm{C} @ @] 1(\mathrm{C}=2 \mathrm{C}([\mathrm{H}])=\mathrm{C}([\mathrm{H}]) \mathrm{C}([\mathrm{H}])=\mathrm{C}([\mathrm{H}]) \mathrm{C}=2[\mathrm{H}]) \mathrm{C} 3=\mathrm{C}([\mathrm{H}]) \mathrm{C}([\mathrm{Cl}]) \\
=\mathrm{C}([\mathrm{H}]) \mathrm{C}([\mathrm{H}])=\mathrm{C} 3[\mathrm{H}]) \mathrm{N}([\mathrm{H}])[\mathrm{H}]) \mathrm{C}([\mathrm{H}])([\mathrm{H}])[\mathrm{H}]\end{array}$ & 2.523 & 2.630 \\
\hline $\operatorname{Tr} 96$ & $\begin{array}{l}\mathrm{O}=\mathrm{C} 1 \mathrm{~N}(\mathrm{C}(=\mathrm{N}[\mathrm{C} @ @] 1(\mathrm{C}=2 \mathrm{C}([\mathrm{H}])=\mathrm{C}([\mathrm{H}]) \mathrm{C}([\mathrm{H}])=\mathrm{C}([\mathrm{H}]) \mathrm{C}=2[\mathrm{H}]) \mathrm{C} 3=\mathrm{C}([\mathrm{H}]) \mathrm{C}(=\mathrm{C}([ \\
\mathrm{H}]) \mathrm{C}([\mathrm{H}])=\mathrm{C} 3[\mathrm{H}]) \mathrm{C} 4=\mathrm{C}([\mathrm{H}]) \mathrm{N}=\mathrm{C}([\mathrm{H}]) \mathrm{C}([\mathrm{H}])=\mathrm{C} 4[\mathrm{H}]) \mathrm{N}([\mathrm{H}])[\mathrm{H}]) \mathrm{C}([\mathrm{H}])([\mathrm{H}])[\mathrm{H}]\end{array}$ & 1.770 & 2.515 \\
\hline $\operatorname{Tr} 97$ & $\begin{array}{l}\mathrm{O}=\mathrm{C} 1 \mathrm{~N}(\mathrm{C}(=\mathrm{N}[\mathrm{C} @ @] 1(/ \mathrm{C} 2=\mathrm{C}(\backslash \mathrm{H}]) \mathrm{C}(=\mathrm{C}([\mathrm{H}]) \mathrm{C}([\mathrm{H}])=\mathrm{C} 2[\mathrm{H}]) \mathrm{C} 3=\mathrm{C}([\mathrm{H}]) \mathrm{N}=\mathrm{C}([\mathrm{H}]) \mathrm{C} \\
([\mathrm{F}])=\mathrm{C} 3[\mathrm{H}])[\mathrm{C} @ @] 4([\mathrm{H}]) \mathrm{C}([\mathrm{H}])([\mathrm{H}]) \mathrm{C} 4([\mathrm{H}])[\mathrm{H}]) \mathrm{N}([\mathrm{H}])[\mathrm{H}]) \mathrm{C}([\mathrm{H}])([\mathrm{H}])[\mathrm{H}]\end{array}$ & 2.796 & 2.135 \\
\hline $\operatorname{Tr} 98$ & $\begin{array}{l}\mathrm{O}=\mathrm{C} 1 \mathrm{~N}(\mathrm{C}(=\mathrm{N}[\mathrm{C} @] 1(/ \mathrm{C} 2=\mathrm{C}(\backslash \mathrm{H}]) \mathrm{C}([\mathrm{H}])=\mathrm{C}([\mathrm{H}]) \mathrm{C}([\mathrm{H}])=\mathrm{C} 2[\mathrm{H}]) \mathrm{C} 3=\mathrm{C}([\mathrm{H}]) \mathrm{C}(=\mathrm{C}([\mathrm{H} \\
]) \mathrm{C}([\mathrm{H}])=\mathrm{C} 3[\mathrm{H}]) \mathrm{C} 4=\mathrm{C}([\mathrm{H}]) \mathrm{C}([\mathrm{H}])=\mathrm{C}([\mathrm{H}]) \mathrm{C}([\mathrm{H}])=\mathrm{C} 4[\mathrm{H}]) \mathrm{N}([\mathrm{H}])[\mathrm{H}]) \mathrm{C}([\mathrm{H}])([\mathrm{H}])[\mathrm{H}]\end{array}$ & 1.481 & 1.335 \\
\hline $\operatorname{Tr} 99$ & $\begin{array}{l}\mathrm{O}=\mathrm{C} 1 \mathrm{~N}(\mathrm{C}(=\mathrm{N}[\mathrm{C} @] 1(\mathrm{C}=2 \mathrm{C}([\mathrm{H}])=\mathrm{C}([\mathrm{H}]) \mathrm{C}([\mathrm{H}])=\mathrm{C}([\mathrm{H}]) \mathrm{C}=2[\mathrm{H}]) \mathrm{C} 3=\mathrm{C}([\mathrm{H}]) \mathrm{C}(=\mathrm{C}([\mathrm{H} \\
]) \mathrm{C}([\mathrm{H}])=\mathrm{C} 3[\mathrm{H}]) \mathrm{C} 4=\mathrm{C}([\mathrm{H}]) \mathrm{C}([\mathrm{H}])=\mathrm{C}(\mathrm{OC}([\mathrm{H}])([\mathrm{H}])[\mathrm{H}]) \mathrm{C}([\mathrm{H}])=\mathrm{C} 4[\mathrm{H}]) \mathrm{N}([\mathrm{H}])[\mathrm{H}]) \mathrm{C} \\
([\mathrm{H}])([\mathrm{H}])[\mathrm{H}]\end{array}$ & 1.420 & 1.864 \\
\hline $\operatorname{Tr} 100$ & $\begin{array}{l}\mathrm{O}=\mathrm{C} 3 \mathrm{~N}([\mathrm{H}])[\mathrm{C} @]([\mathrm{H}])(\mathrm{C}(=\mathrm{O}) \mathrm{N}([\mathrm{H}])[\mathrm{C} @]([\mathrm{H}])([\mathrm{C} @]([\mathrm{H}])(\mathrm{O}[\mathrm{H}]) \mathrm{C}([\mathrm{H}])([\mathrm{H}]) \mathrm{N}([\mathrm{H}] \\
) \mathrm{C}([\mathrm{H}])([\mathrm{H}]) \mathrm{C} 1=\mathrm{C}([\mathrm{H}]) \mathrm{C}(=\mathrm{C}([\mathrm{H}]) \mathrm{C}([\mathrm{H}])=\mathrm{C} 1[\mathrm{H}]) \mathrm{N}(\mathrm{C}([\mathrm{H}])([\mathrm{H}])[\mathrm{H}]) \mathrm{C}([\mathrm{H}])([\mathrm{H}])[\mathrm{H}]) \\
\mathrm{C}([\mathrm{H}])([\mathrm{H}]) \mathrm{C} 2=\mathrm{C}([\mathrm{H}]) \mathrm{C}([\mathrm{H}])=\mathrm{C}([\mathrm{H}]) \mathrm{C}([\mathrm{H}])=\mathrm{C} 2[\mathrm{H}]) \mathrm{C}([\mathrm{H}])([\mathrm{H}]) \mathrm{N} 3 \mathrm{C}([\mathrm{H}])([\mathrm{H}]) \mathrm{C} 4= \\
\mathrm{C}([\mathrm{H}]) \mathrm{C}([\mathrm{H}])=\mathrm{C}([\mathrm{H}]) \mathrm{C}([\mathrm{H}])=\mathrm{C} 4[\mathrm{H}]\end{array}$ & 2.547 & 1.786 \\
\hline $\operatorname{Tr} 101$ & $\begin{array}{l}\mathrm{O}=\mathrm{C} 1 \mathrm{~N}(\mathrm{C}(=\mathrm{N}[\mathrm{C} @ @] 1(/ \mathrm{C} 2=\mathrm{C}(\mathrm{V}[\mathrm{H}]) \mathrm{C}([\mathrm{H}])=\mathrm{C}([\mathrm{H}]) \mathrm{C}([\mathrm{H}])=\mathrm{C} 2[\mathrm{H}]) \mathrm{C} 3=\mathrm{C}([\mathrm{H}]) \mathrm{C}(\mathrm{C \# N} \\
)=\mathrm{C}([\mathrm{H}]) \mathrm{C}([\mathrm{H}])=\mathrm{C} 3[\mathrm{H}]) \mathrm{N}([\mathrm{H}])[\mathrm{H}]) \mathrm{C}([\mathrm{H}])([\mathrm{H}])[\mathrm{H}]\end{array}$ & 2.432 & 2.299 \\
\hline $\operatorname{Tr} 102$ & $\begin{array}{l}\mathrm{O}=\mathrm{C} 1 \mathrm{~N}(\mathrm{C}(=\mathrm{N}[\mathrm{C} @] 1(\mathrm{C}=2 \mathrm{C}([\mathrm{H}])=\mathrm{C}([\mathrm{H}]) \mathrm{C}([\mathrm{H}])=\mathrm{C}([\mathrm{H}]) \mathrm{C}=2[\mathrm{H}]) \mathrm{C} 3=\mathrm{C}([\mathrm{H}]) \mathrm{C}(=\mathrm{C}([\mathrm{H} \\
]) \mathrm{C}([\mathrm{H}])=\mathrm{C} 3[\mathrm{H}]) \mathrm{C} 4=\mathrm{NC}([\mathrm{H}])=\mathrm{C}([\mathrm{H}]) \mathrm{C}([\mathrm{H}])=\mathrm{C} 4[\mathrm{H}]) \mathrm{N}([\mathrm{H}])[\mathrm{H}]) \mathrm{C}([\mathrm{H}])([\mathrm{H}])[\mathrm{H}]\end{array}$ & 1.721 & 2.184 \\
\hline $\operatorname{Tr} 103$ & $\begin{array}{l}\mathrm{O}=\mathrm{C}(\mathrm{N}([\mathrm{C} @ @] 1([\mathrm{H}]) \mathrm{C}([\mathrm{H}])([\mathrm{H}]) \mathrm{C}([\mathrm{H}])([\mathrm{H}]) \mathrm{C}([\mathrm{H}])([\mathrm{H}]) \mathrm{C}([\mathrm{H}])([\mathrm{H}]) \mathrm{C} 1([\mathrm{H}])[\mathrm{H}]) \mathrm{C}([ \\
\mathrm{H}])([\mathrm{H}])[\mathrm{H}]) \mathrm{C}([\mathrm{H}])([\mathrm{H}]) \mathrm{C}([\mathrm{H}])([\mathrm{H}])[\mathrm{C} @([\mathrm{H}])(\mathrm{N} 4 \mathrm{C}(=\mathrm{N} / \mathrm{C} 3=\mathrm{C}([\mathrm{H}]) \mathrm{C}([\mathrm{H}])=\mathrm{C}(\mathrm{O} / \mathrm{C} 2 \\
=\mathrm{C}([\mathrm{H}]) \mathrm{C}([\mathrm{H}])=\mathrm{C}([\mathrm{H}]) \mathrm{C}([\mathrm{H}])=\mathrm{C} 2[\mathrm{H}]) \mathrm{C}([\mathrm{H}])=\mathrm{C} 3 \mathrm{C} 4([\mathrm{H}])[\mathrm{H}]) \mathrm{N}([\mathrm{H}])[\mathrm{H}]) \mathrm{C} 5=\mathrm{C}([\mathrm{H}]) \\
\mathrm{C}([\mathrm{H}])=\mathrm{C}([\mathrm{H}]) \mathrm{C}([\mathrm{H}])=\mathrm{C} 5[\mathrm{H}]\end{array}$ & 2.785 & 2.388 \\
\hline Tr104 & $\mathrm{O}=\mathrm{C}(\mathrm{N}([\mathrm{C} @ @] 1([\mathrm{H}]) \mathrm{C}([\mathrm{H}])([\mathrm{H}]) \mathrm{C}([\mathrm{H}])([\mathrm{H}]) \mathrm{C}([\mathrm{H}])([\mathrm{H}]) \mathrm{C}([\mathrm{H}])([\mathrm{H}]) \mathrm{C} 1([\mathrm{H}])[\mathrm{H}]) \mathrm{C}([$ & 3.638 & 3.271 \\
\hline
\end{tabular}




\begin{tabular}{|c|c|c|c|}
\hline & $\begin{array}{l}\mathrm{H}])([\mathrm{H}]) \mathrm{C} 2=\mathrm{C}([\mathrm{H}]) \mathrm{N}([\mathrm{H}]) \mathrm{N}=\mathrm{C} 2[\mathrm{H}]) \mathrm{C}([\mathrm{H}])([\mathrm{H}]) \mathrm{C}([\mathrm{H}])([\mathrm{H}])[\mathrm{C} @]([\mathrm{H}])(\mathrm{N} 5 \mathrm{C}(=\mathrm{NC}=4 \\
\mathrm{C}([\mathrm{H}])=\mathrm{C}([\mathrm{H}]) \mathrm{C}(\mathrm{O} / \mathrm{C} 3=\mathrm{C}(\mathrm{VH}]) \mathrm{C}([\mathrm{H}])=\mathrm{C}([\mathrm{H}]) \mathrm{C}([\mathrm{H}])=\mathrm{C} 3[\mathrm{H}])=\mathrm{C}([\mathrm{H}]) \mathrm{C}=4 \mathrm{C} 5([\mathrm{H}])[\mathrm{H} \\
]) \mathrm{N}([\mathrm{H}])[\mathrm{H}])[\mathrm{C} @ @ 6([\mathrm{H}]) \mathrm{C}([\mathrm{H}])([\mathrm{H}])\end{array}$ & & \\
\hline $\operatorname{Tr} 105$ & $\begin{array}{l}\mathrm{O}=\mathrm{C}(\mathrm{N}([\mathrm{C} @ @] 1([\mathrm{H}]) \mathrm{C}([\mathrm{H}])([\mathrm{H}]) \mathrm{C}([\mathrm{H}])([\mathrm{H}]) \mathrm{C}([\mathrm{H}])([\mathrm{H}]) \mathrm{C}([\mathrm{H}])([\mathrm{H}]) \mathrm{C} 1([\mathrm{H}])[\mathrm{H}]) \mathrm{C}([ \\
\mathrm{H}])([\mathrm{H}]) \mathrm{C} 2=\mathrm{C}([\mathrm{H}]) \mathrm{N}(/ \mathrm{N}=\mathrm{C} 2 /[\mathrm{H}]) \mathrm{C}([\mathrm{H}])([\mathrm{H}])[\mathrm{H}]) \mathrm{C}([\mathrm{H}])([\mathrm{H}]) \mathrm{C}([\mathrm{H}])([\mathrm{H}])[\mathrm{C} @]([\mathrm{H}])( \\
\mathrm{N} 5 \mathrm{C}(=\mathrm{NC}=4 \mathrm{C}([\mathrm{H}])=\mathrm{C}([\mathrm{H}]) \mathrm{C}(\mathrm{O} / \mathrm{C} 3=\mathrm{C}([\mathrm{H}]) \mathrm{C}([\mathrm{H}])=\mathrm{C}([\mathrm{H}]) \mathrm{C}([\mathrm{H}])=\mathrm{C} 3[\mathrm{H}])=\mathrm{C}([\mathrm{H}]) \mathrm{C} \\
=4 \mathrm{C} 5([\mathrm{H}])[\mathrm{H}]) \mathrm{N}([\mathrm{H}])[\mathrm{H}])[\mathrm{C} @ @] 6([\mathrm{H}\end{array}$ & 3.959 & 3.583 \\
\hline $\operatorname{Tr} 106$ & $\begin{array}{l}\mathrm{O}=\mathrm{C}(\mathrm{N}([\mathrm{C} @ @] 1([\mathrm{H}]) \mathrm{C}([\mathrm{H}])([\mathrm{H}]) \mathrm{C}([\mathrm{H}])([\mathrm{H}]) \mathrm{C}([\mathrm{H}])([\mathrm{H}]) \mathrm{C}([\mathrm{H}])([\mathrm{H}]) \mathrm{C} 1([\mathrm{H}])[\mathrm{H}]) \mathrm{C}([ \\
\mathrm{H}])([\mathrm{H}]) \mathrm{C}([\mathrm{H}])([\mathrm{H}]) \mathrm{C}=2 \mathrm{~N}=\mathrm{C}([\mathrm{S}] \mathrm{C}=2[\mathrm{H}]) \mathrm{C}([\mathrm{H}])([\mathrm{H}])[\mathrm{H}]) \mathrm{C}([\mathrm{H}])([\mathrm{H}]) \mathrm{C}([\mathrm{H}])([\mathrm{H}])[\mathrm{C} \\
@]([\mathrm{H}])(\mathrm{N} 5 \mathrm{C}(=\mathrm{NC}=4 \mathrm{C}([\mathrm{H}])=\mathrm{C}([\mathrm{H}]) \mathrm{C}(\mathrm{O} / \mathrm{C} 3=\mathrm{C}(\backslash[\mathrm{H}]) \mathrm{C}([\mathrm{H}])=\mathrm{C}([\mathrm{H}]) \mathrm{C}([\mathrm{H}])=\mathrm{C} 3[\mathrm{H}])= \\
\mathrm{C}([\mathrm{H}]) \mathrm{C}=4 \mathrm{C} 5([\mathrm{H}])[\mathrm{H}]) \mathrm{N}([\mathrm{H}])[\mathrm{H}])[\mathrm{C}\end{array}$ & 2.975 & 3.351 \\
\hline $\operatorname{Tr} 107$ & $\begin{array}{l}\mathrm{O}=\mathrm{C}(\mathrm{N}([\mathrm{C} @ @] 1([\mathrm{H}]) \mathrm{C}([\mathrm{H}])([\mathrm{H}]) \mathrm{C}([\mathrm{H}])([\mathrm{H}]) \mathrm{C}([\mathrm{H}])([\mathrm{H}]) \mathrm{C}([\mathrm{H}])([\mathrm{H}]) \mathrm{C} 1([\mathrm{H}])[\mathrm{H}])[\mathrm{C} \\
@ @]([\mathrm{H}])(\mathrm{C}=2 \mathrm{~N}=\mathrm{C}([\mathrm{S}] \mathrm{C}=2[\mathrm{H}]) \mathrm{C}([\mathrm{H}])([\mathrm{H}])[\mathrm{H}]) \mathrm{C}([\mathrm{H}])([\mathrm{H}])[\mathrm{H}]) \mathrm{C}([\mathrm{H}])([\mathrm{H}]) \mathrm{C}([\mathrm{H}])([ \\
\mathrm{H}])[\mathrm{C} @]([\mathrm{H}])(\mathrm{N} 5 \mathrm{C}(=\mathrm{NC}=4 \mathrm{C}([\mathrm{H}])=\mathrm{C}([\mathrm{H}]) \mathrm{C}(\mathrm{O} / \mathrm{C} 3=\mathrm{C}(\backslash[\mathrm{H}]) \mathrm{C}([\mathrm{H}])=\mathrm{C}([\mathrm{H}]) \mathrm{C}([\mathrm{H}])=\mathrm{C} \\
3[\mathrm{H}])=\mathrm{C}([\mathrm{H}]) \mathrm{C}=4 \mathrm{C} 5([\mathrm{H}])[\mathrm{H}]) \mathrm{N}([\mathrm{H}])[\mathrm{H}\end{array}$ & 2.983 & 3.308 \\
\hline $\operatorname{Tr} 108$ & $\begin{array}{l}\mathrm{O}=\mathrm{C}(\mathrm{N}([\mathrm{C} @ @] 1([\mathrm{H}]) \mathrm{C}([\mathrm{H}])([\mathrm{H}]) \mathrm{C}([\mathrm{H}])([\mathrm{H}]) \mathrm{C}([\mathrm{H}])([\mathrm{H}]) \mathrm{C}([\mathrm{H}])([\mathrm{H}]) \mathrm{C} 1([\mathrm{H}])[\mathrm{H}]) \mathrm{C}([ \\
\mathrm{H}])([\mathrm{H}]) \mathrm{C}=2 \mathrm{~N}=\mathrm{C}([\mathrm{S}] \mathrm{C}=2[\mathrm{H}]) \mathrm{C}([\mathrm{H}])([\mathrm{H}])[\mathrm{H}]) \mathrm{C}([\mathrm{H}])([\mathrm{H}]) \mathrm{C}([\mathrm{H}])([\mathrm{H}])[\mathrm{C} @]([\mathrm{H}])(\mathrm{N} 5 \\
\mathrm{C}(=\mathrm{NC}=4 \mathrm{C}([\mathrm{H}])=\mathrm{C}([\mathrm{H}]) \mathrm{C}(\mathrm{O} / \mathrm{C} 3=\mathrm{C}(\backslash[\mathrm{H}]) \mathrm{C}([\mathrm{H}])=\mathrm{C}([\mathrm{H}]) \mathrm{C}([\mathrm{H}])=\mathrm{C} 3[\mathrm{H}])=\mathrm{C}([\mathrm{H}]) \mathrm{C}=4 \\
\mathrm{C} 5([\mathrm{H}])[\mathrm{H}]) \mathrm{N}([\mathrm{H}])[\mathrm{H}])[\mathrm{C} @ @] 6([\mathrm{H}]) \mathrm{C}(\end{array}$ & 3.102 & 3.315 \\
\hline $\operatorname{Tr} 109$ & $\begin{array}{l}\mathrm{O}=\mathrm{C}(\mathrm{OC}([\mathrm{H}])([\mathrm{H}])[\mathrm{C} @]([\mathrm{H}])(\mathrm{N} 3 \mathrm{C}(=\mathrm{N} / \mathrm{C} 2=\mathrm{C}(\backslash[\mathrm{H}]) \mathrm{C}([\mathrm{H}])=\mathrm{C}(\mathrm{O} / \mathrm{C} 1=\mathrm{C}(\backslash \mathrm{H}]) \mathrm{C}([\mathrm{H}])= \\
\mathrm{C}([\mathrm{H}]) \mathrm{C}([\mathrm{H}])=\mathrm{C} 1[\mathrm{H}]) \mathrm{C}([\mathrm{H}])=\mathrm{C} 2 \mathrm{C} 3([\mathrm{H}])[\mathrm{H}]) \mathrm{N}([\mathrm{H}])[\mathrm{H}])[\mathrm{C} @] 4([\mathrm{H}]) \mathrm{C}([\mathrm{H}])([\mathrm{H}]) \mathrm{OC}([ \\
\mathrm{H}])([\mathrm{H}]) \mathrm{C}([\mathrm{H}])([\mathrm{H}]) \mathrm{C} 4([\mathrm{H}])[\mathrm{H}]) \mathrm{N}([\mathrm{C} @ @] 5([\mathrm{H}]) \mathrm{C}([\mathrm{H}])([\mathrm{H}]) \mathrm{C}([\mathrm{H}])([\mathrm{H}]) \mathrm{C}([\mathrm{H}])([\mathrm{H}]) \\
\mathrm{C}([\mathrm{H}])([\mathrm{H}]) \mathrm{C} 5([\mathrm{H}])[\mathrm{H}]) \mathrm{C}([\mathrm{H}])([\mathrm{H}]\end{array}$ & 2.983 & 3.667 \\
\hline $\operatorname{Tr} 110$ & $\begin{array}{l}\mathrm{O}=\mathrm{C}(\mathrm{OC}([\mathrm{H}])([\mathrm{H}])[\mathrm{C} @]([\mathrm{H}])(\mathrm{N} 3 \mathrm{C}(=\mathrm{N} / \mathrm{C} 2=\mathrm{C}(\backslash[\mathrm{H}]) \mathrm{C}([\mathrm{H}])=\mathrm{C}(\mathrm{O} / \mathrm{C} 1=\mathrm{C}(\backslash \mathrm{H}]) \mathrm{C}([\mathrm{H}])= \\
\mathrm{C}([\mathrm{H}]) \mathrm{C}([\mathrm{H}])=\mathrm{C} 1[\mathrm{H}]) \mathrm{C}([\mathrm{H}])=\mathrm{C} 2 \mathrm{C} 3([\mathrm{H}])[\mathrm{H}]) \mathrm{N}([\mathrm{H}])[\mathrm{H}]) \mathrm{C} 4=\mathrm{C}([\mathrm{H}]) \mathrm{C}([\mathrm{H}])=\mathrm{C}([\mathrm{H}]) \mathrm{C}( \\
[\mathrm{H}])=\mathrm{C} 4[\mathrm{H}]) \mathrm{N}([\mathrm{C} @ @] 5([\mathrm{H}]) \mathrm{C}([\mathrm{H}])([\mathrm{H}]) \mathrm{C}([\mathrm{H}])([\mathrm{H}]) \mathrm{C}([\mathrm{H}])([\mathrm{H}]) \mathrm{C}([\mathrm{H}])([\mathrm{H}]) \mathrm{C} 5([\mathrm{H}]) \\
[\mathrm{H}]) \mathrm{C}([\mathrm{H}])([\mathrm{H}])[\mathrm{H}]\end{array}$ & 2.106 & 2.807 \\
\hline $\operatorname{Tr} 111$ & 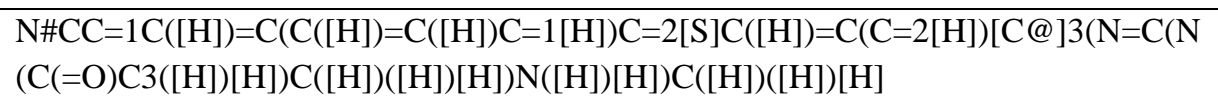 & 2.678 & 2.504 \\
\hline $\operatorname{Tr} 112$ & $\begin{array}{l}\mathrm{N} \# \mathrm{CC}=1 \mathrm{C}([\mathrm{H}])=\mathrm{C}(\mathrm{C}([\mathrm{H}])=\mathrm{C}([\mathrm{H}]) \mathrm{C}=1[\mathrm{H}]) \mathrm{C}=2[\mathrm{~S}] / \mathrm{C}(=\mathrm{C}(/[\mathrm{H}]) \mathrm{C}=2[\mathrm{H}])[\mathrm{C} @] 3(\mathrm{~N}=\mathrm{C}( \\
\mathrm{N}(\mathrm{C}(=\mathrm{O}) \mathrm{C} 3([\mathrm{H}])[\mathrm{H}]) \mathrm{C}([\mathrm{H}])([\mathrm{H}])[\mathrm{H}]) \mathrm{N}([\mathrm{H}])[\mathrm{H}]) \mathrm{C}([\mathrm{H}])([\mathrm{H}])[\mathrm{H}]\end{array}$ & 3.056 & 2.986 \\
\hline $\operatorname{Tr} 113$ & $\begin{array}{l}\mathrm{O}=\mathrm{C} 3 \mathrm{~N}(\mathrm{C}(=\mathrm{N}[\mathrm{C} @](\mathrm{C}=1 \mathrm{C}([\mathrm{H}])=\mathrm{C}([\mathrm{S}] \mathrm{C}=1[\mathrm{H}]) \mathrm{C} 2=\mathrm{C}([\mathrm{H}]) \mathrm{C}(\mathrm{C \# CC}([\mathrm{H}])([\mathrm{H}])[\mathrm{H}])=\mathrm{C}( \\
[\mathrm{H}]) \mathrm{N}=\mathrm{C} 2[\mathrm{H}])(\mathrm{C} 3([\mathrm{H}])[\mathrm{H}]) \mathrm{C}([\mathrm{H}])([\mathrm{H}])[\mathrm{H}]) \mathrm{N}([\mathrm{H}])[\mathrm{H}]) \mathrm{C}([\mathrm{H}])([\mathrm{H}])[\mathrm{H}]\end{array}$ & 3.328 & 2.672 \\
\hline $\operatorname{Tr} 114$ & $\begin{array}{l}\mathrm{O}=\mathrm{C} 4 \mathrm{~N}(\mathrm{C}(=\mathrm{N}[\mathrm{C} @ @](/ \mathrm{C} 1=\mathrm{C}(\backslash[\mathrm{H}]) \mathrm{C}([\mathrm{H}])=\mathrm{C}([\mathrm{H}]) \mathrm{C}(=\mathrm{C} 1[\mathrm{H}]) \mathrm{C} 2=\mathrm{C}([\mathrm{H}]) \mathrm{N}=\mathrm{C}([\mathrm{H}]) \mathrm{C}( \\
[\mathrm{H}])=\mathrm{C} 2[\mathrm{H}])(\mathrm{C} 3=\mathrm{C}([\mathrm{H}]) \mathrm{C}([\mathrm{H}])=\mathrm{C}([\mathrm{H}]) \mathrm{C}([\mathrm{H}])=\mathrm{C} 3[\mathrm{H}]) \mathrm{C} 4([\mathrm{H}])[\mathrm{H}]) \mathrm{N}([\mathrm{H}])[\mathrm{H}]) \mathrm{C}([\mathrm{H}]) \\
([\mathrm{H}])[\mathrm{H}]\end{array}$ & 0.357 & 0.772 \\
\hline $\operatorname{Tr} 115$ & $\begin{array}{l}\mathrm{O}=\mathrm{C} 3 \mathrm{~N}(\mathrm{C}(=\mathrm{N}[\mathrm{C} @](\mathrm{C}=2[\mathrm{~S}] \mathrm{C}(\mathrm{C}=1 \mathrm{C}([\mathrm{H}])=\mathrm{C}(\mathrm{CHCC}([\mathrm{H}])([\mathrm{H}])[\mathrm{H}]) \mathrm{C}([\mathrm{H}])=\mathrm{NC}=1[\mathrm{H}]) \\
=\mathrm{C}([\mathrm{H}]) \mathrm{C}=2[\mathrm{Cl}])(\mathrm{C} 3([\mathrm{H}])[\mathrm{H}]) \mathrm{C}([\mathrm{H}])([\mathrm{H}])[\mathrm{H}]) \mathrm{N}([\mathrm{H}])[\mathrm{H}]) \mathrm{C}([\mathrm{H}])([\mathrm{H}])[\mathrm{H}]\end{array}$ & 4.770 & 4.708 \\
\hline $\operatorname{Tr} 116$ & $\begin{array}{l}\mathrm{O}=\mathrm{C}(\mathrm{O}[\mathrm{C} @] 2([\mathrm{H}])[\mathrm{C} @ @]([\mathrm{H}])(\mathrm{OC} 1=\mathrm{C}(\mathrm{C}(\mathrm{O}[\mathrm{H}])=\mathrm{C}([\mathrm{H}]) \mathrm{C}(\mathrm{O}[\mathrm{H}])=\mathrm{C} 1[\mathrm{H}]) \mathrm{C} 2([\mathrm{H}])[ \\
\mathrm{H}]) \mathrm{C}=3 \mathrm{C}([\mathrm{H}])=\mathrm{C}(\mathrm{O}[\mathrm{H}]) \mathrm{C}(\mathrm{O}[\mathrm{H}])=\mathrm{C}(\mathrm{O}[\mathrm{H}]) \mathrm{C}=3[\mathrm{H}]) \mathrm{C}=4 \mathrm{C}([\mathrm{H}])=\mathrm{C}(\mathrm{O}[\mathrm{H}]) \mathrm{C}(\mathrm{O}[\mathrm{H}])=\mathrm{C}( \\
\mathrm{O}[\mathrm{H}]) \mathrm{C}=4[\mathrm{H}]\end{array}$ & 2.678 & 2.978 \\
\hline
\end{tabular}




\begin{tabular}{|c|c|c|c|}
\hline $\operatorname{Tr} 117$ & $\begin{array}{l}\mathrm{O}=\mathrm{C}(\mathrm{N}([\mathrm{H}]) \mathrm{C}([\mathrm{H}])([\mathrm{H}]) \mathrm{C}([\mathrm{H}])([\mathrm{H}]) \mathrm{C}([\mathrm{H}])([\mathrm{H}]) \mathrm{C}([\mathrm{H}])([\mathrm{H}]) \mathrm{C}([\mathrm{H}])([\mathrm{H}]) \mathrm{C}([\mathrm{H}])([\mathrm{H}]) \mathrm{C} \\
([\mathrm{H}])([\mathrm{H}]) \mathrm{C}([\mathrm{H}])([\mathrm{H}]) \mathrm{C}([\mathrm{H}])([\mathrm{H}]) \mathrm{C}([\mathrm{H}])([\mathrm{H}]) \mathrm{N}([\mathrm{H}]) \mathrm{C}=2 \mathrm{C}=1 \mathrm{C}([\mathrm{H}])=\mathrm{C}([\mathrm{H}]) \mathrm{C}([\mathrm{H}])= \\
\mathrm{C}([\mathrm{H}]) \mathrm{C}=1 \mathrm{~N}=\mathrm{C} 3 \mathrm{C}=2 \mathrm{C}([\mathrm{H}])([\mathrm{H}]) \mathrm{C}([\mathrm{H}])([\mathrm{H}]) \mathrm{C}([\mathrm{H}])([\mathrm{H}]) \mathrm{C} 3([\mathrm{H}]))[\mathrm{H}]) \mathrm{C}=4 \mathrm{OC}=5 \mathrm{C}(\mathrm{C}( \\
=\mathrm{O}) \mathrm{C}=4[\mathrm{H}])=\mathrm{C}(\mathrm{OC}([\mathrm{H}])([\mathrm{H}])[\mathrm{H}]) \mathrm{C}\end{array}$ & 2.208 & 2.264 \\
\hline $\operatorname{Tr} 118$ & $\begin{array}{l}\mathrm{O}=\mathrm{C}(\mathrm{N}([\mathrm{H}]) \mathrm{C}([\mathrm{H}])([\mathrm{H}]) \mathrm{C}([\mathrm{H}])([\mathrm{H}]) \mathrm{C}([\mathrm{H}])([\mathrm{H}]) \mathrm{C}([\mathrm{H}])([\mathrm{H}]) \mathrm{C}([\mathrm{H}])([\mathrm{H}]) \mathrm{C}([\mathrm{H}])([\mathrm{H}]) \mathrm{C} \\
([\mathrm{H}])([\mathrm{H}]) \mathrm{C}([\mathrm{H}])([\mathrm{H}]) \mathrm{C}([\mathrm{H}])([\mathrm{H}]) \mathrm{C}([\mathrm{H}])([\mathrm{H}]) \mathrm{N}([\mathrm{H}]) \mathrm{C} 2=\mathrm{C} 1 \mathrm{C}([\mathrm{H}])=\mathrm{C}([\mathrm{H}]) \mathrm{C}([\mathrm{H}])=\mathrm{C}( \\
[\mathrm{H}]) \mathrm{C} 1=\mathrm{NC} 3=\mathrm{C} 2 \mathrm{C}([\mathrm{H}])([\mathrm{H}]) \mathrm{C}([\mathrm{H}])([\mathrm{H}]) \mathrm{C}([\mathrm{H}])([\mathrm{H}]) \mathrm{C} 3([\mathrm{H}])[\mathrm{H}]) \mathrm{C}=4 \mathrm{OC}=5 \mathrm{C}(\mathrm{C}(=\mathrm{O}) \\
\mathrm{C}=4[\mathrm{H}])=\mathrm{C}(\mathrm{O}[\mathrm{H}]) \mathrm{C}([\mathrm{H}])=\mathrm{C}(\mathrm{OC}([\mathrm{H}\end{array}$ & 2.071 & 1.860 \\
\hline $\operatorname{Tr} 119$ & $\begin{array}{l}\mathrm{O}=\mathrm{C}(\mathrm{N}([\mathrm{H}]) \mathrm{C}([\mathrm{H}])([\mathrm{H}]) \mathrm{C}([\mathrm{H}])([\mathrm{H}]) \mathrm{C}([\mathrm{H}])([\mathrm{H}]) \mathrm{C}([\mathrm{H}])([\mathrm{H}]) \mathrm{C}([\mathrm{H}])([\mathrm{H}]) \mathrm{C}([\mathrm{H}])([\mathrm{H}]) \mathrm{C} \\
([\mathrm{H}])([\mathrm{H}]) \mathrm{C}([\mathrm{H}])([\mathrm{H}]) \mathrm{C}([\mathrm{H}])([\mathrm{H}]) \mathrm{C}([\mathrm{H}])([\mathrm{H}]) \mathrm{N}([\mathrm{H}]) \mathrm{C}=2 \mathrm{C}=1 \mathrm{C}([\mathrm{H}])=\mathrm{C}([\mathrm{H}]) \mathrm{C}([\mathrm{H}])= \\
\mathrm{C}([\mathrm{H}]) \mathrm{C}=1 \mathrm{~N}=\mathrm{C} 3 \mathrm{C}=2 \mathrm{C}([\mathrm{H}])([\mathrm{H}]) \mathrm{C}([\mathrm{H}])([\mathrm{H}]) \mathrm{C}([\mathrm{H}])([\mathrm{H}]) \mathrm{C} 3([\mathrm{H}])[\mathrm{H}]) \mathrm{C}=4 \mathrm{OC}=5 \mathrm{C}(\mathrm{C}( \\
=\mathrm{O}) \mathrm{C}=4[\mathrm{H}])=\mathrm{C}([\mathrm{H}]) \mathrm{C}(\mathrm{OC}([\mathrm{H}])([\mathrm{H}]\end{array}$ & 2.056 & 2.264 \\
\hline $\operatorname{Tr} 120$ & $\begin{array}{l}\mathrm{O}=\mathrm{C} 2 \mathrm{~N}(/ \mathrm{C}(=\mathrm{N} \backslash[\mathrm{H}]) \mathrm{N}([\mathrm{H}])[\mathrm{C} @] 4(\mathrm{C}=1 \mathrm{C}([\mathrm{F}])=\mathrm{C}([\mathrm{H}]) \mathrm{C}([\mathrm{F}])=\mathrm{C}([\mathrm{H}]) \mathrm{C}=1[\mathrm{H}])[\mathrm{C} @ @] \\
2([\mathrm{H}]) \mathrm{C}([\mathrm{H}])([\mathrm{H}]) \mathrm{N}(/ \mathrm{C} 3=\mathrm{N} / \mathrm{C}(\mathrm{OC}([\mathrm{H}])([\mathrm{H}])[\mathrm{H}])=\mathrm{C}([\mathrm{F}]) \mathrm{C}(=\mathrm{N} 3) \mathrm{C}([\mathrm{H}])([\mathrm{H}])[\mathrm{H}]) \mathrm{C} 4([ \\
\mathrm{H}])[\mathrm{H}]) \mathrm{C}([\mathrm{H}])([\mathrm{H}])[\mathrm{H}]\end{array}$ & 4.328 & 4.088 \\
\hline $\operatorname{Tr} 121$ & $\begin{array}{l}\mathrm{O}=\mathrm{C} 2 \mathrm{~N}(/ \mathrm{C}(=\mathrm{N} \backslash[\mathrm{H}]) \mathrm{N}([\mathrm{H}])[\mathrm{C} @] 4(\mathrm{C}=1 \mathrm{C}([\mathrm{F}])=\mathrm{C}([\mathrm{H}]) \mathrm{C}([\mathrm{H}])=\mathrm{C}([\mathrm{Cl}]) \mathrm{C}=1[\mathrm{H}])[\mathrm{C} @ @] \\
2([\mathrm{H}]) \mathrm{C}([\mathrm{H}])([\mathrm{H}]) \mathrm{N}(/ \mathrm{C} 3=\mathrm{N} / \mathrm{C}(\mathrm{OC}([\mathrm{H}])([\mathrm{H}])[\mathrm{H}])=\mathrm{C}([\mathrm{F}]) \mathrm{C}(=\mathrm{N} 3) \mathrm{C}([\mathrm{H}])([\mathrm{H}])[\mathrm{H}]) \mathrm{C} 4([ \\
\mathrm{H}])[\mathrm{H}]) \mathrm{C}([\mathrm{H}])([\mathrm{H}])[\mathrm{H}]\end{array}$ & 3.886 & 4.151 \\
\hline $\operatorname{Tr} 122$ & $\begin{array}{l}\mathrm{O}=\mathrm{C}(\mathrm{N}([\mathrm{H}]) \mathrm{C} 3=\mathrm{C}([\mathrm{H}]) \mathrm{C}([\mathrm{H}])=\mathrm{C} 2 \mathrm{OC}([\mathrm{C} @] 4([\mathrm{C} @ @] 1(\mathrm{~N}=\mathrm{C}(\mathrm{OC} 1([\mathrm{H}])[\mathrm{H}]) \mathrm{N}([\mathrm{H}])[ \\
\mathrm{H}]) \mathrm{C} 2=\mathrm{C} 3[\mathrm{H}]) \mathrm{C}([\mathrm{H}])([\mathrm{H}]) \mathrm{OC} 4([\mathrm{H}])[\mathrm{H}])(\mathrm{C}([\mathrm{H}])([\mathrm{H}])[\mathrm{H}]) \mathrm{C}([\mathrm{H}])([\mathrm{H}])[\mathrm{H}]) \mathrm{C}=5 / \mathrm{N}=\mathrm{C}(/ \\
[\mathrm{H}]) \mathrm{C}([\mathrm{Cl}])=\mathrm{C}([\mathrm{H}]) \mathrm{C}=5[\mathrm{H}]\end{array}$ & 3.418 & 3.050 \\
\hline $\operatorname{Tr} 123$ & $\begin{array}{l}\mathrm{O}=\mathrm{C}(\mathrm{N}([\mathrm{H}]) \mathrm{C} 3=\mathrm{C}([\mathrm{H}]) \mathrm{C}([\mathrm{H}])=\mathrm{C} 2 \mathrm{OC}([\mathrm{H}])([\mathrm{H}])[\mathrm{C} @] 4([\mathrm{C} @ @] 1(\mathrm{~N}=\mathrm{C}(\mathrm{OC} 1([\mathrm{H}])[\mathrm{H}] \\
) \mathrm{N}([\mathrm{H}])[\mathrm{H}]) \mathrm{C} 2=\mathrm{C} 3[\mathrm{H}]) \mathrm{C}([\mathrm{H}])([\mathrm{H}]) \mathrm{C} 4([\mathrm{H}])[\mathrm{H}]) \mathrm{C}=5 / \mathrm{N}=\mathrm{C}(/[\mathrm{H}]) \mathrm{C}([\mathrm{Cl}])=\mathrm{C}([\mathrm{H}]) \mathrm{C}=5[\mathrm{~F} \\
]\end{array}$ & 2.620 & 2.584 \\
\hline $\operatorname{Tr} 124$ & $\begin{array}{l}\mathrm{O}=\mathrm{C}(\mathrm{N}([\mathrm{H}]) \mathrm{C} 3=\mathrm{C}([\mathrm{H}]) \mathrm{C}([\mathrm{H}])=\mathrm{C} 2 \mathrm{OC}([\mathrm{H}])([\mathrm{H}])[\mathrm{C} @] 4([\mathrm{C} @ @] 1(\mathrm{~N}=\mathrm{C}(\mathrm{OC} 1([\mathrm{H}])[\mathrm{H}] \\
) \mathrm{N}([\mathrm{H}])[\mathrm{H}]) \mathrm{C} 2=\mathrm{C} 3[\mathrm{H}]) \mathrm{C}([\mathrm{H}])([\mathrm{H}]) \mathrm{C} 4([\mathrm{H}])[\mathrm{H}]) \mathrm{C}=5 / \mathrm{N}=\mathrm{C}(/[\mathrm{H}]) \mathrm{C}([\mathrm{Cl}])=\mathrm{C}([\mathrm{H}]) \mathrm{C}=5[ \\
\mathrm{H}]\end{array}$ & 2.423 & 2.746 \\
\hline $\operatorname{Tr} 125$ & $\begin{array}{l}{[\mathrm{H}] / \mathrm{C} 4=\mathrm{C}(/ \mathrm{C}([\mathrm{H}])=\mathrm{C} 1 \mathrm{C}(\mathrm{OC}([\mathrm{CC} @ 3([\mathrm{C} @ @] 12 \mathrm{~N}=\mathrm{C}(\mathrm{OC} 2([\mathrm{H}])[\mathrm{H}]) \mathrm{N}([\mathrm{H}])[\mathrm{H}]) \mathrm{C}([\mathrm{H}])} \\
([\mathrm{H}]) \mathrm{OC} 3([\mathrm{H}])[\mathrm{H}])(\mathrm{C}([\mathrm{H}])([\mathrm{H}])[\mathrm{H}]) \mathrm{C}([\mathrm{H}])([\mathrm{H}])[\mathrm{H}])=\mathrm{C} 4[\mathrm{H}]) \mathrm{C} 5=\mathrm{C}([\mathrm{H}]) \mathrm{N}=\mathrm{C}([\mathrm{H}]) \mathrm{C}( \\
\mathrm{OC}([\mathrm{H}])([\mathrm{H}])[\mathrm{H}])=\mathrm{C} 5[\mathrm{H}]\end{array}$ & 2.593 & 2.733 \\
\hline $\operatorname{Tr} 126$ & $\begin{array}{l}{[\mathrm{H}] / \mathrm{C} 4=\mathrm{C}(/ \mathrm{C}([\mathrm{H}])=\mathrm{C} 1 \mathrm{C}(\mathrm{O}[\mathrm{C} @]([\mathrm{H}])([\mathrm{C} @] 3([\mathrm{C} @] 12 \mathrm{~N}=\mathrm{C}(\mathrm{OC} 2([\mathrm{H}])[\mathrm{H}]) \mathrm{N}([\mathrm{H}])[\mathrm{H}])} \\
\mathrm{C}([\mathrm{H}])([\mathrm{H}]) \mathrm{OC} 3([\mathrm{H}])[\mathrm{H}]) \mathrm{C}([\mathrm{H}])([\mathrm{H}]) \mathrm{C}([\mathrm{H}])([\mathrm{H}])[\mathrm{H}])=\mathrm{C} 4[\mathrm{H}]) \mathrm{C} 5=\mathrm{C}([\mathrm{H}]) \mathrm{N}=\mathrm{C}([\mathrm{H}]) \mathrm{C} \\
(\mathrm{C \# CC}([\mathrm{H}])([\mathrm{H}])[\mathrm{H}])=\mathrm{C} 5[\mathrm{H}]\end{array}$ & 2.971 & 2.777 \\
\hline $\operatorname{Tr} 127$ & $\begin{array}{l}\mathrm{H}] / \mathrm{C} 5=\mathrm{C}(/ \mathrm{C}([\mathrm{H}])=\mathrm{C} 1 \mathrm{C}(\mathrm{O}[\mathrm{C} @ @] 4([\mathrm{C} @] 3([\mathrm{C} @ @] 12 \mathrm{~N}=\mathrm{C}(\mathrm{OC} 2([\mathrm{H}])[\mathrm{H}]) \mathrm{N}([\mathrm{H}])[\mathrm{H}] \\
) \mathrm{C}([\mathrm{H}])([\mathrm{H}]) \mathrm{OC} 3([\mathrm{H}])[\mathrm{H}]) \mathrm{C}([\mathrm{H}])([\mathrm{H}]) \mathrm{C}([\mathrm{H}])([\mathrm{H}]) \mathrm{C} 4([\mathrm{H}])[\mathrm{H}])=\mathrm{C} 5[\mathrm{H}]) \mathrm{C} 6=\mathrm{C}([\mathrm{H}]) \mathrm{N} \\
=\mathrm{C}([\mathrm{H}]) \mathrm{C}(\mathrm{C \# CC}([\mathrm{H}])([\mathrm{H}])[\mathrm{H}])=\mathrm{C} 6[\mathrm{H}]\end{array}$ & 2.921 & 4.001 \\
\hline $\operatorname{Tr} 128$ & $\begin{array}{l}{[\mathrm{H}] / \mathrm{C} 4=\mathrm{C}(/ \mathrm{C}([\mathrm{H}])=\mathrm{C} 1 \mathrm{C}(\mathrm{OC}([\mathrm{C} @] 3([\mathrm{C} @ @] 12 \mathrm{~N}=\mathrm{C}(\mathrm{OC} 2([\mathrm{H}])[\mathrm{H}]) \mathrm{N}([\mathrm{H}])[\mathrm{H}]) \mathrm{C}([\mathrm{H}])} \\
([\mathrm{H}]) \mathrm{OC} 3([\mathrm{H}])[\mathrm{H}])(\mathrm{C}([\mathrm{H}])([\mathrm{H}])[\mathrm{H}]) \mathrm{C}([\mathrm{H}])([\mathrm{H}])[\mathrm{H}])=\mathrm{C} 4[\mathrm{H}]) \mathrm{C} 5=\mathrm{C}([\mathrm{H}]) \mathrm{N}=\mathrm{C}([\mathrm{H}]) \mathrm{C}( \\
\mathrm{C \# CC}([\mathrm{H}])([\mathrm{H}])[\mathrm{H}])=\mathrm{C} 5[\mathrm{H}]\end{array}$ & 3.044 & 3.664 \\
\hline $\operatorname{Tr} 129$ & $\begin{array}{l}{[\mathrm{Cl}] \mathrm{C}=5 \mathrm{C}([\mathrm{H}])=\mathrm{C}(/ \mathrm{C} 3=\mathrm{C}(\backslash[\mathrm{H}]) \mathrm{C}([\mathrm{H}])=\mathrm{C} 2 \mathrm{OC}([\mathrm{C} @] 4([\mathrm{C} @ @] 1(\mathrm{~N}=\mathrm{C}(\mathrm{OC} 1([\mathrm{H}])[\mathrm{H}])} \\
\mathrm{N}([\mathrm{H}])[\mathrm{H}]) \mathrm{C} 2=\mathrm{C} 3[\mathrm{H}]) \mathrm{C}([\mathrm{H}])([\mathrm{H}]) \mathrm{OC} 4([\mathrm{H}])[\mathrm{H}])(\mathrm{C}([\mathrm{H}])([\mathrm{H}])[\mathrm{H}]) \mathrm{C}([\mathrm{H}])([\mathrm{H}])[\mathrm{H}]) \mathrm{C}([ \\
\mathrm{H}])=\mathrm{C}([\mathrm{F}]) \mathrm{C}=5[\mathrm{H}]\end{array}$ & 2.726 & 2.396 \\
\hline
\end{tabular}




\begin{tabular}{|c|c|c|c|}
\hline $\operatorname{Tr} 130$ & $\begin{array}{l}\mathrm{O}=\mathrm{C}(\mathrm{N}([\mathrm{H}]) \mathrm{C} 3=\mathrm{C}([\mathrm{H}]) \mathrm{C}([\mathrm{H}])=\mathrm{C} 2 \mathrm{OC}([\mathrm{H}])([\mathrm{H}])[\mathrm{C} @] 4([\mathrm{C} @ @] 1(\mathrm{~N}=\mathrm{C}(\mathrm{OC} 1([\mathrm{H}])[\mathrm{H}] \\
) \mathrm{N}([\mathrm{H}])[\mathrm{H}]) \mathrm{C} 2=\mathrm{C} 3[\mathrm{H}]) \mathrm{C}([\mathrm{H}])([\mathrm{H}]) \mathrm{C} 4([\mathrm{H}])[\mathrm{H}]) \mathrm{C} 5=\mathrm{NC}([\mathrm{H}])=\mathrm{C}([\mathrm{Br}]) \mathrm{C}([\mathrm{H}])=\mathrm{N} 5\end{array}$ & 2.206 & 2.352 \\
\hline $\operatorname{Tr} 131$ & $\begin{array}{l}\mathrm{O}=\mathrm{C}(\mathrm{N}([\mathrm{H}]) \mathrm{C} 3=\mathrm{C}([\mathrm{H}]) \mathrm{C}([\mathrm{H}])=\mathrm{C} 2 \mathrm{OC}([\mathrm{C} @] 4([\mathrm{C} @ @] 1(\mathrm{~N}=\mathrm{C}(\mathrm{OC} 1([\mathrm{H}])[\mathrm{H}]) \mathrm{N}([\mathrm{H}])[ \\
\mathrm{H}]) \mathrm{C} 2=\mathrm{C} 3[\mathrm{H}]) \mathrm{C}([\mathrm{H}])([\mathrm{H}]) \mathrm{OC} 4([\mathrm{H}])[\mathrm{H}])(\mathrm{C}([\mathrm{H}])([\mathrm{H}])[\mathrm{H}]) \mathrm{C}([\mathrm{H}])([\mathrm{H}])[\mathrm{H}]) \mathrm{C} 5=\mathrm{NC}([\mathrm{H} \\
])=\mathrm{C}([\mathrm{Cl}]) \mathrm{C}([\mathrm{H}])=\mathrm{C} 5 \mathrm{C}([\mathrm{H}])([\mathrm{H}])[\mathrm{H}]\end{array}$ & 3.190 & 3.293 \\
\hline $\operatorname{Tr} 132$ & $\begin{array}{l}\mathrm{O}=\mathrm{C}(\mathrm{N}([\mathrm{H}]) \mathrm{C} 3=\mathrm{C}([\mathrm{H}]) \mathrm{C}([\mathrm{H}])=\mathrm{C} 2 \mathrm{OC}([\mathrm{H}])([\mathrm{H}])[\mathrm{C} @] 4([\mathrm{C} @ @] 1(\mathrm{~N}=\mathrm{C}(\mathrm{OC} 1([\mathrm{H}])[\mathrm{H}] \\
) \mathrm{N}([\mathrm{H}])[\mathrm{H}]) \mathrm{C} 2=\mathrm{C} 3[\mathrm{H}]) \mathrm{C}([\mathrm{H}])([\mathrm{H}]) \mathrm{C} 4([\mathrm{H}])[\mathrm{H}]) \mathrm{C}=5 / \mathrm{N}=\mathrm{C}(/[\mathrm{H}]) \mathrm{C}(\mathrm{C \# N})=\mathrm{C}([\mathrm{H}]) \mathrm{C}=5[ \\
\mathrm{H}]\end{array}$ & 2.466 & 2.846 \\
\hline $\operatorname{Tr} 133$ & $\begin{array}{l}\mathrm{O}=\mathrm{C}(\mathrm{N}([\mathrm{H}]) \mathrm{C} 3=\mathrm{C}([\mathrm{H}]) \mathrm{C}([\mathrm{H}])=\mathrm{C} 2 \mathrm{OC}([\mathrm{C} @] 4([\mathrm{C} @ @] 1(\mathrm{~N}=\mathrm{C}(\mathrm{OC} 1([\mathrm{H}])[\mathrm{H}]) \mathrm{N}([\mathrm{H}])[ \\
\mathrm{H}]) \mathrm{C} 2=\mathrm{C} 3[\mathrm{H}]) \mathrm{C}([\mathrm{H}])([\mathrm{H}]) \mathrm{OC} 4([\mathrm{H}])[\mathrm{H}])(\mathrm{C}([\mathrm{H}])([\mathrm{H}])[\mathrm{H}]) \mathrm{C}([\mathrm{H}])([\mathrm{H}])[\mathrm{H}]) \mathrm{C}=5 / \mathrm{N}=\mathrm{C}(/ \\
[\mathrm{H}]) \mathrm{C}(\mathrm{C \# N})=\mathrm{C}([\mathrm{H}]) \mathrm{C}=5[\mathrm{H}]\end{array}$ & 3.143 & 3.150 \\
\hline Tr134 & $\begin{array}{l}{[\mathrm{H}] / \mathrm{C} 5=\mathrm{C}(/ \mathrm{C}([\mathrm{H}])=\mathrm{C} 1 \mathrm{C}(\mathrm{O}[\mathrm{C} @]([\mathrm{H}])([\mathrm{C} @] 3([\mathrm{C} @] 12 \mathrm{~N}=\mathrm{C}(\mathrm{OC} 2([\mathrm{H}])[\mathrm{H}]) \mathrm{N}([\mathrm{H}])[\mathrm{H}])} \\
\mathrm{C}([\mathrm{H}])([\mathrm{H}]) \mathrm{OC} 3([\mathrm{H}])[\mathrm{H}]) \mathrm{C}([\mathrm{H}])([\mathrm{H}]) \mathrm{C} 4=\mathrm{C}([\mathrm{H}]) \mathrm{C}([\mathrm{H}])=\mathrm{C}([\mathrm{H}]) \mathrm{C}([\mathrm{H}])=\mathrm{C} 4[\mathrm{H}])=\mathrm{C} 5[ \\
\mathrm{H}]) \mathrm{C} 6=\mathrm{C}([\mathrm{H}]) \mathrm{N}=\mathrm{C}([\mathrm{H}]) \mathrm{N}=\mathrm{C} 6[\mathrm{H}]\end{array}$ & 3.036 & 2.277 \\
\hline $\operatorname{Tr} 135$ & $\begin{array}{l}{[\mathrm{H}] / \mathrm{C} 5=\mathrm{C}(/ \mathrm{C}([\mathrm{H}])=\mathrm{C} 1 \mathrm{C}(\mathrm{O}[\mathrm{C} @]([\mathrm{H}])([\mathrm{C} @] 3([\mathrm{C} @] 12 \mathrm{~N}=\mathrm{C}(\mathrm{OC} 2([\mathrm{H}])[\mathrm{H}]) \mathrm{N}([\mathrm{H}])[\mathrm{H}])} \\
\mathrm{C}([\mathrm{H}])([\mathrm{H}]) \mathrm{OC} 3([\mathrm{H}])[\mathrm{H}]) \mathrm{C}([\mathrm{H}])([\mathrm{H}]) \mathrm{C} 4=\mathrm{C}([\mathrm{H}]) \mathrm{C}([\mathrm{H}])=\mathrm{C}([\mathrm{H}]) \mathrm{C}([\mathrm{H}])=\mathrm{C} 4[\mathrm{H}])=\mathrm{C} 5[ \\
\mathrm{H}]) \mathrm{C} 6=\mathrm{C}([\mathrm{H}]) \mathrm{N}=\mathrm{C}([\mathrm{H}]) \mathrm{C}(\mathrm{C \# CC}([\mathrm{H}])([\mathrm{H}])[\mathrm{H}])=\mathrm{C} 6[\mathrm{H}]\end{array}$ & 3.148 & 3.325 \\
\hline $\operatorname{Tr} 136$ & $\begin{array}{l}\mathrm{O}=\mathrm{C}(\mathrm{N}([\mathrm{H}]) \mathrm{C} 3=\mathrm{C}([\mathrm{H}]) \mathrm{C}([\mathrm{H}])=\mathrm{C} 2 \mathrm{OC}([\mathrm{C} @] 4([\mathrm{C} @ @] 1(\mathrm{~N}=\mathrm{C}(\mathrm{OC} 1([\mathrm{H}])[\mathrm{H}]) \mathrm{N}([\mathrm{H}])[ \\
\mathrm{H}]) \mathrm{C} 2=\mathrm{C} 3[\mathrm{H}]) \mathrm{C}([\mathrm{H}])([\mathrm{H}]) \mathrm{OC} 4([\mathrm{H}])[\mathrm{H}])(\mathrm{C}([\mathrm{H}])([\mathrm{H}])[\mathrm{H}]) \mathrm{C}([\mathrm{H}])([\mathrm{H}])[\mathrm{H}]) \mathrm{C}=5 / \mathrm{N}=\mathrm{C}(/ \\
[\mathrm{H}]) \mathrm{C}([\mathrm{Br}])=\mathrm{C}([\mathrm{H}]) \mathrm{C}=5[\mathrm{H}]\end{array}$ & 3.991 & 2.644 \\
\hline $\operatorname{Tr} 137$ & $\begin{array}{l}\mathrm{O}=\mathrm{C}(\mathrm{N}([\mathrm{H}]) \mathrm{C} 3=\mathrm{C}([\mathrm{H}]) \mathrm{C}([\mathrm{H}])=\mathrm{C} 2 \mathrm{OC}([\mathrm{C} @] 4([\mathrm{C} @ @] 1(\mathrm{~N}=\mathrm{C}(\mathrm{OC} 1([\mathrm{H}])[\mathrm{H}]) \mathrm{N}([\mathrm{H}])[ \\
\mathrm{H}]) \mathrm{C} 2=\mathrm{C} 3[\mathrm{H}]) \mathrm{C}([\mathrm{H}])([\mathrm{H}]) \mathrm{C} 4([\mathrm{H}])[\mathrm{H}])(\mathrm{C}([\mathrm{H}])([\mathrm{H}])[\mathrm{H}]) \mathrm{C}([\mathrm{H}])([\mathrm{H}])[\mathrm{H}]) \mathrm{C} 5=\mathrm{NC}([\mathrm{H}]) \\
=\mathrm{C}([\mathrm{Cl}]) \mathrm{C}([\mathrm{H}])=\mathrm{C} 5[\mathrm{H}]\end{array}$ & 3.423 & 3.188 \\
\hline $\operatorname{Tr} 138$ & $\begin{array}{l}\mathrm{O}=\mathrm{C}(\mathrm{N}([\mathrm{H}]) \mathrm{C} 3=\mathrm{C}([\mathrm{H}]) \mathrm{C}([\mathrm{H}])=\mathrm{C} 2 \mathrm{OC}([\mathrm{C} @] 4([\mathrm{C} @ @] 1(\mathrm{~N}=\mathrm{C}(\mathrm{OC} 1([\mathrm{H}])[\mathrm{H}]) \mathrm{N}([\mathrm{H}])[ \\
\mathrm{H}]) \mathrm{C} 2=\mathrm{C} 3[\mathrm{H}]) \mathrm{C}([\mathrm{H}])([\mathrm{H}]) \mathrm{C} 4([\mathrm{H}])[\mathrm{H}])(\mathrm{C}([\mathrm{H}])([\mathrm{H}])[\mathrm{H}]) \mathrm{C}([\mathrm{H}])([\mathrm{H}])[\mathrm{H}]) \mathrm{C} 5=\mathrm{NC}([\mathrm{H}]) \\
=\mathrm{C}([\mathrm{F}]) \mathrm{C}([\mathrm{H}])=\mathrm{C} 5[\mathrm{H}]\end{array}$ & 3.039 & 2.963 \\
\hline $\operatorname{Tr} 139$ & $\begin{array}{l}\mathrm{O}=\mathrm{C}(\mathrm{N}([\mathrm{H}]) \mathrm{C} 3=\mathrm{C}([\mathrm{H}]) \mathrm{C}([\mathrm{H}])=\mathrm{C} 2 \mathrm{OC}([\mathrm{C} @] 4([\mathrm{C} @ @] 1(\mathrm{~N}=\mathrm{C}(\mathrm{OC} 1([\mathrm{H}])[\mathrm{H}]) \mathrm{N}([\mathrm{H}])[ \\
\mathrm{H}]) \mathrm{C} 2=\mathrm{C} 3[\mathrm{H}]) \mathrm{C}([\mathrm{H}])([\mathrm{H}]) \mathrm{OC} 4([\mathrm{H}])[\mathrm{H}])(\mathrm{C}([\mathrm{H}])([\mathrm{H}])[\mathrm{H}]) \mathrm{C}([\mathrm{H}])([\mathrm{H}])[\mathrm{H}]) \mathrm{C}=5 / \mathrm{N}=\mathrm{C}(/ \\
[\mathrm{H}]) \mathrm{C}(=\mathrm{NC}=5[\mathrm{H}]) \mathrm{OC}([\mathrm{H}])([\mathrm{H}])[\mathrm{H}]\end{array}$ & 3.101 & 3.481 \\
\hline $\operatorname{Tr} 140$ & $\begin{array}{l}\mathrm{O}=\mathrm{C}(/ \mathrm{C} 1=\mathrm{N} / \mathrm{C}([\mathrm{H}])=\mathrm{C}([\mathrm{Cl}]) \mathrm{C}([\mathrm{H}])=\mathrm{C} 1[\mathrm{H}]) \mathrm{N}([\mathrm{H}]) \mathrm{C} 2=\mathrm{C}([\mathrm{H}]) \mathrm{C}(=\mathrm{C}([\mathrm{F}]) \mathrm{C}([\mathrm{H}])=\mathrm{C} 2[ \\
\mathrm{H}])[\mathrm{C} @] 3(\mathrm{~N}([\mathrm{H}]) \mathrm{C}(=\mathrm{N}[\mathrm{H}]) \mathrm{N}([\mathrm{S}](=\mathrm{O})(=\mathrm{O}) \mathrm{C} 3([\mathrm{H}])[\mathrm{H}]) \mathrm{C}([\mathrm{H}])([\mathrm{H}])[\mathrm{H}]) \mathrm{C}([\mathrm{H}])([\mathrm{H}])[ \\
\mathrm{H}]\end{array}$ & 5.023 & 4.097 \\
\hline $\operatorname{Tr} 141$ & $\begin{array}{l}\mathrm{O}=\mathrm{C}(/ \mathrm{C} 1=\mathrm{N} / \mathrm{C}([\mathrm{H}])=\mathrm{C}(\mathrm{C}([\mathrm{H}])=\mathrm{C} 1[\mathrm{H}]) \mathrm{C}([\mathrm{F}])([\mathrm{F}])[\mathrm{F}]) \mathrm{N}([\mathrm{H}]) \mathrm{C} 2=\mathrm{C}([\mathrm{H}]) \mathrm{C}(=\mathrm{C}([\mathrm{F}]) \mathrm{C}([ \\
\mathrm{H}])=\mathrm{C} 2[\mathrm{H}])[\mathrm{C} @] 3(\mathrm{~N}([\mathrm{H}]) \mathrm{C}(=\mathrm{N}[\mathrm{H}]) \mathrm{N}([\mathrm{S}](=\mathrm{O})(=\mathrm{O}) \mathrm{C} 3([\mathrm{H}])[\mathrm{H}]) \mathrm{C}([\mathrm{H}])([\mathrm{H}])[\mathrm{H}]) \mathrm{C}([\mathrm{H} \\
])([\mathrm{H}])[\mathrm{H}]\end{array}$ & 4.899 & 5.095 \\
\hline $\operatorname{Tr} 142$ & $\begin{array}{l}\mathrm{O}=\mathrm{C}(/ \mathrm{C} 1=\mathrm{N} / \mathrm{C}([\mathrm{H}])=\mathrm{C}(/ \mathrm{N}=\mathrm{C} 1 /[\mathrm{H}]) \mathrm{C}([\mathrm{H}])([\mathrm{H}])[\mathrm{H}]) \mathrm{N}([\mathrm{H}]) \mathrm{C} 2=\mathrm{C}([\mathrm{H}]) \mathrm{C}(=\mathrm{C}([\mathrm{F}]) \mathrm{C}([\mathrm{H} \\
])=\mathrm{C} 2[\mathrm{H}])[\mathrm{C} @] 3(\mathrm{~N}([\mathrm{H}]) \mathrm{C}(=\mathrm{N}[\mathrm{H}]) \mathrm{N}([\mathrm{S}](=\mathrm{O})(=\mathrm{O}) \mathrm{C} 3([\mathrm{H}])[\mathrm{H}]) \mathrm{C}([\mathrm{H}])([\mathrm{H}])[\mathrm{H}]) \mathrm{C}([\mathrm{H}]) \\
([\mathrm{H}])[\mathrm{H}]\end{array}$ & 4.000 & 4.340 \\
\hline $\operatorname{Tr} 143$ & $\begin{array}{l}\mathrm{O}=\mathrm{C}(/ \mathrm{C} 1=\mathrm{N} / \mathrm{C}([\mathrm{H}])=\mathrm{C}(/ \mathrm{N}=\mathrm{C} 1 /[\mathrm{H}]) \mathrm{OC}([\mathrm{H}])([\mathrm{H}])[\mathrm{H}]) \mathrm{N}([\mathrm{H}]) \mathrm{C} 2=\mathrm{C}([\mathrm{H}]) \mathrm{C}(=\mathrm{C}([\mathrm{F}]) \mathrm{C}([ \\
\mathrm{F}])=\mathrm{C} 2[\mathrm{H}])[\mathrm{C} @] 3(\mathrm{~N}([\mathrm{H}]) \mathrm{C}(=\mathrm{N}[\mathrm{H}]) \mathrm{N}([\mathrm{S}](=\mathrm{O})(=\mathrm{O}) \mathrm{C} 3([\mathrm{H}])[\mathrm{H}]) \mathrm{C}([\mathrm{H}])([\mathrm{H}])[\mathrm{H}]) \mathrm{C}([\mathrm{H} \\
])([\mathrm{H}])[\mathrm{H}]\end{array}$ & 4.979 & 5.058 \\
\hline
\end{tabular}




\begin{tabular}{|c|c|c|c|}
\hline $\operatorname{Tr} 144$ & $\begin{array}{l}\mathrm{O}=[\mathrm{S}] 3(=\mathrm{O}) \mathrm{N}(/ \mathrm{C}(=\mathrm{N} \backslash[\mathrm{H}]) \mathrm{N}([\mathrm{H}])[\mathrm{C} @](\mathrm{C}=1[\mathrm{~S}] \mathrm{C}([\mathrm{H}])=\mathrm{C}(\mathrm{C}=1[\mathrm{H}]) \mathrm{C} 2=\mathrm{C}([\mathrm{H}]) \mathrm{C}([\mathrm{H}])= \\
\mathrm{C}([\mathrm{F}]) \mathrm{C}(\mathrm{C \# N})=\mathrm{C} 2[\mathrm{H}])(\mathrm{C} 3([\mathrm{H}])[\mathrm{H}]) \mathrm{C}([\mathrm{H}])([\mathrm{H}])[\mathrm{H}]) \mathrm{C}([\mathrm{H}])([\mathrm{H}])[\mathrm{H}]\end{array}$ & 3.337 & 3.223 \\
\hline $\operatorname{Tr} 145$ & $\begin{array}{l}\mathrm{O}=[\mathrm{S}] 3(=\mathrm{O}) \mathrm{N}(/ \mathrm{C}(=\mathrm{N} \backslash[\mathrm{H}]) \mathrm{N}([\mathrm{H}])[\mathrm{C} @](\mathrm{C}=1[\mathrm{~S}] / \mathrm{C}(=\mathrm{C}(/[\mathrm{H}]) \mathrm{C}=1[\mathrm{Cl}]) \mathrm{C} 2=\mathrm{C}([\mathrm{H}]) \mathrm{N}=\mathrm{C}( \\
[\mathrm{H}]) \mathrm{C}(\mathrm{C \# CC}([\mathrm{H}])([\mathrm{H}])[\mathrm{H}])=\mathrm{C} 2[\mathrm{H}])(\mathrm{C} 3([\mathrm{H}])[\mathrm{H}]) \mathrm{C}([\mathrm{H}])([\mathrm{H}])[\mathrm{H}]) \mathrm{C}([\mathrm{H}])([\mathrm{H}])[\mathrm{H}]\end{array}$ & 4.622 & 4.353 \\
\hline $\operatorname{Tr} 146$ & $\begin{array}{l}\mathrm{O}=\mathrm{C}(/ \mathrm{C} 1=\mathrm{N} / \mathrm{C}([\mathrm{H}])=\mathrm{C}(/ \mathrm{N}=\mathrm{C} 1 /[\mathrm{H}]) \mathrm{OC}([\mathrm{H}])([\mathrm{H}])[\mathrm{H}]) \mathrm{N}([\mathrm{H}]) \mathrm{C} 2=\mathrm{C}([\mathrm{H}]) \mathrm{C}(=\mathrm{C}([\mathrm{F}]) \mathrm{C}([ \\
\mathrm{H}])=\mathrm{C} 2[\mathrm{H}])[\mathrm{C} @] 3(\mathrm{~N}([\mathrm{H}]) \mathrm{C}(=\mathrm{N}[\mathrm{H}]) \mathrm{N}([\mathrm{S}](=\mathrm{O})(=\mathrm{O}) \mathrm{C} 3([\mathrm{H}])[\mathrm{H}]) \mathrm{C}([\mathrm{H}])([\mathrm{H}])[\mathrm{H}]) \mathrm{C}([\mathrm{H} \\
])([\mathrm{H}])[\mathrm{H}]\end{array}$ & 4.785 & 4.708 \\
\hline $\operatorname{Tr} 147$ & $\begin{array}{l}{[\mathrm{F}] \mathrm{C}=1 \mathrm{C}([\mathrm{H}])=\mathrm{C}(\mathrm{C}([\mathrm{H}])=\mathrm{C}([\mathrm{F}]) \mathrm{C}=1[\mathrm{H}]) \mathrm{C}([\mathrm{H}])([\mathrm{H}])[\mathrm{C} @]([\mathrm{H}])(\mathrm{N}([\mathrm{H}]) \mathrm{C}(=\mathrm{O}) \mathrm{C} 2=\mathrm{C}([} \\
\mathrm{H}]) \mathrm{C}(=\mathrm{C}([\mathrm{H}]) \mathrm{C}(=\mathrm{C} 2[\mathrm{H}]) \mathrm{C}([\mathrm{H}])([\mathrm{H}])[\mathrm{H}]) \mathrm{C}(=\mathrm{O}) \mathrm{N}(\mathrm{C}([\mathrm{H}])([\mathrm{H}]) \mathrm{C}([\mathrm{H}])([\mathrm{H}]) \mathrm{C}([\mathrm{H}])([\mathrm{H}] \\
)[\mathrm{H}]) \mathrm{C}([\mathrm{H}])([\mathrm{H}]) \mathrm{C}([\mathrm{H}])([\mathrm{H}]) \mathrm{C}([\mathrm{H}])([\mathrm{H}])[\mathrm{H}])[\mathrm{C} @]([\mathrm{H}])(\mathrm{O}[\mathrm{H}])[\mathrm{C} @] 3([\mathrm{H}]) \mathrm{N}([\mathrm{H}]) \mathrm{C}([ \\
\mathrm{H}])([\mathrm{H}]) \mathrm{C}([\mathrm{H}])([\mathrm{H}]) \mathrm{N}(\mathrm{C} 3([\mathrm{H}])[\end{array}$ & 2.509 & 3.632 \\
\hline $\operatorname{Tr} 148$ & $\begin{array}{l}{[\mathrm{F}] \mathrm{C}=1 \mathrm{C}([\mathrm{H}])=\mathrm{C}(\mathrm{C}([\mathrm{H}])=\mathrm{C}([\mathrm{F}]) \mathrm{C}=1[\mathrm{H}]) \mathrm{C}([\mathrm{H}])([\mathrm{H}])[\mathrm{C} @]([\mathrm{H}])(\mathrm{N}([\mathrm{H}]) \mathrm{C}(=\mathrm{O}) \mathrm{C} 2=\mathrm{C}([} \\
\mathrm{H}]) \mathrm{C}(=\mathrm{C}([\mathrm{H}]) \mathrm{C}(=\mathrm{C} 2[\mathrm{H}]) \mathrm{C}([\mathrm{H}])([\mathrm{H}])[\mathrm{H}]) \mathrm{C}(=\mathrm{O}) \mathrm{N}(\mathrm{C}([\mathrm{H}])([\mathrm{H}]) \mathrm{C}([\mathrm{H}])([\mathrm{H}]) \mathrm{C}([\mathrm{H}])([\mathrm{H}] \\
)[\mathrm{H}]) \mathrm{C}([\mathrm{H}])([\mathrm{H}]) \mathrm{C}([\mathrm{H}])([\mathrm{H}]) \mathrm{C}([\mathrm{H}])([\mathrm{H}])[\mathrm{H}])[\mathrm{C} @]([\mathrm{H}])(\mathrm{O}[\mathrm{H}])[\mathrm{C} @] 3([\mathrm{H}]) \mathrm{N}([\mathrm{H}]) \mathrm{C}([ \\
\mathrm{H}])([\mathrm{H}]) \mathrm{C}([\mathrm{H}])([\mathrm{H}]) \mathrm{N}(\mathrm{C} 3([\mathrm{H}])[\end{array}$ & 2.921 & 3.082 \\
\hline $\operatorname{Tr} 149$ & $\begin{array}{l}{[\mathrm{F}] \mathrm{C}=1 \mathrm{C}([\mathrm{H}])=\mathrm{C}(\mathrm{C}([\mathrm{H}])=\mathrm{C}([\mathrm{F}]) \mathrm{C}=1[\mathrm{H}]) \mathrm{C}([\mathrm{H}])([\mathrm{H}])[\mathrm{C} @]([\mathrm{H}])(\mathrm{N}([\mathrm{H}]) \mathrm{C}(=\mathrm{O}) \mathrm{C} 2=\mathrm{C}([} \\
\mathrm{H}]) \mathrm{C}(=\mathrm{C}([\mathrm{H}]) \mathrm{C}(=\mathrm{C} 2[\mathrm{H}]) \mathrm{C}([\mathrm{H}])([\mathrm{H}])[\mathrm{H}]) \mathrm{C}(=\mathrm{O}) \mathrm{N}(\mathrm{C}([\mathrm{H}])([\mathrm{H}]) \mathrm{C}([\mathrm{H}])([\mathrm{H}]) \mathrm{C}([\mathrm{H}])([\mathrm{H}] \\
)[\mathrm{H}]) \mathrm{C}([\mathrm{H}])([\mathrm{H}]) \mathrm{C}([\mathrm{H}])([\mathrm{H}]) \mathrm{C}([\mathrm{H}])([\mathrm{H}])[\mathrm{H}])[\mathrm{C} @]([\mathrm{H}])(\mathrm{O}[\mathrm{H}])[\mathrm{C} @] 3([\mathrm{H}]) \mathrm{N}([\mathrm{H}]) \mathrm{C}([ \\
\mathrm{H}])([\mathrm{H}]) \mathrm{C}([\mathrm{H}])([\mathrm{H}]) \mathrm{N}(\mathrm{C} 3([\mathrm{H}])[\end{array}$ & 2.398 & 3.082 \\
\hline $\operatorname{Tr} 150$ & $\begin{array}{l}{[\mathrm{F}] \mathrm{C}=1 \mathrm{C}([\mathrm{H}])=\mathrm{C}(\mathrm{C}([\mathrm{H}])=\mathrm{C}([\mathrm{F}]) \mathrm{C}=1[\mathrm{H}]) \mathrm{C}([\mathrm{H}])([\mathrm{H}])[\mathrm{C} @]([\mathrm{H}])(\mathrm{N}([\mathrm{H}]) \mathrm{C}(=\mathrm{O}) \mathrm{C} 2=\mathrm{C}([} \\
\mathrm{H}]) \mathrm{C}(=\mathrm{C}([\mathrm{H}]) \mathrm{C}(=\mathrm{C} 2[\mathrm{H}]) \mathrm{C}([\mathrm{H}])([\mathrm{H}])[\mathrm{H}]) \mathrm{C}(=\mathrm{O}) \mathrm{N}(\mathrm{C}([\mathrm{H}])([\mathrm{H}]) \mathrm{C}([\mathrm{H}])([\mathrm{H}]) \mathrm{C}([\mathrm{H}])([\mathrm{H}] \\
)[\mathrm{H}]) \mathrm{C}([\mathrm{H}])([\mathrm{H}]) \mathrm{C}([\mathrm{H}])([\mathrm{H}]) \mathrm{C}([\mathrm{H}])([\mathrm{H}])[\mathrm{H}])[\mathrm{C} @]([\mathrm{H}])(\mathrm{O}[\mathrm{H}])[\mathrm{C} @] 3([\mathrm{H}]) \mathrm{N}([\mathrm{H}]) \mathrm{C}([ \\
\mathrm{H}])([\mathrm{H}]) \mathrm{C}([\mathrm{H}])([\mathrm{H}]) \mathrm{N}(\mathrm{C} 3([\mathrm{H}])[\end{array}$ & 2.013 & 2.912 \\
\hline $\operatorname{Tr} 151$ & $\begin{array}{l}\mathrm{O}=\mathrm{C}(\mathrm{N}([\mathrm{H}])[\mathrm{C} @]([\mathrm{H}])([\mathrm{C} @ @]([\mathrm{H}])(\mathrm{O}[\mathrm{H}]) \mathrm{C}([\mathrm{H}])([\mathrm{H}])[\mathrm{C} @]([\mathrm{H}])(\mathrm{C}(=\mathrm{O}) \mathrm{N}([\mathrm{H}])[\mathrm{C} @ \\
]([\mathrm{H}])(\mathrm{C}(=\mathrm{O}) \mathrm{N}([\mathrm{H}]) \mathrm{C}([\mathrm{H}])([\mathrm{H}]) \mathrm{C}([\mathrm{H}])(\mathrm{C}([\mathrm{H}])([\mathrm{H}])[\mathrm{H}]) \mathrm{C}([\mathrm{H}])([\mathrm{H}])[\mathrm{H}]) \mathrm{C}([\mathrm{H}])(\mathrm{C}([\mathrm{H}] \\
)([\mathrm{H}])[\mathrm{H}]) \mathrm{C}([\mathrm{H}])([\mathrm{H}])[\mathrm{H}]) \mathrm{C}([\mathrm{H}])([\mathrm{H}])[\mathrm{H}]) \mathrm{C}([\mathrm{H}])([\mathrm{H}]) \mathrm{C}([\mathrm{H}])(\mathrm{C}([\mathrm{H}])([\mathrm{H}])[\mathrm{H}]) \mathrm{C}([\mathrm{H}]) \\
([\mathrm{H}])[\mathrm{H}])[\mathrm{C} @ @]([\mathrm{H}])(\mathrm{N}([\mathrm{H}]\end{array}$ & 5.097 & 4.235 \\
\hline $\operatorname{Tr} 152$ & $\begin{array}{l}\mathrm{O}=\mathrm{C}(\mathrm{N}([\mathrm{H}]) \mathrm{C}([\mathrm{H}])([\mathrm{H}]) \mathrm{C} 1=\mathrm{C}([\mathrm{H}]) \mathrm{C}([\mathrm{H}])=\mathrm{C}([\mathrm{H}]) \mathrm{C}([\mathrm{H}])=\mathrm{C} 1[\mathrm{H}])[\mathrm{C} @]([\mathrm{H}])(\mathrm{OC}([\mathrm{H}] \\
)([\mathrm{H}])[\mathrm{H}]) \mathrm{C}([\mathrm{H}])([\mathrm{H}])[\mathrm{C} @]([\mathrm{H}])(\mathrm{O}[\mathrm{H}])[\mathrm{C} @ @ @]([\mathrm{H}])(\mathrm{N}([\mathrm{H}]) \mathrm{C}(=\mathrm{O}) \mathrm{C} 2=\mathrm{C}([\mathrm{H}]) \mathrm{C}(=\mathrm{C}([ \\
\mathrm{H}]) \mathrm{C}(=\mathrm{C} 2[\mathrm{H}]) \mathrm{C}(=\mathrm{O}) \mathrm{N}([\mathrm{H}])[\mathrm{C} @ @]([\mathrm{H}])(\mathrm{C} 3=\mathrm{C}([\mathrm{H}]) \mathrm{C}([\mathrm{H}])=\mathrm{C}([\mathrm{H}]) \mathrm{C}([\mathrm{H}])=\mathrm{C} 3[\mathrm{H}]) \mathrm{C}( \\
[\mathrm{H}])([\mathrm{H}])[\mathrm{H}]) \mathrm{N}(\mathrm{C}([\mathrm{H}])([\mathrm{H}])[\mathrm{S}](=\mathrm{O})(=\mathrm{O})\end{array}$ & 1.854 & 2.675 \\
\hline $\operatorname{Tr} 153$ & $\begin{array}{l}\mathrm{O}=\mathrm{C}(\mathrm{N}([\mathrm{H}]) \mathrm{C}([\mathrm{H}])([\mathrm{H}]) \mathrm{C}([\mathrm{H}])(\mathrm{C}([\mathrm{H}])([\mathrm{H}])[\mathrm{H}]) \mathrm{C}([\mathrm{H}])([\mathrm{H}])[\mathrm{H}])[\mathrm{C} @]([\mathrm{H}])(\mathrm{OC}([\mathrm{H}])( \\
[\mathrm{H}])[\mathrm{H}]) \mathrm{C}([\mathrm{H}])([\mathrm{H}])[\mathrm{C} @]([\mathrm{H}])(\mathrm{O}[\mathrm{H}])[\mathrm{C} @ @]([\mathrm{H}])(\mathrm{N}([\mathrm{H}]) \mathrm{C}(=\mathrm{O}) \mathrm{C} 1=\mathrm{C}([\mathrm{H}]) \mathrm{C}(=\mathrm{C}([\mathrm{H} \\
]) \mathrm{C}(=\mathrm{C} 1[\mathrm{H}]) \mathrm{C}(=\mathrm{O}) \mathrm{N}([\mathrm{H}])[\mathrm{C} @ @]([\mathrm{H}])(\mathrm{C} 2=\mathrm{C}([\mathrm{H}]) \mathrm{C}([\mathrm{H}])=\mathrm{C}([\mathrm{H}]) \mathrm{C}([\mathrm{H}])=\mathrm{C} 2[\mathrm{H}]) \mathrm{C}([ \\
\mathrm{H}])([\mathrm{H}])[\mathrm{H}]) \mathrm{N}(\mathrm{C}([\mathrm{H}])([\mathrm{H}])[\mathrm{S}](=\mathrm{O})(=\end{array}$ & 2.398 & 2.712 \\
\hline $\operatorname{Tr} 154$ & $\begin{array}{l}\mathrm{O}=\mathrm{C}(\mathrm{N}([\mathrm{H}])[\mathrm{C} @]([\mathrm{H}])(\mathrm{C}(=\mathrm{O}) \mathrm{N}([\mathrm{H}]) \mathrm{C}([\mathrm{H}])([\mathrm{H}]) \mathrm{C} 1=\mathrm{C}([\mathrm{H}]) \mathrm{C}([\mathrm{H}])=\mathrm{C}([\mathrm{H}]) \mathrm{C}([\mathrm{H}])=\mathrm{C} \\
1[\mathrm{H}]) \mathrm{C}([\mathrm{H}])(\mathrm{C}([\mathrm{H}])([\mathrm{H}])[\mathrm{H}]) \mathrm{C}([\mathrm{H}])([\mathrm{H}])[\mathrm{H}])[\mathrm{C} @]([\mathrm{H}])(\mathrm{OC}([\mathrm{H}])([\mathrm{H}])[\mathrm{H}]) \mathrm{C}([\mathrm{H}])([\mathrm{H} \\
])[\mathrm{C} @([\mathrm{H}])(\mathrm{O}[\mathrm{H}])[\mathrm{C} @ @]([\mathrm{H}])(\mathrm{N}([\mathrm{H}]) \mathrm{C}(=\mathrm{O}) \mathrm{C} 2=\mathrm{C}([\mathrm{H}]) \mathrm{C}(=\mathrm{C}([\mathrm{H}]) \mathrm{C}(=\mathrm{C} 2[\mathrm{H}]) \mathrm{C}(=\mathrm{O} \\
) \mathrm{N}([\mathrm{H}])[\mathrm{C} @ @]([\mathrm{H}])(\mathrm{C} 3=\mathrm{C}([\mathrm{H}]) \mathrm{C}([\mathrm{H}])=\end{array}$ & 4.046 & 2.750 \\
\hline Tr155 & $\begin{array}{l}\mathrm{O}=\mathrm{C}(\mathrm{N}([\mathrm{H}])[\mathrm{C} @]([\mathrm{H}])(\mathrm{C}(=\mathrm{O}) \mathrm{N}([\mathrm{H}]) \mathrm{C}([\mathrm{H}])([\mathrm{H}]) \mathrm{C} 1=\mathrm{C}([\mathrm{H}]) \mathrm{C}([\mathrm{H}])=\mathrm{C}([\mathrm{H}]) \mathrm{C}([\mathrm{H}])=\mathrm{C} \\
1[\mathrm{H}]) \mathrm{C}([\mathrm{H}])(\mathrm{C}([\mathrm{H}])([\mathrm{H}])[\mathrm{H}]) \mathrm{C}([\mathrm{H}])([\mathrm{H}])[\mathrm{H}])[\mathrm{C} @]([\mathrm{H}])(\mathrm{OC}([\mathrm{H}])([\mathrm{H}])[\mathrm{H}]) \mathrm{C}([\mathrm{H}])([\mathrm{H} \\
])[\mathrm{C} @]([\mathrm{H}])(\mathrm{O}[\mathrm{H}])[\mathrm{C} @ @]([\mathrm{H}])(\mathrm{N}([\mathrm{H}]) \mathrm{C}(=\mathrm{O}) \mathrm{C} 2=\mathrm{C}([\mathrm{H}]) \mathrm{C}(=\mathrm{C}([\mathrm{H}]) \mathrm{C}(=\mathrm{C} 2[\mathrm{H}]) \mathrm{C}(=\mathrm{O} \\
) \mathrm{N}([\mathrm{H}])[\mathrm{C} @ @]([\mathrm{H}])(\mathrm{C} 3=\mathrm{C}([\mathrm{H}]) \mathrm{C}([\mathrm{H}])=\end{array}$ & 3.125 & 2.794 \\
\hline
\end{tabular}




\begin{tabular}{|c|c|c|c|}
\hline $\operatorname{Tr} 156$ & $\begin{array}{l}\mathrm{O}=\mathrm{C}(\mathrm{N}([\mathrm{H}])[\mathrm{C} @]([\mathrm{H}])(\mathrm{C}(=\mathrm{O}) \mathrm{N}([\mathrm{H}]) \mathrm{C}([\mathrm{H}])([\mathrm{H}]) \mathrm{C}=1 \mathrm{C}([\mathrm{H}])=\mathrm{C}([\mathrm{H}]) \mathrm{C}([\mathrm{H}])=\mathrm{C}([\mathrm{H}]) \mathrm{C} \\
=1[\mathrm{H}]) \mathrm{C}([\mathrm{H}])(\mathrm{C}([\mathrm{H}])([\mathrm{H}])[\mathrm{H}]) \mathrm{C}([\mathrm{H}])([\mathrm{H}])[\mathrm{H}])[\mathrm{C} @]([\mathrm{H}])(\mathrm{OC}([\mathrm{H}])([\mathrm{H}])[\mathrm{H}]) \mathrm{C}([\mathrm{H}])([ \\
\mathrm{H}])[\mathrm{C} @]([\mathrm{H}])(\mathrm{O}[\mathrm{H}])[\mathrm{C} @ @]([\mathrm{H}])(\mathrm{N}([\mathrm{H}]) \mathrm{C}(=\mathrm{O}) \mathrm{C} 2=\mathrm{C}([\mathrm{H}]) \mathrm{C}(=\mathrm{C}([\mathrm{H}]) \mathrm{C}(=\mathrm{C} 2[\mathrm{H}]) \mathrm{C}(= \\
\mathrm{O}) \mathrm{N}([\mathrm{H}])[\mathrm{C} @ @]([\mathrm{H}])(\mathrm{C} 3=\mathrm{C}([\mathrm{H}]) \mathrm{C}([\mathrm{H}])\end{array}$ & 3.097 & 3.773 \\
\hline $\operatorname{Tr} 157$ & $\begin{array}{l}\mathrm{O}=\mathrm{C}(\mathrm{N}([\mathrm{H}])[\mathrm{C} @]([\mathrm{H}])(\mathrm{C}(=\mathrm{O}) \mathrm{N}([\mathrm{H}]) \mathrm{C}([\mathrm{H}])([\mathrm{H}]) \mathrm{C} 1=\mathrm{C}([\mathrm{H}]) \mathrm{C}([\mathrm{H}])=\mathrm{C}([\mathrm{H}]) \mathrm{C}([\mathrm{H}])=\mathrm{C} \\
1[\mathrm{H}]) \mathrm{C}([\mathrm{H}])(\mathrm{C}([\mathrm{H}])([\mathrm{H}])[\mathrm{H}]) \mathrm{C}([\mathrm{H}])([\mathrm{H}])[\mathrm{H}])[\mathrm{C} @]([\mathrm{H}])(\mathrm{OC}([\mathrm{H}])([\mathrm{H}])[\mathrm{H}]) \mathrm{C}([\mathrm{H}])([\mathrm{H} \\
])[\mathrm{C} @([\mathrm{H}])(\mathrm{O}[\mathrm{H}])[\mathrm{C} @ @]([\mathrm{H}])(\mathrm{N}([\mathrm{H}]) \mathrm{C}(=\mathrm{O}) \mathrm{C} 2=\mathrm{C}([\mathrm{H}]) \mathrm{C}(=\mathrm{C}([\mathrm{H}]) \mathrm{C}(=\mathrm{C} 2[\mathrm{H}]) \mathrm{C}(=\mathrm{O} \\
) \mathrm{N}([\mathrm{H}])[\mathrm{C} @ @]([\mathrm{H}])(\mathrm{C} 3=\mathrm{C}([\mathrm{H}]) \mathrm{C}([\mathrm{H}])=\end{array}$ & 3.222 & 3.042 \\
\hline $\operatorname{Tr} 158$ & $\begin{array}{l}\mathrm{O}=\mathrm{C}(\mathrm{N}([\mathrm{H}]) \mathrm{C} 1=\mathrm{C}([\mathrm{H}]) \mathrm{C}([\mathrm{H}])=\mathrm{C}([\mathrm{F}]) \mathrm{C}([\mathrm{H}])=\mathrm{C} 1[\mathrm{H}])[\mathrm{C} @]([\mathrm{H}])(\mathrm{OC}([\mathrm{H}])([\mathrm{H}])[\mathrm{H}]) \mathrm{C}( \\
[\mathrm{H}])([\mathrm{H}])[\mathrm{C} @]([\mathrm{H}])(\mathrm{O}[\mathrm{H}])[\mathrm{C} @ @]([\mathrm{H}])(\mathrm{N}([\mathrm{H}]) \mathrm{C}(=\mathrm{O}) \mathrm{C} 2=\mathrm{C}([\mathrm{H}]) \mathrm{C}(=\mathrm{C}([\mathrm{H}]) \mathrm{C}(=\mathrm{C} 2[\mathrm{H} \\
]) \mathrm{C}(=\mathrm{O}) \mathrm{N}([\mathrm{H}])[\mathrm{C} @ @]([\mathrm{H}])(\mathrm{C} 3=\mathrm{C}([\mathrm{H}]) \mathrm{C}([\mathrm{H}])=\mathrm{C}([\mathrm{H}]) \mathrm{C}([\mathrm{H}])=\mathrm{C} 3[\mathrm{H}]) \mathrm{C}([\mathrm{H}])([\mathrm{H}])[\mathrm{H}] \\
) \mathrm{N}(\mathrm{C}([\mathrm{H}])([\mathrm{H}])[\mathrm{S}](=\mathrm{O})(=\mathrm{O}) \mathrm{C}([\mathrm{H}])([\mathrm{H}])\end{array}$ & 3.000 & 2.988 \\
\hline $\operatorname{Tr} 159$ & $\begin{array}{l}\mathrm{O}=\mathrm{C}(\mathrm{N}([\mathrm{H}]) \mathrm{C} 1=\mathrm{C}([\mathrm{H}]) \mathrm{C}(=\mathrm{C}(\mathrm{C}([\mathrm{H}])=\mathrm{C} 1[\mathrm{H}]) \mathrm{N}(\mathrm{C}([\mathrm{H}])([\mathrm{H}])[\mathrm{H}]) \mathrm{C}([\mathrm{H}])([\mathrm{H}])[\mathrm{H}]) \mathrm{C}(= \\
\mathrm{O}) \mathrm{N}([\mathrm{H}]) \mathrm{C}([\mathrm{H}])([\mathrm{H}]) \mathrm{C}([\mathrm{H}])([\mathrm{H}]) \mathrm{C}([\mathrm{H}])([\mathrm{H}]) \mathrm{OC}([\mathrm{H}])([\mathrm{H}]) \mathrm{C}([\mathrm{H}])([\mathrm{H}])[\mathrm{H}]) \mathrm{N}([\mathrm{H}]) \mathrm{C} 2 \\
=\mathrm{C}([\mathrm{H}]) \mathrm{C}([\mathrm{Cl}])=\mathrm{C}([\mathrm{H}]) \mathrm{C}([\mathrm{H}])=\mathrm{C} 2[\mathrm{H}]\end{array}$ & 1.009 & 1.393 \\
\hline $\operatorname{Tr} 160$ & $\begin{array}{l}\mathrm{O}=\mathrm{C}(\mathrm{N}([\mathrm{H}])[\mathrm{C} @]([\mathrm{H}])(\mathrm{C}(=\mathrm{O}) \mathrm{N}([\mathrm{H}])[\mathrm{C} @]([\mathrm{H}])([\mathrm{C} @]([\mathrm{H}])(\mathrm{O}[\mathrm{H}]) \mathrm{C}([\mathrm{H}])([\mathrm{H}])[\mathrm{C} @]( \\
[\mathrm{H}])(\mathrm{C}(=\mathrm{O}) \mathrm{N}([\mathrm{H}])[\mathrm{C} @]([\mathrm{H}])(\mathrm{C}(=\mathrm{O}) \mathrm{N}([\mathrm{H}])[\mathrm{C} @]([\mathrm{H}])(\mathrm{C}(=\mathrm{O}) \mathrm{N}([\mathrm{H}])[\mathrm{C} @]([\mathrm{H}])(\mathrm{C}(= \\
\mathrm{O}) \mathrm{O}[\mathrm{H}]) \mathrm{C}([\mathrm{H}])([\mathrm{H}]) \mathrm{C} 1=\mathrm{C}([\mathrm{H}]) \mathrm{C}([\mathrm{H}])=\mathrm{C}([\mathrm{H}]) \mathrm{C}([\mathrm{H}])=\mathrm{C} 1[\mathrm{H}]) \mathrm{C}([\mathrm{H}])([\mathrm{H}]) \mathrm{C}([\mathrm{H}])([\mathrm{H}] \\
) \mathrm{C}(=\mathrm{O}) \mathrm{O}[\mathrm{H}]) \mathrm{C}([\mathrm{H}])([\mathrm{H}])[\mathrm{H}]) \mathrm{C}([\mathrm{H}])([\mathrm{H}]\end{array}$ & 4.000 & 4.545 \\
\hline $\operatorname{Tr} 161$ & $\begin{array}{l}\mathrm{O}=\mathrm{C}(\mathrm{N}([\mathrm{H}])[\mathrm{C} @]([\mathrm{H}])(\mathrm{C}(=\mathrm{O}) \mathrm{N}([\mathrm{H}])[\mathrm{C} @]([\mathrm{H}])([\mathrm{C} @]([\mathrm{H}])(\mathrm{O}[\mathrm{H}]) \mathrm{C}([\mathrm{H}])([\mathrm{H}])[\mathrm{C} @]( \\
[\mathrm{H}])(\mathrm{C}(=\mathrm{O}) \mathrm{N}([\mathrm{H}])[\mathrm{C} @]([\mathrm{H}])(\mathrm{C}(=\mathrm{O}) \mathrm{N}([\mathrm{H}])[\mathrm{C} @]([\mathrm{H}])(\mathrm{C}(=\mathrm{O}) \mathrm{N}([\mathrm{H}])[\mathrm{C} @]([\mathrm{H}])(\mathrm{C}(= \\
\mathrm{O}) \mathrm{O}[\mathrm{H}]) \mathrm{C}([\mathrm{H}])([\mathrm{H}]) \mathrm{C} 1=\mathrm{C}([\mathrm{H}]) \mathrm{C}([\mathrm{H}])=\mathrm{C}([\mathrm{H}]) \mathrm{C}([\mathrm{H}])=\mathrm{C} 1[\mathrm{H}]) \mathrm{C}([\mathrm{H}])([\mathrm{H}]) \mathrm{C}([\mathrm{H}])([\mathrm{H}] \\
) \mathrm{C}(=\mathrm{O}) \mathrm{O}[\mathrm{H}]) \mathrm{C}([\mathrm{H}])([\mathrm{H}])[\mathrm{H}]) \mathrm{C}([\mathrm{H}])([\mathrm{H}]\end{array}$ & 3.167 & 3.262 \\
\hline $\operatorname{Tr} 162$ & $\begin{array}{l}\mathrm{O}=\mathrm{C}(\mathrm{N}([\mathrm{H}]) \mathrm{C}(=\mathrm{NC}([\mathrm{H}])([\mathrm{H}]) \mathrm{C} 1=\mathrm{C}([\mathrm{H}]) \mathrm{C}([\mathrm{H}])=\mathrm{C}([\mathrm{H}]) \mathrm{C}([\mathrm{H}])=\mathrm{C} 1[\mathrm{H}]) \mathrm{N}([\mathrm{H}])[\mathrm{H}]) \mathrm{C} \\
=2 \mathrm{C}(=\mathrm{NOC}=2 \mathrm{C}([\mathrm{H}])([\mathrm{H}])[\mathrm{H}]) \mathrm{C} 3=\mathrm{C}([\mathrm{H}]) \mathrm{C}([\mathrm{H}])=\mathrm{C}(\mathrm{OC}([\mathrm{H}])([\mathrm{H}])[\mathrm{H}]) \mathrm{C}([\mathrm{H}])=\mathrm{C} 3[\mathrm{H}]\end{array}$ & 1.398 & 1.566 \\
\hline $\operatorname{Tr} 163$ & $\begin{array}{l}\mathrm{O}=\mathrm{C}(\mathrm{N}([\mathrm{H}]) \mathrm{C}(=\mathrm{NC}([\mathrm{H}])([\mathrm{H}]) \mathrm{C} 1=\mathrm{C}([\mathrm{H}]) \mathrm{C}([\mathrm{Cl}])=\mathrm{C}([\mathrm{Cl}]) \mathrm{C}([\mathrm{H}])=\mathrm{C} 1[\mathrm{H}]) \mathrm{N}([\mathrm{H}])[\mathrm{H}]) \mathrm{C} \\
=2 \mathrm{C}(=\mathrm{NOC}=2 \mathrm{C}([\mathrm{H}])([\mathrm{H}])[\mathrm{H}]) \mathrm{C} 3=\mathrm{C}([\mathrm{H}]) \mathrm{C}([\mathrm{H}])=\mathrm{C}([\mathrm{F}]) \mathrm{C}([\mathrm{H}])=\mathrm{C} 3[\mathrm{H}]\end{array}$ & 1.620 & 2.386 \\
\hline $\operatorname{Tr} 164$ & $\begin{array}{l}\mathrm{O}=\mathrm{C}(\mathrm{N}([\mathrm{H}])[\mathrm{C} @]([\mathrm{H}])(\mathrm{C}(=\mathrm{O}) \mathrm{N}([\mathrm{H}])[\mathrm{C} @]([\mathrm{H}])([\mathrm{C} @ @]([\mathrm{H}])(\mathrm{O}[\mathrm{H}]) \mathrm{C}([\mathrm{H}])([\mathrm{H}])[\mathrm{C} @ \\
]([\mathrm{H}])(\mathrm{C}(=\mathrm{O}) \mathrm{N}([\mathrm{H}])[\mathrm{C} @]([\mathrm{H}])(\mathrm{C}(=\mathrm{O}) \mathrm{N}([\mathrm{H}]) \mathrm{C}([\mathrm{H}])([\mathrm{H}]) \mathrm{C}([\mathrm{H}])(\mathrm{C}([\mathrm{H}])([\mathrm{H}])[\mathrm{H}]) \mathrm{C}([ \\
\mathrm{H}])([\mathrm{H}])[\mathrm{H}]) \mathrm{C}([\mathrm{H}])(\mathrm{C}([\mathrm{H}])([\mathrm{H}])[\mathrm{H}]) \mathrm{C}([\mathrm{H}])([\mathrm{H}])[\mathrm{H}]) \mathrm{C}([\mathrm{H}])([\mathrm{H}])[\mathrm{H}]) \mathrm{C}([\mathrm{H}])([\mathrm{H}]) \mathrm{C}([ \\
\mathrm{H}])(\mathrm{C}([\mathrm{H}])([\mathrm{H}])[\mathrm{H}]) \mathrm{C}([\mathrm{H}])([\mathrm{H}]\end{array}$ & 5.602 & 4.260 \\
\hline $\operatorname{Tr} 165$ & $\begin{array}{l}\mathrm{O}=\mathrm{C}(\mathrm{N}([\mathrm{H}])[\mathrm{C} @]([\mathrm{H}])(\mathrm{C}(=\mathrm{O}) \mathrm{N}([\mathrm{H}])[\mathrm{C} @]([\mathrm{H}])([\mathrm{C} @ @]([\mathrm{H}])(\mathrm{O}[\mathrm{H}]) \mathrm{C}([\mathrm{H}])([\mathrm{H}])[\mathrm{C} @ \\
]([\mathrm{H}])(\mathrm{C}(=\mathrm{O}) \mathrm{N}([\mathrm{H}])[\mathrm{C} @]([\mathrm{H}])(\mathrm{C}(=\mathrm{O}) \mathrm{N}([\mathrm{H}]) \mathrm{C}([\mathrm{H}])([\mathrm{H}]) \mathrm{C}([\mathrm{H}])(\mathrm{C}([\mathrm{H}])([\mathrm{H}])[\mathrm{H}]) \mathrm{C}([ \\
\mathrm{H}])([\mathrm{H}])[\mathrm{H}]) \mathrm{C}([\mathrm{H}])(\mathrm{C}([\mathrm{H}])([\mathrm{H}])[\mathrm{H}]) \mathrm{C}([\mathrm{H}])([\mathrm{H}])[\mathrm{H}]) \mathrm{C}([\mathrm{H}])([\mathrm{H}])[\mathrm{H}]) \mathrm{C}([\mathrm{H}])([\mathrm{H}]) \mathrm{C}([ \\
\mathrm{H}])(\mathrm{C}([\mathrm{H}])([\mathrm{H}])[\mathrm{H}]) \mathrm{C}([\mathrm{H}])([\mathrm{H}]\end{array}$ & 3.959 & 4.297 \\
\hline $\operatorname{Tr} 166$ & $\begin{array}{l}\mathrm{O}=\mathrm{C}(\mathrm{N}([\mathrm{H}])[\mathrm{C} @]([\mathrm{H}])(\mathrm{C}(=\mathrm{O}) \mathrm{N}([\mathrm{H}])[\mathrm{C} @]([\mathrm{H}])([\mathrm{C} @ @]([\mathrm{H}])(\mathrm{O}[\mathrm{H}]) \mathrm{C}([\mathrm{H}])([\mathrm{H}])[\mathrm{C} @ \\
]([\mathrm{H}])(\mathrm{C}(=\mathrm{O}) \mathrm{N}([\mathrm{H}])[\mathrm{C} @]([\mathrm{H}])(\mathrm{C}(=\mathrm{O}) \mathrm{N}([\mathrm{H}]) \mathrm{C}([\mathrm{H}])([\mathrm{H}]) \mathrm{C}([\mathrm{H}])(\mathrm{C}([\mathrm{H}])([\mathrm{H}])[\mathrm{H}]) \mathrm{C}([ \\
\mathrm{H}])([\mathrm{H}])[\mathrm{H}]) \mathrm{C}([\mathrm{H}])(\mathrm{C}([\mathrm{H}])([\mathrm{H}])[\mathrm{H}]) \mathrm{C}([\mathrm{H}])([\mathrm{H}])[\mathrm{H}]) \mathrm{C}([\mathrm{H}])([\mathrm{H}])[\mathrm{H}]) \mathrm{C}([\mathrm{H}])([\mathrm{H}]) \mathrm{C}([ \\
\mathrm{H}])(\mathrm{C}([\mathrm{H}])([\mathrm{H}])[\mathrm{H}]) \mathrm{C}([\mathrm{H}])([\mathrm{H}]\end{array}$ & 3.538 & 4.308 \\
\hline $\operatorname{Tr} 167$ & $\begin{array}{l}\mathrm{O}=[\mathrm{S}](=\mathrm{O})(\mathrm{C}([\mathrm{H}])([\mathrm{H}])[\mathrm{C} @ @]([\mathrm{H}])(\mathrm{C}(=\mathrm{O}) \mathrm{N}([\mathrm{H}])[\mathrm{C} @]([\mathrm{H}])([\mathrm{C} @]([\mathrm{H}])(\mathrm{O}[\mathrm{H}]) \mathrm{C}([\mathrm{H} \\
])([\mathrm{H}])[\mathrm{C} @]([\mathrm{H}])(\mathrm{C}(=\mathrm{O}) \mathrm{N}([\mathrm{H}])[\mathrm{C} @]([\mathrm{H}])(\mathrm{C}(=\mathrm{O}) \mathrm{N}([\mathrm{H}]) \mathrm{C}([\mathrm{H}])([\mathrm{H}]) \mathrm{C} 1=\mathrm{C}([\mathrm{H}]) \mathrm{C}([\mathrm{H}] \\
)=\mathrm{C}([\mathrm{H}]) \mathrm{C}([\mathrm{H}])=\mathrm{C} 1[\mathrm{H}]) \mathrm{C}([\mathrm{H}])(\mathrm{C}([\mathrm{H}])([\mathrm{H}])[\mathrm{H}]) \mathrm{C}([\mathrm{H}])([\mathrm{H}])[\mathrm{H}]) \mathrm{C}([\mathrm{H}])([\mathrm{H}])[\mathrm{H}]) \mathrm{C}([\mathrm{H} \\
])([\mathrm{H}]) \mathrm{C}([\mathrm{H}])(\mathrm{C}([\mathrm{H}])([\mathrm{H}])[\mathrm{H}]) \mathrm{C}\end{array}$ & 1.947 & 2.697 \\
\hline
\end{tabular}




\begin{tabular}{|c|c|c|c|}
\hline $\operatorname{Tr} 168$ & $\begin{array}{l}\mathrm{O}=\mathrm{C}(\mathrm{N}([\mathrm{H}])[\mathrm{C} @]([\mathrm{H}])([\mathrm{C} @]([\mathrm{H}])(\mathrm{O}[\mathrm{H}]) \mathrm{C}([\mathrm{H}])([\mathrm{H}])[\mathrm{C} @]([\mathrm{H}])(\mathrm{C}(=\mathrm{O}) \mathrm{N}([\mathrm{H}])[\mathrm{C} @]( \\
[\mathrm{H}])(\mathrm{C}(=\mathrm{O}) \mathrm{N}([\mathrm{H}]) \mathrm{C}([\mathrm{H}])([\mathrm{H}]) \mathrm{C} 1=\mathrm{C}([\mathrm{H}]) \mathrm{C}([\mathrm{H}])=\mathrm{C}([\mathrm{H}]) \mathrm{C}([\mathrm{H}])=\mathrm{C} 1[\mathrm{H}]) \mathrm{C}([\mathrm{H}])(\mathrm{C}([\mathrm{H}] \\
)([\mathrm{H}])[\mathrm{H}]) \mathrm{C}([\mathrm{H}])([\mathrm{H}])[\mathrm{H}]) \mathrm{C}([\mathrm{H}])([\mathrm{H}])[\mathrm{H}]) \mathrm{C}([\mathrm{H}])([\mathrm{H}]) \mathrm{C}([\mathrm{H}])(\mathrm{C}([\mathrm{H}])([\mathrm{H}])[\mathrm{H}]) \mathrm{C}([\mathrm{H}]) \\
([\mathrm{H}])[\mathrm{H}])[\mathrm{C} @ @]([\mathrm{H}])(\mathrm{N}([\mathrm{H}]) \mathrm{C}(\end{array}$ & 1.504 & 2.056 \\
\hline $\operatorname{Tr} 169$ & $\begin{array}{l}\mathrm{O}=\mathrm{C}(\mathrm{N}([\mathrm{H}])[\mathrm{C} @ @]([\mathrm{H}])(\mathrm{C}(=\mathrm{O}) \mathrm{N}([\mathrm{H}])[\mathrm{C} @]([\mathrm{H}])([\mathrm{C} @]([\mathrm{H}])(\mathrm{O}[\mathrm{H}]) \mathrm{C}([\mathrm{H}])([\mathrm{H}])[\mathrm{C} @ \\
]([\mathrm{H}])(\mathrm{C}(=\mathrm{O}) \mathrm{N}([\mathrm{H}])[\mathrm{C} @]([\mathrm{H}])(\mathrm{C}(=\mathrm{O}) \mathrm{N}([\mathrm{H}]) \mathrm{C}([\mathrm{H}])([\mathrm{H}]) \mathrm{C} 1=\mathrm{C}([\mathrm{H}]) \mathrm{C}([\mathrm{H}])=\mathrm{C}([\mathrm{H}]) \mathrm{C}( \\
[\mathrm{H}])=\mathrm{C} 1[\mathrm{H}]) \mathrm{C}([\mathrm{H}])(\mathrm{C}([\mathrm{H}])([\mathrm{H}])[\mathrm{H}]) \mathrm{C}([\mathrm{H}])([\mathrm{H}])[\mathrm{H}]) \mathrm{C}([\mathrm{H}])([\mathrm{H}])[\mathrm{H}]) \mathrm{C}([\mathrm{H}])([\mathrm{H}]) \mathrm{C}([ \\
\mathrm{H}])(\mathrm{C}([\mathrm{H}])([\mathrm{H}])[\mathrm{H}]) \mathrm{C}([\mathrm{H}])([\mathrm{H}])[\end{array}$ & 4.602 & 2.705 \\
\hline $\operatorname{Tr} 170$ & $\begin{array}{l}\mathrm{O}=\mathrm{C} 1 \mathrm{~N}(/ \mathrm{C}(=\mathrm{N} \backslash[\mathrm{H}]) \mathrm{N}([\mathrm{H}])[\mathrm{C} @] 1(\mathrm{C}([\mathrm{H}])([\mathrm{H}]) \mathrm{C}([\mathrm{H}])([\mathrm{H}])[\mathrm{C} @ @] 2([\mathrm{H}]) \mathrm{C}([\mathrm{H}])([\mathrm{H}]) \\
\mathrm{C}([\mathrm{H}])([\mathrm{H}]) \mathrm{C}([\mathrm{H}])([\mathrm{H}]) \mathrm{C}([\mathrm{H}])([\mathrm{H}]) \mathrm{C} 2([\mathrm{H}])[\mathrm{H}]) \mathrm{C}([\mathrm{H}])([\mathrm{H}])[\mathrm{C} @] 4([\mathrm{H}]) \mathrm{C}([\mathrm{H}])([\mathrm{H}])[ \\
\mathrm{C} @]([\mathrm{H}])(\mathrm{N}([\mathrm{H}]) \mathrm{C}(=\mathrm{O}) \mathrm{C} 3=\mathrm{C}([\mathrm{H}]) \mathrm{C}([\mathrm{H}])=\mathrm{NC}([\mathrm{H}])=\mathrm{C} 3[\mathrm{H}]) \mathrm{C}([\mathrm{H}])([\mathrm{H}]) \mathrm{C}([\mathrm{H}])([\mathrm{H}]) \\
\mathrm{C} 4([\mathrm{H}])[\mathrm{H}]) \mathrm{C}([\mathrm{H}])([\mathrm{H}])[\mathrm{H}]\end{array}$ & 3.409 & 3.300 \\
\hline $\operatorname{Tr} 171$ & $\begin{array}{l}\mathrm{O}=\mathrm{C} 1 \mathrm{~N}(/ \mathrm{C}(=\mathrm{N} \backslash[\mathrm{H}]) \mathrm{N}([\mathrm{H}])[\mathrm{C} @] 1(\mathrm{C}([\mathrm{H}])([\mathrm{H}]) \mathrm{C}([\mathrm{H}])([\mathrm{H}])[\mathrm{C} @ @] 2([\mathrm{H}]) \mathrm{C}([\mathrm{H}])([\mathrm{H}]) \\
\mathrm{C}([\mathrm{H}])([\mathrm{H}]) \mathrm{C}([\mathrm{H}])([\mathrm{H}]) \mathrm{C}([\mathrm{H}])([\mathrm{H}]) \mathrm{C} 2([\mathrm{H}])[\mathrm{H}]) \mathrm{C}([\mathrm{H}])([\mathrm{H}])[\mathrm{C} @] 4([\mathrm{H}]) \mathrm{C}([\mathrm{H}])([\mathrm{H}])[ \\
\mathrm{C} @]([\mathrm{H}])(\mathrm{N}([\mathrm{H}])[\mathrm{C} @ @] 3([\mathrm{H}]) \mathrm{C}([\mathrm{H}])([\mathrm{H}]) \mathrm{C}([\mathrm{H}])([\mathrm{H}]) \mathrm{C}([\mathrm{H}])([\mathrm{H}]) \mathrm{C}([\mathrm{H}])([\mathrm{H}]) \mathrm{C} 3([ \\
\mathrm{H}])[\mathrm{H}]) \mathrm{C}([\mathrm{H}])([\mathrm{H}]) \mathrm{C}([\mathrm{H}])([\mathrm{H}]) \mathrm{C}\end{array}$ & 3.638 & 3.178 \\
\hline $\operatorname{Tr} 172$ & $\begin{array}{l}\mathrm{O}=\mathrm{C} 1 \mathrm{~N}(/ \mathrm{C}(=\mathrm{N} \backslash[\mathrm{H}]) \mathrm{N}([\mathrm{H}])[\mathrm{C} @] 1(\mathrm{C}([\mathrm{H}])([\mathrm{H}]) \mathrm{C}([\mathrm{H}])([\mathrm{H}])[\mathrm{C} @ @] 2([\mathrm{H}]) \mathrm{C}([\mathrm{H}])([\mathrm{H}]) \\
\mathrm{C}([\mathrm{H}])([\mathrm{H}]) \mathrm{C}([\mathrm{H}])([\mathrm{H}]) \mathrm{C}([\mathrm{H}])([\mathrm{H}]) \mathrm{C} 2([\mathrm{H}])[\mathrm{H}]) \mathrm{C}([\mathrm{H}])([\mathrm{H}])[\mathrm{C} @] 4([\mathrm{H}]) \mathrm{C}([\mathrm{H}])([\mathrm{H}])[ \\
\mathrm{C} @]([\mathrm{H}])(\mathrm{N}([\mathrm{H}])[\mathrm{C} @] 3([\mathrm{H}]) \mathrm{C}([\mathrm{H}])([\mathrm{H}]) \mathrm{C}([\mathrm{H}])([\mathrm{H}]) \mathrm{C}([\mathrm{H}])([\mathrm{H}]) \mathrm{C} 3([\mathrm{H}])[\mathrm{H}]) \mathrm{C}([\mathrm{H}])( \\
[\mathrm{H}]) \mathrm{C}([\mathrm{H}])([\mathrm{H}]) \mathrm{C} 4([\mathrm{H}])[\mathrm{H}]) \mathrm{C}(\end{array}$ & 3.367 & 3.160 \\
\hline $\operatorname{Tr} 173$ & $\begin{array}{l}\mathrm{O}=\mathrm{C}(\mathrm{N}([\mathrm{H}])[\mathrm{C} @]([\mathrm{H}])(\mathrm{C}(=\mathrm{O}) \mathrm{N}([\mathrm{H}])[\mathrm{C} @]([\mathrm{H}])([\mathrm{C} @ @]([\mathrm{H}])(\mathrm{O}[\mathrm{H}]) \mathrm{C}([\mathrm{H}])([\mathrm{H}])[\mathrm{C} @ \\
]([\mathrm{H}])(\mathrm{C}(=\mathrm{O}) \mathrm{N}([\mathrm{H}])[\mathrm{C} @]([\mathrm{H}])(\mathrm{C}(=\mathrm{O}) \mathrm{N}([\mathrm{H}]) \mathrm{C}([\mathrm{H}])([\mathrm{H}]) \mathrm{C}([\mathrm{H}])(\mathrm{C}([\mathrm{H}])([\mathrm{H}])[\mathrm{H}]) \mathrm{C}([ \\
\mathrm{H}])([\mathrm{H}])[\mathrm{H}]) \mathrm{C}([\mathrm{H}])(\mathrm{C}([\mathrm{H}])([\mathrm{H}])[\mathrm{H}]) \mathrm{C}([\mathrm{H}])([\mathrm{H}])[\mathrm{H}]) \mathrm{C}([\mathrm{H}])([\mathrm{H}])[\mathrm{H}]) \mathrm{C}([\mathrm{H}])([\mathrm{H}]) \mathrm{C}([ \\
\mathrm{H}])(\mathrm{C}([\mathrm{H}])([\mathrm{H}])[\mathrm{H}]) \mathrm{C}([\mathrm{H}])([\mathrm{H}]\end{array}$ & 3.398 & 3.067 \\
\hline $\operatorname{Tr} 174$ & $\begin{array}{l}\mathrm{O}=\mathrm{C}(\mathrm{N}([\mathrm{H}])[\mathrm{C} @]([\mathrm{H}])(\mathrm{C}(=\mathrm{O}) \mathrm{N}([\mathrm{H}])[\mathrm{C} @]([\mathrm{H}])([\mathrm{C} @ @]([\mathrm{H}])(\mathrm{O}[\mathrm{H}]) \mathrm{C}([\mathrm{H}])([\mathrm{H}])[\mathrm{C} @ \\
]([\mathrm{H}])(\mathrm{C}(=\mathrm{O}) \mathrm{N}([\mathrm{H}])[\mathrm{C} @]([\mathrm{H}])(\mathrm{C}(=\mathrm{O}) \mathrm{N}([\mathrm{H}]) \mathrm{C}([\mathrm{H}])([\mathrm{H}]) \mathrm{C}([\mathrm{H}])(\mathrm{C}([\mathrm{H}])([\mathrm{H}])[\mathrm{H}]) \mathrm{C}([ \\
\mathrm{H}])([\mathrm{H}])[\mathrm{H}]) \mathrm{C}([\mathrm{H}])(\mathrm{C}([\mathrm{H}])([\mathrm{H}])[\mathrm{H}]) \mathrm{C}([\mathrm{H}])([\mathrm{H}])[\mathrm{H}]) \mathrm{C}([\mathrm{H}])([\mathrm{H}])[\mathrm{H}]) \mathrm{C}([\mathrm{H}])([\mathrm{H}]) \mathrm{C}([ \\
\mathrm{H}])(\mathrm{C}([\mathrm{H}])([\mathrm{H}])[\mathrm{H}]) \mathrm{C}([\mathrm{H}])([\mathrm{H}]\end{array}$ & 1.785 & 3.167 \\
\hline $\operatorname{Tr} 175$ & $\begin{array}{l}\mathrm{O}=\mathrm{C}(\mathrm{N}([\mathrm{H}])[\mathrm{C} @]([\mathrm{H}])(\mathrm{C}(=\mathrm{O}) \mathrm{N}([\mathrm{H}])[\mathrm{C} @]([\mathrm{H}])([\mathrm{C} @ @]([\mathrm{H}])(\mathrm{O}[\mathrm{H}]) \mathrm{C}([\mathrm{H}])([\mathrm{H}])[\mathrm{C} @ \\
]([\mathrm{H}])(\mathrm{C}(=\mathrm{O}) \mathrm{N}([\mathrm{H}])[\mathrm{C} @]([\mathrm{H}])(\mathrm{C}(=\mathrm{O}) \mathrm{N}([\mathrm{H}]) \mathrm{C}([\mathrm{H}])([\mathrm{H}]) \mathrm{C}([\mathrm{H}])(\mathrm{C}([\mathrm{H}])([\mathrm{H}])[\mathrm{H}]) \mathrm{C}([ \\
\mathrm{H}])([\mathrm{H}])[\mathrm{H}]) \mathrm{C}([\mathrm{H}])(\mathrm{C}([\mathrm{H}])([\mathrm{H}])[\mathrm{H}]) \mathrm{C}([\mathrm{H}])([\mathrm{H}])[\mathrm{H}]) \mathrm{C}([\mathrm{H}])([\mathrm{H}])[\mathrm{H}]) \mathrm{C}([\mathrm{H}])([\mathrm{H}]) \mathrm{C}([ \\
\mathrm{H}])(\mathrm{C}([\mathrm{H}])([\mathrm{H}])[\mathrm{H}]) \mathrm{C}([\mathrm{H}])([\mathrm{H}]\end{array}$ & 3.201 & 3.103 \\
\hline $\operatorname{Tr} 176$ & $\begin{array}{l}\mathrm{O}=\mathrm{C}(\mathrm{N}([\mathrm{H}])[\mathrm{C} @]([\mathrm{H}])(\mathrm{C}(=\mathrm{O}) \mathrm{N}([\mathrm{H}])[\mathrm{C} @]([\mathrm{H}])([\mathrm{C} @ @]([\mathrm{H}])(\mathrm{O}[\mathrm{H}]) \mathrm{C}([\mathrm{H}])([\mathrm{H}])[\mathrm{C} @ \\
]([\mathrm{H}])(\mathrm{C}(=\mathrm{O}) \mathrm{N}([\mathrm{H}])[\mathrm{C} @]([\mathrm{H}])(\mathrm{C}(=\mathrm{O}) \mathrm{N}([\mathrm{H}]) \mathrm{C}([\mathrm{H}])([\mathrm{H}]) \mathrm{C}([\mathrm{H}])(\mathrm{C}([\mathrm{H}])([\mathrm{H}])[\mathrm{H}]) \mathrm{C}([ \\
\mathrm{H}])([\mathrm{H}])[\mathrm{H}]) \mathrm{C}([\mathrm{H}])(\mathrm{C}([\mathrm{H}])([\mathrm{H}])[\mathrm{H}]) \mathrm{C}([\mathrm{H}])([\mathrm{H}])[\mathrm{H}]) \mathrm{C}([\mathrm{H}])([\mathrm{H}])[\mathrm{H}]) \mathrm{C}([\mathrm{H}])([\mathrm{H}]) \mathrm{C}([ \\
\mathrm{H}])(\mathrm{C}([\mathrm{H}])([\mathrm{H}])[\mathrm{H}]) \mathrm{C}([\mathrm{H}])([\mathrm{H}]\end{array}$ & 2.697 & 3.117 \\
\hline $\operatorname{Tr} 177$ & $\begin{array}{l}\mathrm{O}=\mathrm{C}(\mathrm{N}([\mathrm{H}])[\mathrm{C} @]([\mathrm{H}])(\mathrm{C}(=\mathrm{O}) \mathrm{N}([\mathrm{H}])[\mathrm{C} @]([\mathrm{H}])([\mathrm{C} @ @]([\mathrm{H}])(\mathrm{O}[\mathrm{H}]) \mathrm{C}([\mathrm{H}])([\mathrm{H}])[\mathrm{C} @ \\
]([\mathrm{H}])(\mathrm{C}(=\mathrm{O}) \mathrm{N}([\mathrm{H}])[\mathrm{C} @]([\mathrm{H}])(\mathrm{C}(=\mathrm{O}) \mathrm{N}([\mathrm{H}]) \mathrm{C}([\mathrm{H}])([\mathrm{H}]) \mathrm{C}([\mathrm{H}])(\mathrm{C}([\mathrm{H}])([\mathrm{H}])[\mathrm{H}]) \mathrm{C}([ \\
\mathrm{H}])([\mathrm{H}])[\mathrm{H}]) \mathrm{C}([\mathrm{H}])(\mathrm{C}([\mathrm{H}])([\mathrm{H}])[\mathrm{H}]) \mathrm{C}([\mathrm{H}])([\mathrm{H}])[\mathrm{H}]) \mathrm{C}([\mathrm{H}])([\mathrm{H}])[\mathrm{H}]) \mathrm{C}([\mathrm{H}])([\mathrm{H}]) \mathrm{C}([ \\
\mathrm{H}])(\mathrm{C}([\mathrm{H}])([\mathrm{H}])[\mathrm{H}]) \mathrm{C}([\mathrm{H}])([\mathrm{H}]\end{array}$ & 3.194 & 2.633 \\
\hline $\operatorname{Tr} 178$ & $\begin{array}{l}\mathrm{O}=[\mathrm{S}](=\mathrm{O})(\mathrm{C}([\mathrm{H}])([\mathrm{H}])[\mathrm{C} @]([\mathrm{H}])(\mathrm{N}([\mathrm{H}]) \mathrm{C}(=\mathrm{O})[\mathrm{C} @]([\mathrm{H}])(\mathrm{C}([\mathrm{H}])([\mathrm{H}]) \mathrm{N} 1 \mathrm{~N}=\mathrm{C}(\mathrm{C}([ \\
\mathrm{H}])=\mathrm{C} 1 \mathrm{C}([\mathrm{H}])([\mathrm{H}])[\mathrm{H}]) \mathrm{C}([\mathrm{H}])([\mathrm{H}])[\mathrm{H}]) \mathrm{C}([\mathrm{H}])([\mathrm{H}])[\mathrm{H}]) \mathrm{C}(=\mathrm{O}) \mathrm{N}([\mathrm{H}])[\mathrm{C} @]([\mathrm{H}])([\mathrm{C} \\
@ @]([\mathrm{H}])(\mathrm{O}[\mathrm{H}]) \mathrm{C}([\mathrm{H}])([\mathrm{H}])[\mathrm{C} @]([\mathrm{H}])(\mathrm{C}(=\mathrm{O}) \mathrm{N}([\mathrm{H}])[\mathrm{C} @]([\mathrm{H}])(\mathrm{C}(=\mathrm{O}) \mathrm{N}([\mathrm{H}]) \mathrm{C}([\mathrm{H}] \\
)([\mathrm{H}]) \mathrm{C}([\mathrm{H}])(\mathrm{C}([\mathrm{H}])([\mathrm{H}])[\mathrm{H}]) \mathrm{C}([\mathrm{H}])(\end{array}$ & 2.717 & 3.325 \\
\hline
\end{tabular}




\begin{tabular}{|c|c|c|c|}
\hline $\operatorname{Tr} 179$ & $\begin{array}{l}\mathrm{O}=\mathrm{C}(\mathrm{N}([\mathrm{H}])[\mathrm{C} @]([\mathrm{H}])(\mathrm{C}(=\mathrm{O}) \mathrm{N}([\mathrm{H}])[\mathrm{C} @]([\mathrm{H}])([\mathrm{C} @ @]([\mathrm{H}])(\mathrm{O}[\mathrm{H}]) \mathrm{C}([\mathrm{H}])([\mathrm{H}])[\mathrm{C} @ \\
]([\mathrm{H}])(\mathrm{C}(=\mathrm{O}) \mathrm{N}([\mathrm{H}])[\mathrm{C} @]([\mathrm{H}])(\mathrm{C}(=\mathrm{O}) \mathrm{N}([\mathrm{H}]) \mathrm{C}([\mathrm{H}])([\mathrm{H}]) \mathrm{C}([\mathrm{H}])(\mathrm{C}([\mathrm{H}])([\mathrm{H}])[\mathrm{H}]) \mathrm{C}([ \\
\mathrm{H}])([\mathrm{H}])[\mathrm{H}]) \mathrm{C}([\mathrm{H}])(\mathrm{C}([\mathrm{H}])([\mathrm{H}])[\mathrm{H}]) \mathrm{C}([\mathrm{H}])([\mathrm{H}])[\mathrm{H}]) \mathrm{C}([\mathrm{H}])([\mathrm{H}])[\mathrm{H}]) \mathrm{C}([\mathrm{H}])([\mathrm{H}]) \mathrm{C}([ \\
\mathrm{H}])(\mathrm{C}([\mathrm{H}])([\mathrm{H}])[\mathrm{H}]) \mathrm{C}([\mathrm{H}])([\mathrm{H}]\end{array}$ & 3.495 & 3.455 \\
\hline $\operatorname{Tr} 180$ & $\begin{array}{l}\mathrm{O}=\mathrm{C}(\mathrm{N}([\mathrm{H}])[\mathrm{C} @]([\mathrm{H}])(\mathrm{C}(=\mathrm{O}) \mathrm{N}([\mathrm{H}])[\mathrm{C} @]([\mathrm{H}])([\mathrm{C} @ @]([\mathrm{H}])(\mathrm{O}[\mathrm{H}]) \mathrm{C}([\mathrm{H}])([\mathrm{H}])[\mathrm{C} @ \\
]([\mathrm{H}])(\mathrm{C}(=\mathrm{O}) \mathrm{N}([\mathrm{H}])[\mathrm{C} @]([\mathrm{H}])(\mathrm{C}(=\mathrm{O}) \mathrm{N}([\mathrm{H}]) \mathrm{C}([\mathrm{H}])([\mathrm{H}]) \mathrm{C}([\mathrm{H}])(\mathrm{C}([\mathrm{H}])([\mathrm{H}])[\mathrm{H}]) \mathrm{C}([ \\
\mathrm{H}])([\mathrm{H}])[\mathrm{H}]) \mathrm{C}([\mathrm{H}])(\mathrm{C}([\mathrm{H}])([\mathrm{H}])[\mathrm{H}]) \mathrm{C}([\mathrm{H}])([\mathrm{H}])[\mathrm{H}]) \mathrm{C}([\mathrm{H}])([\mathrm{H}])[\mathrm{H}]) \mathrm{C}([\mathrm{H}])([\mathrm{H}]) \mathrm{C}([ \\
\mathrm{H}])(\mathrm{C}([\mathrm{H}])([\mathrm{H}])[\mathrm{H}]) \mathrm{C}([\mathrm{H}])([\mathrm{H}]\end{array}$ & 3.770 & 3.491 \\
\hline $\operatorname{Tr} 181$ & $\begin{array}{l}\mathrm{O}=\mathrm{C} 1 \mathrm{~N}(/ \mathrm{C}(=\mathrm{N} \backslash[\mathrm{H}]) \mathrm{N}([\mathrm{H}])[\mathrm{C} @] 1(\mathrm{C}([\mathrm{H}])([\mathrm{H}]) \mathrm{C}([\mathrm{H}])([\mathrm{H}])[\mathrm{C} @ @] 2([\mathrm{H}]) \mathrm{C}([\mathrm{H}])([\mathrm{H}]) \\
\mathrm{C}([\mathrm{H}])([\mathrm{H}]) \mathrm{C}([\mathrm{H}])([\mathrm{H}]) \mathrm{C}([\mathrm{H}])([\mathrm{H}]) \mathrm{C} 2([\mathrm{H}])[\mathrm{H}]) \mathrm{C}([\mathrm{H}])([\mathrm{H}])[\mathrm{C} @ @] 4([\mathrm{H}]) \mathrm{C}([\mathrm{H}])([\mathrm{H}] \\
) \mathrm{N}(\mathrm{C}(=\mathrm{O}) \mathrm{C}([\mathrm{H}])([\mathrm{H}])[\mathrm{C} @] 3([\mathrm{H}]) \mathrm{C}([\mathrm{H}])([\mathrm{H}]) \mathrm{C}([\mathrm{H}])([\mathrm{H}]) \mathrm{C}([\mathrm{H}])([\mathrm{H}]) \mathrm{C} 3([\mathrm{H}])[\mathrm{H}]) \mathrm{C}([ \\
\mathrm{H}])([\mathrm{H}]) \mathrm{C} 4([\mathrm{H}])[\mathrm{H}]) \mathrm{C}([\mathrm{H}])([\mathrm{H}]\end{array}$ & 3.432 & 3.124 \\
\hline $\operatorname{Tr} 182$ & $\begin{array}{l}\mathrm{O}=\mathrm{C} 1 \mathrm{~N}(/ \mathrm{C}(=\mathrm{N} /[\mathrm{H}]) \mathrm{N}([\mathrm{H}])[\mathrm{C} @] 1(\mathrm{C}([\mathrm{H}])([\mathrm{H}]) \mathrm{C}([\mathrm{H}])([\mathrm{H}])[\mathrm{C} @ @] 2([\mathrm{H}]) \mathrm{C}([\mathrm{H}])([\mathrm{H}]) \\
\mathrm{C}([\mathrm{H}])([\mathrm{H}]) \mathrm{C}([\mathrm{H}])([\mathrm{H}]) \mathrm{C}([\mathrm{H}])([\mathrm{H}]) \mathrm{C} 2([\mathrm{H}])[\mathrm{H}]) \mathrm{C}([\mathrm{H}])([\mathrm{H}])[\mathrm{C} @] 3([\mathrm{H}]) \mathrm{C}([\mathrm{H}])([\mathrm{H}]) \mathrm{N} \\
(\mathrm{C}(=\mathrm{O}) \mathrm{C}([\mathrm{H}])([\mathrm{H}]) \mathrm{C}([\mathrm{H}])([\mathrm{H}]) \mathrm{C}([\mathrm{H}])([\mathrm{H}]) \mathrm{C}([\mathrm{H}])([\mathrm{H}])[\mathrm{H}]) \mathrm{C}([\mathrm{H}])([\mathrm{H}]) \mathrm{C}([\mathrm{H}])([\mathrm{H}]) \mathrm{C} \\
3([\mathrm{H}])[\mathrm{H}]) \mathrm{C}([\mathrm{H}])([\mathrm{H}])[\mathrm{H}]\end{array}$ & 3.495 & 3.287 \\
\hline $\operatorname{Tr} 183$ & $\begin{array}{l}\mathrm{O}=[\mathrm{S}](=\mathrm{O})(\mathrm{N} 3 \mathrm{C}([\mathrm{H}])([\mathrm{H}])[\mathrm{C} @ @]([\mathrm{H}])(\mathrm{C}([\mathrm{H}])([\mathrm{H}])[\mathrm{C} @] 1(\mathrm{~N}([\mathrm{H}]) \mathrm{C}(=\mathrm{N}[\mathrm{H}]) \mathrm{N}(\mathrm{C} 1= \\
\mathrm{O}) \mathrm{C}([\mathrm{H}])([\mathrm{H}])[\mathrm{H}]) \mathrm{C}([\mathrm{H}])([\mathrm{H}]) \mathrm{C}([\mathrm{H}])([\mathrm{H}])[\mathrm{C} @ @] 2([\mathrm{H}]) \mathrm{C}([\mathrm{H}])([\mathrm{H}]) \mathrm{C}([\mathrm{H}])([\mathrm{H}]) \mathrm{C}([\mathrm{H} \\
])([\mathrm{H}]) \mathrm{C}([\mathrm{H}])([\mathrm{H}]) \mathrm{C} 2([\mathrm{H}])[\mathrm{H}]) \mathrm{C}([\mathrm{H}])([\mathrm{H}]) \mathrm{C}([\mathrm{H}])([\mathrm{H}]) \mathrm{C} 3([\mathrm{H}])[\mathrm{H}]) \mathrm{C}([\mathrm{H}])([\mathrm{H}]) \mathrm{C}([\mathrm{H}]) \\
([\mathrm{H}]) \mathrm{C}([\mathrm{H}])([\mathrm{H}]) \mathrm{C}([\mathrm{H}])([\mathrm{H}])[\end{array}$ & 3.201 & 3.474 \\
\hline $\operatorname{Tr} 184$ & $\begin{array}{l}\mathrm{O}=\mathrm{C} 1 \mathrm{~N}(/ \mathrm{C}(=\mathrm{N} \backslash[\mathrm{H}]) \mathrm{N}([\mathrm{H}])[\mathrm{C} @] 1(\mathrm{C}([\mathrm{H}])([\mathrm{H}]) \mathrm{C}([\mathrm{H}])([\mathrm{H}])[\mathrm{C} @ @] 2([\mathrm{H}]) \mathrm{C}([\mathrm{H}])([\mathrm{H}]) \\
\mathrm{C}([\mathrm{H}])([\mathrm{H}]) \mathrm{C}([\mathrm{H}])([\mathrm{H}]) \mathrm{C}([\mathrm{H}])([\mathrm{H}]) \mathrm{C} 2([\mathrm{H}])[\mathrm{H}]) \mathrm{C}([\mathrm{H}])([\mathrm{H}])[\mathrm{C} @] 5([\mathrm{H}]) \mathrm{C}([\mathrm{H}])([\mathrm{H}])[ \\
\mathrm{C} @]([\mathrm{H}])(\mathrm{N}([\mathrm{H}]) \mathrm{C}(=\mathrm{O}) \mathrm{N}([\mathrm{H}]) \mathrm{C} 3=\mathrm{NC}=4 \mathrm{C}(/ \mathrm{N}=\mathrm{C} 3 /[\mathrm{H}])=\mathrm{C}([\mathrm{H}]) \mathrm{C}([\mathrm{H}])=\mathrm{C}([\mathrm{H}]) \mathrm{C}=4[ \\
\mathrm{H}]) \mathrm{C}([\mathrm{H}])([\mathrm{H}]) \mathrm{C}([\mathrm{H}])([\mathrm{H}]) \mathrm{C} 5([\mathrm{H}])[\mathrm{H}]\end{array}$ & 3.854 & 3.985 \\
\hline $\operatorname{Tr} 185$ & $\begin{array}{l}\mathrm{O}=\mathrm{C} 1 \mathrm{~N}(/ \mathrm{C}(=\mathrm{N} \backslash[\mathrm{H}]) \mathrm{N}([\mathrm{H}])[\mathrm{C} @] 1(\mathrm{C}([\mathrm{H}])([\mathrm{H}]) \mathrm{C}([\mathrm{H}])([\mathrm{H}])[\mathrm{C} @ @] 2([\mathrm{H}]) \mathrm{C}([\mathrm{H}])([\mathrm{H}]) \\
\mathrm{C}([\mathrm{H}])([\mathrm{H}]) \mathrm{C}([\mathrm{H}])([\mathrm{H}]) \mathrm{C}([\mathrm{H}])([\mathrm{H}]) \mathrm{C} 2([\mathrm{H}])[\mathrm{H}]) \mathrm{C}([\mathrm{H}])([\mathrm{H}])[\mathrm{C} @] 4([\mathrm{H}]) \mathrm{C}([\mathrm{H}])([\mathrm{H}])[ \\
\mathrm{C} @]([\mathrm{H}])(\mathrm{N}([\mathrm{H}]) \mathrm{C}(=\mathrm{O}) \mathrm{N}([\mathrm{H}])[\mathrm{C} @ @] 3([\mathrm{H}]) \mathrm{C}([\mathrm{H}])([\mathrm{H}]) \mathrm{C}([\mathrm{H}])([\mathrm{H}]) \mathrm{C}([\mathrm{H}])([\mathrm{H}]) \mathrm{C}([ \\
\mathrm{H}])([\mathrm{H}]) \mathrm{C} 3([\mathrm{H}])[\mathrm{H}]) \mathrm{C}([\mathrm{H}])([\mathrm{H}]) \mathrm{C}\end{array}$ & 2.860 & 3.727 \\
\hline $\operatorname{Tr} 186$ & $\begin{array}{l}\mathrm{O}=\mathrm{C} 1 \mathrm{~N}(/ \mathrm{C}(=\mathrm{N} /[\mathrm{H}]) \mathrm{N}([\mathrm{H}])[\mathrm{C} @] 1(\mathrm{C}([\mathrm{H}])([\mathrm{H}]) \mathrm{C}([\mathrm{H}])([\mathrm{H}])[\mathrm{C} @ @] 2([\mathrm{H}]) \mathrm{C}([\mathrm{H}])([\mathrm{H}]) \\
\mathrm{C}([\mathrm{H}])([\mathrm{H}]) \mathrm{C}([\mathrm{H}])([\mathrm{H}]) \mathrm{C}([\mathrm{H}])([\mathrm{H}]) \mathrm{C}([\mathrm{H}])[\mathrm{H}]) \mathrm{C}([\mathrm{H}])([\mathrm{H}])[\mathrm{C} @] 4([\mathrm{H}]) \mathrm{C}([\mathrm{H}])([\mathrm{H}])[ \\
\mathrm{C} @]([\mathrm{H}])(\mathrm{N}([\mathrm{H}]) \mathrm{C}(=\mathrm{O}) \mathrm{N}([\mathrm{H}]) \mathrm{C} 3=\mathrm{C}(\mathrm{OC}([\mathrm{H}])([\mathrm{H}])[\mathrm{H}]) \mathrm{C}([\mathrm{H}])=\mathrm{C}([\mathrm{H}]) \mathrm{C}([\mathrm{H}])=\mathrm{C} 3[\mathrm{H}] \\
) \mathrm{C}([\mathrm{H}])([\mathrm{H}]) \mathrm{C}([\mathrm{H}])([\mathrm{H}]) \mathrm{C} 4([\mathrm{H}])[\mathrm{H}\end{array}$ & 2.559 & 3.179 \\
\hline $\operatorname{Tr} 187$ & $\begin{array}{l}\mathrm{O}=\mathrm{C} 1 \mathrm{~N}(/ \mathrm{C}(=\mathrm{N} \backslash[\mathrm{H}]) \mathrm{N}([\mathrm{H}])[\mathrm{C} @] 1(\mathrm{C}([\mathrm{H}])([\mathrm{H}]) \mathrm{C}([\mathrm{H}])([\mathrm{H}])[\mathrm{C} @] 2([\mathrm{H}]) \mathrm{C}([\mathrm{H}])([\mathrm{H}]) \mathrm{C}([ \\
\mathrm{H}])([\mathrm{H}]) \mathrm{C}([\mathrm{H}])([\mathrm{H}]) \mathrm{C}([\mathrm{H}])([\mathrm{H}]) \mathrm{C} 2([\mathrm{H}])[\mathrm{H}]) \mathrm{C}([\mathrm{H}])([\mathrm{H}])[\mathrm{C} @] 4([\mathrm{H}]) \mathrm{C}([\mathrm{H}])([\mathrm{H}])[\mathrm{C} @ \\
]([\mathrm{H}])(\mathrm{N}([\mathrm{H}]) \mathrm{C}(=\mathrm{O}) \mathrm{N}([\mathrm{H}]) \mathrm{C} 3=\mathrm{C}([\mathrm{H}]) \mathrm{C}([\mathrm{H}])=\mathrm{C}([\mathrm{Cl}]) \mathrm{C}([\mathrm{H}])=\mathrm{C} 3[\mathrm{H}]) \mathrm{C}([\mathrm{H}])([\mathrm{H}]) \mathrm{C}([ \\
\mathrm{H}])([\mathrm{H}]) \mathrm{C} 4([\mathrm{H}])[\mathrm{H}]) \mathrm{C}([\mathrm{H}])([\mathrm{H}]\end{array}$ & 5.000 & 4.123 \\
\hline $\operatorname{Tr} 188$ & $\begin{array}{l}\mathrm{O}=\mathrm{C} 1 \mathrm{~N}(/ \mathrm{C}(=\mathrm{N} \backslash[\mathrm{H}]) \mathrm{N}([\mathrm{H}])[\mathrm{C} @] 1(\mathrm{C}([\mathrm{H}])([\mathrm{H}]) \mathrm{C}([\mathrm{H}])([\mathrm{H}])[\mathrm{C} @ @] 2([\mathrm{H}]) \mathrm{C}([\mathrm{H}])([\mathrm{H}]) \\
\mathrm{C}([\mathrm{H}])([\mathrm{H}]) \mathrm{C}([\mathrm{H}])([\mathrm{H}]) \mathrm{C}([\mathrm{H}])([\mathrm{H}]) \mathrm{C} 2([\mathrm{H}])[\mathrm{H}]) \mathrm{C}([\mathrm{H}])([\mathrm{H}])[\mathrm{C} @] 4([\mathrm{H}]) \mathrm{C}([\mathrm{H}])([\mathrm{H}])[ \\
\mathrm{C} @([\mathrm{H}])(\mathrm{N}([\mathrm{H}]) \mathrm{C}(=\mathrm{O}) \mathrm{C}([\mathrm{H}])([\mathrm{H}]) \mathrm{C} 3=\mathrm{C}([\mathrm{H}]) \mathrm{C}([\mathrm{H}])=\mathrm{C}([\mathrm{H}]) \mathrm{C}([\mathrm{H}])=\mathrm{C} 3[\mathrm{H}]) \mathrm{C}([\mathrm{H}])( \\
[\mathrm{H}]) \mathrm{C}([\mathrm{H}])([\mathrm{H}]) \mathrm{C} 4([\mathrm{H}])[\mathrm{H}]) \mathrm{C}([\mathrm{H}]\end{array}$ & 2.233 & 2.792 \\
\hline $\operatorname{Tr} 189$ & $\begin{array}{l}\mathrm{O}=\mathrm{C} 1 \mathrm{~N}(/ \mathrm{C}(=\mathrm{N} \backslash[\mathrm{H}]) \mathrm{N}([\mathrm{H}])[\mathrm{C} @ @] 1(\mathrm{C}([\mathrm{H}])([\mathrm{H}])[\mathrm{C} @ @] 2([\mathrm{H}]) \mathrm{C}([\mathrm{H}])([\mathrm{H}]) \mathrm{C}([\mathrm{H}])([\mathrm{H} \\
]) \mathrm{C}([\mathrm{H}])([\mathrm{H}]) \mathrm{C}([\mathrm{H}])([\mathrm{H}]) \mathrm{C} 2([\mathrm{H}])[\mathrm{H}]) \mathrm{C}([\mathrm{H}])([\mathrm{H}]) \mathrm{C}([\mathrm{H}])([\mathrm{H}])[\mathrm{C} @ @] 3([\mathrm{H}]) \mathrm{C}([\mathrm{H}])([ \\
\mathrm{H}]) \mathrm{C}([\mathrm{H}])([\mathrm{H}]) \mathrm{C}([\mathrm{H}])([\mathrm{H}]) \mathrm{C}([\mathrm{H}])([\mathrm{H}]) \mathrm{C} 3([\mathrm{H}])[\mathrm{H}]) \mathrm{C}([\mathrm{H}])([\mathrm{H}]) \mathrm{C} 4=\mathrm{C}([\mathrm{H}]) \mathrm{C}([\mathrm{H}])=\mathrm{C}( \\
\mathrm{C}([\mathrm{H}])=\mathrm{C} 4[\mathrm{H}]) \mathrm{C}([\mathrm{H}])([\mathrm{H}]) \mathrm{N} 5 \mathrm{C}(=\mathrm{O}\end{array}$ & 3.569 & 4.181 \\
\hline
\end{tabular}




\begin{tabular}{|c|c|c|c|}
\hline $\operatorname{Tr} 190$ & $\begin{array}{l}\mathrm{O}=\mathrm{C} 1 \mathrm{~N}(/ \mathrm{C}(=\mathrm{N} /[\mathrm{H}]) \mathrm{N}([\mathrm{H}])[\mathrm{C} @] 1(\mathrm{C}([\mathrm{H}])([\mathrm{H}]) \mathrm{C}([\mathrm{H}])([\mathrm{H}])[\mathrm{C} @ @] 2([\mathrm{H}]) \mathrm{C}([\mathrm{H}])([\mathrm{H}]) \\
\mathrm{C}([\mathrm{H}])([\mathrm{H}]) \mathrm{C}([\mathrm{H}])([\mathrm{H}]) \mathrm{C}([\mathrm{H}])([\mathrm{H}]) \mathrm{C} 2([\mathrm{H}])[\mathrm{H}]) \mathrm{C}([\mathrm{H}])([\mathrm{H}])[\mathrm{C} @ @] 3([\mathrm{H}]) \mathrm{C}([\mathrm{H}])([\mathrm{H}] \\
) \mathrm{N}(\mathrm{C}(=\mathrm{O}) \mathrm{C}([\mathrm{H}])([\mathrm{H}]) \mathrm{C}([\mathrm{H}])([\mathrm{H}]) \mathrm{C}([\mathrm{H}])([\mathrm{H}]) \mathrm{C}([\mathrm{H}])([\mathrm{H}])[\mathrm{H}]) \mathrm{C}([\mathrm{H}])([\mathrm{H}]) \mathrm{C}([\mathrm{H}])([\mathrm{H}]) \\
\mathrm{C} 3([\mathrm{H}])[\mathrm{H}]) \mathrm{C}([\mathrm{H}])([\mathrm{H}])[\mathrm{H}]\end{array}$ & 3.770 & 3.269 \\
\hline $\operatorname{Tr} 191$ & $\begin{array}{l}\mathrm{O}=\mathrm{C} 1 \mathrm{~N}(/ \mathrm{C}(=\mathrm{N} \backslash[\mathrm{H}]) \mathrm{N}([\mathrm{H}])[\mathrm{C} @] 1(\mathrm{C}([\mathrm{H}])([\mathrm{H}]) \mathrm{C}([\mathrm{H}])([\mathrm{H}])[\mathrm{C} @ @] 2([\mathrm{H}]) \mathrm{C}([\mathrm{H}])([\mathrm{H}]) \\
\mathrm{C}([\mathrm{H}])([\mathrm{H}]) \mathrm{C}([\mathrm{H}])([\mathrm{H}]) \mathrm{C}([\mathrm{H}])([\mathrm{H}]) \mathrm{C} 2([\mathrm{H}])[\mathrm{H}]) \mathrm{C}([\mathrm{H}])([\mathrm{H}])[\mathrm{C} @] 4([\mathrm{H}]) \mathrm{C}([\mathrm{H}])([\mathrm{H}]) \mathrm{N} \\
(\mathrm{C}(=\mathrm{O}) \mathrm{C}([\mathrm{H}])([\mathrm{H}])[\mathrm{C} @ 3([\mathrm{H}]) \mathrm{C}([\mathrm{H}])([\mathrm{H}]) \mathrm{C}([\mathrm{H}])([\mathrm{H}]) \mathrm{C}([\mathrm{H}])([\mathrm{H}]) \mathrm{C} 3([\mathrm{H}])[\mathrm{H}]) \mathrm{C}([\mathrm{H}] \\
)([\mathrm{H}]) \mathrm{C}([\mathrm{H}])([\mathrm{H}]) \mathrm{C} 4([\mathrm{H}])[\mathrm{H}])\end{array}$ & 3.013 & 3.754 \\
\hline $\operatorname{Tr} 192$ & $\begin{array}{l}\mathrm{O}=\mathrm{C} 1 \mathrm{~N}(/ \mathrm{C}(=\mathrm{N} \backslash[\mathrm{H}]) \mathrm{N}([\mathrm{H}])[\mathrm{C} @] 1(\mathrm{C}([\mathrm{H}])([\mathrm{H}]) \mathrm{C}([\mathrm{H}])([\mathrm{H}])[\mathrm{C} @ @] 2([\mathrm{H}]) \mathrm{C}([\mathrm{H}])([\mathrm{H}]) \\
\mathrm{C}([\mathrm{H}])([\mathrm{H}]) \mathrm{C}([\mathrm{H}])([\mathrm{H}]) \mathrm{C}([\mathrm{H}])([\mathrm{H}]) \mathrm{C} 2([\mathrm{H}])[\mathrm{H}]) \mathrm{C}([\mathrm{H}])([\mathrm{H}])[\mathrm{C} @] 3([\mathrm{H}]) \mathrm{C}([\mathrm{H}])([\mathrm{H}]) \mathrm{N} \\
(\mathrm{C}(=\mathrm{O}) \mathrm{OC}(\mathrm{C}([\mathrm{H}])([\mathrm{H}])[\mathrm{H}])(\mathrm{C}([\mathrm{H}])([\mathrm{H}])[\mathrm{H}]) \mathrm{C}([\mathrm{H}])([\mathrm{H}])[\mathrm{H}]) \mathrm{C}([\mathrm{H}])([\mathrm{H}]) \mathrm{C}([\mathrm{H}])([\mathrm{H}]) \\
\mathrm{C} 3([\mathrm{H}])[\mathrm{H}]) \mathrm{C}([\mathrm{H}])([\mathrm{H}])[\mathrm{H}]\end{array}$ & 3.538 & 3.231 \\
\hline $\operatorname{Tr} 193$ & $\begin{array}{l}\mathrm{O}=[\mathrm{S}] 2(=\mathrm{O}) \mathrm{N}(/ \mathrm{C}(=\mathrm{N} /[\mathrm{H}]) \mathrm{N}([\mathrm{H}])[\mathrm{C} @] 4(/ \mathrm{C} 1=\mathrm{C}(\backslash \mathrm{H}]) \mathrm{C}([\mathrm{F}])=\mathrm{C}([\mathrm{F}]) \mathrm{C}([\mathrm{H}])=\mathrm{C} 1[\mathrm{~F}])[\mathrm{C} \\
@ @ @ 2([\mathrm{H}]) \mathrm{C}([\mathrm{H}])([\mathrm{H}]) \mathrm{N}(/ \mathrm{C} 3=\mathrm{N} / \mathrm{C}(=\mathrm{C}(/[\mathrm{F}]) \mathrm{C}(=\mathrm{N} 3) \mathrm{OC}([\mathrm{H}])([\mathrm{H}])[\mathrm{H}]) \mathrm{C}([\mathrm{H}])([\mathrm{H}])[\mathrm{H} \\
]) \mathrm{C} 4([\mathrm{H}])[\mathrm{H}]) \mathrm{C}([\mathrm{H}])([\mathrm{H}])[\mathrm{H}]\end{array}$ & 3.495 & 3.809 \\
\hline $\operatorname{Tr} 194$ & $\begin{array}{l}\mathrm{O}=[\mathrm{S}] 2(=\mathrm{O}) \mathrm{N}(/ \mathrm{C}(=\mathrm{N} /[\mathrm{H}]) \mathrm{N}([\mathrm{H}])[\mathrm{C} @] 4(/ \mathrm{C} 1=\mathrm{C}(\backslash \mathrm{H}]) \mathrm{C}([\mathrm{H}])=\mathrm{C}([\mathrm{F}]) \mathrm{C}([\mathrm{H}])=\mathrm{C} 1[\mathrm{~F}])[\mathrm{C} \\
@] 2([\mathrm{H}]) \mathrm{C}([\mathrm{H}])([\mathrm{H}]) \mathrm{N}(/ \mathrm{C} 3=\mathrm{N} / \mathrm{C}(=\mathrm{C}(/[\mathrm{F}]) \mathrm{C}(=\mathrm{N} 3) \mathrm{OC}([\mathrm{H}])([\mathrm{H}])[\mathrm{H}]) \mathrm{C}([\mathrm{H}])([\mathrm{H}])[\mathrm{H}]) \\
\mathrm{C} 4([\mathrm{H}])[\mathrm{H}]) \mathrm{C}([\mathrm{H}])([\mathrm{H}])[\mathrm{H}]\end{array}$ & 3.523 & 4.227 \\
\hline $\operatorname{Tr} 195$ & $\begin{array}{l}\mathrm{O}=[\mathrm{S}] 2(=\mathrm{O}) \mathrm{N}(/ \mathrm{C}(=\mathrm{N} /[\mathrm{H}]) \mathrm{N}([\mathrm{H}])[\mathrm{C} @] 4(/ \mathrm{C} 1=\mathrm{C}(\backslash \mathrm{H}]) \mathrm{C}([\mathrm{F}])=\mathrm{C}([\mathrm{H}]) \mathrm{C}([\mathrm{H}])=\mathrm{C} 1[\mathrm{~F}])[\mathrm{C} \\
@ @] 2([\mathrm{H}]) \mathrm{C}([\mathrm{H}])([\mathrm{H}]) \mathrm{N}(/ \mathrm{C} 3=\mathrm{N} / \mathrm{C}(\mathrm{OC}([\mathrm{H}])([\mathrm{H}])[\mathrm{H}])=\mathrm{C}([\mathrm{H}]) \mathrm{C}(=\mathrm{C} 3[\mathrm{H}]) \mathrm{C}([\mathrm{H}])([\mathrm{H}]) \\
\mathrm{OC}([\mathrm{H}])([\mathrm{H}])[\mathrm{H}]) \mathrm{C} 4([\mathrm{H}])[\mathrm{H}]) \mathrm{C}([\mathrm{H}])([\mathrm{H}])[\mathrm{H}]\end{array}$ & 4.000 & 3.250 \\
\hline Tr196 & $\begin{array}{l}\mathrm{O}=[\mathrm{S}] 2(=\mathrm{O}) \mathrm{N}(/ \mathrm{C}(=\mathrm{N} /[\mathrm{H}]) \mathrm{N}([\mathrm{H}])[\mathrm{C} @ @] 4(/ \mathrm{C} 1=\mathrm{C}(\backslash[\mathrm{H}]) \mathrm{C}([\mathrm{H}])=\mathrm{C}([\mathrm{H}]) \mathrm{C}([\mathrm{H}])=\mathrm{C} 1[\mathrm{H}] \\
)[\mathrm{C} @] 2([\mathrm{H}]) \mathrm{C}([\mathrm{H}])([\mathrm{H}]) \mathrm{N}(/ \mathrm{C} 3=\mathrm{N} / \mathrm{C}(\mathrm{OC}([\mathrm{H}])([\mathrm{H}])[\mathrm{H}])=\mathrm{C}([\mathrm{H}]) \mathrm{C}(=\mathrm{C} 3[\mathrm{H}]) \mathrm{C}([\mathrm{H}])([\mathrm{H}] \\
) \mathrm{OC}([\mathrm{H}])([\mathrm{H}])[\mathrm{H}]) \mathrm{C} 4([\mathrm{H}])[\mathrm{H}]) \mathrm{C}([\mathrm{H}])([\mathrm{H}])[\mathrm{H}]\end{array}$ & 3.569 & 3.199 \\
\hline $\operatorname{Tr} 197$ & $\begin{array}{l}\mathrm{O}=[\mathrm{S}] 2(=\mathrm{O}) \mathrm{N}(/ \mathrm{C}(=\mathrm{N} /[\mathrm{H}]) \mathrm{N}([\mathrm{H}])[\mathrm{C} @ @] 4(/ \mathrm{C} 1=\mathrm{C}(\backslash \mathrm{H}]) \mathrm{C}([\mathrm{H}])=\mathrm{C}([\mathrm{H}]) \mathrm{C}([\mathrm{H}])=\mathrm{C} 1[\mathrm{H}] \\
)[\mathrm{C} @] 2([\mathrm{H}]) \mathrm{C}([\mathrm{H}])([\mathrm{H}]) \mathrm{N}(/ \mathrm{C} 3=\mathrm{N} / \mathrm{C}(\mathrm{OC}([\mathrm{H}])([\mathrm{H}])[\mathrm{H}])=\mathrm{C}([\mathrm{F}]) \mathrm{C}(=\mathrm{N} 3) \mathrm{OC}([\mathrm{H}])([\mathrm{H}])[ \\
\mathrm{H}]) \mathrm{C} 4([\mathrm{H}])[\mathrm{H}]) \mathrm{C}([\mathrm{H}])([\mathrm{H}])[\mathrm{H}]\end{array}$ & 3.523 & 3.583 \\
\hline $\operatorname{Tr} 198$ & $\begin{array}{l}\mathrm{O}=[\mathrm{S}] 2(=\mathrm{O}) \mathrm{N}(/ \mathrm{C}(=\mathrm{N} /[\mathrm{H}]) \mathrm{N}([\mathrm{H}])[\mathrm{C} @ @] 4(/ \mathrm{C} 1=\mathrm{C}(\backslash[\mathrm{H}]) \mathrm{C}([\mathrm{H}])=\mathrm{C}([\mathrm{H}]) \mathrm{C}([\mathrm{H}])=\mathrm{C} 1[\mathrm{H}] \\
)[\mathrm{C} @] 2([\mathrm{H}]) \mathrm{C}([\mathrm{H}])([\mathrm{H}]) \mathrm{N}(/ \mathrm{C} 3=\mathrm{N} / \mathrm{C}(\mathrm{OC}([\mathrm{H}])([\mathrm{H}])[\mathrm{H}])=\mathrm{C}([\mathrm{H}]) \mathrm{C}([\mathrm{H}])=\mathrm{C} 3[\mathrm{H}]) \mathrm{C} 4([\mathrm{H} \\
])[\mathrm{H}]) \mathrm{C}([\mathrm{H}])([\mathrm{H}])[\mathrm{H}]\end{array}$ & 3.066 & 2.664 \\
\hline $\operatorname{Tr} 199$ & $\begin{array}{l}\mathrm{O}=[\mathrm{S}] 2(=\mathrm{O}) \mathrm{N}(/ \mathrm{C}(=\mathrm{N} /[\mathrm{H}]) \mathrm{N}([\mathrm{H}])[\mathrm{C} @ @] 5(/ \mathrm{C} 1=\mathrm{C}(\backslash \mathrm{H}]) \mathrm{C}([\mathrm{H}])=\mathrm{C}([\mathrm{H}]) \mathrm{C}([\mathrm{H}])=\mathrm{C} 1[\mathrm{H}] \\
)[\mathrm{C} @] 2([\mathrm{H}]) \mathrm{C}([\mathrm{H}])([\mathrm{H}]) \mathrm{N}(/ \mathrm{C} 3=\mathrm{N} / \mathrm{C}(=\mathrm{C}(/[\mathrm{F}]) \mathrm{C}(=\mathrm{N} 3) \mathrm{OC}([\mathrm{H}])([\mathrm{H}])[\mathrm{H}])[\mathrm{C} @ @] 4([\mathrm{H}]) \\
\mathrm{C}([\mathrm{H}])([\mathrm{H}]) \mathrm{C} 4([\mathrm{H}])[\mathrm{H}]) \mathrm{C} 5([\mathrm{H}])[\mathrm{H}]) \mathrm{C}([\mathrm{H}])([\mathrm{H}])[\mathrm{H}]\end{array}$ & 3.377 & 3.414 \\
\hline $\operatorname{Tr} 200$ & $\begin{array}{l}\mathrm{O}=[\mathrm{S}] 3(=\mathrm{O}) \mathrm{N}(/ \mathrm{C}(=\mathrm{N} /[\mathrm{H}]) \mathrm{N}([\mathrm{H}])[\mathrm{C} @] 5(/ \mathrm{C} 1=\mathrm{C}(\backslash \mathrm{H}]) \mathrm{C}(=\mathrm{C}([\mathrm{H}]) \mathrm{C}([\mathrm{H}])=\mathrm{C} 1[\mathrm{~F}]) \mathrm{N}([\mathrm{H}] \\
) \mathrm{C}(=\mathrm{O}) \mathrm{C} 2=\mathrm{NC}([\mathrm{H}])=\mathrm{C}([\mathrm{F}]) \mathrm{C}([\mathrm{H}])=\mathrm{C} 2[\mathrm{H}])[\mathrm{C} @] 3([\mathrm{H}]) \mathrm{C}([\mathrm{H}])([\mathrm{H}]) \mathrm{N}(/ \mathrm{C} 4=\mathrm{N} / \mathrm{C}(\mathrm{OC}([ \\
\mathrm{H}])([\mathrm{H}])[\mathrm{H}])=\mathrm{C}([\mathrm{F}]) \mathrm{C}(=\mathrm{N} 4) \mathrm{C}([\mathrm{H}])([\mathrm{H}])[\mathrm{H}]) \mathrm{C} 5([\mathrm{H}])[\mathrm{H}]) \mathrm{C}([\mathrm{H}])([\mathrm{H}])[\mathrm{H}]\end{array}$ & 4.921 & 4.586 \\
\hline $\operatorname{Tr} 201$ & $\begin{array}{l}\mathrm{O}=\mathrm{C}(\mathrm{C}=1 \mathrm{C}([\mathrm{H}])=\mathrm{C}(\mathrm{C}([\mathrm{H}])=\mathrm{C}(\mathrm{C}=1[\mathrm{H}]) \mathrm{C}(=\mathrm{O}) \mathrm{N}([\mathrm{H}])[\mathrm{C} @]([\mathrm{H}])([\mathrm{C} @ @]([\mathrm{H}])(\mathrm{O}[\mathrm{H}]) \\
\mathrm{C}([\mathrm{H}])([\mathrm{H}]) \mathrm{N}([\mathrm{H}])[\mathrm{C} @]([\mathrm{H}])(\mathrm{C}(=\mathrm{O}) \mathrm{N}([\mathrm{H}]) \mathrm{C}([\mathrm{H}])([\mathrm{H}]) \mathrm{C}([\mathrm{H}])(\mathrm{C}([\mathrm{H}])([\mathrm{H}])[\mathrm{H}]) \mathrm{C}([\mathrm{H}] \\
)([\mathrm{H}])[\mathrm{H}])[\mathrm{C} @ @]([\mathrm{H}])(\mathrm{O}[\mathrm{H}]) \mathrm{C}([\mathrm{H}])([\mathrm{H}])[\mathrm{H}]) \mathrm{C}([\mathrm{H}])([\mathrm{H}]) \mathrm{C} 2=\mathrm{C}([\mathrm{H}]) \mathrm{C}([\mathrm{H}])=\mathrm{C}([\mathrm{H}]) \\
\mathrm{C}([\mathrm{H}])=\mathrm{C} 2[\mathrm{H}]) \mathrm{C}([\mathrm{H}])([\mathrm{H}])[\mathrm{H}]) \mathrm{N}([\mathrm{C} @]\end{array}$ & 1.781 & 1.825 \\
\hline $\operatorname{Tr} 202$ & $\begin{array}{l}\mathrm{O}=\mathrm{C}(\mathrm{C}=1 \mathrm{C}([\mathrm{H}])=\mathrm{C}([\mathrm{H}]) \mathrm{C}([\mathrm{H}])=\mathrm{C}(\mathrm{C}=1[\mathrm{H}]) \mathrm{C}(=\mathrm{O}) \mathrm{N}([\mathrm{H}])[\mathrm{C} @]([\mathrm{H}])([\mathrm{C} @ @]([\mathrm{H}])(\mathrm{O}[ \\
\mathrm{H}]) \mathrm{C}([\mathrm{H}])([\mathrm{H}]) \mathrm{N}([\mathrm{H}])[\mathrm{C} @([\mathrm{H}])(\mathrm{C}(=\mathrm{O}) \mathrm{N}([\mathrm{H}]) \mathrm{C}([\mathrm{H}])([\mathrm{H}]) \mathrm{C}([\mathrm{H}])(\mathrm{C}([\mathrm{H}])([\mathrm{H}])[\mathrm{H}]) \mathrm{C}( \\
[\mathrm{H}])([\mathrm{H}])[\mathrm{H}])[\mathrm{C} @ @]([\mathrm{H}])(\mathrm{O}[\mathrm{H}]) \mathrm{C}([\mathrm{H}])([\mathrm{H}])[\mathrm{H}]) \mathrm{C}([\mathrm{H}])([\mathrm{H}]) \mathrm{C} 2=\mathrm{C}([\mathrm{H}]) \mathrm{C}([\mathrm{H}])=\mathrm{C}([\end{array}$ & 1.236 & 1.588 \\
\hline
\end{tabular}




\begin{tabular}{|c|c|c|c|}
\hline & $\mathrm{H}]) \mathrm{C}([\mathrm{H}])=\mathrm{C} 2[\mathrm{H}]) \mathrm{N}([\mathrm{C} @]([\mathrm{H}])(\mathrm{C} 3=\mathrm{C}($ & & \\
\hline $\operatorname{Tr} 203$ & $\begin{array}{l}\mathrm{O}=\mathrm{C}(\mathrm{C}=1 \mathrm{C}([\mathrm{H}])=\mathrm{C}([\mathrm{H}]) \mathrm{C}([\mathrm{H}])=\mathrm{C}(\mathrm{C}=1[\mathrm{H}]) \mathrm{C}(=\mathrm{O}) \mathrm{N}([\mathrm{H}])[\mathrm{C} @]([\mathrm{H}])([\mathrm{C} @ @]([\mathrm{H}])(\mathrm{O}[ \\
\mathrm{H}]) \mathrm{C}([\mathrm{H}])([\mathrm{H}]) \mathrm{N}([\mathrm{H}])[\mathrm{C} @]([\mathrm{H}])(\mathrm{C}(=\mathrm{O}) \mathrm{N}([\mathrm{H}]) \mathrm{C}([\mathrm{H}])([\mathrm{H}]) \mathrm{C}([\mathrm{H}])(\mathrm{C}([\mathrm{H}])([\mathrm{H}])[\mathrm{H}]) \mathrm{C}( \\
[\mathrm{H}])([\mathrm{H}])[\mathrm{H}])[\mathrm{C} @ @]([\mathrm{H}])(\mathrm{O}[\mathrm{H}]) \mathrm{C}([\mathrm{H}])([\mathrm{H}]) \mathrm{C}([\mathrm{H}])([\mathrm{H}]) \mathrm{C}([\mathrm{H}])([\mathrm{H}])[\mathrm{H}]) \mathrm{C}([\mathrm{H}])([\mathrm{H}]) \\
\mathrm{C} 2=\mathrm{C}([\mathrm{H}]) \mathrm{C}([\mathrm{H}])=\mathrm{C}([\mathrm{H}]) \mathrm{C}([\mathrm{H}])=\mathrm{C}\end{array}$ & 2.573 & 2.693 \\
\hline $\operatorname{Tr} 204$ & $\begin{array}{l}\mathrm{O}=\mathrm{C}(\mathrm{C}=1 \mathrm{C}([\mathrm{H}])=\mathrm{C}(\mathrm{C}([\mathrm{H}])=\mathrm{C}(\mathrm{C}=1[\mathrm{H}]) \mathrm{C}(=\mathrm{O}) \mathrm{N}([\mathrm{H}])[\mathrm{C} @]([\mathrm{H}])([\mathrm{C} @ @]([\mathrm{H}])(\mathrm{O}[\mathrm{H}]) \\
\mathrm{C}([\mathrm{H}])([\mathrm{H}]) \mathrm{N}([\mathrm{H}])[\mathrm{C} @]([\mathrm{H}])(\mathrm{C}(=\mathrm{O}) \mathrm{N}([\mathrm{H}]) \mathrm{C}([\mathrm{H}])([\mathrm{H}]) \mathrm{C}([\mathrm{H}])(\mathrm{C}([\mathrm{H}])([\mathrm{H}])[\mathrm{H}]) \mathrm{C}([\mathrm{H}] \\
)([\mathrm{H}])[\mathrm{H}])[\mathrm{C} @ @]([\mathrm{H}])(\mathrm{O}[\mathrm{H}]) \mathrm{C}([\mathrm{H}])([\mathrm{H}]) \mathrm{C}([\mathrm{H}])([\mathrm{H}]) \mathrm{C}([\mathrm{H}])([\mathrm{H}])[\mathrm{H}]) \mathrm{C}([\mathrm{H}])([\mathrm{H}]) \mathrm{C} 2 \\
=\mathrm{C}([\mathrm{H}]) \mathrm{C}([\mathrm{H}])=\mathrm{C}([\mathrm{H}]) \mathrm{C}([\mathrm{H}])=\mathrm{C} 2[\mathrm{H}]\end{array}$ & 4.183 & 2.930 \\
\hline \multicolumn{4}{|c|}{ Test set } \\
\hline Ts1 & $\begin{array}{l}\mathrm{O}=\mathrm{C} 2 \mathrm{~N}([\mathrm{H}])[\mathrm{C} @]([\mathrm{H}])(\mathrm{C}(=\mathrm{O}) \mathrm{N}([\mathrm{H}])[\mathrm{C} @ @]([\mathrm{H}])(\mathrm{C}([\mathrm{H}])([\mathrm{H}]) \mathrm{C}([\mathrm{H}])(\mathrm{C}([\mathrm{H}])([\mathrm{H}])[ \\
\mathrm{H}]) \mathrm{C}([\mathrm{H}])([\mathrm{H}])[\mathrm{H}])[\mathrm{C} @ @]([\mathrm{H}])(\mathrm{O}[\mathrm{H}]) \mathrm{C}([\mathrm{H}])([\mathrm{H}])[\mathrm{C} @([\mathrm{H}])(\mathrm{C}(=\mathrm{O}) \mathrm{N}([\mathrm{H}])[\mathrm{C} @]([\mathrm{H} \\
])(\mathrm{C}(=\mathrm{O}) \mathrm{N}([\mathrm{H}]) \mathrm{C}([\mathrm{H}])([\mathrm{H}]) \mathrm{C}=1 \mathrm{C}([\mathrm{H}])=\mathrm{C}([\mathrm{H}]) \mathrm{C}([\mathrm{H}])=\mathrm{C}([\mathrm{H}]) \mathrm{C}=1[\mathrm{H}]) \mathrm{C}([\mathrm{H}])(\mathrm{C}([\mathrm{H}]) \\
([\mathrm{H}])[\mathrm{H}]) \mathrm{C}([\mathrm{H}])([\mathrm{H}])[\mathrm{H}]) \mathrm{C}([\mathrm{H}])([\mathrm{H}])[\mathrm{H}]) \mathrm{C}([\mathrm{H}])([\mathrm{H}]) \mathrm{C}(=\mathrm{O}) \mathrm{N}([\mathrm{H}]) \mathrm{C}([\mathrm{H}])([\mathrm{H}]) \mathrm{C}([\mathrm{H}]) \\
([\mathrm{H}]) \mathrm{C}([\mathrm{H}])([\mathrm{H}]) \mathrm{C}([\mathrm{H}])([\mathrm{H}]) \mathrm{C}([\mathrm{H}])([\mathrm{H}]) \mathrm{C}([\mathrm{H}])([\mathrm{H}]) \mathrm{OC}(=\mathrm{O}) \mathrm{N}([\mathrm{H}])[\mathrm{C} @ @] 2([\mathrm{H}]) \mathrm{C}([ \\
\mathrm{H}])(\mathrm{C}([\mathrm{H}])([\mathrm{H}])[\mathrm{H}]) \mathrm{C}([\mathrm{H}])([\mathrm{H}])[\mathrm{H}]\end{array}$ & 3.600 & 3.204 \\
\hline Ts2 & $\begin{array}{l}\mathrm{O}=\mathrm{C}(\mathrm{OC}([\mathrm{H}])([\mathrm{H}]) \mathrm{C}=1 \mathrm{~N}=\mathrm{C}(\mathrm{OC}=1 \mathrm{C}([\mathrm{H}])([\mathrm{H}])[\mathrm{H}]) \mathrm{C}([\mathrm{H}])([\mathrm{H}])[\mathrm{H}]) \mathrm{N}([\mathrm{H}])[\mathrm{C} @]([\mathrm{H}]) \\
(\mathrm{C}(=\mathrm{O}) \mathrm{N}([\mathrm{H}])[\mathrm{C} @ @]([\mathrm{H}])(\mathrm{C}([\mathrm{H}])([\mathrm{H}]) \mathrm{C}([\mathrm{H}])(\mathrm{C}([\mathrm{H}])([\mathrm{H}])[\mathrm{H}]) \mathrm{C}([\mathrm{H}])([\mathrm{H}])[\mathrm{H}])[\mathrm{C} @ \\
@]([\mathrm{H}])(\mathrm{O}[\mathrm{H}]) \mathrm{C}([\mathrm{H}])([\mathrm{H}])[\mathrm{C} @]([\mathrm{H}])(\mathrm{C}(=\mathrm{O}) \mathrm{N}([\mathrm{H}])[\mathrm{C} @]([\mathrm{H}])(\mathrm{C}(=\mathrm{O}) \mathrm{N}([\mathrm{H}]) \mathrm{C}([\mathrm{H}])([ \\
\mathrm{H}]) \mathrm{C}([\mathrm{H}])(\mathrm{C}([\mathrm{H}])([\mathrm{H}])[\mathrm{H}]) \mathrm{C}([\mathrm{H}])([\mathrm{H}])[\mathrm{H}]) \mathrm{C}([\mathrm{H}])(\mathrm{C}([\mathrm{H}])([\mathrm{H}])[\mathrm{H}]) \mathrm{C}([\mathrm{H}])([\mathrm{H}])[\mathrm{H}]) \mathrm{C} \\
([\mathrm{H}])([\mathrm{H}])[\mathrm{H}]) \mathrm{C}([\mathrm{H}])([\mathrm{H}])[\mathrm{S}](=\mathrm{O})(=\mathrm{O}) \mathrm{C}([\mathrm{H}])([\mathrm{H}])[\mathrm{H}]\end{array}$ & 5.921 & 3.884 \\
\hline Ts3 & $\begin{array}{l}\mathrm{O}=\mathrm{C}(/ \mathrm{C} 1=\mathrm{C}(([\mathrm{H}]) \mathrm{C}(=\mathrm{C}([\mathrm{H}]) \mathrm{C}(=\mathrm{C} 1[\mathrm{H}]) \mathrm{C} 2=\mathrm{NN}=\mathrm{C}(\mathrm{O} 2)[\mathrm{C} @ @](\mathrm{C}([\mathrm{H}])([\mathrm{H}])[\mathrm{H}])(\mathrm{C}([ \\
\mathrm{H}])([\mathrm{H}]) \mathrm{C}=3 \mathrm{C}([\mathrm{H}])=\mathrm{C}([\mathrm{H}]) \mathrm{C}([\mathrm{H}])=\mathrm{C}([\mathrm{H}]) \mathrm{C}=3[\mathrm{H}])[\mathrm{N}+]([\mathrm{H}])([\mathrm{H}])[\mathrm{H}]) \mathrm{N}(\mathrm{C}([\mathrm{H}])([\mathrm{H}]) \\
[\mathrm{H}])[\mathrm{S}](=\mathrm{O})(=\mathrm{O}) \mathrm{C}([\mathrm{H}])([\mathrm{H}])[\mathrm{H}]) \mathrm{C}([\mathrm{H}])(\mathrm{C}([\mathrm{H}])([\mathrm{H}])[\mathrm{H}]) \mathrm{C}([\mathrm{H}])([\mathrm{H}])[\mathrm{H}]\end{array}$ & 0.783 & 2.741 \\
\hline Ts4 & $\begin{array}{l}\mathrm{O}=\mathrm{C}(\mathrm{N}([\mathrm{H}])[\mathrm{C} @]([\mathrm{H}])([\mathrm{C} @ @](([\mathrm{H}])(\mathrm{O}[\mathrm{H}]) \mathrm{C}([\mathrm{H}])([\mathrm{H}])[\mathrm{C} @]([\mathrm{H}])(\mathrm{C}(=\mathrm{O}) \mathrm{N}([\mathrm{H}])[\mathrm{C} @ \\
]([\mathrm{H}])(\mathrm{C}(=\mathrm{O}) \mathrm{N}([\mathrm{H}]) \mathrm{C}([\mathrm{H}])([\mathrm{H}]) \mathrm{C} 1=\mathrm{C}([\mathrm{H}]) \mathrm{C}([\mathrm{H}])=\mathrm{C}([\mathrm{H}]) \mathrm{C}([\mathrm{H}])=\mathrm{C} 1[\mathrm{H}]) \mathrm{C}([\mathrm{H}])(\mathrm{C}([ \\
\mathrm{H}])([\mathrm{H}])[\mathrm{H}]) \mathrm{C}([\mathrm{H}])([\mathrm{H}])[\mathrm{H}]) \mathrm{C}([\mathrm{H}])([\mathrm{H}])[\mathrm{H}]) \mathrm{C}([\mathrm{H}])([\mathrm{H}]) \mathrm{C}([\mathrm{H}])(\mathrm{C}([\mathrm{H}])([\mathrm{H}])[\mathrm{H}]) \mathrm{C}([ \\
\mathrm{H}])([\mathrm{H}])[\mathrm{H}])[\mathrm{C} @ @]([\mathrm{H}])(\mathrm{N}([\mathrm{H}]) \mathrm{C}(=\mathrm{O}) \mathrm{OC}(\mathrm{C}([\mathrm{H}])([\mathrm{H}])[\mathrm{H}])(\mathrm{C}([\mathrm{H}])([\mathrm{H}])[\mathrm{H}]) \mathrm{C}([\mathrm{H}])( \\
[\mathrm{H}])[\mathrm{H}]) \mathrm{C}([\mathrm{H}])([\mathrm{H}])[\mathrm{S}](=\mathrm{O})(=\mathrm{O}) \mathrm{C}([\mathrm{H}])([\mathrm{H}])[\mathrm{H}]\end{array}$ & 1.947 & 2.860 \\
\hline Ts5 & $\begin{array}{l}\mathrm{O}=\mathrm{C}(\mathrm{OC}([\mathrm{H}])([\mathrm{H}]) \mathrm{C}([\mathrm{H}])=\mathrm{C}([\mathrm{H}])[\mathrm{H}]) \mathrm{N}([\mathrm{H}])[\mathrm{C} @]([\mathrm{H}])(\mathrm{C}(=\mathrm{O}) \mathrm{N}([\mathrm{H}])[\mathrm{C} @]([\mathrm{H}])(\mathrm{C}( \\
=\mathrm{O}) \mathrm{N}([\mathrm{H}])[\mathrm{C} @ @]([\mathrm{H}])(\mathrm{C}([\mathrm{H}])([\mathrm{H}]) \mathrm{C}([\mathrm{H}])(\mathrm{C}([\mathrm{H}])([\mathrm{H}])[\mathrm{H}]) \mathrm{C}([\mathrm{H}])([\mathrm{H}])[\mathrm{H}])[\mathrm{C} @ @]( \\
[\mathrm{H}])(\mathrm{O}[\mathrm{H}]) \mathrm{C}([\mathrm{H}])([\mathrm{H}])[\mathrm{C} @]([\mathrm{H}])(\mathrm{C}(=\mathrm{O}) \mathrm{N}([\mathrm{H}])[\mathrm{C} @([\mathrm{H}])(\mathrm{C}(=\mathrm{O}) \mathrm{N}([\mathrm{H}]) \mathrm{C}([\mathrm{H}])([\mathrm{H}]) \\
\mathrm{C}=1 \mathrm{C}([\mathrm{H}])=\mathrm{C}([\mathrm{H}]) \mathrm{C}([\mathrm{H}])=\mathrm{C}([\mathrm{H}]) \mathrm{C}=1[\mathrm{H}]) \mathrm{C}([\mathrm{H}])(\mathrm{C}([\mathrm{H}])([\mathrm{H}])[\mathrm{H}]) \mathrm{C}([\mathrm{H}])([\mathrm{H}])[\mathrm{H}]) \mathrm{C}( \\
[\mathrm{H}])([\mathrm{H}])[\mathrm{H}]) \mathrm{C}([\mathrm{H}])([\mathrm{H}]) \mathrm{C}(=\mathrm{O}) \mathrm{N}([\mathrm{H}]) \mathrm{C}([\mathrm{H}])([\mathrm{H}]) \mathrm{C}([\mathrm{H}])=\mathrm{C}([\mathrm{H}])[\mathrm{H}]) \mathrm{C}([\mathrm{H}])(\mathrm{C}([\mathrm{H}])( \\
[\mathrm{H}])[\mathrm{H}]) \mathrm{C}([\mathrm{H}])([\mathrm{H}])[\mathrm{H}]\end{array}$ & 3.575 & 3.030 \\
\hline Ts6 & $\begin{array}{l}\mathrm{O}=\mathrm{C} 2 \mathrm{~N}(\mathrm{C}(=\mathrm{N}[\mathrm{C} @] 4(\mathrm{C}=1 \mathrm{C}([\mathrm{H}])=\mathrm{C}([\mathrm{H}]) \mathrm{C}([\mathrm{H}])=\mathrm{C}([\mathrm{H}]) \mathrm{C}=1[\mathrm{H}])[\mathrm{C} @ @] 2([\mathrm{H}]) \mathrm{C}([\mathrm{H}] \\
)([\mathrm{H}]) \mathrm{N}(/ \mathrm{C} 3=\mathrm{N} / \mathrm{C}(=\mathrm{C}(/[\mathrm{F}]) \mathrm{C}(=\mathrm{N} 3) \mathrm{OC}([\mathrm{H}])([\mathrm{H}])[\mathrm{H}]) \mathrm{C}([\mathrm{H}])([\mathrm{H}])[\mathrm{H}]) \mathrm{C} 4([\mathrm{H}])[\mathrm{H}]) \mathrm{N}([ \\
\mathrm{H}])[\mathrm{H}]) \mathrm{C}([\mathrm{H}])([\mathrm{H}])[\mathrm{H}]\end{array}$ & 3.921 & 3.183 \\
\hline Ts7 & $\begin{array}{l}\mathrm{O}=\mathrm{C} 2 \mathrm{~N}(\mathrm{C}(=\mathrm{N}[\mathrm{C} @] 4(\mathrm{C}=1 \mathrm{C}([\mathrm{H}])=\mathrm{C}([\mathrm{H}]) \mathrm{C}([\mathrm{H}])=\mathrm{C}([\mathrm{H}]) \mathrm{C}=1[\mathrm{H}])[\mathrm{C} @ @] 2([\mathrm{H}]) \mathrm{C}([\mathrm{H}] \\
)([\mathrm{H}]) \mathrm{N}(/ \mathrm{C} 3=\mathrm{N} / \mathrm{C}(\mathrm{OC}([\mathrm{H}])([\mathrm{H}]) \mathrm{C}([\mathrm{H}])([\mathrm{H}])[\mathrm{H}])=\mathrm{C}([\mathrm{F}]) \mathrm{C}(=\mathrm{N} 3) \mathrm{OC}([\mathrm{H}])([\mathrm{H}])[\mathrm{H}]) \mathrm{C} 4( \\
[\mathrm{H}])[\mathrm{H}]) \mathrm{N}([\mathrm{H}])[\mathrm{H}]) \mathrm{C}([\mathrm{H}])([\mathrm{H}])[\mathrm{H}]\end{array}$ & 2.863 & 3.643 \\
\hline Ts8 & $\begin{array}{l}\mathrm{O}=\mathrm{C} 2 \mathrm{~N}(\mathrm{C}(=\mathrm{N}[\mathrm{C} @] 4(\mathrm{C}=1 \mathrm{C}([\mathrm{H}])=\mathrm{C}([\mathrm{H}]) \mathrm{C}([\mathrm{H}])=\mathrm{C}([\mathrm{H}]) \mathrm{C}=1[\mathrm{H}])[\mathrm{C} @ @] 2([\mathrm{H}]) \mathrm{C}([\mathrm{H}] \\
)([\mathrm{H}]) \mathrm{N}(/ \mathrm{C} 3=\mathrm{N} / \mathrm{C}(\mathrm{OC}([\mathrm{H}])([\mathrm{H}])[\mathrm{H}])=\mathrm{C}([\mathrm{F}]) \mathrm{C}(=\mathrm{N} 3) \mathrm{C}([\mathrm{H}])([\mathrm{H}]) \mathrm{C}([\mathrm{H}])([\mathrm{H}])[\mathrm{H}]) \mathrm{C} 4([\end{array}$ & 3.796 & 3.456 \\
\hline
\end{tabular}




\begin{tabular}{|c|c|c|c|}
\hline & $\mathrm{H}])[\mathrm{H}]) \mathrm{N}([\mathrm{H}])[\mathrm{H}]) \mathrm{C}([\mathrm{H}])([\mathrm{H}])[\mathrm{H}]$ & & \\
\hline Ts9 & $\begin{array}{l}\mathrm{O}=\mathrm{C} 2 \mathrm{~N}(\mathrm{C}(=\mathrm{N}[\mathrm{C} @] 4(\mathrm{C}=1 \mathrm{C}([\mathrm{H}])=\mathrm{C}([\mathrm{H}]) \mathrm{C}([\mathrm{F}])=\mathrm{C}([\mathrm{H}]) \mathrm{C}=1[\mathrm{~F}])[\mathrm{C} @ @] 2([\mathrm{H}]) \mathrm{C}([\mathrm{H}]) \\
([\mathrm{H}]) \mathrm{N}(/ \mathrm{C} 3=\mathrm{N} / \mathrm{C}(\mathrm{OC}([\mathrm{H}])([\mathrm{H}])[\mathrm{H}])=\mathrm{C}([\mathrm{F}]) \mathrm{C}(=\mathrm{N} 3) \mathrm{C}([\mathrm{H}])([\mathrm{H}]) \mathrm{C}([\mathrm{H}])([\mathrm{H}])[\mathrm{H}]) \mathrm{C} 4([\mathrm{H} \\
])[\mathrm{H}]) \mathrm{N}([\mathrm{H}])[\mathrm{H}]) \mathrm{C}([\mathrm{H}])([\mathrm{H}])[\mathrm{H}]\end{array}$ & 4.276 & 3.931 \\
\hline Ts 10 & $\begin{array}{l}{[\mathrm{F}] / \mathrm{C} 1=\mathrm{C}(/ \mathrm{N}=\mathrm{C}(/ \mathrm{N}=\mathrm{C} 1 / \mathrm{OC}([\mathrm{H}])([\mathrm{H}])[\mathrm{H}]) \mathrm{N} 3 \mathrm{C}([\mathrm{H}])([\mathrm{H}])[\mathrm{C} @ @] 2(\mathrm{~N}=\mathrm{C}(\mathrm{N}(\mathrm{C}(=\mathrm{O})[\mathrm{C}} \\
@] 2([\mathrm{H}]) \mathrm{C} 3([\mathrm{H}])[\mathrm{H}]) \mathrm{C}([\mathrm{H}])([\mathrm{H}])[\mathrm{H}]) \mathrm{N}([\mathrm{H}])[\mathrm{H}]) \mathrm{C} 4=\mathrm{C}([\mathrm{H}]) \mathrm{C}([\mathrm{H}])=\mathrm{C}([\mathrm{F}]) \mathrm{C}([\mathrm{H}])=\mathrm{C} \\
4[\mathrm{~F}]) \mathrm{C}([\mathrm{F}])([\mathrm{F}])[\mathrm{F}]\end{array}$ & 3.495 & 3.203 \\
\hline Ts11 & $\begin{array}{l}\mathrm{O}=\mathrm{C} 2 \mathrm{~N}(\mathrm{C}(=\mathrm{N}[\mathrm{C} @] 4(\mathrm{C}=1 \mathrm{C}([\mathrm{H}])=\mathrm{C}(\mathrm{CHN}) \mathrm{C}([\mathrm{H}])=\mathrm{C}([\mathrm{H}]) \mathrm{C}=1[\mathrm{H}])[\mathrm{C} @ @] 2([\mathrm{H}]) \mathrm{C}([\mathrm{H} \\
])([\mathrm{H}]) \mathrm{N}(/ \mathrm{C} 3=\mathrm{N} / \mathrm{C}(=\mathrm{C}(/[\mathrm{F}]) \mathrm{C}(=\mathrm{N} 3) \mathrm{OC}([\mathrm{H}])([\mathrm{H}])[\mathrm{H}]) \mathrm{C}([\mathrm{H}])([\mathrm{H}])[\mathrm{H}]) \mathrm{C} 4([\mathrm{H}])[\mathrm{H}]) \mathrm{N}([ \\
\mathrm{H}])[\mathrm{H}]) \mathrm{C}([\mathrm{H}])([\mathrm{H}])[\mathrm{H}]\end{array}$ & 3.854 & 3.758 \\
\hline Ts12 & $\begin{array}{l}\mathrm{O}=\mathrm{C}(\mathrm{OC}(\mathrm{C}([\mathrm{H}])([\mathrm{H}])[\mathrm{H}])(\mathrm{C}([\mathrm{H}])([\mathrm{H}])[\mathrm{H}]) \mathrm{C}([\mathrm{H}])([\mathrm{H}])[\mathrm{H}]) \mathrm{N}([\mathrm{H}])[\mathrm{C} @]([\mathrm{H}])(\mathrm{C}(=\mathrm{O}) \\
\mathrm{C}([\mathrm{H}])([\mathrm{H}]) \mathrm{N}([\mathrm{H}])[\mathrm{C} @]([\mathrm{H}])([\mathrm{C} @]([\mathrm{H}])(\mathrm{O}[\mathrm{H}]) \mathrm{C}([\mathrm{H}])([\mathrm{H}])[\mathrm{C} @]([\mathrm{H}])(\mathrm{C}(=\mathrm{O}) \mathrm{N}([\mathrm{H}])[ \\
\mathrm{C} @]([\mathrm{H}])(\mathrm{C}(=\mathrm{O}) \mathrm{N}([\mathrm{H}]) \mathrm{C}([\mathrm{H}])([\mathrm{H}]) \mathrm{C} 1=\mathrm{C}([\mathrm{H}]) \mathrm{C}([\mathrm{H}])=\mathrm{C}([\mathrm{H}]) \mathrm{C}([\mathrm{H}])=\mathrm{C} 1[\mathrm{H}]) \mathrm{C}([\mathrm{H}])( \\
[\mathrm{H}])[\mathrm{H}]) \mathrm{C}([\mathrm{H}])([\mathrm{H}])[\mathrm{H}]) \mathrm{C}([\mathrm{H}])([\mathrm{H}]) \mathrm{C}([\mathrm{H}])(\mathrm{C}([\mathrm{H}])([\mathrm{H}])[\mathrm{H}]) \mathrm{C}([\mathrm{H}])([\mathrm{H}])[\mathrm{H}]) \mathrm{C}([\mathrm{H}])([ \\
\mathrm{H}]) \mathrm{C}(=\mathrm{O}) \mathrm{N}([\mathrm{H}])[\mathrm{H}]\end{array}$ & 0.649 & 2.437 \\
\hline Ts13 & $\begin{array}{l}\mathrm{O}=\mathrm{C}(\mathrm{OC}(\mathrm{C}([\mathrm{H}])([\mathrm{H}])[\mathrm{H}])(\mathrm{C}([\mathrm{H}])([\mathrm{H}])[\mathrm{H}]) \mathrm{C}([\mathrm{H}])([\mathrm{H}])[\mathrm{H}]) \mathrm{N}([\mathrm{H}])[\mathrm{C} @]([\mathrm{H}])(\mathrm{C}(=\mathrm{O}) \\
\mathrm{C}([\mathrm{H}])([\mathrm{H}]) \mathrm{N}([\mathrm{H}])[\mathrm{C} @]([\mathrm{H}])([\mathrm{C} @]([\mathrm{H}])(\mathrm{O}[\mathrm{H}]) \mathrm{C}([\mathrm{H}])([\mathrm{H}])[\mathrm{C} @]([\mathrm{H}])(\mathrm{C}(=\mathrm{O}) \mathrm{N}([\mathrm{H}])[ \\
\mathrm{C} @([\mathrm{H}])(\mathrm{C}(=\mathrm{O}) \mathrm{N}([\mathrm{H}]) \mathrm{C}([\mathrm{H}])([\mathrm{H}]) \mathrm{C} 1=\mathrm{C}([\mathrm{H}]) \mathrm{C}([\mathrm{H}])=\mathrm{C}([\mathrm{H}]) \mathrm{C}([\mathrm{H}])=\mathrm{C} 1[\mathrm{H}]) \mathrm{C}([\mathrm{H}])( \\
\mathrm{C}([\mathrm{H}])([\mathrm{H}])[\mathrm{H}]) \mathrm{C}([\mathrm{H}])([\mathrm{H}])[\mathrm{H}]) \mathrm{C}([\mathrm{H}])([\mathrm{H}])[\mathrm{H}]) \mathrm{C}([\mathrm{H}])([\mathrm{H}]) \mathrm{C}([\mathrm{H}])(\mathrm{C}([\mathrm{H}])([\mathrm{H}])[\mathrm{H}]) \\
\mathrm{C}([\mathrm{H}])([\mathrm{H}])[\mathrm{H}]) \mathrm{C}([\mathrm{H}])([\mathrm{H}]) \mathrm{C}(=\mathrm{O}) \mathrm{N}([\mathrm{H}])[\mathrm{H}]\end{array}$ & 1.504 & 2.485 \\
\hline Ts14 & 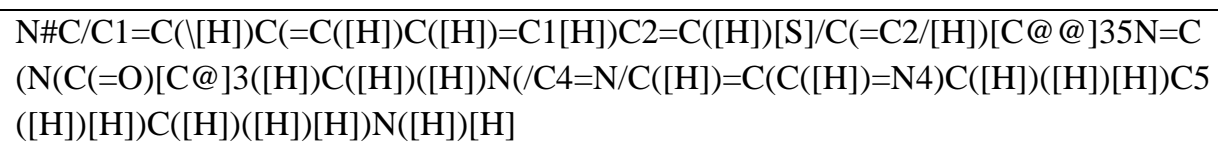 & 4.046 & 3.352 \\
\hline Ts15 & $\begin{array}{l}\mathrm{O}=[\mathrm{S}](=\mathrm{O})(\mathrm{N}(\mathrm{C}=1 \mathrm{C}([\mathrm{H}])=\mathrm{C}(\mathrm{C}([\mathrm{H}])=\mathrm{C}(\mathrm{C}=1[\mathrm{H}]) \mathrm{C}(=\mathrm{O}) \mathrm{N}([\mathrm{H}])[\mathrm{C} @]([\mathrm{H}])(\mathrm{C}([\mathrm{H}])([\mathrm{H}]) \\
\mathrm{N}([\mathrm{H}])[\mathrm{C} @]([\mathrm{H}])(\mathrm{C}(=\mathrm{O}) \mathrm{N}([\mathrm{H}]) \mathrm{C}([\mathrm{H}])([\mathrm{H}]) \mathrm{C}([\mathrm{H}])(\mathrm{C}([\mathrm{H}])([\mathrm{H}])[\mathrm{H}]) \mathrm{C}([\mathrm{H}])([\mathrm{H}])[\mathrm{H}])[ \\
\mathrm{C} @ @]([\mathrm{H}])(\mathrm{OC}([\mathrm{H}])([\mathrm{H}])[\mathrm{H}]) \mathrm{C}([\mathrm{H}])([\mathrm{H}])[\mathrm{H}]) \mathrm{C}([\mathrm{H}])([\mathrm{H}]) \mathrm{C} 2=\mathrm{C}([\mathrm{H}]) \mathrm{C}([\mathrm{H}])=\mathrm{C}([\mathrm{H}]) \\
\mathrm{C}([\mathrm{H}])=\mathrm{C} 2[\mathrm{H}]) \mathrm{C}(=\mathrm{O}) \mathrm{N}([\mathrm{H}])[\mathrm{C} @ @]([\mathrm{H}])(\mathrm{C} 3=\mathrm{C}([\mathrm{H}]) \mathrm{C}([\mathrm{H}])=\mathrm{C}([\mathrm{H}]) \mathrm{C}([\mathrm{H}])=\mathrm{C} 3[\mathrm{H}]) \mathrm{C} \\
([\mathrm{H}])([\mathrm{H}])[\mathrm{H}]) \mathrm{C}([\mathrm{H}])([\mathrm{H}])[\mathrm{H}]) \mathrm{C}([\mathrm{H}])([\mathrm{H}])[\mathrm{H}]\end{array}$ & 3.602 & 2.192 \\
\hline Ts16 & $\begin{array}{l}\mathrm{O}=\mathrm{C}(\mathrm{N}([\mathrm{H}])[\mathrm{C} @]([\mathrm{H}])([\mathrm{C} @ @]([\mathrm{H}])(\mathrm{O}[\mathrm{H}]) \mathrm{C}([\mathrm{H}])([\mathrm{H}])[\mathrm{C} @]([\mathrm{H}])(\mathrm{C}(=\mathrm{O}) \mathrm{N}([\mathrm{H}]) \mathrm{C}([ \\
\mathrm{H}])([\mathrm{H}]) \mathrm{C}([\mathrm{H}])(\mathrm{C}([\mathrm{H}])([\mathrm{H}])[\mathrm{H}]) \mathrm{C}([\mathrm{H}])([\mathrm{H}])[\mathrm{H}]) \mathrm{C}([\mathrm{H}])([\mathrm{H}]) \mathrm{C}([\mathrm{H}])([\mathrm{H}]) \mathrm{O}[\mathrm{H}]) \mathrm{C}([\mathrm{H}]) \\
([\mathrm{H}]) \mathrm{C} 1=\mathrm{C}([\mathrm{H}]) \mathrm{C}([\mathrm{H}])=\mathrm{C}([\mathrm{H}]) \mathrm{C}([\mathrm{H}])=\mathrm{C} 1[\mathrm{H}]) \mathrm{C}([\mathrm{H}])([\mathrm{H}])[\mathrm{C} @]([\mathrm{H}])(\mathrm{N}([\mathrm{H}]) \mathrm{C}(=\mathrm{O}) \mathrm{C} \\
([\mathrm{H}])([\mathrm{H}]) \mathrm{C}([\mathrm{H}])(\mathrm{C}([\mathrm{H}])([\mathrm{H}])[\mathrm{H}]) \mathrm{C}([\mathrm{H}])([\mathrm{H}])[\mathrm{H}]) \mathrm{C}(=\mathrm{O}) \mathrm{O}[\mathrm{H}]\end{array}$ & 1.721 & 1.884 \\
\hline Ts17 & $\begin{array}{l}\mathrm{O}=\mathrm{C}(\mathrm{N}([\mathrm{H}])[\mathrm{C} @ @](([\mathrm{H}])(\mathrm{Cl}=\mathrm{C}([\mathrm{H}]) \mathrm{C}([\mathrm{H}])=\mathrm{C}([\mathrm{H}]) \mathrm{C}([\mathrm{H}])=\mathrm{C} 1[\mathrm{H}]) \mathrm{C}([\mathrm{H}])([\mathrm{H}])[\mathrm{H}]) \mathrm{C} \\
2=\mathrm{C}([\mathrm{H}]) \mathrm{C}(=\mathrm{C}([\mathrm{H}]) \mathrm{C}(=\mathrm{C} 2[\mathrm{H}]) \mathrm{N}(\mathrm{C}([\mathrm{H}])([\mathrm{H}])[\mathrm{H}])[\mathrm{S}](=\mathrm{O})(=\mathrm{O}) \mathrm{C}([\mathrm{H}])([\mathrm{H}])[\mathrm{H}]) \mathrm{C}(=\mathrm{O}) \\
\mathrm{N}([\mathrm{H}])[\mathrm{C} @]([\mathrm{H}])([\mathrm{C} @]([\mathrm{H}])(\mathrm{O}[\mathrm{H}]) \mathrm{C}([\mathrm{H}])([\mathrm{H}]) \mathrm{N}([\mathrm{H}]) \mathrm{C}([\mathrm{H}])([\mathrm{H}]) \mathrm{C} 3=\mathrm{C}([\mathrm{H}]) \mathrm{C} 4=\mathrm{C}( \\
\mathrm{C}([\mathrm{H}])=\mathrm{C} 3[\mathrm{H}]) \mathrm{C}([\mathrm{H}])=\mathrm{C}([\mathrm{H}]) \mathrm{N} 4[\mathrm{H}]) \mathrm{C}([\mathrm{H}])([\mathrm{H}]) \mathrm{C} 5=\mathrm{C}([\mathrm{H}]) \mathrm{C}([\mathrm{H}])=\mathrm{C}([\mathrm{H}]) \mathrm{C}([\mathrm{H}])=\mathrm{C} \\
5[\mathrm{H}]\end{array}$ & 2.638 & 2.171 \\
\hline Ts18 & $\begin{array}{l}\mathrm{O}=\mathrm{C}(\mathrm{N}([\mathrm{H}])[\mathrm{C} @]([\mathrm{H}])(\mathrm{C}=1 \mathrm{~N}=\mathrm{C}(\mathrm{OC}=1 \mathrm{C}([\mathrm{H}])([\mathrm{H}])[\mathrm{H}]) \mathrm{C}([\mathrm{H}])([\mathrm{H}])[\mathrm{H}]) \mathrm{C}([\mathrm{H}])([\mathrm{H}])[ \\
\mathrm{H}]) \mathrm{C}=2 \mathrm{C}([\mathrm{H}])=\mathrm{C}(\mathrm{C}([\mathrm{H}])=\mathrm{C}(\mathrm{C}=2[\mathrm{H}]) \mathrm{N}(\mathrm{C}([\mathrm{H}])([\mathrm{H}])[\mathrm{H}])[\mathrm{S}](=\mathrm{O})(=\mathrm{O}) \mathrm{C}([\mathrm{H}])([\mathrm{H}])[\mathrm{H}]) \\
\mathrm{C}(=\mathrm{O}) \mathrm{N}([\mathrm{H}])[\mathrm{C} @([[\mathrm{H}])([\mathrm{C} @([\mathrm{H}])(\mathrm{O}[\mathrm{H}]) \mathrm{C}([\mathrm{H}])([\mathrm{H}]) \mathrm{N}([\mathrm{H}]) \mathrm{C}([\mathrm{H}])([\mathrm{H}]) \mathrm{C} 3=\mathrm{C}([\mathrm{H}]) \\
\mathrm{C}(\mathrm{OC}([\mathrm{H}])([\mathrm{H}])[\mathrm{H}])=\mathrm{C}([\mathrm{H}]) \mathrm{C}([\mathrm{H}])=\mathrm{C} 3[\mathrm{H}]) \mathrm{C}([\mathrm{H}])([\mathrm{H}]) \mathrm{C} 4=\mathrm{C}([\mathrm{H}]) \mathrm{C}([\mathrm{H}])=\mathrm{C}([\mathrm{H}]) \mathrm{C}([ \\
\mathrm{H}])=\mathrm{C} 4[\mathrm{H}]\end{array}$ & 2.049 & 3.481 \\
\hline Ts19 & $\mathrm{O}=\mathrm{C} 2 \mathrm{~N}([\mathrm{H}]) \mathrm{C} 1=\mathrm{C}([\mathrm{H}]) \mathrm{C}([\mathrm{H}])=\mathrm{C}([\mathrm{H}]) \mathrm{C}([\mathrm{H}])=\mathrm{C} 1 \mathrm{~N}([\mathrm{H}]) \mathrm{C} 3=\mathrm{C} 2 \mathrm{C}([\mathrm{H}])=\mathrm{C}([\mathrm{H}]) \mathrm{C}(=\mathrm{C}$ & 2.458 & 2.897 \\
\hline
\end{tabular}




\begin{tabular}{|c|c|c|c|}
\hline & $3[\mathrm{H}]) \mathrm{C}(=\mathrm{O}) \mathrm{N}([\mathrm{H}]) \mathrm{C} 4=\mathrm{N} / \mathrm{C}(=\mathrm{C}(/[\mathrm{H}]) \mathrm{N} 4[\mathrm{H}]) \mathrm{C} 5=\mathrm{C}([\mathrm{H}]) \mathrm{C}([\mathrm{H}])=\mathrm{NC}([\mathrm{H}])=\mathrm{C} 5[\mathrm{H}]$ & & \\
\hline Ts20 & $\begin{array}{l}\mathrm{O}=\mathrm{C} 2 \mathrm{~N}([\mathrm{H}]) \mathrm{C} 1=\mathrm{C}([\mathrm{H}]) \mathrm{C}([\mathrm{H}])=\mathrm{C}([\mathrm{H}]) \mathrm{C}([\mathrm{H}])=\mathrm{C} 1 \mathrm{~N}([\mathrm{H}]) \mathrm{C} 3=\mathrm{C} 2 \mathrm{C}([\mathrm{H}])=\mathrm{C}([\mathrm{H}]) \mathrm{C}(=\mathrm{C} \\
3[\mathrm{H}]) \mathrm{C}(=\mathrm{O}) \mathrm{N}([\mathrm{H}]) \mathrm{C} 4=\mathrm{C}([\mathrm{H}]) \mathrm{C}([\mathrm{H}])=\mathrm{C}([\mathrm{F}]) \mathrm{C}([\mathrm{H}])=\mathrm{C} 4[\mathrm{H}]\end{array}$ & 2.676 & 1.392 \\
\hline Ts21 & $\begin{array}{l}\mathrm{O}=\mathrm{C} 2 \mathrm{~N}([\mathrm{H}]) \mathrm{C} 1=\mathrm{C}([\mathrm{H}]) \mathrm{C}([\mathrm{H}])=\mathrm{C}([\mathrm{H}]) \mathrm{C}([\mathrm{H}])=\mathrm{C} 1 \mathrm{~N}([\mathrm{H}]) \mathrm{C}=3 / \mathrm{C} 2=\mathrm{C}(/[\mathrm{H}]) \mathrm{C}([\mathrm{H}])=\mathrm{C}( \\
\mathrm{C}=3[\mathrm{H}]) \mathrm{C}(=\mathrm{O}) \mathrm{N}([\mathrm{H}]) \mathrm{C} 4=\mathrm{NC}=5 \mathrm{C}(\mathrm{N} 4[\mathrm{H}])=\mathrm{C}([\mathrm{H}]) \mathrm{N}=\mathrm{C}([\mathrm{H}]) \mathrm{C}=5[\mathrm{H}]\end{array}$ & 2.510 & 1.849 \\
\hline Ts22 & $\begin{array}{l}\mathrm{O}=\mathrm{C}(\mathrm{N}([\mathrm{H}]) \mathrm{C}(=\mathrm{NC}([\mathrm{H}])([\mathrm{H}]) \mathrm{C} 1=\mathrm{C}([\mathrm{H}]) \mathrm{C}(=\mathrm{C}([\mathrm{H}]) \mathrm{C}(=\mathrm{C} 1[\mathrm{H}]) \mathrm{C}([\mathrm{H}])([\mathrm{H}])[\mathrm{H}]) \mathrm{C}([\mathrm{H}] \\
)([\mathrm{H}])[\mathrm{H}]) \mathrm{N}([\mathrm{H}])[\mathrm{H}]) \mathrm{C}=2 \mathrm{C}(=\mathrm{NOC}=2 \mathrm{C}([\mathrm{H}])([\mathrm{H}])[\mathrm{H}]) \mathrm{C} 3=\mathrm{C}([\mathrm{H}]) \mathrm{C}([\mathrm{H}])=\mathrm{C}(\mathrm{OC}([\mathrm{H}])([ \\
\mathrm{H}])[\mathrm{H}]) \mathrm{C}([\mathrm{H}])=\mathrm{C} 3[\mathrm{H}]\end{array}$ & 2.553 & 1.826 \\
\hline Ts23 & $\begin{array}{l}\mathrm{O}=\mathrm{C}(\mathrm{N}([\mathrm{H}]) \mathrm{C}(=\mathrm{NC}([\mathrm{H}])([\mathrm{H}]) \mathrm{C} 1=\mathrm{C}([\mathrm{H}]) \mathrm{C}([\mathrm{Cl}])=\mathrm{C}([\mathrm{H}]) \mathrm{C}([\mathrm{Cl}])=\mathrm{C} 1[\mathrm{H}]) \mathrm{N}([\mathrm{H}])[\mathrm{H}]) \mathrm{C} \\
=2 \mathrm{C}(=\mathrm{N}[\mathrm{S}] \mathrm{C}=2 \mathrm{C}([\mathrm{H}])([\mathrm{H}])[\mathrm{H}]) \mathrm{C} 3=\mathrm{C}([\mathrm{H}]) \mathrm{C}([\mathrm{H}])=\mathrm{C}(\mathrm{OC}([\mathrm{H}])([\mathrm{H}])[\mathrm{H}]) \mathrm{C}([\mathrm{H}])=\mathrm{C} 3[\mathrm{H} \\
]\end{array}$ & 2.538 & 2.559 \\
\hline Ts24 & $\begin{array}{l}\mathrm{O}=\mathrm{C}(\mathrm{N}([\mathrm{H}]) \mathrm{C}(=\mathrm{NC}([\mathrm{H}])([\mathrm{H}]) \mathrm{C}=1 \mathrm{C}([\mathrm{H}])=\mathrm{C}([\mathrm{H}]) \mathrm{C}([\mathrm{H}])=\mathrm{C} 2 \mathrm{C}=1 \mathrm{C}([\mathrm{H}])=\mathrm{C}([\mathrm{H}]) \mathrm{C}([\mathrm{H}] \\
)=\mathrm{C} 2[\mathrm{H}]) \mathrm{N}([\mathrm{H}])[\mathrm{H}]) \mathrm{C}=3 \mathrm{C}(=\mathrm{NOC}=3 \mathrm{C}([\mathrm{H}])([\mathrm{H}])[\mathrm{H}]) \mathrm{C} 4=\mathrm{C}([\mathrm{H}]) \mathrm{C}([\mathrm{H}])=\mathrm{C}([\mathrm{H}]) \mathrm{C}([\mathrm{H}]) \\
=\mathrm{C} 4[\mathrm{H}]\end{array}$ & 1.553 & 1.122 \\
\hline Ts25 & $\begin{array}{l}{[\mathrm{H}] \mathrm{C}=5 \mathrm{C}(\mathrm{O}[\mathrm{H}])=\mathrm{C}(\mathrm{O} / \mathrm{C} 2=\mathrm{C}(\backslash[\mathrm{H}]) \mathrm{C}(\mathrm{O}[\mathrm{H}])=\mathrm{C} 1 \mathrm{OC} 3=\mathrm{C}(\mathrm{O} / \mathrm{C} 1=\mathrm{C} 2 \backslash[\mathrm{H}]) \mathrm{C}(\mathrm{O}[\mathrm{H}])=\mathrm{C}([\mathrm{H}} \\
]) \mathrm{C}(\mathrm{O}[\mathrm{H}])=\mathrm{C} 3 \mathrm{O} / \mathrm{C} 4=\mathrm{C}(\backslash[\mathrm{H}]) \mathrm{C}(\mathrm{O}[\mathrm{H}])=\mathrm{C}([\mathrm{H}]) \mathrm{C}(\mathrm{O}[\mathrm{H}])=\mathrm{C} 4[\mathrm{H}]) \mathrm{C}(\mathrm{O}[\mathrm{H}])=\mathrm{C}([\mathrm{H}]) \mathrm{C}=5 \mathrm{O} \\
{[\mathrm{H}]}\end{array}$ & 1.143 & 1.019 \\
\hline Ts26 & $\begin{array}{l}{[\mathrm{F}] \mathrm{C}=1 \mathrm{C}([\mathrm{H}])=\mathrm{C}(\mathrm{C}([\mathrm{H}])=\mathrm{C}([\mathrm{F}]) \mathrm{C}=1[\mathrm{H}]) \mathrm{C}([\mathrm{H}])([\mathrm{H}])[\mathrm{C} @]([\mathrm{H}])(\mathrm{N}([\mathrm{H}]) \mathrm{C}(=\mathrm{O}) \mathrm{C}=2 \mathrm{C}([} \\
\mathrm{H}])=\mathrm{C}(\mathrm{C}([\mathrm{H}])=\mathrm{C}(\mathrm{C}=2[\mathrm{H}]) \mathrm{C}([\mathrm{H}])([\mathrm{H}])[\mathrm{H}]) \mathrm{C}(=\mathrm{O}) \mathrm{N}(\mathrm{C}([\mathrm{H}])([\mathrm{H}]) \mathrm{C}([\mathrm{H}])([\mathrm{H}]) \mathrm{C}([\mathrm{H}])([ \\
\mathrm{H}])[\mathrm{H}]) \mathrm{C}([\mathrm{H}])([\mathrm{H}]) \mathrm{C}([\mathrm{H}])([\mathrm{H}]) \mathrm{C}([\mathrm{H}])([\mathrm{H}])[\mathrm{H}])[\mathrm{C} @]([\mathrm{H}])(\mathrm{O}[\mathrm{H}])[\mathrm{C} @] 3([\mathrm{H}]) \mathrm{N}([\mathrm{H}]) \\
\mathrm{C}([\mathrm{H}])([\mathrm{H}]) \mathrm{C}([\mathrm{H}])([\mathrm{H}]) \mathrm{N}(\mathrm{C} 3([\mathrm{H}])[\mathrm{H}])[\mathrm{S}](=\mathrm{O})(=\mathrm{O}) \mathrm{C} 4=\mathrm{C}([\mathrm{H}]) \mathrm{C}([\mathrm{H}])=\mathrm{C}([\mathrm{H}]) \mathrm{C}([\mathrm{H}])= \\
\mathrm{C} 4[\mathrm{H}]\end{array}$ & 4.523 & 3.732 \\
\hline Ts27 & $\begin{array}{l}{[\mathrm{F}] \mathrm{C}=1 \mathrm{C}([\mathrm{H}])=\mathrm{C}(\mathrm{C}([\mathrm{H}])=\mathrm{C}([\mathrm{F}]) \mathrm{C}=1[\mathrm{H}]) \mathrm{C}([\mathrm{H}])([\mathrm{H}])[\mathrm{C} @]([\mathrm{H}])(\mathrm{N}([\mathrm{H}]) \mathrm{C}(=\mathrm{O}) \mathrm{C} 2=\mathrm{C}([} \\
\mathrm{H}]) \mathrm{C}(=\mathrm{C}([\mathrm{H}]) \mathrm{C}(=\mathrm{C} 2[\mathrm{H}]) \mathrm{C}(=\mathrm{O}) \mathrm{N} 3[\mathrm{C} @]([\mathrm{H}])(\mathrm{C}([\mathrm{H}])([\mathrm{H}]) \mathrm{C}([\mathrm{H}])([\mathrm{H}]) \mathrm{C} 3([\mathrm{H}])[\mathrm{H}]) \mathrm{C}([ \\
\mathrm{H}])([\mathrm{H}]) \mathrm{OC}([\mathrm{H}])([\mathrm{H}])[\mathrm{H}]) \mathrm{C}([\mathrm{H}])([\mathrm{H}])[\mathrm{H}])[\mathrm{C} @]([\mathrm{H}])(\mathrm{O}[\mathrm{H}])[\mathrm{C} @] 4([\mathrm{H}]) \mathrm{N}([\mathrm{H}]) \mathrm{C}([\mathrm{H}] \\
)([\mathrm{H}]) \mathrm{C}([\mathrm{H}])([\mathrm{H}]) \mathrm{N}(\mathrm{C} 4([\mathrm{H}])[\mathrm{H}])[\mathrm{S}](=\mathrm{O})(=\mathrm{O}) \mathrm{C}([\mathrm{H}])([\mathrm{H}])[\mathrm{H}]\end{array}$ & 3.886 & 3.934 \\
\hline Ts 28 & $\begin{array}{l}{[\mathrm{F}] \mathrm{C}=1 \mathrm{C}([\mathrm{H}])=\mathrm{C}(\mathrm{C}([\mathrm{H}])=\mathrm{C}([\mathrm{F}]) \mathrm{C}=1[\mathrm{H}]) \mathrm{C}([\mathrm{H}])([\mathrm{H}])[\mathrm{C} @]([\mathrm{H}])(\mathrm{N}([\mathrm{H}]) \mathrm{C}(=\mathrm{O}) \mathrm{C} 2=\mathrm{C}([} \\
\mathrm{H}]) \mathrm{C}(=\mathrm{C}([\mathrm{H}]) \mathrm{C}(=\mathrm{C} 2[\mathrm{H}]) \mathrm{C}(=\mathrm{O}) \mathrm{N} 3[\mathrm{C} @]([\mathrm{H}])(\mathrm{C}([\mathrm{H}])([\mathrm{H}]) \mathrm{C}([\mathrm{H}])([\mathrm{H}]) \mathrm{C} 3([\mathrm{H}])[\mathrm{H}]) \mathrm{C}([ \\
\mathrm{H}])([\mathrm{H}]) \mathrm{OC}([\mathrm{H}])([\mathrm{H}])[\mathrm{H}]) \mathrm{C}([\mathrm{H}])([\mathrm{H}])[\mathrm{H}])[\mathrm{C} @]([\mathrm{H}])(\mathrm{O}[\mathrm{H}])[\mathrm{C} @] 4([\mathrm{H}]) \mathrm{N}([\mathrm{H}]) \mathrm{C}([\mathrm{H}] \\
)([\mathrm{H}]) \mathrm{C}([\mathrm{H}])([\mathrm{H}]) \mathrm{N}(\mathrm{C} 4([\mathrm{H}])[\mathrm{H}])[\mathrm{S}](=\mathrm{O})(=\mathrm{O}) \mathrm{C}([\mathrm{H}])([\mathrm{H}]) \mathrm{C} 5=\mathrm{C}([\mathrm{H}]) \mathrm{C}([\mathrm{H}])=\mathrm{C}([\mathrm{H}]) \mathrm{C} \\
([\mathrm{H}])=\mathrm{C} 5[\mathrm{H}]\end{array}$ & 5.000 & 3.962 \\
\hline Ts29 & $\begin{array}{l}\mathrm{O}=\mathrm{C}(\mathrm{N}([\mathrm{H}])[\mathrm{C} @ @]([\mathrm{H}])(\mathrm{Cl}=\mathrm{C}([\mathrm{H}]) \mathrm{C}([\mathrm{H}])=\mathrm{C}([\mathrm{H}]) \mathrm{C}([\mathrm{H}])=\mathrm{C} 1[\mathrm{H}]) \mathrm{C}([\mathrm{H}])([\mathrm{H}])[\mathrm{H}]) \mathrm{C} \\
2=\mathrm{C}([\mathrm{H}]) \mathrm{C}(=\mathrm{C}([\mathrm{H}]) \mathrm{C}(=\mathrm{C} 2[\mathrm{H}]) \mathrm{N}(\mathrm{C}([\mathrm{H}])([\mathrm{H}])[\mathrm{H}])[\mathrm{S}](=\mathrm{O})(=\mathrm{O}) \mathrm{C}([\mathrm{H}])([\mathrm{H}])[\mathrm{H}]) \mathrm{C}(=\mathrm{O}) \\
\mathrm{N}([\mathrm{H}])[\mathrm{C} @]([\mathrm{H}])([\mathrm{C} @ @]([\mathrm{H}])(\mathrm{N}=[\mathrm{N}+]=[\mathrm{N}- \\
]) \mathrm{C}([\mathrm{H}])([\mathrm{H}])[\mathrm{C} @ @]([\mathrm{H}])(\mathrm{OC}([\mathrm{H}])([\mathrm{H}])[\mathrm{H}]) \mathrm{C}(=\mathrm{O}) \mathrm{N}([\mathrm{H}])[\mathrm{C} @([\mathrm{H}])(\mathrm{C}(=\mathrm{O}) \mathrm{N}([\mathrm{H}]) \mathrm{C} \\
([\mathrm{H}])([\mathrm{H}]) \mathrm{C} 3=\mathrm{C}([\mathrm{H}]) \mathrm{C}([\mathrm{H}])=\mathrm{C}([\mathrm{H}]) \mathrm{C}([\mathrm{H}])=\mathrm{C} 3[\mathrm{H}]) \mathrm{C}([\mathrm{H}])(\mathrm{C}([\mathrm{H}])([\mathrm{H}])[\mathrm{H}]) \mathrm{C}([\mathrm{H}])([\mathrm{H} \\
])[\mathrm{H}]) \mathrm{C}([\mathrm{H}])([\mathrm{H}]) \mathrm{O} / \mathrm{C} 4=\mathrm{C}([\mathrm{H}]) \mathrm{C}([\mathrm{F}])=\mathrm{C}([\mathrm{H}]) \mathrm{C}([\mathrm{F}])=\mathrm{C} 4[\mathrm{H}]\end{array}$ & 3.155 & 3.807 \\
\hline Ts 30 & $\begin{array}{l}\mathrm{O}=\mathrm{C}(\mathrm{N}([\mathrm{H}])[\mathrm{C} @ @](([\mathrm{H}])(\mathrm{Cl}=\mathrm{C}([\mathrm{H}]) \mathrm{C}([\mathrm{H}])=\mathrm{C}([\mathrm{H}]) \mathrm{C}([\mathrm{H}])=\mathrm{C} 1[\mathrm{H}]) \mathrm{C}([\mathrm{H}])([\mathrm{H}])[\mathrm{H}]) \mathrm{C} \\
2=\mathrm{C}([\mathrm{H}]) \mathrm{C}(=\mathrm{C}([\mathrm{H}]) \mathrm{C}(=\mathrm{C} 2[\mathrm{H}]) \mathrm{N}(\mathrm{C}([\mathrm{H}])([\mathrm{H}])[\mathrm{H}])[\mathrm{S}](=\mathrm{O})(=\mathrm{O}) \mathrm{C}([\mathrm{H}])([\mathrm{H}])[\mathrm{H}]) \mathrm{C}(=\mathrm{O}) \\
\mathrm{N}([\mathrm{H}])[\mathrm{C} @]([\mathrm{H}])([\mathrm{C} @ @]([\mathrm{H}])(\mathrm{O}[\mathrm{H}]) \mathrm{C}([\mathrm{H}])([\mathrm{H}])[\mathrm{C} @ @]([\mathrm{H}])(\mathrm{OC}([\mathrm{H}])([\mathrm{H}]) \mathrm{C}([\mathrm{H}])( \\
[\mathrm{H}])[\mathrm{H}]) \mathrm{C}(=\mathrm{O}) \mathrm{N}([\mathrm{H}])[\mathrm{C} @]([\mathrm{H}])(\mathrm{C}(=\mathrm{O}) \mathrm{N}([\mathrm{H}]) \mathrm{C}([\mathrm{H}])([\mathrm{H}]) \mathrm{C} 3=\mathrm{C}([\mathrm{H}]) \mathrm{C}([\mathrm{H}])=\mathrm{C}([\mathrm{H}]) \\
\mathrm{C}([\mathrm{H}])=\mathrm{C} 3[\mathrm{H}]) \mathrm{C}([\mathrm{H}])(\mathrm{C}([\mathrm{H}])([\mathrm{H}])[\mathrm{H}]) \mathrm{C}([\mathrm{H}])([\mathrm{H}])[\mathrm{H}]) \mathrm{C}([\mathrm{H}])([\mathrm{H}]) \mathrm{O} / \mathrm{C} 4=\mathrm{C}(\backslash \mathrm{H}]) \mathrm{C}([ \\
\mathrm{F}])=\mathrm{C}([\mathrm{H}]) \mathrm{C}([\mathrm{F}])=\mathrm{C} 4[\mathrm{H}]\end{array}$ & 4.678 & 3.400 \\
\hline
\end{tabular}




\begin{tabular}{|c|c|c|c|}
\hline Ts31 & $\begin{array}{l}\mathrm{O}=\mathrm{C}(\mathrm{N}([\mathrm{H}])[\mathrm{C} @ @]([\mathrm{H}])(\mathrm{C} 1=\mathrm{C}([\mathrm{H}]) \mathrm{C}([\mathrm{H}])=\mathrm{C}([\mathrm{H}]) \mathrm{C}([\mathrm{H}])=\mathrm{C} 1[\mathrm{H}]) \mathrm{C}([\mathrm{H}])([\mathrm{H}])[\mathrm{H}]) \mathrm{C} \\
=2 \mathrm{C}([\mathrm{H}])=\mathrm{C}(\mathrm{C}([\mathrm{H}])=\mathrm{C}(\mathrm{C}=2[\mathrm{H}]) \mathrm{N}(\mathrm{C}([\mathrm{H}])([\mathrm{H}])[\mathrm{H}])[\mathrm{S}](=\mathrm{O})(=\mathrm{O}) \mathrm{C}([\mathrm{H}])([\mathrm{H}])[\mathrm{H}]) \mathrm{C}(= \\
\mathrm{O}) \mathrm{N}([\mathrm{H}])[\mathrm{C} @([\mathrm{H}])([\mathrm{C} @ @]([\mathrm{H}])(\mathrm{O}[\mathrm{H}]) \mathrm{C}([\mathrm{H}])([\mathrm{H}])[\mathrm{C} @ @]([\mathrm{H}])(\mathrm{OC}([\mathrm{H}])([\mathrm{H}])[\mathrm{C} @ \\
@] 3([\mathrm{H}]) \mathrm{C}([\mathrm{H}])([\mathrm{H}]) \mathrm{C} 3([\mathrm{H}])[\mathrm{H}]) \mathrm{C}(=\mathrm{O}) \mathrm{N}([\mathrm{H}])[\mathrm{C} @]([\mathrm{H}])(\mathrm{C}(=\mathrm{O}) \mathrm{N}([\mathrm{H}]) \mathrm{C}([\mathrm{H}])([\mathrm{H}]) \mathrm{C} \\
4=\mathrm{C}([\mathrm{H}]) \mathrm{C}([\mathrm{H}])=\mathrm{C}([\mathrm{H}]) \mathrm{C}([\mathrm{H}])=\mathrm{C} 4[\mathrm{H}]) \mathrm{C}([\mathrm{H}])(\mathrm{C}([\mathrm{H}])([\mathrm{H}])[\mathrm{H}]) \mathrm{C}([\mathrm{H}])([\mathrm{H}])[\mathrm{H}]) \mathrm{C}([\mathrm{H}] \\
)([\mathrm{H}]) \mathrm{O} / \mathrm{C} 5=\mathrm{C}(([\mathrm{H}]) \mathrm{C}([\mathrm{F}])=\mathrm{C}([\mathrm{H}]) \mathrm{C}([\mathrm{F}])=\mathrm{C} 5[\mathrm{H}]\end{array}$ & 4.886 & 5.030 \\
\hline Ts32 & $\begin{array}{l}\mathrm{O}=\mathrm{C} 1 \mathrm{C} 4=\mathrm{C}(\mathrm{O} / \mathrm{C}(=\mathrm{C} 1 / \mathrm{C}([\mathrm{H}])([\mathrm{H}]) \mathrm{C}([\mathrm{H}])=\mathrm{C}(\mathrm{C}([\mathrm{H}])([\mathrm{H}])[\mathrm{H}]) \mathrm{C}([\mathrm{H}])([\mathrm{H}])[\mathrm{H}]) \mathrm{C}=2 \mathrm{C}([ \\
\mathrm{H}])=\mathrm{C}([\mathrm{H}]) \mathrm{C}(\mathrm{O}[\mathrm{H}])=\mathrm{C}([\mathrm{H}]) \mathrm{C}=2 \mathrm{O}[\mathrm{H}]) \mathrm{C} 3=\mathrm{C}(\mathrm{OC}(\mathrm{C}([\mathrm{H}])=\mathrm{C} 3[\mathrm{H}])(\mathrm{C}([\mathrm{H}])([\mathrm{H}])[\mathrm{H}]) \mathrm{C}([ \\
\mathrm{H}])([\mathrm{H}])[\mathrm{H}]) \mathrm{C}([\mathrm{H}])=\mathrm{C} 4 \mathrm{O}[\mathrm{H}]\end{array}$ & 0.193 & 1.433 \\
\hline Ts33 & $\begin{array}{l}\mathrm{O}=\mathrm{C}(\mathrm{OC}(\mathrm{C}([\mathrm{H}])([\mathrm{H}])[\mathrm{H}])(\mathrm{C}([\mathrm{H}])([\mathrm{H}])[\mathrm{H}]) \mathrm{C}([\mathrm{H}])([\mathrm{H}])[\mathrm{H}]) \mathrm{N}([\mathrm{H}])[\mathrm{C} @]([\mathrm{H}])(\mathrm{C}(=\mathrm{O}) \\
\mathrm{N}([\mathrm{H}])[\mathrm{C} @]([\mathrm{H}])([\mathrm{C} @]([\mathrm{H}])(\mathrm{O}[\mathrm{H}]) \mathrm{C}([\mathrm{H}])([\mathrm{H}])[\mathrm{C} @]([\mathrm{H}])(\mathrm{C}(=\mathrm{O}) \mathrm{N}([\mathrm{H}])[\mathrm{C} @]([\mathrm{H}])( \\
\mathrm{C}(=\mathrm{O}) \mathrm{N}([\mathrm{H}]) \mathrm{C}([\mathrm{H}])([\mathrm{H}]) \mathrm{C} 1=\mathrm{C}([\mathrm{H}]) \mathrm{C}([\mathrm{H}])=\mathrm{C}([\mathrm{H}]) \mathrm{C}([\mathrm{H}])=\mathrm{C} 1[\mathrm{H}]) \mathrm{C}([\mathrm{H}])(\mathrm{C}([\mathrm{H}])([\mathrm{H}] \\
)[\mathrm{H}]) \mathrm{C}([\mathrm{H}])([\mathrm{H}])[\mathrm{H}]) \mathrm{C}([\mathrm{H}])([\mathrm{H}])[\mathrm{H}]) \mathrm{C}([\mathrm{H}])([\mathrm{H}]) \mathrm{C}([\mathrm{H}])(\mathrm{C}([\mathrm{H}])([\mathrm{H}])[\mathrm{H}]) \mathrm{C}([\mathrm{H}])([\mathrm{H}]) \\
[\mathrm{H}]) \mathrm{C}([\mathrm{H}])([\mathrm{H}]) \mathrm{C}(=\mathrm{O}) \mathrm{N}([\mathrm{H}])[\mathrm{H}]\end{array}$ & 1.504 & 2.330 \\
\hline Ts34 & $\begin{array}{l}\mathrm{O}=\mathrm{C} 3 \mathrm{~N}([\mathrm{H}])[\mathrm{C} @]([\mathrm{H}])(\mathrm{C}(=\mathrm{O}) \mathrm{N}([\mathrm{H}])[\mathrm{C} @]([\mathrm{H}])([\mathrm{C} @]([\mathrm{H}])(\mathrm{O}[\mathrm{H}]) \mathrm{C}([\mathrm{H}])([\mathrm{H}]) \mathrm{N}([\mathrm{H}] \\
) \mathrm{C}([\mathrm{H}])([\mathrm{H}]) \mathrm{C} 1=\mathrm{C}([\mathrm{H}]) \mathrm{C}(=\mathrm{C}([\mathrm{H}]) \mathrm{C}([\mathrm{H}])=\mathrm{C} 1[\mathrm{H}]) \mathrm{N}(\mathrm{C}([\mathrm{H}])([\mathrm{H}])[\mathrm{H}]) \mathrm{C}([\mathrm{H}])([\mathrm{H}])[\mathrm{H}]) \\
\mathrm{C}([\mathrm{H}])([\mathrm{H}]) \mathrm{C} 2=\mathrm{C}([\mathrm{H}]) \mathrm{C}([\mathrm{H}])=\mathrm{C}([\mathrm{H}]) \mathrm{C}([\mathrm{H}])=\mathrm{C} 2[\mathrm{H}]) \mathrm{C}([\mathrm{H}])([\mathrm{H}]) \mathrm{N} 3 / \mathrm{C} 4=\mathrm{C}(\mathrm{V}]) \mathrm{C}([ \\
\mathrm{H}])=\mathrm{C}([\mathrm{H}]) \mathrm{C}([\mathrm{H}])=\mathrm{C} 4[\mathrm{H}]\end{array}$ & 1.087 & 1.850 \\
\hline Ts35 & $\begin{array}{l}\mathrm{O}=\mathrm{C} 3 \mathrm{~N}([\mathrm{H}])[\mathrm{C} @]([\mathrm{H}])(\mathrm{C}(=\mathrm{O}) \mathrm{N}([\mathrm{H}])[\mathrm{C} @]([\mathrm{H}])([\mathrm{C} @]([\mathrm{H}])(\mathrm{O}[\mathrm{H}]) \mathrm{C}([\mathrm{H}])([\mathrm{H}]) \mathrm{N}([\mathrm{H}] \\
) \mathrm{C}([\mathrm{H}])([\mathrm{H}]) \mathrm{C} 1=\mathrm{C}([\mathrm{H}]) \mathrm{C}(=\mathrm{C}([\mathrm{H}]) \mathrm{C}([\mathrm{H}])=\mathrm{C} 1[\mathrm{H}]) \mathrm{N}(\mathrm{C}([\mathrm{H}])([\mathrm{H}])[\mathrm{H}]) \mathrm{C}([\mathrm{H}])([\mathrm{H}])[\mathrm{H}]) \\
\mathrm{C}([\mathrm{H}])([\mathrm{H}]) \mathrm{C} 2=\mathrm{C}([\mathrm{H}]) \mathrm{C}([\mathrm{H}])=\mathrm{C}([\mathrm{H}]) \mathrm{C}([\mathrm{H}])=\mathrm{C} 2[\mathrm{H}]) \mathrm{C}([\mathrm{H}])([\mathrm{H}]) \mathrm{N} 3 \mathrm{C}([\mathrm{H}])([\mathrm{H}]) \mathrm{C} 4= \\
\mathrm{C}([\mathrm{H}]) \mathrm{C}([\mathrm{H}])=\mathrm{C}([\mathrm{F}]) \mathrm{C}([\mathrm{F}])=\mathrm{C} 4[\mathrm{H}]\end{array}$ & 2.565 & 2.361 \\
\hline Ts36 & $\begin{array}{l}\mathrm{O}=\mathrm{C} 3 \mathrm{~N}([\mathrm{H}])[\mathrm{C} @]([\mathrm{H}])(\mathrm{C}(=\mathrm{O}) \mathrm{N}([\mathrm{H}])[\mathrm{C} @]([\mathrm{H}])([\mathrm{C} @]([\mathrm{H}])(\mathrm{O}[\mathrm{H}]) \mathrm{C}([\mathrm{H}])([\mathrm{H}]) \mathrm{N}([\mathrm{H}] \\
) \mathrm{C}([\mathrm{H}])([\mathrm{H}]) \mathrm{C} 1=\mathrm{C}([\mathrm{H}]) \mathrm{C}(=\mathrm{C}([\mathrm{H}]) \mathrm{C}([\mathrm{H}])=\mathrm{C} 1[\mathrm{H}]) \mathrm{N}(\mathrm{C}([\mathrm{H}])([\mathrm{H}])[\mathrm{H}]) \mathrm{C}([\mathrm{H}])([\mathrm{H}])[\mathrm{H}]) \\
\mathrm{C}([\mathrm{H}])([\mathrm{H}]) \mathrm{C} 2=\mathrm{C}([\mathrm{H}]) \mathrm{C}([\mathrm{H}])=\mathrm{C}([\mathrm{H}]) \mathrm{C}([\mathrm{H}])=\mathrm{C} 2[\mathrm{H}]) \mathrm{C}([\mathrm{H}])([\mathrm{H}]) \mathrm{N} 3 \mathrm{C}([\mathrm{H}])([\mathrm{H}]) \mathrm{C} 4= \\
\mathrm{C}([\mathrm{H}]) \mathrm{C}([\mathrm{H}])=\mathrm{C}([\mathrm{F}]) \mathrm{C}([\mathrm{H}])=\mathrm{C} 4[\mathrm{~F}]\end{array}$ & 2.062 & 2.049 \\
\hline Ts37 & $\begin{array}{l}\mathrm{O}=\mathrm{C}(\mathrm{N}([\mathrm{H}])[\mathrm{C} @ @]([\mathrm{H}])(\mathrm{C}([\mathrm{H}])([\mathrm{H}]) \mathrm{C} 1=\mathrm{C}([\mathrm{H}]) \mathrm{C}([\mathrm{H}])=\mathrm{C}([\mathrm{H}]) \mathrm{C}([\mathrm{H}])=\mathrm{C} 1[\mathrm{H}])[\mathrm{C} @] \\
([\mathrm{H}])(\mathrm{O}[\mathrm{H}]) \mathrm{C}([\mathrm{H}])([\mathrm{H}]) \mathrm{N}([\mathrm{H}]) \mathrm{C}([\mathrm{H}])([\mathrm{H}]) \mathrm{C} 2=\mathrm{C}([\mathrm{H}]) \mathrm{C}([\mathrm{H}])=\mathrm{C}([\mathrm{H}]) \mathrm{C}(=\mathrm{C} 2[\mathrm{H}]) \mathrm{N}(\mathrm{C}( \\
[\mathrm{H}])([\mathrm{H}])[\mathrm{H}]) \mathrm{C}([\mathrm{H}])([\mathrm{H}])[\mathrm{H}])[\mathrm{C} @ 3(\mathrm{~N}([\mathrm{H}]) \mathrm{C}(=\mathrm{O}) \mathrm{N}(\mathrm{C} 3([\mathrm{H}])[\mathrm{H}]) \mathrm{C}([\mathrm{H}])([\mathrm{H}]) \mathrm{C} 4=\mathrm{C}([ \\
\mathrm{H}]) \mathrm{C}([\mathrm{H}])=\mathrm{C}([\mathrm{H}]) \mathrm{C}([\mathrm{H}])=\mathrm{C} 4[\mathrm{H}]) \mathrm{C}([\mathrm{H}])([\mathrm{H}]) \mathrm{C}=5 \mathrm{C}([\mathrm{H}])=\mathrm{C}([\mathrm{H}]) \mathrm{C}([\mathrm{H}])=\mathrm{C}([\mathrm{H}]) \mathrm{C}=5[ \\
\mathrm{H}]\end{array}$ & 1.301 & 1.184 \\
\hline Ts38 & $\begin{array}{l}\mathrm{O}=\mathrm{C} 1 \mathrm{~N}(\mathrm{C}(=\mathrm{N}[\mathrm{C} @ @] 1(\mathrm{C}=2 \mathrm{C}([\mathrm{H}])=\mathrm{C}([\mathrm{H}]) \mathrm{C}([\mathrm{H}])=\mathrm{C}([\mathrm{H}]) \mathrm{C}=2[\mathrm{H}]) \mathrm{C} 3=\mathrm{C}([\mathrm{H}]) \mathrm{C}(=\mathrm{C}([ \\
\mathrm{H}]) \mathrm{C}([\mathrm{H}])=\mathrm{C} 3[\mathrm{H}]) \mathrm{C} 4=\mathrm{C}([\mathrm{H}]) \mathrm{N}=\mathrm{C}([\mathrm{H}]) \mathrm{C}([\mathrm{F}])=\mathrm{C} 4[\mathrm{H}]) \mathrm{N}([\mathrm{H}])[\mathrm{H}]) \mathrm{C}([\mathrm{H}])([\mathrm{H}])[\mathrm{H}]\end{array}$ & 2.538 & 2.740 \\
\hline Ts39 & $\begin{array}{l}\mathrm{O}=\mathrm{C} 1 \mathrm{~N}(\mathrm{C}(=\mathrm{N}[\mathrm{C} @ @] 1(\mathrm{C}=2 \mathrm{C}([\mathrm{H}])=\mathrm{C}([\mathrm{H}]) \mathrm{C}([\mathrm{H}])=\mathrm{C}([\mathrm{H}]) \mathrm{C}=2[\mathrm{H}]) \mathrm{C} 3=\mathrm{C}([\mathrm{H}]) \mathrm{C}(=\mathrm{C}([ \\
\mathrm{H}]) \mathrm{C}([\mathrm{H}])=\mathrm{C} 3[\mathrm{H}]) \mathrm{C} 4=\mathrm{C}([\mathrm{H}]) \mathrm{C}(\mathrm{OC}([\mathrm{H}])([\mathrm{H}])[\mathrm{H}])=\mathrm{C}([\mathrm{H}]) \mathrm{C}([\mathrm{H}])=\mathrm{C} 4[\mathrm{H}]) \mathrm{N}([\mathrm{H}])[\mathrm{H}]) \\
\mathrm{C}([\mathrm{H}])([\mathrm{H}])[\mathrm{H}]\end{array}$ & 1.222 & 2.632 \\
\hline Ts 40 & $\begin{array}{l}\mathrm{O}=\mathrm{C} 1 \mathrm{~N}(\mathrm{C}(=\mathrm{N}[\mathrm{C} @ @] 1(\mathrm{C}=2 \mathrm{C}([\mathrm{H}])=\mathrm{C}(\mathrm{C}([\mathrm{H}])=\mathrm{C}([\mathrm{H}]) \mathrm{C}=2[\mathrm{H}]) \mathrm{C} 3=\mathrm{C}([\mathrm{H}]) \mathrm{C}([\mathrm{Cl}])=\mathrm{C}( \\
[\mathrm{H}]) \mathrm{N}=\mathrm{C} 3[\mathrm{H}])[\mathrm{C} @ @] 4([\mathrm{H}]) \mathrm{C}([\mathrm{H}])([\mathrm{H}]) \mathrm{C} 4([\mathrm{H}])[\mathrm{H}]) \mathrm{N}([\mathrm{H}])[\mathrm{H}]) \mathrm{C}([\mathrm{H}])([\mathrm{H}])[\mathrm{H}]\end{array}$ & 3.678 & 2.896 \\
\hline Ts41 & $\begin{array}{l}\mathrm{O}=\mathrm{C} 1 \mathrm{~N}(\mathrm{C}(=\mathrm{N}[\mathrm{C} @] 1(\mathrm{C}=2 \mathrm{C}([\mathrm{H}])=\mathrm{C}([\mathrm{H}]) \mathrm{C}([\mathrm{H}])=\mathrm{C}([\mathrm{H}]) \mathrm{C}=2[\mathrm{H}]) \mathrm{C} 3=\mathrm{C}([\mathrm{H}]) \mathrm{C}([\mathrm{F}])=\mathrm{C} \\
([\mathrm{H}]) \mathrm{C}([\mathrm{H}])=\mathrm{C} 3[\mathrm{H}]) \mathrm{N}([\mathrm{H}])[\mathrm{H}]) \mathrm{C}([\mathrm{H}])([\mathrm{H}])[\mathrm{H}]\end{array}$ & 2.143 & 1.637 \\
\hline Ts42 & $\begin{array}{l}\mathrm{O}=\mathrm{C} 1 \mathrm{~N}(\mathrm{C}(=\mathrm{N}[\mathrm{C} @ @] 1(/ \mathrm{C} 2=\mathrm{C}(\backslash \mathrm{H}]) \mathrm{C}([\mathrm{H}])=\mathrm{C}([\mathrm{H}]) \mathrm{C}([\mathrm{H}])=\mathrm{C} 2[\mathrm{H}]) \mathrm{C} 3=\mathrm{C}([\mathrm{H}]) \mathrm{C}(=\mathrm{C}([ \\
\mathrm{H}]) \mathrm{C}([\mathrm{H}])=\mathrm{C} 3[\mathrm{H}]) \mathrm{C} 4=\mathrm{C}([\mathrm{H}]) \mathrm{C}(\mathrm{OC}([\mathrm{H}])([\mathrm{H}]) \mathrm{C}([\mathrm{H}])([\mathrm{H}])[\mathrm{H}])=\mathrm{C}([\mathrm{H}]) \mathrm{C}([\mathrm{H}])=\mathrm{C} 4[\mathrm{H}] \\
) \mathrm{N}([\mathrm{H}])[\mathrm{H}]) \mathrm{C}([\mathrm{H}])([\mathrm{H}])[\mathrm{H}]\end{array}$ & 2.337 & 2.238 \\
\hline
\end{tabular}




\begin{tabular}{|c|c|c|c|}
\hline Ts43 & $\begin{array}{l}\mathrm{O}=\mathrm{C} 1 \mathrm{~N}(\mathrm{C}(=\mathrm{N}[\mathrm{C} @ @] 1(\mathrm{C}=2 \mathrm{C}([\mathrm{H}])=\mathrm{C}([\mathrm{H}]) \mathrm{C}([\mathrm{H}])=\mathrm{C}([\mathrm{H}]) \mathrm{C}=2[\mathrm{H}]) \mathrm{C} 3=\mathrm{C}([\mathrm{H}]) \mathrm{C}(=\mathrm{C}([ \\
\mathrm{H}]) \mathrm{C}([\mathrm{H}])=\mathrm{C} 3[\mathrm{H}]) \mathrm{C}([\mathrm{H}])([\mathrm{H}])[\mathrm{H}]) \mathrm{N}([\mathrm{H}])[\mathrm{H}]) \mathrm{C}([\mathrm{H}])([\mathrm{H}])[\mathrm{H}]\end{array}$ & 2.260 & 2.310 \\
\hline Ts44 & $\begin{array}{l}\mathrm{O}=\mathrm{C}(\mathrm{N}([\mathrm{C} @ @] 1([\mathrm{H}]) \mathrm{C}([\mathrm{H}])([\mathrm{H}]) \mathrm{C}([\mathrm{H}])([\mathrm{H}]) \mathrm{C}([\mathrm{H}])([\mathrm{H}]) \mathrm{C}([\mathrm{H}])([\mathrm{H}]) \mathrm{C} 1([\mathrm{H}])[\mathrm{H}]) \mathrm{C}([ \\
\mathrm{H}])([\mathrm{H}]) \mathrm{C} 2=\mathrm{C}(\mathrm{N}([\mathrm{H}]) \mathrm{N}=\mathrm{C} 2[\mathrm{H}]) \mathrm{C}([\mathrm{H}])([\mathrm{H}])[\mathrm{H}]) \mathrm{C}([\mathrm{H}])([\mathrm{H}]) \mathrm{C}([\mathrm{H}])([\mathrm{H}])[\mathrm{C} @]([\mathrm{H}])( \\
\mathrm{N} 5 \mathrm{C}(=\mathrm{NC}=4 \mathrm{C}([\mathrm{H}])=\mathrm{C}([\mathrm{H}]) \mathrm{C}(\mathrm{O} / \mathrm{C} 3=\mathrm{C}(\mathrm{VH}]) \mathrm{C}([\mathrm{H}])=\mathrm{C}([\mathrm{H}]) \mathrm{C}([\mathrm{H}])=\mathrm{C} 3[\mathrm{H}])=\mathrm{C}([\mathrm{H}]) \mathrm{C} \\
=4 \mathrm{C} 5([\mathrm{H}])[\mathrm{H}]) \mathrm{N}([\mathrm{H}])[\mathrm{H}])[\mathrm{C} @ @] 6([\mathrm{H}]) \mathrm{C}([\mathrm{H}])([\mathrm{H}]) \mathrm{C}([\mathrm{H}])([\mathrm{H}]) \mathrm{C}([\mathrm{H}])([\mathrm{H}]) \mathrm{C}([\mathrm{H}])([ \\
\mathrm{H}]) \mathrm{C} 6([\mathrm{H}])[\mathrm{H}]\end{array}$ & 3.409 & 3.164 \\
\hline Ts45 & $\begin{array}{l}\mathrm{O}=\mathrm{C}(\mathrm{N}([\mathrm{C} @ @] 1([\mathrm{H}]) \mathrm{C}([\mathrm{H}])([\mathrm{H}]) \mathrm{C}([\mathrm{H}])([\mathrm{H}]) \mathrm{C}([\mathrm{H}])([\mathrm{H}]) \mathrm{C}([\mathrm{H}])([\mathrm{H}]) \mathrm{Cl}([\mathrm{H}])[\mathrm{H}]) \mathrm{C}([ \\
\mathrm{H}])([\mathrm{H}])[\mathrm{H}]) \mathrm{C}([\mathrm{H}])([\mathrm{H}]) \mathrm{C}([\mathrm{H}])([\mathrm{H}])[\mathrm{C} @([\mathrm{H}])(\mathrm{N} 4 \mathrm{C}(=\mathrm{N} / \mathrm{C} 3=\mathrm{C}([\mathrm{H}]) \mathrm{C}([\mathrm{H}])=\mathrm{C}(\mathrm{OC}= \\
2 \mathrm{C}([\mathrm{H}])=\mathrm{C}([\mathrm{H}]) \mathrm{C}([\mathrm{H}])=\mathrm{C}([\mathrm{H}]) \mathrm{C}=2[\mathrm{H}]) \mathrm{C}([\mathrm{H}])=\mathrm{C} 3 \mathrm{C} 4([\mathrm{H}])[\mathrm{H}]) \mathrm{N}([\mathrm{H}])[\mathrm{H}])[\mathrm{C} @] 5([\mathrm{H}] \\
) \mathrm{C}([\mathrm{H}])([\mathrm{H}]) \mathrm{OC}([\mathrm{H}])([\mathrm{H}]) \mathrm{C}([\mathrm{H}])([\mathrm{H}]) \mathrm{C} 5([\mathrm{H}])[\mathrm{H}]\end{array}$ & 2.914 & 3.847 \\
\hline Ts46 & $\begin{array}{l}\mathrm{O}=\mathrm{C}(\mathrm{OC}([\mathrm{H}])([\mathrm{H}])[\mathrm{C} @]([\mathrm{H}])(\mathrm{N} 3 \mathrm{C}(=\mathrm{N} / \mathrm{C} 2=\mathrm{C}(\mathrm{VH}]) \mathrm{C}([\mathrm{H}])=\mathrm{C}(\mathrm{O} / \mathrm{C} 1=\mathrm{C}(\mathrm{V}[\mathrm{H}]) \mathrm{C}([\mathrm{H}])= \\
\mathrm{C}([\mathrm{H}]) \mathrm{C}([\mathrm{H}])=\mathrm{C} 1[\mathrm{H}]) \mathrm{C}([\mathrm{H}])=\mathrm{C} 2 \mathrm{C} 3([\mathrm{H}])[\mathrm{H}]) \mathrm{N}([\mathrm{H}])[\mathrm{H}])[\mathrm{C} @ @] 4([\mathrm{H}]) \mathrm{C}([\mathrm{H}])([\mathrm{H}]) \mathrm{C}( \\
[\mathrm{H}])([\mathrm{H}]) \mathrm{C}([\mathrm{H}])([\mathrm{H}]) \mathrm{C}([\mathrm{H}])([\mathrm{H}]) \mathrm{C} 4([\mathrm{H}])[\mathrm{H}]) \mathrm{N}([\mathrm{H}])[\mathrm{C} @ @] 5([\mathrm{H}]) \mathrm{C}([\mathrm{H}])([\mathrm{H}]) \mathrm{C}([\mathrm{H}]) \\
([\mathrm{H}]) \mathrm{C}([\mathrm{H}])([\mathrm{H}]) \mathrm{C}([\mathrm{H}])([\mathrm{H}]) \mathrm{C} 5([\mathrm{H}])[\mathrm{H}]\end{array}$ & 3.222 & 2.706 \\
\hline Ts47 & $\begin{array}{l}\mathrm{O}=\mathrm{C} 3 \mathrm{~N}(\mathrm{C}(=\mathrm{N}[\mathrm{C} @](\mathrm{C}=1 \mathrm{C}([\mathrm{H}])=\mathrm{C}([\mathrm{S}] \mathrm{C}=1[\mathrm{Cl}]) \mathrm{C}=2 \mathrm{C}([\mathrm{H}])=\mathrm{C}(\mathrm{C \# CC}([\mathrm{H}])([\mathrm{H}])[\mathrm{H}]) \mathrm{C} \\
([\mathrm{H}])=\mathrm{NC}=2[\mathrm{H}])(\mathrm{C} 3([\mathrm{H}])[\mathrm{H}]) \mathrm{C}([\mathrm{H}])([\mathrm{H}])[\mathrm{H}]) \mathrm{N}([\mathrm{H}])[\mathrm{H}]) \mathrm{C}([\mathrm{H}])([\mathrm{H}])[\mathrm{H}]\end{array}$ & 4.097 & 3.896 \\
\hline Ts48 & 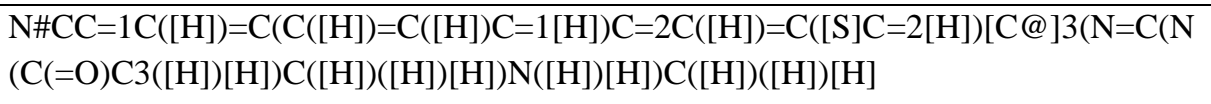 & 3.244 & 1.873 \\
\hline Ts49 & $\begin{array}{l}\mathrm{O}=\mathrm{C}(\mathrm{O}[\mathrm{C} @] 2([\mathrm{H}])[\mathrm{C} @ @]([\mathrm{H}])(\mathrm{OC} 1=\mathrm{C}(\mathrm{C}(\mathrm{O}[\mathrm{H}])=\mathrm{C}([\mathrm{H}]) \mathrm{C}(\mathrm{O}[\mathrm{H}])=\mathrm{C} 1[\mathrm{H}]) \mathrm{C} 2([\mathrm{H}])[ \\
\mathrm{H}]) \mathrm{C} 3=\mathrm{C}([\mathrm{H}]) \mathrm{C}(\mathrm{O}[\mathrm{H}])=\mathrm{C}(\mathrm{O}[\mathrm{H}]) \mathrm{C}([\mathrm{H}])=\mathrm{C} 3[\mathrm{H}]) \mathrm{C}=4 \mathrm{C}([\mathrm{H}])=\mathrm{C}(\mathrm{O}[\mathrm{H}]) \mathrm{C}(\mathrm{O}[\mathrm{H}])=\mathrm{C}(\mathrm{O}[ \\
\mathrm{H}]) \mathrm{C}=4[\mathrm{H}]\end{array}$ & 1.276 & 2.728 \\
\hline Ts50 & $\begin{array}{l}\mathrm{O}=\mathrm{C}(\mathrm{N}([\mathrm{H}]) \mathrm{C} 3=\mathrm{C}([\mathrm{H}]) \mathrm{C}([\mathrm{H}])=\mathrm{C} 2 \mathrm{OC}([\mathrm{C} @] 4([\mathrm{C} @ @] 1(\mathrm{~N}=\mathrm{C}(\mathrm{OC} 1([\mathrm{H}])[\mathrm{H}]) \mathrm{N}([\mathrm{H}])[ \\
\mathrm{H}]) \mathrm{C} 2=\mathrm{C} 3[\mathrm{H}]) \mathrm{C}([\mathrm{H}])([\mathrm{H}]) \mathrm{OC} 4([\mathrm{H}])[\mathrm{H}])(\mathrm{C}([\mathrm{H}])([\mathrm{H}])[\mathrm{H}]) \mathrm{C}([\mathrm{H}])([\mathrm{H}])[\mathrm{H}]) \mathrm{C}=5 / \mathrm{N}=\mathrm{C}(/ \\
[\mathrm{H}]) \mathrm{C}([\mathrm{Cl}])=\mathrm{C}([\mathrm{H}]) \mathrm{C}=5[\mathrm{~F}]\end{array}$ & 3.288 & 2.888 \\
\hline Ts51 & $\begin{array}{l}{[\mathrm{H}] / \mathrm{C} 4=\mathrm{C}(/ \mathrm{C}([\mathrm{H}])=\mathrm{C} 1 \mathrm{C}(\mathrm{OC}([\mathrm{H}])([\mathrm{H}])[\mathrm{C} @] 3([\mathrm{C} @ @] 12 \mathrm{~N}=\mathrm{C}(\mathrm{OC} 2([\mathrm{H}])[\mathrm{H}]) \mathrm{N}([\mathrm{H}])[} \\
\mathrm{H}]) \mathrm{C}([\mathrm{H}])([\mathrm{H}]) \mathrm{OC} 3([\mathrm{H}])[\mathrm{H}])=\mathrm{C} 4[\mathrm{H}]) \mathrm{C} 5=\mathrm{C}([\mathrm{H}]) \mathrm{N}=\mathrm{C}([\mathrm{H}]) \mathrm{C}(\mathrm{C \# CC}([\mathrm{H}])([\mathrm{H}])[\mathrm{H}])=\mathrm{C} \\
5[\mathrm{H}]\end{array}$ & 2.910 & 3.616 \\
\hline Ts52 & $\begin{array}{l}\mathrm{O}=\mathrm{C}(\mathrm{N}([\mathrm{H}]) \mathrm{C} 3=\mathrm{C}([\mathrm{H}]) \mathrm{C}([\mathrm{H}])=\mathrm{C} 2 \mathrm{OC}([\mathrm{C} @] 4([\mathrm{C} @ @] 1(\mathrm{~N}=\mathrm{C}(\mathrm{OC} 1([\mathrm{H}])[\mathrm{H}]) \mathrm{N}([\mathrm{H}])[ \\
\mathrm{H}]) \mathrm{C} 2=\mathrm{C} 3[\mathrm{H}]) \mathrm{C}([\mathrm{H}])([\mathrm{H}]) \mathrm{OC} 4([\mathrm{H}])[\mathrm{H}])(\mathrm{C}([\mathrm{H}])([\mathrm{H}])[\mathrm{H}]) \mathrm{C}([\mathrm{H}])([\mathrm{H}])[\mathrm{H}]) \mathrm{C} 5=\mathrm{NC}([\mathrm{H} \\
])=\mathrm{C}([\mathrm{Br}]) \mathrm{C}([\mathrm{H}])=\mathrm{N} 5\end{array}$ & 3.078 & 2.657 \\
\hline Ts53 & $\begin{array}{l}\mathrm{O}=\mathrm{C}(\mathrm{N}([\mathrm{H}]) \mathrm{C} 3=\mathrm{C}([\mathrm{H}]) \mathrm{C}([\mathrm{H}])=\mathrm{C} 2 \mathrm{OC}([\mathrm{H}])([\mathrm{H}])[\mathrm{C} @ @] 4([\mathrm{C} @ @] 1(\mathrm{~N}=\mathrm{C}(\mathrm{OC} 1([\mathrm{H}])[ \\
\mathrm{H}]) \mathrm{N}([\mathrm{H}])[\mathrm{H}]) \mathrm{C} 2=\mathrm{C} 3[\mathrm{H}]) \mathrm{C}([\mathrm{H}])([\mathrm{H}]) \mathrm{C} 4([\mathrm{H}])[\mathrm{H}]) \mathrm{C} 5=\mathrm{NC}([\mathrm{H}])=\mathrm{C}([\mathrm{Cl}]) \mathrm{C}([\mathrm{H}])=\mathrm{C} 5 \mathrm{C}( \\
[\mathrm{H}])([\mathrm{H}])[\mathrm{H}]\end{array}$ & 2.504 & 2.395 \\
\hline Ts54 & $\begin{array}{l}\mathrm{O}=\mathrm{C}(/ \mathrm{C} 1=\mathrm{N} / \mathrm{C}([\mathrm{H}])=\mathrm{C}(\mathrm{C}([\mathrm{H}])=\mathrm{C} 1[\mathrm{H}]) \mathrm{C}([\mathrm{H}])([\mathrm{H}])[\mathrm{H}]) \mathrm{N}([\mathrm{H}]) \mathrm{C} 2=\mathrm{C}([\mathrm{H}]) \mathrm{C}(=\mathrm{C}([\mathrm{F}]) \mathrm{C} \\
([\mathrm{H}])=\mathrm{C} 2[\mathrm{H}])[\mathrm{C} @] 3(\mathrm{~N}([\mathrm{H}]) \mathrm{C}(=\mathrm{N}[\mathrm{H}]) \mathrm{N}([\mathrm{S}](=\mathrm{O})(=\mathrm{O}) \mathrm{C} 3([\mathrm{H}])[\mathrm{H}]) \mathrm{C}([\mathrm{H}])([\mathrm{H}])[\mathrm{H}]) \mathrm{C}([ \\
\mathrm{H}])([\mathrm{H}])[\mathrm{H}]\end{array}$ & 4.523 & 4.046 \\
\hline Ts55 & $\begin{array}{l}\mathrm{O}=\mathrm{C}(/ \mathrm{C} 1=\mathrm{N} / \mathrm{C}([\mathrm{H}])=\mathrm{C}([\mathrm{F}]) \mathrm{C}([\mathrm{H}])=\mathrm{C} 1[\mathrm{H}]) \mathrm{N}([\mathrm{H}]) \mathrm{C} 2=\mathrm{C}([\mathrm{F}]) \mathrm{C}(=\mathrm{C}([\mathrm{F}]) \mathrm{C}([\mathrm{H}])=\mathrm{C} 2[\mathrm{H}] \\
)[\mathrm{C} @] 3(\mathrm{~N}([\mathrm{H}]) \mathrm{C}(=\mathrm{N}[\mathrm{H}]) \mathrm{N}([\mathrm{S}](=\mathrm{O})(=\mathrm{O}) \mathrm{C} 3([\mathrm{H}])[\mathrm{H}]) \mathrm{C}([\mathrm{H}])([\mathrm{H}])[\mathrm{H}]) \mathrm{C}([\mathrm{H}])([\mathrm{H}])[\mathrm{H}]\end{array}$ & 4.312 & 4.509 \\
\hline Ts56 & $\begin{array}{l}\mathrm{O}=[\mathrm{S}] 3(=\mathrm{O}) \mathrm{N}(/ \mathrm{C}(=\mathrm{N} \backslash[\mathrm{H}]) \mathrm{N}([\mathrm{H}])[\mathrm{C} @](\mathrm{C}=1[\mathrm{~S}] \mathrm{C}([\mathrm{H}])=\mathrm{C}(\mathrm{C}=1[\mathrm{H}]) \mathrm{C} 2=\mathrm{C}([\mathrm{H}]) \mathrm{C}([\mathrm{H}])= \\
\mathrm{C}([\mathrm{H}]) \mathrm{C}(\mathrm{CHN})=\mathrm{C} 2[\mathrm{H}])(\mathrm{C} 3([\mathrm{H}])[\mathrm{H}]) \mathrm{C}([\mathrm{H}])([\mathrm{H}])[\mathrm{H}]) \mathrm{C}([\mathrm{H}])([\mathrm{H}])[\mathrm{H}]\end{array}$ & 3.592 & 3.122 \\
\hline
\end{tabular}




\begin{tabular}{|c|c|c|c|}
\hline Ts57 & $\begin{array}{l}\mathrm{O}=\mathrm{C}(\mathrm{N}([\mathrm{H}])[\mathrm{C} @] 1([\mathrm{H}]) \mathrm{C}([\mathrm{H}])([\mathrm{H}]) \mathrm{C} 1([\mathrm{H}])[\mathrm{H}])[\mathrm{C} @]([\mathrm{H}])(\mathrm{OC}([\mathrm{H}])([\mathrm{H}])[\mathrm{H}]) \mathrm{C}([\mathrm{H}]) \\
([\mathrm{H}])[\mathrm{C} @]([\mathrm{H}])(\mathrm{O}[\mathrm{H}])[\mathrm{C} @ @]([\mathrm{H}])(\mathrm{N}([\mathrm{H}]) \mathrm{C}(=\mathrm{O}) \mathrm{C}=2 \mathrm{C}([\mathrm{H}])=\mathrm{C}([\mathrm{H}]) \mathrm{C}([\mathrm{H}])=\mathrm{C}(\mathrm{C}=2[ \\
\mathrm{H}]) \mathrm{C}(=\mathrm{O}) \mathrm{N}(\mathrm{C}([\mathrm{H}])([\mathrm{H}]) \mathrm{C}([\mathrm{H}])([\mathrm{H}]) \mathrm{C}([\mathrm{H}])([\mathrm{H}])[\mathrm{H}]) \mathrm{C}([\mathrm{H}])([\mathrm{H}]) \mathrm{C}([\mathrm{H}])([\mathrm{H}]) \mathrm{C}([\mathrm{H}])([ \\
\mathrm{H}])[\mathrm{H}]) \mathrm{C}([\mathrm{H}])([\mathrm{H}]) \mathrm{O} / \mathrm{C} 3=\mathrm{C}(\backslash \mathrm{H}]) \mathrm{C}([\mathrm{F}])=\mathrm{C}([\mathrm{H}]) \mathrm{C}([\mathrm{F}])=\mathrm{C} 3[\mathrm{H}]\end{array}$ & 2.658 & 2.105 \\
\hline Ts58 & $\begin{array}{l}\mathrm{O}=\mathrm{C}(\mathrm{N}([\mathrm{H}]) \mathrm{C}(=\mathrm{NC}([\mathrm{H}])([\mathrm{H}]) \mathrm{C} 1=\mathrm{C}([\mathrm{H}]) \mathrm{C}([\mathrm{Cl}])=\mathrm{C}(\mathrm{C}([\mathrm{Cl}])=\mathrm{C} 1[\mathrm{H}]) \mathrm{N}([\mathrm{H}])[\mathrm{S}](=\mathrm{O})(= \\
\mathrm{O}) \mathrm{C}([\mathrm{H}])([\mathrm{H}])[\mathrm{H}]) \mathrm{N}([\mathrm{H}])[\mathrm{H}]) \mathrm{C}=2 \mathrm{C}(=\mathrm{N}[\mathrm{S}] \mathrm{C}=2 \mathrm{C}([\mathrm{H}])([\mathrm{H}])[\mathrm{H}]) \mathrm{C} 3=\mathrm{C}([\mathrm{H}]) \mathrm{C}([\mathrm{H}])=\mathrm{C}( \\
\mathrm{OC}([\mathrm{H}])([\mathrm{H}])[\mathrm{H}]) \mathrm{C}([\mathrm{H}])=\mathrm{C} 3[\mathrm{H}]\end{array}$ & 2.553 & 4.081 \\
\hline Ts59 & $\begin{array}{l}\mathrm{O}=\mathrm{C}(\mathrm{N}([\mathrm{H}])[\mathrm{C} @](([\mathrm{H}])(\mathrm{C}(=\mathrm{O}) \mathrm{N}([\mathrm{H}])[\mathrm{C} @]([\mathrm{H}])(\mathrm{C}(=\mathrm{O}) \mathrm{O}[\mathrm{H}]) \mathrm{C}([\mathrm{H}])([\mathrm{H}]) \mathrm{C} 1=\mathrm{C}([\mathrm{H}]) \\
\mathrm{C}([\mathrm{H}])=\mathrm{C}([\mathrm{H}]) \mathrm{C}([\mathrm{H}])=\mathrm{C} 1[\mathrm{H}]) \mathrm{C}([\mathrm{H}])([\mathrm{H}]) \mathrm{C}([\mathrm{H}])([\mathrm{H}]) \mathrm{C}(=\mathrm{O}) \mathrm{O}[\mathrm{H}])[\mathrm{C} @ @]([\mathrm{H}])(\mathrm{N}([ \\
\mathrm{H}]) \mathrm{C}(=\mathrm{O})[\mathrm{C} @ @]([\mathrm{H}])(\mathrm{N}([\mathrm{H}]) \mathrm{C}([\mathrm{H}])([\mathrm{H}])[\mathrm{C} @ @]([\mathrm{H}])(\mathrm{O}[\mathrm{H}]) \mathrm{C}(=\mathrm{O})[\mathrm{C} @ @]([\mathrm{H}])(\mathrm{N}( \\
[\mathrm{H}]) \mathrm{C}(=\mathrm{O})[\mathrm{C} @ @]([\mathrm{H}])(\mathrm{N}([\mathrm{H}]) \mathrm{C}(=\mathrm{O})[\mathrm{C} @ @]([\mathrm{H}])(\mathrm{N}([\mathrm{H}]) \mathrm{C}(=\mathrm{O})[\mathrm{C} @ @]([\mathrm{H}])(\mathrm{N}([\mathrm{H}] \\
)[\mathrm{H}]) \mathrm{C}([\mathrm{H}])([\mathrm{H}]) \mathrm{C}([\mathrm{H}])([\mathrm{H}]) \mathrm{C}(=\mathrm{O}) \mathrm{O}[\mathrm{H}]) \mathrm{C}([\mathrm{H}])([\mathrm{H}]) \mathrm{C}([\mathrm{H}])(\mathrm{C}([\mathrm{H}])([\mathrm{H}])[\mathrm{H}]) \mathrm{C}([\mathrm{H}])([ \\
\mathrm{H}])[\mathrm{H}]) \mathrm{C}([\mathrm{H}])([\mathrm{H}]) \mathrm{C}(=\mathrm{O}) \mathrm{O}[\mathrm{H}]) \mathrm{C}([\mathrm{H}])([\mathrm{H}]) \mathrm{C}([\mathrm{H}])(\mathrm{C}([\mathrm{H}])([\mathrm{H}])[\mathrm{H}]) \mathrm{C}([\mathrm{H}])([\mathrm{H}])[\mathrm{H}]) \mathrm{C} \\
([\mathrm{H}])([\mathrm{H}])[\mathrm{H}]) \mathrm{C}([\mathrm{H}])(\mathrm{C}([\mathrm{H}])([\mathrm{H}])[\mathrm{H}]) \mathrm{C}([\mathrm{H}])([\mathrm{H}])[\mathrm{H}] \sim\end{array}$ & 5.523 & 6.540 \\
\hline Ts60 & $\begin{array}{l}\mathrm{O}=[\mathrm{S}](=\mathrm{O})(\mathrm{C}([\mathrm{H}])([\mathrm{H}]) \mathrm{C}([\mathrm{H}])([\mathrm{H}])[\mathrm{C} @ @]([\mathrm{H}])(\mathrm{C}(=\mathrm{O}) \mathrm{N}([\mathrm{H}])[\mathrm{C} @]([\mathrm{H}])([\mathrm{C} @]([\mathrm{H}]) \\
(\mathrm{O}[\mathrm{H}]) \mathrm{C}([\mathrm{H}])([\mathrm{H}])[\mathrm{C} @]([\mathrm{H}])(\mathrm{C}(=\mathrm{O}) \mathrm{N}([\mathrm{H}])[\mathrm{C} @]([\mathrm{H}])(\mathrm{C}(=\mathrm{O}) \mathrm{N}([\mathrm{H}]) \mathrm{C}([\mathrm{H}])([\mathrm{H}]) \mathrm{C}= \\
\mathrm{C}([\mathrm{H}]) \mathrm{C}([\mathrm{H}])=\mathrm{C}([\mathrm{H}]) \mathrm{C}([\mathrm{H}])=\mathrm{C} 1[\mathrm{H}]) \mathrm{C}([\mathrm{H}])(\mathrm{C}([\mathrm{H}])([\mathrm{H}])[\mathrm{H}]) \mathrm{C}([\mathrm{H}])([\mathrm{H}])[\mathrm{H}]) \mathrm{C}([\mathrm{H}])([ \\
\mathrm{H}])[\mathrm{H}]) \mathrm{C}([\mathrm{H}])([\mathrm{H}]) \mathrm{C}([\mathrm{H}])(\mathrm{C}([\mathrm{H}])([\mathrm{H}])[\mathrm{H}]) \mathrm{C}([\mathrm{H}])([\mathrm{H}])[\mathrm{H}]) \mathrm{N}([\mathrm{H}]) \mathrm{C}(=\mathrm{O})[\mathrm{C} @ @([\mathrm{H}] \\
)(\mathrm{N}([\mathrm{H}]) \mathrm{C}(=\mathrm{O}) \mathrm{OC}(\mathrm{C}([\mathrm{H}])([\mathrm{H}])[\mathrm{H}])(\mathrm{C}([\mathrm{H}])([\mathrm{H}])[\mathrm{H}]) \mathrm{C}([\mathrm{H}])([\mathrm{H}])[\mathrm{H}]) \mathrm{C}([\mathrm{H}])(\mathrm{C}([\mathrm{H}])([ \\
\mathrm{H}])[\mathrm{H}]) \mathrm{C}([\mathrm{H}])([\mathrm{H}])[\mathrm{H}]) \mathrm{C}([\mathrm{H}])([\mathrm{H}])[\mathrm{H}]\end{array}$ & 4.097 & 3.591 \\
\hline Ts61 & $\begin{array}{l}\mathrm{O}=\mathrm{C}(\mathrm{N}([\mathrm{H}])[\mathrm{C} @]([\mathrm{H}])([\mathrm{C} @]([\mathrm{H}])(\mathrm{O}[\mathrm{H}]) \mathrm{C}([\mathrm{H}])([\mathrm{H}])[\mathrm{C} @]([\mathrm{H}])(\mathrm{C}(=\mathrm{O}) \mathrm{N}([\mathrm{H}])[\mathrm{C} @]( \\
[\mathrm{H}])(\mathrm{C}(=\mathrm{O}) \mathrm{N}([\mathrm{H}]) \mathrm{C}([\mathrm{H}])([\mathrm{H}]) \mathrm{C} 1=\mathrm{C}([\mathrm{H}]) \mathrm{C}([\mathrm{H}])=\mathrm{C}([\mathrm{H}]) \mathrm{C}([\mathrm{H}])=\mathrm{C} 1[\mathrm{H}]) \mathrm{C}([\mathrm{H}])([\mathrm{H}])[ \\
\mathrm{H}]) \mathrm{C}([\mathrm{H}])([\mathrm{H}])[\mathrm{H}]) \mathrm{C}([\mathrm{H}])([\mathrm{H}]) \mathrm{C}([\mathrm{H}])(\mathrm{C}([\mathrm{H}])([\mathrm{H}])[\mathrm{H}]) \mathrm{C}([\mathrm{H}])([\mathrm{H}])[\mathrm{H}])[\mathrm{C} @ @]([\mathrm{H}])( \\
\mathrm{N}([\mathrm{H}]) \mathrm{C}(=\mathrm{O}) \mathrm{OC}(\mathrm{C}([\mathrm{H}])([\mathrm{H}])[\mathrm{H}])(\mathrm{C}([\mathrm{H}])([\mathrm{H}])[\mathrm{H}]) \mathrm{C}([\mathrm{H}])([\mathrm{H}])[\mathrm{H}]) \mathrm{C}(=\mathrm{O}) \mathrm{N}([\mathrm{H}])[\mathrm{H}]\end{array}$ & 0.649 & 2.008 \\
\hline Ts62 & $\begin{array}{l}\mathrm{O}=\mathrm{C} 1 \mathrm{~N}(/ \mathrm{C}(=\mathrm{N} \backslash[\mathrm{H}]) \mathrm{N}([\mathrm{H}])[\mathrm{C} @] 1(\mathrm{C}([\mathrm{H}])([\mathrm{H}]) \mathrm{C}([\mathrm{H}])([\mathrm{H}])[\mathrm{C} @] 2([\mathrm{H}]) \mathrm{C}([\mathrm{H}])([\mathrm{H}]) \mathrm{C}([ \\
\mathrm{H}])([\mathrm{H}]) \mathrm{C}([\mathrm{H}])([\mathrm{H}]) \mathrm{C}([\mathrm{H}])([\mathrm{H}]) \mathrm{C} 2([\mathrm{H}])[\mathrm{H}]) \mathrm{C}([\mathrm{H}])([\mathrm{H}])[\mathrm{C} @] 4([\mathrm{H}]) \mathrm{C}([\mathrm{H}])([\mathrm{H}])[\mathrm{C} @ \\
]([\mathrm{H}])(\mathrm{N}([\mathrm{H}]) \mathrm{C}(=\mathrm{O}) \mathrm{C} 3=\mathrm{C}([\mathrm{H}]) \mathrm{C}([\mathrm{H}])=\mathrm{C}([\mathrm{H}]) \mathrm{C}([\mathrm{H}])=\mathrm{C} 3[\mathrm{H}]) \mathrm{C}([\mathrm{H}])([\mathrm{H}]) \mathrm{C}([\mathrm{H}])([\mathrm{H}]) \\
\mathrm{C} 4([\mathrm{H}])[\mathrm{H}]) \mathrm{C}([\mathrm{H}])([\mathrm{H}])[\mathrm{H}]\end{array}$ & 3.886 & 3.523 \\
\hline Ts63 & $\begin{array}{l}\mathrm{O}=\mathrm{C} 1 \mathrm{~N}(/ \mathrm{C}(=\mathrm{N} \backslash[\mathrm{H}]) \mathrm{N}([\mathrm{H}])[\mathrm{C} @] 1(\mathrm{C}([\mathrm{H}])([\mathrm{H}]) \mathrm{C}([\mathrm{H}])([\mathrm{H}])[\mathrm{C} @ @] 2([\mathrm{H}]) \mathrm{C}([\mathrm{H}])([\mathrm{H}]) \\
\mathrm{C}([\mathrm{H}])([\mathrm{H}]) \mathrm{C}([\mathrm{H}])([\mathrm{H}]) \mathrm{C}([\mathrm{H}])([\mathrm{H}]) \mathrm{C} 2([\mathrm{H}])[\mathrm{H}]) \mathrm{C}([\mathrm{H}])([\mathrm{H}])[\mathrm{C} @] 4([\mathrm{H}]) \mathrm{C}([\mathrm{H}])([\mathrm{H}])[ \\
\mathrm{C} @([\mathrm{H}])(\mathrm{N}([\mathrm{H}]) \mathrm{C}([\mathrm{H}])([\mathrm{H}])[\mathrm{C} @] 3([\mathrm{H}]) \mathrm{C}([\mathrm{H}])([\mathrm{H}]) \mathrm{C} 3([\mathrm{H}])[\mathrm{H}]) \mathrm{C}([\mathrm{H}])([\mathrm{H}]) \mathrm{C}([\mathrm{H}])( \\
[\mathrm{H}]) \mathrm{C} 4([\mathrm{H}])[\mathrm{H}]) \mathrm{C}([\mathrm{H}])([\mathrm{H}])[\mathrm{H}]\end{array}$ & 3.319 & 3.124 \\
\hline Ts64 & $\begin{array}{l}\mathrm{O}=\mathrm{C} 1 \mathrm{~N}(/ \mathrm{C}(=\mathrm{N} /[\mathrm{H}]) \mathrm{N}([\mathrm{H}])[\mathrm{C} @] 1(\mathrm{C}([\mathrm{H}])([\mathrm{H}]) \mathrm{C}([\mathrm{H}])([\mathrm{H}])[\mathrm{C} @ @] 2([\mathrm{H}]) \mathrm{C}([\mathrm{H}])([\mathrm{H}]) \\
\mathrm{C}([\mathrm{H}])([\mathrm{H}]) \mathrm{C}([\mathrm{H}])([\mathrm{H}]) \mathrm{C}([\mathrm{H}])([\mathrm{H}]) \mathrm{C} 2([\mathrm{H}])[\mathrm{H}]) \mathrm{C}([\mathrm{H}])([\mathrm{H}])[\mathrm{C} @] 4([\mathrm{H}]) \mathrm{C}([\mathrm{H}])([\mathrm{H}])[ \\
\mathrm{C} @]([\mathrm{H}])(\mathrm{N}([\mathrm{H}]) \mathrm{C}(=\mathrm{O}) \mathrm{N}([\mathrm{H}]) \mathrm{C} 3=\mathrm{C}([\mathrm{H}]) \mathrm{C}([\mathrm{H}])=\mathrm{C}(\mathrm{OC}([\mathrm{H}])([\mathrm{H}])[\mathrm{H}]) \mathrm{C}([\mathrm{H}])=\mathrm{C} 3[\mathrm{H}] \\
) \mathrm{C}([\mathrm{H}])([\mathrm{H}]) \mathrm{C}([\mathrm{H}])([\mathrm{H}]) \mathrm{C} 4([\mathrm{H}])[\mathrm{H}]) \mathrm{C}([\mathrm{H}])([\mathrm{H}])[\mathrm{H}]\end{array}$ & 5.000 & 3.179 \\
\hline Ts65 & $\begin{array}{l}\mathrm{O}=\mathrm{C} 1 \mathrm{~N}(/ \mathrm{C}(=\mathrm{N} \backslash[\mathrm{H}]) \mathrm{N}([\mathrm{H}])[\mathrm{C} @] 1(\mathrm{C}([\mathrm{H}])([\mathrm{H}]) \mathrm{C}([\mathrm{H}])([\mathrm{H}])[\mathrm{C} @] 2([\mathrm{H}]) \mathrm{C}([\mathrm{H}])([\mathrm{H}]) \mathrm{C}([ \\
\mathrm{H}])([\mathrm{H}]) \mathrm{C}([\mathrm{H}])([\mathrm{H}]) \mathrm{C}([\mathrm{H}])([\mathrm{H}]) \mathrm{C} 2([\mathrm{H}])[\mathrm{H}]) \mathrm{C}([\mathrm{H}])([\mathrm{H}])[\mathrm{C} @ @] 3([\mathrm{H}]) \mathrm{C}([\mathrm{H}])([\mathrm{H}]) \mathrm{N}( \\
\mathrm{C}(=\mathrm{O}) \mathrm{OC}(\mathrm{C}([\mathrm{H}])([\mathrm{H}])[\mathrm{H}])(\mathrm{C}([\mathrm{H}])([\mathrm{H}])[\mathrm{H}]) \mathrm{C}([\mathrm{H}])([\mathrm{H}])[\mathrm{H}]) \mathrm{C}([\mathrm{H}])([\mathrm{H}]) \mathrm{C}([\mathrm{H}])([\mathrm{H}]) \mathrm{C} \\
3([\mathrm{H}])[\mathrm{H}]) \mathrm{C}([\mathrm{H}])([\mathrm{H}])[\mathrm{H}]\end{array}$ & 3.387 & 3.981 \\
\hline Ts66 & $\begin{array}{l}\mathrm{O}=[\mathrm{S}] 2(=\mathrm{O}) \mathrm{N}(/ \mathrm{C}(=\mathrm{N} /[\mathrm{H}]) \mathrm{N}([\mathrm{H}])[\mathrm{C} @] 4(/ \mathrm{C} 1=\mathrm{C}(\backslash \mathrm{H}]) \mathrm{C}([\mathrm{H}])=\mathrm{C}([\mathrm{H}]) \mathrm{C}([\mathrm{H}])=\mathrm{C} 1[\mathrm{H}])[ \\
\mathrm{C} @ @] 2([\mathrm{H}]) \mathrm{C}([\mathrm{H}])([\mathrm{H}]) \mathrm{N}(/ \mathrm{C} 3=\mathrm{N} / \mathrm{C}(=\mathrm{C}(/[\mathrm{F}]) \mathrm{C}(=\mathrm{N} 3) \mathrm{OC}([\mathrm{H}])([\mathrm{H}])[\mathrm{H}]) \mathrm{C}([\mathrm{H}])([\mathrm{H}])[ \\
\mathrm{H}]) \mathrm{C} 4([\mathrm{H}])[\mathrm{H}]) \mathrm{C}([\mathrm{H}])([\mathrm{H}])[\mathrm{H}]\end{array}$ & 3.292 & 2.984 \\
\hline Ts67 & $\mathrm{O}=[\mathrm{S}] 2(=\mathrm{O}) \mathrm{N}(/ \mathrm{C}(=\mathrm{N} /[\mathrm{H}]) \mathrm{N}([\mathrm{H}])[\mathrm{C} @] 4(/ \mathrm{C} 1=\mathrm{C}(\backslash[\mathrm{H}]) \mathrm{C}([\mathrm{H}])=\mathrm{C}([\mathrm{H}]) \mathrm{C}([\mathrm{H}])=\mathrm{C} 1[\mathrm{~F}])[$ & 3.721 & 3.109 \\
\hline
\end{tabular}




\begin{tabular}{|c|c|c|c|}
\hline & $\begin{array}{l}\mathrm{C} @ @] 2([\mathrm{H}]) \mathrm{C}([\mathrm{H}])([\mathrm{H}]) \mathrm{N}(/ \mathrm{C} 3=\mathrm{N} / \mathrm{C}(=\mathrm{C}(/[\mathrm{F}]) \mathrm{C}(=\mathrm{N} 3) \mathrm{OC}([\mathrm{H}])([\mathrm{H}])[\mathrm{H}]) \mathrm{C}([\mathrm{H}])([\mathrm{H}])[ \\
\mathrm{H}]) \mathrm{C} 4([\mathrm{H}])[\mathrm{H}]) \mathrm{C}([\mathrm{H}])([\mathrm{H}])[\mathrm{H}]\end{array}$ & & \\
\hline Ts68 & $\begin{array}{l}\mathrm{O}=[\mathrm{S}] 2(=\mathrm{O}) \mathrm{N}(/ \mathrm{C}(=\mathrm{N} /[\mathrm{H}]) \mathrm{N}([\mathrm{H}])[\mathrm{C} @ @] 4(/ \mathrm{C} 1=\mathrm{C}(\backslash \mathrm{H}]) \mathrm{C}([\mathrm{H}])=\mathrm{C}([\mathrm{H}]) \mathrm{C}([\mathrm{H}])=\mathrm{C} 1[\mathrm{H}] \\
)[\mathrm{C} @] 2([\mathrm{H}]) \mathrm{C}([\mathrm{H}])([\mathrm{H}]) \mathrm{N}(/ \mathrm{C} 3=\mathrm{N} / \mathrm{C}(\mathrm{OC}([\mathrm{H}])([\mathrm{H}])[\mathrm{H}])=\mathrm{C}([\mathrm{H}]) \mathrm{C}(=\mathrm{C} 3[\mathrm{H}]) \mathrm{C}([\mathrm{H}])([\mathrm{H}] \\
) \mathrm{OC}([\mathrm{H}])([\mathrm{H}]) \mathrm{C}([\mathrm{H}])([\mathrm{H}])[\mathrm{H}]) \mathrm{C} 4([\mathrm{H}])[\mathrm{H}]) \mathrm{C}([\mathrm{H}])([\mathrm{H}])[\mathrm{H}]\end{array}$ & 3.215 & 3.236 \\
\hline Ts69 & $\begin{array}{l}\mathrm{O}=[\mathrm{S}] 2(=\mathrm{O}) \mathrm{N}(/ \mathrm{C}(=\mathrm{N} /[\mathrm{H}]) \mathrm{N}([\mathrm{H}])[\mathrm{C} @ @] 4(/ \mathrm{C} 1=\mathrm{C}(\mathrm{V}[\mathrm{H}]) \mathrm{C}([\mathrm{H}])=\mathrm{C}([\mathrm{H}]) \mathrm{C}([\mathrm{H}])=\mathrm{C} 1[\mathrm{H}] \\
)[\mathrm{C} @] 2([\mathrm{H}]) \mathrm{C}([\mathrm{H}])([\mathrm{H}]) \mathrm{N}(/ \mathrm{C} 3=\mathrm{N} / \mathrm{C}(=\mathrm{C}(/[\mathrm{F}]) \mathrm{C}(=\mathrm{N} 3) \mathrm{OC}([\mathrm{H}])([\mathrm{H}])[\mathrm{H}]) \mathrm{C}([\mathrm{H}])([\mathrm{H}]) \mathrm{C} \\
([\mathrm{H}])([\mathrm{H}])[\mathrm{H}]) \mathrm{C} 4([\mathrm{H}])[\mathrm{H}]) \mathrm{C}([\mathrm{H}])([\mathrm{H}])[\mathrm{H}]\end{array}$ & 3.086 & 3.195 \\
\hline Ts70 & $\begin{array}{l}\mathrm{O}=\mathrm{C}(\mathrm{C}=1 \mathrm{C}([\mathrm{H}])=\mathrm{C}([\mathrm{H}]) \mathrm{C}([\mathrm{H}])=\mathrm{C}(\mathrm{C}=1[\mathrm{H}]) \mathrm{C}(=\mathrm{O}) \mathrm{N}([\mathrm{H}])[\mathrm{C} @]([\mathrm{H}])([\mathrm{C} @ @]([\mathrm{H}])(\mathrm{O}[ \\
\mathrm{H}]) \mathrm{C}([\mathrm{H}])([\mathrm{H}]) \mathrm{N}([\mathrm{H}])[\mathrm{C} @]([\mathrm{H}])(\mathrm{C}(=\mathrm{O}) \mathrm{N}([\mathrm{H}]) \mathrm{C}([\mathrm{H}])([\mathrm{H}]) \mathrm{C}([\mathrm{H}])(\mathrm{C}([\mathrm{H}])([\mathrm{H}])[\mathrm{H}]) \mathrm{C}( \\
[\mathrm{H}])([\mathrm{H}])[\mathrm{H}])[\mathrm{C} @ @]([\mathrm{H}])(\mathrm{O}[\mathrm{H}]) \mathrm{C}([\mathrm{H}])([\mathrm{H}]) \mathrm{C}([\mathrm{H}])([\mathrm{H}]) \mathrm{C}([\mathrm{H}])([\mathrm{H}])[\mathrm{H}]) \mathrm{C}([\mathrm{H}])([\mathrm{H}]) \\
\mathrm{C} 2=\mathrm{C}([\mathrm{H}]) \mathrm{C}([\mathrm{H}])=\mathrm{C}([\mathrm{H}]) \mathrm{C}([\mathrm{H}])=\mathrm{C} 2[\mathrm{H}]) \mathrm{N}([\mathrm{C} @]([\mathrm{H}])(\mathrm{C} 3=\mathrm{C}([\mathrm{H}]) \mathrm{C}([\mathrm{H}])=\mathrm{C}([\mathrm{H}]) \mathrm{C}([ \\
\mathrm{H}])=\mathrm{C} 3[\mathrm{H}]) \mathrm{C}([\mathrm{H}])([\mathrm{H}])[\mathrm{H}]) \mathrm{C}([\mathrm{H}])([\mathrm{H}])[\mathrm{H}]\end{array}$ & 1.280 & 1.661 \\
\hline \multicolumn{4}{|c|}{ Calibration set } \\
\hline Calib1 & $\begin{array}{l}\mathrm{O}=\mathrm{C}(\mathrm{OC}([\mathrm{H}])([\mathrm{H}]) \mathrm{C}([\mathrm{H}])([\mathrm{H}]) \mathrm{C}([\mathrm{H}])=\mathrm{C}([\mathrm{H}])[\mathrm{H}]) \mathrm{N}([\mathrm{H}])[\mathrm{C} @]([\mathrm{H}])(\mathrm{C}(=\mathrm{O}) \mathrm{N}([\mathrm{H}])[\mathrm{C} \\
@]([\mathrm{H}])(\mathrm{C}(=\mathrm{O}) \mathrm{N}([\mathrm{H}])[\mathrm{C} @ @]([\mathrm{H}])(\mathrm{C}([\mathrm{H}])([\mathrm{H}]) \mathrm{C}([\mathrm{H}])(\mathrm{C}([\mathrm{H}])([\mathrm{H}])[\mathrm{H}]) \mathrm{C}([\mathrm{H}])([\mathrm{H}])[ \\
\mathrm{H}])[\mathrm{C} @ @]([\mathrm{H}])(\mathrm{O}[\mathrm{H}]) \mathrm{C}([\mathrm{H}])([\mathrm{H}])[\mathrm{C} @]([\mathrm{H}])(\mathrm{C}(=\mathrm{O}) \mathrm{N}([\mathrm{H}])[\mathrm{C} @]([\mathrm{H}])(\mathrm{C}(=\mathrm{O}) \mathrm{N}([\mathrm{H}]) \\
\mathrm{C}([\mathrm{H}])([\mathrm{H}]) \mathrm{C}=1 \mathrm{C}([\mathrm{H}])=\mathrm{C}([\mathrm{H}]) \mathrm{C}([\mathrm{H}])\end{array}$ & 3.212 & 3.066 \\
\hline Calib2 & $\begin{array}{l}\mathrm{O}=\mathrm{C}(\mathrm{OC}([\mathrm{H}])([\mathrm{H}]) \mathrm{C}([\mathrm{H}])([\mathrm{H}]) \mathrm{C}([\mathrm{H}])([\mathrm{H}]) \mathrm{C}([\mathrm{H}])=\mathrm{C}([\mathrm{H}])[\mathrm{H}]) \mathrm{N}([\mathrm{H}])[\mathrm{C} @]([\mathrm{H}])(\mathrm{C}(= \\
\mathrm{O}) \mathrm{N}([\mathrm{H}])[\mathrm{C} @]([\mathrm{H}])(\mathrm{C}(=\mathrm{O}) \mathrm{N}([\mathrm{H}])[\mathrm{C} @ @]([\mathrm{H}])(\mathrm{C}([\mathrm{H}])([\mathrm{H}]) \mathrm{C}([\mathrm{H}])(\mathrm{C}([\mathrm{H}])([\mathrm{H}])[\mathrm{H}]) \mathrm{C} \\
([\mathrm{H}])([\mathrm{H}])[\mathrm{H}])[\mathrm{C} @ @]([\mathrm{H}])(\mathrm{O}[\mathrm{H}]) \mathrm{C}([\mathrm{H}])([\mathrm{H}])[\mathrm{C} @]([\mathrm{H}])(\mathrm{C}(=\mathrm{O}) \mathrm{N}([\mathrm{H}])[\mathrm{C} @]([\mathrm{H}])(\mathrm{C}( \\
=\mathrm{O}) \mathrm{N}([\mathrm{H}]) \mathrm{C}([\mathrm{H}])([\mathrm{H}]) \mathrm{C}=1 \mathrm{C}([\mathrm{H}])=\mathrm{C}\end{array}$ & 2.950 & 3.103 \\
\hline Calib3 & $\begin{array}{l}\mathrm{O}=\mathrm{C}(/ \mathrm{C} 1=\mathrm{C}(\mathrm{VH}]) \mathrm{C}([\mathrm{H}])=\mathrm{C}([\mathrm{H}]) \mathrm{C}(=\mathrm{C} 1[\mathrm{H}]) \mathrm{C}([\mathrm{H}])([\mathrm{H}]) \mathrm{N} 4 \mathrm{C}(=\mathrm{NC}=3 \mathrm{C}([\mathrm{H}])=\mathrm{C}([\mathrm{H}]) \mathrm{C} \\
(\mathrm{OC}=2 \mathrm{C}([\mathrm{H}])=\mathrm{C}([\mathrm{H}]) \mathrm{C}([\mathrm{H}])=\mathrm{C}([\mathrm{H}]) \mathrm{C}=2[\mathrm{H}])=\mathrm{C}([\mathrm{H}]) \mathrm{C}=3 \mathrm{C} 4([\mathrm{H}])[\mathrm{H}]) \mathrm{N}([\mathrm{H}])[\mathrm{H}]) \mathrm{N}(\mathrm{C} \\
([\mathrm{H}])([\mathrm{H}])[\mathrm{H}]) \mathrm{C} 5([\mathrm{H}]) \mathrm{C}([\mathrm{H}])([\mathrm{H}]) \mathrm{C}([\mathrm{H}])([\mathrm{H}]) \mathrm{C}([\mathrm{H}])([\mathrm{H}]) \mathrm{C}([\mathrm{H}])([\mathrm{H}]) \mathrm{C} 5([\mathrm{H}])[\mathrm{H}]\end{array}$ & 2.801 & 2.815 \\
\hline Calib4 & $\begin{array}{l}\mathrm{O}=\mathrm{C}(\mathrm{O}[\mathrm{H}]) \mathrm{C}([\mathrm{H}])([\mathrm{H}]) \mathrm{C}([\mathrm{H}])([\mathrm{H}])[\mathrm{C} @ @]([\mathrm{H}])(\mathrm{C}(=\mathrm{O}) \mathrm{N}([\mathrm{H}])[\mathrm{C} @]([\mathrm{H}])(\mathrm{C}(=\mathrm{O}) \mathrm{N}([ \\
\mathrm{H}])[\mathrm{C} @]([\mathrm{H}])(\mathrm{C}(=\mathrm{O}) \mathrm{N}([\mathrm{H}])[\mathrm{C} @]([\mathrm{H}])([\mathrm{C} @ @]([\mathrm{H}])(\mathrm{O}[\mathrm{H}]) \mathrm{C}([\mathrm{H}])([\mathrm{H}])[\mathrm{C} @]([\mathrm{H}])(\mathrm{C} \\
(=\mathrm{O}) \mathrm{N}([\mathrm{H}])[\mathrm{C} @]([\mathrm{H}])(\mathrm{C}(=\mathrm{O}) \mathrm{N}([\mathrm{H}])[\mathrm{C} @([\mathrm{H}])(\mathrm{C}(=\mathrm{O}) \mathrm{N}([\mathrm{H}])[\mathrm{C} @]([\mathrm{H}])(\mathrm{C}(=\mathrm{O}) \mathrm{O}[\mathrm{H}] \\
) \mathrm{C}([\mathrm{H}])([\mathrm{H}]) \mathrm{C} 1=\mathrm{C}([\mathrm{H}]) \mathrm{C}([\mathrm{H}])=\mathrm{C}([\mathrm{H}]) \mathrm{C}([\mathrm{H}]\end{array}$ & 4.796 & 4.688 \\
\hline Calib5 & $\begin{array}{l}\mathrm{O}=\mathrm{C}(\mathrm{N}([\mathrm{H}])[\mathrm{C} @](([\mathrm{H}])([\mathrm{C} @ @]([\mathrm{H}])(\mathrm{O}[\mathrm{H}]) \mathrm{C}([\mathrm{H}])([\mathrm{H}])[\mathrm{C} @]([\mathrm{H}])(\mathrm{C}(=\mathrm{O}) \mathrm{N}([\mathrm{H}])[\mathrm{C} @ \\
]([\mathrm{H}])(\mathrm{C}(=\mathrm{O}) \mathrm{N}([\mathrm{H}]) \mathrm{C}([\mathrm{H}])([\mathrm{H}]) \mathrm{C} 1=\mathrm{C}([\mathrm{H}]) \mathrm{C}([\mathrm{H}])=\mathrm{C}([\mathrm{H}]) \mathrm{C}([\mathrm{H}])=\mathrm{C} 1[\mathrm{H}]) \mathrm{C}([\mathrm{H}])(\mathrm{C}([ \\
\mathrm{H}])([\mathrm{H}])[\mathrm{H}]) \mathrm{C}([\mathrm{H}])([\mathrm{H}])[\mathrm{H}]) \mathrm{C}([\mathrm{H}])([\mathrm{H}])[\mathrm{H}]) \mathrm{C}([\mathrm{H}])([\mathrm{H}]) \mathrm{C}([\mathrm{H}])(\mathrm{C}([\mathrm{H}])([\mathrm{H}])[\mathrm{H}]) \mathrm{C}([ \\
\mathrm{H}])([\mathrm{H}])[\mathrm{H}])[\mathrm{C} @ @]([\mathrm{H}])(\mathrm{N}([\mathrm{H}]) \mathrm{C}\end{array}$ & 4.027 & 3.717 \\
\hline Calib6 & $\begin{array}{l}\mathrm{O}=\mathrm{C}(\mathrm{N}([\mathrm{H}])[\mathrm{C} @(([\mathrm{H}])([\mathrm{C} @ @]([\mathrm{H}])(\mathrm{O}[\mathrm{H}]) \mathrm{C}([\mathrm{H}])([\mathrm{H}])[\mathrm{C} @]([\mathrm{H}])(\mathrm{C}(=\mathrm{O}) \mathrm{N}([\mathrm{H}])[\mathrm{C} @ \\
]([\mathrm{H}])(\mathrm{C}(=\mathrm{O}) \mathrm{N}([\mathrm{H}]) \mathrm{C}([\mathrm{H}])([\mathrm{H}]) \mathrm{C} 1=\mathrm{C}([\mathrm{H}]) \mathrm{C}([\mathrm{H}])=\mathrm{C}([\mathrm{H}]) \mathrm{C}([\mathrm{H}])=\mathrm{C} 1[\mathrm{H}]) \mathrm{C}([\mathrm{H}])(\mathrm{C}([ \\
\mathrm{H}])([\mathrm{H}])[\mathrm{H}]) \mathrm{C}([\mathrm{H}])([\mathrm{H}])[\mathrm{H}]) \mathrm{C}([\mathrm{H}])([\mathrm{H}]) \mathrm{C}([\mathrm{H}])(\mathrm{C}([\mathrm{H}])([\mathrm{H}])[\mathrm{H}]) \mathrm{C}([\mathrm{H}])([\mathrm{H}])[\mathrm{H}]) \mathrm{C}([ \\
\mathrm{H}])([\mathrm{H}]) \mathrm{C}([\mathrm{H}])(\mathrm{C}([\mathrm{H}])([\mathrm{H}])[\mathrm{H}])\end{array}$ & 0.979 & 3.121 \\
\hline Calib7 & $\begin{array}{l}\mathrm{O}=\mathrm{C} 2 \mathrm{~N}(\mathrm{C}(=\mathrm{N}[\mathrm{C} @] 4(\mathrm{C}=1 \mathrm{C}([\mathrm{H}])=\mathrm{C}([\mathrm{H}]) \mathrm{C}([\mathrm{F}])=\mathrm{C}([\mathrm{H}]) \mathrm{C}=1[\mathrm{H}])[\mathrm{C} @ @] 2([\mathrm{H}]) \mathrm{C}([\mathrm{H}]) \\
([\mathrm{H}]) \mathrm{N}(/ \mathrm{C} 3=\mathrm{N} / \mathrm{C}(\mathrm{OC}([\mathrm{H}])([\mathrm{H}])[\mathrm{H}])=\mathrm{C}([\mathrm{F}]) \mathrm{C}(=\mathrm{N} 3) \mathrm{OC}([\mathrm{H}])([\mathrm{H}])[\mathrm{H}]) \mathrm{C} 4([\mathrm{H}])[\mathrm{H}]) \mathrm{N}([ \\
\mathrm{H}])[\mathrm{H}]) \mathrm{C}([\mathrm{H}])([\mathrm{H}])[\mathrm{H}]\end{array}$ & 4.357 & 3.957 \\
\hline Calib8 & $\begin{array}{l}\mathrm{O}=\mathrm{C} 2 \mathrm{~N}(\mathrm{C}(=\mathrm{N}[\mathrm{C} @] 4(\mathrm{C}=1 \mathrm{C}([\mathrm{F}])=\mathrm{C}([\mathrm{H}]) \mathrm{C}([\mathrm{F}])=\mathrm{C}([\mathrm{H}]) \mathrm{C}=1[\mathrm{~F}])[\mathrm{C} @ @] 2([\mathrm{H}]) \mathrm{C}([\mathrm{H}])) \\
[\mathrm{H}]) \mathrm{N}(/ \mathrm{C} 3=\mathrm{N} / \mathrm{C}(=\mathrm{C}(/[\mathrm{F}]) \mathrm{C}(=\mathrm{N} 3) \mathrm{OC}([\mathrm{H}])([\mathrm{H}])[\mathrm{H}]) \mathrm{C}([\mathrm{H}])([\mathrm{H}])[\mathrm{H}]) \mathrm{C} 4([\mathrm{H}])[\mathrm{H}]) \mathrm{N}([\mathrm{H}]\end{array}$ & 3.824 & 4.008 \\
\hline
\end{tabular}




\begin{tabular}{|c|c|c|c|}
\hline & )$[\mathrm{H}]) \mathrm{C}([\mathrm{H}])([\mathrm{H}])[\mathrm{H}]$ & & \\
\hline Calib9 & $\begin{array}{l}\mathrm{O}=\mathrm{C} 2 \mathrm{~N}(\mathrm{C}(=\mathrm{N}[\mathrm{C} @] 4(/ \mathrm{Cl}=\mathrm{C}(\backslash[\mathrm{H}]) \mathrm{C}([\mathrm{Cl}])=\mathrm{C}([\mathrm{H}]) \mathrm{C}([\mathrm{H}])=\mathrm{C} 1[\mathrm{H}])[\mathrm{C} @ @] 2([\mathrm{H}]) \mathrm{C}([\mathrm{H}] \\
)([\mathrm{H}]) \mathrm{N}(/ \mathrm{C} 3=\mathrm{N} / \mathrm{C}(\mathrm{OC}([\mathrm{H}])([\mathrm{H}])[\mathrm{H}])=\mathrm{C}([\mathrm{F}]) \mathrm{C}(=\mathrm{N} 3) \mathrm{C}([\mathrm{H}])([\mathrm{H}])[\mathrm{H}]) \mathrm{C} 4([\mathrm{H}])[\mathrm{H}]) \mathrm{N}([ \\
\mathrm{H}])[\mathrm{H}]) \mathrm{C}([\mathrm{H}])([\mathrm{H}])[\mathrm{H}]\end{array}$ & 3.409 & 3.183 \\
\hline Calib10 & $\begin{array}{l}\mathrm{O}=\mathrm{C} 2 \mathrm{~N}(\mathrm{C}(=\mathrm{N}[\mathrm{C} @] 5(\mathrm{C}=1 \mathrm{C}([\mathrm{F}])=\mathrm{C}([\mathrm{H}]) \mathrm{C}([\mathrm{H}])=\mathrm{C}([\mathrm{F}]) \mathrm{C}=1[\mathrm{H}])[\mathrm{C} @ @] 2([\mathrm{H}]) \mathrm{C}([\mathrm{H}]) \\
([\mathrm{H}]) \mathrm{N}(/ \mathrm{C} 3=\mathrm{N} / \mathrm{C}(\mathrm{OC}([\mathrm{H}])([\mathrm{H}])[\mathrm{H}])=\mathrm{C}([\mathrm{Cl}]) \mathrm{C}(=\mathrm{N} 3)[\mathrm{C} @] 4([\mathrm{H}]) \mathrm{C}([\mathrm{H}])([\mathrm{H}]) \mathrm{C} 4([\mathrm{H}])[ \\
\mathrm{H}]) \mathrm{C} 5([\mathrm{H}])[\mathrm{H}]) \mathrm{N}([\mathrm{H}])[\mathrm{H}]) \mathrm{C}([\mathrm{H}])([\mathrm{H}])[\mathrm{H}]\end{array}$ & 4.187 & 4.330 \\
\hline Calib11 & $\begin{array}{l}\mathrm{O}=\mathrm{C} 2 \mathrm{~N}(\mathrm{C}(=\mathrm{N}[\mathrm{C} @] 5(\mathrm{C}=1 \mathrm{C}([\mathrm{H}])=\mathrm{C}(\mathrm{CHN}) \mathrm{C}([\mathrm{H}])=\mathrm{C}([\mathrm{H}]) \mathrm{C}=1[\mathrm{H}])[\mathrm{C} @ @] 2([\mathrm{H}]) \mathrm{C}([\mathrm{H} \\
])([\mathrm{H}]) \mathrm{N}(/ \mathrm{C} 3=\mathrm{N} / \mathrm{C}(=\mathrm{C}(/[\mathrm{F}]) \mathrm{C}(=\mathrm{N} 3)[\mathrm{C} @ @] 4([\mathrm{H}]) \mathrm{C}([\mathrm{H}])([\mathrm{H}]) \mathrm{C} 4([\mathrm{H}])[\mathrm{H}]) \mathrm{C}([\mathrm{H}])([\mathrm{H}] \\
)[\mathrm{H}]) \mathrm{C} 5([\mathrm{H}])[\mathrm{H}]) \mathrm{N}([\mathrm{H}])[\mathrm{H}]) \mathrm{C}([\mathrm{H}])([\mathrm{H}])[\mathrm{H}]\end{array}$ & 3.367 & 3.126 \\
\hline Calib12 & $\begin{array}{l}{[\mathrm{F}] \mathrm{C}=1 \mathrm{C}([\mathrm{H}])=\mathrm{C}([\mathrm{F}]) \mathrm{C}(=\mathrm{C}([\mathrm{H}]) \mathrm{C}=1[\mathrm{H}])[\mathrm{C} @ @] 24 \mathrm{~N}=\mathrm{C}(\mathrm{N}(\mathrm{C}(=\mathrm{O})[\mathrm{C} @] 2([\mathrm{H}]) \mathrm{C}([\mathrm{H}])} \\
([\mathrm{H}]) \mathrm{N}(/ \mathrm{C} 3=\mathrm{N} / \mathrm{C}(=\mathrm{C}(/[\mathrm{F}]) \mathrm{C}(=\mathrm{N} 3) \mathrm{OC}([\mathrm{H}])([\mathrm{H}])[\mathrm{H}]) \mathrm{C}([\mathrm{H}])([\mathrm{H}]) \mathrm{O}[\mathrm{H}]) \mathrm{C} 4([\mathrm{H}])[\mathrm{H}]) \mathrm{C}([ \\
\mathrm{H}])([\mathrm{H}])[\mathrm{H}]) \mathrm{N}([\mathrm{H}])[\mathrm{H}]\end{array}$ & 3.796 & 3.608 \\
\hline Calib13 & $\begin{array}{l}\mathrm{O}=\mathrm{C} 2 \mathrm{~N}(\mathrm{C}(=\mathrm{N}[\mathrm{C} @] 4(\mathrm{C}=1 \mathrm{C}([\mathrm{F}])=\mathrm{C}([\mathrm{H}]) \mathrm{C}([\mathrm{F}])=\mathrm{C}([\mathrm{F}]) \mathrm{C}=1[\mathrm{H}])[\mathrm{C} @ @] 2([\mathrm{H}]) \mathrm{C}([\mathrm{H}])( \\
[\mathrm{H}]) \mathrm{N}(/ \mathrm{C} 3=\mathrm{N} / \mathrm{C}(\mathrm{OC}([\mathrm{H}])([\mathrm{H}])[\mathrm{H}])=\mathrm{C}([\mathrm{F}]) \mathrm{C}(=\mathrm{N} 3) \mathrm{C}([\mathrm{H}])([\mathrm{H}]) \mathrm{C}([\mathrm{H}])([\mathrm{H}])[\mathrm{H}]) \mathrm{C} 4([\mathrm{H}] \\
)[\mathrm{H}]) \mathrm{N}([\mathrm{H}])[\mathrm{H}]) \mathrm{C}([\mathrm{H}])([\mathrm{H}])[\mathrm{H}]\end{array}$ & 4.252 & 4.506 \\
\hline Calib14 & $\begin{array}{l}\mathrm{O}=\mathrm{C}(/ \mathrm{C} 1=\mathrm{N} / \mathrm{C}([\mathrm{H}])=\mathrm{C}([\mathrm{F}]) \mathrm{C}([\mathrm{H}])=\mathrm{C} 1[\mathrm{H}]) \mathrm{N}([\mathrm{H}]) \mathrm{C} 2=\mathrm{C}([\mathrm{H}]) \mathrm{C}(=\mathrm{C}([\mathrm{F}]) \mathrm{C}([\mathrm{H}])=\mathrm{C} 2[\mathrm{H} \\
])[\mathrm{C} @] 3(\mathrm{~N}([\mathrm{H}]) \mathrm{C}(=\mathrm{N}[\mathrm{H}]) \mathrm{N}([\mathrm{S}](=\mathrm{O})(=\mathrm{O}) \mathrm{C} 3([\mathrm{H}])[\mathrm{H}]) \mathrm{C}([\mathrm{H}])([\mathrm{H}])[\mathrm{H}]) \mathrm{C}([\mathrm{H}])([\mathrm{H}])[\mathrm{H}]\end{array}$ & 4.312 & 4.159 \\
\hline Calib15 & $\begin{array}{l}\mathrm{O}=[\mathrm{S}] 3(=\mathrm{O}) \mathrm{N}(\mathrm{C}=2 \mathrm{C}([\mathrm{H}])=\mathrm{C}(\mathrm{C}([\mathrm{H}])=\mathrm{Cl} / \mathrm{C}(=\mathrm{C}(/[\mathrm{H}]) \mathrm{N}(\mathrm{Cl}=2) \mathrm{C}([\mathrm{H}])([\mathrm{H}]) \mathrm{C} 3([\mathrm{H}])[\mathrm{H}] \\
) \mathrm{C}([\mathrm{H}])([\mathrm{H}]) \mathrm{C}([\mathrm{H}])([\mathrm{H}])[\mathrm{H}]) \mathrm{C}(=\mathrm{O}) \mathrm{N}([\mathrm{H}])[\mathrm{C} @]([\mathrm{H}])(\mathrm{C}([\mathrm{H}])([\mathrm{H}]) \mathrm{N}([\mathrm{H}])[\mathrm{C} @]([\mathrm{H}])( \\
\mathrm{C}(=\mathrm{O}) \mathrm{N}([\mathrm{H}]) \mathrm{C}([\mathrm{H}])([\mathrm{H}]) \mathrm{C}([\mathrm{H}])(\mathrm{C}([\mathrm{H}])([\mathrm{H}])[\mathrm{H}]) \mathrm{C}([\mathrm{H}])([\mathrm{H}])[\mathrm{H}])[\mathrm{C} @ @]([\mathrm{H}])(\mathrm{O}[\mathrm{H}]) \\
\mathrm{C}([\mathrm{H}])([\mathrm{H}]) \mathrm{C}([\mathrm{H}])([\mathrm{H}]) \mathrm{C}([\mathrm{H}])([\mathrm{H}\end{array}$ & 5.398 & 4.593 \\
\hline Calib16 & $\begin{array}{l}\mathrm{O}=\mathrm{C}(\mathrm{N}([\mathrm{H}])[\mathrm{C} @ @](([\mathrm{H}])(\mathrm{C}=1 \mathrm{C}([\mathrm{H}])=\mathrm{C}([\mathrm{H}]) \mathrm{C}([\mathrm{H}])=\mathrm{C}([\mathrm{H}]) \mathrm{C}=1[\mathrm{H}]) \mathrm{C}([\mathrm{H}])([\mathrm{H}])[\mathrm{H}]) \\
\mathrm{C} 2=\mathrm{C}([\mathrm{H}]) \mathrm{C}(=\mathrm{C}([\mathrm{H}]) \mathrm{C}(=\mathrm{C} 2[\mathrm{H}]) \mathrm{N}(\mathrm{C}([\mathrm{H}])([\mathrm{H}])[\mathrm{H}])[\mathrm{S}](=\mathrm{O})(=\mathrm{O}) \mathrm{C}([\mathrm{H}])([\mathrm{H}])[\mathrm{H}]) \mathrm{C}= \\
\mathrm{O}) \mathrm{N}([\mathrm{H}])[\mathrm{C} @]([\mathrm{H}])([\mathrm{C} @]([\mathrm{H}])(\mathrm{O}[\mathrm{H}]) \mathrm{C}([\mathrm{H}])([\mathrm{H}]) \mathrm{N}([\mathrm{H}]) \mathrm{C}([\mathrm{H}])([\mathrm{H}]) \mathrm{C} 3=\mathrm{C}([\mathrm{H}]) \mathrm{C} 4= \\
\mathrm{C}(\mathrm{C}([\mathrm{H}])=\mathrm{C} 3[\mathrm{H}]) \mathrm{C}([\mathrm{H}])=\mathrm{C}([\mathrm{H}]) \mathrm{N} 4[\mathrm{~S}](=\end{array}$ & 3.569 & 3.680 \\
\hline Calib17 & $\begin{array}{l}\mathrm{O}=\mathrm{C}(\mathrm{OC}([\mathrm{H}])([\mathrm{H}]) \mathrm{C}=1 \mathrm{~N}=\mathrm{C}(\mathrm{OC}=1 \mathrm{C}([\mathrm{H}])([\mathrm{H}])[\mathrm{H}]) \mathrm{C}([\mathrm{H}])([\mathrm{H}])[\mathrm{H}]) \mathrm{N}([\mathrm{H}])[\mathrm{C} @]([\mathrm{H}]) \\
(\mathrm{C}(=\mathrm{O}) \mathrm{N}([\mathrm{H}])[\mathrm{C} @ @]([\mathrm{H}])(\mathrm{C}([\mathrm{H}])([\mathrm{H}]) \mathrm{C} 2=\mathrm{C}([\mathrm{H}]) \mathrm{C}([\mathrm{H}])=\mathrm{C}([\mathrm{H}]) \mathrm{C}([\mathrm{H}])=\mathrm{C} 2[\mathrm{H}])[\mathrm{C} \\
@]([\mathrm{H}])(\mathrm{O}[\mathrm{H}]) \mathrm{C}([\mathrm{H}])([\mathrm{H}]) \mathrm{N}([\mathrm{H}]) \mathrm{C}([\mathrm{H}])([\mathrm{H}]) \mathrm{C} 3=\mathrm{C}([\mathrm{H}]) \mathrm{C}(\mathrm{OC}([\mathrm{H}])([\mathrm{H}])[\mathrm{H}])=\mathrm{C}([\mathrm{H}]) \\
\mathrm{C}([\mathrm{H}])=\mathrm{C} 3[\mathrm{H}]) \mathrm{C}([\mathrm{H}])([\mathrm{H}])[\mathrm{S}](=\mathrm{O})(=\mathrm{O})\end{array}$ & 0.824 & 2.441 \\
\hline Calib18 & $\begin{array}{l}\mathrm{O}=\mathrm{C}(\mathrm{N}([\mathrm{H}])[\mathrm{C} @ @](([\mathrm{H}])(\mathrm{C}=1 \mathrm{C}([\mathrm{H}])=\mathrm{C}([\mathrm{H}]) \mathrm{C}([\mathrm{H}])=\mathrm{C}([\mathrm{H}]) \mathrm{C}=1[\mathrm{H}]) \mathrm{C}([\mathrm{H}])([\mathrm{H}])[\mathrm{H}]) \\
\mathrm{C} 2=\mathrm{C}([\mathrm{H}]) \mathrm{C}(=\mathrm{C}([\mathrm{H}]) \mathrm{C}(=\mathrm{C} 2[\mathrm{H}]) \mathrm{N}(\mathrm{C}([\mathrm{H}])([\mathrm{H}])[\mathrm{H}])[\mathrm{S}](=\mathrm{O})(=\mathrm{O}) \mathrm{C}([\mathrm{H}])([\mathrm{H}])[\mathrm{H}]) \mathrm{C}(= \\
\mathrm{O}) \mathrm{N}([\mathrm{H}])[\mathrm{C} @]([\mathrm{H}])([\mathrm{C} @]([\mathrm{H}])(\mathrm{O}[\mathrm{H}]) \mathrm{C}([\mathrm{H}])([\mathrm{H}]) \mathrm{N}([\mathrm{H}]) \mathrm{C}([\mathrm{H}])([\mathrm{H}]) \mathrm{C} 3=\mathrm{C}([\mathrm{H}]) \mathrm{C}(\mathrm{O} \\
\mathrm{C}([\mathrm{H}])([\mathrm{H}])[\mathrm{H}])=\mathrm{C}([\mathrm{H}]) \mathrm{C}([\mathrm{H}])=\mathrm{C} 3[\mathrm{H}])\end{array}$ & 2.038 & 2.070 \\
\hline Calib19 & $\begin{array}{l}\mathrm{O}=\mathrm{C}(\mathrm{OC}([\mathrm{H}])([\mathrm{H}]) \mathrm{C}=1 \mathrm{~N}=\mathrm{C}(\mathrm{OC}=1 \mathrm{C}([\mathrm{H}])([\mathrm{H}])[\mathrm{H}]) \mathrm{C}([\mathrm{H}])([\mathrm{H}])[\mathrm{H}]) \mathrm{N}([\mathrm{H}])[\mathrm{C} @]([\mathrm{H}]) \\
(\mathrm{C}(=\mathrm{O}) \mathrm{N}([\mathrm{H}])[\mathrm{C} @([\mathrm{H}])([\mathrm{C} @ @]([\mathrm{H}])(\mathrm{O}[\mathrm{H}]) \mathrm{C}([\mathrm{H}])([\mathrm{H}])[\mathrm{C} @]([\mathrm{H}])(\mathrm{C}(=\mathrm{O}) \mathrm{N}([\mathrm{H}])[\mathrm{C} \\
@]([\mathrm{H}])(\mathrm{C}(=\mathrm{O}) \mathrm{N}([\mathrm{H}]) \mathrm{C}(\mathrm{C}([\mathrm{H}])([\mathrm{H}])[\mathrm{H}])(\mathrm{C}([\mathrm{H}])([\mathrm{H}])[\mathrm{H}]) \mathrm{C}([\mathrm{H}])([\mathrm{H}])[\mathrm{H}]) \mathrm{C}([\mathrm{H}])(\mathrm{C}([ \\
\mathrm{H}])([\mathrm{H}])[\mathrm{H}]) \mathrm{C}([\mathrm{H}])([\mathrm{H}])[\mathrm{H}]) \mathrm{C}([\mathrm{H}]\end{array}$ & 5.921 & 4.009 \\
\hline Calib20 & $\begin{array}{l}\mathrm{O}=\mathrm{C}(\mathrm{N}([\mathrm{H}]) \mathrm{C}(=\mathrm{NC}([\mathrm{H}])([\mathrm{H}]) \mathrm{C} 1=\mathrm{C}([\mathrm{H}]) \mathrm{C}([\mathrm{Cl}])=\mathrm{C}([\mathrm{H}]) \mathrm{C}([\mathrm{Cl}])=\mathrm{C} 1[\mathrm{H}]) \mathrm{N}([\mathrm{H}])[\mathrm{H}]) \mathrm{C} \\
2=\mathrm{C}(\mathrm{N}(/ \mathrm{N}=\mathrm{C} 2 / \mathrm{C}([\mathrm{H}])([\mathrm{H}])[\mathrm{H}]) \mathrm{C}([\mathrm{H}])([\mathrm{H}])[\mathrm{H}]) \mathrm{C} 3=\mathrm{C}([\mathrm{H}]) \mathrm{C}([\mathrm{H}])=\mathrm{C}(\mathrm{OC}([\mathrm{H}])([\mathrm{H}])[\mathrm{H} \\
]) \mathrm{C}([\mathrm{H}])=\mathrm{C} 3[\mathrm{H}]\end{array}$ & 1.398 & 1.196 \\
\hline
\end{tabular}




\begin{tabular}{|c|c|c|c|}
\hline Calib21 & $\begin{array}{l}\mathrm{O}=\mathrm{C} 2 \mathrm{~N}([\mathrm{H}]) \mathrm{C} 1=\mathrm{C}([\mathrm{H}]) \mathrm{C}([\mathrm{H}])=\mathrm{C}([\mathrm{H}]) \mathrm{C}([\mathrm{H}])=\mathrm{C} 1 \mathrm{~N}([\mathrm{H}]) \mathrm{C}=3 / \mathrm{C} 2=\mathrm{C}(/[\mathrm{H}]) \mathrm{C}([\mathrm{H}])=\mathrm{C}( \\
\mathrm{C}=3[\mathrm{H}]) \mathrm{C}(=\mathrm{O}) \mathrm{N}([\mathrm{H}]) \mathrm{C} 4=\mathrm{C}([\mathrm{H}]) \mathrm{C}([\mathrm{H}])=\mathrm{NC}([\mathrm{H}])=\mathrm{C} 4[\mathrm{H}]\end{array}$ & 2.530 & 2.605 \\
\hline Calib22 & $\begin{array}{l}\mathrm{O}=\mathrm{C} 2 \mathrm{~N}([\mathrm{H}]) \mathrm{C}=1 \mathrm{C}([\mathrm{H}])=\mathrm{C}([\mathrm{H}]) \mathrm{C}([\mathrm{H}])=\mathrm{C}([\mathrm{H}]) \mathrm{C}=1 \mathrm{~N}([\mathrm{H}]) \mathrm{C} 3=\mathrm{C} 2 \mathrm{C}([\mathrm{H}])=\mathrm{C}([\mathrm{H}]) \mathrm{C}(= \\
\mathrm{C} 3[\mathrm{H}]) \mathrm{C}(=\mathrm{O}) \mathrm{N}([\mathrm{H}]) \mathrm{C} 4=\mathrm{N} / \mathrm{C}(=\mathrm{C}(/[\mathrm{H}]) \mathrm{N} 4[\mathrm{H}]) \mathrm{C} 5=\mathrm{C}([\mathrm{H}]) \mathrm{C}([\mathrm{H}])=\mathrm{C}([\mathrm{F}]) \mathrm{C}([\mathrm{H}])=\mathrm{C} 5[\mathrm{H} \\
]\end{array}$ & 2.533 & 2.778 \\
\hline Calib23 & $\begin{array}{l}\mathrm{O}=\mathrm{C}(\mathrm{N}([\mathrm{H}]) \mathrm{C}(=\mathrm{NC}([\mathrm{H}])([\mathrm{H}]) \mathrm{C} 1=\mathrm{C}([\mathrm{H}]) \mathrm{C}(=\mathrm{C}([\mathrm{H}]) \mathrm{C}([\mathrm{Cl}])=\mathrm{C} 1[\mathrm{H}]) \mathrm{C} 2=\mathrm{C}([\mathrm{H}]) \mathrm{N}=\mathrm{C}([ \\
\mathrm{H}]) \mathrm{C}([\mathrm{H}])=\mathrm{C} 2[\mathrm{H}]) \mathrm{N}([\mathrm{H}])[\mathrm{H}]) \mathrm{C}=3 \mathrm{C}(=\mathrm{NOC}=3 \mathrm{C}([\mathrm{H}])([\mathrm{H}])[\mathrm{H}]) \mathrm{C} 4=\mathrm{C}([\mathrm{H}]) \mathrm{C}([\mathrm{H}])=\mathrm{C}( \\
\mathrm{OC}([\mathrm{H}])([\mathrm{H}])[\mathrm{H}]) \mathrm{C}([\mathrm{H}])=\mathrm{C} 4[\mathrm{H}]\end{array}$ & 1.745 & 1.995 \\
\hline Calib24 & $\begin{array}{l}\mathrm{O}=\mathrm{C}(\mathrm{N}([\mathrm{H}]) \mathrm{C}(=\mathrm{NC}([\mathrm{H}])([\mathrm{H}]) \mathrm{C} 1=\mathrm{C}([\mathrm{H}]) \mathrm{C}(=\mathrm{C}([\mathrm{H}]) \mathrm{C}([\mathrm{Cl}])=\mathrm{C} 1[\mathrm{H}]) \mathrm{C}=2 \mathrm{C}([\mathrm{H}])=\mathrm{C}([\mathrm{H} \\
])[\mathrm{S}] \mathrm{C}=2[\mathrm{H}]) \mathrm{N}([\mathrm{H}])[\mathrm{H}]) \mathrm{C}=3 \mathrm{C}(=\mathrm{NOC}=3 \mathrm{C}([\mathrm{H}])([\mathrm{H}])[\mathrm{H}]) \mathrm{C} 4=\mathrm{C}([\mathrm{H}]) \mathrm{C}([\mathrm{H}])=\mathrm{C}(\mathrm{OC}([\mathrm{H} \\
])([\mathrm{H}])[\mathrm{H}]) \mathrm{C}([\mathrm{H}])=\mathrm{C} 4[\mathrm{H}]\end{array}$ & 1.921 & 2.576 \\
\hline Calib25 & $\begin{array}{l}\mathrm{O}=\mathrm{C}(\mathrm{N}([\mathrm{H}]) \mathrm{C}(=\mathrm{NC}([\mathrm{H}])([\mathrm{H}]) \mathrm{C} 1=\mathrm{C}([\mathrm{H}]) \mathrm{C}(=\mathrm{C}([\mathrm{H}]) \mathrm{C}([\mathrm{Cl}])=\mathrm{C} 1[\mathrm{H}]) \mathrm{C}([\mathrm{H}])=\mathrm{C}([\mathrm{H}])[\mathrm{H} \\
]) \mathrm{N}([\mathrm{H}])[\mathrm{H}]) \mathrm{C}=2 \mathrm{C}(=\mathrm{NOC}=2 \mathrm{C}([\mathrm{H}])([\mathrm{H}])[\mathrm{H}]) \mathrm{C} 3=\mathrm{C}([\mathrm{H}]) \mathrm{C}([\mathrm{H}])=\mathrm{C}(\mathrm{OC}([\mathrm{H}])([\mathrm{H}])[\mathrm{H}]) \\
\mathrm{C}([\mathrm{H}])=\mathrm{C} 3[\mathrm{H}]\end{array}$ & 2.367 & 2.071 \\
\hline Calib26 & $\begin{array}{l}\mathrm{O}=\mathrm{C}(\mathrm{N}([\mathrm{H}]) \mathrm{C}(=\mathrm{NC}([\mathrm{H}])([\mathrm{H}]) \mathrm{C} 1=\mathrm{C}([\mathrm{H}]) \mathrm{C}([\mathrm{Cl}])=\mathrm{C}(\mathrm{O}[\mathrm{H}]) \mathrm{C}([\mathrm{Cl}])=\mathrm{C} 1[\mathrm{H}]) \mathrm{N}([\mathrm{H}])[\mathrm{H}]) \\
\mathrm{C}=2 \mathrm{C}(=\mathrm{NOC}=2 \mathrm{C}([\mathrm{H}])([\mathrm{H}])[\mathrm{H}]) \mathrm{C} 3=\mathrm{C}([\mathrm{H}]) \mathrm{C}([\mathrm{H}])=\mathrm{C}(\mathrm{OC}([\mathrm{H}])([\mathrm{H}])[\mathrm{H}]) \mathrm{C}([\mathrm{H}])=\mathrm{C} 3[\mathrm{H} \\
]\end{array}$ & 2.319 & 2.491 \\
\hline Calib27 & $\begin{array}{l}\mathrm{O}=\mathrm{C}(\mathrm{N}([\mathrm{H}]) \mathrm{C}(=\mathrm{NC}([\mathrm{H}])([\mathrm{H}]) \mathrm{Cl}=\mathrm{C}([\mathrm{H}]) \mathrm{C}([\mathrm{Cl}])=\mathrm{C}([\mathrm{H}]) \mathrm{C}([\mathrm{Cl}])=\mathrm{C} 1[\mathrm{H}]) \mathrm{N}([\mathrm{H}])[\mathrm{H}]) \mathrm{C} \\
=2 \mathrm{C}(=\mathrm{NOC}=2 \mathrm{C}([\mathrm{H}])([\mathrm{H}])[\mathrm{H}]) \mathrm{C} 3=\mathrm{C}([\mathrm{H}]) \mathrm{C}([\mathrm{H}])=\mathrm{C}([\mathrm{H}]) \mathrm{C}([\mathrm{H}])=\mathrm{C} 3[\mathrm{H}]\end{array}$ & 1.409 & 2.198 \\
\hline Calib28 & $\begin{array}{l}\mathrm{O}=\mathrm{C}(\mathrm{N}([\mathrm{H}]) \mathrm{C}(=\mathrm{NC}([\mathrm{H}])([\mathrm{H}]) \mathrm{C} 1=\mathrm{C}([\mathrm{H}]) \mathrm{C}([\mathrm{Br}])=\mathrm{C}([\mathrm{H}]) \mathrm{C}([\mathrm{Cl}])=\mathrm{C} 1[\mathrm{H}]) \mathrm{N}([\mathrm{H}])[\mathrm{H}]) \mathrm{C} \\
=2 \mathrm{C}(=\mathrm{NOC}=2 \mathrm{C}([\mathrm{H}])([\mathrm{H}])[\mathrm{H}]) \mathrm{C} 3=\mathrm{C}([\mathrm{H}]) \mathrm{C}([\mathrm{H}])=\mathrm{C}(\mathrm{OC}([\mathrm{H}])([\mathrm{H}])[\mathrm{H}]) \mathrm{C}([\mathrm{H}])=\mathrm{C} 3[\mathrm{H}]\end{array}$ & 2.569 & 2.072 \\
\hline Calib29 & $\begin{array}{l}\mathrm{O}=\mathrm{C}(\mathrm{N}([\mathrm{H}]) \mathrm{C}(=\mathrm{NC}([\mathrm{H}])([\mathrm{H}]) \mathrm{C} 1=\mathrm{C}([\mathrm{H}]) \mathrm{C}([\mathrm{Cl}])=\mathrm{C}(\mathrm{C}([\mathrm{Cl}])=\mathrm{C} 1[\mathrm{H}]) \mathrm{N}([\mathrm{H}]) \mathrm{C}(=\mathrm{O}) \mathrm{C}([ \\
\mathrm{H}])([\mathrm{H}]) \mathrm{N}([\mathrm{H}]) \mathrm{C}([\mathrm{H}])([\mathrm{H}]) \mathrm{C}([\mathrm{H}])([\mathrm{H}])[\mathrm{H}]) \mathrm{N}([\mathrm{H}])[\mathrm{H}]) \mathrm{C}=2 \mathrm{C}(=\mathrm{N}[\mathrm{S}] \mathrm{C}=2 \mathrm{C}([\mathrm{H}])([\mathrm{H}])[ \\
\mathrm{H}]) \mathrm{C} 3=\mathrm{C}([\mathrm{H}]) \mathrm{C}([\mathrm{H}])=\mathrm{C}(\mathrm{OC}([\mathrm{H}])([\mathrm{H}])[\mathrm{H}]) \mathrm{C}([\mathrm{H}])=\mathrm{C} 3[\mathrm{H}]\end{array}$ & 4.000 & 3.805 \\
\hline Calib30 & $\begin{array}{l}{[\mathrm{H}] \mathrm{C}=2 \mathrm{C}(\mathrm{O}[\mathrm{H}])=\mathrm{C}([\mathrm{H}]) \mathrm{C}=1 \mathrm{OC}=3 \mathrm{C}(\mathrm{OC}=1 \mathrm{C}=2 \mathrm{O}[\mathrm{H}])=\mathrm{C}([\mathrm{H}]) \mathrm{C}(\mathrm{O}[\mathrm{H}])=\mathrm{C} 4 \mathrm{OC} 5=\mathrm{C}(\mathrm{O}} \\
\mathrm{C}=34) \mathrm{C}(\mathrm{O}[\mathrm{H}])=\mathrm{C}([\mathrm{H}]) \mathrm{C}(\mathrm{O}[\mathrm{H}])=\mathrm{C} 5[\mathrm{H}]\end{array}$ & 1.097 & 1.459 \\
\hline Calib31 & $\begin{array}{l}{[\mathrm{F}] \mathrm{C}=1 \mathrm{C}([\mathrm{H}])=\mathrm{C}(\mathrm{C}([\mathrm{H}])=\mathrm{C}([\mathrm{F}]) \mathrm{C}=1[\mathrm{H}]) \mathrm{C}([\mathrm{H}])([\mathrm{H}])[\mathrm{C} @]([\mathrm{H}])(\mathrm{N}([\mathrm{H}]) \mathrm{C}(=\mathrm{O}) \mathrm{C} 2=\mathrm{C}([} \\
\mathrm{H}]) \mathrm{C}(=\mathrm{C}([\mathrm{H}]) \mathrm{C}(=\mathrm{C} 2[\mathrm{H}]) \mathrm{C}([\mathrm{H}])([\mathrm{H}])[\mathrm{H}]) \mathrm{C}(=\mathrm{O}) \mathrm{N}(\mathrm{C}([\mathrm{H}])([\mathrm{H}]) \mathrm{C}([\mathrm{H}])([\mathrm{H}]) \mathrm{C}([\mathrm{H}])([\mathrm{H}] \\
)[\mathrm{H}]) \mathrm{C}([\mathrm{H}])([\mathrm{H}]) \mathrm{C}([\mathrm{H}])([\mathrm{H}]) \mathrm{C}([\mathrm{H}])([\mathrm{H}])[\mathrm{H}])[\mathrm{C} @]([\mathrm{H}])(\mathrm{O}[\mathrm{H}])[\mathrm{C} @ 3([\mathrm{H}]) \mathrm{N}([\mathrm{H}]) \mathrm{C}([ \\
\mathrm{H}])([\mathrm{H}]) \mathrm{C}([\mathrm{H}])([\mathrm{H}]) \mathrm{N}(\mathrm{C} 3([\mathrm{H}])[\end{array}$ & 5.000 & 3.113 \\
\hline Calib32 & $\begin{array}{l}{[\mathrm{F}] \mathrm{C}=1 \mathrm{C}([\mathrm{H}])=\mathrm{C}(\mathrm{C}([\mathrm{H}])=\mathrm{C}([\mathrm{F}]) \mathrm{C}=1[\mathrm{H}]) \mathrm{C}([\mathrm{H}])([\mathrm{H}])[\mathrm{C} @]([\mathrm{H}])(\mathrm{N}([\mathrm{H}]) \mathrm{C}(=\mathrm{O}) \mathrm{C} 2=\mathrm{C}([} \\
\mathrm{H}]) \mathrm{C}(=\mathrm{C}([\mathrm{H}]) \mathrm{C}(=\mathrm{C} 2[\mathrm{H}]) \mathrm{C}([\mathrm{H}])([\mathrm{H}])[\mathrm{H}]) \mathrm{C}(=\mathrm{O}) \mathrm{N}(\mathrm{C}([\mathrm{H}])([\mathrm{H}]) \mathrm{C}([\mathrm{H}])([\mathrm{H}]) \mathrm{C}([\mathrm{H}])([\mathrm{H}] \\
)[\mathrm{H}]) \mathrm{C}([\mathrm{H}])([\mathrm{H}]) \mathrm{C}([\mathrm{H}])([\mathrm{H}]) \mathrm{C}([\mathrm{H}])([\mathrm{H}])[\mathrm{H}])[\mathrm{C} @]([\mathrm{H}])(\mathrm{O}[\mathrm{H}])[\mathrm{C} @ 3([\mathrm{H}]) \mathrm{N}([\mathrm{H}]) \mathrm{C}([ \\
\mathrm{H}])([\mathrm{H}]) \mathrm{C}([\mathrm{H}])([\mathrm{H}]) \mathrm{N}(\mathrm{C} 3([\mathrm{H}])[\end{array}$ & 4.301 & 3.425 \\
\hline Calib33 & $\begin{array}{l}\mathrm{O}=\mathrm{C} 3 \mathrm{~N}(\mathrm{C}([\mathrm{H}])([\mathrm{H}]) \mathrm{C}([\mathrm{H}])([\mathrm{H}]) \mathrm{C}=5 \mathrm{C}([\mathrm{H}])=\mathrm{C}(\mathrm{OC}=2 \mathrm{C}(\mathrm{OC}([\mathrm{H}])([\mathrm{H}])[\mathrm{H}])=\mathrm{C}([\mathrm{H}]) \mathrm{C}= \\
1 \mathrm{~N}=\mathrm{C}(\mathrm{N}(\mathrm{C}([\mathrm{H}])([\mathrm{H}]) \mathrm{C}=1 \mathrm{C}=2[\mathrm{H}])[\mathrm{C} @ @]([\mathrm{H}])(\mathrm{C}([\mathrm{H}])([\mathrm{H}]) \mathrm{C} 3([\mathrm{H}])[\mathrm{H}])[\mathrm{C} @ @] 4([\mathrm{H} \\
]) \mathrm{C}([\mathrm{H}])([\mathrm{H}]) \mathrm{C}([\mathrm{H}])([\mathrm{H}]) \mathrm{C}([\mathrm{H}])([\mathrm{H}]) \mathrm{C}([\mathrm{H}])([\mathrm{H}]) \mathrm{C} 4([\mathrm{H}])[\mathrm{H}]) \mathrm{N}([\mathrm{H}])[\mathrm{H}]) \mathrm{C}([\mathrm{H}])=\mathrm{C}([ \\
\mathrm{H}]) \mathrm{C}=5[\mathrm{H}])[\mathrm{C} @] 6([\mathrm{H}]) \mathrm{C}([\mathrm{H}])([\mathrm{H}]) \mathrm{C}([\mathrm{H}\end{array}$ & 3.921 & 3.938 \\
\hline Calib34 & $\begin{array}{l}\mathrm{O}=\mathrm{C}(\mathrm{N}([\mathrm{H}])[\mathrm{C} @ @]([\mathrm{H}])(\mathrm{C} 1=\mathrm{C}([\mathrm{H}]) \mathrm{C}([\mathrm{H}])=\mathrm{C}([\mathrm{H}]) \mathrm{C}([\mathrm{H}])=\mathrm{C} 1[\mathrm{H}]) \mathrm{C}([\mathrm{H}])([\mathrm{H}])[\mathrm{H}]) \mathrm{C} \\
2=\mathrm{C}([\mathrm{H}]) \mathrm{C}(=\mathrm{C}([\mathrm{H}]) \mathrm{C}(=\mathrm{C} 2[\mathrm{H}]) \mathrm{N}(\mathrm{C}([\mathrm{H}])([\mathrm{H}])[\mathrm{H}])[\mathrm{S}](=\mathrm{O})(=\mathrm{O}) \mathrm{C}([\mathrm{H}])([\mathrm{H}])[\mathrm{H}]) \mathrm{C}(=\mathrm{O}) \\
\mathrm{N}([\mathrm{H}])[\mathrm{C} @]([\mathrm{H}])([\mathrm{C} @]]([\mathrm{H}])(\mathrm{O}[\mathrm{H}]) \mathrm{C}([\mathrm{H}])([\mathrm{H}])[\mathrm{C} @ @]([\mathrm{H}])(\mathrm{OC}([\mathrm{H}])([\mathrm{H}])[\mathrm{H}]) \mathrm{C}(=\mathrm{O}\end{array}$ & 2.678 & 3.264 \\
\hline
\end{tabular}




\begin{tabular}{|c|c|c|c|}
\hline & ) $\mathrm{N}([\mathrm{H}])[\mathrm{C} @]([\mathrm{H}])(\mathrm{C}(=\mathrm{O}) \mathrm{N}([\mathrm{H}]) \mathrm{C}([\mathrm{H}])$ & & \\
\hline Calib35 & $\begin{array}{l}\mathrm{O}=\mathrm{C}(\mathrm{N}([\mathrm{H}])[\mathrm{C} @ @]([\mathrm{H}])(\mathrm{C} 1=\mathrm{C}([\mathrm{H}]) \mathrm{C}([\mathrm{H}])=\mathrm{C}([\mathrm{H}]) \mathrm{C}([\mathrm{H}])=\mathrm{C} 1[\mathrm{H}]) \mathrm{C}([\mathrm{H}])([\mathrm{H}])[\mathrm{H}]) \mathrm{C} \\
2=\mathrm{C}([\mathrm{H}]) \mathrm{C}(=\mathrm{C}([\mathrm{H}]) \mathrm{C}(=\mathrm{C} 2[\mathrm{H}]) \mathrm{N}(\mathrm{C}([\mathrm{H}])([\mathrm{H}])[\mathrm{H}])[\mathrm{S}](=\mathrm{O})(=\mathrm{O}) \mathrm{C}([\mathrm{H}])([\mathrm{H}])[\mathrm{H}]) \mathrm{C}(=\mathrm{O}) \\
\mathrm{N}([\mathrm{H}])[\mathrm{C} @]([\mathrm{H}])([\mathrm{C} @ @]([\mathrm{H}])(\mathrm{O}[\mathrm{H}]) \mathrm{C}([\mathrm{H}])([\mathrm{H}])[\mathrm{C} @ @]([\mathrm{H}])(\mathrm{OC}([\mathrm{H}])([\mathrm{H}]) \mathrm{C}([\mathrm{H}]) \\
=\mathrm{C}([\mathrm{H}])[\mathrm{H}]) \mathrm{C}(=\mathrm{O}) \mathrm{N}([\mathrm{H}])[\mathrm{C} @([\mathrm{H}])(\mathrm{C}(=\end{array}$ & 4.481 & 3.325 \\
\hline Calib36 & $\begin{array}{l}\mathrm{O}=\mathrm{C} 1 \mathrm{C}([\mathrm{H}])=\mathrm{C}(\mathrm{OC}=2 / \mathrm{C} 1=\mathrm{C}(/ \mathrm{O}[\mathrm{H}]) \mathrm{C}([\mathrm{H}])=\mathrm{C}(\mathrm{O}[\mathrm{H}]) \mathrm{C}=2[\mathrm{H}]) \mathrm{C} 3=\mathrm{C}(\mathrm{O}[\mathrm{H}]) \mathrm{C}([\mathrm{H}])=\mathrm{C} \\
(\mathrm{O}[\mathrm{H}]) \mathrm{C}([\mathrm{H}])=\mathrm{C} 3[\mathrm{H}]\end{array}$ & 0.083 & 0.503 \\
\hline Calib37 & $\begin{array}{l}\mathrm{O}=\mathrm{C} 3 \mathrm{~N}([\mathrm{H}])[\mathrm{C} @]([\mathrm{H}])(\mathrm{C}(=\mathrm{O}) \mathrm{N}([\mathrm{H}])[\mathrm{C} @]([\mathrm{H}])([\mathrm{C} @]([\mathrm{H}])(\mathrm{O}[\mathrm{H}]) \mathrm{C}([\mathrm{H}])([\mathrm{H}]) \mathrm{N}([\mathrm{H}] \\
) \mathrm{C}([\mathrm{H}])([\mathrm{H}]) \mathrm{C} 1=\mathrm{C}([\mathrm{H}]) \mathrm{C}(=\mathrm{C}([\mathrm{H}]) \mathrm{C}([\mathrm{H}])=\mathrm{C} 1[\mathrm{H}]) \mathrm{C}(\mathrm{C}([\mathrm{H}])([\mathrm{H}])[\mathrm{H}])(\mathrm{C}([\mathrm{H}])([\mathrm{H}])[\mathrm{H}]) \\
\mathrm{C}([\mathrm{H}])([\mathrm{H}])[\mathrm{H}]) \mathrm{C}([\mathrm{H}])([\mathrm{H}]) \mathrm{C} 2=\mathrm{C}([\mathrm{H}]) \mathrm{C}([\mathrm{F}])=\mathrm{C}([\mathrm{H}]) \mathrm{C}([\mathrm{F}])=\mathrm{C} 2[\mathrm{H}]) \mathrm{C}([\mathrm{H}])([\mathrm{H}]) \mathrm{N} 3 \mathrm{C} \\
([\mathrm{H}])([\mathrm{H}]) \mathrm{C} 4=\mathrm{C}([\mathrm{H}]) \mathrm{C}([\mathrm{F}])=\mathrm{C}([\mathrm{H}\end{array}$ & 4.699 & 3.185 \\
\hline Calib38 & $\begin{array}{l}\mathrm{O}=\mathrm{C} 3 \mathrm{~N}(\mathrm{C}([\mathrm{H}])([\mathrm{H}])[\mathrm{C} @ @]([\mathrm{H}])(\mathrm{C}(=\mathrm{O}) \mathrm{N}([\mathrm{H}])[\mathrm{C} @]([\mathrm{H}])([\mathrm{C} @]([\mathrm{H}])(\mathrm{O}[\mathrm{H}]) \mathrm{C}([\mathrm{H}])([ \\
\mathrm{H}]) \mathrm{N}([\mathrm{H}]) \mathrm{C}([\mathrm{H}])([\mathrm{H}]) \mathrm{C} 1=\mathrm{C}([\mathrm{H}]) \mathrm{C}(=\mathrm{C}([\mathrm{H}]) \mathrm{C}([\mathrm{H}])=\mathrm{C} 1[\mathrm{H}]) \mathrm{N}(\mathrm{C}([\mathrm{H}])([\mathrm{H}])[\mathrm{H}]) \mathrm{C}([\mathrm{H}]) \\
([\mathrm{H}])[\mathrm{H}]) \mathrm{C}([\mathrm{H}])([\mathrm{H}]) \mathrm{C} 2=\mathrm{C}([\mathrm{H}]) \mathrm{C}([\mathrm{H}])=\mathrm{C}([\mathrm{H}]) \mathrm{C}([\mathrm{H}])=\mathrm{C} 2[\mathrm{H}]) \mathrm{C} 3([\mathrm{H}])[\mathrm{H}]) \mathrm{C}([\mathrm{H}])([\mathrm{H} \\
]) \mathrm{C} 4=\mathrm{C}([\mathrm{H}]) \mathrm{C}([\mathrm{H}])=\mathrm{C}([\mathrm{H}]) \mathrm{C}([\mathrm{H}])=\mathrm{C}\end{array}$ & 1.659 & 1.724 \\
\hline Calib39 & $\begin{array}{l}\mathrm{O}=\mathrm{C} 3 \mathrm{~N}([\mathrm{H}])[\mathrm{C} @]([\mathrm{H}])(\mathrm{C}(=\mathrm{O}) \mathrm{N}([\mathrm{H}])[\mathrm{C} @]([\mathrm{H}])([\mathrm{C} @]([\mathrm{H}])(\mathrm{O}[\mathrm{H}]) \mathrm{C}([\mathrm{H}])([\mathrm{H}]) \mathrm{N}([\mathrm{H}] \\
) \mathrm{C}([\mathrm{H}])([\mathrm{H}]) \mathrm{C} 1=\mathrm{C}([\mathrm{H}]) \mathrm{C}(=\mathrm{C}([\mathrm{H}]) \mathrm{C}([\mathrm{H}])=\mathrm{C} 1[\mathrm{H}]) \mathrm{N}(\mathrm{C}([\mathrm{H}])([\mathrm{H}])[\mathrm{H}]) \mathrm{C}([\mathrm{H}])([\mathrm{H}])[\mathrm{H}]) \\
\mathrm{C}([\mathrm{H}])([\mathrm{H}]) \mathrm{C} 2=\mathrm{C}([\mathrm{H}]) \mathrm{C}([\mathrm{H}])=\mathrm{C}([\mathrm{H}]) \mathrm{C}([\mathrm{H}])=\mathrm{C} 2[\mathrm{H}]) \mathrm{C}([\mathrm{H}])([\mathrm{H}]) \mathrm{N} 3 \mathrm{C}([\mathrm{H}])([\mathrm{H}]) \mathrm{C}([\mathrm{H} \\
])([\mathrm{H}]) \mathrm{C} 4=\mathrm{C}([\mathrm{H}]) \mathrm{C}([\mathrm{H}])=\mathrm{C}([\mathrm{H}]) \mathrm{C}([\end{array}$ & 1.167 & 1.823 \\
\hline Calib40 & $\begin{array}{l}\mathrm{O}=\mathrm{C} 1 \mathrm{~N}(\mathrm{C}(=\mathrm{N}[\mathrm{C} @] 1(\mathrm{C}=2 \mathrm{C}([\mathrm{H}])=\mathrm{C}([\mathrm{H}]) \mathrm{C}([\mathrm{H}])=\mathrm{C}([\mathrm{H}]) \mathrm{C}=2[\mathrm{H}]) \mathrm{C} 3=\mathrm{C}([\mathrm{H}]) \mathrm{C}(=\mathrm{C}([\mathrm{H} \\
]) \mathrm{C}([\mathrm{H}])=\mathrm{C} 3[\mathrm{H}]) \mathrm{C} 4=\mathrm{C}([\mathrm{H}]) \mathrm{C}([\mathrm{H}])=\mathrm{NC}([\mathrm{H}])=\mathrm{C} 4[\mathrm{H}]) \mathrm{N}([\mathrm{H}])[\mathrm{H}]) \mathrm{C}([\mathrm{H}])([\mathrm{H}])[\mathrm{H}]\end{array}$ & 1.420 & 2.309 \\
\hline Calib41 & $\begin{array}{l}\mathrm{O}=\mathrm{C} 1 \mathrm{~N}(\mathrm{C}(=\mathrm{N}[\mathrm{C} @ @] 1(\mathrm{C}=2 \mathrm{C}([\mathrm{H}])=\mathrm{C}([\mathrm{H}]) \mathrm{C}([\mathrm{H}])=\mathrm{C}([\mathrm{H}]) \mathrm{C}=2[\mathrm{H}]) \mathrm{C} 3=\mathrm{C}([\mathrm{H}]) \mathrm{C}(=\mathrm{C}([ \\
\mathrm{H}]) \mathrm{C}([\mathrm{H}])=\mathrm{C} 3[\mathrm{H}]) \mathrm{C} 4=\mathrm{C}([\mathrm{H}]) \mathrm{N}=\mathrm{C}([\mathrm{H}]) \mathrm{C}(=\mathrm{C} 4[\mathrm{H}]) \mathrm{C}([\mathrm{H}])([\mathrm{H}])[\mathrm{H}]) \mathrm{N}([\mathrm{H}])[\mathrm{H}]) \mathrm{C}([\mathrm{H}]) \\
([\mathrm{H}])[\mathrm{H}]\end{array}$ & 2.367 & 2.645 \\
\hline Calib42 & $\begin{array}{l}\mathrm{O}=\mathrm{C} 1 \mathrm{~N}(\mathrm{C}(=\mathrm{N}[\mathrm{C} @ @] 1(/ \mathrm{C} 2=\mathrm{C}(\mathrm{VH}]) \mathrm{C}([\mathrm{H}])=\mathrm{C}([\mathrm{H}]) \mathrm{C}([\mathrm{H}])=\mathrm{C} 2[\mathrm{H}]) \mathrm{C} 3=\mathrm{C}([\mathrm{H}]) \mathrm{C}(=\mathrm{C}([ \\
\mathrm{H}]) \mathrm{C}([\mathrm{H}])=\mathrm{C} 3[\mathrm{H}]) \mathrm{C} 4=\mathrm{C}([\mathrm{H}]) \mathrm{N}=\mathrm{C}([\mathrm{H}]) \mathrm{C}(\mathrm{C \# N})=\mathrm{C} 4[\mathrm{H}]) \mathrm{N}([\mathrm{H}])[\mathrm{H}]) \mathrm{C}([\mathrm{H}])([\mathrm{H}])[\mathrm{H}]\end{array}$ & 2.328 & 2.634 \\
\hline Calib43 & $\begin{array}{l}\mathrm{O}=\mathrm{C}(\mathrm{N}([\mathrm{C} @] 1([\mathrm{H}]) \mathrm{C}([\mathrm{H}])([\mathrm{H}]) \mathrm{C}([\mathrm{H}])([\mathrm{H}]) \mathrm{C}([\mathrm{H}])([\mathrm{H}]) \mathrm{C}([\mathrm{H}])([\mathrm{H}]) \mathrm{C} 1([\mathrm{H}])[\mathrm{H}]) \mathrm{C}([\mathrm{H} \\
])([\mathrm{H}]) \mathrm{C}=2 \mathrm{~N}=\mathrm{C}([\mathrm{S}] \mathrm{C}=2[\mathrm{H}]) \mathrm{C}([\mathrm{H}])([\mathrm{H}]) \mathrm{OC}([\mathrm{H}])([\mathrm{H}]))[\mathrm{H}]) \mathrm{C}([\mathrm{H}])([\mathrm{H}]) \mathrm{C}([\mathrm{H}])([\mathrm{H}])[\mathrm{C} \\
@]([\mathrm{H}])(\mathrm{N} 5 \mathrm{C}(=\mathrm{N} / \mathrm{C} 4=\mathrm{C}(\backslash[\mathrm{H}]) \mathrm{C}([\mathrm{H}])=\mathrm{C}(\mathrm{O} / \mathrm{C} 3=\mathrm{C}(\backslash \mathrm{H}]) \mathrm{C}([\mathrm{H}])=\mathrm{C}([\mathrm{H}]) \mathrm{C}([\mathrm{H}])=\mathrm{C} 3[\mathrm{H}]) \\
\mathrm{C}([\mathrm{H}])=\mathrm{C} 4 \mathrm{C} 5([\mathrm{H}])[\mathrm{H}]) \mathrm{N}([\mathrm{H}])[\mathrm{H}])[\end{array}$ & 3.886 & 3.501 \\
\hline Calib44 & $\begin{array}{l}\mathrm{O}=\mathrm{C} 3 \mathrm{~N}(\mathrm{C}(=\mathrm{N}[\mathrm{C} @](\mathrm{C}=2[\mathrm{~S}] \mathrm{C}([\mathrm{H}])=\mathrm{C}(/ \mathrm{C} 1=\mathrm{C}(\backslash[\mathrm{H}]) \mathrm{C}(\mathrm{C \# CC}([\mathrm{H}])([\mathrm{H}])[\mathrm{H}])=\mathrm{C}([\mathrm{H}]) \mathrm{N} \\
=\mathrm{C} 1[\mathrm{H}]) \mathrm{C}=2[\mathrm{H}])(\mathrm{C} 3([\mathrm{H}])[\mathrm{H}]) \mathrm{C}([\mathrm{H}])([\mathrm{H}])[\mathrm{H}]) \mathrm{N}([\mathrm{H}])[\mathrm{H}]) \mathrm{C}([\mathrm{H}])([\mathrm{H}])[\mathrm{H}]\end{array}$ & 4.108 & 3.728 \\
\hline Calib45 & $\begin{array}{l}\mathrm{O}=\mathrm{C}(\mathrm{N}([\mathrm{H}]) \mathrm{C}([\mathrm{H}])([\mathrm{H}]) \mathrm{C}([\mathrm{H}])([\mathrm{H}]) \mathrm{C}([\mathrm{H}])([\mathrm{H}]) \mathrm{C}([\mathrm{H}])([\mathrm{H}]) \mathrm{C}([\mathrm{H}])([\mathrm{H}]) \mathrm{C}([\mathrm{H}])([\mathrm{H}]) \mathrm{C} \\
([\mathrm{H}])([\mathrm{H}]) \mathrm{C}([\mathrm{H}])([\mathrm{H}]) \mathrm{C}([\mathrm{H}])([\mathrm{H}]) \mathrm{C}([\mathrm{H}])([\mathrm{H}]) \mathrm{N}([\mathrm{H}]) \mathrm{C} 2=\mathrm{C} 1 \mathrm{C}([\mathrm{H}])=\mathrm{C}([\mathrm{H}]) \mathrm{C}([\mathrm{H}])=\mathrm{C}( \\
[\mathrm{H}]) \mathrm{C} 1=\mathrm{NC} 3=\mathrm{C} 2 \mathrm{C}([\mathrm{H}])([\mathrm{H}]) \mathrm{C}([\mathrm{H}])([\mathrm{H}]) \mathrm{C}([\mathrm{H}])([\mathrm{H}]) \mathrm{C} 3([\mathrm{H}])[\mathrm{H}]) \mathrm{C}=4 \mathrm{OC}=5 \mathrm{C}(\mathrm{C}(=\mathrm{O}) \\
\mathrm{C}=4[\mathrm{H}])=\mathrm{C}(\mathrm{O}[\mathrm{H}]) \mathrm{C}([\mathrm{H}])=\mathrm{C}(\mathrm{O}[\mathrm{H}])\end{array}$ & 1.975 & 1.955 \\
\hline Calib46 & $\begin{array}{l}\mathrm{O}=\mathrm{C}(\mathrm{N}([\mathrm{H}]) \mathrm{C}([\mathrm{H}])([\mathrm{H}]) \mathrm{C}([\mathrm{H}])([\mathrm{H}]) \mathrm{C}([\mathrm{H}])([\mathrm{H}]) \mathrm{C}([\mathrm{H}])([\mathrm{H}]) \mathrm{C}([\mathrm{H}])([\mathrm{H}]) \mathrm{C}([\mathrm{H}])([\mathrm{H}]) \mathrm{C} \\
([\mathrm{H}])([\mathrm{H}]) \mathrm{C}([\mathrm{H}])([\mathrm{H}]) \mathrm{C}([\mathrm{H}])([\mathrm{H}]) \mathrm{C}([\mathrm{H}])([\mathrm{H}]) \mathrm{N}([\mathrm{H}]) \mathrm{C} 2=\mathrm{C} 1 \mathrm{C}([\mathrm{H}])=\mathrm{C}([\mathrm{H}]) \mathrm{C}([\mathrm{Cl}])=\mathrm{C} \\
([\mathrm{H}]) \mathrm{C} 1=\mathrm{NC} 3=\mathrm{C} 2 \mathrm{C}([\mathrm{H}])([\mathrm{H}]) \mathrm{C}([\mathrm{H}])([\mathrm{H}]) \mathrm{C}([\mathrm{H}])([\mathrm{H}]) \mathrm{C} 3([\mathrm{H}])[\mathrm{H}]) \mathrm{C}=4 \mathrm{OC}=5 \mathrm{C}(\mathrm{C}(=\mathrm{O} \\
) \mathrm{C}=4[\mathrm{H}])=\mathrm{C}([\mathrm{H}]) \mathrm{C}(\mathrm{OC}([\mathrm{H}])([\mathrm{H}])\end{array}$ & 1.374 & 2.116 \\
\hline Calib47 & $\mathrm{O}=[\mathrm{S}] 3(=\mathrm{O}) \mathrm{N}(\mathrm{C}(=\mathrm{N}[\mathrm{C} @ @](\mathrm{C}=1[\mathrm{~S}] / \mathrm{C}(=\mathrm{C}(/[\mathrm{H}]) \mathrm{C}=1[\mathrm{Cl}]) \mathrm{C} 2=\mathrm{C}([\mathrm{H}]) \mathrm{C}(\mathrm{C \# CC}([\mathrm{H}])([$ & 4.620 & 4.484 \\
\hline
\end{tabular}




\begin{tabular}{|c|c|c|c|}
\hline & $\mathrm{H}])[\mathrm{H}])=\mathrm{C}([\mathrm{H}]) \mathrm{N}=\mathrm{C} 2[\mathrm{H}])(\mathrm{C}([\mathrm{H}])([\mathrm{H}])[\mathrm{H}]) \mathrm{C} 3([\mathrm{H}])[\mathrm{H}]) \mathrm{N}([\mathrm{H}])[\mathrm{H}]) \mathrm{C}([\mathrm{H}])([\mathrm{H}])[\mathrm{H}]$ & & \\
\hline Calib48 & $\begin{array}{l}\mathrm{O}=\mathrm{C}(\mathrm{N}([\mathrm{H}]) \mathrm{C} 3=\mathrm{C}([\mathrm{H}]) \mathrm{C}([\mathrm{H}])=\mathrm{C} 2 \mathrm{OC}([\mathrm{C} @] 4([\mathrm{C} @ @] 1(\mathrm{~N}=\mathrm{C}(\mathrm{OC} 1([\mathrm{H}])[\mathrm{H}]) \mathrm{N}([\mathrm{H}])[ \\
\mathrm{H}]) \mathrm{C} 2=\mathrm{C} 3[\mathrm{H}]) \mathrm{C}([\mathrm{H}])([\mathrm{H}]) \mathrm{OC} 4([\mathrm{H}])[\mathrm{H}])(\mathrm{C}([\mathrm{H}])([\mathrm{H}])[\mathrm{H}]) \mathrm{C}([\mathrm{H}])([\mathrm{H}])[\mathrm{H}]) \mathrm{C}=5 / \mathrm{N}=\mathrm{C}(/ \\
[\mathrm{H}]) \mathrm{C}([\mathrm{F}])=\mathrm{C}([\mathrm{H}]) \mathrm{C}=5[\mathrm{H}]\end{array}$ & 2.932 & 2.825 \\
\hline Calib49 & $\begin{array}{l}{[\mathrm{H}] / \mathrm{C} 4=\mathrm{C}(/ \mathrm{C}([\mathrm{H}])=\mathrm{C} 1 \mathrm{C}(\mathrm{O}[\mathrm{C} @]([\mathrm{H}])([\mathrm{C} @] 3([\mathrm{C} @] 12 \mathrm{~N}=\mathrm{C}(\mathrm{OC} 2([\mathrm{H}])[\mathrm{H}]) \mathrm{N}([\mathrm{H}])[\mathrm{H}])} \\
\mathrm{C}([\mathrm{H}])([\mathrm{H}]) \mathrm{OC} 3([\mathrm{H}])[\mathrm{H}]) \mathrm{C}([\mathrm{H}])([\mathrm{H}]) \mathrm{C}([\mathrm{H}])([\mathrm{H}]) \mathrm{C}([\mathrm{H}])([\mathrm{H}])[\mathrm{H}])=\mathrm{C} 4[\mathrm{H}]) \mathrm{C} 5=\mathrm{C}([\mathrm{H}]) \\
\mathrm{N}=\mathrm{C}([\mathrm{H}]) \mathrm{C}(\mathrm{C \# CC}([\mathrm{H}])([\mathrm{H}])[\mathrm{H}])=\mathrm{C} 5[\mathrm{H}]\end{array}$ & 3.056 & 2.813 \\
\hline Calib50 & $\begin{array}{l}\mathrm{F}] \mathrm{C}([\mathrm{F}])([\mathrm{F}]) \mathrm{C}([\mathrm{H}])([\mathrm{H}]) \mathrm{C}([\mathrm{H}])([\mathrm{H}])[\mathrm{C} @] 3([\mathrm{H}]) \mathrm{O} / \mathrm{C} 1=\mathrm{C}(\backslash \mathrm{H}]) \mathrm{C}([\mathrm{H}])=\mathrm{C}(\mathrm{C}([\mathrm{H}])=\mathrm{C} \\
1[\mathrm{C} @ @] 2(\mathrm{~N}=\mathrm{C}(\mathrm{OC} 2([\mathrm{H}])[\mathrm{H}]) \mathrm{N}([\mathrm{H}])[\mathrm{H}])[\mathrm{C} @] 34 \mathrm{C}([\mathrm{H}])([\mathrm{H}]) \mathrm{OC} 4([\mathrm{H}])[\mathrm{H}]) \mathrm{C} 5=\mathrm{C}([\mathrm{H} \\
]) \mathrm{N}=\mathrm{C}([\mathrm{H}]) \mathrm{C}(\mathrm{C \# CC}([\mathrm{H}])([\mathrm{H}])[\mathrm{H}])=\mathrm{C} 5[\mathrm{H}]\end{array}$ & 2.061 & 2.233 \\
\hline Calib51 & $\begin{array}{l}\mathrm{O}=\mathrm{C}(\mathrm{N}([\mathrm{H}]) \mathrm{C} 3=\mathrm{C}([\mathrm{H}]) \mathrm{C}([\mathrm{H}])=\mathrm{C} 2 \mathrm{OC}([\mathrm{H}])([\mathrm{H}])[\mathrm{C} @ @] 4([\mathrm{C} @ @] 1(\mathrm{~N}=\mathrm{C}(\mathrm{OC} 1([\mathrm{H}])[ \\
\mathrm{H}]) \mathrm{N}([\mathrm{H}])[\mathrm{H}]) \mathrm{C} 2=\mathrm{C} 3[\mathrm{H}]) \mathrm{C}([\mathrm{H}])([\mathrm{H}]) \mathrm{C} 4([\mathrm{H}])[\mathrm{H}]) \mathrm{C} 5=\mathrm{NC}([\mathrm{H}])=\mathrm{C}(\mathrm{CHN}) \mathrm{C}([\mathrm{H}])=\mathrm{C} 5 \mathrm{C} \\
([\mathrm{H}])([\mathrm{H}])[\mathrm{H}]\end{array}$ & 2.668 & 2.520 \\
\hline Calib52 & $\begin{array}{l}\mathrm{O}=\mathrm{C}(\mathrm{N}([\mathrm{H}]) \mathrm{C} 3=\mathrm{C}([\mathrm{H}]) \mathrm{C}([\mathrm{H}])=\mathrm{C} 2 \mathrm{OC}([\mathrm{C} @] 4([\mathrm{C} @ @] 1(\mathrm{~N}=\mathrm{C}(\mathrm{OC} 1([\mathrm{H}])[\mathrm{H}]) \mathrm{N}([\mathrm{H}])[ \\
\mathrm{H}]) \mathrm{C} 2=\mathrm{C} 3[\mathrm{H}]) \mathrm{C}([\mathrm{H}])([\mathrm{H}]) \mathrm{C} 4([\mathrm{H}])[\mathrm{H}])(\mathrm{C}([\mathrm{H}])([\mathrm{H}])[\mathrm{H}]) \mathrm{C}([\mathrm{H}])([\mathrm{H}])[\mathrm{H}]) \mathrm{C} 5=\mathrm{NC}([\mathrm{H}]) \\
=\mathrm{C}(/ \mathrm{N}=\mathrm{C} 5 /[\mathrm{H}]) \mathrm{OC}([\mathrm{H}])([\mathrm{H}])[\mathrm{H}]\end{array}$ & 3.213 & 3.362 \\
\hline Calib53 & $\begin{array}{l}\mathrm{O}=\mathrm{C}(\mathrm{N}([\mathrm{H}]) \mathrm{C} 3=\mathrm{C}([\mathrm{H}]) \mathrm{C}([\mathrm{H}])=\mathrm{C} 2 \mathrm{OC}([\mathrm{C} @ @] 4([\mathrm{C} @] 1(\mathrm{~N}=\mathrm{C}(\mathrm{OC} 1([\mathrm{H}])[\mathrm{H}]) \mathrm{N}([\mathrm{H}])[ \\
\mathrm{H}]) \mathrm{C} 2=\mathrm{C} 3[\mathrm{H}]) \mathrm{C}([\mathrm{H}])([\mathrm{H}]) \mathrm{OC} 4([\mathrm{H}])[\mathrm{H}])(\mathrm{C}([\mathrm{H}])([\mathrm{H}])[\mathrm{H}]) \mathrm{C}([\mathrm{H}])([\mathrm{H}])[\mathrm{H}]) \mathrm{C}=5 / \mathrm{N}=\mathrm{C}(/ \\
[\mathrm{H}]) \mathrm{C}([\mathrm{Cl}])=\mathrm{C}([\mathrm{H}]) \mathrm{C}=5[\mathrm{H}]\end{array}$ & 0.520 & 1.689 \\
\hline Calib54 & $\begin{array}{l}{[\mathrm{F}] \mathrm{C}=1 \mathrm{C}([\mathrm{H}])=\mathrm{C}(\mathrm{C}([\mathrm{H}])=\mathrm{C}([\mathrm{F}]) \mathrm{C}=1[\mathrm{H}]) \mathrm{C}([\mathrm{H}])([\mathrm{H}])[\mathrm{C} @]([\mathrm{H}])(\mathrm{N}([\mathrm{H}]) \mathrm{C}(=\mathrm{O}) \mathrm{C} 2=\mathrm{C}([} \\
\mathrm{H}]) \mathrm{C}(=\mathrm{C}([\mathrm{H}]) \mathrm{C}(=\mathrm{C} 2[\mathrm{H}]) \mathrm{C}([\mathrm{H}])([\mathrm{H}])[\mathrm{H}]) \mathrm{C}(=\mathrm{O}) \mathrm{N}(\mathrm{C}([\mathrm{H}])([\mathrm{H}]) \mathrm{C}([\mathrm{H}])([\mathrm{H}]) \mathrm{C}([\mathrm{H}])([\mathrm{H}] \\
)[\mathrm{H}]) \mathrm{C}([\mathrm{H}])([\mathrm{H}]) \mathrm{C}([\mathrm{H}])([\mathrm{H}]) \mathrm{C}([\mathrm{H}])([\mathrm{H}])[\mathrm{H}])[\mathrm{C} @]([\mathrm{H}])(\mathrm{O}[\mathrm{H}])[\mathrm{C} @] 3([\mathrm{H}]) \mathrm{N}([\mathrm{H}]) \mathrm{C}([ \\
\mathrm{H}])([\mathrm{H}]) \mathrm{C}([\mathrm{H}])([\mathrm{H}]) \mathrm{N}(\mathrm{C} 3([\mathrm{H}])[\end{array}$ & 1.237 & 2.964 \\
\hline Calib55 & $\begin{array}{l}{[\mathrm{F}] \mathrm{C}=1 \mathrm{C}([\mathrm{H}])=\mathrm{C}(\mathrm{C}([\mathrm{H}])=\mathrm{C}([\mathrm{F}]) \mathrm{C}=1[\mathrm{H}]) \mathrm{C}([\mathrm{H}])([\mathrm{H}])[\mathrm{C} @]([\mathrm{H}])(\mathrm{N}([\mathrm{H}]) \mathrm{C}(=\mathrm{O}) \mathrm{C} 2=\mathrm{C}([} \\
\mathrm{H}]) \mathrm{C}(=\mathrm{C}([\mathrm{H}]) \mathrm{C}(=\mathrm{C} 2[\mathrm{H}]) \mathrm{C}([\mathrm{H}])([\mathrm{H}])[\mathrm{H}]) \mathrm{C}(=\mathrm{O}) \mathrm{N}(\mathrm{C}([\mathrm{H}])([\mathrm{H}]) \mathrm{C}([\mathrm{H}])([\mathrm{H}]) \mathrm{C}([\mathrm{H}])([\mathrm{H}] \\
)[\mathrm{H}]) \mathrm{C}([\mathrm{H}])([\mathrm{H}]) \mathrm{C}([\mathrm{H}])([\mathrm{H}]) \mathrm{C}([\mathrm{H}])([\mathrm{H}])[\mathrm{H}])[\mathrm{C} @]([\mathrm{H}])(\mathrm{O}[\mathrm{H}])[\mathrm{C} @] 3([\mathrm{H}]) \mathrm{N}([\mathrm{H}]) \mathrm{C}([ \\
\mathrm{H}])([\mathrm{H}]) \mathrm{C}([\mathrm{H}])([\mathrm{H}]) \mathrm{N}(\mathrm{C} 3([\mathrm{H}])[\end{array}$ & 1.921 & 2.770 \\
\hline Calib56 & $\begin{array}{l}\mathrm{O}=\mathrm{C}(\mathrm{N}([\mathrm{H}])[\mathrm{C} @] 1([\mathrm{H}]) \mathrm{C}([\mathrm{H}])([\mathrm{H}]) \mathrm{C} 1([\mathrm{H}])[\mathrm{H}])[\mathrm{C} @]([\mathrm{H}])(\mathrm{OC}([\mathrm{H}])([\mathrm{H}])[\mathrm{H}]) \mathrm{C}([\mathrm{H}]) \\
([\mathrm{H}])[\mathrm{C} @]([\mathrm{H}])(\mathrm{O}[\mathrm{H}])[\mathrm{C} @ @]([\mathrm{H}])(\mathrm{N}([\mathrm{H}]) \mathrm{C}(=\mathrm{O}) \mathrm{C} 2=\mathrm{C}([\mathrm{H}]) \mathrm{C}(=\mathrm{C}([\mathrm{H}]) \mathrm{C}(=\mathrm{C} 2[\mathrm{H}]) \mathrm{C}( \\
=\mathrm{O}) \mathrm{N}([\mathrm{H}])[\mathrm{C} @ @]([\mathrm{H}])(\mathrm{C} 3=\mathrm{C}([\mathrm{H}]) \mathrm{C}([\mathrm{H}])=\mathrm{C}([\mathrm{H}]) \mathrm{C}([\mathrm{H}])=\mathrm{C} 3[\mathrm{H}]) \mathrm{C}([\mathrm{H}])([\mathrm{H}])[\mathrm{H}]) \mathrm{N}( \\
\mathrm{C}([\mathrm{H}])([\mathrm{H}])[\mathrm{S}](=\mathrm{O})(=\mathrm{O}) \mathrm{C}([\mathrm{H}])([\mathrm{H}])[\mathrm{H}]\end{array}$ & 1.745 & 2.845 \\
\hline Calib57 & $\begin{array}{l}\mathrm{O}=\mathrm{C}(\mathrm{N}([\mathrm{H}]) \mathrm{C}(=\mathrm{NC}([\mathrm{H}])([\mathrm{H}]) \mathrm{C} 1=\mathrm{C}([\mathrm{H}]) \mathrm{C}([\mathrm{Cl}])=\mathrm{C}([\mathrm{Cl}]) \mathrm{C}([\mathrm{H}])=\mathrm{C} 1[\mathrm{H}]) \mathrm{N}([\mathrm{H}])[\mathrm{H}]) \mathrm{C} \\
=2 \mathrm{C}(=\mathrm{NOC}=2 \mathrm{C}([\mathrm{H}])([\mathrm{H}])[\mathrm{H}]) \mathrm{C} 3=\mathrm{C}([\mathrm{H}]) \mathrm{C}([\mathrm{H}])=\mathrm{C}(\mathrm{OC}([\mathrm{H}])([\mathrm{H}])[\mathrm{H}]) \mathrm{C}([\mathrm{H}])=\mathrm{C} 3[\mathrm{H}]\end{array}$ & 2.041 & 2.297 \\
\hline Calib58 & $\begin{array}{l}\mathrm{O}=\mathrm{C}(\mathrm{N}([\mathrm{H}])[\mathrm{C} @]([\mathrm{H}])([\mathrm{C} @]([\mathrm{H}])(\mathrm{O}[\mathrm{H}]) \mathrm{C}([\mathrm{H}])([\mathrm{H}])[\mathrm{C} @]([\mathrm{H}])(\mathrm{C}(=\mathrm{O}) \mathrm{N}([\mathrm{H}])[\mathrm{C} @]( \\
[\mathrm{H}])(\mathrm{C}(=\mathrm{O}) \mathrm{N}([\mathrm{H}]) \mathrm{C}([\mathrm{H}])([\mathrm{H}]) \mathrm{C} 1=\mathrm{C}([\mathrm{H}]) \mathrm{C}([\mathrm{H}])=\mathrm{C}([\mathrm{H}]) \mathrm{C}([\mathrm{H}])=\mathrm{C} 1[\mathrm{H}]) \mathrm{C}([\mathrm{H}])(\mathrm{C}([\mathrm{H}] \\
)([\mathrm{H}])[\mathrm{H}]) \mathrm{C}([\mathrm{H}])([\mathrm{H}])[\mathrm{H}]) \mathrm{C}([\mathrm{H}])([\mathrm{H}])[\mathrm{H}]) \mathrm{C}([\mathrm{H}])([\mathrm{H}]) \mathrm{C}([\mathrm{H}])(\mathrm{C}([\mathrm{H}])([\mathrm{H}])[\mathrm{H}]) \mathrm{C}([\mathrm{H}]) \\
([\mathrm{H}])[\mathrm{H}])[\mathrm{C} @]([\mathrm{H}])(\mathrm{N}([\mathrm{H}]) \mathrm{C}(=\end{array}$ & 4.229 & 2.813 \\
\hline Calib59 & $\begin{array}{l}\mathrm{O}=\mathrm{C}(\mathrm{O}[\mathrm{H}]) \mathrm{C}([\mathrm{H}])([\mathrm{H}]) \mathrm{C}([\mathrm{H}])([\mathrm{H}])[\mathrm{C} @]([\mathrm{H}])(\mathrm{N}([\mathrm{H}])[\mathrm{H}]) \mathrm{C}(=\mathrm{O}) \mathrm{N}([\mathrm{H}])[\mathrm{C} @ @]([\mathrm{H}])( \\
\mathrm{C}(=\mathrm{O}) \mathrm{N}([\mathrm{H}])[\mathrm{C} @]([\mathrm{H}])(\mathrm{C}(=\mathrm{O}) \mathrm{N}([\mathrm{H}])[\mathrm{C} @]([\mathrm{H}])([\mathrm{C} @ @]([\mathrm{H}])(\mathrm{O}[\mathrm{H}]) \mathrm{C}([\mathrm{H}])([\mathrm{H}])[\mathrm{C} \\
@]([\mathrm{H}])(\mathrm{C}(=\mathrm{O}) \mathrm{N}([\mathrm{H}])[\mathrm{C} @]([\mathrm{H}])(\mathrm{C}(=\mathrm{O}) \mathrm{N}([\mathrm{H}])[\mathrm{C} @]([\mathrm{H}])(\mathrm{C}(=\mathrm{O}) \mathrm{N}([\mathrm{H}])[\mathrm{C} @ @]([\mathrm{H}]) \\
(\mathrm{C}(=\mathrm{O}) \mathrm{O}[\mathrm{H}]) \mathrm{C}([\mathrm{H}])([\mathrm{H}]) \mathrm{C} 1=\mathrm{C}([\mathrm{H}]) \mathrm{C}([\mathrm{H}])=\end{array}$ & 4.796 & 4.594 \\
\hline
\end{tabular}




\begin{tabular}{|c|c|c|c|}
\hline Calib60 & $\begin{array}{l}\mathrm{O}=\mathrm{C}(\mathrm{N}([\mathrm{H}])[\mathrm{C} @]([\mathrm{H}])(\mathrm{C}(=\mathrm{O}) \mathrm{N}([\mathrm{H}])[\mathrm{C} @]([\mathrm{H}])([\mathrm{C} @ @]([\mathrm{H}])(\mathrm{O}[\mathrm{H}]) \mathrm{C}([\mathrm{H}])([\mathrm{H}])[\mathrm{C} @ \\
]([\mathrm{H}])(\mathrm{C}(=\mathrm{O}) \mathrm{N}([\mathrm{H}])[\mathrm{C} @]([\mathrm{H}])(\mathrm{C}(=\mathrm{O}) \mathrm{N}([\mathrm{H}]) \mathrm{C}([\mathrm{H}])([\mathrm{H}]) \mathrm{C}([\mathrm{H}])(\mathrm{C}([\mathrm{H}])([\mathrm{H}])[\mathrm{H}]) \mathrm{C}([ \\
\mathrm{H}])([\mathrm{H}])[\mathrm{H}]) \mathrm{C}([\mathrm{H}])(\mathrm{C}([\mathrm{H}])([\mathrm{H}])[\mathrm{H}]) \mathrm{C}([\mathrm{H}])([\mathrm{H}])[\mathrm{H}]) \mathrm{C}([\mathrm{H}])([\mathrm{H}])[\mathrm{H}]) \mathrm{C}([\mathrm{H}])([\mathrm{H}]) \mathrm{C}([ \\
\mathrm{H}])(\mathrm{C}([\mathrm{H}])([\mathrm{H}])[\mathrm{H}]) \mathrm{C}([\mathrm{H}])([\mathrm{H}]\end{array}$ & 2.327 & 3.115 \\
\hline Calib61 & $\begin{array}{l}\mathrm{O}=\mathrm{C} 1 \mathrm{~N}(/ \mathrm{C}(=\mathrm{N} \backslash[\mathrm{H}]) \mathrm{N}([\mathrm{H}])[\mathrm{C} @] 1(\mathrm{C}([\mathrm{H}])([\mathrm{H}]) \mathrm{C}([\mathrm{H}])([\mathrm{H}])[\mathrm{C} @] 2([\mathrm{H}]) \mathrm{C}([\mathrm{H}])([\mathrm{H}]) \mathrm{C}([ \\
\mathrm{H}])([\mathrm{H}]) \mathrm{C}([\mathrm{H}])([\mathrm{H}]) \mathrm{C}([\mathrm{H}])([\mathrm{H}]) \mathrm{C} 2([\mathrm{H}])[\mathrm{H}]) \mathrm{C}([\mathrm{H}])([\mathrm{H}])[\mathrm{C} @] 4([\mathrm{H}]) \mathrm{C}([\mathrm{H}])([\mathrm{H}])[\mathrm{C} @ \\
]([\mathrm{H}])(\mathrm{N}([\mathrm{H}]) \mathrm{C}(=\mathrm{O}) \mathrm{N}([\mathrm{H}]) \mathrm{C} 3=\mathrm{C}([\mathrm{H}]) \mathrm{C}([\mathrm{H}])=\mathrm{C}(\mathrm{CHN}) \mathrm{C}([\mathrm{H}])=\mathrm{C} 3[\mathrm{H}]) \mathrm{C}([\mathrm{H}])([\mathrm{H}]) \mathrm{C}([ \\
\mathrm{H}])([\mathrm{H}]) \mathrm{C} 4([\mathrm{H}])[\mathrm{H}]) \mathrm{C}([\mathrm{H}])([\mathrm{H}])\end{array}$ & 4.523 & 4.248 \\
\hline Calib62 & $\begin{array}{l}\mathrm{O}=\mathrm{C} 1 \mathrm{~N}(/ \mathrm{C}(=\mathrm{N} \backslash[\mathrm{H}]) \mathrm{N}([\mathrm{H}])[\mathrm{C} @] 1(\mathrm{C}([\mathrm{H}])([\mathrm{H}]) \mathrm{C}([\mathrm{H}])([\mathrm{H}])[\mathrm{C} @] 2([\mathrm{H}]) \mathrm{C}([\mathrm{H}])([\mathrm{H}]) \mathrm{C}([ \\
\mathrm{H}])([\mathrm{H}]) \mathrm{C}([\mathrm{H}])([\mathrm{H}]) \mathrm{C}([\mathrm{H}])([\mathrm{H}]) \mathrm{C} 2([\mathrm{H}])[\mathrm{H}]) \mathrm{C}([\mathrm{H}])([\mathrm{H}])[\mathrm{C} @] 4([\mathrm{H}]) \mathrm{C}([\mathrm{H}])([\mathrm{H}])[\mathrm{C} @ \\
]([\mathrm{H}])(\mathrm{N}([\mathrm{H}]) \mathrm{C}(=\mathrm{O}) \mathrm{N}([\mathrm{H}]) \mathrm{C} 3=\mathrm{C}([\mathrm{H}]) \mathrm{C}([\mathrm{H}])=\mathrm{C}([\mathrm{H}]) \mathrm{C}([\mathrm{H}])=\mathrm{C} 3[\mathrm{H}]) \mathrm{C}([\mathrm{H}])([\mathrm{H}]) \mathrm{C}([\mathrm{H} \\
])([\mathrm{H}]) \mathrm{C} 4([\mathrm{H}])[\mathrm{H}]) \mathrm{C}([\mathrm{H}])([\mathrm{H}])\end{array}$ & 3.796 & 3.673 \\
\hline Calib63 & $\begin{array}{l}\mathrm{O}=\mathrm{C} 1 \mathrm{~N}(/ \mathrm{C}(=\mathrm{N} /[\mathrm{H}]) \mathrm{N}([\mathrm{H}])[\mathrm{C} @] 1(\mathrm{C}([\mathrm{H}])([\mathrm{H}]) \mathrm{C}([\mathrm{H}])([\mathrm{H}])[\mathrm{C} @ @] 2([\mathrm{H}]) \mathrm{C}([\mathrm{H}])([\mathrm{H}]) \\
\mathrm{C}([\mathrm{H}])([\mathrm{H}]) \mathrm{C}([\mathrm{H}])([\mathrm{H}]) \mathrm{C}([\mathrm{H}])([\mathrm{H}]) \mathrm{C} 2([\mathrm{H}])[\mathrm{H}]) \mathrm{C}([\mathrm{H}])([\mathrm{H}])[\mathrm{C} @] 3([\mathrm{H}]) \mathrm{C}([\mathrm{H}])([\mathrm{H}]) \mathrm{N} \\
(\mathrm{C}([\mathrm{H}])([\mathrm{H}]) \mathrm{C}([\mathrm{H}])([\mathrm{H}]) \mathrm{C} 3([\mathrm{H}])[\mathrm{H}]) \mathrm{C}([\mathrm{H}])([\mathrm{H}]) \mathrm{C}([\mathrm{H}])([\mathrm{H}]) \mathrm{C}([\mathrm{H}])([\mathrm{H}]) \mathrm{C}([\mathrm{H}])([\mathrm{H}]) \\
\mathrm{C}([\mathrm{H}])([\mathrm{H}])[\mathrm{H}]) \mathrm{C}([\mathrm{H}])([\mathrm{H}])\end{array}$ & 3.119 & 2.925 \\
\hline Calib64 & $\begin{array}{l}\mathrm{O}=[\mathrm{S}] 2(=\mathrm{O}) \mathrm{N}(/ \mathrm{C}(=\mathrm{N} /[\mathrm{H}]) \mathrm{N}([\mathrm{H}])[\mathrm{C} @] 4(/ \mathrm{C} 1=\mathrm{C}(\backslash[\mathrm{H}]) \mathrm{C}([\mathrm{F}])=\mathrm{C}([\mathrm{F}]) \mathrm{C}([\mathrm{H}])=\mathrm{C} 1[\mathrm{~F}])[\mathrm{C} \\
@] 2([\mathrm{H}]) \mathrm{C}([\mathrm{H}])([\mathrm{H}]) \mathrm{N}(/ \mathrm{C} 3=\mathrm{N} / \mathrm{C}(\mathrm{OC}([\mathrm{H}])([\mathrm{H}])[\mathrm{H}])=\mathrm{C}([\mathrm{H}]) \mathrm{C}(=\mathrm{C} 3[\mathrm{H}]) \mathrm{C}([\mathrm{H}])([\mathrm{H}]) \mathrm{O} \\
\mathrm{C}([\mathrm{H}])([\mathrm{H}])[\mathrm{H}]) \mathrm{C} 4([\mathrm{H}])[\mathrm{H}]) \mathrm{C}([\mathrm{H}])([\mathrm{H}])[\mathrm{H}]\end{array}$ & 4.155 & 4.368 \\
\hline \multicolumn{4}{|c|}{ External set } \\
\hline Ext1 & $\begin{array}{l}\mathrm{O}=\mathrm{C} 2 \mathrm{~N}([\mathrm{H}])[\mathrm{C} @]([\mathrm{H}])(\mathrm{C}(=\mathrm{O}) \mathrm{N}([\mathrm{H}])[\mathrm{C} @ @]([\mathrm{H}])(\mathrm{C}([\mathrm{H}])([\mathrm{H}]) \mathrm{C}([\mathrm{H}])(\mathrm{C}([\mathrm{H}])([\mathrm{H}])) \\
\mathrm{H}]) \mathrm{C}([\mathrm{H}])([\mathrm{H}])[\mathrm{H}])[\mathrm{C} @ @](([\mathrm{H}])(\mathrm{O}[\mathrm{H}]) \mathrm{C}([\mathrm{H}])([\mathrm{H}])[\mathrm{C} @]([\mathrm{H}])(\mathrm{C}(=\mathrm{O}) \mathrm{N}([\mathrm{H}])[\mathrm{C} @]([\mathrm{H} \\
])(\mathrm{C}(=\mathrm{O}) \mathrm{N}([\mathrm{H}]) \mathrm{C}([\mathrm{H}])([\mathrm{H}]) \mathrm{C}=1 \mathrm{C}([\mathrm{H}])=\mathrm{C}([\mathrm{H}]) \mathrm{C}([\mathrm{H}])=\mathrm{C}([\mathrm{H}]) \mathrm{C}=1[\mathrm{H}]) \mathrm{C}([\mathrm{H}])(\mathrm{C}([\mathrm{H}]) \\
([\mathrm{H}])[\mathrm{H}]) \mathrm{C}([\mathrm{H}])([\mathrm{H}])[\mathrm{H}]) \mathrm{C}([\mathrm{H}])([\mathrm{H}])[\mathrm{H}]) \mathrm{C}([\mathrm{H}])([\mathrm{H}]) \mathrm{C}(=\mathrm{O}) \mathrm{N}([\mathrm{H}]) \mathrm{C}([\mathrm{H}])([\mathrm{H}]) \mathrm{C}([\mathrm{H}]) \\
([\mathrm{H}]) \mathrm{C}([\mathrm{H}])([\mathrm{H}]) \mathrm{C}([\mathrm{H}])([\mathrm{H}]) \mathrm{C}([\mathrm{H}])([\mathrm{H}]) \mathrm{C}([\mathrm{H}])([\mathrm{H}]) \mathrm{OC}(=\mathrm{O}) \mathrm{N}([\mathrm{H}])[\mathrm{C} @ @] 2([\mathrm{H}]) \mathrm{C}([ \\
\mathrm{H}])(\mathrm{C}([\mathrm{H}])([\mathrm{H}])[\mathrm{H}]) \mathrm{C}([\mathrm{H}])([\mathrm{H}])[\mathrm{H}]\end{array}$ & 3.600 & 3.204 \\
\hline Ext2 & $\begin{array}{l}\mathrm{O}=\mathrm{C}(\mathrm{OC}([\mathrm{H}])([\mathrm{H}]) \mathrm{C}=1 \mathrm{~N}=\mathrm{C}(\mathrm{OC}=1 \mathrm{C}([\mathrm{H}])([\mathrm{H}])[\mathrm{H}]) \mathrm{C}([\mathrm{H}])([\mathrm{H}])[\mathrm{H}]) \mathrm{N}([\mathrm{H}])[\mathrm{C} @]([\mathrm{H}]) \\
(\mathrm{C}(=\mathrm{O}) \mathrm{N}([\mathrm{H}])[\mathrm{C} @ @](([\mathrm{H}])(\mathrm{C}([\mathrm{H}])([\mathrm{H}]) \mathrm{C}([\mathrm{H}])(\mathrm{C}([\mathrm{H}])([\mathrm{H}])[\mathrm{H}]) \mathrm{C}([\mathrm{H}])([\mathrm{H}])[\mathrm{H}])[\mathrm{C} @ \\
@]([\mathrm{H}])(\mathrm{O}[\mathrm{H}]) \mathrm{C}([\mathrm{H}])([\mathrm{H}])[\mathrm{C} @]([\mathrm{H}])(\mathrm{C}(=\mathrm{O}) \mathrm{N}([\mathrm{H}])[\mathrm{C} @]([\mathrm{H}])(\mathrm{C}(=\mathrm{O}) \mathrm{N}([\mathrm{H}]) \mathrm{C}([\mathrm{H}])([ \\
\mathrm{H}]) \mathrm{C}([\mathrm{H}])(\mathrm{C}([\mathrm{H}])([\mathrm{H}])[\mathrm{H}]) \mathrm{C}([\mathrm{H}])([\mathrm{H}])[\mathrm{H}]) \mathrm{C}([\mathrm{H}])(\mathrm{C}([\mathrm{H}])([\mathrm{H}])[\mathrm{H}]) \mathrm{C}([\mathrm{H}])([\mathrm{H}])[\mathrm{H}]) \mathrm{C} \\
([\mathrm{H}])([\mathrm{H}])[\mathrm{H}]) \mathrm{C}([\mathrm{H}])([\mathrm{H}])[\mathrm{S}](=\mathrm{O})(=\mathrm{O}) \mathrm{C}([\mathrm{H}])([\mathrm{H}])[\mathrm{H}]\end{array}$ & 5.921 & 3.884 \\
\hline Ext3 & $\begin{array}{l}\mathrm{O}=\mathrm{C}(/ \mathrm{C} 1=\mathrm{C}(([\mathrm{H}]) \mathrm{C}(=\mathrm{C}([\mathrm{H}]) \mathrm{C}(=\mathrm{C} 1[\mathrm{H}]) \mathrm{C} 2=\mathrm{NN}=\mathrm{C}(\mathrm{O} 2)[\mathrm{C} @ @](\mathrm{C}([\mathrm{H}])([\mathrm{H}])[\mathrm{H}])(\mathrm{C}([ \\
\mathrm{H}])([\mathrm{H}]) \mathrm{C}=3 \mathrm{C}([\mathrm{H}])=\mathrm{C}([\mathrm{H}]) \mathrm{C}([\mathrm{H}])=\mathrm{C}([\mathrm{H}]) \mathrm{C}=3[\mathrm{H}])[\mathrm{N}+]([\mathrm{H}])([\mathrm{H}])[\mathrm{H}]) \mathrm{N}(\mathrm{C}([\mathrm{H}])([\mathrm{H}]) \\
[\mathrm{H}])[\mathrm{S}](=\mathrm{O})(=\mathrm{O}) \mathrm{C}([\mathrm{H}])([\mathrm{H}])[\mathrm{H}]) \mathrm{C}([\mathrm{H}])(\mathrm{C}([\mathrm{H}])([\mathrm{H}])[\mathrm{H}]) \mathrm{C}([\mathrm{H}])([\mathrm{H}])[\mathrm{H}]\end{array}$ & 0.783 & 2.741 \\
\hline Ext4 & $\begin{array}{l}\mathrm{O}=\mathrm{C}(\mathrm{N}([\mathrm{H}])[\mathrm{C} @]([\mathrm{H}])([\mathrm{C} @ @]([\mathrm{H}])(\mathrm{O}[\mathrm{H}]) \mathrm{C}([\mathrm{H}])([\mathrm{H}])[\mathrm{C} @]([\mathrm{H}])(\mathrm{C}(=\mathrm{O}) \mathrm{N}([\mathrm{H}])[\mathrm{C} @ \\
]([\mathrm{H}])(\mathrm{C}(=\mathrm{O}) \mathrm{N}([\mathrm{H}]) \mathrm{C}([\mathrm{H}])([\mathrm{H}]) \mathrm{Cl}=\mathrm{C}([\mathrm{H}]) \mathrm{C}([\mathrm{H}])=\mathrm{C}([\mathrm{H}]) \mathrm{C}([\mathrm{H}])=\mathrm{C} 1[\mathrm{H}]) \mathrm{C}([\mathrm{H}])(\mathrm{C}([ \\
\mathrm{H}])([\mathrm{H}])[\mathrm{H}]) \mathrm{C}([\mathrm{H}])([\mathrm{H}])[\mathrm{H}]) \mathrm{C}([\mathrm{H}])([\mathrm{H}])[\mathrm{H}]) \mathrm{C}([\mathrm{H}])([\mathrm{H}]) \mathrm{C}([\mathrm{H}])(\mathrm{C}([\mathrm{H}])([\mathrm{H}])[\mathrm{H}]) \mathrm{C}([ \\
\mathrm{H}])([\mathrm{H}])[\mathrm{H}])[\mathrm{C} @ @]([\mathrm{H}])(\mathrm{N}([\mathrm{H}]) \mathrm{C}(=\mathrm{O}) \mathrm{OC}(\mathrm{C}([\mathrm{H}])([\mathrm{H}])[\mathrm{H}])(\mathrm{C}([\mathrm{H}])([\mathrm{H}])[\mathrm{H}]) \mathrm{C}([\mathrm{H}])( \\
[\mathrm{H}])[\mathrm{H}]) \mathrm{C}([\mathrm{H}])([\mathrm{H}])[\mathrm{S}](=\mathrm{O})(=\mathrm{O}) \mathrm{C}([\mathrm{H}])([\mathrm{H}])[\mathrm{H}]\end{array}$ & 1.947 & 2.860 \\
\hline Ext5 & $\begin{array}{l}\mathrm{O}=\mathrm{C}(\mathrm{OC}([\mathrm{H}])([\mathrm{H}]) \mathrm{C}([\mathrm{H}])=\mathrm{C}([\mathrm{H}])[\mathrm{H}]) \mathrm{N}([\mathrm{H}])[\mathrm{C} @]([\mathrm{H}])(\mathrm{C}(=\mathrm{O}) \mathrm{N}([\mathrm{H}])[\mathrm{C} @]([\mathrm{H}])(\mathrm{C}( \\
=\mathrm{O}) \mathrm{N}([\mathrm{H}])[\mathrm{C} @ @([\mathrm{H}])(\mathrm{C}([\mathrm{H}])([\mathrm{H}]) \mathrm{C}([\mathrm{H}])(\mathrm{C}([\mathrm{H}])([\mathrm{H}])[\mathrm{H}]) \mathrm{C}([\mathrm{H}])([\mathrm{H}])[\mathrm{H}])[\mathrm{C} @ @]( \\
[\mathrm{H}])(\mathrm{O}[\mathrm{H}]) \mathrm{C}([\mathrm{H}])([\mathrm{H}])[\mathrm{C} @]([\mathrm{H}])(\mathrm{C}(=\mathrm{O}) \mathrm{N}([\mathrm{H}])[\mathrm{C} @]([\mathrm{H}])(\mathrm{C}(=\mathrm{O}) \mathrm{N}([\mathrm{H}]) \mathrm{C}([\mathrm{H}])([\mathrm{H}]) \\
\mathrm{C}=1 \mathrm{C}([\mathrm{H}])=\mathrm{C}([\mathrm{H}]) \mathrm{C}([\mathrm{H}])=\mathrm{C}([\mathrm{H}]) \mathrm{C}=1[\mathrm{H}]) \mathrm{C}([\mathrm{H}])(\mathrm{C}([\mathrm{H}])([\mathrm{H}])[\mathrm{H}]) \mathrm{C}([\mathrm{H}])([\mathrm{H}])[\mathrm{H}]) \mathrm{C}( \\
[\mathrm{H}])([\mathrm{H}])[\mathrm{H}]) \mathrm{C}([\mathrm{H}])([\mathrm{H}]) \mathrm{C}(=\mathrm{O}) \mathrm{N}([\mathrm{H}]) \mathrm{C}([\mathrm{H}])([\mathrm{H}]) \mathrm{C}([\mathrm{H}])=\mathrm{C}([\mathrm{H}])[\mathrm{H}]) \mathrm{C}([\mathrm{H}])(\mathrm{C}([\mathrm{H}])(\end{array}$ & 3.575 & 3.030 \\
\hline
\end{tabular}




\begin{tabular}{|c|c|c|c|}
\hline & $[\mathrm{H}])[\mathrm{H}]) \mathrm{C}([\mathrm{H}])([\mathrm{H}])[\mathrm{H}]$ & & \\
\hline Ext6 & $\begin{array}{l}\mathrm{O}=\mathrm{C} 2 \mathrm{~N}(\mathrm{C}(=\mathrm{N}[\mathrm{C} @] 4(\mathrm{C}=1 \mathrm{C}([\mathrm{H}])=\mathrm{C}([\mathrm{H}]) \mathrm{C}([\mathrm{H}])=\mathrm{C}([\mathrm{H}]) \mathrm{C}=1[\mathrm{H}])[\mathrm{C} @ @] 2([\mathrm{H}]) \mathrm{C}([\mathrm{H}] \\
)([\mathrm{H}]) \mathrm{N}(/ \mathrm{C} 3=\mathrm{N} / \mathrm{C}(=\mathrm{C}(/[\mathrm{F}]) \mathrm{C}(=\mathrm{N} 3) \mathrm{OC}([\mathrm{H}])([\mathrm{H}])[\mathrm{H}]) \mathrm{C}([\mathrm{H}])([\mathrm{H}])[\mathrm{H}]) \mathrm{C} 4([\mathrm{H}])[\mathrm{H}]) \mathrm{N}([ \\
\mathrm{H}])[\mathrm{H}]) \mathrm{C}([\mathrm{H}])([\mathrm{H}])[\mathrm{H}]\end{array}$ & 3.921 & 3.183 \\
\hline Ext7 & $\begin{array}{l}\mathrm{O}=\mathrm{C} 2 \mathrm{~N}(\mathrm{C}(=\mathrm{N}[\mathrm{C} @] 4(\mathrm{C}=1 \mathrm{C}([\mathrm{H}])=\mathrm{C}([\mathrm{H}]) \mathrm{C}([\mathrm{H}])=\mathrm{C}([\mathrm{H}]) \mathrm{C}=1[\mathrm{H}])[\mathrm{C} @ @] 2([\mathrm{H}]) \mathrm{C}([\mathrm{H}] \\
)([\mathrm{H}]) \mathrm{N}(/ \mathrm{C} 3=\mathrm{N} / \mathrm{C}(\mathrm{OC}([\mathrm{H}])([\mathrm{H}]) \mathrm{C}([\mathrm{H}])([\mathrm{H}])[\mathrm{H}])=\mathrm{C}([\mathrm{F}]) \mathrm{C}(=\mathrm{N} 3) \mathrm{OC}([\mathrm{H}])([\mathrm{H}])[\mathrm{H}]) \mathrm{C} 4( \\
[\mathrm{H}])[\mathrm{H}]) \mathrm{N}([\mathrm{H}])[\mathrm{H}]) \mathrm{C}([\mathrm{H}])([\mathrm{H}])[\mathrm{H}]\end{array}$ & 2.863 & 3.643 \\
\hline Ext8 & $\begin{array}{l}\mathrm{O}=\mathrm{C} 2 \mathrm{~N}(\mathrm{C}(=\mathrm{N}[\mathrm{C} @] 4(\mathrm{C}=1 \mathrm{C}([\mathrm{H}])=\mathrm{C}([\mathrm{H}]) \mathrm{C}([\mathrm{H}])=\mathrm{C}([\mathrm{H}]) \mathrm{C}=1[\mathrm{H}])[\mathrm{C} @ @] 2([\mathrm{H}]) \mathrm{C}([\mathrm{H}] \\
)([\mathrm{H}]) \mathrm{N}(/ \mathrm{C} 3=\mathrm{N} / \mathrm{C}(\mathrm{OC}([\mathrm{H}])([\mathrm{H}])[\mathrm{H}])=\mathrm{C}([\mathrm{F}]) \mathrm{C}(=\mathrm{N} 3) \mathrm{C}([\mathrm{H}])([\mathrm{H}]) \mathrm{C}([\mathrm{H}])([\mathrm{H}])[\mathrm{H}]) \mathrm{C} 4([ \\
\mathrm{H}])[\mathrm{H}]) \mathrm{N}([\mathrm{H}])[\mathrm{H}]) \mathrm{C}([\mathrm{H}])([\mathrm{H}])[\mathrm{H}]\end{array}$ & 3.796 & 3.456 \\
\hline Ext9 & $\begin{array}{l}\mathrm{O}=\mathrm{C} 2 \mathrm{~N}(\mathrm{C}(=\mathrm{N}[\mathrm{C} @] 4(\mathrm{C}=1 \mathrm{C}([\mathrm{H}])=\mathrm{C}([\mathrm{H}]) \mathrm{C}([\mathrm{F}])=\mathrm{C}([\mathrm{H}]) \mathrm{C}=1[\mathrm{~F}])[\mathrm{C} @ @] 2([\mathrm{H}]) \mathrm{C}([\mathrm{H}]) \\
([\mathrm{H}]) \mathrm{N}(/ \mathrm{C} 3=\mathrm{N} / \mathrm{C}(\mathrm{OC}([\mathrm{H}])([\mathrm{H}])[\mathrm{H}])=\mathrm{C}([\mathrm{F}]) \mathrm{C}(=\mathrm{N} 3) \mathrm{C}([\mathrm{H}])([\mathrm{H}]) \mathrm{C}([\mathrm{H}])([\mathrm{H}])[\mathrm{H}]) \mathrm{C} 4([\mathrm{H} \\
])[\mathrm{H}]) \mathrm{N}([\mathrm{H}])[\mathrm{H}]) \mathrm{C}([\mathrm{H}])([\mathrm{H}])[\mathrm{H}]\end{array}$ & 4.276 & 3.931 \\
\hline Ext10 & $\begin{array}{l}{[\mathrm{F}] / \mathrm{C} 1=\mathrm{C}(/ \mathrm{N}=\mathrm{C}(/ \mathrm{N}=\mathrm{C} 1 / \mathrm{OC}([\mathrm{H}])([\mathrm{H}])[\mathrm{H}]) \mathrm{N} 3 \mathrm{C}([\mathrm{H}])([\mathrm{H}])[\mathrm{C} @ @] 2(\mathrm{~N}=\mathrm{C}(\mathrm{N}(\mathrm{C}(=\mathrm{O})[\mathrm{C}} \\
@] 2([\mathrm{H}]) \mathrm{C} 3([\mathrm{H}])[\mathrm{H}]) \mathrm{C}([\mathrm{H}])([\mathrm{H}])[\mathrm{H}]) \mathrm{N}([\mathrm{H}])[\mathrm{H}]) \mathrm{C} 4=\mathrm{C}([\mathrm{H}]) \mathrm{C}([\mathrm{H}])=\mathrm{C}([\mathrm{F}]) \mathrm{C}([\mathrm{H}])=\mathrm{C} \\
4[\mathrm{~F}]) \mathrm{C}([\mathrm{F}])([\mathrm{F}])[\mathrm{F}]\end{array}$ & 3.495 & 3.203 \\
\hline Ext11 & $\begin{array}{l}\mathrm{O}=\mathrm{C} 2 \mathrm{~N}(\mathrm{C}(=\mathrm{N}[\mathrm{C} @] 4(\mathrm{C}=1 \mathrm{C}([\mathrm{H}])=\mathrm{C}(\mathrm{CHN}) \mathrm{C}([\mathrm{H}])=\mathrm{C}([\mathrm{H}]) \mathrm{C}=1[\mathrm{H}])[\mathrm{C} @ @] 2([\mathrm{H}]) \mathrm{C}([\mathrm{H} \\
])([\mathrm{H}]) \mathrm{N}(/ \mathrm{C} 3=\mathrm{N} / \mathrm{C}(=\mathrm{C}(/[\mathrm{F}]) \mathrm{C}(=\mathrm{N} 3) \mathrm{OC}([\mathrm{H}])([\mathrm{H}])[\mathrm{H}]) \mathrm{C}([\mathrm{H}])([\mathrm{H}])[\mathrm{H}]) \mathrm{C} 4([\mathrm{H}])[\mathrm{H}]) \mathrm{N}([ \\
\mathrm{H}])[\mathrm{H}]) \mathrm{C}([\mathrm{H}])([\mathrm{H}])[\mathrm{H}]\end{array}$ & 3.854 & 3.758 \\
\hline Ext12 & $\begin{array}{l}\mathrm{O}=\mathrm{C}(\mathrm{OC}(\mathrm{C}([\mathrm{H}])([\mathrm{H}])[\mathrm{H}])(\mathrm{C}([\mathrm{H}])([\mathrm{H}])[\mathrm{H}]) \mathrm{C}([\mathrm{H}])([\mathrm{H}])[\mathrm{H}]) \mathrm{N}([\mathrm{H}])[\mathrm{C} @]([\mathrm{H}])(\mathrm{C}(=\mathrm{O}) \\
\mathrm{C}([\mathrm{H}])([\mathrm{H}]) \mathrm{N}([\mathrm{H}])[\mathrm{C} @]([\mathrm{H}])([\mathrm{C} @]([\mathrm{H}])(\mathrm{O}[\mathrm{H}]) \mathrm{C}([\mathrm{H}])([\mathrm{H}])[\mathrm{C} @]([\mathrm{H}])(\mathrm{C}(=\mathrm{O}) \mathrm{N}([\mathrm{H}])[ \\
\mathrm{C} @]([\mathrm{H}])(\mathrm{C}(=\mathrm{O}) \mathrm{N}([\mathrm{H}]) \mathrm{C}([\mathrm{H}])([\mathrm{H}]) \mathrm{C} 1=\mathrm{C}([\mathrm{H}]) \mathrm{C}([\mathrm{H}])=\mathrm{C}([\mathrm{H}]) \mathrm{C}([\mathrm{H}])=\mathrm{C} 1[\mathrm{H}]) \mathrm{C}([\mathrm{H}])( \\
[\mathrm{H}])[\mathrm{H}]) \mathrm{C}([\mathrm{H}])([\mathrm{H}])[\mathrm{H}]) \mathrm{C}([\mathrm{H}])([\mathrm{H}]) \mathrm{C}([\mathrm{H}])(\mathrm{C}([\mathrm{H}])([\mathrm{H}])[\mathrm{H}]) \mathrm{C}([\mathrm{H}])([\mathrm{H}])[\mathrm{H}]) \mathrm{C}([\mathrm{H}])([ \\
\mathrm{H}]) \mathrm{C}(=\mathrm{O}) \mathrm{N}([\mathrm{H}])[\mathrm{H}]\end{array}$ & 0.649 & 2.437 \\
\hline Ext13 & $\begin{array}{l}\mathrm{O}=\mathrm{C}(\mathrm{OC}(\mathrm{C}([\mathrm{H}])([\mathrm{H}])[\mathrm{H}])(\mathrm{C}([\mathrm{H}])([\mathrm{H}])[\mathrm{H}]) \mathrm{C}([\mathrm{H}])([\mathrm{H}])[\mathrm{H}]) \mathrm{N}([\mathrm{H}])[\mathrm{C} @]([\mathrm{H}])(\mathrm{C}(=\mathrm{O}) \\
\mathrm{C}([\mathrm{H}])([\mathrm{H}]) \mathrm{N}([\mathrm{H}])[\mathrm{C} @]([\mathrm{H}])([\mathrm{C} @]([\mathrm{H}])(\mathrm{O}[\mathrm{H}]) \mathrm{C}([\mathrm{H}])([\mathrm{H}])[\mathrm{C} @]([\mathrm{H}])(\mathrm{C}(=\mathrm{O}) \mathrm{N}([\mathrm{H}])[ \\
\mathrm{C} @([\mathrm{H}])(\mathrm{C}(=\mathrm{O}) \mathrm{N}([\mathrm{H}]) \mathrm{C}([\mathrm{H}])([\mathrm{H}]) \mathrm{C} 1=\mathrm{C}([\mathrm{H}]) \mathrm{C}([\mathrm{H}])=\mathrm{C}([\mathrm{H}]) \mathrm{C}([\mathrm{H}])=\mathrm{C} 1[\mathrm{H}]) \mathrm{C}([\mathrm{H}])( \\
\mathrm{C}([\mathrm{H}])([\mathrm{H}])[\mathrm{H}]) \mathrm{C}([\mathrm{H}])([\mathrm{H}])[\mathrm{H}]) \mathrm{C}([\mathrm{H}])([\mathrm{H}])[\mathrm{H}]) \mathrm{C}([\mathrm{H}])([\mathrm{H}]) \mathrm{C}([\mathrm{H}])(\mathrm{C}([\mathrm{H}])([\mathrm{H}])[\mathrm{H}]) \\
\mathrm{C}([\mathrm{H}])([\mathrm{H}])[\mathrm{H}]) \mathrm{C}([\mathrm{H}])([\mathrm{H}]) \mathrm{C}(=\mathrm{O}) \mathrm{N}([\mathrm{H}])[\mathrm{H}]\end{array}$ & 1.504 & 2.485 \\
\hline Ext14 & 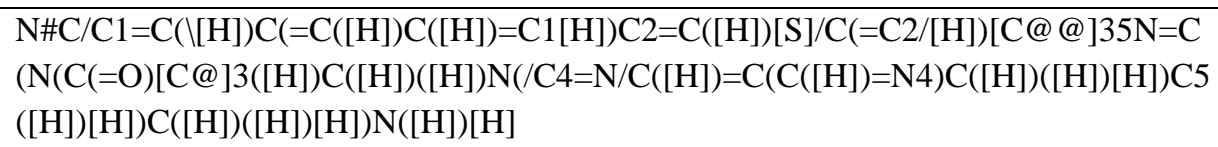 & 4.046 & 3.352 \\
\hline Ext15 & $\begin{array}{l}\mathrm{O}=[\mathrm{S}](=\mathrm{O})(\mathrm{N}(\mathrm{C}=1 \mathrm{C}([\mathrm{H}])=\mathrm{C}(\mathrm{C}([\mathrm{H}])=\mathrm{C}(\mathrm{C}=1[\mathrm{H}]) \mathrm{C}(=\mathrm{O}) \mathrm{N}([\mathrm{H}])[\mathrm{C} @]([\mathrm{H}])(\mathrm{C}([\mathrm{H}])([\mathrm{H}]) \\
\mathrm{N}([\mathrm{H}])[\mathrm{C} @([\mathrm{H}])(\mathrm{C}(=\mathrm{O}) \mathrm{N}([\mathrm{H}]) \mathrm{C}([\mathrm{H}])([\mathrm{H}]) \mathrm{C}([\mathrm{H}])(\mathrm{C}([\mathrm{H}])([\mathrm{H}])[\mathrm{H}]) \mathrm{C}([\mathrm{H}])([\mathrm{H}])[\mathrm{H}])[ \\
\mathrm{C} @ @]([\mathrm{H}])(\mathrm{OC}([\mathrm{H}])([\mathrm{H}])[\mathrm{H}]) \mathrm{C}([\mathrm{H}])([\mathrm{H}])[\mathrm{H}]) \mathrm{C}([\mathrm{H}])([\mathrm{H}]) \mathrm{C} 2=\mathrm{C}([\mathrm{H}]) \mathrm{C}([\mathrm{H}])=\mathrm{C}([\mathrm{H}]) \\
\mathrm{C}([\mathrm{H}])=\mathrm{C} 2[\mathrm{H}]) \mathrm{C}(=\mathrm{O}) \mathrm{N}([\mathrm{H}])[\mathrm{C} @ @]([\mathrm{H}])(\mathrm{C} 3=\mathrm{C}([\mathrm{H}]) \mathrm{C}([\mathrm{H}])=\mathrm{C}([\mathrm{H}]) \mathrm{C}([\mathrm{H}])=\mathrm{C} 3[\mathrm{H}]) \mathrm{C} \\
([\mathrm{H}])([\mathrm{H}])[\mathrm{H}]) \mathrm{C}([\mathrm{H}])([\mathrm{H}])[\mathrm{H}]) \mathrm{C}([\mathrm{H}])([\mathrm{H}])[\mathrm{H}]\end{array}$ & 3.602 & 2.192 \\
\hline Ext16 & $\begin{array}{l}\mathrm{O}=\mathrm{C}(\mathrm{N}([\mathrm{H}])[\mathrm{C} @]([\mathrm{H}])([\mathrm{C} @ @]([\mathrm{H}])(\mathrm{O}[\mathrm{H}]) \mathrm{C}([\mathrm{H}])([\mathrm{H}])[\mathrm{C} @]([\mathrm{H}])(\mathrm{C}(=\mathrm{O}) \mathrm{N}([\mathrm{H}]) \mathrm{C}([ \\
\mathrm{H}])([\mathrm{H}]) \mathrm{C}([\mathrm{H}])(\mathrm{C}([\mathrm{H}])([\mathrm{H}])[\mathrm{H}]) \mathrm{C}([\mathrm{H}])([\mathrm{H}])[\mathrm{H}]) \mathrm{C}([\mathrm{H}])([\mathrm{H}]) \mathrm{C}([\mathrm{H}])([\mathrm{H}]) \mathrm{O}[\mathrm{H}]) \mathrm{C}([\mathrm{H}]) \\
([\mathrm{H}]) \mathrm{C} 1=\mathrm{C}([\mathrm{H}]) \mathrm{C}([\mathrm{H}])=\mathrm{C}([\mathrm{H}]) \mathrm{C}([\mathrm{H}])=\mathrm{C} 1[\mathrm{H}]) \mathrm{C}([\mathrm{H}])([\mathrm{H}])[\mathrm{C} @]([\mathrm{H}])(\mathrm{N}([\mathrm{H}]) \mathrm{C}(=\mathrm{O}) \mathrm{C} \\
([\mathrm{H}])([\mathrm{H}]) \mathrm{C}([\mathrm{H}])(\mathrm{C}([\mathrm{H}])([\mathrm{H}])[\mathrm{H}]) \mathrm{C}([\mathrm{H}])([\mathrm{H}])[\mathrm{H}]) \mathrm{C}(=\mathrm{O}) \mathrm{O}[\mathrm{H}]\end{array}$ & 1.721 & 1.884 \\
\hline Ext17 & $\begin{array}{l}\mathrm{O}=\mathrm{C}(\mathrm{N}([\mathrm{H}])[\mathrm{C} @ @(([\mathrm{H}])(\mathrm{Cl}=\mathrm{C}([\mathrm{H}]) \mathrm{C}([\mathrm{H}])=\mathrm{C}([\mathrm{H}]) \mathrm{C}([\mathrm{H}])=\mathrm{C} 1[\mathrm{H}]) \mathrm{C}([\mathrm{H}])([\mathrm{H}])[\mathrm{H}]) \mathrm{C} \\
2=\mathrm{C}([\mathrm{H}]) \mathrm{C}(=\mathrm{C}([\mathrm{H}]) \mathrm{C}(=\mathrm{C} 2[\mathrm{H}]) \mathrm{N}(\mathrm{C}([\mathrm{H}])([\mathrm{H}])[\mathrm{H}])[\mathrm{S}](=\mathrm{O})(=\mathrm{O}) \mathrm{C}([\mathrm{H}])([\mathrm{H}])[\mathrm{H}]) \mathrm{C}(=\mathrm{O})\end{array}$ & 2.638 & 2.171 \\
\hline
\end{tabular}




\begin{tabular}{|c|c|c|c|}
\hline & $\begin{array}{l}\mathrm{N}([\mathrm{H}])[\mathrm{C} @]([\mathrm{H}])([\mathrm{C} @]([\mathrm{H}])(\mathrm{O}[\mathrm{H}]) \mathrm{C}([\mathrm{H}])([\mathrm{H}]) \mathrm{N}([\mathrm{H}]) \mathrm{C}([\mathrm{H}])([\mathrm{H}]) \mathrm{C} 3=\mathrm{C}([\mathrm{H}]) \mathrm{C} 4=\mathrm{C}( \\
\mathrm{C}([\mathrm{H}])=\mathrm{C} 3[\mathrm{H}]) \mathrm{C}([\mathrm{H}])=\mathrm{C}([\mathrm{H}]) \mathrm{N} 4[\mathrm{H}]) \mathrm{C}([\mathrm{H}])([\mathrm{H}]) \mathrm{C} 5=\mathrm{C}([\mathrm{H}]) \mathrm{C}([\mathrm{H}])=\mathrm{C}([\mathrm{H}]) \mathrm{C}([\mathrm{H}])=\mathrm{C} \\
5[\mathrm{H}]\end{array}$ & & \\
\hline Ext18 & $\begin{array}{l}\mathrm{O}=\mathrm{C}(\mathrm{N}([\mathrm{H}])[\mathrm{C} @]([\mathrm{H}])(\mathrm{C}=1 \mathrm{~N}=\mathrm{C}(\mathrm{OC}=1 \mathrm{C}([\mathrm{H}])([\mathrm{H}])[\mathrm{H}]) \mathrm{C}([\mathrm{H}])([\mathrm{H}])[\mathrm{H}]) \mathrm{C}([\mathrm{H}])([\mathrm{H}])[ \\
\mathrm{H}]) \mathrm{C}=2 \mathrm{C}([\mathrm{H}])=\mathrm{C}(\mathrm{C}([\mathrm{H}])=\mathrm{C}(\mathrm{C}=2[\mathrm{H}]) \mathrm{N}(\mathrm{C}([\mathrm{H}])([\mathrm{H}])[\mathrm{H}])[\mathrm{S}](=\mathrm{O})(=\mathrm{O}) \mathrm{C}([\mathrm{H}])([\mathrm{H}])[\mathrm{H}]) \\
\mathrm{C}(=\mathrm{O}) \mathrm{N}([\mathrm{H}])[\mathrm{C} @]([\mathrm{H}])([\mathrm{C} @([\mathrm{H}])(\mathrm{O}[\mathrm{H}]) \mathrm{C}([\mathrm{H}])([\mathrm{H}]) \mathrm{N}([\mathrm{H}]) \mathrm{C}([\mathrm{H}])([\mathrm{H}]) \mathrm{C} 3=\mathrm{C}([\mathrm{H}]) \\
\mathrm{C}(\mathrm{OC}([\mathrm{H}])([\mathrm{H}])[\mathrm{H}])=\mathrm{C}([\mathrm{H}]) \mathrm{C}([\mathrm{H}])=\mathrm{C} 3[\mathrm{H}]) \mathrm{C}([\mathrm{H}])([\mathrm{H}]) \mathrm{C} 4=\mathrm{C}([\mathrm{H}]) \mathrm{C}([\mathrm{H}])=\mathrm{C}([\mathrm{H}]) \mathrm{C}([ \\
\mathrm{H}])=\mathrm{C} 4[\mathrm{H}]\end{array}$ & 2.049 & 3.481 \\
\hline Ext19 & $\begin{array}{l}\mathrm{O}=\mathrm{C} 2 \mathrm{~N}([\mathrm{H}]) \mathrm{C} 1=\mathrm{C}([\mathrm{H}]) \mathrm{C}([\mathrm{H}])=\mathrm{C}([\mathrm{H}]) \mathrm{C}([\mathrm{H}])=\mathrm{C} 1 \mathrm{~N}([\mathrm{H}]) \mathrm{C} 3=\mathrm{C} 2 \mathrm{C}([\mathrm{H}])=\mathrm{C}([\mathrm{H}]) \mathrm{C}(=\mathrm{C} \\
3[\mathrm{H}]) \mathrm{C}(=\mathrm{O}) \mathrm{N}([\mathrm{H}]) \mathrm{C} 4=\mathrm{N} / \mathrm{C}(=\mathrm{C}(/[\mathrm{H}]) \mathrm{N} 4[\mathrm{H}]) \mathrm{C} 5=\mathrm{C}([\mathrm{H}]) \mathrm{C}([\mathrm{H}])=\mathrm{NC}([\mathrm{H}])=\mathrm{C} 5[\mathrm{H}]\end{array}$ & 2.458 & 2.897 \\
\hline Ext20 & $\begin{array}{l}\mathrm{O}=\mathrm{C} 2 \mathrm{~N}([\mathrm{H}]) \mathrm{C} 1=\mathrm{C}([\mathrm{H}]) \mathrm{C}([\mathrm{H}])=\mathrm{C}([\mathrm{H}]) \mathrm{C}([\mathrm{H}])=\mathrm{C} 1 \mathrm{~N}([\mathrm{H}]) \mathrm{C} 3=\mathrm{C} 2 \mathrm{C}([\mathrm{H}])=\mathrm{C}([\mathrm{H}]) \mathrm{C}(=\mathrm{C} \\
3[\mathrm{H}]) \mathrm{C}(=\mathrm{O}) \mathrm{N}([\mathrm{H}]) \mathrm{C} 4=\mathrm{C}([\mathrm{H}]) \mathrm{C}([\mathrm{H}])=\mathrm{C}([\mathrm{F}]) \mathrm{C}([\mathrm{H}])=\mathrm{C} 4[\mathrm{H}]\end{array}$ & 2.676 & 1.392 \\
\hline Ext21 & $\begin{array}{l}\mathrm{O}=\mathrm{C} 2 \mathrm{~N}([\mathrm{H}]) \mathrm{C} 1=\mathrm{C}([\mathrm{H}]) \mathrm{C}([\mathrm{H}])=\mathrm{C}([\mathrm{H}]) \mathrm{C}([\mathrm{H}])=\mathrm{C} 1 \mathrm{~N}([\mathrm{H}]) \mathrm{C}=3 / \mathrm{C} 2=\mathrm{C}(/[\mathrm{H}]) \mathrm{C}([\mathrm{H}])=\mathrm{C}( \\
\mathrm{C}=3[\mathrm{H}]) \mathrm{C}(=\mathrm{O}) \mathrm{N}([\mathrm{H}]) \mathrm{C} 4=\mathrm{NC}=5 \mathrm{C}(\mathrm{N} 4[\mathrm{H}])=\mathrm{C}([\mathrm{H}]) \mathrm{N}=\mathrm{C}([\mathrm{H}]) \mathrm{C}=5[\mathrm{H}]\end{array}$ & 2.510 & 1.849 \\
\hline Ext22 & $\begin{array}{l}\mathrm{O}=\mathrm{C}(\mathrm{N}([\mathrm{H}]) \mathrm{C}(=\mathrm{NC}([\mathrm{H}])([\mathrm{H}]) \mathrm{C} 1=\mathrm{C}([\mathrm{H}]) \mathrm{C}(=\mathrm{C}([\mathrm{H}]) \mathrm{C}(=\mathrm{C} 1[\mathrm{H}]) \mathrm{C}([\mathrm{H}])([\mathrm{H}])[\mathrm{H}]) \mathrm{C}([\mathrm{H}] \\
)([\mathrm{H}])[\mathrm{H}]) \mathrm{N}([\mathrm{H}])[\mathrm{H}]) \mathrm{C}=2 \mathrm{C}(=\mathrm{NOC}=2 \mathrm{C}([\mathrm{H}])([\mathrm{H}])[\mathrm{H}]) \mathrm{C} 3=\mathrm{C}([\mathrm{H}]) \mathrm{C}([\mathrm{H}])=\mathrm{C}(\mathrm{OC}([\mathrm{H}])([ \\
\mathrm{H}])[\mathrm{H}]) \mathrm{C}([\mathrm{H}])=\mathrm{C} 3[\mathrm{H}]\end{array}$ & 2.553 & 1.826 \\
\hline Ext23 & $\begin{array}{l}\mathrm{O}=\mathrm{C}(\mathrm{N}([\mathrm{H}]) \mathrm{C}(=\mathrm{NC}([\mathrm{H}])([\mathrm{H}]) \mathrm{Cl}=\mathrm{C}([\mathrm{H}]) \mathrm{C}([\mathrm{Cl}])=\mathrm{C}([\mathrm{H}]) \mathrm{C}([\mathrm{Cl}])=\mathrm{C} 1[\mathrm{H}]) \mathrm{N}([\mathrm{H}])[\mathrm{H}]) \mathrm{C} \\
=2 \mathrm{C}(=\mathrm{N}[\mathrm{S}] \mathrm{C}=2 \mathrm{C}([\mathrm{H}])([\mathrm{H}])[\mathrm{H}]) \mathrm{C} 3=\mathrm{C}([\mathrm{H}]) \mathrm{C}([\mathrm{H}])=\mathrm{C}(\mathrm{OC}([\mathrm{H}])([\mathrm{H}])[\mathrm{H}]) \mathrm{C}([\mathrm{H}])=\mathrm{C} 3[\mathrm{H} \\
]\end{array}$ & 2.538 & 2.559 \\
\hline Ext24 & $\begin{array}{l}\mathrm{O}=\mathrm{C}(\mathrm{N}([\mathrm{H}]) \mathrm{C}(=\mathrm{NC}([\mathrm{H}])([\mathrm{H}]) \mathrm{C}=1 \mathrm{C}([\mathrm{H}])=\mathrm{C}([\mathrm{H}]) \mathrm{C}([\mathrm{H}])=\mathrm{C} 2 \mathrm{C}=1 \mathrm{C}([\mathrm{H}])=\mathrm{C}([\mathrm{H}]) \mathrm{C}([\mathrm{H}] \\
)=\mathrm{C} 2[\mathrm{H}]) \mathrm{N}([\mathrm{H}])[\mathrm{H}]) \mathrm{C}=3 \mathrm{C}(=\mathrm{NOC}=3 \mathrm{C}([\mathrm{H}])([\mathrm{H}])[\mathrm{H}]) \mathrm{C} 4=\mathrm{C}([\mathrm{H}]) \mathrm{C}([\mathrm{H}])=\mathrm{C}([\mathrm{H}]) \mathrm{C}([\mathrm{H}]) \\
=\mathrm{C} 4[\mathrm{H}]\end{array}$ & 1.553 & 1.122 \\
\hline Ext25 & $\begin{array}{l}{[\mathrm{H}] \mathrm{C}=5 \mathrm{C}(\mathrm{O}[\mathrm{H}])=\mathrm{C}(\mathrm{O} / \mathrm{C} 2=\mathrm{C}(\backslash[\mathrm{H}]) \mathrm{C}(\mathrm{O}[\mathrm{H}])=\mathrm{C} 1 \mathrm{OC} 3=\mathrm{C}(\mathrm{O} / \mathrm{C} 1=\mathrm{C} 2 \backslash[\mathrm{H}]) \mathrm{C}(\mathrm{O}[\mathrm{H}])=\mathrm{C}([\mathrm{H}} \\
]) \mathrm{C}(\mathrm{O}[\mathrm{H}])=\mathrm{C} 3 \mathrm{O} / \mathrm{C} 4=\mathrm{C}(\backslash[\mathrm{H}]) \mathrm{C}(\mathrm{O}[\mathrm{H}])=\mathrm{C}([\mathrm{H}]) \mathrm{C}(\mathrm{O}[\mathrm{H}])=\mathrm{C} 4[\mathrm{H}]) \mathrm{C}(\mathrm{O}[\mathrm{H}])=\mathrm{C}([\mathrm{H}]) \mathrm{C}=5 \mathrm{O} \\
{[\mathrm{H}]}\end{array}$ & 1.143 & 1.019 \\
\hline Ext26 & $\begin{array}{l}{[\mathrm{F}] \mathrm{C}=1 \mathrm{C}([\mathrm{H}])=\mathrm{C}(\mathrm{C}([\mathrm{H}])=\mathrm{C}([\mathrm{F}]) \mathrm{C}=1[\mathrm{H}]) \mathrm{C}([\mathrm{H}])([\mathrm{H}])[\mathrm{C} @]([\mathrm{H}])(\mathrm{N}([\mathrm{H}]) \mathrm{C}(=\mathrm{O}) \mathrm{C}=2 \mathrm{C}([} \\
\mathrm{H}])=\mathrm{C}(\mathrm{C}([\mathrm{H}])=\mathrm{C}(\mathrm{C}=2[\mathrm{H}]) \mathrm{C}([\mathrm{H}])([\mathrm{H}])[\mathrm{H}]) \mathrm{C}(=\mathrm{O}) \mathrm{N}(\mathrm{C}([\mathrm{H}])([\mathrm{H}]) \mathrm{C}([\mathrm{H}])([\mathrm{H}]) \mathrm{C}([\mathrm{H}])([ \\
\mathrm{H}])[\mathrm{H}]) \mathrm{C}([\mathrm{H}])([\mathrm{H}]) \mathrm{C}([\mathrm{H}])([\mathrm{H}]) \mathrm{C}([\mathrm{H}])([\mathrm{H}])[\mathrm{H}])[\mathrm{C} @]([\mathrm{H}])(\mathrm{O}[\mathrm{H}])[\mathrm{C} @] 3([\mathrm{H}]) \mathrm{N}([\mathrm{H}]) \\
\mathrm{C}([\mathrm{H}])([\mathrm{H}]) \mathrm{C}([\mathrm{H}])([\mathrm{H}]) \mathrm{N}(\mathrm{C} 3([\mathrm{H}])[\mathrm{H}])[\mathrm{S}](=\mathrm{O})(=\mathrm{O}) \mathrm{C} 4=\mathrm{C}([\mathrm{H}]) \mathrm{C}([\mathrm{H}])=\mathrm{C}([\mathrm{H}]) \mathrm{C}([\mathrm{H}])= \\
\mathrm{C} 4[\mathrm{H}]\end{array}$ & 4.523 & 3.732 \\
\hline Ext27 & $\begin{array}{l}{[\mathrm{F}] \mathrm{C}=1 \mathrm{C}([\mathrm{H}])=\mathrm{C}(\mathrm{C}([\mathrm{H}])=\mathrm{C}([\mathrm{F}]) \mathrm{C}=1[\mathrm{H}]) \mathrm{C}([\mathrm{H}])([\mathrm{H}])[\mathrm{C} @]([\mathrm{H}])(\mathrm{N}([\mathrm{H}]) \mathrm{C}(=\mathrm{O}) \mathrm{C} 2=\mathrm{C}([} \\
\mathrm{H}]) \mathrm{C}(=\mathrm{C}([\mathrm{H}]) \mathrm{C}(=\mathrm{C} 2[\mathrm{H}]) \mathrm{C}(=\mathrm{O}) \mathrm{N} 3[\mathrm{C} @]([\mathrm{H}])(\mathrm{C}([\mathrm{H}])([\mathrm{H}]) \mathrm{C}([\mathrm{H}])([\mathrm{H}]) \mathrm{C} 3([\mathrm{H}])[\mathrm{H}]) \mathrm{C}([ \\
\mathrm{H}])([\mathrm{H}]) \mathrm{OC}([\mathrm{H}])([\mathrm{H}])[\mathrm{H}]) \mathrm{C}([\mathrm{H}])([\mathrm{H}])[\mathrm{H}])[\mathrm{C} @]([\mathrm{H}])(\mathrm{O}[\mathrm{H}])[\mathrm{C} @] 4([\mathrm{H}]) \mathrm{N}([\mathrm{H}]) \mathrm{C}([\mathrm{H}] \\
)([\mathrm{H}]) \mathrm{C}([\mathrm{H}])([\mathrm{H}]) \mathrm{N}(\mathrm{C} 4([\mathrm{H}])[\mathrm{H}])[\mathrm{S}](=\mathrm{O})(=\mathrm{O}) \mathrm{C}([\mathrm{H}])([\mathrm{H}])[\mathrm{H}]\end{array}$ & 3.886 & 3.934 \\
\hline Ext28 & $\begin{array}{l}{[\mathrm{F}] \mathrm{C}=1 \mathrm{C}([\mathrm{H}])=\mathrm{C}(\mathrm{C}([\mathrm{H}])=\mathrm{C}([\mathrm{F}]) \mathrm{C}=1[\mathrm{H}]) \mathrm{C}([\mathrm{H}])([\mathrm{H}])[\mathrm{C} @]([\mathrm{H}])(\mathrm{N}([\mathrm{H}]) \mathrm{C}(=\mathrm{O}) \mathrm{C} 2=\mathrm{C}([} \\
\mathrm{H}]) \mathrm{C}(=\mathrm{C}([\mathrm{H}]) \mathrm{C}(=\mathrm{C} 2[\mathrm{H}]) \mathrm{C}(=\mathrm{O}) \mathrm{N} 3[\mathrm{C} @]([\mathrm{H}])(\mathrm{C}([\mathrm{H}])([\mathrm{H}]) \mathrm{C}([\mathrm{H}])([\mathrm{H}]) \mathrm{C} 3([\mathrm{H}])[\mathrm{H}]) \mathrm{C}([ \\
\mathrm{H}])([\mathrm{H}]) \mathrm{OC}([\mathrm{H}])([\mathrm{H}])[\mathrm{H}]) \mathrm{C}([\mathrm{H}])([\mathrm{H}])[\mathrm{H}])[\mathrm{C} @]([\mathrm{H}])(\mathrm{O}[\mathrm{H}])[\mathrm{C} @] 4([\mathrm{H}]) \mathrm{N}([\mathrm{H}]) \mathrm{C}([\mathrm{H}] \\
)([\mathrm{H}]) \mathrm{C}([\mathrm{H}])([\mathrm{H}]) \mathrm{N}(\mathrm{C} 4([\mathrm{H}])[\mathrm{H}])[\mathrm{S}](=\mathrm{O})(=\mathrm{O}) \mathrm{C}([\mathrm{H}])([\mathrm{H}]) \mathrm{C} 5=\mathrm{C}([\mathrm{H}]) \mathrm{C}([\mathrm{H}])=\mathrm{C}([\mathrm{H}]) \mathrm{C} \\
([\mathrm{H}])=\mathrm{C} 5[\mathrm{H}]\end{array}$ & 5.000 & 3.962 \\
\hline Ext29 & $\begin{array}{l}\mathrm{O}=\mathrm{C}(\mathrm{N}([\mathrm{H}])[\mathrm{C} @ @](([\mathrm{H}])(\mathrm{Cl}=\mathrm{C}([\mathrm{H}]) \mathrm{C}([\mathrm{H}])=\mathrm{C}([\mathrm{H}]) \mathrm{C}([\mathrm{H}])=\mathrm{C} 1[\mathrm{H}]) \mathrm{C}([\mathrm{H}])([\mathrm{H}])[\mathrm{H}]) \mathrm{C} \\
2=\mathrm{C}([\mathrm{H}]) \mathrm{C}(=\mathrm{C}([\mathrm{H}]) \mathrm{C}(=\mathrm{C} 2[\mathrm{H}]) \mathrm{N}(\mathrm{C}([\mathrm{H}])([\mathrm{H}])[\mathrm{H}])[\mathrm{S}](=\mathrm{O})(=\mathrm{O}) \mathrm{C}([\mathrm{H}])([\mathrm{H}])[\mathrm{H}]) \mathrm{C}(=\mathrm{O}) \\
\mathrm{N}([\mathrm{H}])[\mathrm{C} @]([\mathrm{H}])([\mathrm{C} @ @]([\mathrm{H}])(\mathrm{N}=[\mathrm{N}+]=[\mathrm{N}-\end{array}$ & 3.155 & 3.807 \\
\hline
\end{tabular}




\begin{tabular}{|c|c|c|c|}
\hline & $\begin{array}{l}]) \mathrm{C}([\mathrm{H}])([\mathrm{H}])[\mathrm{C} @ @]([\mathrm{H}])(\mathrm{OC}([\mathrm{H}])([\mathrm{H}])[\mathrm{H}]) \mathrm{C}(=\mathrm{O}) \mathrm{N}([\mathrm{H}])[\mathrm{C} @]([\mathrm{H}])(\mathrm{C}(=\mathrm{O}) \mathrm{N}([\mathrm{H}]) \mathrm{C} \\
([\mathrm{H}])([\mathrm{H}]) \mathrm{C} 3=\mathrm{C}([\mathrm{H}]) \mathrm{C}([\mathrm{H}])=\mathrm{C}([\mathrm{H}]) \mathrm{C}([\mathrm{H}])=\mathrm{C} 3[\mathrm{H}]) \mathrm{C}([\mathrm{H}])(\mathrm{C}([\mathrm{H}])([\mathrm{H}])[\mathrm{H}]) \mathrm{C}([\mathrm{H}])([\mathrm{H} \\
])[\mathrm{H}]) \mathrm{C}([\mathrm{H}])([\mathrm{H}]) \mathrm{O} / \mathrm{C} 4=\mathrm{C}(\backslash[\mathrm{H}]) \mathrm{C}([\mathrm{F}])=\mathrm{C}([\mathrm{H}]) \mathrm{C}([\mathrm{F}])=\mathrm{C} 4[\mathrm{H}]\end{array}$ & & \\
\hline Ext30 & $\begin{array}{l}\mathrm{O}=\mathrm{C}(\mathrm{N}([\mathrm{H}])[\mathrm{C} @ @]([\mathrm{H}])(\mathrm{C} 1=\mathrm{C}([\mathrm{H}]) \mathrm{C}([\mathrm{H}])=\mathrm{C}([\mathrm{H}]) \mathrm{C}([\mathrm{H}])=\mathrm{C} 1[\mathrm{H}]) \mathrm{C}([\mathrm{H}])([\mathrm{H}])[\mathrm{H}]) \mathrm{C} \\
2=\mathrm{C}([\mathrm{H}]) \mathrm{C}(=\mathrm{C}([\mathrm{H}]) \mathrm{C}(=\mathrm{C} 2[\mathrm{H}]) \mathrm{N}(\mathrm{C}([\mathrm{H}])([\mathrm{H}])[\mathrm{H}])[\mathrm{S}](=\mathrm{O})(=\mathrm{O}) \mathrm{C}([\mathrm{H}])([\mathrm{H}])[\mathrm{H}]) \mathrm{C}(=\mathrm{O}) \\
\mathrm{N}([\mathrm{H}])[\mathrm{C} @]([\mathrm{H}])([\mathrm{C} @ @]([\mathrm{H}])(\mathrm{O}[\mathrm{H}]) \mathrm{C}([\mathrm{H}])([\mathrm{H}])[\mathrm{C} @ @(([\mathrm{H}])(\mathrm{OC}([\mathrm{H}])([\mathrm{H}]) \mathrm{C}([\mathrm{H}])( \\
[\mathrm{H}])[\mathrm{H}]) \mathrm{C}(=\mathrm{O}) \mathrm{N}([\mathrm{H}])[\mathrm{C} @]([\mathrm{H}])(\mathrm{C}(=\mathrm{O}) \mathrm{N}([\mathrm{H}]) \mathrm{C}([\mathrm{H}])([\mathrm{H}]) \mathrm{C} 3=\mathrm{C}([\mathrm{H}]) \mathrm{C}([\mathrm{H}])=\mathrm{C}([\mathrm{H}]) \\
\mathrm{C}([\mathrm{H}])=\mathrm{C} 3[\mathrm{H}]) \mathrm{C}([\mathrm{H}])(\mathrm{C}([\mathrm{H}])([\mathrm{H}])[\mathrm{H}]) \mathrm{C}([\mathrm{H}])([\mathrm{H}])[\mathrm{H}]) \mathrm{C}([\mathrm{H}])([\mathrm{H}]) \mathrm{O} / \mathrm{C} 4=\mathrm{C}(\backslash \mathrm{H}]) \mathrm{C}([ \\
\mathrm{F}])=\mathrm{C}([\mathrm{H}]) \mathrm{C}([\mathrm{F}])=\mathrm{C} 4[\mathrm{H}]\end{array}$ & 4.678 & 3.400 \\
\hline Ext31 & $\begin{array}{l}\mathrm{O}=\mathrm{C}(\mathrm{N}([\mathrm{H}])[\mathrm{C} @ @]([\mathrm{H}])(\mathrm{C} 1=\mathrm{C}([\mathrm{H}]) \mathrm{C}([\mathrm{H}])=\mathrm{C}([\mathrm{H}]) \mathrm{C}([\mathrm{H}])=\mathrm{C} 1[\mathrm{H}]) \mathrm{C}([\mathrm{H}])([\mathrm{H}])[\mathrm{H}]) \mathrm{C} \\
=2 \mathrm{C}([\mathrm{H}])=\mathrm{C}(\mathrm{C}([\mathrm{H}])=\mathrm{C}(\mathrm{C}=2[\mathrm{H}]) \mathrm{N}(\mathrm{C}([\mathrm{H}])([\mathrm{H}])[\mathrm{H}])[\mathrm{S}](=\mathrm{O})(=\mathrm{O}) \mathrm{C}([\mathrm{H}])([\mathrm{H}])[\mathrm{H}]) \mathrm{C}(= \\
\mathrm{O}) \mathrm{N}([\mathrm{H}])[\mathrm{C} @]([\mathrm{H}])([\mathrm{C} @ @]([\mathrm{H}])(\mathrm{O}[\mathrm{H}]) \mathrm{C}([\mathrm{H}])([\mathrm{H}])[\mathrm{C} @ @]([\mathrm{H}])(\mathrm{OC}([\mathrm{H}])([\mathrm{H}])[\mathrm{C} @ \\
@] 3([\mathrm{H}]) \mathrm{C}([\mathrm{H}])([\mathrm{H}]) \mathrm{C} 3([\mathrm{H}])[\mathrm{H}]) \mathrm{C}(=\mathrm{O}) \mathrm{N}([\mathrm{H}])[\mathrm{C} @]([\mathrm{H}])(\mathrm{C}(=\mathrm{O}) \mathrm{N}([\mathrm{H}]) \mathrm{C}([\mathrm{H}])([\mathrm{H}]) \mathrm{C} \\
4=\mathrm{C}([\mathrm{H}]) \mathrm{C}([\mathrm{H}])=\mathrm{C}([\mathrm{H}]) \mathrm{C}([\mathrm{H}])=\mathrm{C} 4[\mathrm{H}]) \mathrm{C}([\mathrm{H}])(\mathrm{C}([\mathrm{H}])([\mathrm{H}])[\mathrm{H}]) \mathrm{C}([\mathrm{H}])([\mathrm{H}])[\mathrm{H}]) \mathrm{C}([\mathrm{H}] \\
)([\mathrm{H}]) \mathrm{O} / \mathrm{C} 5=\mathrm{C}([\mathrm{H}]) \mathrm{C}([\mathrm{F}])=\mathrm{C}([\mathrm{H}]) \mathrm{C}([\mathrm{F}])=\mathrm{C} 5[\mathrm{H}]\end{array}$ & 4.886 & 5.030 \\
\hline Ext32 & $\begin{array}{l}\mathrm{O}=\mathrm{C} 1 \mathrm{C} 4=\mathrm{C}(\mathrm{O} / \mathrm{C}(=\mathrm{C} 1 / \mathrm{C}([\mathrm{H}])([\mathrm{H}]) \mathrm{C}([\mathrm{H}])=\mathrm{C}(\mathrm{C}([\mathrm{H}])([\mathrm{H}])[\mathrm{H}]) \mathrm{C}([\mathrm{H}])([\mathrm{H}])[\mathrm{H}]) \mathrm{C}=2 \mathrm{C}([ \\
\mathrm{H}])=\mathrm{C}([\mathrm{H}]) \mathrm{C}(\mathrm{O}[\mathrm{H}])=\mathrm{C}([\mathrm{H}]) \mathrm{C}=2 \mathrm{O}[\mathrm{H}]) \mathrm{C} 3=\mathrm{C}(\mathrm{OC}(\mathrm{C}([\mathrm{H}])=\mathrm{C} 3[\mathrm{H}])(\mathrm{C}([\mathrm{H}])([\mathrm{H}])[\mathrm{H}]) \mathrm{C}([ \\
\mathrm{H}])([\mathrm{H}])[\mathrm{H}]) \mathrm{C}([\mathrm{H}])=\mathrm{C} 4 \mathrm{O}[\mathrm{H}]\end{array}$ & 0.193 & 1.433 \\
\hline Ext33 & $\begin{array}{l}\mathrm{O}=\mathrm{C}(\mathrm{OC}(\mathrm{C}([\mathrm{H}])([\mathrm{H}])[\mathrm{H}])(\mathrm{C}([\mathrm{H}])([\mathrm{H}])[\mathrm{H}]) \mathrm{C}([\mathrm{H}])([\mathrm{H}])[\mathrm{H}]) \mathrm{N}([\mathrm{H}])[\mathrm{C} @]([\mathrm{H}])(\mathrm{C}(=\mathrm{O}) \\
\mathrm{N}([\mathrm{H}])[\mathrm{C} @]([\mathrm{H}])([\mathrm{C} @]([\mathrm{H}])(\mathrm{O}[\mathrm{H}]) \mathrm{C}([\mathrm{H}])([\mathrm{H}])[\mathrm{C} @([\mathrm{H}])(\mathrm{C}(=\mathrm{O}) \mathrm{N}([\mathrm{H}])[\mathrm{C} @]([\mathrm{H}])( \\
\mathrm{C}(=\mathrm{O}) \mathrm{N}([\mathrm{H}]) \mathrm{C}([\mathrm{H}])([\mathrm{H}]) \mathrm{C} 1=\mathrm{C}([\mathrm{H}]) \mathrm{C}([\mathrm{H}])=\mathrm{C}([\mathrm{H}]) \mathrm{C}([\mathrm{H}])=\mathrm{C} 1[\mathrm{H}]) \mathrm{C}([\mathrm{H}])(\mathrm{C}([\mathrm{H}])([\mathrm{H}] \\
)[\mathrm{H}]) \mathrm{C}([\mathrm{H}])([\mathrm{H}])[\mathrm{H}]) \mathrm{C}([\mathrm{H}])([\mathrm{H}])[\mathrm{H}]) \mathrm{C}([\mathrm{H}])([\mathrm{H}]) \mathrm{C}([\mathrm{H}])(\mathrm{C}([\mathrm{H}])([\mathrm{H}])[\mathrm{H}]) \mathrm{C}([\mathrm{H}])([\mathrm{H}]) \\
[\mathrm{H}]) \mathrm{C}([\mathrm{H}])([\mathrm{H}]) \mathrm{C}(=\mathrm{O}) \mathrm{N}([\mathrm{H}])[\mathrm{H}]\end{array}$ & 1.504 & 2.330 \\
\hline Ext34 & $\begin{array}{l}\mathrm{O}=\mathrm{C} 3 \mathrm{~N}([\mathrm{H}])[\mathrm{C} @]([\mathrm{H}])(\mathrm{C}(=\mathrm{O}) \mathrm{N}([\mathrm{H}])[\mathrm{C} @]([\mathrm{H}])([\mathrm{C} @]([\mathrm{H}])(\mathrm{O}[\mathrm{H}]) \mathrm{C}([\mathrm{H}])([\mathrm{H}]) \mathrm{N}([\mathrm{H}] \\
) \mathrm{C}([\mathrm{H}])([\mathrm{H}]) \mathrm{C} 1=\mathrm{C}([\mathrm{H}]) \mathrm{C}(=\mathrm{C}([\mathrm{H}]) \mathrm{C}([\mathrm{H}])=\mathrm{C} 1[\mathrm{H}]) \mathrm{N}(\mathrm{C}([\mathrm{H}])([\mathrm{H}])[\mathrm{H}]) \mathrm{C}([\mathrm{H}])([\mathrm{H}])[\mathrm{H}]) \\
\mathrm{C}([\mathrm{H}])([\mathrm{H}]) \mathrm{C} 2=\mathrm{C}([\mathrm{H}]) \mathrm{C}([\mathrm{H}])=\mathrm{C}([\mathrm{H}]) \mathrm{C}([\mathrm{H}])=\mathrm{C} 2[\mathrm{H}]) \mathrm{C}([\mathrm{H}])([\mathrm{H}]) \mathrm{N} 3 / \mathrm{C} 4=\mathrm{C}([\mathrm{H}]) \mathrm{C}([ \\
\mathrm{H}])=\mathrm{C}([\mathrm{H}]) \mathrm{C}([\mathrm{H}])=\mathrm{C} 4[\mathrm{H}]\end{array}$ & 1.087 & 1.850 \\
\hline Ext35 & $\begin{array}{l}\mathrm{O}=\mathrm{C} 3 \mathrm{~N}([\mathrm{H}])[\mathrm{C} @]([\mathrm{H}])(\mathrm{C}(=\mathrm{O}) \mathrm{N}([\mathrm{H}])[\mathrm{C} @]([\mathrm{H}])([\mathrm{C} @]([\mathrm{H}])(\mathrm{O}[\mathrm{H}]) \mathrm{C}([\mathrm{H}])([\mathrm{H}]) \mathrm{N}([\mathrm{H}] \\
) \mathrm{C}([\mathrm{H}])([\mathrm{H}]) \mathrm{C} 1=\mathrm{C}([\mathrm{H}]) \mathrm{C}(=\mathrm{C}([\mathrm{H}]) \mathrm{C}([\mathrm{H}])=\mathrm{C} 1[\mathrm{H}]) \mathrm{N}(\mathrm{C}([\mathrm{H}])([\mathrm{H}])[\mathrm{H}]) \mathrm{C}([\mathrm{H}])([\mathrm{H}])[\mathrm{H}]) \\
\mathrm{C}([\mathrm{H}])([\mathrm{H}]) \mathrm{C} 2=\mathrm{C}([\mathrm{H}]) \mathrm{C}([\mathrm{H}])=\mathrm{C}([\mathrm{H}]) \mathrm{C}([\mathrm{H}])=\mathrm{C} 2[\mathrm{H}]) \mathrm{C}([\mathrm{H}])([\mathrm{H}]) \mathrm{N} 3 \mathrm{C}([\mathrm{H}])([\mathrm{H}]) \mathrm{C} 4= \\
\mathrm{C}([\mathrm{H}]) \mathrm{C}([\mathrm{H}])=\mathrm{C}([\mathrm{F}]) \mathrm{C}([\mathrm{F}])=\mathrm{C} 4[\mathrm{H}]\end{array}$ & 2.565 & 2.361 \\
\hline Ext36 & $\begin{array}{l}\mathrm{O}=\mathrm{C} 3 \mathrm{~N}([\mathrm{H}])[\mathrm{C} @]([\mathrm{H}])(\mathrm{C}(=\mathrm{O}) \mathrm{N}([\mathrm{H}])[\mathrm{C} @]([\mathrm{H}])([\mathrm{C} @]([\mathrm{H}])(\mathrm{O}[\mathrm{H}]) \mathrm{C}([\mathrm{H}])([\mathrm{H}]) \mathrm{N}([\mathrm{H}] \\
) \mathrm{C}([\mathrm{H}])([\mathrm{H}]) \mathrm{C} 1=\mathrm{C}([\mathrm{H}]) \mathrm{C}(=\mathrm{C}([\mathrm{H}]) \mathrm{C}([\mathrm{H}])=\mathrm{C} 1[\mathrm{H}]) \mathrm{N}(\mathrm{C}([\mathrm{H}])([\mathrm{H}])[\mathrm{H}]) \mathrm{C}([\mathrm{H}])([\mathrm{H}])[\mathrm{H}]) \\
\mathrm{C}([\mathrm{H}])([\mathrm{H}]) \mathrm{C} 2=\mathrm{C}([\mathrm{H}]) \mathrm{C}([\mathrm{H}])=\mathrm{C}([\mathrm{H}]) \mathrm{C}([\mathrm{H}])=\mathrm{C} 2[\mathrm{H}]) \mathrm{C}([\mathrm{H}])([\mathrm{H}]) \mathrm{N} 3 \mathrm{C}([\mathrm{H}])([\mathrm{H}]) \mathrm{C} 4= \\
\mathrm{C}([\mathrm{H}]) \mathrm{C}([\mathrm{H}])=\mathrm{C}([\mathrm{F}]) \mathrm{C}([\mathrm{H}])=\mathrm{C} 4[\mathrm{~F}]\end{array}$ & 2.062 & 2.049 \\
\hline Ext37 & $\begin{array}{l}\mathrm{O}=\mathrm{C}(\mathrm{N}([\mathrm{H}])[\mathrm{C} @ @](([\mathrm{H}])(\mathrm{C}([\mathrm{H}])([\mathrm{H}]) \mathrm{C} 1=\mathrm{C}([\mathrm{H}]) \mathrm{C}([\mathrm{H}])=\mathrm{C}([\mathrm{H}]) \mathrm{C}([\mathrm{H}])=\mathrm{C} 1[\mathrm{H}])[\mathrm{C} @] \\
([\mathrm{H}])(\mathrm{O}[\mathrm{H}]) \mathrm{C}([\mathrm{H}])([\mathrm{H}]) \mathrm{N}([\mathrm{H}]) \mathrm{C}([\mathrm{H}])([\mathrm{H}]) \mathrm{C} 2=\mathrm{C}([\mathrm{H}]) \mathrm{C}([\mathrm{H}])=\mathrm{C}([\mathrm{H}]) \mathrm{C}(=\mathrm{C} 2[\mathrm{H}]) \mathrm{N}(\mathrm{C}( \\
[\mathrm{H}])([\mathrm{H}])[\mathrm{H}]) \mathrm{C}([\mathrm{H}])([\mathrm{H}])[\mathrm{H}])[\mathrm{C} @] 3(\mathrm{~N}([\mathrm{H}]) \mathrm{C}(=\mathrm{O}) \mathrm{N}(\mathrm{C} 3([\mathrm{H}])[\mathrm{H}]) \mathrm{C}([\mathrm{H}])([\mathrm{H}]) \mathrm{C} 4=\mathrm{C}([ \\
\mathrm{H}]) \mathrm{C}([\mathrm{H}])=\mathrm{C}([\mathrm{H}]) \mathrm{C}([\mathrm{H}])=\mathrm{C} 4[\mathrm{H}]) \mathrm{C}([\mathrm{H}])([\mathrm{H}]) \mathrm{C}=5 \mathrm{C}([\mathrm{H}])=\mathrm{C}([\mathrm{H}]) \mathrm{C}([\mathrm{H}])=\mathrm{C}([\mathrm{H}]) \mathrm{C}=5[ \\
\mathrm{H}]\end{array}$ & 1.301 & 1.184 \\
\hline Ext38 & $\begin{array}{l}\mathrm{O}=\mathrm{C} 1 \mathrm{~N}(\mathrm{C}(=\mathrm{N}[\mathrm{C} @ @] 1(\mathrm{C}=2 \mathrm{C}([\mathrm{H}])=\mathrm{C}([\mathrm{H}]) \mathrm{C}([\mathrm{H}])=\mathrm{C}([\mathrm{H}]) \mathrm{C}=2[\mathrm{H}]) \mathrm{C} 3=\mathrm{C}([\mathrm{H}]) \mathrm{C}(=\mathrm{C}([ \\
\mathrm{H}]) \mathrm{C}([\mathrm{H}])=\mathrm{C} 3[\mathrm{H}]) \mathrm{C} 4=\mathrm{C}([\mathrm{H}]) \mathrm{N}=\mathrm{C}([\mathrm{H}]) \mathrm{C}([\mathrm{F}])=\mathrm{C} 4[\mathrm{H}]) \mathrm{N}([\mathrm{H}])[\mathrm{H}]) \mathrm{C}([\mathrm{H}])([\mathrm{H}])[\mathrm{H}]\end{array}$ & 2.538 & 2.740 \\
\hline Ext39 & $\begin{array}{l}\mathrm{O}=\mathrm{C} 1 \mathrm{~N}(\mathrm{C}(=\mathrm{N}[\mathrm{C} @ @] 1(\mathrm{C}=2 \mathrm{C}([\mathrm{H}])=\mathrm{C}([\mathrm{H}]) \mathrm{C}([\mathrm{H}])=\mathrm{C}([\mathrm{H}]) \mathrm{C}=2[\mathrm{H}]) \mathrm{C} 3=\mathrm{C}([\mathrm{H}]) \mathrm{C}(=\mathrm{C}([ \\
\mathrm{H}]) \mathrm{C}([\mathrm{H}])=\mathrm{C} 3[\mathrm{H}]) \mathrm{C} 4=\mathrm{C}([\mathrm{H}]) \mathrm{C}(\mathrm{OC}([\mathrm{H}])([\mathrm{H}])[\mathrm{H}])=\mathrm{C}([\mathrm{H}]) \mathrm{C}([\mathrm{H}])=\mathrm{C} 4[\mathrm{H}]) \mathrm{N}([\mathrm{H}])[\mathrm{H}])\end{array}$ & 1.222 & 2.632 \\
\hline
\end{tabular}




\begin{tabular}{|c|c|c|c|}
\hline & $\mathrm{C}([\mathrm{H}])([\mathrm{H}])[\mathrm{H}]$ & & \\
\hline Ext40 & $\begin{array}{l}\mathrm{O}=\mathrm{C} 1 \mathrm{~N}(\mathrm{C}(=\mathrm{N}[\mathrm{C} @ @] 1(\mathrm{C}=2 \mathrm{C}([\mathrm{H}])=\mathrm{C}(\mathrm{C}([\mathrm{H}])=\mathrm{C}([\mathrm{H}]) \mathrm{C}=2[\mathrm{H}]) \mathrm{C} 3=\mathrm{C}([\mathrm{H}]) \mathrm{C}([\mathrm{Cl}])=\mathrm{C}( \\
[\mathrm{H}]) \mathrm{N}=\mathrm{C} 3[\mathrm{H}])[\mathrm{C} @ @] 4([\mathrm{H}]) \mathrm{C}([\mathrm{H}])([\mathrm{H}]) \mathrm{C} 4([\mathrm{H}])[\mathrm{H}]) \mathrm{N}([\mathrm{H}])[\mathrm{H}]) \mathrm{C}([\mathrm{H}])([\mathrm{H}])[\mathrm{H}]\end{array}$ & 3.678 & 2.896 \\
\hline Ext41 & $\begin{array}{l}\mathrm{O}=\mathrm{C} 1 \mathrm{~N}(\mathrm{C}(=\mathrm{N}[\mathrm{C} @] 1(\mathrm{C}=2 \mathrm{C}([\mathrm{H}])=\mathrm{C}([\mathrm{H}]) \mathrm{C}([\mathrm{H}])=\mathrm{C}([\mathrm{H}]) \mathrm{C}=2[\mathrm{H}]) \mathrm{C} 3=\mathrm{C}([\mathrm{H}]) \mathrm{C}([\mathrm{F}])=\mathrm{C} \\
([\mathrm{H}]) \mathrm{C}([\mathrm{H}])=\mathrm{C} 3[\mathrm{H}]) \mathrm{N}([\mathrm{H}])[\mathrm{H}]) \mathrm{C}([\mathrm{H}])([\mathrm{H}])[\mathrm{H}]\end{array}$ & 2.143 & 1.637 \\
\hline Ext42 & $\begin{array}{l}\mathrm{O}=\mathrm{C} 1 \mathrm{~N}(\mathrm{C}(=\mathrm{N}[\mathrm{C} @ @] 1(/ \mathrm{C} 2=\mathrm{C}(\mathrm{VH}]) \mathrm{C}([\mathrm{H}])=\mathrm{C}([\mathrm{H}]) \mathrm{C}([\mathrm{H}])=\mathrm{C} 2[\mathrm{H}]) \mathrm{C} 3=\mathrm{C}([\mathrm{H}]) \mathrm{C}(=\mathrm{C}([ \\
\mathrm{H}]) \mathrm{C}([\mathrm{H}])=\mathrm{C} 3[\mathrm{H}]) \mathrm{C} 4=\mathrm{C}([\mathrm{H}]) \mathrm{C}(\mathrm{OC}([\mathrm{H}])([\mathrm{H}]) \mathrm{C}([\mathrm{H}])([\mathrm{H}])[\mathrm{H}])=\mathrm{C}([\mathrm{H}]) \mathrm{C}([\mathrm{H}])=\mathrm{C} 4[\mathrm{H}] \\
) \mathrm{N}([\mathrm{H}])[\mathrm{H}]) \mathrm{C}([\mathrm{H}])([\mathrm{H}])[\mathrm{H}]\end{array}$ & 2.337 & 2.238 \\
\hline Ext43 & $\begin{array}{l}\mathrm{O}=\mathrm{C} 1 \mathrm{~N}(\mathrm{C}(=\mathrm{N}[\mathrm{C} @ @] 1(\mathrm{C}=2 \mathrm{C}([\mathrm{H}])=\mathrm{C}([\mathrm{H}]) \mathrm{C}([\mathrm{H}])=\mathrm{C}([\mathrm{H}]) \mathrm{C}=2[\mathrm{H}]) \mathrm{C} 3=\mathrm{C}([\mathrm{H}]) \mathrm{C}(=\mathrm{C}([ \\
\mathrm{H}]) \mathrm{C}([\mathrm{H}])=\mathrm{C} 3[\mathrm{H}]) \mathrm{C}([\mathrm{H}])([\mathrm{H}])[\mathrm{H}]) \mathrm{N}([\mathrm{H}])[\mathrm{H}]) \mathrm{C}([\mathrm{H}])([\mathrm{H}])[\mathrm{H}]\end{array}$ & 2.260 & 2.310 \\
\hline Ext44 & $\begin{array}{l}\mathrm{O}=\mathrm{C}(\mathrm{N}([\mathrm{C} @ @] 1([\mathrm{H}]) \mathrm{C}([\mathrm{H}])([\mathrm{H}]) \mathrm{C}([\mathrm{H}])([\mathrm{H}]) \mathrm{C}([\mathrm{H}])([\mathrm{H}]) \mathrm{C}([\mathrm{H}])([\mathrm{H}]) \mathrm{C} 1([\mathrm{H}])[\mathrm{H}]) \mathrm{C}([ \\
\mathrm{H}])([\mathrm{H}]) \mathrm{C} 2=\mathrm{C}(\mathrm{N}([\mathrm{H}]) \mathrm{N}=\mathrm{C} 2[\mathrm{H}]) \mathrm{C}([\mathrm{H}])([\mathrm{H}]))[\mathrm{H}]) \mathrm{C}([\mathrm{H}])([\mathrm{H}]) \mathrm{C}([\mathrm{H}])([\mathrm{H}])[\mathrm{C} @]([\mathrm{H}])( \\
\mathrm{N} 5 \mathrm{C}(=\mathrm{NC}=4 \mathrm{C}([\mathrm{H}])=\mathrm{C}([\mathrm{H}]) \mathrm{C}(\mathrm{O} / \mathrm{C} 3=\mathrm{C}([\mathrm{H}]) \mathrm{C}([\mathrm{H}])=\mathrm{C}([\mathrm{H}]) \mathrm{C}([\mathrm{H}])=\mathrm{C} 3[\mathrm{H}])=\mathrm{C}([\mathrm{H}]) \mathrm{C} \\
=4 \mathrm{C} 5([\mathrm{H}])[\mathrm{H}]) \mathrm{N}([\mathrm{H}])[\mathrm{H}])[\mathrm{C} @ @] 6([\mathrm{H}]) \mathrm{C}([\mathrm{H}])([\mathrm{H}]) \mathrm{C}([\mathrm{H}])([\mathrm{H}]) \mathrm{C}([\mathrm{H}])([\mathrm{H}]) \mathrm{C}([\mathrm{H}])([ \\
\mathrm{H}]) \mathrm{C} 6([\mathrm{H}])[\mathrm{H}]\end{array}$ & 3.409 & 3.164 \\
\hline Ext 45 & $\begin{array}{l}\mathrm{O}=\mathrm{C}(\mathrm{N}([\mathrm{C} @ @] 1([\mathrm{H}]) \mathrm{C}([\mathrm{H}])([\mathrm{H}]) \mathrm{C}([\mathrm{H}])([\mathrm{H}]) \mathrm{C}([\mathrm{H}])([\mathrm{H}]) \mathrm{C}([\mathrm{H}])([\mathrm{H}]) \mathrm{C} 1([\mathrm{H}])[\mathrm{H}]) \mathrm{C}([ \\
\mathrm{H}])([\mathrm{H}])[\mathrm{H}]) \mathrm{C}([\mathrm{H}])([\mathrm{H}]) \mathrm{C}([\mathrm{H}])([\mathrm{H}])[\mathrm{C} @]([\mathrm{H}])(\mathrm{N} 4 \mathrm{C}(=\mathrm{N} / \mathrm{C} 3=\mathrm{C}([\mathrm{H}]) \mathrm{C}([\mathrm{H}])=\mathrm{C}(\mathrm{OC}= \\
2 \mathrm{C}([\mathrm{H}])=\mathrm{C}([\mathrm{H}]) \mathrm{C}([\mathrm{H}])=\mathrm{C}([\mathrm{H}]) \mathrm{C}=2[\mathrm{H}]) \mathrm{C}([\mathrm{H}])=\mathrm{C} 3 \mathrm{C} 4([\mathrm{H}])) \mathrm{H}]) \mathrm{N}([\mathrm{H}])[\mathrm{H}])[\mathrm{C} @] 5([\mathrm{H}] \\
) \mathrm{C}([\mathrm{H}])([\mathrm{H}]) \mathrm{OC}([\mathrm{H}])([\mathrm{H}]) \mathrm{C}([\mathrm{H}])([\mathrm{H}]) \mathrm{C}([\mathrm{H}])[\mathrm{H}]\end{array}$ & 2.914 & 3.847 \\
\hline Ext46 & $\begin{array}{l}\mathrm{O}=\mathrm{C}(\mathrm{OC}([\mathrm{H}])([\mathrm{H}])[\mathrm{C} @]([\mathrm{H}])(\mathrm{N} 3 \mathrm{C}(=\mathrm{N} / \mathrm{C} 2=\mathrm{C}(\backslash[\mathrm{H}]) \mathrm{C}([\mathrm{H}])=\mathrm{C}(\mathrm{O} / \mathrm{C} 1=\mathrm{C}(\mathrm{V}[\mathrm{H}]) \mathrm{C}([\mathrm{H}])= \\
\mathrm{C}([\mathrm{H}]) \mathrm{C}([\mathrm{H}])=\mathrm{C} 1[\mathrm{H}]) \mathrm{C}([\mathrm{H}])=\mathrm{C} 2 \mathrm{C} 3([\mathrm{H}])[\mathrm{H}]) \mathrm{N}([\mathrm{H}])[\mathrm{H}])[\mathrm{C} @ @] 4([\mathrm{H}]) \mathrm{C}([\mathrm{H}])([\mathrm{H}]) \mathrm{C}( \\
[\mathrm{H}])([\mathrm{H}]) \mathrm{C}([\mathrm{H}])([\mathrm{H}]) \mathrm{C}([\mathrm{H}])([\mathrm{H}]) \mathrm{C} 4([\mathrm{H}])[\mathrm{H}]) \mathrm{N}([\mathrm{H}])[\mathrm{C} @ @] 5([\mathrm{H}]) \mathrm{C}([\mathrm{H}])([\mathrm{H}]) \mathrm{C}([\mathrm{H}]) \\
([\mathrm{H}]) \mathrm{C}([\mathrm{H}])([\mathrm{H}]) \mathrm{C}([\mathrm{H}])([\mathrm{H}]) \mathrm{C} 5([\mathrm{H}])[\mathrm{H}]\end{array}$ & 3.222 & 2.706 \\
\hline Ext47 & $\begin{array}{l}\mathrm{O}=\mathrm{C} 3 \mathrm{~N}(\mathrm{C}(=\mathrm{N}[\mathrm{C} @](\mathrm{C}=1 \mathrm{C}([\mathrm{H}])=\mathrm{C}([\mathrm{S}] \mathrm{C}=1[\mathrm{Cl}]) \mathrm{C}=2 \mathrm{C}([\mathrm{H}])=\mathrm{C}(\mathrm{C \# CC}([\mathrm{H}])([\mathrm{H}])[\mathrm{H}]) \mathrm{C} \\
([\mathrm{H}])=\mathrm{NC}=2[\mathrm{H}])(\mathrm{C} 3([\mathrm{H}])[\mathrm{H}]) \mathrm{C}([\mathrm{H}])([\mathrm{H}])[\mathrm{H}]) \mathrm{N}([\mathrm{H}])[\mathrm{H}]) \mathrm{C}([\mathrm{H}])([\mathrm{H}])[\mathrm{H}]\end{array}$ & 4.097 & 3.896 \\
\hline Ext 48 & 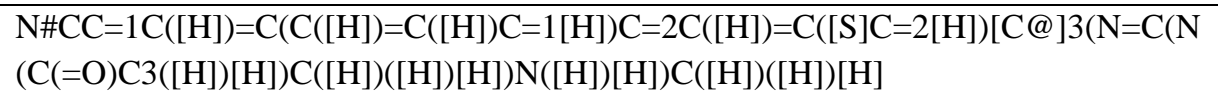 & 3.244 & 1.873 \\
\hline Ext 49 & $\begin{array}{l}\mathrm{O}=\mathrm{C}(\mathrm{O}[\mathrm{C} @] 2([\mathrm{H}])[\mathrm{C} @ @]([\mathrm{H}])(\mathrm{OC} 1=\mathrm{C}(\mathrm{C}(\mathrm{O}[\mathrm{H}])=\mathrm{C}([\mathrm{H}]) \mathrm{C}(\mathrm{O}[\mathrm{H}])=\mathrm{C} 1[\mathrm{H}]) \mathrm{C} 2([\mathrm{H}])[ \\
\mathrm{H}]) \mathrm{C} 3=\mathrm{C}([\mathrm{H}]) \mathrm{C}(\mathrm{O}[\mathrm{H}])=\mathrm{C}(\mathrm{O}[\mathrm{H}]) \mathrm{C}([\mathrm{H}])=\mathrm{C} 3[\mathrm{H}]) \mathrm{C}=4 \mathrm{C}([\mathrm{H}])=\mathrm{C}(\mathrm{O}[\mathrm{H}]) \mathrm{C}(\mathrm{O}[\mathrm{H}])=\mathrm{C}(\mathrm{O}[ \\
\mathrm{H}]) \mathrm{C}=4[\mathrm{H}]\end{array}$ & 1.276 & 2.728 \\
\hline Ext50 & $\begin{array}{l}\mathrm{O}=\mathrm{C}(\mathrm{N}([\mathrm{H}]) \mathrm{C} 3=\mathrm{C}([\mathrm{H}]) \mathrm{C}([\mathrm{H}])=\mathrm{C} 2 \mathrm{OC}([\mathrm{C} @] 4([\mathrm{C} @ @] 1(\mathrm{~N}=\mathrm{C}(\mathrm{OC} 1([\mathrm{H}])[\mathrm{H}]) \mathrm{N}([\mathrm{H}])[ \\
\mathrm{H}]) \mathrm{C} 2=\mathrm{C} 3[\mathrm{H}]) \mathrm{C}([\mathrm{H}])([\mathrm{H}]) \mathrm{OC} 4([\mathrm{H}])[\mathrm{H}])(\mathrm{C}([\mathrm{H}])([\mathrm{H}])[\mathrm{H}]) \mathrm{C}([\mathrm{H}])([\mathrm{H}])[\mathrm{H}]) \mathrm{C}=5 / \mathrm{N}=\mathrm{C}(/ \\
[\mathrm{H}]) \mathrm{C}([\mathrm{Cl}])=\mathrm{C}([\mathrm{H}]) \mathrm{C}=5[\mathrm{~F}]\end{array}$ & 3.288 & 2.888 \\
\hline Ext51 & $\begin{array}{l}{[\mathrm{H}] / \mathrm{C} 4=\mathrm{C}(/ \mathrm{C}([\mathrm{H}])=\mathrm{C} 1 \mathrm{C}(\mathrm{OC}([\mathrm{H}])([\mathrm{H}])[\mathrm{C} @] 3([\mathrm{C} @ @] 12 \mathrm{~N}=\mathrm{C}(\mathrm{OC} 2([\mathrm{H}])[\mathrm{H}]) \mathrm{N}([\mathrm{H}])[} \\
\mathrm{H}]) \mathrm{C}([\mathrm{H}])([\mathrm{H}]) \mathrm{OC} 3([\mathrm{H}])[\mathrm{H}])=\mathrm{C} 4[\mathrm{H}]) \mathrm{C} 5=\mathrm{C}([\mathrm{H}]) \mathrm{N}=\mathrm{C}([\mathrm{H}]) \mathrm{C}(\mathrm{C \# CC}([\mathrm{H}])([\mathrm{H}])[\mathrm{H}])=\mathrm{C} \\
5[\mathrm{H}]\end{array}$ & 2.910 & 3.616 \\
\hline Ext52 & $\begin{array}{l}\mathrm{O}=\mathrm{C}(\mathrm{N}([\mathrm{H}]) \mathrm{C} 3=\mathrm{C}([\mathrm{H}]) \mathrm{C}([\mathrm{H}])=\mathrm{C} 2 \mathrm{OC}([\mathrm{C} @] 4([\mathrm{C} @ @] 1(\mathrm{~N}=\mathrm{C}(\mathrm{OC} 1([\mathrm{H}])[\mathrm{H}]) \mathrm{N}([\mathrm{H}])[ \\
\mathrm{H}]) \mathrm{C} 2=\mathrm{C} 3[\mathrm{H}]) \mathrm{C}([\mathrm{H}])([\mathrm{H}]) \mathrm{OC} 4([\mathrm{H}])[\mathrm{H}])(\mathrm{C}([\mathrm{H}])([\mathrm{H}])[\mathrm{H}]) \mathrm{C}([\mathrm{H}])([\mathrm{H}])[\mathrm{H}]) \mathrm{C} 5=\mathrm{NC}([\mathrm{H} \\
])=\mathrm{C}([\mathrm{Br}]) \mathrm{C}([\mathrm{H}])=\mathrm{N} 5\end{array}$ & 3.078 & 2.657 \\
\hline Ext53 & $\begin{array}{l}\mathrm{O}=\mathrm{C}(\mathrm{N}([\mathrm{H}]) \mathrm{C} 3=\mathrm{C}([\mathrm{H}]) \mathrm{C}([\mathrm{H}])=\mathrm{C} 2 \mathrm{OC}([\mathrm{H}])([\mathrm{H}])[\mathrm{C} @ @] 4([\mathrm{C} @ @] 1(\mathrm{~N}=\mathrm{C}(\mathrm{OC} 1([\mathrm{H}])[ \\
\mathrm{H}]) \mathrm{N}([\mathrm{H}])[\mathrm{H}]) \mathrm{C} 2=\mathrm{C} 3[\mathrm{H}]) \mathrm{C}([\mathrm{H}])([\mathrm{H}]) \mathrm{C} 4([\mathrm{H}])[\mathrm{H}]) \mathrm{C} 5=\mathrm{NC}([\mathrm{H}])=\mathrm{C}([\mathrm{Cl}]) \mathrm{C}([\mathrm{H}])=\mathrm{C} 5 \mathrm{C}(\end{array}$ & 2.504 & 2.395 \\
\hline
\end{tabular}




\begin{tabular}{|c|c|c|c|}
\hline & $[\mathrm{H}])([\mathrm{H}])[\mathrm{H}]$ & & \\
\hline Ext54 & $\begin{array}{l}\mathrm{O}=\mathrm{C}(/ \mathrm{C} 1=\mathrm{N} / \mathrm{C}([\mathrm{H}])=\mathrm{C}(\mathrm{C}([\mathrm{H}])=\mathrm{C} 1[\mathrm{H}]) \mathrm{C}([\mathrm{H}])([\mathrm{H}])[\mathrm{H}]) \mathrm{N}([\mathrm{H}]) \mathrm{C} 2=\mathrm{C}([\mathrm{H}]) \mathrm{C}(=\mathrm{C}([\mathrm{F}]) \mathrm{C} \\
([\mathrm{H}])=\mathrm{C} 2[\mathrm{H}])[\mathrm{C} @] 3(\mathrm{~N}([\mathrm{H}]) \mathrm{C}(=\mathrm{N}[\mathrm{H}]) \mathrm{N}([\mathrm{S}](=\mathrm{O})(=\mathrm{O}) \mathrm{C} 3([\mathrm{H}])[\mathrm{H}]) \mathrm{C}([\mathrm{H}])([\mathrm{H}])[\mathrm{H}]) \mathrm{C}([ \\
\mathrm{H}])([\mathrm{H}])[\mathrm{H}]\end{array}$ & 4.523 & 4.046 \\
\hline Ext55 & $\begin{array}{l}\mathrm{O}=\mathrm{C}(/ \mathrm{C} 1=\mathrm{N} / \mathrm{C}([\mathrm{H}])=\mathrm{C}([\mathrm{F}]) \mathrm{C}([\mathrm{H}])=\mathrm{C} 1[\mathrm{H}]) \mathrm{N}([\mathrm{H}]) \mathrm{C} 2=\mathrm{C}([\mathrm{F}]) \mathrm{C}(=\mathrm{C}([\mathrm{F}]) \mathrm{C}([\mathrm{H}])=\mathrm{C} 2[\mathrm{H}] \\
)[\mathrm{C} @ 3(\mathrm{~N}([\mathrm{H}]) \mathrm{C}(=\mathrm{N}[\mathrm{H}]) \mathrm{N}([\mathrm{S}](=\mathrm{O})(=\mathrm{O}) \mathrm{C} 3([\mathrm{H}])[\mathrm{H}]) \mathrm{C}([\mathrm{H}])([\mathrm{H}])[\mathrm{H}]) \mathrm{C}([\mathrm{H}])([\mathrm{H}])[\mathrm{H}]\end{array}$ & 4.312 & 4.509 \\
\hline Ext56 & $\begin{array}{l}\mathrm{O}=[\mathrm{S}] 3(=\mathrm{O}) \mathrm{N}(/ \mathrm{C}(=\mathrm{N} \backslash[\mathrm{H}]) \mathrm{N}([\mathrm{H}])[\mathrm{C} @](\mathrm{C}=1[\mathrm{~S}] \mathrm{C}([\mathrm{H}])=\mathrm{C}(\mathrm{C}=1[\mathrm{H}]) \mathrm{C} 2=\mathrm{C}([\mathrm{H}]) \mathrm{C}([\mathrm{H}])= \\
\mathrm{C}([\mathrm{H}]) \mathrm{C}(\mathrm{C \# N})=\mathrm{C} 2[\mathrm{H}])(\mathrm{C} 3([\mathrm{H}])[\mathrm{H}]) \mathrm{C}([\mathrm{H}])([\mathrm{H}])[\mathrm{H}]) \mathrm{C}([\mathrm{H}])([\mathrm{H}])[\mathrm{H}]\end{array}$ & 3.592 & 3.122 \\
\hline Ext57 & $\begin{array}{l}\mathrm{O}=\mathrm{C}(\mathrm{N}([\mathrm{H}])[\mathrm{C} @] 1([\mathrm{H}]) \mathrm{C}([\mathrm{H}])([\mathrm{H}]) \mathrm{C} 1([\mathrm{H}])[\mathrm{H}])[\mathrm{C} @]([\mathrm{H}])(\mathrm{OC}([\mathrm{H}])([\mathrm{H}])[\mathrm{H}]) \mathrm{C}([\mathrm{H}]) \\
([\mathrm{H}])[\mathrm{C} @]([\mathrm{H}])(\mathrm{O}[\mathrm{H}])[\mathrm{C} @ @]([\mathrm{H}])(\mathrm{N}([\mathrm{H}]) \mathrm{C}(=\mathrm{O}) \mathrm{C}=2 \mathrm{C}([\mathrm{H}])=\mathrm{C}([\mathrm{H}]) \mathrm{C}([\mathrm{H}])=\mathrm{C}(\mathrm{C}=2[ \\
\mathrm{H}]) \mathrm{C}(=\mathrm{O}) \mathrm{N}(\mathrm{C}([\mathrm{H}])([\mathrm{H}]) \mathrm{C}([\mathrm{H}])([\mathrm{H}]) \mathrm{C}([\mathrm{H}])([\mathrm{H}])[\mathrm{H}]) \mathrm{C}([\mathrm{H}])([\mathrm{H}]) \mathrm{C}([\mathrm{H}])([\mathrm{H}]) \mathrm{C}([\mathrm{H}])([ \\
\mathrm{H}])[\mathrm{H}]) \mathrm{C}([\mathrm{H}])([\mathrm{H}]) \mathrm{O} / \mathrm{C} 3=\mathrm{C}(\mathrm{V}[\mathrm{H}]) \mathrm{C}([\mathrm{F}])=\mathrm{C}([\mathrm{H}]) \mathrm{C}([\mathrm{F}])=\mathrm{C} 3[\mathrm{H}]\end{array}$ & 2.658 & 2.105 \\
\hline Ext58 & $\begin{array}{l}\mathrm{O}=\mathrm{C}(\mathrm{N}([\mathrm{H}]) \mathrm{C}(=\mathrm{NC}([\mathrm{H}])([\mathrm{H}]) \mathrm{C} 1=\mathrm{C}([\mathrm{H}]) \mathrm{C}([\mathrm{Cl}])=\mathrm{C}(\mathrm{C}([\mathrm{Cl}])=\mathrm{C} 1[\mathrm{H}]) \mathrm{N}([\mathrm{H}])[\mathrm{S}](=\mathrm{O})(= \\
\mathrm{O}) \mathrm{C}([\mathrm{H}])([\mathrm{H}])[\mathrm{H}]) \mathrm{N}([\mathrm{H}])[\mathrm{H}]) \mathrm{C}=2 \mathrm{C}(=\mathrm{N}[\mathrm{S}] \mathrm{C}=2 \mathrm{C}([\mathrm{H}])([\mathrm{H}])[\mathrm{H}]) \mathrm{C} 3=\mathrm{C}([\mathrm{H}]) \mathrm{C}([\mathrm{H}])=\mathrm{C}( \\
\mathrm{OC}([\mathrm{H}])([\mathrm{H}])[\mathrm{H}]) \mathrm{C}([\mathrm{H}])=\mathrm{C} 3[\mathrm{H}]\end{array}$ & 2.553 & 4.081 \\
\hline Ext59 & $\begin{array}{l}\mathrm{O}=\mathrm{C}(\mathrm{N}([\mathrm{H}])[\mathrm{C} @]([\mathrm{H}])(\mathrm{C}(=\mathrm{O}) \mathrm{N}([\mathrm{H}])[\mathrm{C} @]([\mathrm{H}])(\mathrm{C}(=\mathrm{O}) \mathrm{O}[\mathrm{H}]) \mathrm{C}([\mathrm{H}])([\mathrm{H}]) \mathrm{C} 1=\mathrm{C}([\mathrm{H}]) \\
\mathrm{C}([\mathrm{H}])=\mathrm{C}([\mathrm{H}]) \mathrm{C}([\mathrm{H}])=\mathrm{C} 1[\mathrm{H}]) \mathrm{C}([\mathrm{H}])([\mathrm{H}]) \mathrm{C}([\mathrm{H}])([\mathrm{H}]) \mathrm{C}(=\mathrm{O}) \mathrm{O}[\mathrm{H}])[\mathrm{C} @ @]([\mathrm{H}])(\mathrm{N}([ \\
\mathrm{H}]) \mathrm{C}(=\mathrm{O})[\mathrm{C} @ @]([\mathrm{H}])(\mathrm{N}([\mathrm{H}]) \mathrm{C}([\mathrm{H}])([\mathrm{H}])[\mathrm{C} @ @]([\mathrm{H}])(\mathrm{O}[\mathrm{H}]) \mathrm{C}(=\mathrm{O})[\mathrm{C} @ @]([\mathrm{H}])(\mathrm{N}( \\
[\mathrm{H}]) \mathrm{C}(=\mathrm{O})[\mathrm{C} @ @]([\mathrm{H}])(\mathrm{N}([\mathrm{H}]) \mathrm{C}(=\mathrm{O})[\mathrm{C} @ @]([\mathrm{H}])(\mathrm{N}([\mathrm{H}]) \mathrm{C}(=\mathrm{O})[\mathrm{C} @ @]([\mathrm{H}])(\mathrm{N}([\mathrm{H}] \\
)[\mathrm{H}]) \mathrm{C}([\mathrm{H}])([\mathrm{H}]) \mathrm{C}([\mathrm{H}])([\mathrm{H}]) \mathrm{C}(=\mathrm{O}) \mathrm{O}[\mathrm{H}]) \mathrm{C}([\mathrm{H}])([\mathrm{H}]) \mathrm{C}([\mathrm{H}])(\mathrm{C}([\mathrm{H}])([\mathrm{H}])[\mathrm{H}]) \mathrm{C}([\mathrm{H}])([ \\
\mathrm{H}])[\mathrm{H}]) \mathrm{C}([\mathrm{H}])([\mathrm{H}]) \mathrm{C}(=\mathrm{O}) \mathrm{O}[\mathrm{H}]) \mathrm{C}([\mathrm{H}])([\mathrm{H}]) \mathrm{C}([\mathrm{H}])(\mathrm{C}([\mathrm{H}])([\mathrm{H}])[\mathrm{H}]) \mathrm{C}([\mathrm{H}])([\mathrm{H}])[\mathrm{H}]) \mathrm{C} \\
([\mathrm{H}])([\mathrm{H}])[\mathrm{H}]) \mathrm{C}([\mathrm{H}])(\mathrm{C}([\mathrm{H}])([\mathrm{H}])[\mathrm{H}]) \mathrm{C}([\mathrm{H}])([\mathrm{H}])[\mathrm{H}] \sim\end{array}$ & 5.523 & 6.540 \\
\hline
\end{tabular}

${ }^{1}$ Experimental inhibitory activity; ${ }^{2}$ Predicted inhibitory activity 